\title{
THE EFFECTS OF PERMANGANATE AND OTHER SELECTED CHEMICAL INHIBITORS ON SURFACE AND CRACK TIP CORROSION
}

\author{
A Dissertation \\ Presented to the Faculty of the School of Engineering and Applied Science \\ University of Virginia
}

In Partial Fulfillment

of the Requirements for the Degree

Doctor of Philosophy (Materials Science and Engineering)

by

Samuel B. Madden

August 2014 


\section{APPROVAL SHEET}

The dissertation is submitted in partial fulfillment of the requirements for the degree of Doctor of Philosophy (Materials Science and Engineering)

Samuel B. Madden

(Author)

This dissertation has been read and approved by the examining Committee:

Dr. John R. Scully

(Dissertation Advisor)

Dr. Elizabeth J. Opila

(Committee Chairwoman)

Dr. Gary M. Koenig

(Committee Member)

Dr. James T. Burns

(Committee Member)

Dr. Robert G. Kelly

(Committee Member)

Accepted for the School of Engineering and Applied Science:

$$
\text { Dr. James H. Aylor }
$$

Dean, School of Engineering and Applied

Science

May 2014 


\begin{abstract}
Environmental concern over the use of very effective but highly toxic chromate ionic inhibitors has led to the search for new chemical inhibitors that can protect Al-base precipitation age hardened alloys such as 2024-T351 and AA7075-T6, from corrosion including environmentally assisted cracking. These alloys are commonly used in aerospace applications. Presented in this dissertation are investigations to understand the mechanisms by which alternative ionic solution phase inhibitors function to inhibit corrosion. Extensive comparison to chromate is made. The work presented focuses on both surface corrosion as well as the inhibition of electrochemical processes that can lead to accelerated environmentally assisted crack growth, particularly those that can be explored in simulated film rupture repassivation tests.
\end{abstract}

The inhibition of corrosion on AA2024-T351 in NaCl solutions, mitigated by either in-situ permanganate ions $\left(\mathrm{MnO}_{4}^{-}\right)$or permanganate pretreatment, was examined. Both room temperature pretreatments and solution phase additions were studied as a function of inhibitor concentration. The roles of the inhibitor during anodic and cathodic polarizations were investigated. Inhibition of corrosion at the open circuit corrosion potential (OCP) under conditions where anodic and cathodic reactions are coupled was also examined. The oxidation states of the manganese oxides that contributed to protection were determined using potentiometric electrochemical reduction and in-situ Raman spectroscopy. The thermodynamics of the Mn-water system was also considered over a range of 
concentrations. Permanganate was shown to be both an anodic and cathodic inhibitor, as well as an inhibitor of copper replating at OCP.

The inhibition of corrosion on AA2024-T351 in NaCl solutions, mitigated by ceriumpermanganate combined inhibitor pretreatments, was examined next. Room temperature pretreatments were studied as a function of total inhibitor concentrations ranging from 10 to $50 \mathrm{mM}$. Pretreated samples were exposed to $50 \mathrm{mM} \mathrm{NaCl}$ solutions. The roles of the inhibitor during anodic and cathodic polarization were also investigated. The KouteckyLevich correction was applied to experimental cathodic E- $i$ data. Inhibition of open circuit corrosion under conditions where anodic and cathodic reactions are coupled was also examined. The thermodynamics of the $\mathrm{Ce}$ - and $\mathrm{Mn}$-water systems was also considered over a range of concentrations. There was no indication of the formation of a combined $\mathrm{Mn}-\mathrm{Ce}$ molecular species and no reference to such a species was found in the literature. The cerium-permanganate combination was determined to provide nearly the same level of cathodic inhibition seen during the single inhibitor application of permanganate. Cerium alone was inferior to permanganate as a cathodic inhibitor. In addition to strong cathodic inhibition, the combination of $\mathrm{Ce}$ and $\mathrm{Mn}$ increased inhibition of open circuit corrosion compared to single inhibitor pretreatments as determined by copper redeposition assessment. However, anodic inhibition was not observed with the inhibitors when used alone or when combined.

The scratch depassivation technique was utilized on AA2024-T351, AA7075-T6, and $99.999 \%$ aluminum in molybdate $\left(\mathrm{MoO}_{4}{ }^{2-}\right)$ permanganate $\left(\mathrm{MnO}_{4}{ }^{-}\right)$and chromate $\left(\mathrm{CrO}_{4}{ }^{2-}\right)$ containing $\mathrm{NaCl}$ solutions to examine electrochemical repassivation kinetics over a range of applied potentials and inhibitor concentrations. Environmental fatigue crack propagation 
is indicated to be inhibited below a critical cyclic loading frequency. The significance of this frequency can be understood in the context of an oxide repassivation time constant. Therefore, repassivation is pertinent to the behavior of crack tips which are depassivated by plastic deformation during mechanical loading under fatigue conditions. Scratch repassivation current vs. time and single frequency impedance measurements were taken in the region of frequency response dominated by surface capacitance. Current decay and capacitance measurements enabled the observation of the effects of inhibitor additions on film formation and oxide growth as protective oxide films, electrically modeled as capacitors, formed on the scratched electrode surface. Impedance measurements overcame the shortcomings of incomplete inventory of anodic oxidation current densities typical of aluminum repassivation at coupled electrode surfaces. Chromate was found to suppress scratched electrode current transients at high potentials on both AA2024-T351 and AA7075-T6. Capacitance measurements suggested the rapid formation and growth of an oxide at enhanced rates when $\mathrm{CrO}_{4}{ }^{2-}$ was in solution as compared to an uninhibited $\mathrm{NaCl}$ solution. Molybdate did not suppress the transient film rupture repassivation current density in AA2024-T351 or AA7075-T6. Impedance measurements in the presence of molybdate do not indicate the enhanced formation and growth of a passive film in the case of molybdate. The observed environmental fatigue crack propagation (EFCP) inhibition due to chromate may be due to enhanced repassivation and subsequently limited hydrogen production and uptake supported by oxide film barrier effects. The observed ECFP reduction with $\mathrm{MoO}_{4}{ }^{2-}$ is discussed in light of the observed lack of an effect on repassivation kinetics. The buffer capacity of molybdate may affect the local solution chemistry and this chemical effect may also promote EFCP inhibition. Permanganate was 
found to suppress the transient current density of AA7075 after scratch depassivation but not in the case of AA2024. $\mathrm{MnO}_{4}{ }^{-}$was also found to positively affect interfacial impedance suggesting improved barrier protection for both AA7075 and AA2024. These findings suggest some promise for the inhibition of EFCP with chromate, molybdate, and permanganate.

Single frequency impedance, Rutherford backscattering spectrometry (RBS) and elastic recoil detection (ERD) analysis of abrasion-repassivated Al-Mg-Zn-Cu alloy (AA7075T6) surfaces give the first direct measurement of enhanced repassivation, inhibitor species incorporation, followed by reduced hydrogen uptake into an $\mathrm{Al}-\mathrm{Zn}-\mathrm{Mg}-\mathrm{Cu}$ alloy in a combined experiment. Abrasion was used to simulate film rupture and repassivation. Repassivation was investigated with chromate, molybdate, and permanganate inhibitor additions to aqueous sodium chloride solutions. Chromate $(0.1 \mathrm{M})$ was found to be an effective inhibitor of hydrogen uptake during in-situ surface abrasion in $1.0 \mathrm{M} \mathrm{NaCl}$ solution. The evidence presented provides insight into the processes responsible for inhibition of EFCP of AA7075 controlled by hydrogen ingress at depassivated crack tips. Chromate is shown to be capable of enhancing repassivation, forming a compact oxide barrier and retarding hydrogen uptake into the fracture process zones of crack tips. Moreover, these findings provide a new methodology through which corrosion inhibitors may be evaluated for SCC and EFCP inhibitor function.

Ultimately, this thesis extends the scientific understanding of inhibitors and their functions, from surface corrosion inhibition to inhibition of environmentally assisted cracking governed by processes at depassivated crack tips. This research enables better corrosion 
inhibitor selection based on more informative and pertinent test methods for both surface as well as environmental cracking corrosion phenomena. 


\section{Acknowledgements}

I would first like to thank my advisor, Dr. John Scully, for providing me with the opportunity and the support to pursue my degree. Working together has been a privilege, and the experience has been challenging and very rewarding. I would also like to thank my committee: Dr. Opila, Dr. Kelly, Dr. Burns, and Dr. Koenig for their advice and guidance. I thank Dr. Ulgut for his support in the development of several critical research tools that enabled this study, as well as Dr. Goncharova of the UWO and Dr. Naab and Dr. Toader of the MIBL for their help and guidance at their respective accelerator facilities.

I would also like to thank Dr. King and Dr. Horton for their support and guidance here at CESE. Thanks for being great colleagues as well as great friends. Thanks to my colleagues within CESE and MSE for their support and thanks to my friends in and around Charlottesville for many stress relieving evenings.

Most of all I would like to thank my family, both at home in Massachusetts and here in Charlottesville. Caitlin, I love you. Thanks for staying by my side when the hours were long and the end seemed far away. Thank you to James and Lindsey for making me feel at home in Charlottesville. And thanks to my parents and my brother for their endless encouragement and love. 


\section{Table of Contents}

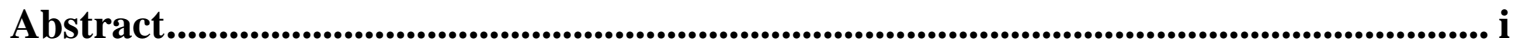

Acknowledgements ...................................................................................................... vi

Table of Contents ......................................................................................................................... vii

List of Tables .............................................................................................................. xii

List of Figures............................................................................................................. xiii

List of Symbols ......................................................................................................... xxviii

1 Introduction: Background, Significance and Objectives .......................................1

$1.1 \quad$ AA2024 and AA7075 Metallurgy ......................................................

1.2 AA2024 and AA7075 Corrosion Susceptibility ...........................................

1.3 Corrosion and Corrosion Protection of Precipitation Age Hardened

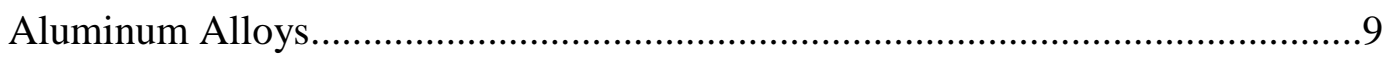

1.3.1 Inhibition of Crack Tip Processes that lead to Hydrogen Embrittlement ..................................................................................... 12

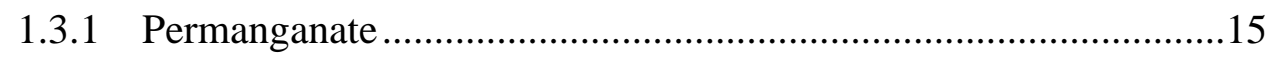

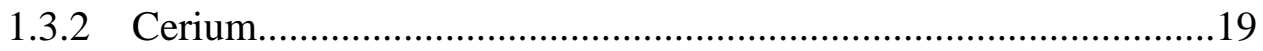

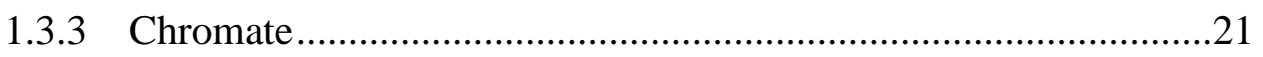

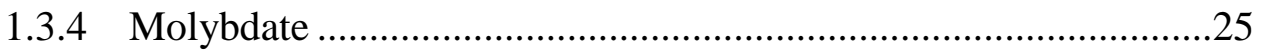

$1.4 \quad$ Inhibitor Activity at Relevant Material Length Scales ..............................27

1.5 Critical Unresolved Issues for Surface Corrosion Protection by Single Ionic

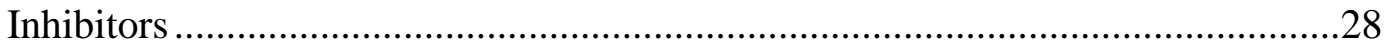

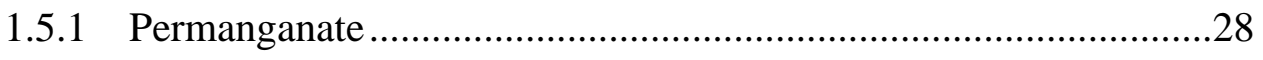

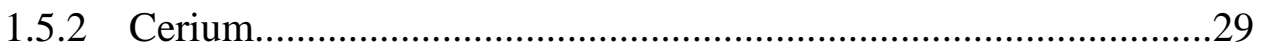

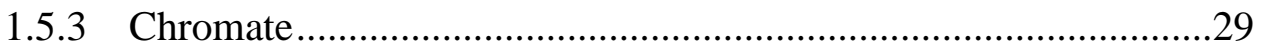

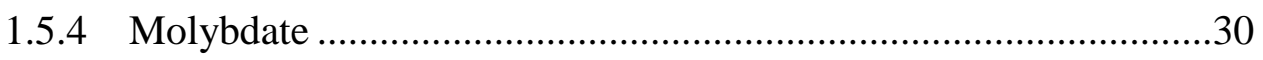

1.6 Critical Unresolved Issues for Binary Inhibitor Combinations ..................30

1.7 Critical Unresolved Issues for Crack Growth Inhibition .............................31

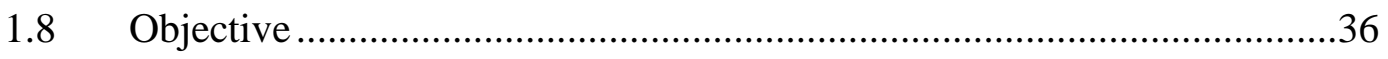

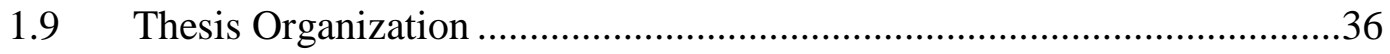

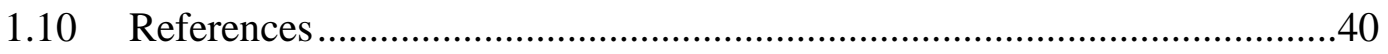

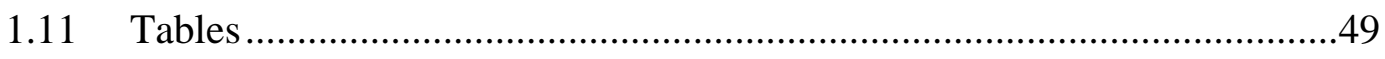

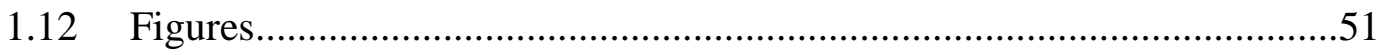




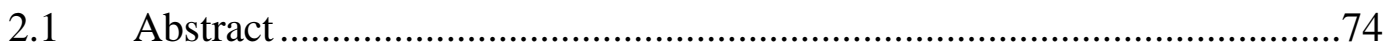

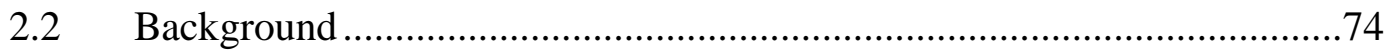

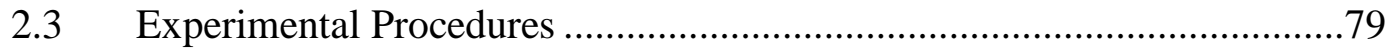

2.3.1 Materials and Test Electrolytes....................................................79

2.3.2 Open Circuit Measurements and Electrochemical Impedance

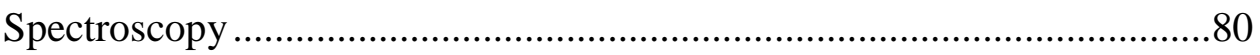

2.3.3 Microscopic Analysis after Open Circuit Exposures......................81

2.3.4 Anodic and Cathodic Cyclic Potentiodynamic Polarization Scans 82

2.3.5 Cyclic Voltammetry Tests to Assay Copper Redeposition.............83

2.3.6 Raman Spectroscopy..................................................................84

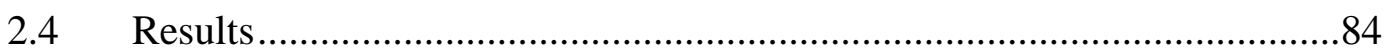

2.4.1 Effects of Permanganate during OCP exposures of AA2024 in 0.05

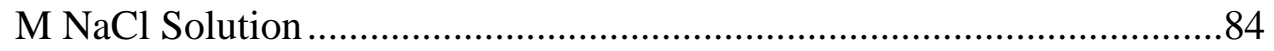

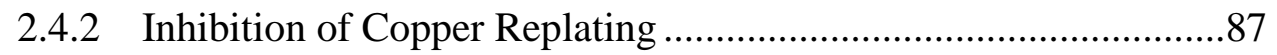

2.4.3 Anodic Inhibition by Permanganate Ions ......................................8

2.4.3.1 Localized Corrosion Behavior on Pretreated 2024-T351 88

2.4.3.2 Localized Corrosion Behavior of AA2024-T351 with Permanganate in Solution ...................................................... 89

2.4.4 Investigation of Cathodic Inhibition with $\mathrm{KMnO}_{4} \ldots \ldots \ldots \ldots \ldots \ldots \ldots \ldots . . . . .90$

2.4.5 Manganese Oxide Valence State Determination ............................94

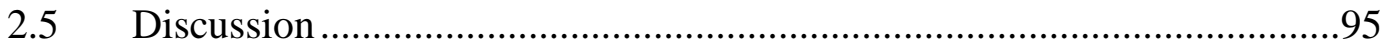

2.5.1 Oxidation States of Manganese ……………………………........95

2.5.2 Modes of $\mathrm{MnO}_{4}^{-}$Inhibition: Anodic ................................................96

2.5.3 Modes of $\mathrm{MnO}_{4}$ - Inhibition: Cathodic .............................................98

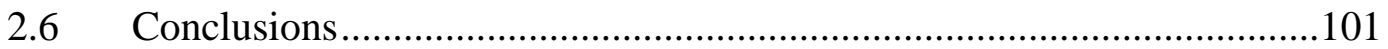

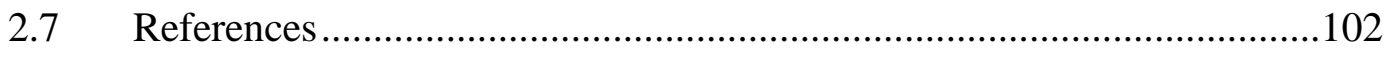

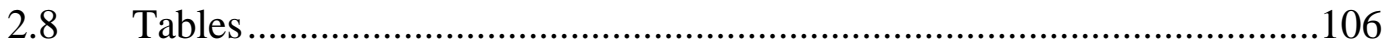

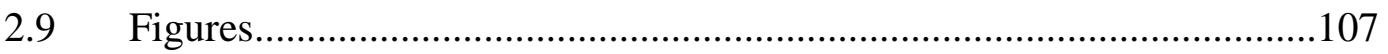


3 Corrosion Inhibition of AA2024-T351 Using Cerium-Permanganate

Combinations..................................................................................................123

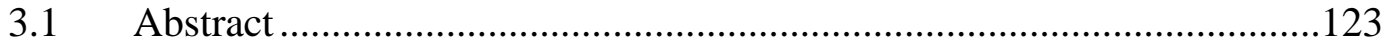

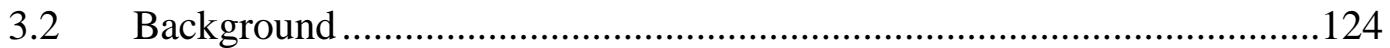

3.3 Experimental Procedures ................................................................128

3.3.1 Materials and Test Electrolytes...................................................128

3.3.2 Open Circuit Measurements During Open Circuit Exposure Periods 129

3.3.3 Microscopic Analysis after Open Circuit Exposures

130

3.3.4 Anodic and Cathodic Cyclic Potentiodynamic Polarization Scans 131

3.3.5 Cyclic Voltammetry Tests to Assay Copper Redeposition...........132

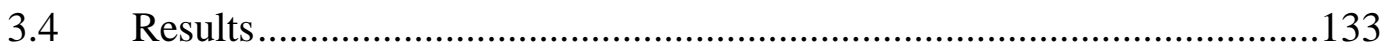

3.4.1 Effects of Cerium-Permanganate Pretreatment on OCP Exposures of AA2024 in $50 \mathrm{mM} \mathrm{NaCl}$ Solution...................................................133

3.4.2 Open Circuit Behavior of Pretreated AA2024-T351 ....................136

3.4.3 Inhibition of Copper Replating ....................................................137

3.4.4 Anodic Inhibition of AA2024-T351 by Cerium-Permanganate

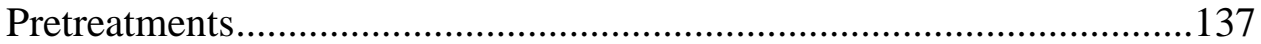

3.4.5 Investigation of Cathodic Inhibition with Cerium-Permanganate

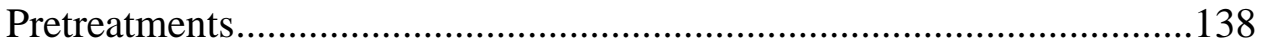

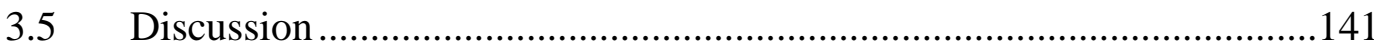

3.5.1 0Open circuit and Anodic Inhibition by Cerium Permanganate

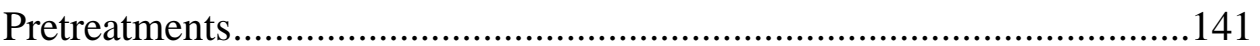

3.5.2 Diffusion Limited Current Density Inhibition by CeriumPermanganate Pretreatments ...................................................................142

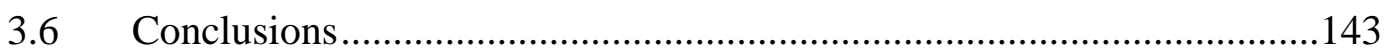

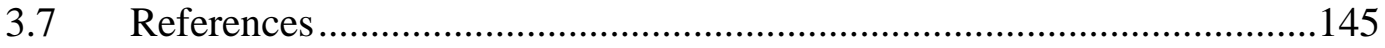

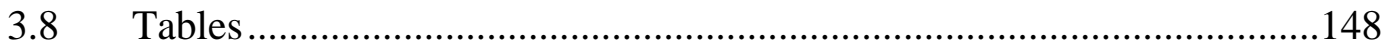

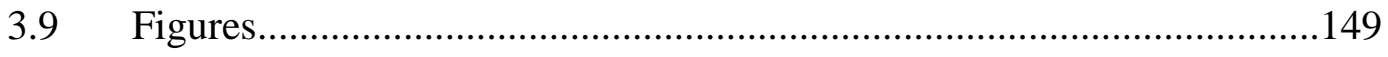

4 Effects of Chromate and Molybdate Ions on Scratch Repassivation Behavior of Precipitation Hardened Aluminum Alloys....................................................................170

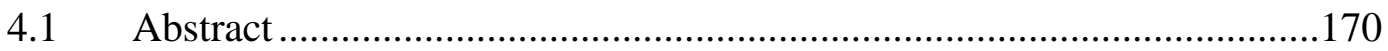




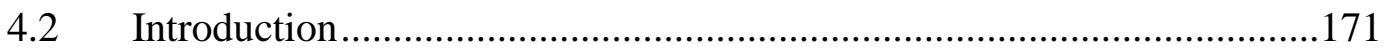

4.2.1 Methods to Study Repassivation...............................................176

4.2.2 Repassivation Studies on Aluminum with Ionic Inhibiting

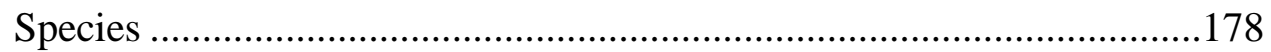

4.2.3 Finite Cathodic Limited Repassivation Studies - The Role of Inhibitors on the Crack Flank ...............................................................181

4.2.4 Complications in Scratch Repassivation Testing .........................182

4.2.5 Novel Method for Inhibitor Testing During Scratch Repassivation Testing 183

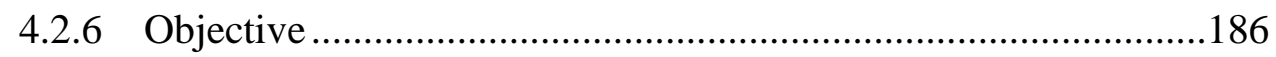

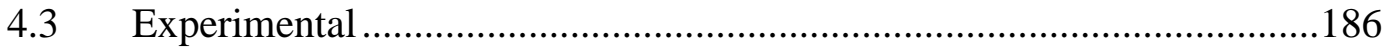

4.3.1 Net Current Collection for Scratch Depassivation........................186

4.3.2 Finite Cathodic Coupled Crack tip and Crack Flank ....................189

4.3.3 Impedance Measurement of the Scratch Depassivated Surface ..189

4.3.4 Additional Methods ......................................................................193

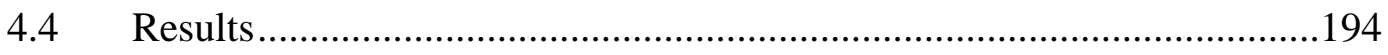

4.4.1 The Inhibition of Pitting Potential as Determined by Epit ...........194

4.4.2 The Inhibitor Buffer Capacity .........................................................196

4.4.3 The Effect of Inhibitors on Open Circuit Potential during Scratch

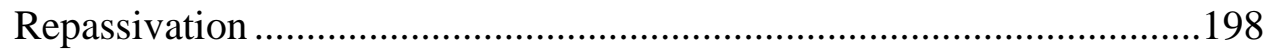

4.4.4 Crack Flank - Crack Tip Galvanic Couple Behavior and The Inhibition of Cathodic Reactions Critical to Crack Chemistry .................200

4.4.5 The Inhibition of Net Current Density During Scratch Repassivation 201

4.4.6 The Effect of Chromate, Molybdate, and Permanganate on Surface Impedance Changes During Scratch Repassivation in NaCl Solutions...207

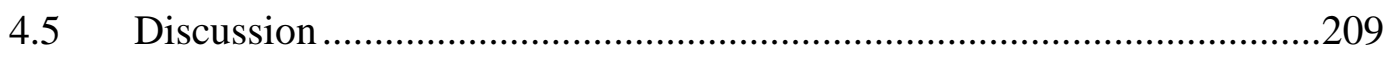

4.5.1 Effect of Selected Inhibitor additions on Current Transient Behavior of AA2024 and AA7075. .209

4.5.2 Comparison of Current Transient Behavior of AA2024 and AA7075 211

4.5.3 Effect of Chloride Concentration, $\mathrm{pH}$, and Potential .211 
4.5.4 Effects Relevant to EFCP and Implications Toward Understanding

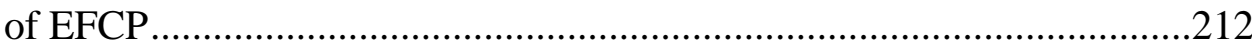

4.5.5 Alternative Mechanisms of EFCP Inhibition...........................215

4.5.6 Remaining Concerns for Repassivation as it Relates to SCC and EFCP 215

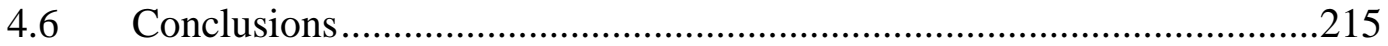

4.7 Acknowledgements ...................................................................216

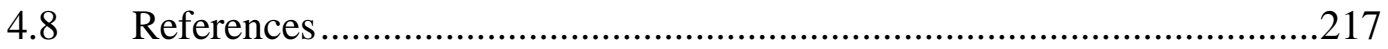

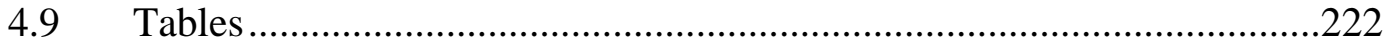

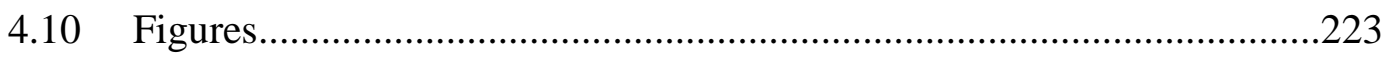

5 Direct Evidence for Enhanced Repassivation, Incorporation of Inhibitor Species, and Reduced Hydrogen Uptake in an Al-Zn-Mg-Cu Alloy with Selected Ionic

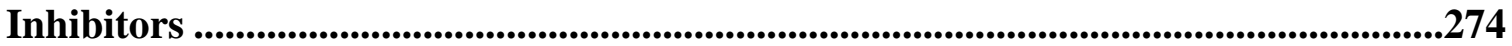

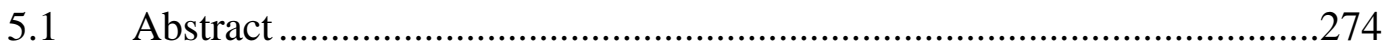

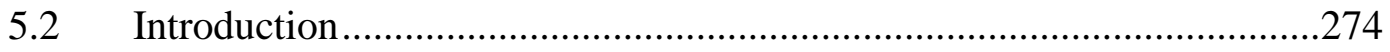

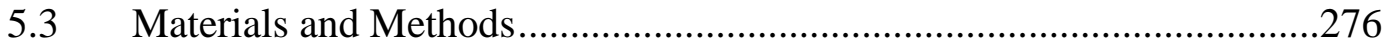

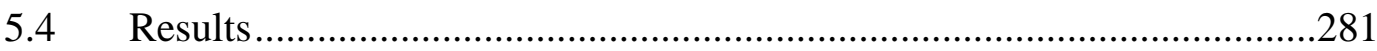

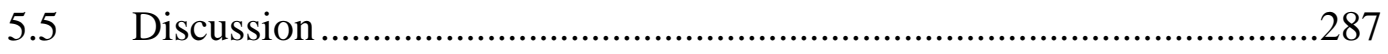

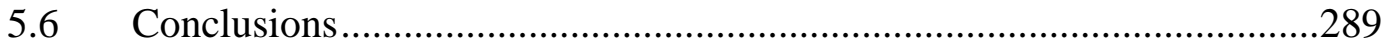

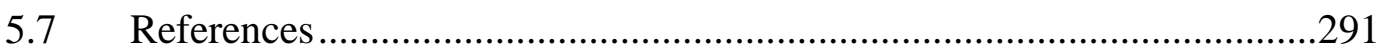

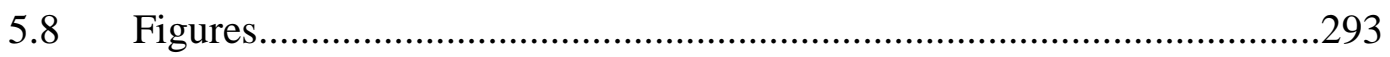

6 Thesis Conclusions and Suggested Future Work..................................................318

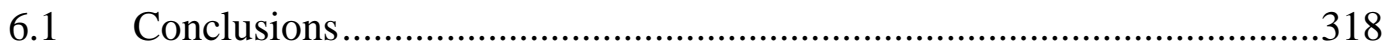

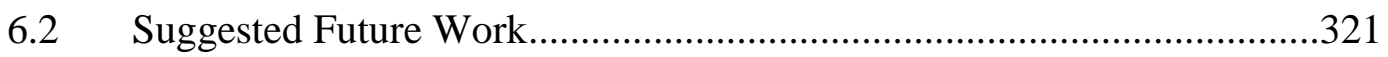

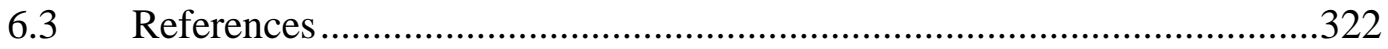




\section{List of Tables}

Table 1.1. Corrosion potentials for intermetallic compounds common in aluminum alloys.

Table 1.2 Intermetallic particle statistics for AA2024 in sheet, rod and wire forms as well as AA7075-T6 sheet.

Table 1.3 Inhibitor road map showing areas of strength in the literature and areas where needs, gaps, and opportunities have been addressed in this thesis. Some fields are not applicable due to inhibitor limitations.

Table 2.1. Chemical composition of AA2024-T351 (wt. \%) The T4 designation indicates slight cold working of T351.... 106

Table 2.2.Compilation of EDS data from AA2024-T351 intermetallic particles in Figure 2.3 , before and after pretreatment in $0.1 \mathrm{M} \mathrm{KMnO}_{4}$ 106

Table 3.1.Chemical composition of AA2024-T351 (wt. \%) The T4 designation indicates

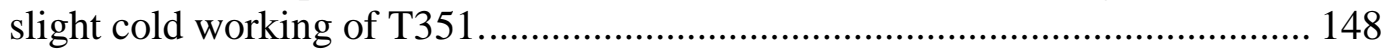

Table 3.2. Compilation of EDS data for AA2024-T351 intermetallic particles in Figures 1 and 2, before and after pretreatment in .1 $\mathrm{M} \mathrm{KMnO}_{4}$

Table 4.1. Chemical composition of AA2024-T351 and AA7075-T6 (wt. \%). AA2024 chemical composition measured with ICP-OES. AA7075 ingot metallurgy provided by Alcoa................................................................................ 222

Table 4.2. Fitting parameters for $99.999 \%$ aluminum at $-0.8 \mathrm{~V}$ (SCE) ....................... 222 


\section{List of Figures}

Figure 1.1 Binary solid solubility as a function of temperature for several of the most common alloying elements in aluminum alloys.

Figure 1.2. Natural aging (A) and artificial aging (B) time and hardness curves for binary $\mathrm{Al}-\mathrm{Cu}$ alloys showing age hardening due to formation of copper rich G-P zones in the natural case and transitional precipitates in the artificial case. In the artificial case the growth of the transitional precipitates at long aging times results in the conversion to equilibrium phase precipitates that are generally not coherent with the aluminum matrix leading to a decrease in hardness. 52

Figure 1.3. (A) TTT curve for AA2024 showing initial stages and aging progression of several strengthening precipitate phases. (B) Scanning electron microscope secondary electron image of coarse constituent particles in AA2024-T4. ........... 53

Figure 1.4 Backscattered electron detector image of AA2024-T351 sheet with corresponding intermetallic selection (A). AA2024-T4 rod - $13 \mathrm{~mm}$ diameter (B),

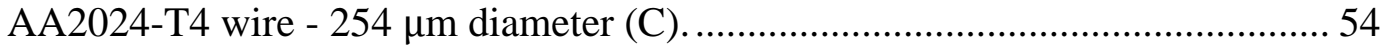

Figure 1.5. Backscatter SEM image of AA7075-T6 with accompanying EDS mapping conducted at $15 \mathrm{kV}$ accelerating voltage. B, C, D, E, and F are EDS maps of Mg, $\mathrm{Fe}, \mathrm{Cu}, \mathrm{Zn}$, and $\mathrm{Al}$ respectively.

Figure 1.6. AA2024-T351 sheet, AA2024-T4 rod - 13 mm diameter, AA2024-T4 wire $254 \mu \mathrm{m}$ diameter particle statistics. ............................................................ 56

Figure 1.7. AA7075-T6 sheet particle radius statistics assuming spherical particles....... 56

Figure 1.8. Potential-pH equilibrium (Pourbaix) diagram for the $\mathrm{Al}-\mathrm{H}_{2} \mathrm{O}$ system at $25^{\circ} \mathrm{C}$

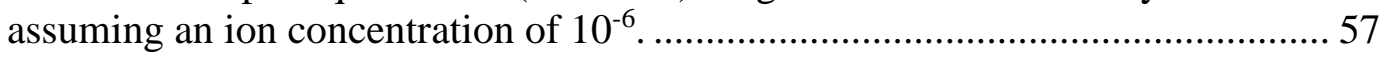

Figure 1.9. Galvanic couple between copper rich intermetallic and aluminum matrix (A). Precipitation free zone (PFZ) and accompanying grain boundary with large precipitate particles in an Al-Zn-Mg alloy.................................................. 58

Figure 1.10. The E-log(i) behavior of stationary electrodes of $99.998 \% \mathrm{Al}, \mathrm{S}-\mathrm{Al}_{2} \mathrm{CuMg}$, AA2024-T3, $\theta-\mathrm{Al}_{2} \mathrm{Cu}, \mathrm{Al}_{20} \mathrm{Cu}_{2}(\mathrm{MnFe})_{3}$, and $99.999 \% \mathrm{Cu}$ in $0.1 \mathrm{M} \mathrm{Na}_{2} \mathrm{SO}_{4}+0.005$ $\mathrm{M} \mathrm{NaCl}$ at ambient aeration. (a) cathodic (including anodic $\mathrm{S}-\mathrm{Al}_{2} \mathrm{CuMg}$ curve) and (b) anodic; all recorded after a $2 \mathrm{hr}$ OCP 59

Figure 1.11. (A) The effect of relative humidity on the SCC velocity of AA7075-T651. (B) The effect of chloride concentration on SC crack velocity.....

Figure 1.12. Effect of copper solid solution content on pitting and repassivation potential of aluminum-copper binary alloys.

Figure 1.13. Effect of aging time on stress corrosion crack velocity in alloys with greater than 1 wt. \% $\mathrm{Cu}$ (AA7075 and 7178) and less than 1 wt. \% Cu (AA7079). ........ 62

Figure 1.14. Fatigue crack growth rate comparison for AA2024 and AA7075. 63 
Figure 1.15. Crack tip pH with crack tip growth rate in EAC of AA7075 and AA7050 in a bulk solution of $0.05 \mathrm{M} \mathrm{NaCl}$ and $0.5 \mathrm{M} \mathrm{Na}_{2} \mathrm{CrO}_{4}$.

Figure 1.16. Crack tip $\mathrm{pH}$ with applied potential for 7xxx series aluminum alloys in $\mathrm{Cl}^{-}$ containing electrolyte.

Figure 1.17. Illustration of complex multidimensional systems and properties that effect SCC and EFCP. The presence of the embrittling environment at the crack tip, the anodic and cathodic electrochemical reactions that lead to hydrogen production, and diffusion of hydrogen into the fracture process zone are all important steps in hydrogen environmental embrittlement. The second diagram illustrates the complex processes occurring at the tip of the crack. 65

Figure 1.18. Effect of surface concentration of hydrogen on concentration gradient of hydrogen in a semi-infinite plate provided to show the possibility of lowering the internal hydrogen concentration bellow the critical concentration through the use of an inhibiting ion which lowers $\mathrm{C}_{\mathrm{H}}^{*}$, 66

Figure 1.19. Comparison between a repassivation event collected from a dynamically strained electrode and that of a scratch repassivation. Peak currents have been normalized for emphasis. 67

Figure 1.20. Crack growth rates for full immersion molybdate containing sodium chloride solutions as compared to sodium chloride alone $f_{\text {Crit }}$ is on the order of $10 \mathrm{~Hz} . . .68$

Figure 1.21. Crack growth rates for full immersion chromate containing sodium chloride solutions as compared to sodium chloride alone. $f_{\text {Crit }}$ is on the order of $100 \mathrm{~Hz}$. 68

Figure 1.22. The E-pH diagrams for permanganate at 3 concentrations, $0.1 \mathrm{M}, 0.05 \mathrm{M}$ and $0.005 \mathrm{M}$ with chlorine held at $0.05 \mathrm{M}$. OCP for AA2024 is approximately $-0.55 \mathrm{~V}$ (SCE)

Figure 1.23: Aqueous cerium E-pH diagram showing the stability and region of predominance of $\mathrm{Ce}^{4+}$ oxides over a large range of $\mathrm{pH}$.

Figure 1.24. (a) E-pH diagram in $\mathrm{H}_{2} \mathrm{O}$ at $25^{\circ} \mathrm{C}$ for $.1 \mathrm{M}$ chromate and $1 \mathrm{M}$ chloride showing the stability of chromium oxide over a large region of potential and $\mathrm{pH}$. (b) Speciation of chromium-chloride system at previously stated concentrations at -0.8 $\mathrm{V}(\mathrm{SCE})$.

Figure 1.25. Buffer capacity of $200 \mathrm{~mL}$ of $0.5 \mathrm{M} \mathrm{Na}_{2} \mathrm{CrO}_{4}+0.5 \mathrm{M} \mathrm{NaCl}$. 71

Figure 1.26. (a) E-pH diagram for $0.1 \mathrm{M}$ molybdate and $1 \mathrm{M}$ chloride solution showing the stability of molybdenum oxide over a large region of potential and $\mathrm{pH}$ with high potential stability in the low $\mathrm{pH}$ region. (b) Speciation of molybdate-chloride system at previously stated concentrations at $-0.8 \mathrm{~V}$ (SCE) .............................. 72

Figure 1.27 Relevant length scales over which inhibitors play a role. Source: University of Virginia, CESE, courtesy of Matthew R. Begley and Richard P. Gangloff, Hongbo Cong and J.R. Scully..... 73

Figure 1.28. After a scratch the bare metallic electrode will undergo oxide nucleation and an increase in oxide surface coverage, followed by thickening of the oxide film as repassivation occurs. $\mathrm{D}$ is the thickness of the oxide layer...... 73 
Figure 2.1.(a) E-pH diagram for $0.005 \mathrm{M}$ permanganate with addition of $0.05 \mathrm{M}$ chloride. (b) Speciation diagrams for $0.005 \mathrm{M} \mathrm{MnO}_{4}^{-}+0.05 \mathrm{M} \mathrm{Cl}^{-}$at $-0.5 \mathrm{~V}_{\text {sce, }}$, the open circuit of AA-2024.AA2024. E-pH and speciation diagrams were calculated using Medusa software ${ }^{[1]}$. 107

Figure 2.2. (a) As-polished (1200 grit) AA2024-T351 and subsequent surface damage caused by 24 hour exposure to aerated $0.05 \mathrm{M} \mathrm{NaCl}$ solution. (b) The damaged specimen image is distorted laterally but includes both a secondary electron image (left) and a backscattered electron image (right), which clearly shows intermetallic trenching. 107

Figure 2.3. (a) AA2024-T351 polished to 1200 grit with specific EDS sites labeled 1-8 as reported in Table 2.2. (b) Same sample and area as (a) after pretreatment for 24 hours in $0.1 \mathrm{M} \mathrm{KMnO}_{4}$. 108

Figure 2.4. (a) AA2024-T351 LT surface pretreated with $0.1 \mathrm{M} \mathrm{KMnO}_{4}$ for 24 hours (secondary electron image). (b) AA2024-T351 pretreated with $0.05 \mathrm{M} \mathrm{KMnO}_{4}$ for 24 hours (secondary electron image). (c) AA2024-T351 pretreated with $0.005 \mathrm{M}$ $\mathrm{KMnO}_{4}$ for 24 hours (backscattered image). 108

Figure 2.5. (a) AA2024-T351 LT surface exposed to aerated $0.05 \mathrm{M} \mathrm{NaCl}$ for 24 hours after pretreatment in $0.1 \mathrm{M} \mathrm{KMnO}_{4}$ for 24 hours. (b) AA2024-T351 exposed to $0.05 \mathrm{M} \mathrm{NaCl}$ for 24 hours after pretreatment in $0.05 \mathrm{M} \mathrm{KMnO}_{4}$ for 24 hours. (c) AA2024-T351 exposed to $0.05 \mathrm{M} \mathrm{NaCl}$ for 24 hours after pretreatment in $0.005 \mathrm{M}$ $\mathrm{KMnO}_{4}$ for 24 hours. 109

Figure 2.6. (a) AA2024-T351 LT surface exposed to aerated $0.05 \mathrm{M} \mathrm{NaCl}$ with $0.1 \mathrm{M}$ $\mathrm{KMnO}_{4}$ in-situ for 24 hours. (b) AA2024-T351 exposed to $0.05 \mathrm{M} \mathrm{NaCl}$ with 0.05 $\mathrm{M} \mathrm{KMnO}_{4}$ in-situ for 24 hours. (c) AA2024-T351 exposed to $0.05 \mathrm{M} \mathrm{NaCl}$ with $0.005 \mathrm{M} \mathrm{KMnO}_{4}$ in-situ for 24 hours. 109

Figure 2.7. AA2024-T351 LT surface exposed to aerated $0.05 \mathrm{M} \mathrm{NaCl}$ solution with 0.05 $\mathrm{M} \mathrm{KMnO}_{4}$ in-situ for 24 hours. The manganese oxide caps were removed after the exposure with a stiff bristled brush. 110

Figure 2.8. EIS of AA2024-T351 pretreated with 0.1 M KMnO4 for 1 and 24 hours (a) and exposed to $0.05 \mathrm{M} \mathrm{NaCl}$ with $0.1 \mathrm{M} \mathrm{KMnO} 4$ in solution for 1 and 24 hours (b). 110

Figure 2.9. The OCP was recorded for one hour after 24 hours at OCP in $0.05 \mathrm{M}$ sodium chloride solution with inhibitor added in-situ at the concentrations reported. ... 111

Figure 2.10. OCP measurements for pretreated AA2024-T351 after treatment for 24 hours in varying concentrations of $\mathrm{KMnO}_{4}$. OCP measured in $0.05 \mathrm{~m} \mathrm{NaCl}$ solution. Experiments have been run 10 times and show great reproducibility. 111

Figure 2.11. (a) CVs of AA2024-T351 etched for 24 hours in $0.05 \mathrm{M} \mathrm{NaCl}$ solution with varying inhibitor concentration in solution, $0.4 \mathrm{M}, 0.05 \mathrm{M}, 0.005 \mathrm{M} \mathrm{KMnO}_{4}$, and no inhibitor. (b) CVs of AA2024-T351 etched in $0.05 \mathrm{M} \mathrm{NaCl}$ solution for 24 hours after pretreatment for 24 hours in varying inhibitor concentration: $0.1 \mathrm{M}, 0.05 \mathrm{M}$, $0.005 \mathrm{M} \mathrm{KMnO}_{4}$. 112 
Figure 2.12. The relative intensities of the $\mathrm{Cu}^{1+}$ reduction peak on the AA2024-T351 surface when corroded with the $\mathrm{MnO}_{4}{ }^{-}$ion in solution for 24 hours in $0.05 \mathrm{M} \mathrm{NaCl}$ (a) and with $\mathrm{MnO}_{4}^{-}$inhibitor pretreatment followed by exposure for 24 hours in $0.05 \mathrm{M} \mathrm{NaCl}$ (b). All CV tests run 5 times. Error bars indicate one standard deviation. 112

Figure 2.13. The anodic polarization behavior for AA2024-T351 after pretreatment in $\mathrm{KMnO}_{4}$ for 24 hours. Scan run in $0.05 \mathrm{M}$ sodium chloride solution. (a) Pretreatment in $0.1 \mathrm{M} \mathrm{KMnO}_{4}$, (b) Pretreatment in $0.05 \mathrm{M} \mathrm{KMnO}_{4}$, (c) Pretreatment in $0.005 \mathrm{M}$ $\mathrm{KMnO}_{4}$

Figure 2.14. The anodic polarization behavior for AA2024-T351 after 24 hours of exposure to $0.05 \mathrm{M}$ sodium chloride solution with the inhibitor in-situ. Scans conducted in $0.05 \mathrm{M}$ sodium chloride solution with varied inhibitor concentrations of (a) $0.1 \mathrm{M}$

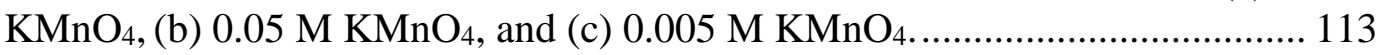

Figure 2.15. SEM backscattered image of multiple phase ingot with large S-phase regions. The large light colored regions of the micrographs are S-phase and indicate the higher copper content and corresponding higher Z. (a) Sample as polished. (b) Sphase held potentiostatically in $0.1 \mathrm{M} \mathrm{MnO}_{4}^{-}$and $0.05 \mathrm{M} \mathrm{Cl}^{-}$solution for 200 seconds. Note the growth of manganese oxide over the S-phase particles. (c) Sphase held potentiostatically in $0.05 \mathrm{M} \mathrm{Cl}^{-}$solution for 200 seconds. While (a) and (b) represent the same region of the ingot, (c) does not. However, the region of severe damage is in the center of an S-phase particle. Insert of EDS line scan shows the enrichment of copper in proximity of visible $S$-phase damage. 114

Figure 2.16. AA2024 exposed to $0.005 \mathrm{M} \mathrm{KMnO}_{4}+0.05 \mathrm{M} \mathrm{NaCl}$ for 24 hours and then anodically polarized to potentials above the pitting potential $(0.3 \mathrm{~V}$ vs. SCE $)$ in fresh $0.005 \mathrm{M} \mathrm{KMnO}_{4}+0.05 \mathrm{M} \mathrm{NaCl}$ solution. 114

Figure 2.17. Cumulative probability of pitting as a function of potential for both pretreatment (a) and in-situ solution phase (b) permanganate inhibitor concentration. Probability of Pitting in $0.05 \mathrm{M} \mathrm{NaCl}$....................................... 115

Figure 2.18. The average difference between pitting and OCP for Mn pretreatment (a) and in-situ solution phase additions (b), as a function of inhibitor concentration. The error bars indicate one standard deviation. 115

Figure 2.19. Cathodic E vs $\log (i)$ polarization curves for AA2024-T4 (a) and high purity copper (b) samples measured at various rotation rates from $100 \mathrm{rpm}$ to 2500 in $\mathrm{pH}$ 8.4 borate buffer. Solutions were aerated naturally. 116

Figure 2.20. Cathodic polarization behavior of AA2024-T4 and high purity copper samples ground to 1200 grit, tested at $1000 \mathrm{rpm}$ in $\mathrm{pH} 8.4$ borate buffer solution after $100 \mathrm{~h}$ pretreatment (a) and run at $1500 \mathrm{rpm}$ in $0.05 \mathrm{M} \mathrm{NaCl}$ solution with in-situ inhibitor additions (b). Rotation rate has been kept constant and inhibitor concentration is tested at different levels. All data collected under natural aeration. 116

Figure 2.21. Potentiodynamic scan of high purity copper with Mn-oxide deposited at a potential of $-0.5 \mathrm{~V}(\mathrm{SCE})$ run in $\mathrm{pH} 8.4$ borate buffer. 117 
Figure 2.22. (a) Total current at $-1.0 \mathrm{~V}$ (SCE) for AA2024-T4 and pure copper compared with the Levich prediction $\left(\mathrm{i}_{\mathrm{d}}\right)$ in $\mathrm{pH} 8.4$ borate buffer solution. (b) Diffusion limited current extracted from $i_{\text {total }}$ at $-1.0 \mathrm{~V}$ (SCE) for AA2024-T4 and pure copper compared with the Levich prediction in $\mathrm{pH} 8.4$ borate buffer. Data extracted from Figure 2.20. 117

Figure 2.23. Total cathodic current at $-1.0 \mathrm{~V}$ (SCE) for AA2024-T4 for the inhibitor pretreatment tests in inhibitor free environment (a) and with the inhibitor present in-situ (b). Note that (a) and (b) have an order of magnitude difference in the ordinate. Total current densities for pure copper, bare AA2024-T4 and the Levich prediction $i_{d}$ values are included for comparison. Data extracted from Figure 2.20.

118

Figure 2.24. Diffusion limited current density extracted from $i_{\text {total }}$ at $-1.0 \mathrm{~V}$ (SCE) for AA2024-T4 after pretreatment at various inhibitor concentration compared with pure copper, bare AA2024-T4, and the Levich prediction. Tests were conducted in borate buffer solution at $\mathrm{pH}$ 8.4. Data extracted from Figure 2.23. 118

Figure 2.25. (A) Extracted activation controlled current density over a range of potentials for high purity $\mathrm{Cu}, \mathrm{AA} 2024-\mathrm{T} 351$, and AA2024-T351 pretreated in $0.05 \mathrm{M} \mathrm{MnO}_{4}^{-}$ and $0.1 \mathrm{M} \mathrm{MnO}_{4}^{-}$. (B) Calculated copper coverage, @CuKaeshe , at $-0.6 \mathrm{~V}$ using Equation [15]. $\mathrm{Cu}$ (1), AA2024-T351(2), and AA2024-T351 pretreated in $0.05 \mathrm{M}$ $\mathrm{MnO}_{4}^{-}$(3) and in $0.1 \mathrm{M} \mathrm{MnO}_{4}^{-}$(4). 119

Figure 2.26. Constant current discharge potential vs. time plots on of electrodeposited

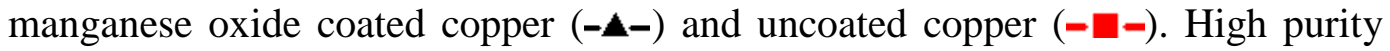
Copper with Mn oxide deposited at $-0.5 \mathrm{~V}$ SCE from $0.1 \mathrm{M} \mathrm{KMnO}_{4}$ with a $\mathrm{pH}$ of 6. Galvanostatic reduction performed at $-20 \mathrm{~mA} / \mathrm{cm}^{2}$ in $\mathrm{pH} 8.4$ borate buffer. The red dotted line (..) indicates the reversible potential of $\mathbf{M n O 4}-+4 H+3 e-\leftrightarrow$ MnO2 + 2H20 at $0.54 \mathrm{~V}$ vs SCE. The blue dashed (--) line indicates the reversible potential for $\mathbf{M n O 2}+\mathbf{H 2 O}+\boldsymbol{e}-\leftrightarrow \boldsymbol{M n O O H}+\boldsymbol{O H}-$ at $0.187 \mathrm{~V}$ vs $\mathrm{SCE}$. The green dash-dotted line $(-\cdot-)$ indicates the reversible potential for $\mathbf{M n O O H}+\boldsymbol{H} \mathbf{2 O}+\boldsymbol{e}-\leftrightarrow \boldsymbol{M n}(\boldsymbol{O H}) \mathbf{2}+\boldsymbol{O H}-$ at $-0.385 \mathrm{~V}$ vs SCE. 120

Figure 2.27. a) Raman spectra for AA2024-T351 (no Raman response), AA2024-T351 pretreated in $0.005 \mathrm{M} \mathrm{KMnO}_{4}$, copper pretreated in $0.1 \mathrm{M} \mathrm{KMnO}_{4}$ at $-0.5 \mathrm{~V}$ (SCE), manganese metal held potentiostatically at $0.35 \mathrm{~V}$ (SCE), and $\mathrm{MnO}_{2}$ powder. b) Background subtracted peaks showing three peaks at 630,573 , and $506 \mathrm{~cm}^{-1} . .121$

Figure 2.28. (a) Gueshi model showing effect of IMC special geometry in AA2024-T351 on modeled ORR kinetics and actual data values. (b) A schematic showing effect of decreasing active area in an inactive matrix.

Figure 2.29. (a) Cathodic reaction rate on high purity copper with and without electrodeposited manganese oxide film. (b) Membrane modeling of cathodic reaction kinetics from Equation [18] in mass transport and mixed ORR regime for high purity copper with a manganese oxide film grown on its surface from $0.1 \mathrm{M}$ $\mathrm{KMnO}_{4}$ solution at $-0.5 \mathrm{~V}(\mathrm{SCE})$. 
Figure 3.1.(a) The E-pH behavior of $.05 \mathrm{M} \mathrm{Ce}^{3+}$ in aqueous solution. (b) The concentrations of cerium species in the cerium- $\mathrm{H}_{2} \mathrm{O}$ system at $-0.5 \mathrm{~V} \mathrm{SCE}$, near the open circuit potential of AA2024. 149

Figure 3.2. (a) The E-pH behavior of $0.05 \mathrm{M} \mathrm{MnO}_{4}^{-}$in aqueous solution. (b) The concentrations of manganese species in the manganese- $\mathrm{H}_{2} \mathrm{O}$ system at $-0.5 \mathrm{~V} \mathrm{SCE}$, near the open circuit potential of AA2024. 150

Figure 3.3. Concentrations of species in the cerium-chloride- $\mathrm{H}_{2} \mathrm{O}$ system at $0.78 \mathrm{~V}$ (SCE), the $\mathrm{E}^{\mathrm{o}}$ of Equation [3.2]. The cerium oxide species $\left(\mathrm{CeO}_{2}\right)$ is stable above $\mathrm{pH} 3.3$ at this potential.

Figure 3.4. (a) The as-polished (1200 grit) AA2024-T351 and the subsequent surface damage caused by 24 hour exposure to $0.05 \mathrm{M} \mathrm{NaCl}$ solution in a naturally aerated state at room temperature. (b) The damaged specimen image is compressed horizontally to include a secondary electron and backscattered electron image. 152

Figure 3.5. (a) AA2024-T351 pretreated with $0.01 \mathrm{M} \mathrm{Ce}^{3+}$ for $100 \mathrm{hr}$ and $0.04 \mathrm{M} \mathrm{MnO}_{4}^{-}$ for $24 \mathrm{hr}$ (secondary electron image). (b) Same pretreatment times with inhibitor concentrations of $0.025 \mathrm{M} \mathrm{Ce}^{3+}$ and $0.025 \mathrm{MnO}_{4}^{-}$. (c) Same pretreatment times with inhibitor concentrations of $0.04 \mathrm{M} \mathrm{Ce}^{3+}$ and $0.01 \mathrm{MnO}_{4}^{-}$. (d) Same pretreatment times with inhibitor concentrations of $0.05 \mathrm{M} \mathrm{Ce}^{3}$. (e) Same pretreatment time with inhibitor concentration of $0.05 \mathrm{MnO}_{4}^{-}$ 153

Figure 3.6. (a) AA2024-T351 with indication of EDS sites labeled 1-8. (b) Same sample and area as (a) Sample has been pretreated for 24 hours in $0.1 \mathrm{M} \mathrm{KMnO}_{4}$. Indication of EDS sites labeled 1-8. 154

Figure 3.7. (a) Intermetallic particle 1 from Figure 3.6 (b). Intermetallic particle 5 from Figure 3.6. The left spectrum is taken from an untreated sample, the right, a pretreated sample. 155

Figure 3.8. EDS at $15 \mathrm{kV}$ accelerating potential of AA2024-T351 pretreated in $10 \mathrm{mM}$ $\mathrm{Ce}^{3+}+40 \mathrm{mM} \mathrm{MnO}_{4}^{-}(\mathrm{a}), 25 \mathrm{mM} \mathrm{Ce}^{3+}+24 \mathrm{mM} \mathrm{MnO}_{4}^{-}$(b), $40 \mathrm{mM} \mathrm{Ce}^{3+}+10 \mathrm{mM}$ $\mathrm{MnO}_{4}^{-}$. All cerium pretreatments lasted for 100 hours. All permanganate pretreatments lasted for 24 hours. 156

Figure 3.9. (a) AA2024-T351 pretreated with $0.01 \mathrm{M} \mathrm{Ce}^{3+}$ for $100 \mathrm{hrs}$ and $0.04 \mathrm{M} \mathrm{MnO}_{4}^{-}$ for 24 hours and exposed to $0.05 \mathrm{M} \mathrm{Cl}^{-}$for $24 \mathrm{~h}$ (secondary electron image). (b) Same pretreatment with inhibitor concentrations of $0.025 \mathrm{M} \mathrm{Ce}^{3+}$ and $0.025 \mathrm{MnO}_{4}^{-}$ and exposed to $0.05 \mathrm{M} \mathrm{Cl}^{-}$for $24 \mathrm{~h}$ (c) Same pretreatment with inhibitor concentrations of $0.04 \mathrm{M} \mathrm{Ce}^{3+}$ and $0.01 \mathrm{MnO}_{4}{ }^{-}$and exposed to $0.05 \mathrm{M} \mathrm{Cl}^{-}$for $24 \mathrm{~h}$. (d) Same pretreatment with inhibitor concentrations of $0.05 \mathrm{M} \mathrm{Ce}^{3+}$ and exposed to $0.05 \mathrm{M} \mathrm{Cl}^{-}$for $24 \mathrm{~h}$.(e) Same pretreatment with inhibitor concentrations of 0.05 $\mathrm{MnO}_{4}{ }^{-}$and exposed to $0.05 \mathrm{M} \mathrm{Cl}^{-}$for $24 \mathrm{~h}$. Samples were not cleaned post exposure. 158

Figure 3.10. EDS at $15 \mathrm{kV}$ accelerating potential of AA2024-T351 exposed for 24 hours in $50 \mathrm{mM} \mathrm{NaCl}$ after pretreatment in $10 \mathrm{mM} \mathrm{Ce} 3++40 \mathrm{mM} \mathrm{MnO} 4-(\mathrm{a}), 25 \mathrm{mM}$ $\mathrm{Ce} 3++24$ mM MnO4- (b). All cerium pretreatments lasted for 100 hours. All permanganate pretreatments lasted for 24 hours. 159 
Figure 3.11. Surface damage revealed by abrasive removal of cerium oxide after pretreatment in $0.05 \mathrm{M} \mathrm{Ce}^{3+}$ for $100 \mathrm{~h}$ and subsequent exposure to $0.05 \mathrm{M} \mathrm{NaCl}$ for 24 hours. 161

Figure 3.12. Open circuit test of pretreated AA2024-T351 in $50 \mathrm{mM} \mathrm{NaCl}$ solution with ambient aeration. Test included $100 \mathrm{~h}$ pretreatment in $\mathrm{Ce}^{3+}$ followed by $24 \mathrm{~h}$ pretreatment in $\mathrm{MnO}_{4}{ }^{-}$at the indicated concentrations. Pretreatments are $\mathrm{Cl}$ - free. 162

Figure 3.13. CVs of pretreated AA2024-T351 after a 24 hour exposure in $50 \mathrm{mM} \mathrm{NaCl}$ solution. 162

Figure 3.14. The relative intensities of the $\mathrm{Cu}^{1+}$ reduction peak on the AA2024-T351 surface pretreated in cerium for $100 \mathrm{~h}$ and subsequently in permanganate for $24 \mathrm{~h}$ followed by exposure for $24 \mathrm{~h}$ in $0.05 \mathrm{M} \mathrm{NaCl}$ are shown. Combined inhibitor concentrations tested had ratios of cerium to permanganate of 0.2 and 0.8 with a total inhibitor concentration of $0.05 \mathrm{M}$. Results are compared to permanganate pretreatments and cerium pretreatments. A large decrease in the copper surface coverage is evident for the combined inhibitor pretreatment over either single inhibitor pretreatment. 163

Figure 3.15. Anodic E-logi behavior of AA2024 with cerium-permanganate pretreatments at stated concentrations tested in $5 \mathrm{mM}$ sodium chloride. 164

Figure 3.16. Cumulative probability of pitting on AA 2024-T351 as a function of potential for both permanganate- cerium combinations and in-situ solution phase permanganate inhibitor concentration. Combined inhibitor concentrations tested had ratios of cerium to permanganate of $0, .2, .5, .8$, and 1 with a total inhibitor concentration of $0.05 \mathrm{M}$. Cumulative probability of pitting in $0.05 \mathrm{M} \mathrm{NaCl}$ solution. 165

Figure 3.17. Cathodic E vs $\log (i)$ data for AA2024-T4 (a) and high purity copper (b) samples run at various rotation rates from $100 \mathrm{rpm}$ to 2500 in $\mathrm{pH} 8.4$ borate buffer. Solutions were aerated naturally and no pretreatment was performed.............. 166

Figure 3.18. Rotating disk cathodic polarizations experiments on AA2024-T351 at a rotation rate of $2500 \mathrm{rpm}$ in $\mathrm{pH} 8.4$ borate buffer. Combined inhibitor concentrations tested had ratios of cerium to permanganate of $0.2,0.5$, and 0.8 with a total inhibitor concentration of $0.05 \mathrm{M}$. Total current densities for bare AA2024, cerium treated, and permanganate treated have been included for reference..... 166

Figure 3.19. (a) RPM Ramp 500 to 2500 (increments of 500) of AA2024-T351 in pH 8.4 borate buffer after stated pretreatments When manganese deposits are present it reacts electrochemically effecting the current density at each rotation rate. (b) RPM Ramp 500 to 2500 (increments of 500) of high purity copper in $\mathrm{pH} 8.4$ borate buffer after stated pretreatments. 167

Figure 3.20. Diffusion limited current density on high purity copper compared to AA2024$\mathrm{T} 351$ at rotation rates from 500 to $2500 \mathrm{rpm}$ in $\mathrm{pH} 8.4$ borate buffer. Combined inhibitor concentrations tested had ratios of cerium to permanganate of 4,1 , and 0.2 with a total inhibitor concentration of $0.05 \mathrm{M}$. 168 
Figure 3.21. Diffusion limited current density on high purity copper at rotation rates from 500 to $2500 \mathrm{rpm}$ in $\mathrm{pH} 8.4$ borate buffer. Combined inhibitor concentrations tested had ratios of cerium to permanganate of 4,1 , and 0.2 with a total inhibitor concentration of $.05 \mathrm{M}$. 168

Figure 3.22. Schematic of different cathodic reaction inhibition mechanisms for the reduction of permanganate, the chemical precipitation of cerium hydroxide, and the effect of dual inhibitor pretreatment. 169

Figure 4.1. Diagram illustrating crack tip processes that lead to the embrittlement of precipitation age hardened aluminum alloys in the fracture process zone through the uptake of hydrogen from proton and water reduction. Hydrolysis of dissolving aluminum can exacerbate hydrogen production and uptake by increasing $\mathrm{H}^{+}$ concentration and raising $\mathrm{E}_{\mathrm{H}+} / \mathrm{E}_{\mathrm{H} 2}$. Inhibitors are thought to act through the passivation of the fractured surfaces, producing a decrease in dissolution and subsequently hydrolysis while providing a hydrogen permeation barrier. Buffering effects may also influence the crack tip environment. 223

Figure 4.2. Crack growth rates for full immersion molybdate containing sodium chloride solutions as compared to sodium chloride alone $f_{\text {Crit }}$ is on the order of $10 \mathrm{~Hz}$. 224

Figure 4.3. Crack growth rates for full immersion chromate containing sodium chloride solutions as compared to sodium chloride alone. $f_{\text {Crit }}$ is on the order of $100 \mathrm{~Hz} .224$

Figure 4.4. Effect of surface concentration from 0.5 to 0.1 hydrogen atoms per solvent atom on concentration gradient of hydrogen in a semi-infinite plate provided to show the possibility of lowering the internal hydrogen concentration bellow the critical concentration through the use of an inhibiting ion which lowers $\mathrm{C}_{\mathrm{H} \text {,s. } . ~}^{*} 225$

Figure 4.5. (a) E-pH diagram in $\mathrm{H}_{2} \mathrm{O}$ at $25{ }^{\circ} \mathrm{C}$ for $0.1 \mathrm{M}$ chromate and $1 \mathrm{M}$ chloride showing the stability of chromium oxide over a large region of potential and $\mathrm{pH}$. (b) Speciation of chromium-chloride system at previously stated concentrations at $-0.8 \mathrm{~V}(\mathrm{SCE})$. 226

Figure 4.6. (a) E-pH diagram for $0.1 \mathrm{M}$ molybdate and $1 \mathrm{M}$ chloride solution showing the stability of molybdenum oxide over a large region of potential and $\mathrm{pH}$ with high potential stability in the low $\mathrm{pH}$ region. (b) Speciation of molybdate-chloride

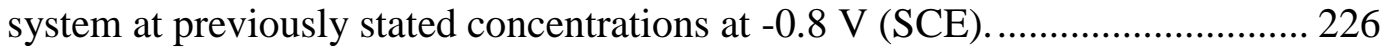

Figure 4.7. The effect of potential on the transient exponential behavior of anodic current decay as characterized by the constant $\beta$ on the high purity aluminum scratched in $0.085 \mathrm{M} \mathrm{NaCl}$ with the addition of reported inhibitors with a concentration of 0.08 M. $\left(\beta=2.3 \beta^{\prime}\right)$

Figure 4.8. Effect of chromate on the open circuit potential of aluminum after it was depassivated in a guillotine experiment. Line $a$ was conducted in $0.1 \mathrm{M} \mathrm{NaCl}$ solution while $b$ and $c$ were conducted in 0.01 and $1.0 \mathrm{M} \mathrm{Na}_{2} \mathrm{CrO}_{4}$ solutions, respectively. 228

Figure 4.9. Schematic of an oxide covered surface after a scratch. The bare metallic electrode undergoes oxide nucleation and an increase in oxide surface coverage, 
followed by thickening of the oxide film as repassivation occurs. The thickness of the oxide layer is $\mathrm{d}_{\mathrm{ox}}$.

Figure 4.10. Theoretical model Bode impedance for depassivated and passive aluminum electrodes. Values were determined assuming a passivated $R_{\text {film }}$ value of $10^{4} \Omega-\mathrm{cm}^{2}$,

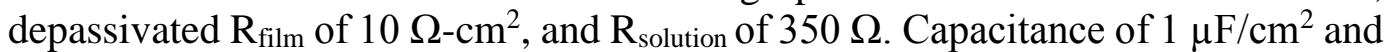
$100 \mu \mathrm{F} / \mathrm{cm}^{2}$ were used for the passivated and bare electrodes, respectively. Values corrected for electrode surface area of $2 \times 10^{-4} \mathrm{~cm}^{2}$. 230

Figure 4.11. In the region where the slope of $\log |\mathrm{Z}|$ vs $\log \omega$ is equal to -1 the relationship $C=1 / 2 \pi f|\mathrm{Z}|$ holds true. 231

Figure 4.12. Schematic of scratched electrode cell with wiring diagram for data capture devices. A PAR 273A was used with corrware for easy control of range changes. A SCE was used unless otherwise noted. Data acquisition rate of 10,000 to 20,000 points a second was utilized. DAQ was a National Instruments rack mounted PXI Express module. 232

Figure 4.13. Detailed 3-D scratch topography for calculating area of exposed electrode during scratched electrode testing. SEM image of AA2024 scratched in aqueous $0.5 \mathrm{M} \mathrm{CrO}_{4}{ }^{2-}+0.05 \mathrm{M} \mathrm{NaCl}$ with a $\mathrm{pH}$ of 6.5 . Accelerating voltage of $2 \mathrm{kV}$ was used in secondary electron mode with a working distance of $11 \mathrm{~mm}$. 233

Figure 4.14. Schematic of split cell design. Galvanic current is measured with a zero resistance ammeter (ZRA) while the potential of the scratch depassivated surface coupled through the salt bridge to the cathode cell is monitored with a saturated calomel reference electrode. 234

Figure 4.15. The standard UI for the ESA400 software suite from Gamry Instruments, Inc. 235

Figure 4.16. The Debug UI for the modified ESA400 software suite. 236

Figure 4.17. Mathcad Fourier transforms script for analysis of single frequency impedance measurements written by Burak Ulgut and edited by the author..... 239

Figure 4.18. Anodic inhibitors of interest for EFCP inhibition. Potentiodynamic scan of AA2024-T351 in molybdate, permanganate, and chromate solutions containing $0.05 \mathrm{M} \mathrm{NaCl}$ solution. Lines to the left of polarization curves indicate scratch potentials investigated ranging from $-0.8 \mathrm{~V}$ to $-0.3 \mathrm{~V}$ (SCE). Solutions were deaerated. 240

Figure 4.19. Effect of $0.1 \mathrm{M} \mathrm{CrO}_{4}{ }^{2-}$ on the anodic E-logi behavior of AA7075 in aqueous $1 \mathrm{M} \mathrm{Cl}^{-}$with an adjusted $\mathrm{pH}$ of 3 . All runs in duplicate. 241

Figure 4.20. Effect of $0.1 \mathrm{M} \mathrm{CrO}_{4}{ }^{2-}$ on the anodic E-logi behavior of AA7075 in aqueous $1 \mathrm{M} \mathrm{Cl}^{-}$with an adjusted $\mathrm{pH}$ of 6.5. All runs in duplicate. 241

Figure 4.21. Effect of $0.1 \mathrm{M} \mathrm{MoO}_{4}{ }^{2-}$ on the anodic E-logi behavior of AA7075 in aqueous $1 \mathrm{M} \mathrm{Cl}^{-}$with an adjusted $\mathrm{pH}$ of 3 . All runs in duplicate. 242

Figure 4.22. Effect of $0.1 \mathrm{M} \mathrm{MoO}_{4}{ }^{2-}$ on the anodic E-logi behavior of AA7075 in aqueous $1 \mathrm{M} \mathrm{Cl}^{-}$with an adjusted $\mathrm{pH}$ of 6.5. All runs in duplicate. 242 
Figure 4.23. Effect of 0.1 $\mathrm{M} \mathrm{MoO}_{4}{ }^{2-}$ on the anodic E-logi behavior of AA7075 in aqueous $1 \mathrm{M} \mathrm{Cl}^{-}$with an adjusted $\mathrm{pH}$ of 9 , the natural $\mathrm{pH}$ of the molybdate solution as prepared. All runs in duplicate. 243

Figure 4.24. Effect of $0.1 \mathrm{M} \mathrm{MnO}_{4}{ }^{-}$on the anodic E-logi behavior of AA7075 in aqueous $1 \mathrm{M} \mathrm{Cl}^{-}$with an adjusted $\mathrm{pH}$ of 9 . All runs in duplicate. 243

Figure 4.25. Effect of $0.1 \mathrm{M} \mathrm{MnO}_{4}{ }^{-}$on the anodic E-logi behavior of AA7075 in aqueous $1 \mathrm{M} \mathrm{Cl}^{-}$with an adjusted $\mathrm{pH}$ of 6.5. All runs in duplicate. 244

Figure 4.26. Effect of $0.1 \mathrm{M} \mathrm{MnO}_{4}^{-}$on the anodic E-logi behavior of AA7075 in aqueous $1 \mathrm{M} \mathrm{Cl}^{-}$with an adjusted $\mathrm{pH}$ of 9 . 244

Figure 4.27. Molybdate buffer capacity as determined by Al-Ghamdi. Initial $\mathrm{pH}$ was 9.3 for $0.1 \mathrm{M} \mathrm{MoO}_{4}{ }^{2-}$ solutions. "Solution $\mathrm{pH}$ from titration of $(0.01 \mathrm{M}, 0.05 \mathrm{M}$, and $0.1 \mathrm{M}$ ) sodium molybdate $+0.6 \mathrm{M} \mathrm{NaCl}$ with $0.25 \mathrm{~mL}$ of $1 \mathrm{M} \mathrm{HCl}$ reported as $\mathrm{HCl}$ concentration in mM. Molybdate concentration varied from 0.1 to $0.01 \mathrm{M}$ and results are compared against uninhibited $0.6 \mathrm{M} \mathrm{NaCl}$. Molybdate chemical reactions are shown with relevant predominant species in the measured $\mathrm{pH}$ range."

Figure 4.28. Chromate buffer capacity. The capacity of $0.5 \mathrm{M} \mathrm{CrO}_{4}{ }^{2-}+0.05 \mathrm{M} \mathrm{NaCl}$ to buffer $\mathrm{pH}$ with addition of $\mathrm{H}_{2} \mathrm{SO}_{4} .0 .20 \mathrm{~L}$ of $0.5 \mathrm{M} \mathrm{CrO}_{4}{ }^{2-}+0.05 \mathrm{M} \mathrm{NaCl}$ solution were titrated with $1 \mathrm{~N} \mathrm{H}_{2} \mathrm{SO}_{4}$. 246

Figure 4.29. Buffer capacity of $200 \mathrm{~mL}$ of $0.1 \mathrm{M} \mathrm{AlCl}_{3}+0.1 \mathrm{M} \mathrm{Na}_{2} \mathrm{Cr}_{2} \mathrm{O}_{7}$ or $0.1 \mathrm{M}$ $\mathrm{Na}_{2} \mathrm{MoO}_{4}$. Solution was titrated with $0.1 \mathrm{M} \mathrm{NaOH}$. Values of $\mathrm{kPa}$ are taken from the literature. Actual $\mathrm{kPa}$, calculated from inflection of titration curve, was $\mathrm{pKa}_{1}$ of 3.95 and a $\mathrm{pKa}_{2}$ of 6.58 for chromate and $\mathrm{pKa}_{1}$ of 3.9 and a $\mathrm{pKa}_{2}$ of 6.59 for molybdate. Also shown, the titration of $0.1 \mathrm{M} \mathrm{AlCl}_{3}$ into $200 \mathrm{ml}$ of $1.0 \mathrm{M} \mathrm{MoO}_{4}{ }^{2-}$.

Figure 4.30. The effect of $\mathrm{pH}(\mathrm{A})$ and $\mathrm{Cl}^{-}$concentration (B) on the open circuit potential of scratched AA2024 in aqueous $0.05 \mathrm{M} \mathrm{NaCl}$. Chloride concentration had little effect on the transient recovery of the OCP while $\mathrm{pH}$ had a marked effect on $d E / d t$ as well as affecting the steady state OCP at longer times. Both low and high $\mathrm{pH}$ appeared to lower the OCP at longer times below that of the $\mathrm{pH} 6.5$ experiment. However, the $\mathrm{pH} 10$ resting OCP was almost $200 \mathrm{mV}$ below the $\mathrm{pH} 4$. 248

Figure 4.31. The effect of $0.05 \mathrm{M} \mathrm{CrO}_{4}{ }^{2-}, \mathrm{MoO}_{4}{ }^{2-}$, and $\mathrm{MnO}_{4}{ }^{-}$on the open circuit potential of scratched AA2024 in pH 6.5 aqueous $0.05 \mathrm{M} \mathrm{NaCl}$. The inhibitors mediate the potential drop that occurs on the electrode after the surface oxide has been removed. The effect ranks with the strength of the oxidizing nature of each ion with the highest potentials reached by permanganate, followed by chromate and then molybdate. Open circuit potentials of passivated surfaces in the deaerated solutions are indicated by dotted lines. 249

Figure 4.32. The effect of $\mathrm{CrO}_{4}{ }^{2-}, \mathrm{MoO}_{4}{ }^{2-}$ and $\mathrm{MnO}_{4}$ - concentration on the open circuit potential of scratched AA2024 in $\mathrm{pH} 6.5$ aqueous $0.05 \mathrm{M} \mathrm{NaCl}$. The inhibitors mediate the potential drop that occurs on the electrode after the surface oxide has been removed. 250 
Figure 4.33. The effect of $0.1 \mathrm{M} \mathrm{CrO}_{4}{ }^{2-}$ and $\mathrm{MoO}_{4}{ }^{2-}$ on the scratch repassivation behavior of AA7075 when the inhibitors are confined to a much larger cathode in aqueous 1 $\mathrm{M} \mathrm{NaCl}$ with an adjusted $\mathrm{pH}$ 6.5. Also shown, is the OCP of the passivated 7075 electrolyte system moments before the scratch occurs.

Figure 4.34. Effect of $0.1 \mathrm{M} \mathrm{CrO}_{4}{ }^{2-}, \mathrm{MoO}_{4}{ }^{2-}$, and $\mathrm{MnO}_{4}{ }^{2-}$ on the cathodic E-logi behavior of AA7075 in aqueous $1 \mathrm{M} \mathrm{Cl}^{-}$with an adjusted $\mathrm{pH}$ of 3 and 6.5. 252

Figure 4.35 . $i_{\text {net }}$ vs. time behavior of $99.999 \% \mathrm{Al}$ in $1 \mathrm{M} \mathrm{NaCl}$ at $\mathrm{pH} 6.5$ potentiostatically held at $-0.8 \mathrm{~V} \mathrm{SCE}$. The same data is plotted in both formats. 253

Figure 4.36. Scratch of $99.999 \% \mathrm{Al}$ in $1 \mathrm{M} \mathrm{NaCl}$ at $\mathrm{pH} 6.5$ potentiostatically held at -0.8 $\mathrm{V}$ SCE. The maximum ohmic potential drop at the working electrode is $0.3 \mathrm{~V}$ increasing the hydrogen over-potential. 253

Figure 4.37. Current transients from $99.999 \%$ aluminum scratched electrodes. Potentials from -0.5 to $-0.8 \mathrm{~V}$ (SCE) are shown at three chloride concentrations from 0.05 to $1 \mathrm{M}$. The $\mathrm{pH}$ for all tests was 6.5 . 254

Figure 4.38. Calculated IR limit as compared to peak net current density of scratched 99.999\% aluminum electrodes in various chloride concentrations at time zero after scratch. 255

Figure 4.39. Net current transients from AA7075 (a) and AA2024 (b) in aqueous $1 \mathrm{M} \mathrm{NaCl}$ with $\mathrm{pH}$ adjusted to 6.5 with $\mathrm{NaOH}$. Scratch potentials vary from -0.5 to $-0.8 \mathrm{~V}$ (SCE). Current transients from AA7075 (c) and AA2024 (d) in aqueous $1 \mathrm{M} \mathrm{NaCl}$ with $\mathrm{pH}$ adjusted to 6.5 . Scratch potentials vary from -0.5 to $-0.8 \mathrm{~V}$ (SCE) for (c) and -0.4 to $-0.8 \mathrm{~V}$ (SCE) for (d)..... 256

Figure 4.40. (a) Scratched electrode net anodic current densities for AA7075-T6 in 0.05 M $\mathrm{NaCl}$ solution with $\mathrm{pH}$ 6.5. (b) Calculated IR limit as compared to peak current density of scratched AA7075 electrodes at time zero after scratch. IR limit is calculated for an aqueous $0.05 \mathrm{M} \mathrm{NaCl}$ solution, a $0.1 \mathrm{M} \mathrm{CrO}_{4}{ }^{2-}$ and $0.05 \mathrm{M} \mathrm{NaCl}$ solution, and $0.1 \mathrm{M} \mathrm{MoO}_{4}{ }^{2-}$ and $0.05 \mathrm{M} \mathrm{NaCl}$ solution. (c) and (d) show scratched electrode current transients for AA7075-T6 with $0.1 \mathrm{M} \mathrm{MoO}_{4}{ }^{2-}$ and $0.1 \mathrm{M} \mathrm{CrO}_{4}{ }^{2-}$ additions, respectfully. 257

Figure 4.41. (a) Scratched electrode charge passed for AA7075-T6 in 0.05 M NaCl solution with a $\mathrm{pH}$ of 6.5 and $0.1 \mathrm{M} \mathrm{CrO}_{4}{ }^{2-}$ and $0.05 \mathrm{M} \mathrm{NaCl}$ solution. (b) Scratched electrode charge passed for AA2024-T351 in $0.05 \mathrm{M} \mathrm{NaCl}$ solution with a $\mathrm{pH}$ of 6.5 and $0.1 \mathrm{M} \mathrm{Na}_{2} \mathrm{MoO}_{4}$ and $0.05 \mathrm{M} \mathrm{NaCl}$ solution. Integration of current over three periods from 0.05 milliseconds to 0.01 second, 0.01 to 0.1 second, and 0.1 to 1 second. 258

Figure 4.42. (a) Scratched electrode net anodic current densities for AA2024-T6 in 0.05 M $\mathrm{NaCl}$ solution with $\mathrm{pH}$ 6.5. (b) Calculated IR limit as compared to peak current density of scratched AA2024 electrodes at time zero after scratch. IR limit is calculated for an aqueous $0.05 \mathrm{M} \mathrm{NaCl}$ solution, $0.1 \mathrm{M} \mathrm{CrO}_{4}{ }^{2-}$ and $0.05 \mathrm{M} \mathrm{NaCl}$ solution, and $0.1 \mathrm{M} \mathrm{MoO}_{4}{ }^{2-}$ and $0.05 \mathrm{M} \mathrm{NaCl}$ solution. (c) and (d) show scratched electrode current transients for AA2024-T351 with $0.1 \mathrm{M} \mathrm{MoO}_{4}{ }^{2-}$ and $0.1 \mathrm{M} \mathrm{CrO}_{4}{ }^{2-}$ additions, respectfully. 259 
Figure 4.43. (a) Scratched electrode charge passed for AA2024-T351 in $0.05 \mathrm{M} \mathrm{NaCl}$ solution with a pH of 6.5 and $0.1 \mathrm{M} \mathrm{CrO}_{4}{ }^{2-}$ and $0.05 \mathrm{M} \mathrm{NaCl}$ solution. (b) Scratched electrode charge passed for AA2024-T351 in $0.05 \mathrm{M} \mathrm{NaCl}$ solution with a $\mathrm{pH}$ of 6.5 and $0.1 \mathrm{M} \mathrm{MoO}_{4}{ }^{2-}$ and $0.05 \mathrm{M} \mathrm{NaCl}$ solution. Integration of current over three periods from 0.05 milliseconds to 0.01 second, 0.01 to 0.1 second, and 0.1 to 1 second. 260

Figure 4.44. (a) Scratched electrode net anodic current densities for AA7075-T6 in $1 \mathrm{M}$ $\mathrm{NaCl}$ solution with $\mathrm{pH}$ 6.5. Effect of addition of $0.1 \mathrm{M} \mathrm{MoO}_{4}{ }^{2-}, 0.1 \mathrm{M} \mathrm{MnO}_{4}^{-}$, and $0.1 \mathrm{M} \mathrm{CrO}_{4}{ }^{2-}$ additions are also presented. 261

Figure 4.45. (a) Scratched electrode net anodic current densities for AA2024-T351 in $1 \mathrm{M}$ $\mathrm{NaCl}$ solution with $\mathrm{pH}$ 6.5. (b) (c) and (d) show scratched electrode current transients for AA7075-T6 with 0.1 $\mathrm{M} \mathrm{CrO}_{4}{ }^{2-}, 0.1 \mathrm{M} \mathrm{MoO}_{4}{ }^{2-}$, and $0.1 \mathrm{M} \mathrm{MnO}_{4}{ }^{-}$ additions, respectfully. 262

Figure 4.46 Single frequency impedance at $1000 \mathrm{~Hz}$ and phase angle data for scratched AA2024 in $1 \mathrm{M} \mathrm{NaCl}$ with and without the addition of inhibiting $\mathrm{CrO}_{4}{ }^{2-}, \mathrm{MoO}_{4}{ }^{2-}$, and $\mathrm{MnO}_{4}{ }^{-}$anions with the concentration of $0.1 \mathrm{M} . \mathrm{pH}$ was adjusted to 6.5 for all solutions. 263

Figure 4.47 Fast single frequency impedance and phase data for scratched AA2024 in $1 \mathrm{M}$ $\mathrm{NaCl}$ with and without the addition of inhibiting $\mathrm{CrO}_{4}{ }^{2-}, \mathrm{MoO}_{4}{ }^{2-}$, and $\mathrm{MnO}_{4}{ }^{-}$anions with the concentration of $0.1 \mathrm{M}$. $\mathrm{pH}$ was adjusted to 3 for all solutions. 264

Figure 4.48 Fast single frequency impedance and phase data for scratched AA7075 in $1 \mathrm{M}$ $\mathrm{NaCl}$ with and without the addition of inhibiting $\mathrm{CrO}_{4}{ }^{2-}, \mathrm{MoO}_{4}{ }^{2-}$, and $\mathrm{MnO}_{4}{ }^{-}$anion with concentration of $0.1 \mathrm{M}$. pH was adjusted to 6.5 for all solutions. 265

Figure 4.49 Fast single frequency impedance and phase data for scratched AA7075 in $1 \mathrm{M}$ $\mathrm{NaCl}$ with and without the addition of inhibiting $\mathrm{CrO}_{4}{ }^{2-}, \mathrm{MoO}_{4}{ }^{2-}$, and $\mathrm{MnO}_{4}{ }^{-}$anion with concentration of $0.1 \mathrm{M}$. $\mathrm{pH}$ was adjusted to 3 for all solutions. 266

Figure 4.50 Logarithmic constant $m$ plotted vs potential for passivating oxide film growth on AA2024 (A) and AA7075 (B) in $1 \mathrm{M} \mathrm{NaCl}, 0.05 \mathrm{M} \mathrm{NaCl}$, and $0.05 \mathrm{M} \mathrm{NaCl}$ with $0.1 \mathrm{M} \mathrm{MoO}_{4}{ }^{2-}$ and $0.1 \mathrm{M} \mathrm{CrO}_{4}{ }^{2-}$ inhibitor additions. Constant $m$ is obtained by logarithmic modeling of current transients according to Equation 4.11 which was modified from Equation 4.3. 267

Figure 4.51. E- $\log i$ behavior of 99.999 wt \% aluminum as a function of time after a mechanical depassivation event. E-logi behavior in $1 \mathrm{M}, 0.5 \mathrm{M}$, and $0.05 \mathrm{M} \mathrm{NaCl}$ are shown in $\mathrm{A}, \mathrm{B}$, and $\mathrm{C}$ respectively. Data gathered from scratch transients shown in Figure 4.37 a-c. 268

Figure 4.52. E-logi behavior of AA7075 and AA2024 as a function of time after a mechanical depassivation event. AA7075 and A2024 E-logi behavior in $1 \mathrm{M} \mathrm{NaCl}$ is shown in $\mathrm{A}$ and $\mathrm{B}$ respectively and in $0.05 \mathrm{M} \mathrm{NaCl}$ in $\mathrm{C}$ and $\mathrm{D}$ respectively. Data gathered from scratch transients shown in Figure 4.39. 269

Figure 4.53. The effect to $0.1 \mathrm{M} \mathrm{MoO}_{4}{ }^{2-}$ and $\mathrm{CrO}_{4}{ }^{2-}$ on the E-logi behavior of AA7075 as a function of time after a mechanical depassivation event. AA7075 E-logi behavior 
in $0.1 \mathrm{MoO}_{4}{ }^{2-}$ and $0.05 \mathrm{M} \mathrm{NaCl}$ is shown in $\mathrm{B}$ and in $0.1 \mathrm{CrO}_{4}{ }^{2-}$ and $0.05 \mathrm{M} \mathrm{NaCl}$

in C. Data gathered from scratch transients shown in Figure 4.40 ................... 270

Figure 4.54. The effect to $0.1 \mathrm{M} \mathrm{MoO}_{4}{ }^{2-}$ and $\mathrm{CrO}_{4}{ }^{2-}$ on the E-logi behavior of AA2024 as a function of time after a mechanical depassivation event. AA2024 E-logi behavior in $0.1 \mathrm{MoO}_{4}{ }^{2-}$ and $0.05 \mathrm{M} \mathrm{NaCl}$ is shown in $\mathrm{B}$ and in $0.1 \mathrm{CrO}_{4}{ }^{2-}$ and $0.05 \mathrm{M} \mathrm{NaCl}$ in C. Data gathered from scratch transients shown in Figure 4.42. 271

Figure 4.55. The effect to $0.1 \mathrm{M} \mathrm{MoO}_{4}{ }^{2-}$ and $\mathrm{CrO}_{4}{ }^{2-}$ on the E-logi behavior of AA7075 as a function of time after a mechanical depassivation event. AA7075 E-logi behavior in $0.1 \mathrm{MoO}_{4}{ }^{2-}$ and $0.1 \mathrm{M} \mathrm{NaCl}$ is shown in $\mathrm{B}$ and in $0.1 \mathrm{CrO}_{4}{ }^{2-}$ and $0.1 \mathrm{M} \mathrm{NaCl}$ in C. Data gathered from scratch transients shown in Figure 4.42. Anodic potentiodynamic polarization curves show the high current densities that persist after a depassivation event. 272

Figure 4.56. Oxide thickness calculated from impedance measurements shown in Figure 4.48 for AA7075-T6 scratch depassivated in $1.0 \mathrm{M} \mathrm{NaCl}$ and $0.1 \mathrm{M} \mathrm{CrO}_{4}{ }^{2-}$ solution at $-0.8 \mathrm{~V}$ (SCE). 273

Figure 5.1. Theoretical electrochemical impedance spectroscopy of a completely passivated and completely active aluminum surface using well established data from the literature (A). Example of experimental electrochemical impedance spectroscopy conducted 15 minutes after scratch depassivation of an area of $8 \times 10^{-}$ $4 \mathrm{~cm}^{2}$. The red arrow indicated the trend expected of the single frequency impedance data collected during scratch testing. The phase data shows that at $1 \mathrm{kHz}$ after 15 minutes the frequency is well within the capacitive range.................. 293

Figure 5.2. Schematic of Michigan ion beam lab RBS chamber................................... 294

Figure 5.3. Al-Zn-Mg-Cu alloy depassivated in $1 \mathrm{M} \mathrm{NaCl}$ with and without $0.1 \mathrm{M} \mathrm{CrO}_{4}{ }^{2-}$ and $0.1 \mathrm{M} \mathrm{MoO}_{4}{ }^{2-}$ with diamond tip scribe showing lower $i_{\text {net }}$ vs.time and enhanced barrier properties of the oxide film in the presence of chromate and molybdate. 294

Figure 5.4. Backscattered electron image of AA7075 ground to 1200 grit in ethanol. Image taken with an accelerating voltage of $15 \mathrm{kV}$ and a working distance of $12 \mathrm{~mm} .295$

Figure 5.5. EDS map of AA7075 ground to 1200 grit in ethanol. An accelerating voltage of $15 \mathrm{kV}$ a. 296

Figure 5.6. Secondary electron image of AA7075 held potentiostatically at $-0.8 \mathrm{~V}$ (SCE) and abraded with 800 grit $\mathrm{SiC}$ paper in $0.1 \mathrm{M}$ chromate $+1 \mathrm{M}$ sodium chloride. The sample was then held potentiostatically at $-0.8 \mathrm{~V}$ (SCE) for $1 \mathrm{~h}$ after abrasion. Sample imaging occurred several weeks after RBS and ERD analysis upon return of the samples. Image taken with an accelerating voltage of $15 \mathrm{kV}$ and a working distance of $10 \mathrm{~mm}$. 297

Figure 5.7. EDS map of AA7075 abraded in $0.1 \mathrm{M}$ chromate $+1 \mathrm{M}$ sodium chloride and held potentiostatically at $-0.8 \mathrm{~V}$ (SCE) for $1 \mathrm{~h}$. 298

Figure 5.8. Secondary electron image of AA7075 held potentiostatically at $-0.8 \mathrm{~V}$ (SCE) and abraded with 800 grit $\mathrm{SiC}$ paper in $0.1 \mathrm{M}$ molybdate $+1 \mathrm{M}$ sodium chloride. 
Held potentiostatically at $-0.8 \mathrm{~V}$ (SCE) for $1 \mathrm{~h}$ after abrasion. Sample imaging occurred several weeks after RBS and ERD analysis upon return of the samples. Image taken with an accelerating voltage of $15 \mathrm{kV}$ and a working distance of 10 $\mathrm{mm}$. 299

Figure 5.9. EDS map of AA7075 abraded in 0.1 M molybdate + $1 \mathrm{M}$ sodium chloride and held potentiostatically at $-0.8 \mathrm{~V}(\mathrm{SCE})$ for $1 \mathrm{~h}$. 300

Figure 5.10. Secondary electron image of AA7075 held potentiostatically at -0.8 V (SCE) and abraded with 800 grit $\mathrm{SiC}$ paper in $0.1 \mathrm{M}$ permanganate $+1 \mathrm{M}$ sodium chloride. Held potentiostatically at $-0.8 \mathrm{~V}$ (SCE) for $1 \mathrm{~h}$ after abrasion. Sample imaging occurred several weeks after RBS and ERD analysis upon return of the samples. Image taken with an accelerating voltage of $15 \mathrm{kV}$ and a working distance of 10 $\mathrm{mm}$. 301

Figure 5.11. EDS map of AA7075 abraded in 0.1 M permanganate $+1 \mathrm{M}$ sodium chloride and held potentiostatically at $-0.8 \mathrm{~V}$ (SCE) for $1 \mathrm{~h}$. 302

Figure 5.12. RBS spectra and simulation of $\mathrm{Al}-\mathrm{Zn}-\mathrm{Mg}-\mathrm{Cu}$ ground in aqueous $1 \mathrm{M}$ chloride solution with chromate, molybdate, and permanganate. As received and $10 \mathrm{~h} 0.1 \mathrm{M}$ $\mathrm{NaOH}$ samples for reference. 303

Figure 5.13. Detailed view of chromium, molybdenum, and manganese enriched oxides. 304

Figure 5.14. ERD spectra and simulation of $\mathrm{Al}-\mathrm{Zn}-\mathrm{Mg}-\mathrm{Cu}$ ground in aqueous $1 \mathrm{M}$ chloride solution with chromate and molybdate additions. As received and $10 \mathrm{~h} 0.1 \mathrm{M} \mathrm{NaOH}$ samples for reference. 305

Figure 5.15. Model systems showing atomic concentration depth profiles for AA075 as received exposed to ethanol and humid air (a), exposed for $10 \mathrm{~h}$ to $0.1 \mathrm{M} \mathrm{NaOH}$ (b), and ground in $1 \mathrm{M}$ chloride (c). 306

Figure 5.16. Model systems showing atomic concentration depth profiles for AA075 ground in $1 \mathrm{M}$ chloride with $0.05 \mathrm{M} \mathrm{Cr}_{2} \mathrm{O}_{7}{ }^{2-}, 0.1 \mathrm{M} \mathrm{MoO}_{4}{ }^{2-}$ and $0.1 \mathrm{M} \mathrm{MnO}_{4}^{-}$. 307

Figure 5.17. Hydrogen depth profile of all samples showing protective nature of chromate. Hydrogen concentration depth profiles for AA7075-T6 abraded in $1.0 \mathrm{M} \mathrm{NaCl}$ with $0.1 \mathrm{M} \mathrm{CrO}_{4}{ }^{2-}$ are reduced to level similar to those seen in a similar sample ground in ethanol and exposed to humid lab air. 308

Figure 5.18. RBS spectra and simulation of $\mathrm{Al}-\mathrm{Zn}-\mathrm{Mg}-\mathrm{Cu}$ ground in aqueous $1 \mathrm{M}$ chloride solution with chromate, molybdate, and permanganate additions. As-received (ground in ethanol and exposed to humid lab air) and $10 \mathrm{~h} 0.1 \mathrm{M} \mathrm{NaOH}$ samples for reference. RBS results from UWO. Detailed view of chromium, molybdenum, and manganese enriched oxides. 310

Figure 5.19. ERD spectra and simulation of $\mathrm{Al}-\mathrm{Zn}-\mathrm{Mg}-\mathrm{Cu}$ ground in aqueous $1 \mathrm{M}$ chloride solution with chromate, molybdate, and permanganate additions. As-received (ground in ethanoland exposed to humid lab air) and $10 \mathrm{~h} 0.1 \mathrm{M} \mathrm{NaOH}$ samples for reference. 
Figure 5.20. Model systems showing atomic concentration depth profiles for AA075 as received exposed to ethanol and humid air, exposed for $10 \mathrm{~h}$ to $0.1 \mathrm{M} \mathrm{NaOH}$, and ground in $1 \mathrm{M}$ chloride with $0.05 \mathrm{M} \mathrm{Cr}_{2} \mathrm{O}_{7}{ }^{2-}, 0.1 \mathrm{M} \mathrm{MoO}_{4}{ }^{2-}$ and $0.1 \mathrm{M} \mathrm{MnO}_{4}^{-}$.

Figure 5.21. Hydrogen depth profile of all samples showing protective nature of chromate. Hydrogen concentration depth profiles for AA7075-T6 abraded in $1.0 \mathrm{M} \mathrm{NaCl}$ with $0.1 \mathrm{M} \mathrm{CrO}_{4}{ }^{2-}$ are reduced to level similar to those seen in a similar sample ground in ethanol and exposed to humid lab air. 315

Figure 5.22. Projected diffusion concentration profiles for hydrogen diffusion through bulk AA7075 316

Figure 5.23. Effect of injected vacancy concentration on $\mathrm{D}_{\text {eff }}$ in the vacancy effected zone. 316

Figure 5.24. Projected diffusion concentration profiles for hydrogen-vacancy complexes through AA7075 over 3600 seconds. 


\section{List of Symbols}

$\beta$

$\beta_{\mathrm{a}}$

C

$C_{H}$

$\mathrm{C}_{\mathrm{O}}$

CV

D

$d a / d N$

$\Delta K$

$\mathrm{E}_{\mathrm{app}}$

$\mathrm{E}_{\text {couple }}$

ESCE

$\mathrm{E}^{0}$

EAC

EDS

EFCP

EIS

ERD

$E_{P Z C}$

$f$

$f$

F anodic Tafel slope

cathodic Tafel slope

capacitance

Hydrogen concentration

Concentration of $\mathrm{O}_{2}$ in water

cyclic voltammetry

Diffusion coefficient

crack growth rate

stress intensity range

applied potential

galvanic couple potential

potential measured vs. SCE

equilibrium half-cell potential

environmentally assisted cracking

energy-dispersive $x$-ray spectroscopy

environmental fatigue crack propagation

electrochemical impedance spectroscopy

elastic recoil detection

potential of zero charge

fatigue loading frequency

electrochemical impedance frequency

Faraday's constant 


\begin{tabular}{|c|c|}
\hline$F P Z$ & fracture process zone \\
\hline$H$ & hydrogen \\
\hline$H E E$ & hydrogen environmental embrittlement \\
\hline$i$ & current density $\left(\mathrm{A} / \mathrm{cm}^{2}\right)$ \\
\hline$I G C$ & intergranular corrosion \\
\hline$i_{0}$ & exchange current density \\
\hline I & current (Amps) \\
\hline$i_{\mathrm{lim}}$ & limited current density \\
\hline$i_{\text {tot }}$ & total current density \\
\hline$i_{c t}$ & charge transfer current density \\
\hline$i_{D}$ & diffusion limited current density \\
\hline$M$ & Molar \\
\hline$n$ & number of electrons transferred in a reaction \\
\hline$N R A$ & nuclear reaction analysis \\
\hline$O C P$ & open circuit potential \\
\hline ORR & oxygen reduction reaction \\
\hline$R$ & universal gas constant \\
\hline$R$ & resistance $(\Omega)$ \\
\hline$R D E$ & rotating disk electrode \\
\hline SIMS & secondary ion mass spectrometry \\
\hline SIRMS & synchrotron infrared spectroscopy \\
\hline$U I$ & user interface \\
\hline XANES & X-ray absorption near edge structure \\
\hline$X P S$ & X-ray photoelectron spectroscopy \\
\hline
\end{tabular}




$\begin{array}{ll}Z R A & \text { zero resistance ammeter } \\ \sigma & \text { conductivity }(\mathrm{S} / \mathrm{m}) \\ \rho & \text { resistivity }(\Omega \cdot \mathrm{m}) \\ \mathrm{Q} & \text { charge } \\ t & \text { time } \\ t_{\text {repass }} & \text { repassivation time } \\ \mathrm{T} & \text { temperature } \\ v & \text { viscosity } \\ \eta & \text { over-potential } \\ |\mathrm{Z}| & \text { impedance magnitude }\end{array}$




\section{Introduction: Background, Significance and Objectives}

\subsection{AA2024 and AA7075 Metallurgy}

Aluminum alloys are used widely in the aerospace industry because of their advantageous strength/weight ratios ${ }^{[1,2]}$. Precipitation hardened aluminum alloys in particular are widely used in aerospace load bearing structures such as fuselage and loaded wing structures ${ }^{[1,3]}$. The elements that are most commonly used as alloying elements for increasing the strength of aluminum are copper, magnesium, silicon, and zinc ${ }^{[1]}$. Their equilibrium solid solubility as a function of temperature can be found in Figure $1.1^{[1]}$. The decrease in solubility from higher temperatures to lower temperatures provides one mechanism for the significant increase in hardness and strength achieved after solution heat treatment and subsequent precipitation age hardening $^{[1]}$. The precipitation hardening can be seen in Figure 1.2 and results from the formation of coherent GP zones in the natural aging case (A) and transitional precipitates in the artificial ageing case (B); both of which resist the movement of dislocations through the lattice. These strengthening particles are submicrometer in diameter $^{[4]}$. At long times in Figure $1.2(\mathrm{~B})$, it can be seen that the artificial aging process results in the conversion of transitional precipitates to equilibrium phase precipitates which tend to lose coherence with the lattice resulting in a softening of the material. The materials used in this work will either be naturally aged in the T351 and T4 tempers or artificially aged in the T6 temper, both of which can be considered peak aged ${ }^{[1]}$. the An example of a TTT curve for AA2024 is shown in Figure $1.3(\mathrm{~A})^{[5]}$. 
The precipitation age hardened alloys examined in this work, AA2024 and AA7075, also suffer from the formation of unwanted coarse grain boundary precipitates during the aging process. Due to heterogeneous nucleation, many of these particles nucleate along grain boundaries which results in the local depletion of solute along these boundaries. Among these particles in no particular order are the S-phase $\left(\mathrm{Al}_{2} \mathrm{CuMg}\right) \theta$ - phase $\left(\mathrm{Al}_{2} \mathrm{Cu}\right)$ in AA2024 ${ }^{[6]}$ and , $\eta$-phase $\left(\mathrm{MgZn}_{2}\right)$ and $\mathrm{Al}_{7} \mathrm{Cu}_{2} \mathrm{Fe}$ in $\mathrm{AA} 7075^{[4,7,8]}$.

As coarse constituent particles S-phase in AA2024 is usually spherical with AlCu and Al$\mathrm{Cu}-\mathrm{Mn}-\mathrm{Fe}$ type particles tending to be larger and blocky ${ }^{[9]}$. In $\mathrm{AA} 7075$ the $\mathrm{Al}_{7} \mathrm{Cu}_{2} \mathrm{Fe}$ phase tends to be comparatively large and irregularly shaped ${ }^{[4]}$. These coarse constituents can be up to around $10 \mu \mathrm{m}$ in radius. An example of the coarse constituent particles found in AA2024-T4 temper can be seen in Figure 1.3 (B) where the lighter regions of the micrograph correspond to coarse constituent particles rich in copper in this case. These coarse constituent particles are generally recognized as fracture nucleation sites due to their brittle nature. Cracks can develop within the brittle particles which can lead to equivalent initial flaw sizes (EIFS) large enough to significantly affect the useful lifetime of the structure. The course constituents also play a major role in the poor corrosion resistance of these alloys through galvanic coupling with the solute lean matrix ${ }^{[6]}$. Scanning electron microscopy (SEM) of AA2024 sheet, rod, and wire stocks used for this work are provided in Figure 1.4, while SEM and EDS mapping of AA7075 sheet can be found in Figure 1.5. Constituent particle size distribution analysis for AA2024 can be found in Figure 1.6 and can be found for AA7075 in Figure 1.7. Average particle size and nearest neighbor distance can be found in Table 1.2. This data is presented to help provide some understanding of the nature of the alloys of interest in this work. This distribution does not 
include the nanometer scale precipitates that are responsible for precipitation age hardening effects in the alloys which would require TEM level investigation.

\subsection{AA2024 and AA7075 Corrosion Susceptibility}

Commercially pure aluminum exhibits excellent corrosion resistance due to the effective barrier properties of the aluminum oxide/hydroxide surface films that are relatively stable over a wide range of $\mathrm{pH}$ and potential (Figure 1.8) ${ }^{[1,10]}$. However, in the presence of aggressive halide species such as chloride the protective barrier oxide can break down locally resulting in aluminum oxidation to $\mathrm{Al}^{3+}$, with a large thermodynamic driving force due to the reactions low Nernst potential ${ }^{[11]}$. Some aluminum alloys also exhibit excellent corrosion resistance. The $5 \mathrm{xxx}$ series $\mathrm{Al}-\mathrm{Mg}$ alloys are a good example of a corrosion resistant aluminum alloy, so long as they are unsensitized, being used widely in marine environments $^{[1]}$. However, precipitation hardened aluminum alloys containing copper, magnesium, iron, and/or zinc such as AA2024 and AA7075, those most commonly used in the aerospace industry due to their strength, are highly susceptible to corrosion ${ }^{[1,12]}$. Alloys containing copper experience the worst corrosion resistance among aluminum alloys ${ }^{[10]}$. Several microstructural and metallurgical factors are at fault for this susceptibility. Local galvanic coupling between the intermetallic phases in the form of micrometer scale constituent particles and the surrounding aluminum matrix provides one driving force for the poor corrosion resistance of these alloys $[6,9,13-18]$. Another is the presence of a precipitate free zone (PFZ) that can arise from the diffusion of solute to the grain boundary and the depletion of precipitate nucleation sites in proximity to the grain 
boundary ${ }^{[10,18]}$. This is known to lead to large precipitated intermetallic phases located primarily on grain boundaries. A schematic of the local galvanic cells present in these alloys is shown in Figure 1.9 (A) while the PFZ can be seen in Figure $1.9(\mathrm{~B})^{[10]}$.

Localized corrosion of alloys such as AA2024 and 7075 results from the presence of intermetallic compounds in a solute lean $\alpha$-Al matrix that are either noble with respect to their surrounding matrix or that tend to be more corrosion susceptible and therefore initiation sites for pitting ${ }^{[6,9]}$. Known chemistries and corrosion potentials of several relevant intermetallic phases that are commonly found in these aluminum alloys can be found in Table $1.1^{[8]}$ while selected E- $\log (\mathrm{i})$ behavior can be found in Figure $1.10^{[9]}$ These intermetallic particles can cause two types of corrosion issues. The first issue arises from the combination of cathodically active particles constituent particles adjacent to solute depleted areas surrounding precipitate particles. This situation results in a galvanic couple developing between a high-potential cathodic particle, typically rich in copper in these alloys such as $\mathrm{Al}_{2} \mathrm{Cu}$ whose E- $\log (\mathrm{i})$ behavior is seen in Figure 1.10, and a low-potential area of solute depletion represented in Figure 1.10 by the aluminum anodic E-log(i) behavior. This results in a strong local galvanic couple that can drive corrosion of the solute depleted zone. The solute depleted zone is prone to breakdown due to the effect of the decrease solid solution copper content as seen in Figure 1.12. The second issue arises from the presence of more active phases such as $\mathrm{S}$-phase $\left(\mathrm{Al}_{2} \mathrm{MgCu}\right)$ in AA2024, $\eta$-phase $\left(\mathrm{MgZn}_{2}\right)$ in $\mathrm{AA} 7075$, and $\beta$-phase $\left(\mathrm{Al}_{2} \mathrm{Mg}_{3}\right)$ in $5 \mathrm{xxx}$ series alloys ${ }^{[1]}$. For example, the most detrimental intermetallic in AA2024 is the S-phase particle. S-phase has a breakdown potential well below the open circuit potential (OCP) of AA2024 as seen in Figure $1.10^{[9]}$. Particles such as $\mathrm{Al}_{2} \mathrm{CuMg}$ can be detrimental to the corrosion resistance of the alloy 
because of their tendency to pit and dealloy at the open circuit potential ${ }^{[17]}$. The breakdown potential seen in Figure $1.10^{[9]}$ may actually be in a critical dealloying potential for the alloy. This process has many damaging consequences including the creation of catalytic high surface area copper-enriched intermetallic remnants and the associated deposition of copper on the alloy surface ${ }^{[19]}$, both of which result in increased oxygen reduction reaction rates. This increase in ORR supports increased aluminum matrix dissolution in the form of localized trenching around the intermetallics and also larger pit growth that can produce nucleation sites for subsequent crack growth ${ }^{[20-22]}$. The nucleation of inter granular corrosion (IGC) sites typically arises from solute depletion, deaeration of boundaries with active phases, as well as a specific local chemistry often characterized by a low $\mathrm{pH}$. However, most of this work only identifies metallurgical culprits and establishes framework that explain how pitting and IGC occur.

Subcritical crack growth including stress corrosion cracking (SCC), intergranular stress corrosion cracking (IGSCC), and corrosion fatigue are also important modes of degradation in these alloys all of which do not occur in dry gas environments ${ }^{[1,23]}$. With the introduction of water vapor into a variety of gases, including $\mathrm{H}_{2}, \mathrm{~N}_{2}$, and argon, subcrititcal crack growth is observed to increase the crack growth rate indicting that an aqueous environment encourages these modes of degradation ${ }^{[1]}$. Chloride content in aqueous solutions increases the rate of subcritical crack growth in $\mathrm{Al}-\mathrm{Cu}-\mathrm{Mg}$ and $\mathrm{Al}-\mathrm{Zn}-$ $\mathrm{Mg}-\mathrm{Cu}$ alloys as seen in Figure $1.11(\mathrm{~B})^{[23]}$. These facts strongly suggest that the electrochemical corrosion behavior of these alloys in the relevant environments is critical to understanding subcritical crack growth. This will likely remain the case for hydrogen induced cracking since hydrogen production is coupled with anodic dissolution. 
Intergranular corrosion is also an important mode of degradation in the $\mathrm{Al}-\mathrm{Cu}-\mathrm{Mg}$ and $\mathrm{Al}-$ $\mathrm{Zn}-\mathrm{Mg}-\mathrm{Cu}$ alloy systems. Stress corrosion cracking (SCC) in these alloy systems is chiefly along these intergranular paths with intergranular corrosion often a key indicator of susceptibility to intergranular stress corrosion cracking (IGSCC). In IGSCC both mechanical and electrochemical driving forces are present and this underscores the importance of electrochemical factors in even hydrogen controlled processes. In these copper containing alloys it is thought that both of the corrosion mechanisms described previously contribute to environmentally assisted cracking (EAC) ${ }^{[1]}$. Both AA2024 and, more controversially, AA7075 are thought to suffer EAC growth due in some part to the effects of copper on local electrochemical cells and local breakdown along grain boundaries ${ }^{[24]}$. In AA2024, copper depletion in the PFZ results in the first type of corrosion susceptibility described in the previous paragraph; namely cathodic copper-rich particles coupled to copper-lean Al-matrix in the PFZ. This results in intergranular attack and/or EAC. In the case of AA7075 a different mechanism is at play, more akin to the second issue described above; the presence of active intermetallic phase particles. Figure 1.13 demonstrates the effect of aging time on the SCC resistance of several alloys including AA7075 with varying copper contents. As the aging time of the copper-rich alloys increases the susceptibility to SCC is drastically decreased. One explanation for this behavior involves an increase in the copper content of the intragranular $\eta$ and $\eta^{\prime}$ $\left(\mathrm{Mg}\left[\mathrm{Zn}_{\mathrm{x}} \mathrm{Cu}_{\mathrm{y}} \mathrm{Al}_{\mathrm{z}}\right]_{2}\right)$ phases with aging time. This increase in copper content ennobles the particles and decreases the potential driving force associated with this electrochemical couple with the surrounding solute lean matrix ${ }^{[24,25]}$. This acts to decrease the susceptibility of the overaged alloy to intergranular corrosion and EAC. However, this is a controversial 
topic as there are other proposed mechanisms including a change in deformation mode (shearing to looping) resulting from accelerated particle growth related to increased copper content and grain boundaries acting as active corrosion pathways through the dissolution of $\mathrm{MgZn}_{2}$ precipitates $^{[24,26]}$.

Environmental fatigue crack propagation $(\mathrm{EFCP})$ or corrosion fatigue is another important mode of degradation in these alloy systems. However, unlike SCC, this mode of cracking tends to occur with no relationship to high angle grain boundary direction and actually tends to follow a transgranular path ${ }^{[1,12]}$. The fatigue crack growth behavior of AA2024 and AA7075 in similar tempers to those used in this work is shown in Figure $1.14^{[1]}$. AA2024 shows better fatigue resistance over the whole $d a / d N-\Delta K$ range than AA7075. However, an increase in the copper content of AA7075 can increase the alloys resistance ${ }^{[1]}$.

The driving force for EFCP and IGSCC in these aluminum alloys is a controversial topic ${ }^{[26,}$ 27]. While some maintain that EFCP and IGSCC are driven by the intense local dissolution of aluminum at the crack tip, it is more probable that they results from hydrogen environmental embrittlement $(\mathrm{HEE})^{[27,28]}$. The production and delivery of hydrogen between the environment local to the crack tip and the fracture process zone (FPZ) is a complex multi-step, multi-phenomenon process. There are several potential limiting factors that could produce a rate determining step that would control the embrittlement of the FPZ and the rate at which cracking could occur per cycle. The source of hydrogen in the local crack tip environment is undoubtedly related to the reduction of water in the aqueous solution at the crack tip and thus, the rate of crack growth in relation to the production and adsorption of the embrittling environment is an important factor. Another important factor is the rate of both chemical and electrochemical reactions that can produce 
atomic hydrogen in the local crack tip environment, namely the local anodic dissolution of aluminum coupled to the hydrogen evolution reaction (HER) and also the hydrolysis of water by $\mathrm{Al}^{3+}$ ions. The hydrolysis of cations leads to acidification. Acidification raises the hydrogen overpotential and leads to proton reduction. The local crack tip chemistry is thus a very important factor. Figure $1.15^{[24]}$ shows the local $\mathrm{pH}$ of the crack tip at specific crack growth rates in 7xxxx series alloys in chromate and chloride containing electrolytes. As the crack tip growth rate increases the $\mathrm{pH}$ of the crack tip decreases. The $\mathrm{pH}$ is proportional to the concentration of $\mathrm{Al}^{3+}$ ions resulting from the hydrolysis of the $\mathrm{Al}^{3+}$ ions as the crack progresses. Figure $1.16^{[24]}$ shows the $\mathrm{pH}$ of the crack tip as a function of applied potential. Again, the crack tip $\mathrm{pH}$ is proportional to the concentration of $\mathrm{Al}^{3+}$. As the applied potential is increased the $\mathrm{pH}$ of the crack tip electrolyte decreased ${ }^{[24]}$. This effect is due to an increase in local anodic reaction rate and an increase in the ratio of anodic to local cathodic reaction. The cathodic reaction occurs remotely. The production and adsorption rates of atomic hydrogen and the subsequent diffusion of atomic hydrogen from the crack tip to the FPZ ${ }^{[28]}$ are both potential limiting factors in the embrittlement of the FPZ. The schematic show in Figure 1.17 illustrates these important processes. The concentration of hydrogen in the fracture process zone must reach a critical concentration $\left(\mathrm{C}_{\mathrm{H}, \mathrm{Crit}}\right)$ at which the cohesive strength in the region of high stress is decreased sufficiently to result in fracture ${ }^{[29]}$. A concentration depth profile of hydrogen is shown in Figure 1.18 for several effective surface hydrogen concentrations. A simple one dimensional semiinfinite diffusion expression was used to predict hydrogen concentration as a function of depth. Understanding how inhibitors can affect hydrogen concentrations in the fracture process zone is critical to understanding EFCP mechanisms. 
The AA2024 and AA7075 alloy systems are susceptible to corrosion and environmentally influenced crack growth. They remain widely used in the aerospace industry despite corrosion concerns. Huge efforts have been undertaken to help reduce the effect of the environment on these alloys. This dissertation will focus on one of the protection strategies possible in this class of alloys.

\subsection{Corrosion and Corrosion Protection of Precipitation Age Hardened Aluminum Alloys}

Many forms of corrosion protection have been implemented to address the corrosion

susceptibility issues in precipitation age hardened aluminum alloys ${ }^{[30-33]}$. Ionic inhibitors packaged and released from coatings and pretreatments represent one key approach. Hexavalent chromates are currently recognized as one of if not the most effective chemical species used to mitigate corrosion on alloys such as AA2024 and AA7075 [34-39]. Unfortunately, due to their being a known human carcinogen, OSHA has imposed strict new $\mathrm{Cr}(\mathrm{VI})$ exposure limits ${ }^{[40]}$. The additional costs incurred providing an adequate working environment and allowing for the proper disposal of $\mathrm{CrO}_{4}{ }^{2-}$ have led to efforts to find new non-toxic replacements. One aspect of current research in the field of corrosion inhibition has focused on cationic and anionic inhibitors. One specific category is oxoanions of the form $\mathrm{MeO}_{4}{ }^{(\mathrm{n}-8)}$ (such as molybdates, tungstates, and vanadates) ${ }^{[33,41-50]}$, structural analogues of hexavalent chromium. In this work, several potential chromate replacement inhibitors were evaluated for their effectiveness in limiting corrosion susceptibility. Many of these inhibitors have attributes of chromate. For instance chromate 
is a soluble high valence state oxoanion $\left(\mathrm{CrO}_{4}{ }^{2-}\right)$ which is capable of being reduced to a sparingly soluble lower valence state oxide or hydroxide $\left(\mathrm{Cr}_{2} \mathrm{O}_{3}\right)$. Molybdate $\left(\mathrm{MoO}_{4}{ }^{2-}\right)$ and permanganate $\left(\mathrm{MnO}_{4}{ }^{-}\right)$are both reducible to sparingly soluble oxides and/or hydroxides $\left(\mathrm{MoO}_{2}\right.$ and $\left.\mathrm{MnO}_{2}\right)$

The inhibitors have been evaluated based on traditional corrosion phenomena such as anodic inhibition of pitting potential $\left(\mathrm{E}_{\mathrm{pit}}\right)^{[33]}$ and dealloying of active phases ${ }^{[51]}$, as well as inhibition of the oxygen reduction reaction (ORR) on the matrix and on IMC particles ${ }^{[51,}$ ${ }^{52]}$. Dissolution and trenching around particles has also been examined.

Inhibitors have been assessed mechanistically and examined for their ability to prevent constituent particle attack. In addition to these methods we have examined the potential to augment inhibitor function through the use of multiple inhibitors concurrently.

Examination of the interactions that can occur in multi-inhibitor systems will lead to new inhibitor combinations that are intelligently engineered to maximize synergistic effects, or additive effects, and to eliminate antagonistic effects. There are several types of synergistic effects that are possible including forming a complex ion species as a result of the presence of both inhibitors or oxidizing a lower oxidation state species to a more inhibitive form (helper chemistry). An additive effect such as filling in deposited oxide gaps over cathodic sites would also lead to improvement in inhibitor functionality.

Chambers performed a high throughput analysis of 28 single and binary inhibitor mixtures ${ }^{[53]}$. He used a flourometric assay to monitor the concentration of $\mathrm{Al}^{3+}$ in solution to gauge corrosion inhibition. His method worked well as a brute force analysis of synergistic vs. antagonistic combinatorial inhibitor behavior using a few screening metrics. 
Chambers found synergistic relationships between $\mathrm{CeCl}_{3} / \mathrm{Na}_{2} \mathrm{MoO}_{4}, \mathrm{LaCl}_{3} / \mathrm{Na}_{2} \mathrm{MoO}_{4}$, and $\mathrm{NaVO}_{3} / \mathrm{Na}_{3} \mathrm{PO}_{4}$ and he found an antagonistic relationship between $\mathrm{CeCl}_{3} / \mathrm{NaVO}_{3}$. Cook used electrochemical impedance spectroscopy (EIS) to examine the benefit of combinations on the electrochemical impedance magnitude of AA2024 and included some analysis of pit depths ${ }^{[54]}$. Neither Cook nor Chambers examined the function of the systems at a submicrometer or molecular level that would provide insight into why some worked well together and some did not. By examining the electrochemical behavior and observing the interaction at a microscopic level, it is possible to describe obstacles to designing functional inhibitor combinations and provide insight into why and how inhibitors work together in hopes of directing future research on combinatorial inhibitor selection.

In addition to the traditional inhibitor metrics and combined inhibitor properties new test methods are in need of development to examine inhibitor metrics thought to be important to the inhibition of SCC and EFCP. There are several ways through which inhibitors may affect the concentration of hydrogen $\left(\mathrm{C}_{\mathrm{H}}\right)$ in the fracture process zone. There are a number of stages in the hydrogen controlled IGSCC and/or EFCP process that inhibitors might interfere with. One would be the creation of a barrier oxide at the crack tip that was a permeation barrier to hydrogen adsorption or absorption. This mechanism has a direct effect on the delivery of hydrogen to the FPZ through the creation of a hydrogen barrier. One indirect measure of an inhibitors ability to enhance barrier oxide properties is the inhibitors ability to prevent pitting by raising the pitting potential ( $\left.\mathrm{E}_{\mathrm{pit}}\right)$. Pitting occurs when the barrier oxide breaks down locally causing the anodic dissolution of the underlying substrate ${ }^{[55]}$. An increase in the potential at which this process occurs indicates a stabilizing effect on barrier oxide protection which, in the case of aluminum oxide, would 
also provide a hydrogen diffusion barrier. Other mechanisms of inhibition can affect the production of hydrogen in the local crack tip electrolyte. The first of these would be the chemical buffering capability of an inhibitor. If an inhibitor can buffer the $\mathrm{pH}$ of the crack tip the natural aluminum oxide may become more thermodynamically stable enabling it to act as a hydrogen permeation barrier. Another mechanism would be to limit the cathodic reaction kinetics on the local cathode sites galvanically coupled to the anodic reactions occurring in the anoxic crack tip decreasing the rate of the anodic dissolution and thus the concentration of $\mathrm{Al}^{3+}$ ions available for hydrolysis.

\subsubsection{Inhibition of Crack Tip Processes that lead to Hydrogen Embrittlement}

Most inhibitor studies have been limited to investigation of their effects on surface corrosion $^{[33-36,44,46,51,52,56-59]}$. Inhibitors interactions with EAC mechanisms have only been studied in a small number of publications in the last 25 years ${ }^{[27,29,60,61]}$. The ability of corrosion inhibitors to effectively reduce the embrittlement of precipitation age hardened aluminum alloys in crack-tip environments requires more background information as it is not a topic that has been fully investigated before in the literature. To begin a review of oxide film rupture literature is required. There are many methods that have been used to examine repassivaiton behavior including fractured thin film ${ }^{[62-64]}$, fractured tensile bar ${ }^{[65]}$, laser depassivation ${ }^{[66]}$, guillotined wire $^{[67]}$, continuous abrasion ${ }^{[68]}$, and scratch depassivation ${ }^{[69-71]}$. This type of testing has been performed on stainless steel 304L ${ }^{[71,72]}$, titanium alloys ${ }^{[62,73-75]}$, and aluminum alloys ${ }^{[76-78]}$. 
Generally these studies have looked to explore crack growth through dissolution rates at the crack $\operatorname{tip}^{[74]}$ by quantifying potential and current values as depassivated electrode surfaces repassivate. Modeling of scratch current transients as a function of time in these studies are typically of the form $i=i_{o}(t / \tau)^{-m}$ where $i$ is the current density after some peak current density, $i_{o}, t$ is time since $i_{o}, \tau$ is a characteristic time constant, and $m$ is a logarithmic constant. This equation describes the logarithmic nature of the current decay. It is thought that as dislocations pile up they will eventually rupture the surface oxide leading to a current peak and a repassivation event ${ }^{[74]}$. The assumption here is that the metallic phase is ductile while the oxide is a brittle ionic bound crystalline phase. The slip offset in the underlying metal produces displacement not accommodated by the oxide. The oxide is abruptly ruptured by the emerging slip step. Figure 1.19 illustrates the hypothesized relationship between a scratch repassivation event and a repassivation event on a dynamically strained electrode. The HER occurs at a higher rate on bare aluminum and the overpotential increases both increasing the concentration of hydrogen available for absorption into the fracture process zone.

It is suggested in the literature that film rupture events lead to increased rates of hydrogen production, uptake and hydrogen embrittlement as well as enhanced anodic dissolution in the crack tip ${ }^{[74,79,80]}$. The ability of a stable film to regrow after film rupture is critical because it reduces the production of atomic hydrogen by limiting dissolution and hydrolysis of metal ions. The $\mathrm{Al}_{2} \mathrm{O}_{3}$ oxide is a hydrogen permeation barrier which limits hydrogen ingress ${ }^{[81]}$. The reduction in atomic hydrogen penetrating the surface of the metal decreases the amount of embrittling absorbed atomic hydrogen in the fracture process zone and the improved repassivated surface also slows anodic dissolution of $\mathrm{Al}$ 
(schematic in Figure 1.17). The crack flank plays an important role as it is an important site for coupled cathodic reactions. Thus, understanding effects of inhibitors on repassivation, buffering, oxide formation, and crack flank behavior are crucial to understanding the effects of inhibitors on cyclic or static load environmental crack growth.

Figure 1.20 and 1.21 and show the dependence of fatigue crack growth rate in AA7075 on loading frequency at a constant $\Delta \mathrm{K}$ with and without two inhibitors, chromate and molybdate. The inhibitors affect the $d a / \mathrm{dN}$ values suppressing them at low loading frequencies. The effect dissipates as the loading frequency is increased. The growth of inhibitor assisted oxides at the crack tip has been rationalized to be responsible for the frequency dependence of $\mathrm{da} / \mathrm{dN}$ in the low frequency regime of fatigue crack growth. $f_{\text {CritUB }}$ (as seen in Figure 1.20 and 1.21) has been rationalized as the frequency at which an oxide barrier is disrupted faster than it can repassivate or regrow to prevent $\mathrm{Al}^{3+}$ dissolution from bare aluminum and the production, adsorption and absorption of $\mathrm{H}_{\mathrm{ads}}$ into the lattice. The critical frequency will be different for different $\Delta \mathrm{K}$ and $\mathrm{R}$ which alter the crack tip strain rate. At low to moderate frequencies up to the critical frequency upper bound $f_{\text {CritUB }}$ electrochemical corrosion inhibitors such as chromate and molybdate have been shown to reduce $\mathrm{da} / \mathrm{dN}$ to levels below that seen in moist air and/or full immersion chloride environments ${ }^{[79,80]}$. This indicates that the inhibitors are able to negate the effects of the environment on fatigue crack growth rate. The role of inhibitors is speculated by Warner and Gasem with no direct evidence of enhanced repassivation, incorporation of species at the tip in oxide, or evidence of reduction in hydrogen uptake.

Several inhibitors will be evaluated for their ability to effect repassivating oxide film characteristics as well as any other function they might have with respect to local 
environmental conditions such as crack tip $\mathrm{pH}$ buffering. Ultimately, direct evidence of reduced hydrogen uptake in the presence of inhibitors is investigated. The following sections provide background information on each inhibitor.

\subsubsection{Permanganate}

Permanganate based coating products have been in commercial production for many years and several patents exist ${ }^{[82,83]}$. Previous scientific studies of $\mathrm{MnO}_{4}^{-}$on AA2024 have focused on open circuit electrochemical properties, barrier layer properties, exposure tests such as ASTM B-117 ${ }^{[84]}$ neutral salt spray testing, as well as on the oxidation states of protective manganese oxides ${ }^{[85-87]}$. The mechanism by which $\mathrm{MnO}_{4}^{-}$inhibits corrosion on AA2024 has been shown to be the growth of manganese oxides over copper-rich intermetallic particles ${ }^{[87]}$. However, the detail of what precisely the inhibitor does is unclear from the electrochemical perspective.

It is useful at this point to review the electrochemical and chemical dynamics of the aqueous-manganese system. Permanagate $\left(\mathrm{Mn}^{+7}\right)$ is a very strong oxidizer due to its high potential as seen in Figure 1.22. When permanganate is added to solution in contact with AA2024-T351 at a lower potential, it will be reduced and undergo several possible electrochemical reactions. Over all ranges of $\mathrm{pH}, \mathrm{MnO}_{4}^{-}$will likely be reduced to manganese dioxide by the following electrochemical $1 \frac{1}{2}$ cell reaction. 


$$
\begin{gathered}
\mathbf{M n O}_{4}{ }^{-}+\mathbf{4} \mathbf{H}^{+}+\mathbf{3} \boldsymbol{e}^{-} \leftrightarrow \mathbf{M n O}_{2}+\mathbf{2} \mathbf{H}_{2} \mathbf{O} \\
E(V v S S C E)=1.451-0.0788 p H+0.0197 \log \left(\mathrm{MnO}_{4}{ }^{-}\right)
\end{gathered}
$$

Where $\mathrm{pH}$ is in standard $\mathrm{pH}$ units and $\mathrm{MnO}_{4}{ }^{-}$is in molar concentration. Several additional electrochemical $1 / 2$ cell reactions describe further reducion of the oxide to $I V, I I I$, and $I I$ states.

$$
\begin{gathered}
\mathbf{2} \mathbf{M n O}_{\mathbf{2}}+\mathbf{4} \boldsymbol{H}^{+}+\mathbf{2} \boldsymbol{e}^{-} \leftrightarrow \mathbf{M n}_{\mathbf{2}} \boldsymbol{O}_{\mathbf{3}}+\mathbf{2} \boldsymbol{H}_{\mathbf{2}} \boldsymbol{O} \\
E(V v S S C E)=0.773-0.0591 p H \\
\mathbf{3} \mathbf{M n}_{\mathbf{2}} \boldsymbol{O}_{\mathbf{3}}+\mathbf{4} \boldsymbol{H}^{+}+\mathbf{2} \boldsymbol{e}^{-} \leftrightarrow \mathbf{2} \mathbf{M n}_{\mathbf{3}} \boldsymbol{O}_{\mathbf{4}}+\mathbf{2} \boldsymbol{H}_{\mathbf{2}} \boldsymbol{O} \\
E(V v s S C E)=0.448-0.0591 p H \\
\mathbf{M n}_{\mathbf{3}} \boldsymbol{O}_{\mathbf{4}}+\mathbf{2} \boldsymbol{H}^{+}+\mathbf{2} \boldsymbol{e}^{-} \leftrightarrow \mathbf{3 M n O}+\boldsymbol{H}_{\mathbf{2}} \boldsymbol{O} \\
E(V v S S C E)=0.001-0.0591 p H
\end{gathered}
$$

At low $\mathrm{pH}$ and low potential the oxides will disolve as $\mathrm{Mn}^{2+}$. However, at higher $\mathrm{pH}$ above 8, a stable $\mathrm{Mn}(\mathrm{II})$ hydroxyde is prevalent on E-pH diagrams.

The equilibrium speciation predictions as a function of pHcan be seen in Figure 1.22. These speciation diagrams indicate predominante species ratios at $-0.5 \mathrm{~V}_{\text {sce, }}$, approximatly the value of the observed OCP for AA2024-T351 in 0.05 M sodium chloride solution with the permanganate ion in solution. It can be seen that above a $\mathrm{pH}$ of 8.5 , the stable $\mathrm{Mn}(\mathrm{II})$ species is $\mathrm{Mn}(\mathrm{OH})_{2}$. This suggests that this species could deposit electrochemically and remain stable at high $\mathrm{pH}$ cathodic sites such as copper rich constituent particles. The copper rich intermetallic particles experience a higher $\mathrm{pH}$ environment because of preferential reduction of $\mathrm{O}_{2 \text { (gas) }}$ over copper containing particles due to a higher exchange current density and more conductive oxide than aluminum oxide ${ }^{[56]}$. 
Inhibition of localized corrosion (pitting) on AA2024 has been shown ${ }^{[86]}$. However, the effect of manganese oxides on the oxygen reduction reaction (ORR) has not been reported in the literature. Moreover, fundamental understanding of the mechanisms that lead to anodic inhibition is lacking. Release and transport of manganese species for active inhibition has not been studied. The manganese system has multiple valence states between +7 and +2 which, in a similar way to the chromate system, can provide mechanisms for transport of the soluble oxidizing inhibiting species (as shown in Figure 1.22) to sites for electrochemical reduction leading to the potential for self-healing capabilities. Initially, soluble permanganate, manganese in the +7 valence state, can be electrochemically deposited on $\mathrm{Cu}$ rich intermetallic sites. The $\mathrm{MnO}_{2}$ and $\mathrm{MnO}\left(\mathrm{MnOH}_{2}\right)$ are relatively insoluble as shown in Figure 1.22. However, incomplete $\mathrm{MnO}_{4}{ }^{-}$reduction could lead to a reservoir of the Mn(VII) species trapped in a lower valence state oxide available for later use. Release by chemical dissolution of the $\mathrm{Mn}^{2+}$ species is possible from the oxide caps if a solution is present at a $\mathrm{pH}$ lower than approximately 8 according to chemical equilibria and dependency of Mn hydroxide solubility on $\mathrm{pH}$. However, when a cathodic reaction occurs above a finite rate over a $\mathrm{Cu}$-rich site, the $\mathrm{pH}$ will rise and the $\mathrm{Mn}^{2+}$ can again be chemically deposited over the active cathodic site as $\mathrm{Mn}(\mathrm{OH})_{2}$ or $\mathrm{MnO}$. In this manner the manganese species could actively provide corrosion protection. X-ray photoelectron spectroscopy (XPS), Raman, and X-ray absorption near edge structure (XANES studies) on platinum, gold, and additional aluminum alloy substrates with manganese deposits have also been conducted, mainly to elucidate identity and oxide stoichiometry after a given amount of reduction ${ }^{[88-95]}$. These papers illustrate the difficulty in definitive determination of oxide valence states in manganese oxides. No reliable in situ 
surface analytical probe has clearly and unambiguously determined the oxidation state of electrodeposited manganese oxides.

In Chapter 2 we examined both pretreatments of AA2024 with permanganate, to examine the effects of the protective manganese oxide barriers, and exposures in the presence of the permanganate ion with no pretreatment to distinguish the effects of the soluble ion from the manganese oxides on corrosion behavior. Open circuit full immersion exposure experiments were conducted. Examinations of the resulting surface chemical compositions and deposit morphologies were conducted. Severity of copper replating was explored to measure inhibition of $\mathrm{Cu}$ rich phase as well as matrix dealloying. Mixed inhibition investigations were complimented with anodic and cathodic polarizations experiments. Pitting potentials were determined and oxygen reduction reaction (ORR) kinetics were extracted using the Koutecky-Levich correction ${ }^{[56]}$. Theoretical modeling with Gueshi and membrane models was performed to analyze oxygen reduction reaction kinetics obtained from rotating disk electrode (RDE) experiments. Electron transport through the solid/electrolyte-interface was also characterized. Raman spectroscopy and galvanostatic reductions were used to attempt to characterize the valence states of protective manganese oxides. The work on permanganate in Chapter 2 will help towards designing better permanganate based corrosion inhibiting coatings and to provide a comprehensive understanding of the mechanisms of permanganate inhibition including the valence state of electrochemically deposited manganese oxides.

To date, no literature has been discovered regarding the effectiveness of permanganate as an inhibitor of intergranular corrosion, $\mathrm{SCC}$, or EFCP in precipitation age hardened aluminum alloys. There is one account of the inhibition of SCC by permanganate in mild 
steel when it is added to an electrolyte of $35 \% \mathrm{NaOH}$ which would otherwise lead to caustic cracking of the mild steel ${ }^{[96]}$. The inhibition was attributed to the oxidative nature of the inhibitor pushing the free corrosion potential past the critical range. Potentiostatic cracking experiments in the critical potential range were not affected by the addition of permanganate. In principle permanganate could be forecasted to be an effective inhibitor of EAC on 2xxx and 7xxx series aluminum alloys if its addition led to a rapidly reduced barrier film that ultimately affected hydrogen uptake. However, to date, there is no evidence in the literature that permanganate has been used as an inhibitor of EAC in aluminum alloys. Unlike chromate and molybdate, it is not a buffer, and so is in some respects not as well suited for controlling crack tip chemistry ${ }^{[97]}$.

Table 1.3 provides a road map of the literature findings as they relate to permanganate and the many forms of corrosion that have been discussed, highlighting gaps that have been filled by this work.

\subsubsection{Cerium}

Cerium has been studied as a corrosion inhibitor on precipitation age hardened aluminum alloys ${ }^{[33,52,57-60,98-104]}$. The mechanism of corrosion inhibition operates through a chemical reaction of $\mathrm{Ce}^{3+}$ with hydroxide anions in high $\mathrm{pH}$ areas (cathodic reaction sites) ${ }^{[105]}$. A precipitate is formed that acts as a barrier layer providing corrosion protection. Cerium has been found to reduce cathodic reaction kinetics on precipitation age hardened aluminum alloys ${ }^{[33,52]}$. Generally, the precipitate is found on the surface in the Ce(III) 
hydroxide, however, the precipitate depends on the $\mathrm{pH}$ and available oxidizers in solution $^{[103]}$. Anodic inhibition has not been reported in the literature, however, cerium was found to be a potent inhibitor of copper replating, a useful measurement of open circuit $\operatorname{corrosion}^{[33]}$.

High throughput analysis of binary inhibitor combinations was performed by Chambers and Cook ${ }^{[53,54]}$. They found the combination of $\mathrm{CeCl}_{3}$ with $\mathrm{Na}_{2} \mathrm{MoO}_{4}$ to be an effective inhibitor combination. Wilson ${ }^{[106]}$ of the CESE group has completed further analysis of this system although, at present the results are not yet published. No examination of $\mathrm{Ce}^{+3}$ and $\mathrm{MnO}_{4}{ }^{-}$has been reported in the literature.

In Chapter 2 we will be interested in the ability of permanganate to combine with cerium to augment the protection provided by either as a single inhibitor. This is of interest not only because of the potential to improve permanganate inhibition, but also because understanding the fundamentals of how inhibitors work when combined together can lead to better selection of inhibitor combinations.

The permanganate-cerium system has been chosen for the focus in this study based in part on a concept patented by Martin Kendig ${ }^{[107]}$ in which permanganate is used to oxidize $\mathrm{Cr}^{3+}$ to $\mathrm{Cr}^{6+}$ to gain the protection of chromate without having to use the common strontium chromate inhibitor ${ }^{[108]}$. In this study, $\mathrm{Ce}^{3+}$ may be oxidized to $\mathrm{Ce}^{4+}$ in solution by permanganate. This might provide better protection since $\mathrm{Ce}^{4+}$ is stable over a wider $\mathrm{pH}$ range, albeit at oxidizing potentials ${ }^{[99]}$. The cerium E-pH diagram can be found in Figure 1.23 . 
Purported inhibition by cerium of IGSCC of Al-Li-Cu 8xxx alloys has been observed ${ }^{[60,}$ ${ }^{109]}$. Davo suggests that cerium can function as an inhibitor of IGSCC in 8xxx series alloys in two ways. The first is through the growth of a protective oxide film over the entire surface of the test specimen which was found to decrease pit density and thus crack initiation site density. Some evidence of a decrease in surface pit damage was presented; however, the cerium did not have an effect on $\mathrm{E}_{\mathrm{pit}}$ nor was any evidence of crack growth rate inhibition presented. Surface damage alone was correlated to IGSCC. The second claimed mechanism was through the inhibition of anodic dissolution of T-phase precipitates at the grain boundaries, however, no evidence was provided for this claim. Cerium has been excluded from the repassivation study in this thesis because it does not exhibit what is thought to be one of the most important preliminary characteristics of an inhibitor in relation to inhibition of transgranular EFCP; namely, the ability to increase the pitting potential.

Table 1.3 provides a road map of the literature findings as they relate to cerium and the many forms of corrosion that have been discussed, highlighting gaps that have been filled by this work.

\subsubsection{Chromate}

The inhibition of corrosion of AA2024 and 7075 by chromate and chromate conversion coatings is well documented ${ }^{[34-39]}$. Chromate can either be included in a primer as a pigment addition or it can be used to form a chromate conversion coating (CCC). When it 
is used as a primer pigment low solubility salts such as $\mathrm{SrCrO}_{4}$ and $\mathrm{BaCrO}_{4}$ are selected for inclusion as a pigment in the primer layer with the low solubility acting to reduce osmotic blistering. ${ }^{[110]}$ The chromate is available to slowly leach from the coating providing $\mathrm{Cr}(\mathrm{VI})$ that can be reduced onto any bare aluminum surface as a $\mathrm{Cr}(\mathrm{III})$ barrier oxide. When a chromate conversion coating is prepared, chromate is reduced electrochemically to a thick $\mathrm{Cr}$ (III) rich barrier oxide in the presence of $\mathrm{HF}$, which acts to remove $\mathrm{Al}_{2} \mathrm{O}_{3}$ on the aluminum surface so as to activate the aluminum surface. One interpretation proposes that the reduction proceeds through a sol-gel mechanism including the formation of polymerized $\mathrm{Cr}(\mathrm{III})$ entrapping $\mathrm{Cr}(\mathrm{VI})$ which can then be released to provide further protection of the substrate ${ }^{[37,111]}$. Chromate has not been studied as a component in a binary inhibitor combination due to the fact that it is being phased out as an inhibitor. There is a patent on the effects of using trivalent chrome with a strong oxidizer to generate hexavalent chrome; however, since the industry is moving away from hexavalent chrome we have chosen to exclude it from binary inhibitor combinations ${ }^{[107]}$.

To understand how and why chromate can act as an effective inhibitor of pitting corrosion, cathodic reaction kinetics, IGSCC, IGC, and environmental fatigue crack propagation (EFCP), a brief overview of its thermodynamics is required. Chromate exists as a +6 valence state oxoanions in aqueous solutions. One mechanism through which it can inhibit corrosion and EAC is by being reduced to its more stable $\mathrm{Cr}(\mathrm{III})$ oxide or hydroxide, creating a sparingly soluble barrier that protects the underlying substrate from local attack. The E-pH behavior of chromate is shown in Figure 1.24. Chromate can be reduced to a $\mathrm{Cr}^{3+}$ oxide that is stable over a much wider range of potential and $\mathrm{pH}$ in comparison to aluminum oxide. Aluminum oxide is only stable in a narrow band while $\mathrm{Cr}_{2} \mathrm{O}_{3}$ is stable at 
$-0.8 \mathrm{~V}_{\mathrm{SCE}}$ from $\mathrm{pH} 4$ to 14 as shown in the speciation diagram in Figure 1.24(b). In aqueous chloride containing solutions chromate has been found to be an excellent corrosion inhibitor ${ }^{[112-114]}$, especially for localized corrosion by reducing pit nucleation occurrence and by decreasing pit growth kinetics ${ }^{[113,115]}$. This reduction in pitting is attributed to its ability to stabilize barrier oxides through either electrochemical reduction and incorporation into the oxide film or through competitive adsorption with $\mathrm{Cl}^{-}$as an adsorbing ion without a three dimensional oxide ${ }^{[55]}$. It has also been found to be an inhibitor of cathodic reaction kinetics ${ }^{[115,116]}$. The mechanism of protection is a matter of debate; however, it is generally acknowledged that hexavalent chromate is reduced to the trivalent state forming a chromium (III) hydroxide following the overall or full cell reaction given in Equation 1.5.

$$
2 \mathrm{Al}+\mathrm{Cr}_{2} \mathrm{O}_{7}^{2-}+\mathrm{H}^{+}+\mathrm{H}_{2} \mathrm{O}=\mathrm{Al}_{2} \mathrm{O}_{3}+2 \mathrm{Cr}(\mathrm{OH})_{3}
$$

It is suggested that the reduction is incomplete resulting in significant reservoirs of hexavalent chrome being trapped in the hydroxide film which can subsequently leach into solution providing a self-healing mechanism ${ }^{[117,118]}$.

The understanding of how chromate inhibits EAC is not understood nearly as well as its surface corrosion inhibition. There is evidence in the literature of its effectiveness based on reduction in typical cracking type metrics and suggestions of mechanistic understanding $[29,61,80,119]$. Lui found a drastic decrease in crack propagation rate during fatigue testing of AA7075-T76 at low $\Delta \mathrm{K}$ when chromate was introduced to the $3.5 \% \mathrm{NaCl}$ test electrolyte. Their analysis was that the oxide barrier film damage produced at the crack tip at low $\Delta \mathrm{K}$ levels was quickly repaired in a chemical process due to the presence of chromate. Their experimental results were interesting, however the mechanistic 
understanding of inhibitor effect on crack tip conditions was lacking. Gasem found large frequency dependent decreases in crack propagation rates in constant $\Delta \mathrm{K}$ fatigue testing of AA7075-T651 ${ }^{[29]}$. His findings are presented in Figure 4.3. He found crack tip propagation inhibition was sensitive to frequency, $\Delta \mathrm{K}$, and chromate concentration and hypothesized that either chromate migration to the crack tip or a process in which high strain rates disrupted a barrier oxide faster than it could repassivate, dominated the chromates inhibitive behavior. He then went on to show that due to enhanced migration and mixing of ionic species in the crack at high loading frequencies, the former hypothesis regarding transport limited inhibition could be discarded. His conclusion was that chromate functions in a competitive repassivation process with film rupture caused by high cyclic crack tip strain rate. However, measurements of the effect of chromate on the repassivation of aluminum alloys are limited to observations of the free corrosion potential in the presence of chromate after mechanical depassivation ${ }^{[78,120,121]}$. Of course, a key unspoken part of this process is that the repassivated film limits dissolution hydrolysis and coupled hydrogen production followed by uptake. To the author's knowledge, no evidence of the inhibition of hydrogen uptake during an oxide film rupture event in the presence of chromate is published in the literature and limited electrochemical analysis of repassivation in the presence of chromate is available. Nor is such data available for any other inhibitor.

Other possible mechanisms for crack growth rate inhibition exist including arguments having to do with $\mathrm{pH}$ buffering of the crack tip as well as cathodic inhibition mechanisms that affect the galvanically coupled crack-flank and crack-tip leading to a decrease in anodically dissolved $\mathrm{Al}^{3+}$ at the crack-tip. Chromate is a buffer and its buffer capacity is shown in Figure 1.25 $5^{[24]}$, so it is possible that the $\mathrm{pH}$ in the crack is buffered to some degree. 
Both alternative mechanisms may play some role in EAC susceptibility. A buffered crack tip may make the native aluminum oxide more thermodynamically favorable changing repassivation kinetics. Progress towards explaining the mechanisms behind the inhibition of EFCP by chromate is presented in Chapter 4 along with comparisons to the inhibition provided by permanganate and molybdate.

Table 1.3 provides a road map of the literature findings as they relate to chromate and the many forms of corrosion that have been discussed, highlighting needs, gaps, and opportunities that have been addressed by this work.

\subsubsection{Molybdate}

Molybdate is an oxoanion with a similar structure to chromate, $\mathrm{MeO}_{4}{ }^{2-}$ with a valence of +6. Figure 1.26 (a) shows molybdenum's E-pH behavior with its stable $\mathrm{Mo}^{4+}$ oxide over all $\mathrm{pH}$ values. At $-0.8 \mathrm{~V}_{\mathrm{SCE}}$ the $\mathrm{Mo}(\mathrm{IV})$ oxide is stable from a $\mathrm{pH}$ of 4 to 12 as shown in Figure 1.26(b). At low $\mathrm{pH}$, molybdenum oxides exhibit a region of thermodynamic stability that extends to high potentials. The surface corrosion inhibition properties of molybdenum and molybdate have been studied on aluminum alloys and other metals such as carbon steel $[33,49,52]$. Molybdate has been found to be a potent anodic inhibitor, increasing the pitting potential of AA2024 significantly ${ }^{[33]}$. The mechanism is reported to be the incorporation of $\mathrm{MoO}_{4}{ }^{2-}$ into barrier films blocking the adsorption of $\mathrm{Cl}^{-}$. Molybdate was also found to be a potent inhibitor of the oxygen reduction reaction (ORR) and copper replating ${ }^{[33]}$. Molybdate was found to be an effective cathodic inhibitor only if it was 
present in solution suggesting that no physically precipitated species was present or a film was ineffective in blocking the $\mathrm{ORR}^{[52]}$. Additionally, molybdenum has been used as an alloying element via ion implantation in high purity aluminum resulting in an increase in the pitting potential resulting from the solid solution incorporation of molybdate into the aluminum matrix ${ }^{[50]}$. Anionic addition of molybdate to chloride containing solutions showed increased pitting potential and inhibition of the oxygen reduction reaction (ORR) on AA2024 and AA7075 ${ }^{[33,79]}$. Molybdate has been studied as an addition to binary inhibitor combinations extensively by Wilson ${ }^{[106]}$.

There is no literature available concerning the inhibition of SCC and IGSCC by molybdate on aluminum alloys. There is one report detailing increases in fatigue life cycles for HS4340 steel with the addition of molybdate to moist air environments ${ }^{[122]}$. Molybdate has also been shown to be an inhibitor of EFCP ${ }^{[79,119]}$. However, the mechanism of inhibition of EFCP is unclear. Proposed mechanisms include oxide film repassivation augmentation and $\mathrm{pH}$ buffering ${ }^{[79]}$. In Chapter 3 we will examine the ability of Mo to stabilize and repassivate a scratch depassivated oxide barrier on $\mathrm{Al}-\mathrm{Mg}-\mathrm{Zn}-\mathrm{Cu}$ alloys. In Chapter 5 we will go on to examine the effects of molybdate on the uptake of hydrogen into the fracture process zone.

Table 1.3 provides a road map of inhibitor information available in the literature and needs, gaps, and opportunities that have been addressed by this work. 


\subsection{Inhibitor Activity at Relevant Material Length Scales}

Corrosion inhibitors protect alloy systems from numerous degradation modes over a range of length scales. At a macroscopic level they can affect coating performance properties such as coating adhesion, blistering, and polymer degradation. At the microscopic level, corrosion inhibitors protect alloy systems from pit growth and block cathodic reaction surface sites such as copper rich intermetallic particles. At the nanoscale they can affect metastable pitting initiation/stabilization at oxide film defects and affect hydrogen diffusion through native oxide barriers. Inhibitors must effectively function in systems that range in length scales from meters to angstroms, protecting alloy systems from macro-scale coating failures while also inhibiting processes such as hydrogen embrittlement that occur at the nano-scale (Figure 1.27). It is difficult to comprehensively probe each length scale in any study.

Figure 1.17 illustrates the multidimensional environments in which inhibitors must function. Surface corrosion such as pitting, IMC trenching, cathodic corrosion, as well as the hydrogen evolution and oxygen reduction reactions may all be affected to a greater extent or less by corrosion inhibitors. They also may provide protection from crack tip processes that lead to the embrittlement of precipitation age hardened aluminum alloys. They may inhibit embrittlement in the fracture process zone by limiting the uptake of hydrogen. Hydrolysis of dissolving aluminum can exacerbate hydrogen production and uptake. Inhibitors are thought to act through the passivation of the fractured surfaces, producing a decrease in hydrolysis while providing a hydrogen permeation barrier. Buffering effects may also influence the crack tip environment. The coarse constituent 
particles affect the rates of oxygen reduction reaction couple to the aluminum matrix. The fine intermetallic compounds also affect the hydrogen embrittlement of the material by acting as hydrogen traps ${ }^{[26]}$. Several inhibitors and inhibitor systems are evaluated over for their effectiveness throughout this wide range of process and length and time scales.

\subsection{Critical Unresolved Issues for Surface Corrosion Protection by Single Ionic Inhibitors}

\subsubsection{Permanganate}

One critical issue not resolved in the literature for the permanganate system is the understanding of the permanganate species responsible for inhibition of cathodic reactions on intermetallic particles and anodic reactions at pits. The valence state of $\mathrm{Mn}$ as it is deposited on the AA2024 substrate at intermetallic compounds and copper rich sites has

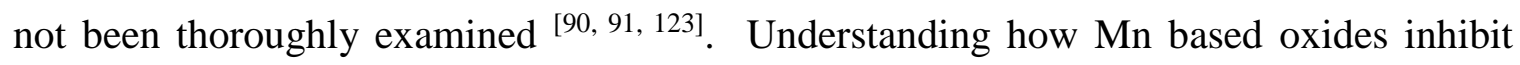
pitting most effectively as well as which Mn based oxides are readily deposited on the heterogeneous substrate is critical to understand and optimize any potential inhibitor coating system. Determining the ability of permanganate to protect the S-phase from dealloying is critical. Determining the molecular species of the deposited manganese will also be vital to determining the ability of manganese coatings to self-heal or release soluble Mn species to the crack tip. To have self-healing properties manganese in a coating must be stored in a stable state yet be able to be released into an aqueous environment in some soluble form when corrosive conditions are encountered. Once released the aqueous 
species must be reduced in a way that limits the rate of corrosion. Understanding the reaction mechanisms that might lead to any self-healing properties of the manganese deposits will be critical to controlling this process. Work done and needs, gaps and opportunities are summarized by Table 1.1 .

\subsubsection{Cerium}

The cerium single inhibitor system has been carefully analyzed in the literature and no further analysis of its ability to inhibit corrosion of precipitation age hardened aluminum alloys will be presented here ${ }^{[33,52,57-60,98-104]}$. Cerium was found to be a potent cathodic inhibitor that functions through the precipitation of a cerium oxide/hydroxide uniformly

over the surface of the alloy providing a barrier to oxygen reduction ${ }^{[105]}$. The inhibitor was found to have no anodic inhibition properties ${ }^{[33]}$. No further study was conducted to assess cerium as a single inhibitor.

\subsubsection{Chromate}

The literature on the inhibition of corrosion on precipitation age hardened aluminum alloys

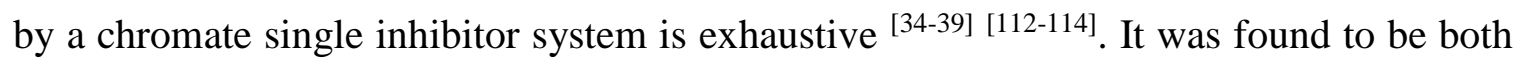
an anodic and cathodic inhibitor functioning through the incorporation of $\mathrm{CrO}_{4}{ }^{2-}$ into the native oxide with some reduction to the $\mathrm{Cr}(\mathrm{III})$ species as well as competitive adsorption 
of $\mathrm{CrO}_{4}{ }^{2-}$. No further analysis of its ability to inhibit surface type corrosion as a single inhibitor of precipitation age hardened aluminum alloys will be presented here.

\subsubsection{Molybdate}

The molybdate single inhibitor system has been carefully analyzed in the literature and no further analysis of its ability to inhibit surface type corrosion of precipitation age hardened aluminum alloys will be presented here. Molybdate functions as a potent anodic and cathodic inhibitor which is thought to operate at open circuit through the competitive adsorption with $\mathrm{Cl}^{-[33,49,52]}$.

\subsection{Critical Unresolved Issues for Binary Inhibitor Combinations}

Understanding how two inhibitors function when combined is critical to optimizing inhibitor systems. The inhibitors have the possibility of having synergistic effects on one another, additive effects, or potentially antagonistic effects. It is also possible that it will be some combination of the above in OCP, anodic, or cathodic inhibitor regimes. Defining what interactions are occurring and how they manifest themselves microscopically and electrochemically will enable more intelligently designed inhibitor systems to be developed. For instance there are several types of synergistic effects that are possible including forming a complex combined ionic species or by oxidizing a lower oxidation

state species to a more inhibitive form. Another effect would be a change from a chemically precipitated species to a more effective electrochemically deposited species. A 
binary combination might provide a cathodic inhibitor component and an anodic inhibitor component. An additive effect such as filling in oxide deposit gaps over cathodic sites is also possible.

The permanganate-cerium system has been selected for closer study in this thesis for several reasons. The first is the potential for the system to have interesting synergistic behavior through the chemical interactions of the two inhibitors in solution. The second is that several other possible combinations including cerium-molybdenum systems have already been analyzed by another member of the $\mathrm{CESE}^{[106]}$. That work is not published but it is underway. The combination of inhibitors with chromate was rejected because of the need to remove chromate from service. The primary purpose for a binary system was to find a replacement for chromate unlikely to be achieved by a single drop-in replacement.

With this in mind the permanganate-cerium system will be the only binary system analyzed in this work. The first unresolved issue is to determine the effect of the binary inhibitor system on the electrochemical corrosion system. Another is to determine the mechanism of any inhibition found. Understanding and describing the mechanisms that lead to synergistic, antagonistic, or additive effects is critical to advance the understanding of multi-inhibitor protection schemes.

\subsection{Critical Unresolved Issues for Crack Growth Inhibition}

Three single inhibitors have been selected for study as crack growth inhibitors. The first and most obvious is chromate due to its wide acceptance as a potent inhibitor of crack 
growth and the lack of information available on precise function in the literature ${ }^{[29]}$. Understanding how chromate effects repassivation metrics such as net current during repassivation, oxide barrier properties, and absorbed hydrogen concentrations has not been addressed in the literature. The second, molybdate, has been chosen because of its anodic inhibition characteristics and the availability of crack growth literature for precipitation age hardened aluminum alloys in the presence of molybdate. The mechanistic understanding of how molybdate effects EFCP is also lacking ${ }^{[27]}$. The third is permanganate, which has been selected due to the anodic inhibition that will be demonstrated in this thesis. Thus it passes early screening as a potential beneficial crack tip inhibitor.

One key challenge is to develop better methods to assess the action of inhibitors during EAC and EFCP. There are many difficult issues to overcome when addressing crack tip environments. The challenge of monitoring electrochemical and chemical effects at a crack tip, respectively has been tackled by Kolman ${ }^{[74]}$ and Cooper ${ }^{[24]}$ which has provided the framework to examine a simulated crack tip type environment. The premise stated by Gasem $^{[29]}$ and Warner ${ }^{[27]}$ is that oxide rupture in the occluded crack-tip leads to aluminum dissolution, hydrolysis of $\mathrm{Al}^{3+}$, IR drop, hydrogen production, hydrogen uptake all prior to repassivation of the surface. However, evidence for the enhanced repassivation of aluminum surfaces in the presence of inhibitors is lacking in the literature and is a central speculation in the mechanisms proposed in their work.

Chapter 4 attempts to determine what the effect of inhibitors is on scratch repassivation of AA2024 and AA7075 in hopes of developing a mechanistic understanding of inhibitor effects on repassivation electrochemistry at the crack tip. As noted previously, $f_{C r i t U B}$ has been rationalized as the frequency at which an oxide barrier is disrupted faster than it can 
regrow. By examining the kinetics of oxide regrowth, the potential for a relationship between $f_{\text {Crit }}$ and $\tau_{\text {repass }}$ can be examined. In the past $\tau_{\text {repass }}$ has been observed by recording the transient current decay observed after scratching a metallic electrode or fracturing a metallic thin film ${ }^{[69,124]}$. However, the addition of inhibitors to the repassivation process creates difficulty in using current transients because of complications in tracking all of the sources of current as seen in Equation 1.6 ${ }^{[75]}$. Moreover, where the addition of oxidizing inhibitors can add greatly to the cathodic components of the equation. In Equation 1.6, $i_{\text {net }}$ is the total current measured by a potentiostat, $i_{\text {crack tip }}^{\text {anodic }}$ is the anodic current generated from the dissolution of aluminum, that cathodic terms represent the current from the reduction of oxygen, the evolution of hydrogen, or the reduction of an inhibitor, and the $C \frac{d V}{d t}$ term represents the capacitive current generated from the rapid changes in potential, $V$, produced from IR drop ${ }^{[125,126]}$.

$$
i_{\text {net }}=i_{\text {crack tip }}^{\text {anodic }}-\left(i_{\text {crack tip }}^{\text {cathodic }}+i_{\text {passive surface }}^{\text {cathodic }}\right) \pm C \frac{d V}{d t}
$$

Of interest is the effect of the ionic inhibitor on each of these, yet $i_{\text {net }}$, is what is routinely measured. Therefore, a single frequency impedance approach is used to measure $\mathrm{C}_{\mathrm{ox}}$ as a unique measure of repassivation. The well-known relationship between oxide thickness $\left(d_{o x}\right)$ and capacitance $\left(C_{o x}\right)$ will be used to monitor oxide growth (Chapter 4$)$. This relationship is shown in Equation 1.7 where $A$ is the area of the electrode, $\varepsilon$ is the permittivity of the oxide, and $\varepsilon_{o}$ is the permittivity of free space. One advantage of using the capacitance of the oxide to probe its thickness is the high frequency range at which the capacitance data is acquired, roughly $1000 \mathrm{~Hz}$. 


$$
C_{o x}=\frac{\varepsilon \varepsilon_{o} A}{d_{o x}}
$$

Several stages of growth are predicted after scratching a metallic electrode, as shown in Figure 1.28. The nucleation of oxide on the bare metallic surface will have an effect on the capacitance described by Equation 1.8 for a parallel circuit where $\theta$ is the fraction of bare metallic electrode.

$$
C_{\text {total }}=\theta C_{\text {bare }}+(1-\theta) C_{o x}
$$

The measurement of $C_{\text {total }}$ will be enabled by EIS at $1000 \mathrm{~Hz}$. Initially $\theta=1$ and $\mathrm{C}_{\text {bare }}$ is measured. The method relies on the fact that $C_{b a r e}$ is much larger than $C_{o x}$ for aluminum. $C_{\text {bare }}$ will initially be on the order of the double layer or Helmholtz capacitance which is between 40 and $100 \mu \mathrm{F} / \mathrm{cm}^{2}{ }^{[127-129]}$ when $\theta=1$ and the $\mathrm{Al}_{2} \mathrm{O}_{3}$ oxide capacitance is on the order of $1-2 \mu \mathrm{F} / \mathrm{cm}^{2}{ }^{[130]}$ when $\theta=0$. EIS measurements described in detail in Chapter 4 will be used to access the capacitive data. Therefore, $\mathrm{C}_{\text {total }}$ during repassivation should decrease from $40 \mu \mathrm{F} / \mathrm{cm}^{2}$ to $2 \mu \mathrm{F} / \mathrm{cm}^{2}$ during a scratch repassivation event as long as 1000 $\mathrm{Hz}$ is in the correct frequency range to be dominated by this parameter.

Monitoring the kinetics of oxide film growth at short time scales has the potential to shed light on how anodic dissolution and/or hydrogen embrittlement at the crack tip occurs and which inhibitors can be implemented to best imped it. The $f_{\text {Crit }}$ of a system is related to the repassivation of the crack tip and thus repassivation kinetics on bare electrodes should be related through the repassivation time constant, $\tau_{\text {repassivation }} \tau_{\text {repassivation }}$ will be examined from $i_{\text {net }}$ analysis but also from the impedance magnitude. By examining the rate of oxide growth on a freshly scratch sample, $\tau_{\text {repassivation }}$ can be determined for a range of inhibitor concentrations providing a fast method for down selection of effective inhibitors of EFCP. 
The well-known relationship between oxide thickness and capacitance provides an opportunity to explore the processes that effect crack growth. One challenging aspect of accessing these processes will be the rapid acquisition of highly accurate capacitance data. During the process of film nucleation and growth there should be a decrease in the oxide capacitance of the system first through the change of $\theta$ from 1 to 0 and then through its inverse proportionality with the oxide thickness $\left(d_{o x}\right)$. By monitoring this capacitance and comparing it to data collected from EFCP tests the mechanism can be studied.

We want to establish whether $f_{\text {Crit }}$ is related to the time to form the first layer of an oxide film, many layers of oxide, or to adsorb an inhibitor onto the surface. Is there a critical oxide thickness that is reached at $f_{C r i t}$ or is it related to the elimination of defects after the film has grown?

In addition to observing the growth of oxides over a scratch test specimen, other opportunities for development of crack tip type inhibition will be addressed. Additional modes of crack tip type inhibition such as crack tip solution buffering or effects of inhibitor of crack flank cathodic reaction kinetics will be examined, as well as hydrogen overpotentials that result from different inhibitor additions. Inhibitors that can naturally act as $\mathrm{pH}$ buffers can indirectly affect the adsorption of atomic hydrogen by decreasing the concentration of $\mathrm{H}^{+}$in solution, stabilizing the aluminum oxide, and decreasing the HER production rate. Reductions in hydrogen evolution over-potential can also be affected by inhibitors that affect the OCP of the system. This effect could be revealed by examining the uncoupled OCP of the electrode when an anode is scratched. Direct evidence of the inhibition of hydrogen absorption into the substrate by a corrosion inhibitor is addressed as well as the effects of inhibitors on the cathodic reactions occurring on the crack flank. 
The effect of the inhibitor on the cathodic reaction at the crack tip will be addressed through galvanic coupled scratch experiments.

\subsection{Objective}

The objective of this thesis is to address the needs and gaps in current ionic inhibitor understanding as they relate to both surface corrosion and environmental assisted crack tip inhibition, and to develop inhibitors that may one day replace chromate. Both single and binary inhibitors have been selected for corrosion inhibition; from separating inhibitor deposit effects from ionic effects to the effect of deposit valence on the cathodic reaction rates observed. The second objective is to help develop methods and methodology to assess ionic inhibitor impact on EAC and EFCP. This investigation was focused on fundamental processes at play in crack tip chemical and electrochemical systems in order to better assess the impact that anionic inhibitors may have on crack susceptibility.

\subsection{Thesis Organization}

This thesis is organized by the needs, gaps and opportunities and/or critical issues pertaining to inhibition of corrosion and corrosion influenced cracking of aerospace aluminum alloys.

Chapter 2 examines the ability of permanganate to act as a single ionic inhibitor of surface type corrosion of AA2024. The inhibition of corrosion on AA2024-T351 in $\mathrm{NaCl}$ 
solutions, mitigated by either in-situ permanganate ions $\left(\mathrm{MnO}_{4}^{-}\right)$or permanganate pretreatment, was examined. There is a lack of info on this topic and this provides a foundation for crack tip studies. Both room temperature pretreatment and solution phase additions were studied as a function of inhibitor concentration. The roles of the inhibitor during anodic and cathodic polarizations were investigated. Inhibition of corrosion at open circuit corrosion potential (OCP) under conditions where anodic and cathodic reactions are coupled were also examined. The oxidation states of the manganese oxides that contributed to protection were determined using potentiometric electrochemical reduction and in-situ Raman spectroscopy. The thermodynamics of the Mn-water system was also considered over a range of concentrations. Permanganate is shown to be both an anodic and cathodic inhibitor, and an inhibitor of copper replating at OCP.

Chapter 3 examines the ability of permanganate and cerium to act synergistically, additively, separately, as well as antagonistically in a combined inhibitor profile. Room temperature pretreatments were studied as a function of total inhibitor concentration from $10 \mathrm{mM}$ to $50 \mathrm{mM}$. Pretreated samples were exposed to $50 \mathrm{mM} \mathrm{NaCl}$ solutions. The roles of the inhibitor during anodic and cathodic polarizations were investigated. Inhibition of open circuit corrosion under conditions where anodic and cathodic reactions are coupled was also examined. The Koutecky-Levich correction was applied to experimental cathodic E- $i$ data. The thermodynamics of the Ce-water and Mn-water systems was also considered over a range of concentrations. There was no indication of a combined species and no reference to such a species in the literature was found. The cerium-permanganate combination was determined to provide nearly the same level of cathodic inhibition seen in the single inhibitor application of permanganate. No improvement in cathodic reaction 
kinetics over the single inhibitor was observed. Cerium alone was inferior as a cathodic inhibitor. In addition to strong cathodic inhibition, the combination increased inhibition of open circuit corrosion compared to single inhibitor pretreatments as assessed by copper redeposition. However, anodic inhibition was not observed for the binary combination.

Chapter 4 examines the effects of permanganate, chromate, and molybdate on scratch depassivated aluminum alloy electrodes fundamentals to elucidate the effects of inhibitors on the relevant electrochemical reactions occurring in an occluded crack tip environments. The events at crack tips are well known to govern the processes controlling SCC and EFCP in this alloy system. Scratch depassivation of AA2024-T351, AA7075-T6, and 99.999\% aluminum in permanganate $\left(\mathrm{MnO}_{4}^{-}\right)$, molybdate $\left(\mathrm{MoO}_{4}{ }^{2-}\right)$, and chromate $\left(\mathrm{CrO}_{4}{ }^{2-}\right)$ containing $\mathrm{NaCl}$ solutions was used to examine electrochemical repassivation kinetics over a range of potentials and inhibitor concentrations. However, a complete assessment of all $\mathrm{Cl}^{-}$levels, $\mathrm{pH}$ levels, and inhibitor concentrations has not been attempted. Chromate was found to suppress scratched electrode current transients at anodic potentials under certain conditions on both AA2024-T351 and AA7075-T6. Molybdate did not suppress transient current density in AA2024-T351 or AA7075-T6. Permanganate acted by quickly lowering total current density into the cathodic regime. Chapter 4 also presents a novel technique suitable for assessing inhibitor function during repassivation. A fast single frequency impedance method for scratch depassivated aluminum alloy electrodes is presented. Impedance data enabling assessment of the growth kinetics of barrier films at short times after scratch depassivation are shown for the first time. Chromate is found to be superior to molybdate or permanganate in quickly improving the barrier properties of the regrown oxide film which is likely to have an effect on the absorption of hydrogen that leads to 
embrittlement and EAC. Chapter 4 also presents the effects of aqueous inhibitor additions to galvanically coupled aluminum alloys exposed to crack tip and crack flank solutions, where a mechanical depassivation event at a simulated crack tip results in the flow of current from the cathodic crack flank to the anodic crack tip. The inhibition of the cathodic reaction at crack flank areas is demonstrated for molybdate, chromate and permanganate.

Chapter 5 presents Rutherford backscattering spectrometry (RBS) and elastic recoil detection (ERD) analysis of abrasion-repassivated Al-Mg-Zn-Cu alloy (AA7075-T651) surfaces, giving the first direct evidence of inhibitor species incorporation into repassivating oxides and inhibition of hydrogen uptake into AA7075 as a result of ionic inhibitor additions to aqueous chloride solutions. The effect is only seen in the case of chromate. Other functions of molybdate are discussed. The evidence presented lends new insight into the processes occurring at the crack tip in cyclic cracking, where EFCP is slowed, as well as static crack growth. The inhibiting effect of chromate is discussed in connection with the FPZ of EFCP. Moreover, these findings demonstrating a new metric through which corrosion inhibitors should be evaluated. 
$1.10 \quad$ References

1. J. R. Davis \& Associates. and ASM International. Handbook Committee., Aluminum and aluminum alloys. ASM specialty handbook (Materials Park, $\mathrm{OH}$ : ASM International, 1993). p. iii.

2. M.F. Ashby, Materials selection in mechanical design. 3rd ed (Amsterdam ; Boston: Butterworth-Heinemann, 2005). p. xiv.

3. J.D. Edwards, F.C. Frary, Z. Jeffries, and Aluminum Company of America., The aluminum industry. 1st ed. Chemical engineering series (New York London,: McGraw-Hill, 1930.

4. N. Birbilis, M.K. Cavanaugh, and R.G. Buchheit, "Electrochemical behavior and localized corrosion associated with A17Cu2Fe particles in aluminum alloy 7075T651," Corrosion Science 48, 12 (2006): p. 4202.

5. J.T. Staley, "Quench factor analysis of aluminium alloys," Materials Science and Technology 3, 11 (1987): p. 923.

6. R.P. Wei, C.M. Liao, and M. Gao, "A transmission electron microscopy study of constituent-particle-induced corrosion in 7075-T6 and 2024-T3 aluminum alloys," Metall Mater Trans A 29, 4 (1998): p. 1153.

7. J.A. Wert, "Identification of precipitates in $7075 \mathrm{Al}$ after high-temperature aging," Scripta Metallurgica 15, 4 (1981): p. 445.

8. N. Birbilis and R.G. Buchheit, "Electrochemical Characteristics of Intermetallic Phases in Aluminum Alloys: An Experimental Survey and Discussion," Journal of The Electrochemical Society 152, 4 (2005): p. B140.

9. G.O. Ilevbare, O. Schneider, R.G. Kelly, and J.R. Scully, "In situ confocal laser scanning microscopy of AA 2024-T3 corrosion metrology - I. Localized corrosion of particles," Journal of the Electrochemical Society 151, 8 (2004): p. B453.

10. I.J. Polmear, Light alloys: from traditional alloys to nanocrystals. 4th ed (Oxford ; Burlington, MA: Elsevier/Butterworth-Heinemann, 2006). p. xiv.

11. M. Pourbaix, Atlas of electrochemical equilibria in aqueous solutions. 2d English ed (Houston, Tex.: National Association of Corrosion Engineers, 1974). p. 644 p.

12. A. Handbook, "Volume 13B," Corrosion: Materials (2005).

13. O. Schneider, G.O. Ilevbare, J.R. Scully, and R.G. Kelly, "In situ confocal laser scanning microscopy of AA 2024-T3 corrosion metrology - II. Trench formation around particles," Journal of the Electrochemical Society 151, 8 (2004): p. B465.

14. J.R. Scully, T.O. Knight, R.G. Buchheit, and D.E. Peebles, "Electrochemical characteristics of the $\mathrm{Al}_{2} \mathrm{Cu}, \mathrm{Al}_{3} \mathrm{Ta}$ and $\mathrm{Al}_{3} \mathrm{Zr}$ intermetallic phases and their relevancy to the localized corrosion of Al alloys," Corrosion Science 35, 1-4 (1993): p. 185.

15. G.S. Chen, "Microconstituent-induced pitting corrosion in aluminum alloy 2024T3," CORROSION 52, 01 (1996).

16. D.G. Harlow and R.P. Wei, "A probability model for the growth of corrosion pits in aluminum alloys induced by constituent particles," Engineering Fracture Mechanics 59, 3 (1998): p. 305. 
17. R.G. Buchheit, R.P. Grant, P.F. Hlava, B. Mckenzie, and G.L. Zender, "Local dissolution phenomena associated with $\mathrm{S}$ phase $\left(\mathrm{Al}_{2} \mathrm{CuMg}\right)$ particles in aluminum alloy 2024-T3," Journal of the Electrochemical Society 144, 8 (1997): p. 2621.

18. D.A. Little, B.J. Connolly, and J.R. Scully, "An electrochemical framework to explain the intergranular stress corrosion behavior in two $\mathrm{Al}-\mathrm{Cu}-\mathrm{Mg}-\mathrm{Ag}$ alloys as a function of aging," Corrosion Science 49, 2 (2007): p. 347.

19. R.G. Buchheit, R.K. Boger, M.C. Carroll, R.M. Leard, C. Paglia, and J.L. Searles, "The electrochemistry of intermetallic particles and localized corrosion in Al alloys," Jom-J Min Met Mat S 53, 7 (2001): p. 29.

20. S. Kim, J.T. Burns, and R.P. Gangloff, "Fatigue crack formation and growth from localized corrosion in $\mathrm{Al}-\mathrm{Zn}-\mathrm{Mg}-\mathrm{Cu}$," Engineering Fracture Mechanics 76, 5 (2009): p. 651.

21. R.M. Pidaparti and R.R. Patel, "Correlation between corrosion pits and stresses in Al alloys," Mater Lett 62, 30 (2008): p. 4497.

22. K.K. Sankaran, R. Perez, and K.V. Jata, "Effects of pitting corrosion on the fatigue behavior of aluminum alloy 7075-T6: modeling and experimental studies," Mat Sci Eng a-Struct 297, 1-2 (2001): p. 223.

23. M.O. Speidel, "Stress-Corrosion Cracking of Aluminum-Alloys," Metall Trans A 6, 4 (1975): p. 631.

24. K.R. Cooper, Chemistry and Electrochemistry of Environment-assisted Cracking of an Al-Zn-Mg-Cu Alloy: University of Virginia, 2001.

25. P. Doig, P.E.J. Flewitt, and J.W. Edington, "The Stress Corrosion Susceptibility of 7075 Al-Zn-Mg-Cu Alloys Tempered from T6 to an Overaged T7X," CORROSION 33, 6 (1977): p. 217.

26. R.P. Gangloff and M.B. Ives, eds. Environment-Induced Cracking of Metals. 1989, NACE: Houston, TX. 55.

27. J.S. Warner, Inhibition of environmental fatigue crack propagation in agehardenable aluminum alloys, in MSE PhD Thesis. 2010, University of Virginia: Charlottesville.

28. N.J.H. Holroyd and D. Hardie, "Factors controlling crack velocity in 7000 series aluminium alloys during fatigue in an aggressive environment," Corrosion Science 23, 6 (1983): p. 527.

29. Z.M. Gasem, Frequency dependant environmental fatigue crack propagation in the 7XXX alloy/aqueous chloride system., in MSE PhD Thesis. 1999, University of Virginia: Charlottesville.

30. C.M. Reddy, Q.S. Yu, C.E. Moffitt, D.M. Wieliczka, W. Johnson, J.E. Deffeyes, and H.K. Yasuda, "Improved corrosion protection of aluminum alloys by system approach interface engineering: Part 1 - Alclad 2024-T3," Corrosion 56, 8 (2000): p. 819.

31. D. Zhu and W.J. van Ooij, "Corrosion protection of AA 2024-T3 by bis-[3(triethoxysilyl)propyl]tetrasulfide in neutral sodium chloride solution. Part 1: corrosion of AA 2024-T3," Corrosion Science 45, 10 (2003): p. 2163.

32. K.A. Yasakau, M.L. Zheludkevich, S.V. Lamaka, and M.G.S. Ferreira, "Mechanism of Corrosion Inhibition of AA2024 by Rare-Earth Compounds," The Journal of Physical Chemistry B 110, 11 (2006): p. 5515. 
33. M.A. Jakab, F. Presuel-Moreno, and J.R. Scully, "Critical Concentrations Associated with Cobalt, Cerium, and Molybdenum Inhibition of AA2024-T3 Corrosion: Delivery from Al-Co-Ce(-Mo) Alloys," CORROSION 61, 3 (2005): p. 246.

34. G.O. Ilevbare and J.R. Scully, "Mass-transport-limited oxygen reduction reaction on AA2024-T3 and selected intermetallic compounds in chromate-containing solutions," Corrosion 57, 2 (2001): p. 134.

35. G.O. Ilevbare and J.R. Scully, "Oxygen Reduction Reaction Kinetics on Chromate Conversion Coated $\mathrm{Al}-\mathrm{Cu}, \mathrm{Al}-\mathrm{Cu}-\mathrm{Mg}$, and $\mathrm{Al}-\mathrm{Cu}-\mathrm{Mn}-\mathrm{Fe}$ Intermetallic Compounds," Journal of The Electrochemical Society 148, 5 (2001): p. B196.

36. G.O. Ilevbare, J.R. Scully, J. Yuan, and R.G. Kelly, "Inhibition of pitting corrosion on aluminum alloy 2024-T3: Effect of soluble chromate additions vs chromate conversion coating," Corrosion 56, 3 (2000): p. 227.

37. M.W. Kendig and R.G. Buchheit, "Corrosion inhibition of aluminum and aluminum alloys by soluble chromates, chromate coatings, and chromate-free coatings," Corrosion 59, 5 (2003): p. 379.

38. Y. Yoon and R.G. Buchheit, "Dissolution Behavior of $\mathrm{Al}_{2} \mathrm{CuMg}$ (S Phase) in Chloride and Chromate Conversion Coating Solutions," Journal of the Electrochemical Society 153, 5 (2006): p. B151.

39. W. Zhang, B. Hurley, and R.G. Buchheit, "Characterization of Chromate Conversion Coating Formation and Breakdown Using Electrode Arrays," Journal of The Electrochemical Society 149, 8 (2002): p. B357.

40. OSHA, IMIS Code Number 0689 Chromium (VI) (Hexavalent Chromium). 2006.

41. R.L. Twite and G.P. Bierwagen, "Review of alternatives to chromate for corrosion protection of aluminum aerospace alloys," Progress in Organic Coatings 33, 2 (1998): p. 91.

42. W.D. Robertson, "Molybdate and Tungstate as Corrosion Inhibitors and the Mechanism of Inhibition," Journal of The Electrochemical Society 98, 3 (1951): p. 94.

43. K.D. Ralston, S. Chrisanti, T.L. Young, and R.G. Buchheit, "Corrosion inhibition of aluminum alloy 2024-T3 by aqueous vanadium species," Journal of the Electrochemical Society 155, 7 (2008): p. C350.

44. M. Iannuzzi and G.S. Frankel, "Inhibition of Aluminum Alloy 2024 Corrosion by Vanadates: An In Situ Atomic Force Microscopy Scratching Investigation," Corrosion 63, 7 (2007): p. 672.

45. M. Iannuzzi and G.S. Frankel, "Mechanisms of corrosion inhibition of AA2024-T3 by vanadates," Corrosion Science 49, 5 (2007): p. 2371.

46. M. Iannuzzi, J. Kovac, and G.S. Frankel, "A study of the mechanisms of corrosion inhibition of AA2024-T3 by vanadates using the split cell technique," Electrochimica Acta 52, 12 (2007): p. 4032.

47. M. Iannuzzi, T. Young, and G.S. Frankel, "Aluminum alloy corrosion inhibition by vanadates," Journal of the Electrochemical Society 153, 12 (2006): p. B533.

48. K.D. Ralston, T.L. Young, and R.G. Buchheit, "Electrochemical Evaluation of Constituent Intermetallics in Aluminum Alloy 2024-T3 Exposed to Aqueous Vanadate Inhibitors," Journal of the Electrochemical Society 156, 4 (2009): p. C135. 
49. E.A. Lizlovs, "Molybdates as Corrosion-Inhibitors in Presence of Chlorides," Corrosion 32, 7 (1976): p. 263.

50. A.H. Alsaffar, V. Ashworth, A.K.O. Bairamov, D.J. Chivers, W.A. Grant, and R.P.M. Procter, "Effect of Molybdenum Ion-Implantation on the General and Pitting Corrosion Behavior of Pure Aluminum and a High-Strength AluminumAlloy," Corrosion Science 20, 1 (1980): p. 127.

51. S.B. Madden and J.R. Scully, "Inhibition of AA2024-T351 Corrosion Using Permanganate," Journal of The Electrochemical Society 161, 3 (2014): p. C162.

52. M.A. Jakab, F. Presuel-Moreno, and J.R. Scully, "Effect of Molybdate, Cerium, and Cobalt Ions on the Oxygen Reduction Reaction on AA2024-T3 and Selected Intermetallics," Journal of The Electrochemical Society 153, 7 (2006): p. B244.

53. B.D. Chambers and S.R. Taylor, "The high throughput assessment of aluminium alloy corrosion using fluorometric methods. Part II - A combinatorial study of corrosion inhibitors and synergistic combinations," Corrosion Science 49, 3 (2007): p. 1597.

54. R.L. Cook and S.R. Taylor, "Pigment-derived inhibitors for aluminum alloy 2024T3," Corrosion 56, 3 (2000): p. 321.

55. E. McCafferty, Introduction to corrosion science (New York: Springer, 2010). p. xvi.

56. M.A. Jakab, D.A. Little, and J.R. Scully, "Experimental and Modeling Studies of the Oxygen Reduction Reaction on AA2024-T3," Journal of The Electrochemical Society 152, 8 (2005): p. B311.

57. M.A. Jakab, F. Presuel-Moreno, and J.R. Scully, "Critical Concentrations Associated with Cobalt, Cerium, and Molybdenum Inhibition of AA2024-T3 Corrosion: Delivery from Al-Co-Ce(-Mo) Alloys , March 2005," CORROSION 61, 03 (2005).

58. A.J. Aldykewicz, H.S. Isaacs, and A.J. Davenport, "The Investigation of Cerium as a Cathodic Inhibitor for Aluminum-Copper Alloys," Journal of The Electrochemical Society 142, 10 (1995): p. 3342.

59. F. Andreatta, M.E. Druart, A. Lanzutti, M. Lekka, D. Cossement, M.G. Olivier, and L. Fedrizzi, "Localized corrosion inhibition by cerium species on clad AA2024 aluminium alloy investigated by means of electrochemical micro-cell," Corrosion Science 65, 0 (2012): p. 376.

60. B. Davó, A. Conde, and J.J. de Damborenea, "Inhibition of stress corrosion cracking of alloy AA8090 T-8171 by addition of rare earth salts," Corrosion Science 47, 5 (2005): p. 1227.

61. X.F. Liu, S.J. Huang, and H.C. Gu, "Crack growth behaviour of high strength aluminium alloy in $3.5 \% \mathrm{NaCl}$ solution with corrosion inhibiting pigments," International Journal of Fatigue 24, 7 (2002): p. 803.

62. D.G. Kolman and J.R. Scully, "Limitations of Potentiostatic Repassivation Techniques and Their Relationship to the Applicability of the High-Field Approximation to the Repassivation of Titanium," Journal of the Electrochemical Society 142, 7 (1995): p. 2179.

63. G.S. Frankel, C.V. Jahnes, V. Brusic, and A.J. Davenport, "Repassivation Transients Measured with the Breaking-Electrode Technique on Aluminum ThinFilm Samples," Journal of the Electrochemical Society 142, 7 (1995): p. 2290. 
64. G.S. Frankel, B.M. Rush, C.V. Jahnes, C.E. Farrell, A.J. Davenport, and H.S. Isaacs, "Repassivation Transients Measured with Thin Film Breaking Electrodes," Journal of The Electrochemical Society 138, 2 (1991): p. 643.

65. R.P. Frankenthal, J. Kruger, and Electrochemical Society., Passivity of metals : (proceedings of the fourth International Symposium on Passivity). The Corrosion monograph series (Princeton, N.J.: Electrochemical Society, 1978). p. xviii.

66. R. Oltra and G.M. Indrianjafy, "Depassivation Experiments by Laser-PulseInduced Spallation," Journal of The Electrochemical Society 139, 9 (1992): p. L78.

67. G.T. Burstein and R.J. Cinderey, "Evolution of the corrosion potential of repassivating aluminium surfaces," Corrosion Science 33, 3 (1992): p. 475.

68. N.D. Tomashov and L.P. Vershinina, "Kinetics of some electrode processes on a continuously renewed surface of solid metal," Electrochimica Acta 15, 4 (1970): p. 501.

69. D.G. Kolman and J.R. Scully, "On the repassivation behavior of high-purity titanium and selected alpha, beta, and beta+alpha titanium alloys in aqueous chloride solutions," Journal of the Electrochemical Society 143, 6 (1996): p. 1847.

70. G.T. Burstein and D.H. Davies, "The Electrochemical Behavior of Scratched Iron Surfaces in Aqueous Solutions," Journal of The Electrochemical Society 128, 1 (1981): p. 33.

71. G.T. Burstein and P.I. Marshall, "Growth of Passivating Films on Scratched 3041 Stainless-Steel in Alkaline-Solution," Corrosion Science 23, 2 (1983): p. 125.

72. R.S. Lillard, G. Vasquez, and D.F. Bahr, "The Kinetics of Anodic Dissolution and Repassivation on Stainless Steel 304L in Solutions Containing Nitrate," Journal of the Electrochemical Society 158, 6 (2011): p. C194.

73. D.G. Kolman and J.R. Scully, "On the Repassivation Behavior of High-Purity Titanium and Selected alpha, beta, and beta + alpha Titanium Alloys in Aqueous Chloride Solutions," Journal of The Electrochemical Society 143, 6 (1996): p. 1847.

74. D.G. Kolman, M.A. Gaudett, and J.R. Scully, "Modeling of anodic current transients resulting from oxide rupture of plastically strained beta+alpha titanium," Journal of the Electrochemical Society 145, 6 (1998): p. 1829.

75. D.G. Kolman and J.R. Scully, "An assessment of the crack tip potential of betatitanium alloys during hydrogen environmentally assisted crack propagation based on crack tip and passive surface electrochemical measurements," Corrosion Science 42, 11 (2000): p. 1863.

76. B.W. Madsen and T.A. Adler, "Passivation and repassivation kinetics of ironaluminum alloys in $1 \mathrm{~N} \mathrm{H} 2 \mathrm{SO} 4$ using potential step and scratch tests," Wear 171, 1-2 (1994): p. 215.

77. F.P. Ford, G.T. Burstein, and T.P. Hoar, "Bare Surface Reaction Rates and Their Relation to Environment Controlled Cracking of Aluminum Alloys," Journal of The Electrochemical Society 127, 6 (1980): p. 1325.

78. C. Devicharan, M.J. Vasquez, P.H. Gary, and R.C. Clive, "Studies on the repassivation behavior of aluminum and aluminum alloy exposed to chromate solutions," Surface and Interface Analysis 35, 2 (2003): p. 226. 
79. J.S. Warner, S. Kim, and R.P. Gangloff, "Molybdate inhibition of environmental fatigue crack propagation in $\mathrm{Al}-\mathrm{Zn}-\mathrm{Mg}-\mathrm{Cu}$," International Journal of Fatigue 31, 11-12 (2009): p. 1952.

80. Z.M. Gasem, Rate-Limiting Process in Environmental Fatigue Crack Propagation in 7000-Series Aluminum Alloys in TMS-AIME, R.H. Jones, Editor. 2001: Warrendale PA. p. 501.

81. G.A. Young Jr and J.R. Scully, "The diffusion and trapping of hydrogen in high purity aluminum," Acta Materialia 46, 18 (1998): p. 6337.

82. J.W. Bibber, US Patent 48956081990.

83. J.W. Bibber, US Patent 55542311996.

84. ASTM, B-117 - Standard Practice for Operating Salt Spray (Fog) Apparatus, in ASTM. 2009.

85. J.W. Bibber, A Chrome Free Conversion Coating and Sealant for Aluminum and Its Alloys, in CORROSION 99. 1999, NACE International: San Antonio, Tx.

86. M. Crouse, K.L. Vasanth, A.E. Miller, and M.G. Pujar, Evaluation of Potassium Permanganate $\left(\mathrm{KMnO}_{4}\right)$ as a Green Corrosion Inhibitor/Sealant for Anodized Al 2024 and Al 6061 at Different pH Values, in CORROSION 2002. 2002, NACE International: Denver, Co.

87. S.A. Kulinich, M. Farzaneh, and X.W. Du, "Growth of corrosion-resistant manganese oxide coatings on an aluminum alloy," Inorg Mater+ 43, 9 (2007): p. 956.

88. F. Buciuman, F. Patcas, R. Craciun, and D.R.T. Zahn, "Vibrational spectroscopy of bulk and supported manganese oxides," Physical Chemistry Chemical Physics 1, 1 (1999): p. 185

89. I. Danilidis, A.J. Davenport, and J.M. Sykes, "Characterisation by X-ray absorption near-edge spectroscopy of $\mathrm{KMnO} 4-$ based no-rinse conversion coatings on $\mathrm{Al}$ and Al alloys," Corrosion Science 49, 4 (2007): p. 1981.

90. I. Danilidis, J. Hunter, G.M. Scamans, and J.M. Sykes, "Effects of inorganic additions on the performance of manganese-based conversion treatments," Corrosion Science 49, 3 (2007): p. 1559.

91. I. Danilidis, J. Hunter, G.M. Scamans, and J.M. Sykes, "Effect of silica nanoparticles on the performance of manganese-based conversion treatments," Corrosion Science 50, 11 (2008): p. 3038.

92. B.A.L. Demishima, T. Ohtsuka, H. Konno, and N. Sato, "XPS Study of the $\mathrm{MnO}_{2}$ Electrode in Borate Solution during the Discharge Process," Electrochimica Acta 36, 9 (1991): p. 1485.

93. B.A.L. Demishima, T. Ohtsuka, and N. Sato, "A Study of the Discharge Process of Manganese Oxide in Borate Solution Using Insitu Techniques," Electrochimica Acta 38, 2-3 (1993): p. 341.

94. L.M. Gassa, H.T. Mishima, B.A.L. deMishima, and J.R. Vilche, "An electrochemical impedance spectroscopy study of electrodeposited manganese oxide films in borate buffers," Electrochimica Acta 42, 11 (1997): p. 1717.

95. W.H. Ho and S.K. Yen, "Characterization of Electrolytic Manganese Oxide Coating on Pt for Lithium Battery Applications," Journal of The Electrochemical Society 152, 3 (2005): p. A506. 
96. M.J. Humphries and R.N. Parkins, "Stress-corrosion cracking of mild steels in sodium hydroxide solutions containing various additional substances," Corrosion Science 7, 11 (1967): p. 747.

97. D.D. Perrin and International Union of Pure and Applied Chemistry. Commission on Electroanalytical Chemistry., Dissociation constants of inorganic acids and bases in aqueous solution (London,: Butterworths, 1969). p. v.

98. D. Ho, N. Brack, J. Scully, T. Markley, M. Forsyth, and B. Hinton, "Cerium Dibutylphosphate as a Corrosion Inhibitor for AA2024-T3 Aluminum Alloys," Journal of The Electrochemical Society 153, 9 (2006): p. B392.

99. A.J. Aldykiewicz, A.J. Davenport, and H.S. Isaacs, "Studies of the formation of cerium-rich protective films using x-ray absorption near-edge spectroscopy and rotating disk electrode methods," Journal of the Electrochemical Society 143, 1 (1996): p. 147.

100. X. Yu and G. Li, "XPS study of cerium conversion coating on the anodized 2024 aluminum alloy," Journal of Alloys and Compounds 364, 1-2 (2004): p. 193.

101. M.L. Zheludkevich, R. Serra, M.F. Montemor, K.A. Yasakau, I.M.M. Salvado, and M.G.S. Ferreira, "Nanostructured sol-gel coatings doped with cerium nitrate as pretreatments for AA2024-T3: Corrosion protection performance," Electrochimica Acta 51, 2 (2005): p. 208.

102. F.J. Presuel-Moreno, M.A. Jakab, and J.R. Scully, "Inhibition of the oxygen reduction reaction on copper with cobalt, cerium, and molybdate ions," Journal of the Electrochemical Society 152, 9 (2005): p. B376.

103. S.A. Hayes, P. Yu, T.J. O'Keefe, M.J. O'Keefe, and J.O. Stoffer, "The phase stability of cerium species in aqueous systems - I. E-pH diagram for the Ce-HClO4H2O system," Journal of the Electrochemical Society 149, 12 (2002): p. C623.

104. A.S. Hamdy, A.M. Beccaria, and P. Traverso, "Corrosion protection of aluminium metal-matrix composites by cerium conversion coatings," Surface and Interface Analysis 34, 1 (2002): p. 171.

105. A.J. Davenport, H.S. Isaacs, and M.W. Kendig, "Xanes Investigation of the Role of Cerium Compounds as Corrosion-Inhibitors for Aluminum," Corrosion Science 32, 5-6 (1991): p. 653.

106. A. Wilson, in MSE. 2014, University of Virginia: Charlottesville.

107. M.W. Kendig, Chromate-Genrating Corrosion Inhibitor 2010, US 7691498 B2: United States.

108. M. Kendig, M. Cunningham, and R. Evans, "A 'Smart' Corrosion-Inhibiting Pigment Based on a Chemical Reaction," ECS Transactions 24, 1 (2010): p. 27.

109. B. Davo and J.J. de Damborenea, "Use of rare earth salts as electrochemical corrosion inhibitors for an Al-Li-Cu (8090) alloy in 3.56\% NaCl," Electrochimica Acta 49, 27 (2004): p. 4957.

110. J. Sinko, "Challenges of chromate inhibitor pigments replacement in organic coatings," Progress in Organic Coatings 42, 3-4 (2001): p. 267.

111. L. Xia and R.L. McCreery, "Chemistry of a chromate conversion coating on aluminum alloy AA2024-T3 probed by vibrational spectroscopy," Journal of the Electrochemical Society 145, 9 (1998): p. 3083. 
112. H. Böhni and H.H. Uhlig, "Environmental Factors Affecting the Critical Pitting Potential of Aluminum," Journal of The Electrochemical Society 116, 7 (1969): p. 906.

113. S.T. Pride, J.R. Scully, and J.L. Hudson, "Metastable Pitting of Aluminum and Criteria for the Transition to Stable Pit Growth," Journal of the Electrochemical Society 141, 11 (1994): p. 3028.

114. R.P. Frankenthal, "The inhibition of pitting and stress-corrosion cracking of $\mathrm{Mg}-\mathrm{Al}$ alloys by NO3-," Corrosion Science 7, 1 (1967): p. 61.

115. J. Zhao, G. Frankel, and R.L. McCreery, "Corrosion protection of untreated AA2024-T3 in chloride solution by a chromate conversion coating monitored with Raman spectroscopy," Journal of the Electrochemical Society 145, 7 (1998): p. 2258.

116. A. Sehgal, D. Lu, and G.S. Frankel, "Pitting in Aluminum Thin Films: Supersaturation and Effects of Dichromate Ions," Journal of The Electrochemical Society 145, 8 (1998): p. 2834.

117. P.L. Hagans and C.M. Haas, "Influence of Metallurgy on the Protective Mechanism of Chromium-Based Conversion Coatings on Aluminum-Copper Alloys," Surface and Interface Analysis 21, 2 (1994): p. 65.

118. K. Asami, M. Oki, G.E. Thompson, G.C. Wood, and V. Ashworth, "Composition of the near surface regions of conversion coated aluminium," Electrochimica Acta 32, 2 (1987): p. 337.

119. X.F. Liu, S.J. Huang, and H.C. Gu, "The effect of corrosion inhibiting pigments on environmentally assisted cracking of high strength aluminum alloy," Corrosion Science 45, 9 (2003): p. 1921.

120. R.J. Cinderey and G.T. Burstein, "The effects of chromate on the transient repassivation potential of aluminium in chloride solution," Corrosion Science 33, 3 (1992): p. 493.

121. P. Schmutz and G.S. Frankel, "Influence of dichromate ions on corrosion of pure aluminum and AA2024-T3 in $\mathrm{NaCl}$ solution studied by AFM scratching," Journal of the Electrochemical Society 146, 12 (1999): p. 4461.

122. V.S. Agarwala, "Multipurpose Corrosion Inhibitors for Aerospace Materials in Naval Environments " REPORT NO. NADC-87034-60 (1987).

123. V. Moutarlier, M.P. Gigandet, B. Normand, and J. Pagetti, "EIS characterisation of anodic films formed on 2024 aluminium alloy, in sulphuric acid containing molybdate or permanganate species," Corrosion Science 47, 4 (2005): p. 937.

124. G.T. Burstein and D.H. Davies, "The Effects of Anions on the Behavior of Scratched Iron Electrodes in Aqueous-Solutions," Corrosion Science 20, 10 (1980): p. 1143.

125. R.G. Kelly and R.C. Newman, "Confirmation of the Applicability of Scratched Electrode Techniques for the Determination of Bare Surface Current Densities," Journal of the Electrochemical Society 137, 1 (1990): p. 357.

126. P.D. Bastek, R.C. Newman, and R.G. Kelly, "Measurement of Passive Film Effects on Scratched Electrode Behavior," Journal of the Electrochemical Society 140, 7 (1993): p. 1884.

127. J.R. Scully, D.C. Silverman, and M.W. Kendig, Electrochemical Impedance: Analysis and Interpretation. Stp (Philadelphia: ASTM, 1993. 
128. ASTM, Characterization of the Corrosion of Aluminum Thin Films using Electrochemical Impedance Methods in Electrochemical Impedance: Analysis and Interpretation. 1993: Philadelphia. p. 276.

129. C.H. Hamann, A. Hamnett, and W. Vielstich, Electrochemistry (Weinheim ; New York: Wiley-VCH, 1998). p. xvii.

130. J.R. Scully, R.P. Frankenthal, K.J. Hanson, D.J. Siconolfi, and J.D. Sinclair, "Localized Corrosion of Sputtered Aluminum and Al-0.5-Percent Cu Alloy ThinFilms in Aqueous $\mathrm{Hf}$ Solution .2. Inhibition by $\mathrm{CO}_{2}$," Journal of the Electrochemical Society 137, 5 (1990): p. 1373. 


\section{$1.11 \quad$ Tables}

Table 1.1. Corrosion potentials for intermetallic compounds common in aluminum alloys.

\begin{tabular}{|c|c|c|c|c|c|}
\hline \multirow[b]{2}{*}{ Stoichiometry } & \multirow[b]{2}{*}{ Phase } & \multicolumn{3}{|c|}{ Corrosion potential $\left(\mathrm{mV}_{\mathrm{SCE}}\right)$} & \multirow[b]{2}{*}{ Note } \\
\hline & & $0.01 \mathrm{M}$ & $0.1 \mathrm{M}$ & $0.6 \mathrm{M}$ & \\
\hline $\mathrm{Al}_{3} \mathrm{Fe}$ & $\beta$ & -493 & -539 & -566 & \\
\hline $\mathrm{Al}_{2} \mathrm{Cu}$ & $\theta$ & -592 & -665 & -695 & \\
\hline $\mathrm{Al}_{3} \mathrm{Zr}$ & $\beta$ & -752 & -776 & -801 & \\
\hline $\mathrm{Al}_{6} \mathrm{Mn}$ & - & -839 & -779 & -913 & \\
\hline $\mathrm{Al}_{3} \mathrm{Ti}$ & $\beta$ & -620 & -603 & -799 & \\
\hline $\mathrm{Al}_{32} \mathrm{Zn}_{49}$ & $\mathbf{T}^{\prime}$ & -1009 & -1004 & -1063 & \\
\hline $\mathrm{Mg}_{2} \mathrm{Al}_{3}$ & $\beta$ & -1124 & -1013 & -1162 & \\
\hline $\mathrm{MgZn}_{2}$ & $\mathbf{M}, \eta$ & -1001 & -1029 & -1095 & \\
\hline $\mathrm{Mg}_{2} \mathrm{Si}$ & $\beta$ & -1355 & -1538 & -1536 & \\
\hline $\mathrm{Al}_{7} \mathrm{Cu}_{2} \mathrm{Fe}$ & - & -549 & -551 & -654 & \\
\hline $\mathrm{Mg}(\mathrm{A} 1 \mathrm{Cu})$ & - & -898 & -943 & -936 & \\
\hline $\mathrm{Al}_{2} \mathrm{CuMg}$ & $\mathrm{S}$ & -956 & -883 & -1061 & \\
\hline $\mathrm{Al}_{20} \mathrm{Cu}_{2} \mathrm{Mn}_{3}$ & - & -550 & -565 & -617 & \\
\hline $\mathrm{Al}_{12} \mathrm{Mn}_{3} \mathrm{Si}$ & - & -890 & -810 & -858 & \\
\hline A1 (99.9999) & - & -679 & -823 & -849 & A \\
\hline $\mathrm{Cu}(99.9)$ & - & -177 & -232 & -220 & A \\
\hline Si (99.9995) & $\alpha$ & -450 & -441 & -452 & A \\
\hline $\mathrm{Mg}(99.9)$ & - & -1601 & -1586 & -1688 & A \\
\hline Mn (99.9) & - & -1315 & -1323 & -1318 & A \\
\hline $\mathrm{Cr}(99.0)$ & - & -495 & -506 & -571 & A \\
\hline $\mathrm{Zn}(99.99)$ & - & -985 & -1000 & -1028 & A \\
\hline $\mathrm{A} 1-2 \% \mathrm{Cu}$ & $\alpha$ & -813 & -672 & -744 & B \\
\hline $\mathrm{A} 1-4 \% \mathrm{Cu}$ & $\alpha$ & -750 & -602 & -642 & B \\
\hline $7 X 75$ Matrix & - & -699 & -799 & -812 & $\mathbf{M}$ \\
\hline AA $7075-T 651$ & - & -816 & -965 & -1180 & $\mathrm{x}$ \\
\hline
\end{tabular}

Notes:

A. Pure metal were obtained from Alfa-Aeser and tested using the microcell method.

B. These specimens are homogeneous solid solutions and tested using the microcell method.

M. The phase denoted as $7 \times 75$ matrix is the particle-free matrix-phase of AA 7474

$\mathrm{X}$. Tests upon AA7075-T651 were done on bulk specimens using conventional electrochemical methods and an electrode area of $1 \mathrm{~cm}^{2}$

Reproduced with permission of JECS 
Table 1.2 Intermetallic particle statistics for AA2024 in sheet, rod and wire forms as well as AA7075-T6 sheet.

\begin{tabular}{lrrrrr}
\hline AA2024 & Avg. NND $(\mu \mathrm{m})$ & $\mathrm{SD}(\mu \mathrm{m})$ & Avg. $r(\mu \mathrm{m})$ & $\mathrm{SD}(\mu \mathrm{m})$ & Area $\%$ \\
& & & & & \\
\hline T351 sheet & 5.021 & 3.625 & 0.661 & 0.659 & 1.995 \\
T4 rod & 2.745 & 4.585 & 0.782 & 0.538 & 0.787 \\
T4 wire & 2.294 & 1.082 & 0.468 & 0.307 & 4.978 \\
\hline AA7075 & & & & & \\
\hline T-6 Sheet & 3.064 & 2.408 & 0.808 & 0.966 & 1.724
\end{tabular}

Table 1.3 Inhibitor road map showing areas of strength in the literature (Lit.) and areas where needs, gaps, and opportunities have been addressed in this thesis (Thesis). Some fields are not applicable (NA) due to inhibitor limitations.

\begin{tabular}{llllll}
\hline & $\begin{array}{l}\text { Surface } \\
\text { Corrosion }\end{array}$ & $\begin{array}{l}\text { Multiple } \\
\text { Inhibitor } \\
\text { Function }\end{array}$ & $\begin{array}{l}\text { Traditional } \\
\text { SCC \& EFCP }\end{array}$ & Repassivation & $\begin{array}{l}\text { Hydrogen } \\
\text { Uptake }\end{array}$ \\
\hline Chromate & Lit. & NA & Lit. & Thesis & Thesis \\
Molybdate & Lit. & Lit. & Lit. & Thesis & Thesis \\
Permanganate & Thesis & Thesis & No Lit. & Thesis & Thesis \\
Cerium & Lit. & Thesis & NA & NA & NA
\end{tabular}




\subsection{Figures}

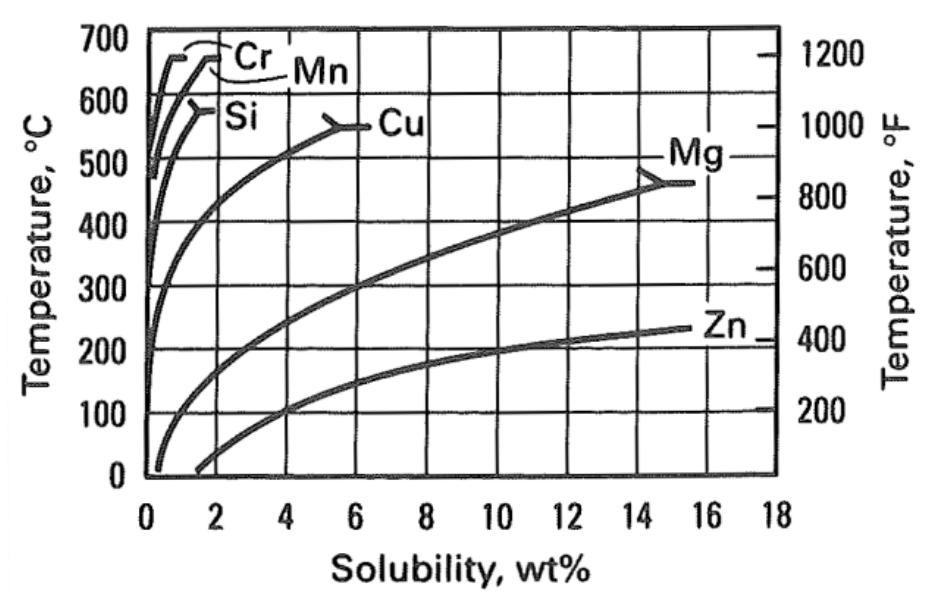

Figure 1.1 Binary solid solubility as a function of temperature for several of the most common alloying elements in aluminum alloys.

I.J. Polmear, Light alloys: from traditional alloys to nanocrystals. 4th ed (Oxford) Burlington, MA: Elsevier/Butterworth-Heinemann, 2006). p. xiv. Reproduced with permission from Elsevier. 


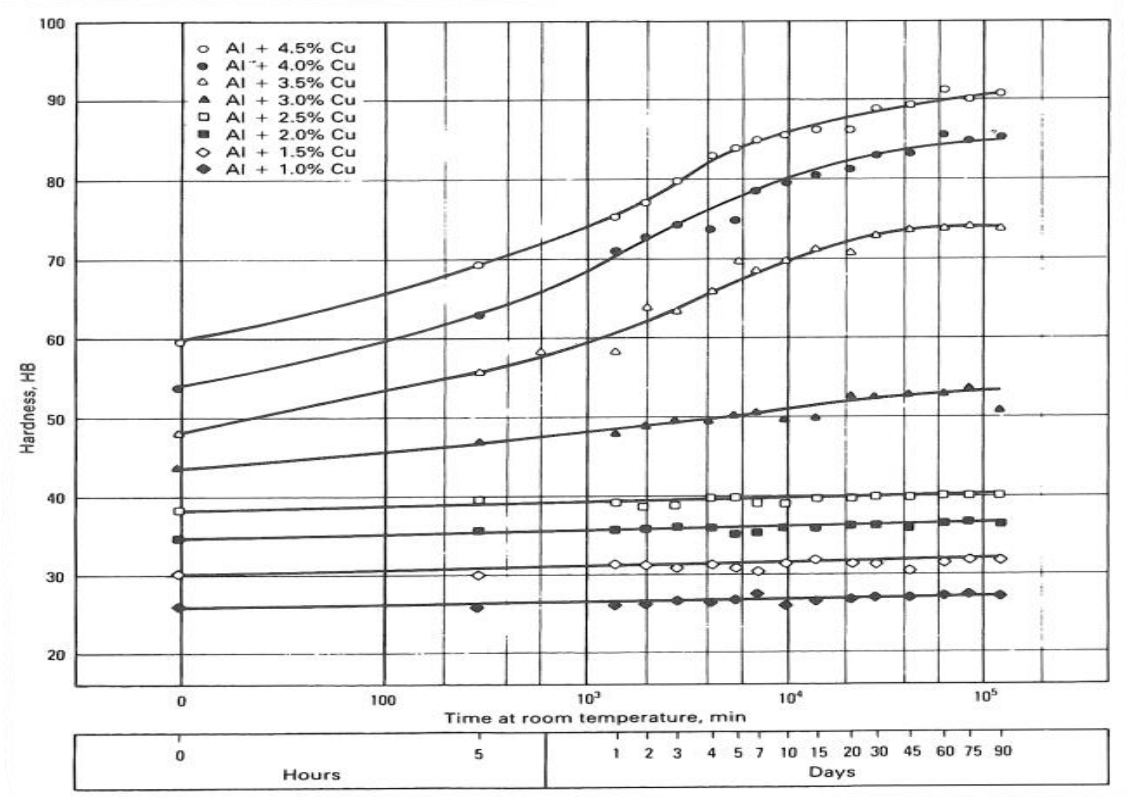

A

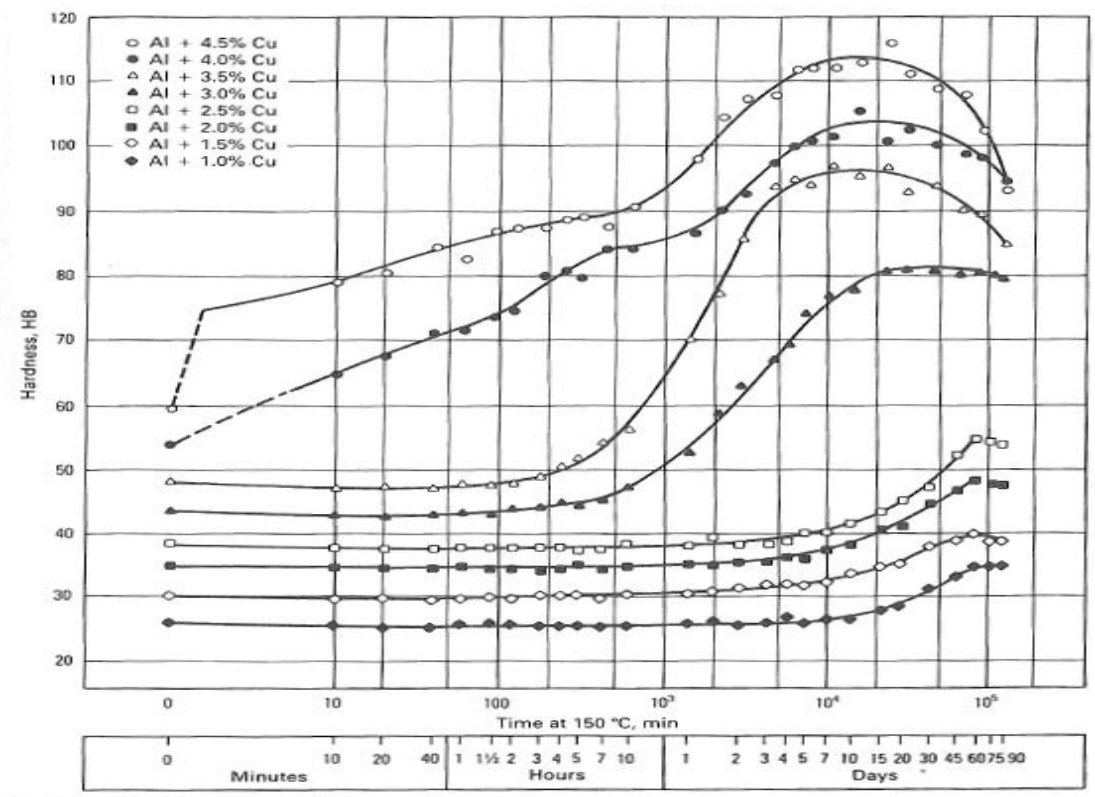

B

Figure 1.2. Natural aging (A) and artificial aging (B) time and hardness curves for binary $\mathrm{Al}-\mathrm{Cu}$ alloys showing age hardening due to formation of copper rich G-P zones in the natural case and transitional precipitates in the artificial case. In the artificial case the growth of the transitional precipitates at long aging times results in the conversion to equilibrium phase precipitates that are generally not coherent with the aluminum matrix leading to a decrease in hardness.

J. R. Davis \& Associates. and ASM International. Handbook Committee, Aluminum and aluminum alloys. ASM specialty handbook (Materials Park, OH: ASM International, 1993). p. iii. Reproduced with permission of ASM International. 


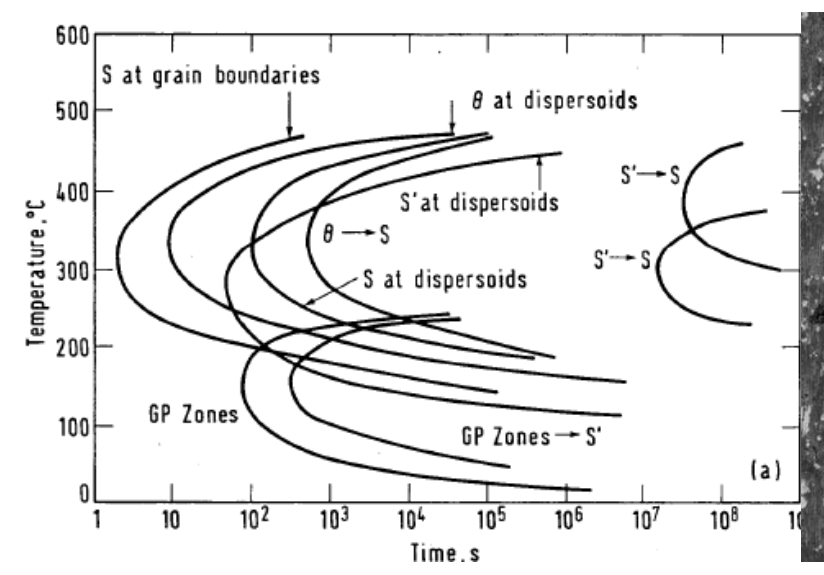

A

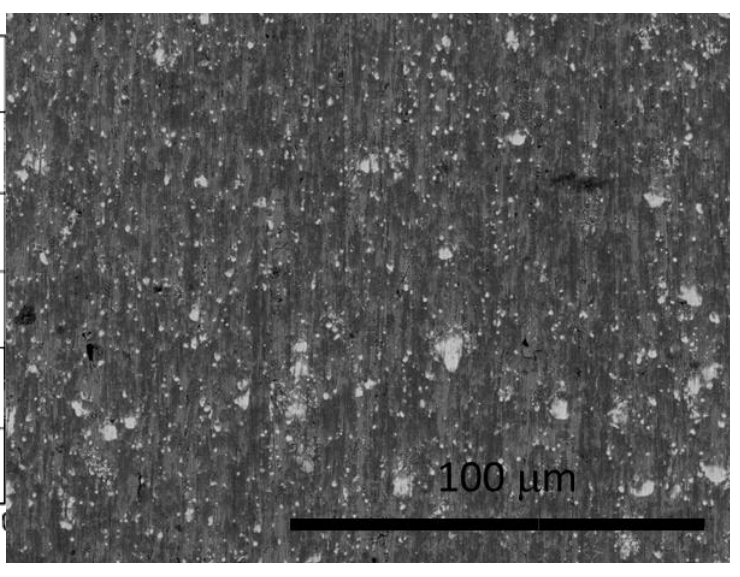

B

Figure 1.3. (A) TTT curve for AA2024 showing initial stages and aging progression of several strengthening precipitate phases. (B) Scanning electron microscope secondary electron image of coarse constituent particles in AA2024-T4. 

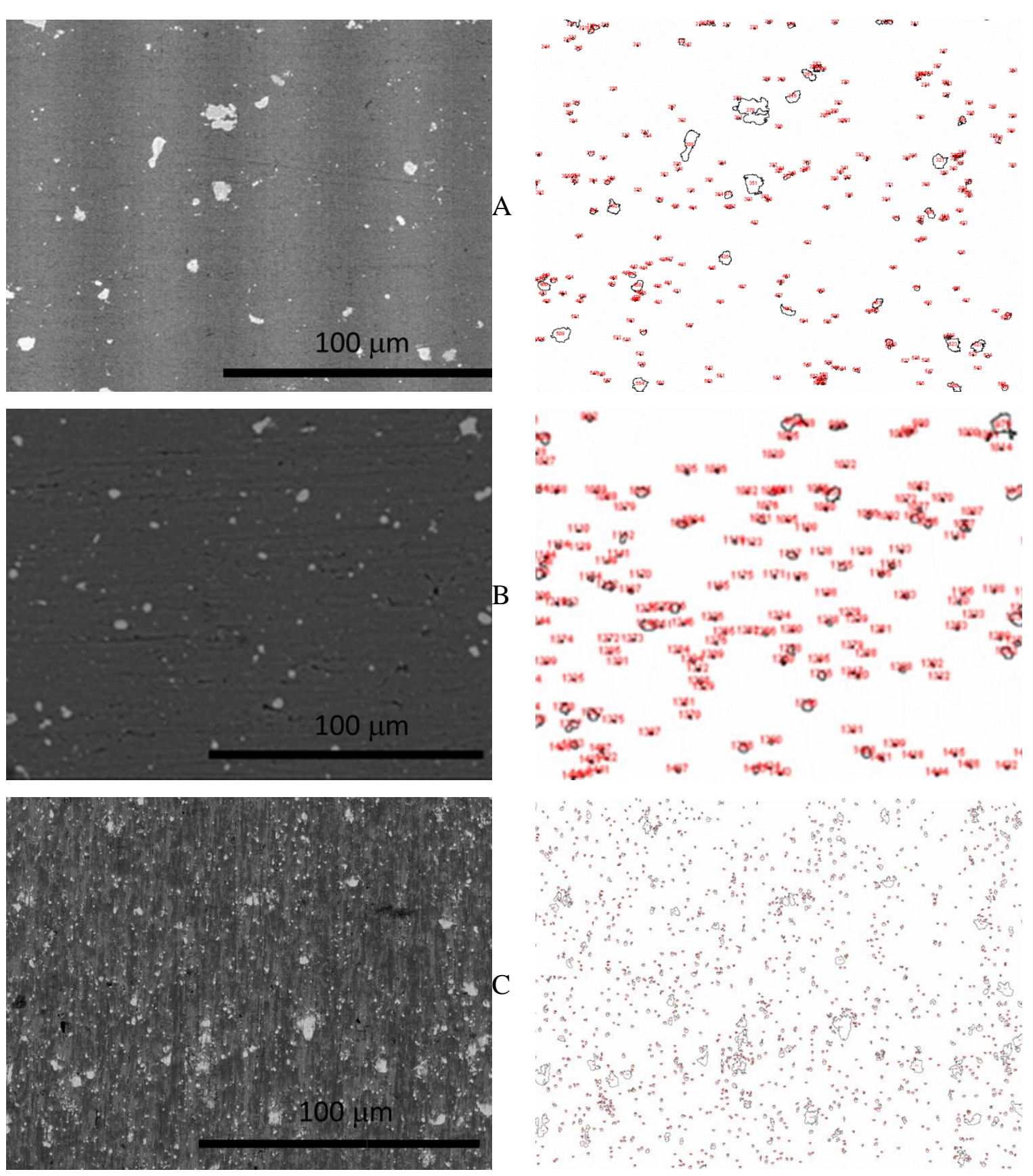

Figure 1.4 Backscattered electron detector image of AA2024-T351 sheet with corresponding intermetallic selection (A). AA2024-T4 rod - $13 \mathrm{~mm}$ diameter (B), AA2024-T4 wire - $254 \mu \mathrm{m}$ diameter (C). 


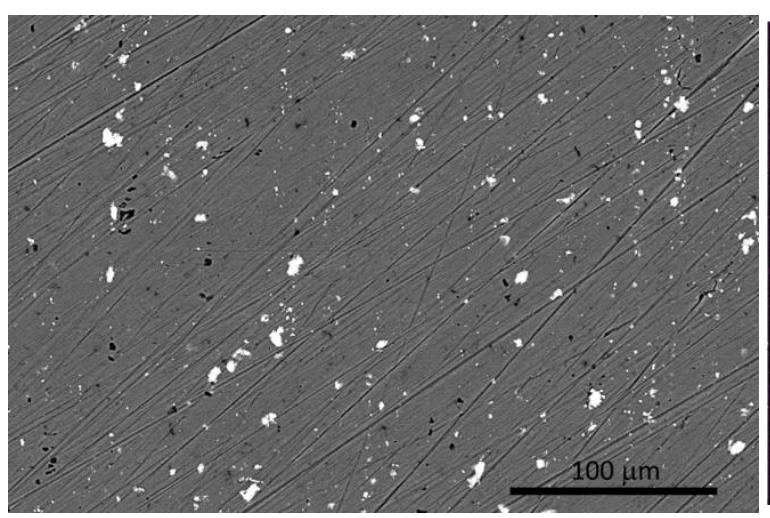

A

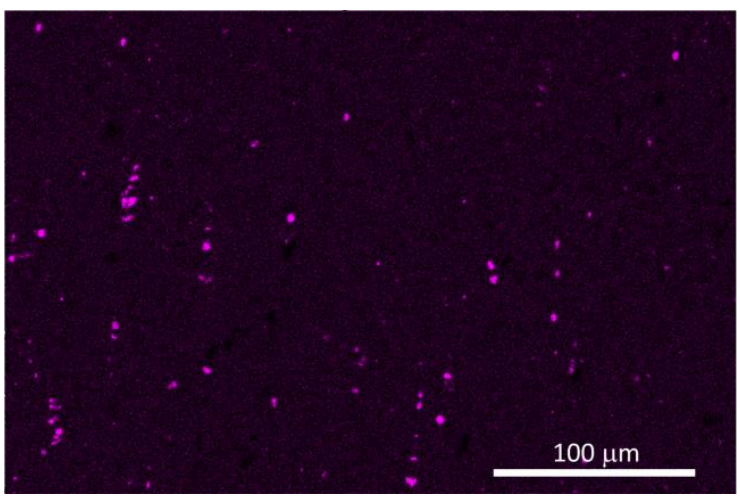

B

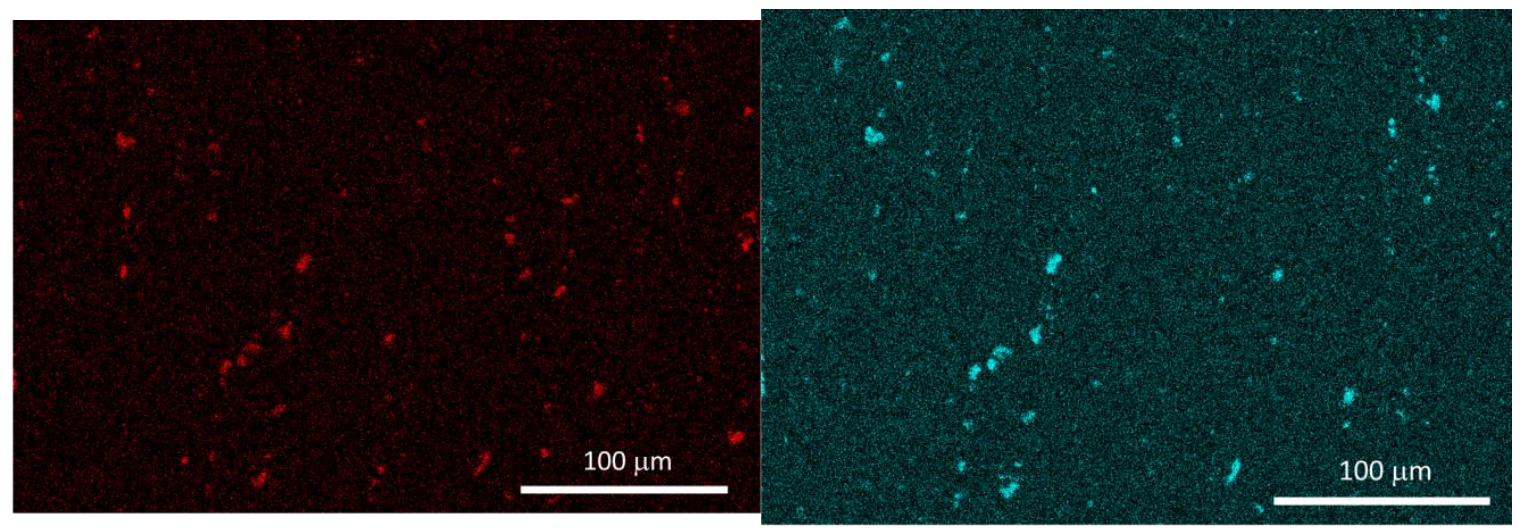

$\mathrm{C}$

$\mathrm{D}$

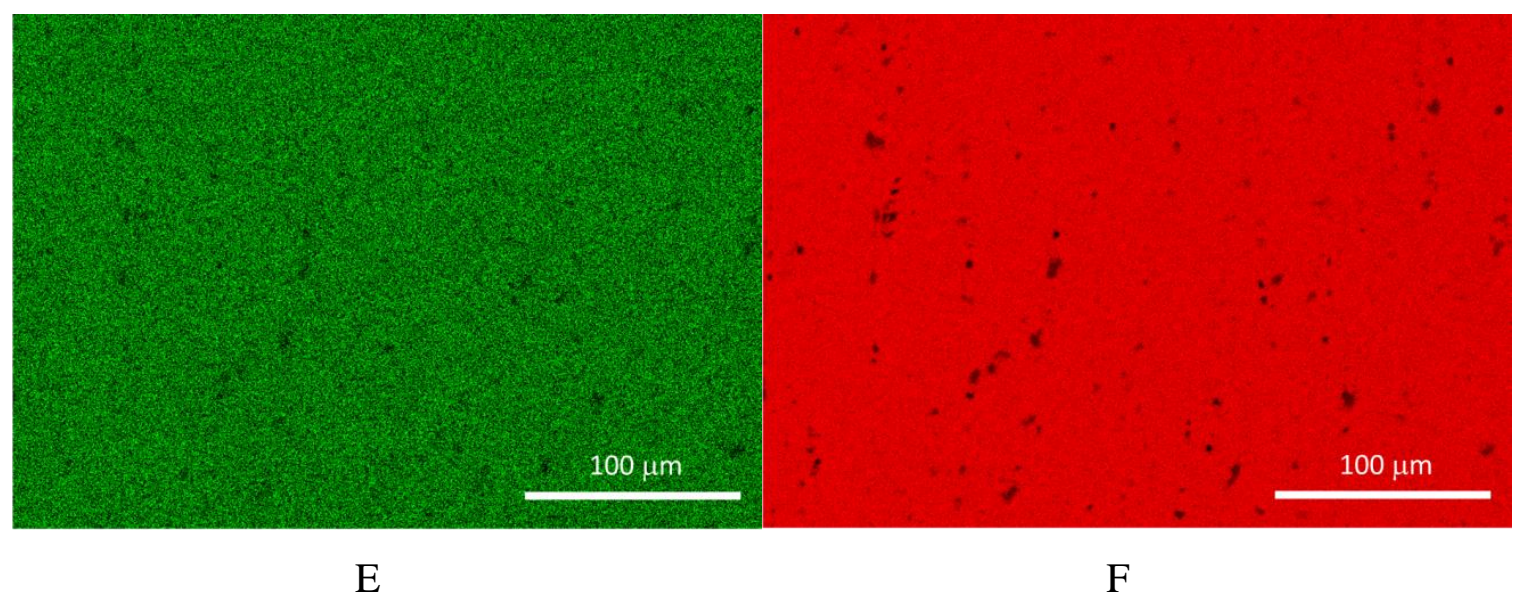

Figure 1.5. Backscatter SEM image of AA7075-T6 with accompanying EDS mapping conducted at $15 \mathrm{kV}$ accelerating voltage. B, C, D, E, and F are EDS maps of Mg, Fe, $\mathrm{Cu}, \mathrm{Zn}$, and $\mathrm{Al}$ respectively. 


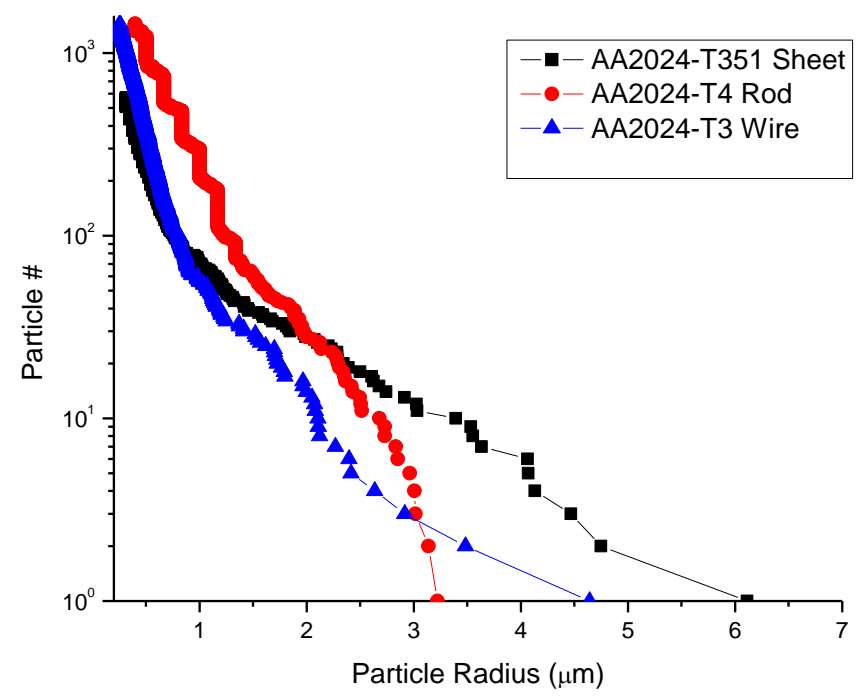

Figure 1.6. AA2024-T351 sheet, AA2024-T4 rod - 13 mm diameter, AA2024-T4 wire $254 \mu \mathrm{m}$ diameter particle statistics.

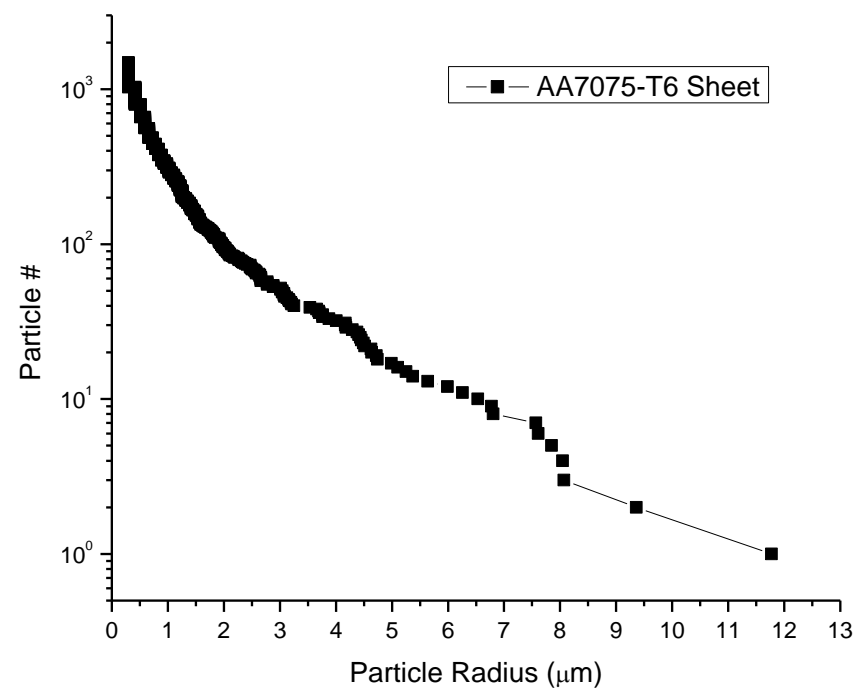

Figure 1.7. AA7075-T6 sheet particle radius statistics assuming spherical particles. 


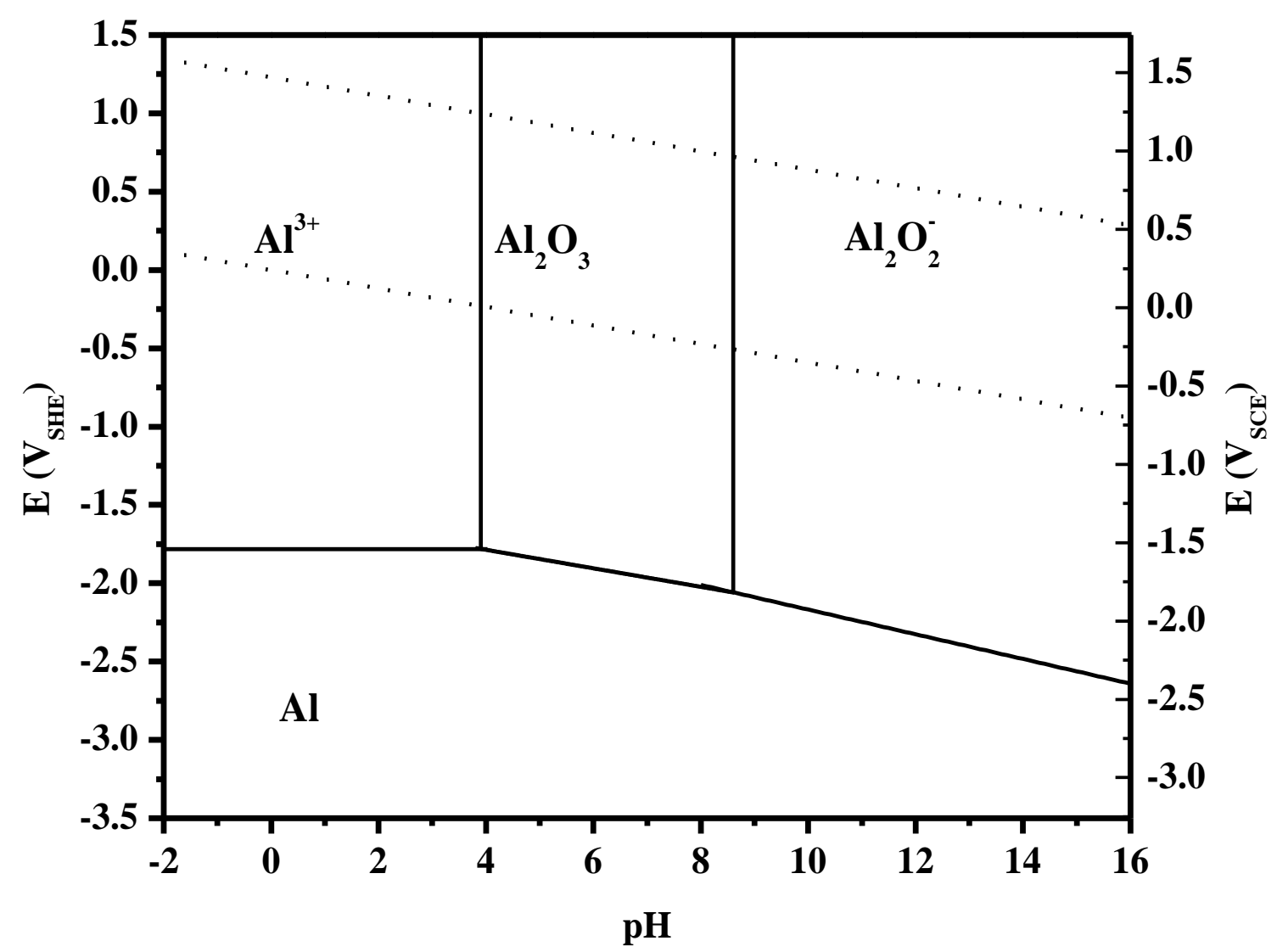

Figure 1.8. Potential-pH equilibrium (Pourbaix) diagram for the $\mathrm{Al}-\mathrm{H}_{2} \mathrm{O}$ system at $25^{\circ} \mathrm{C}$ assuming an ion concentration of $10^{-6}$.

Potential values are for the standard hydrogen electrode (SHE) scale.

Reproduced with permission from M. Pourbaix, Atlas of Electrochemical Equilibrium in Aqueous Solutions, NACE International (1974) p. 171 


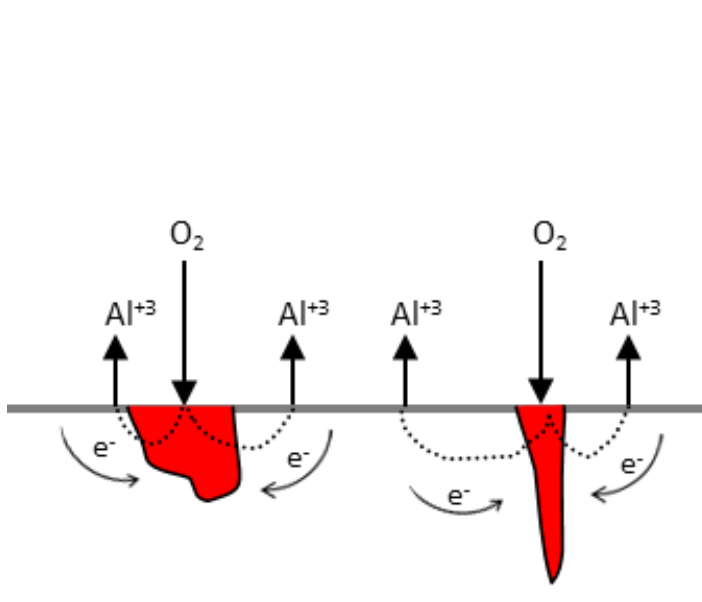

A

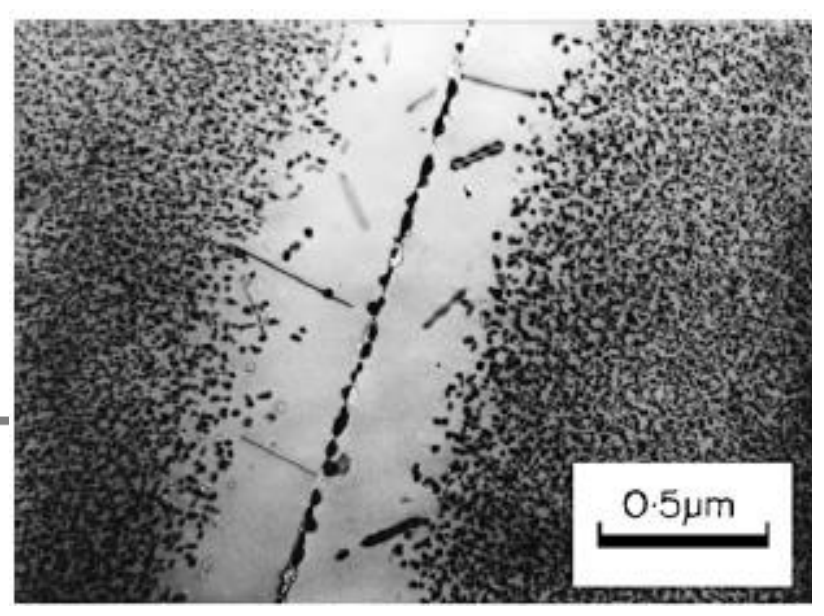

B

Figure 1.9. Galvanic couple between copper rich intermetallic and aluminum matrix (A). Precipitation free zone (PFZ) and accompanying grain boundary with large precipitate particles in an Al-Zn-Mg alloy.

Reproduced with permission from Elsevier. 

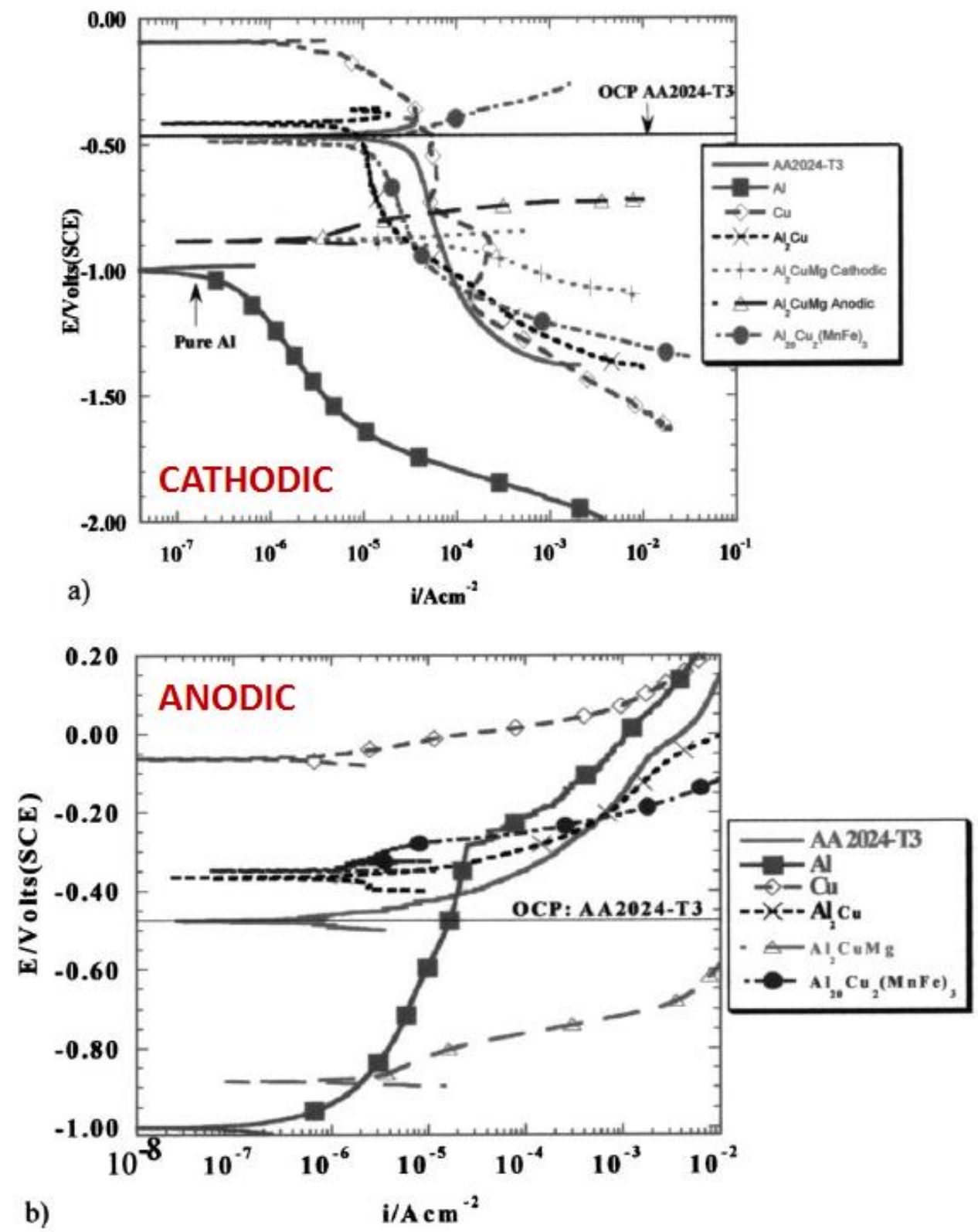

Figure 1.10. The E- $\log (\mathrm{i})$ behavior of stationary electrodes of $99.998 \% \mathrm{Al}, \mathrm{S}-\mathrm{Al}_{2} \mathrm{CuMg}$, AA2024-T3, $\theta-\mathrm{Al}_{2} \mathrm{Cu}, \mathrm{Al}_{20} \mathrm{Cu}_{2}(\mathrm{MnFe})_{3}$, and $99.999 \% \mathrm{Cu}$ in $0.1 \mathrm{M} \mathrm{Na}_{2} \mathrm{SO}_{4}+0.005 \mathrm{M}$ $\mathrm{NaCl}$ at ambient aeration. (a) cathodic (including anodic $\mathrm{S}-\mathrm{Al}_{2} \mathrm{CuMg}$ curve) and (b) anodic; all recorded after a $2 \mathrm{hr}$ OCP Reproduced with permission from J. Elec. Soc. 

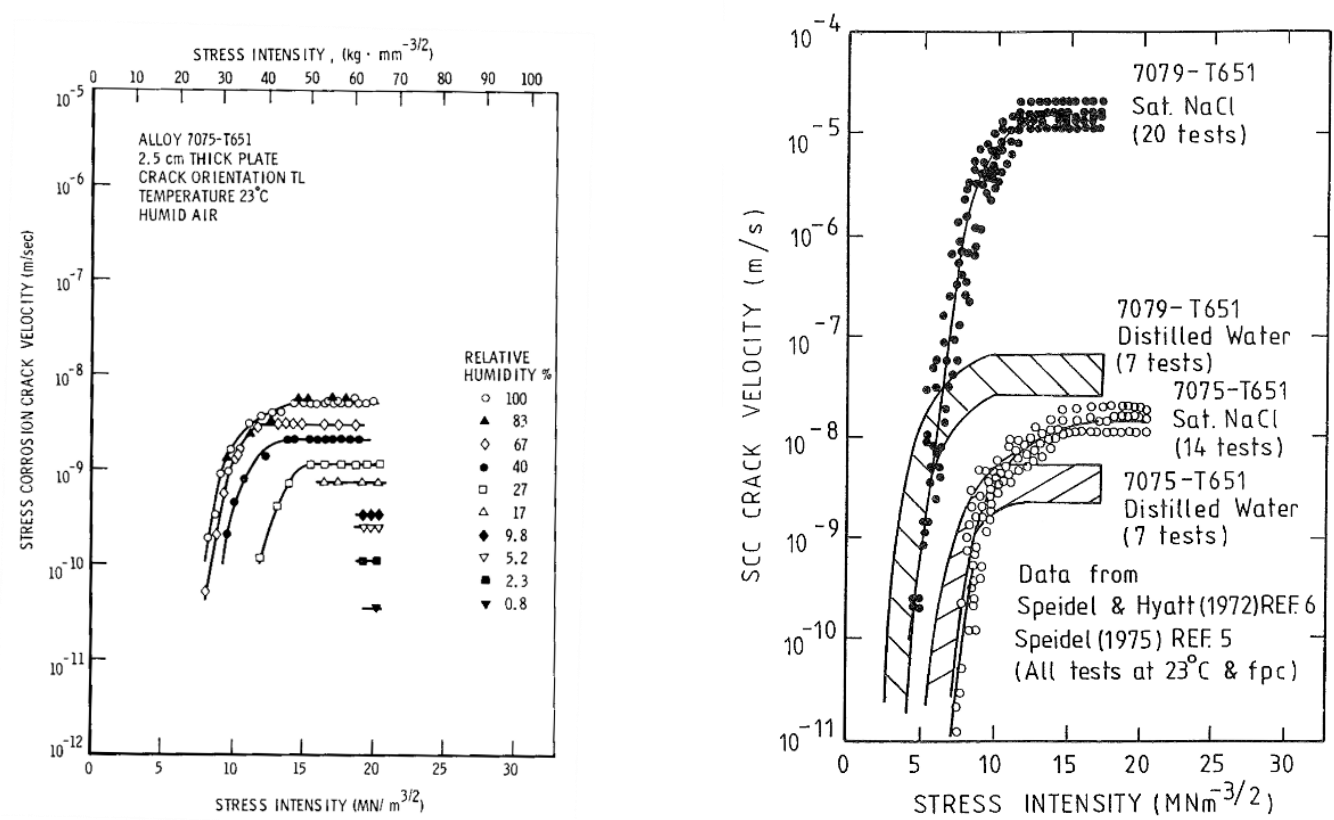

A

Figure 1.11. (A) The effect of relative humidity on the SCC velocity of AA7075-T651. (B) The effect of chloride concentration on SC crack velocity.

M.O. Speidel, "Stress-Corrosion Cracking of Aluminum-Alloys," Metall Trans A 6, 4 (1975): p. 631.Reproduced with permission from Metall Trans. 


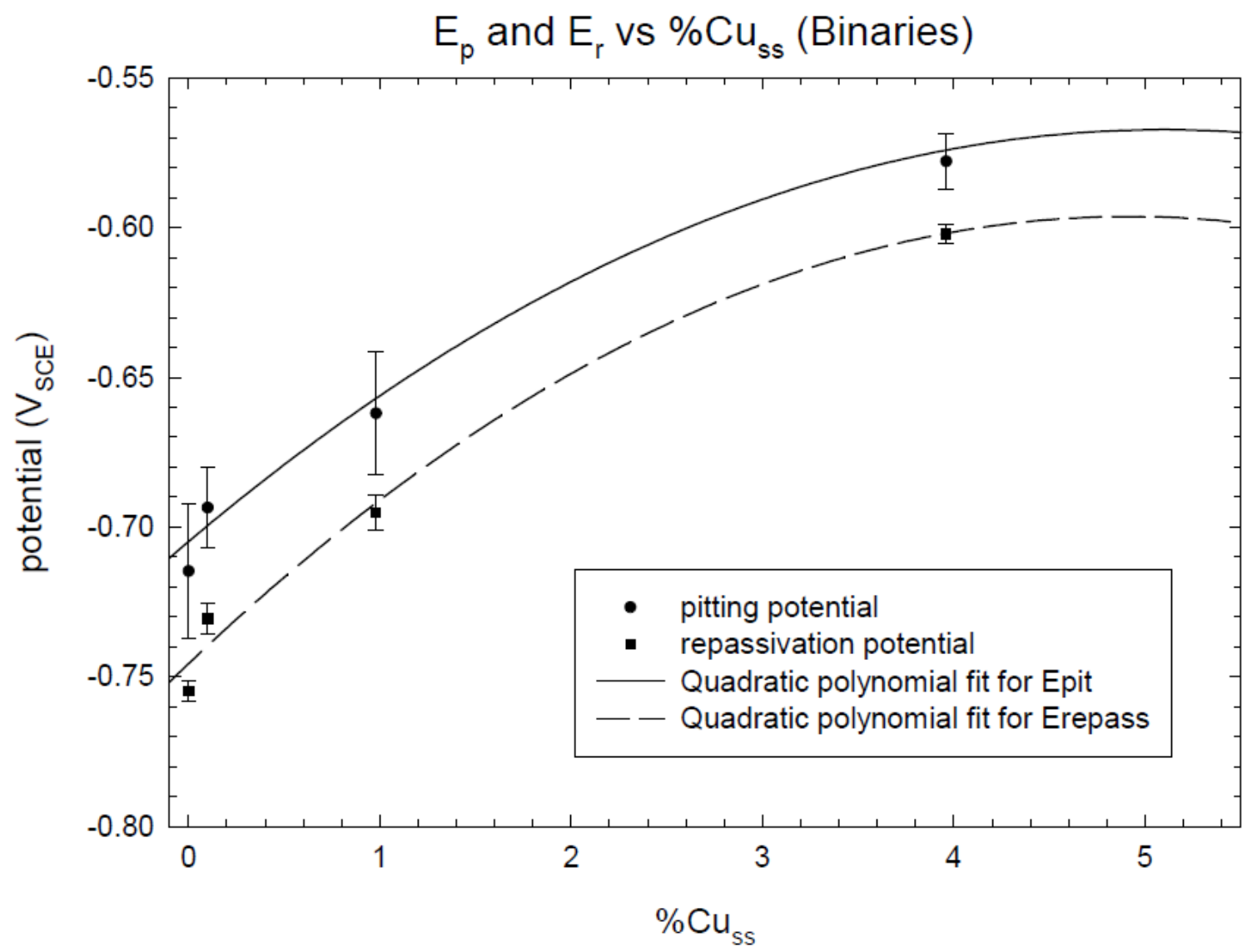

Figure 1.12. Effect of copper solid solution content on pitting and repassivation potential of aluminum-copper binary alloys. 


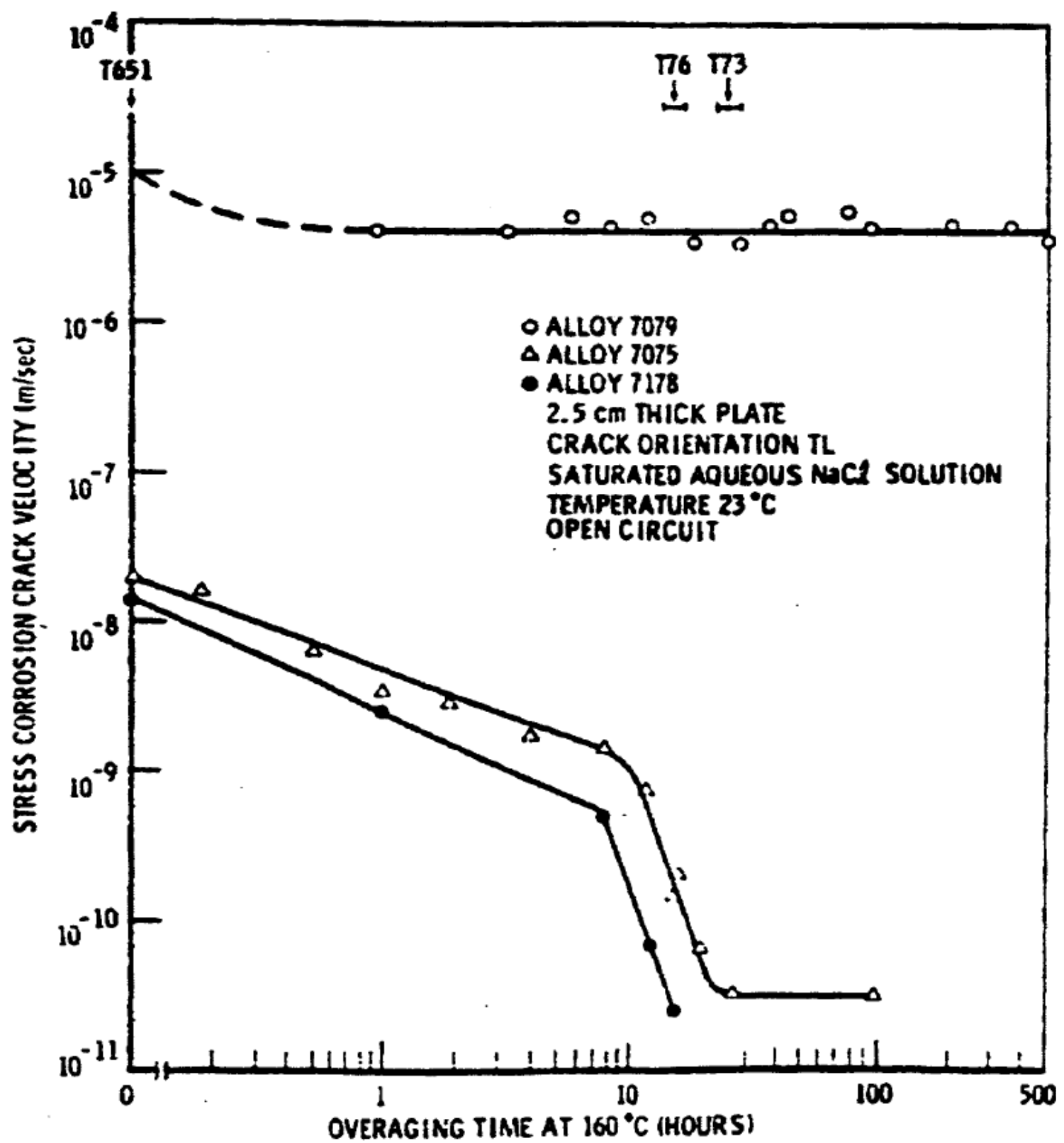

Figure 1.13. Effect of aging time on stress corrosion crack velocity in alloys with greater than 1 wt. \% Cu (AA7075 and 7178) and less than 1 wt. \% Cu (AA7079). 


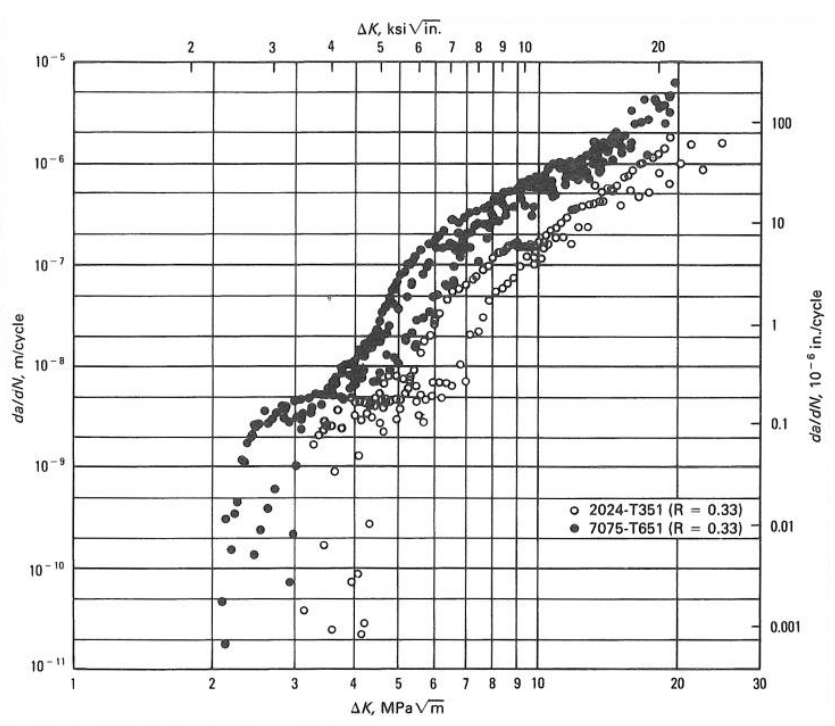

Figure 1.14. Fatigue crack growth rate comparison for AA2024 and AA7075. Reproduced with permission of ASM International.

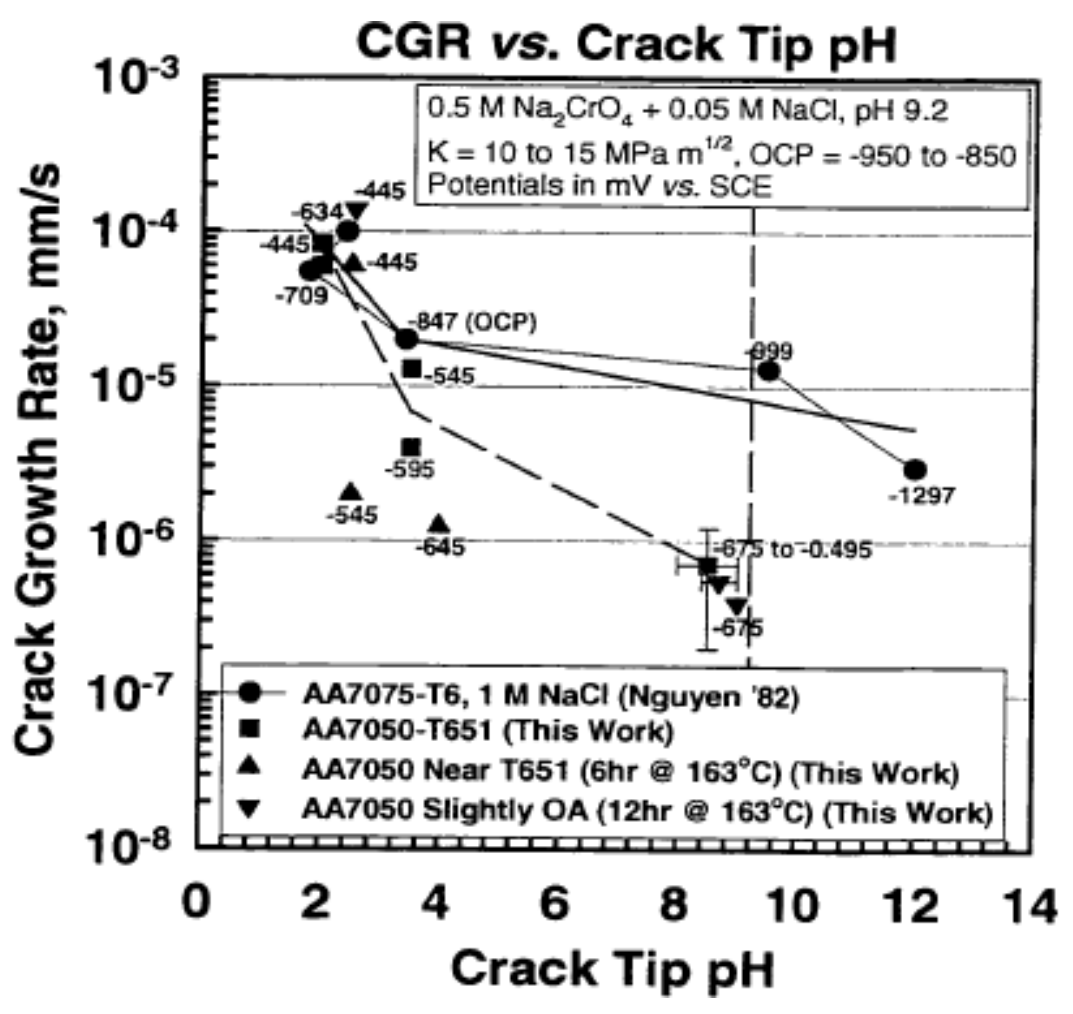

Figure 1.15. Crack tip $\mathrm{pH}$ with crack tip growth rate in EAC of AA7075 and AA7050 in a bulk solution of $0.05 \mathrm{M} \mathrm{NaCl}$ and $0.5 \mathrm{M} \mathrm{Na}_{2} \mathrm{CrO}_{4}$

Reproduced with permission of UVA 


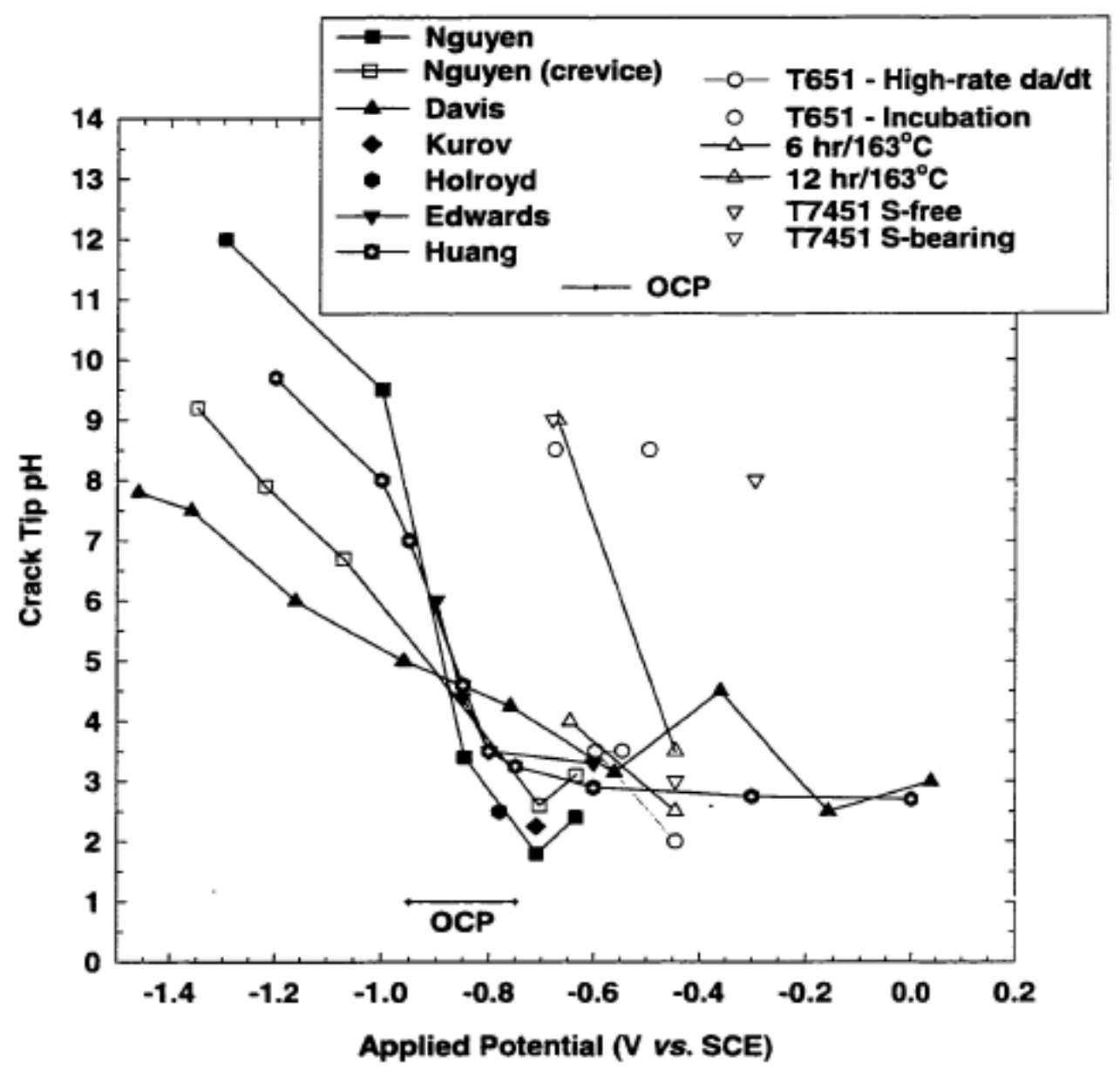

Figure 1.16. Crack tip $\mathrm{pH}$ with applied potential for 7xxx series aluminum alloys in $\mathrm{Cl}^{-}$ containing electrolyte.

Reproduced with permission of UVA 

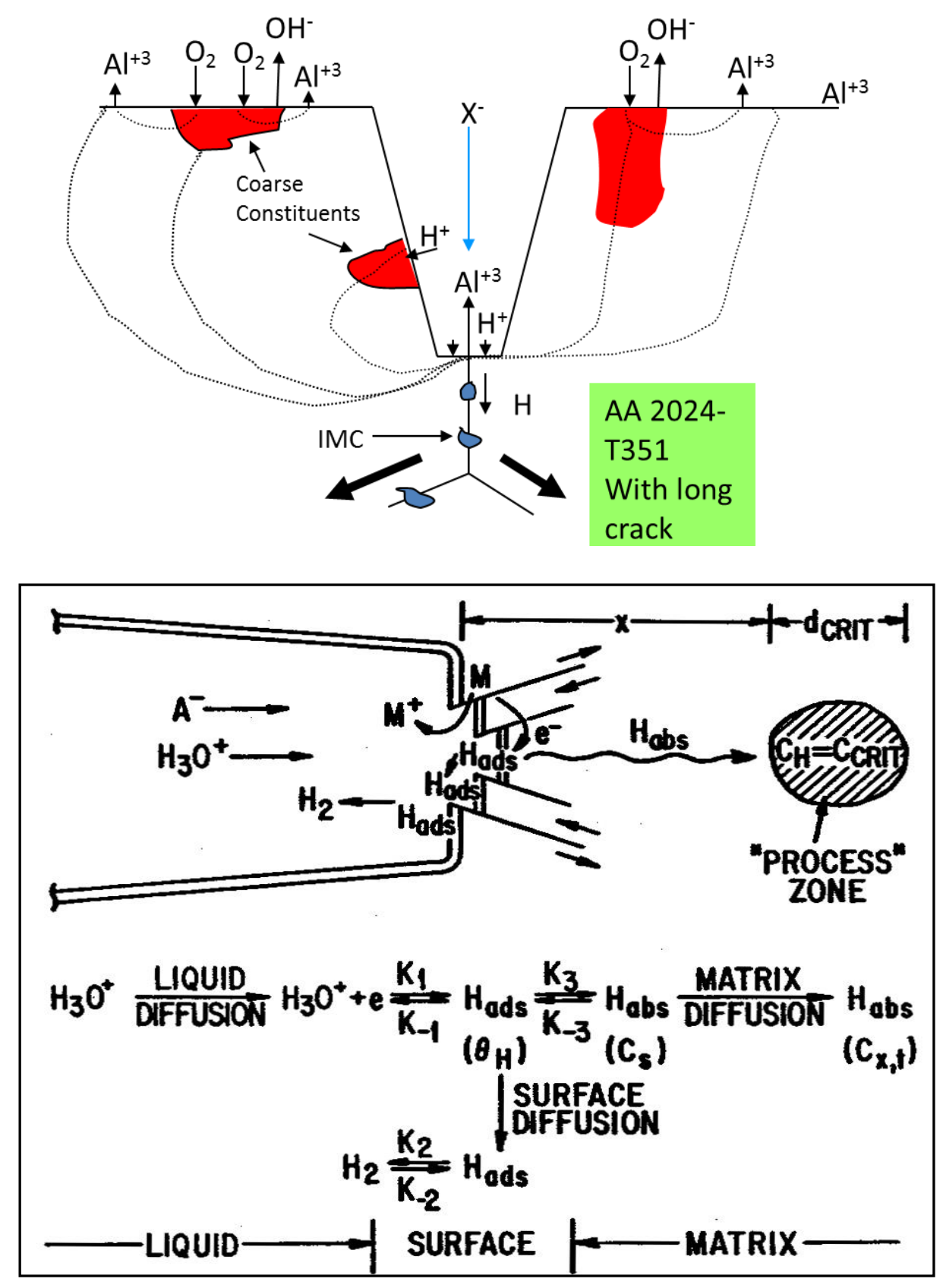

Figure 1.17. Illustration of complex multidimensional systems and properties that effect SCC and EFCP. The presence of the embrittling environment at the crack tip, the anodic and cathodic electrochemical reactions that lead to hydrogen production, and diffusion of hydrogen into the fracture process zone are all important steps in hydrogen environmental embrittlement. The second diagram illustrates the complex processes occurring at the tip of the crack.

Reproduced with permission of Ford 


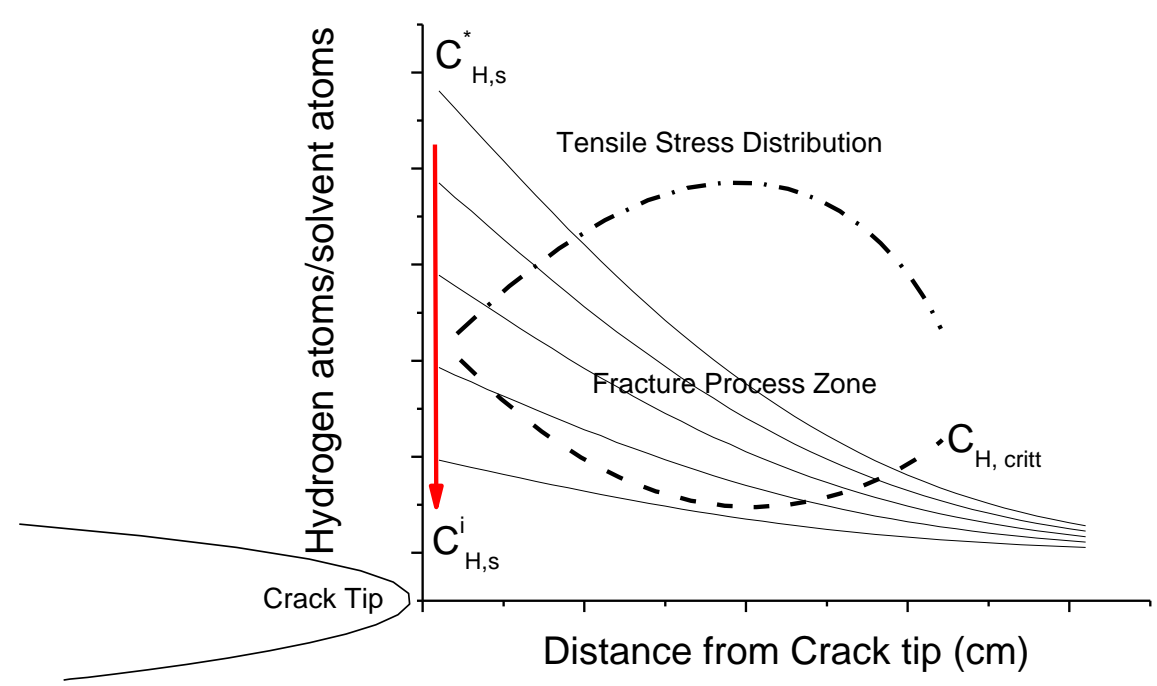

Figure 1.18. Effect of surface concentration of hydrogen on concentration gradient of hydrogen in a semi-infinite plate provided to show the possibility of lowering the internal hydrogen concentration bellow the critical concentration through the use of an inhibiting ion which lowers $\mathrm{C}_{\mathrm{H}, \mathrm{s}}^{*}$. 


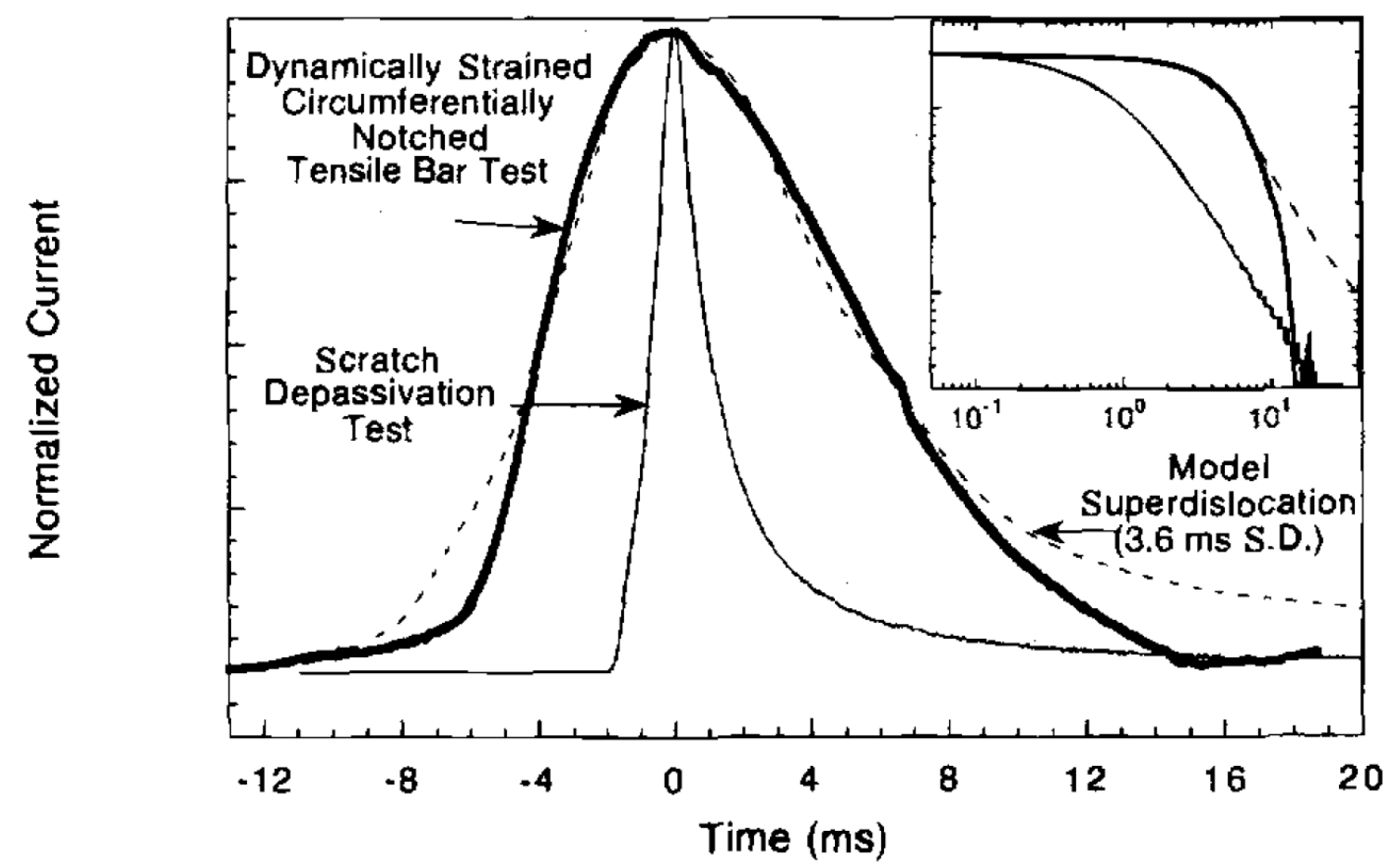

Figure 1.19. Comparison between a repassivation event collected from a dynamically strained electrode and that of a scratch repassivation. Peak currents have been normalized for emphasis.

Reproduced with permission of UVA 

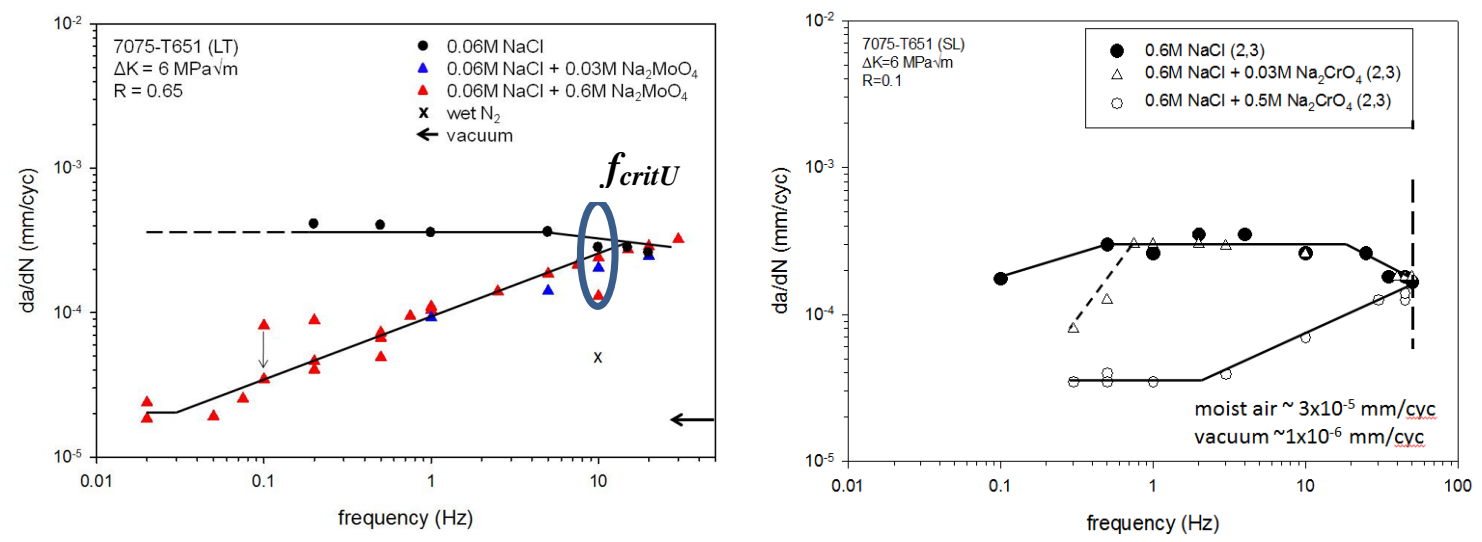

Figure 1.20. Crack growth rates for full Figure 1.21. Crack growth rates for full immersion molybdate containing sodium immersion chromate containing sodium chloride solutions as compared to sodium chloride solutions as compared to sodium chloride alone $f_{\text {Crit }}$ is on the order of $10 \mathrm{~Hz}$. chloride alone. $f_{\text {Crit }}$ is on the order of 100 $\mathrm{Hz}$.

J.S. Warner, S. Kim, and R.P. Gangloff, Z.M. Gasem, Rate-Limiting Process in "Molybdate inhibition of environmental Environmental Fatigue Crack Propagation in fatigue crack propagation in Al-Zn-Mg-Cu," 7000-Series Aluminum Alloys in TMS-AIME, International Journal of Fatigue 31, 11-12 R.H. Jones, Editor. 2001: Warrendale PA. p. (2009): p. 1952. Reproduced with permission. 501. Reproduced with permission. 

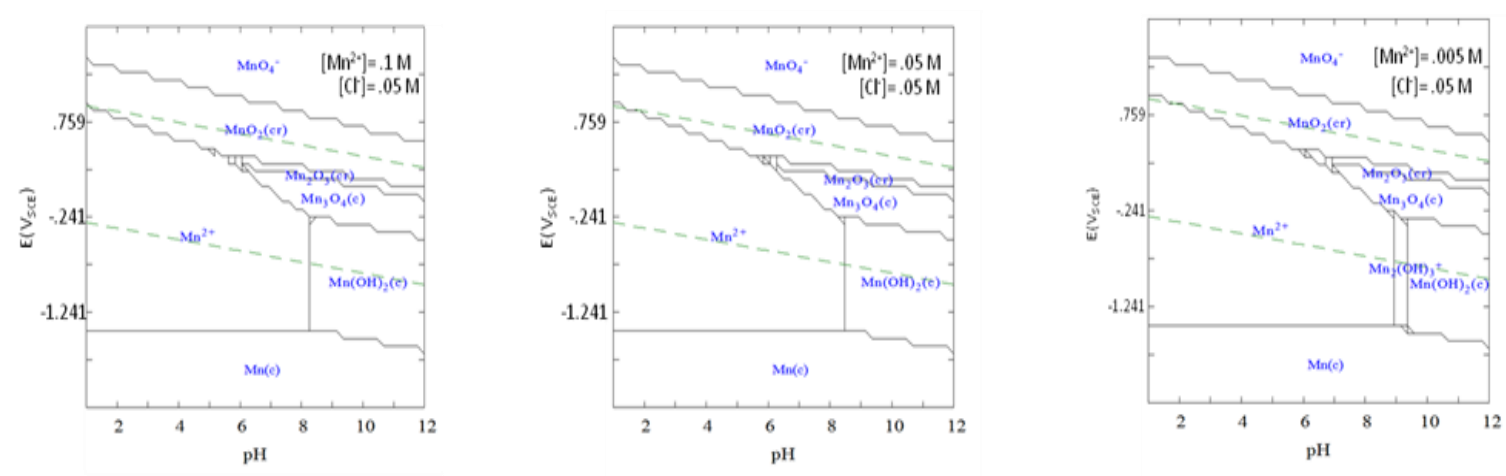

Figure 1.22. The E-pH diagrams for permanganate at 3 concentrations, $0.1 \mathrm{M}, 0.05 \mathrm{M}$ and $0.005 \mathrm{M}$ with chlorine held at $0.05 \mathrm{M}$. OCP for AA2024 is approximately $-0.55 \mathrm{~V}$ (SCE).

I. Puigdomenech, Hydra/Medusa Chemical Equilibrium Database and Plotting Software. 2004, freely downloadable software at www.kemi.kth.se/medusa/: KTH Royal Institute of Technology

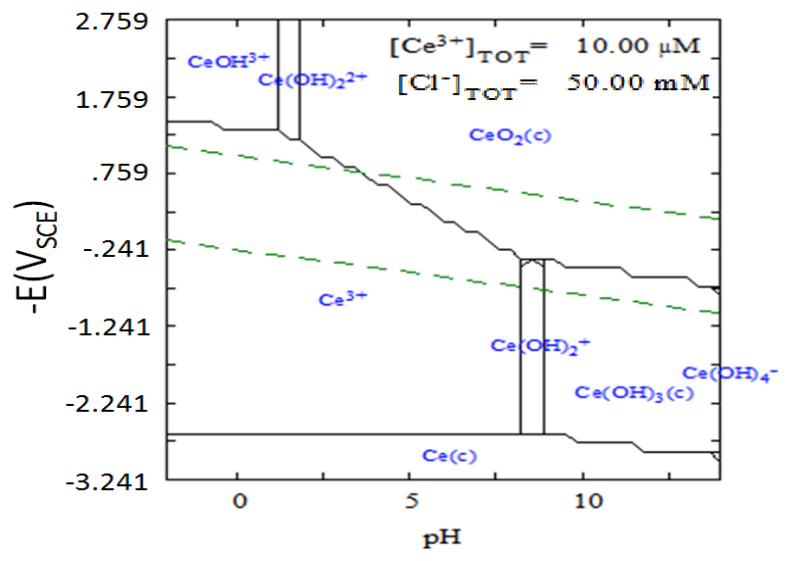

Figure 1.23: Aqueous cerium E-pH diagram showing the stability and region of predominance of $\mathrm{Ce}^{4+}$ oxides over a large range of $\mathrm{pH}$.

I. Puigdomenech, Hydra/Medusa Chemical Equilibrium Database and Plotting Software. 2004, freely downloadable software at www.kemi.kth.se/medusa/: KTH Royal Institute of Technology 
a)

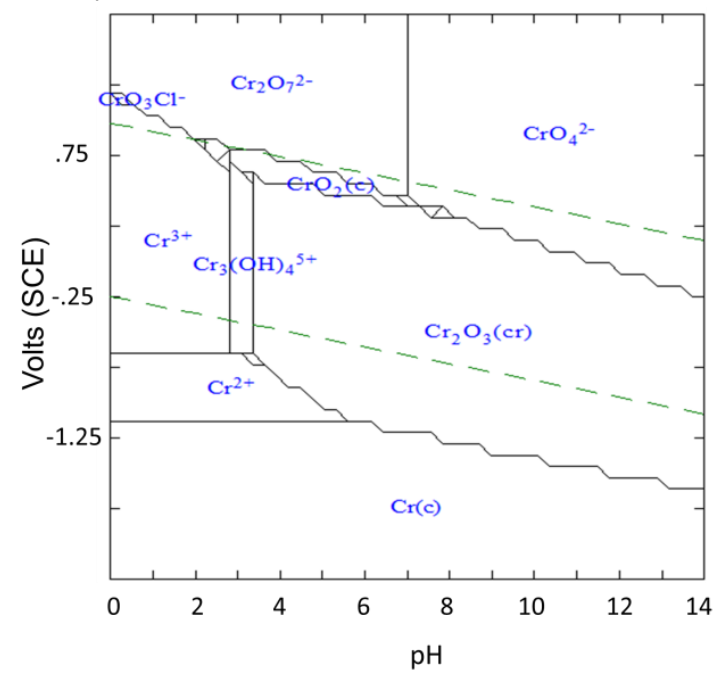

b)

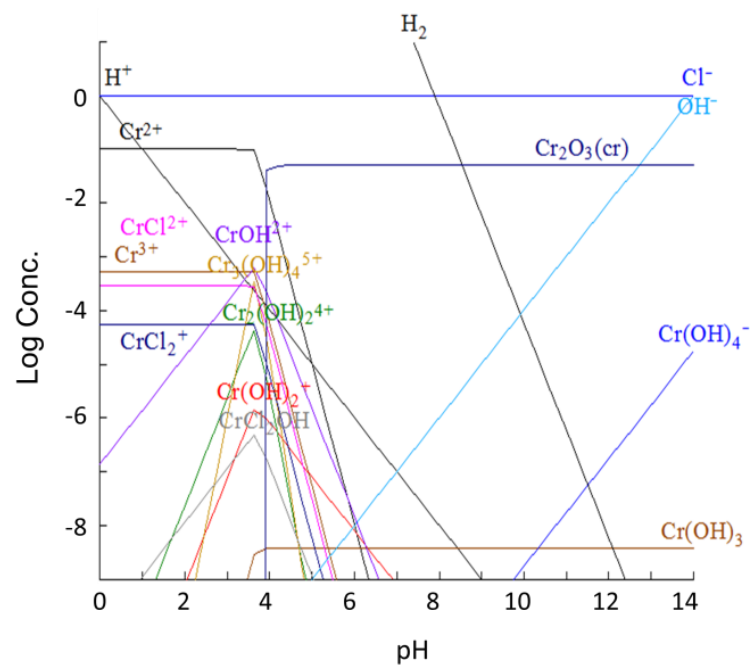

Figure 1.24. (a) E-pH diagram in $\mathrm{H}_{2} \mathrm{O}$ at $25{ }^{\circ} \mathrm{C}$ for $.1 \mathrm{M}$ chromate and $1 \mathrm{M}$ chloride showing the stability of chromium oxide over a large region of potential and $\mathrm{pH}$. (b) Speciation of chromium-chloride system at previously stated concentrations at $-0.8 \mathrm{~V}$ (SCE).

I. Puigdomenech, Hydra/Medusa Chemical Equilibrium Database and Plotting Software. 2004, freely downloadable software at www.kemi.kth.se/medusa/: KTH Royal Institute of Technology 


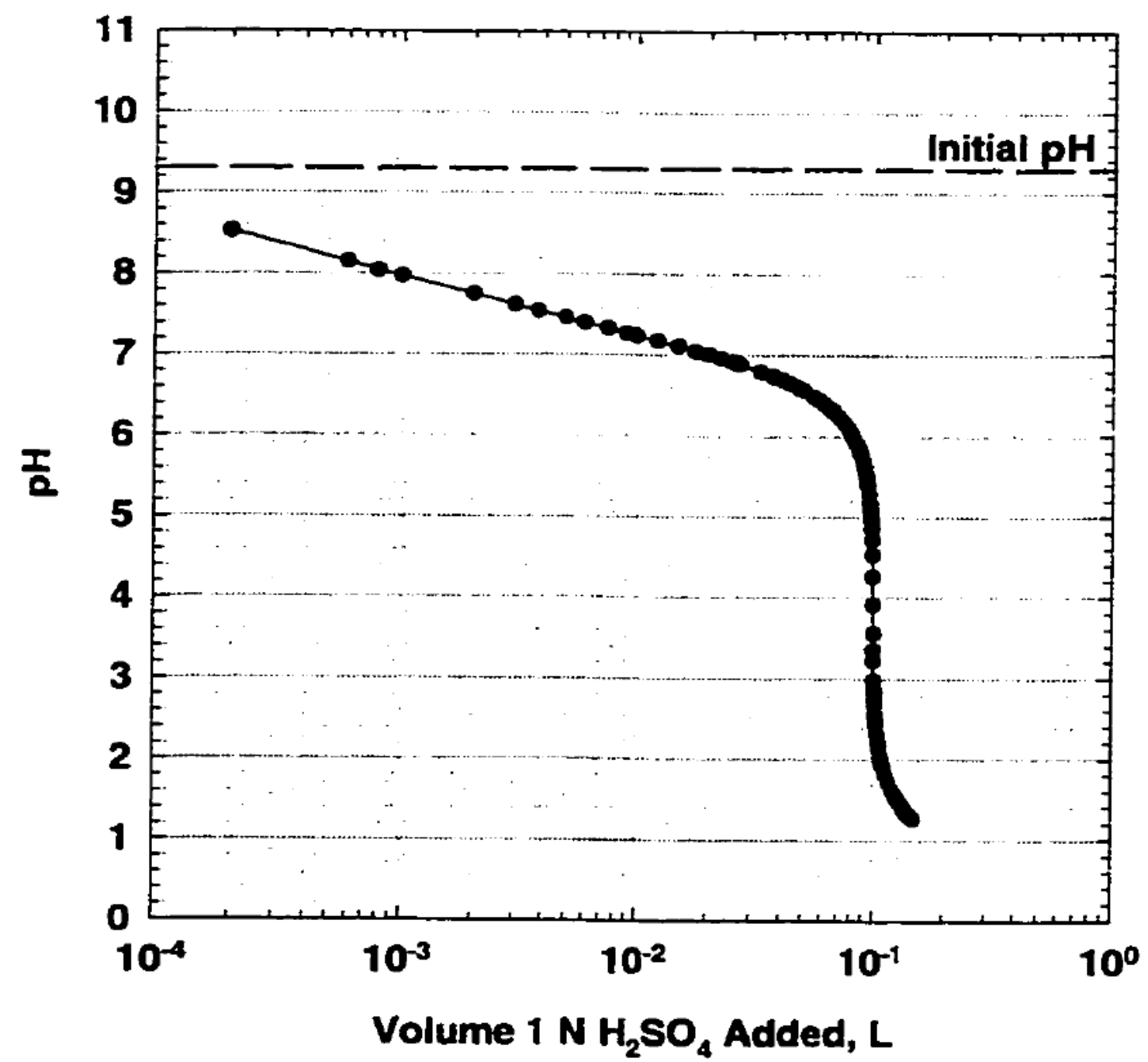

Figure 1.25. Buffer capacity of $200 \mathrm{~mL}$ of $0.5 \mathrm{M} \mathrm{Na}_{2} \mathrm{CrO}_{4}+0.5 \mathrm{M} \mathrm{NaCl}$. Reproduced with permission of UVA 

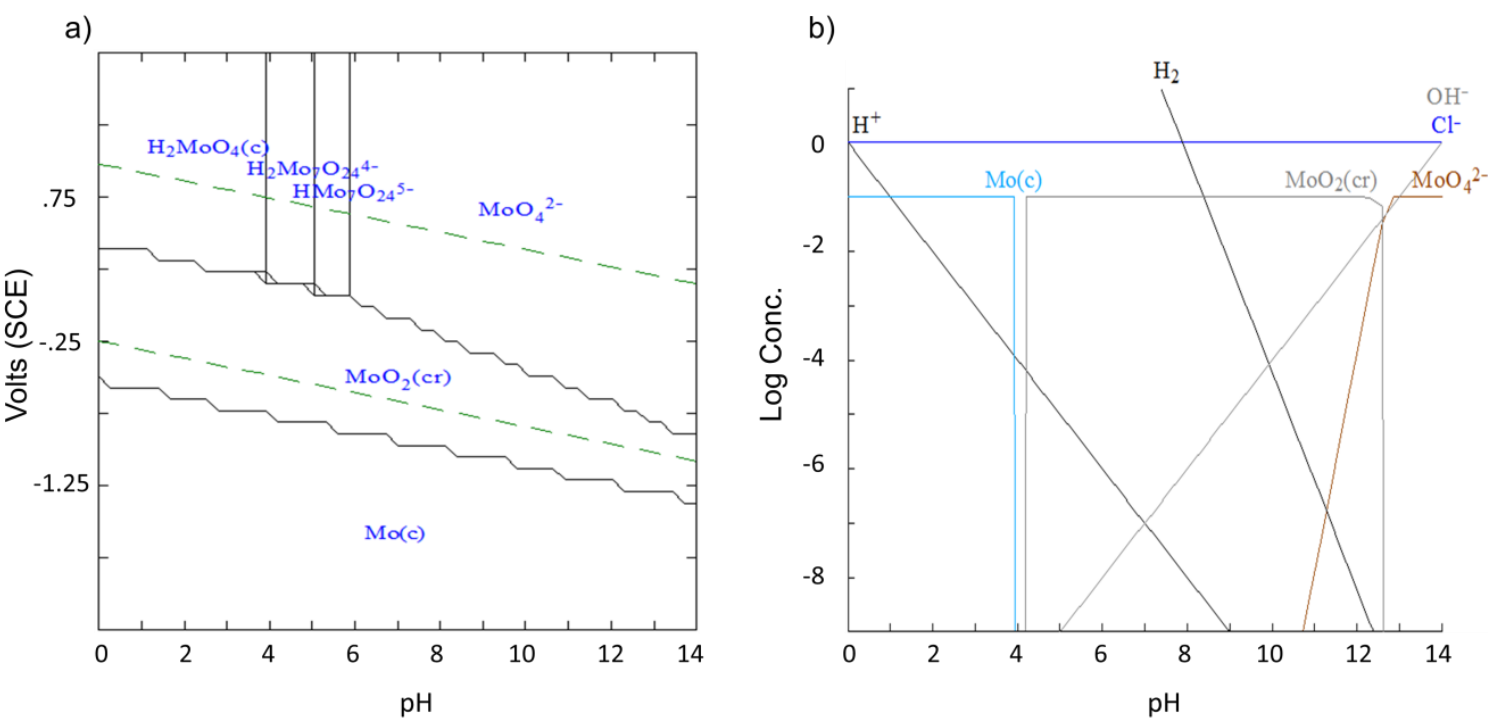

Figure 1.26. (a) E-pH diagram for $0.1 \mathrm{M}$ molybdate and $1 \mathrm{M}$ chloride solution showing the stability of molybdenum oxide over a large region of potential and $\mathrm{pH}$ with high potential stability in the low $\mathrm{pH}$ region. (b) Speciation of molybdate-chloride system at previously stated concentrations at $-0.8 \mathrm{~V}$ (SCE).

I. Puigdomenech, Hydra/Medusa Chemical Equilibrium Database and Plotting Software. 2004, freely downloadable software at www.kemi.kth.se/medusa/: KTH Royal Institute of Technology 


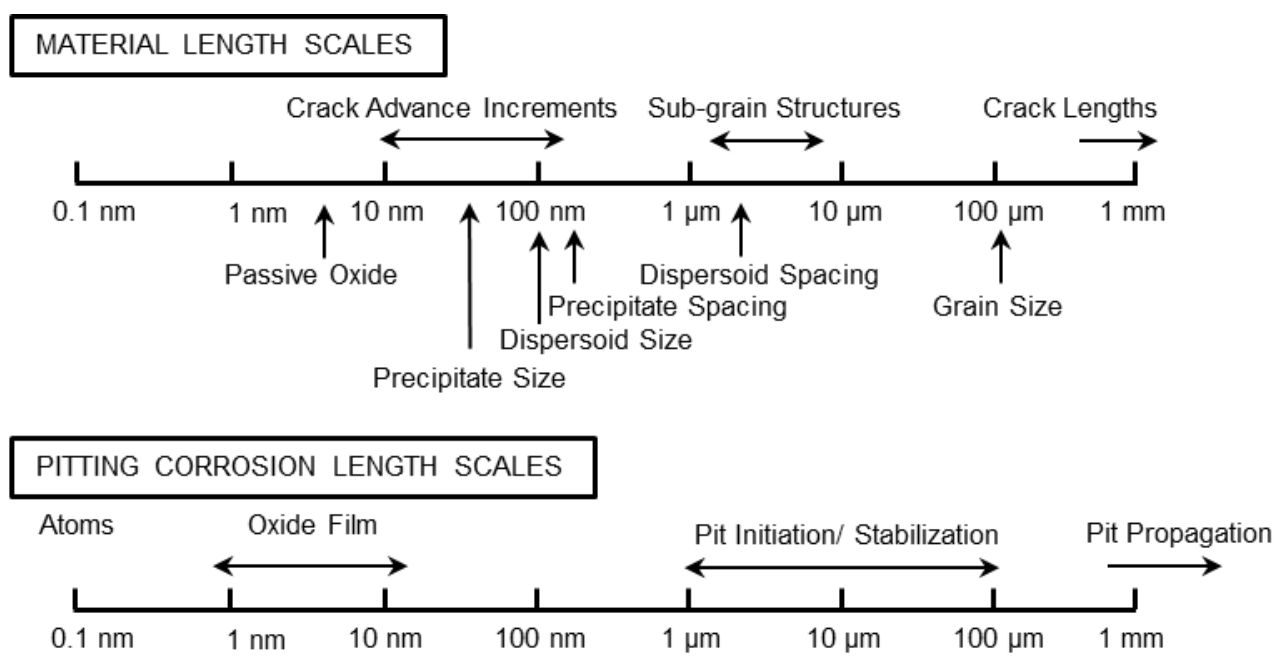

Figure 1.27 Relevant length scales over which inhibitors play a role. Source: University of Virginia, CESE, courtesy of Matthew R. Begley and Richard P. Gangloff, Hongbo Cong and J.R. Scully

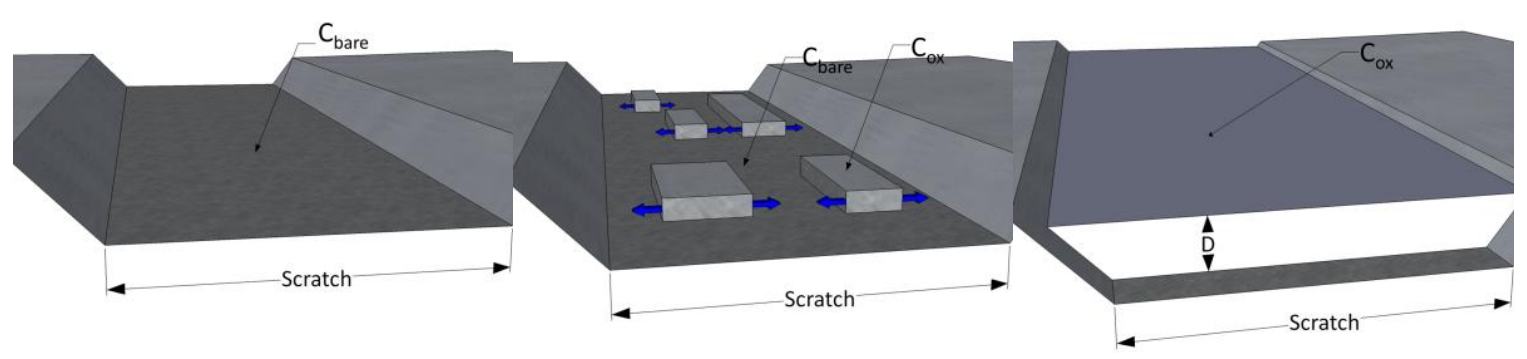

Figure 1.28. After a scratch the bare metallic electrode will undergo oxide nucleation and an increase in oxide surface coverage, followed by thickening of the oxide film as repassivation occurs. $\mathrm{D}$ is the thickness of the oxide layer. 


\section{Corrosion Inhibition of AA2024-T351 Using Permanganate}

\subsection{Abstract}

The inhibition of corrosion on AA2024-T351 in NaCl solutions, mitigated by either in-situ permanganate ions $\left(\mathrm{MnO}_{4}^{-}\right)$or permanganate pretreatment, was examined. Both room temperature pretreatment and solution phase additions were studied as a function of inhibitor concentration. The roles of the inhibitor during anodic and cathodic polarizations were investigated. Inhibition of corrosion at open circuit corrosion potential (OCP) under conditions where anodic and cathodic reactions are coupled was also examined. The oxidation states of the manganese oxides that contributed to protection were determined using potentiometric electrochemical reduction and in-situ Raman spectroscopy. The thermodynamics of the Mn-water system was also considered over a range of concentrations. Permanganate was shown to be both an anodic and cathodic inhibitor, and an inhibitor of copper replating at OCP.

\subsection{Background}

Precipitation hardened aluminum alloys containing copper and iron are highly susceptible to corrosion due to local galvanic coupling between intermetallic phases and the surrounding aluminum matrix ${ }^{[1-7]}$. Multiple forms of corrosion protection have been implemented to address this issue ${ }^{[8-10]}$. Hexavalent chromates are currently recognized as the most effective chemical species used to mitigate corrosion on alloys such as AA2024 ${ }^{[11-16]}$. OSHA in 2006 imposed strict new $\mathrm{Cr}(\mathrm{VI})$ exposure limits due to their carcinogenic 
properties ${ }^{[17]}$. The additional costs incurred providing an adequate working environment and allowing for the proper disposal of $\mathrm{CrO}_{4}{ }^{2-}$ have led to efforts to find new, non-toxic replacements.

Localized corrosion of alloys, such as AA2024-T351, results from the presence of intermetallic compounds in a solute lean $\alpha$-Al matrix that are either noble with respect to their surrounding matrix or particles that act as initiation sites for pitting ${ }^{[1,4]}$. Particles such as $\mathrm{Al}_{2} \mathrm{CuMg}$ and $\mathrm{Al}_{2} \mathrm{Cu}$ can also be detrimental to the corrosion resistance of the alloy because of their tendency to dealloy at the OCP ${ }^{[7]}$. This process has many damaging consequences including the creation of catalytic high surface area copper enriched intermetallic remnants and the associated deposition of copper on the alloy surface ${ }^{[18]}$, both of which result in increased oxygen reduction reaction (ORR) rates. This increase in ORR supports increased aluminum matrix dissolution in the form of localized trenching around the intermetallics and pit initiation and growth that can produce nucleation sites for subsequent crack growth ${ }^{[19-21]}$. Effective inhibition of these constituent-particle-related damage modes in this class of alloys is of strong interest.

One aspect of current research in the field of corrosion inhibition has focused on oxoanions of the form $\mathrm{MeO}_{4}{ }^{(\mathrm{n}-8)}$ (such as molybdates, tungstates, and vanadates, where $n$ is the oxidation state of the central atom) ${ }^{[22-32]}$, analogues of hexavalent chromium. In this study, permanganate $\left(\mathrm{MnO}_{4}^{-}\right)$has been evaluated as a potential replacement for $\mathrm{CrO}_{4}{ }^{2-}$.

Permanagate is a strong oxidizer due to its high equilibrium potential as seen in Figure 2.1(a). E-pH and speciation diagrams were calculated using Medusa software ${ }^{[33]}$. Permanganate will be spontaneously reduced when added to solution in contact with 
AA2024-T351 at its OCP. Serveral possible electrochemical reactions are possible. Over all ranges of $\mathrm{pH}, \mathrm{MnO}_{4}^{-}$in the +7 valence state will likely first be reduced to manganese (IV) dioxide by the following electrochemical half cell reaction ${ }^{[34]}$.

$$
\mathrm{MnO}_{4}{ }^{-}+4 \mathrm{H}^{+}+3 \mathrm{e}^{-} \leftrightarrow \mathrm{MnO}_{2}+2 \mathrm{H}_{2} \mathrm{O}
$$

Several additional electrochemical reactions can further reduce the $\operatorname{Mn}(I V)$ oxide to $\operatorname{Mn}(I I I)$, a mixed valence oxide, and finaly the $\operatorname{Mn}(I I)$ state ${ }^{[34]}$.

$$
\begin{gathered}
2 \mathrm{MnO}_{2}+\mathrm{H}_{2} \mathrm{O}+2 e^{-} \leftrightarrow \mathrm{Mn}_{2} \mathrm{O}_{3}+2 \mathrm{OH}^{-} \\
3 \mathrm{Mn}_{2} \mathrm{O}_{3}+\mathrm{H}_{2} \mathrm{O}+2 e^{-} \leftrightarrow 2 \mathrm{Mn}_{3} \mathrm{O}_{4}+2 \mathrm{OH}^{-} \\
\mathrm{Mn}_{3} \mathrm{O}_{4}+2 \mathrm{H}^{+}+2 e^{-} \leftrightarrow 3 \mathrm{MnO}+\mathrm{H}_{2} \mathrm{O}
\end{gathered}
$$

At low $\mathrm{pH}$ and low potentials the oxides will disolve as $\mathrm{Mn}^{2+}$. However, at higher $\mathrm{pH}$, above 8 , a stable hydroxide exists in an insoluble form ${ }^{[34]}$.

The predicted equilibrium speciation behavior can be seen in Figure 2.1(b). These speciation diagrams indicate predominant species at $-0.5 \mathrm{~V}_{\text {sce. }}$ This potential is approximatly the value of the observed OCP for AA2024-T351 in 0.05 M sodium chloride solution with the permanganate ion in solution. The predominant $\mathrm{Mn}(\mathrm{II})$ species is $\mathrm{Mn}(\mathrm{OH})_{2}$ at $\mathrm{pH}$ levels above 8.5. Half-cell reactions [9] through [12] substantiate that $\mathrm{Mn}(\mathrm{II})$ could deposit electrochemically and remain stable at high $\mathrm{pH}$ cathodic sites such as those present over copper rich constituent particles ${ }^{[11,35]}$. 
Permanganate based coating products have been in commercial production for many years and several patents exist ${ }^{[36,37]}$. Previous scientific studies of $\mathrm{MnO}_{4}^{-}$on AA2024 have focused on open circuit electrochemical properties, barrier layer properties, lab exposure tests such as ASTM B-117 ${ }^{[38]}$ neutral salt spray testing, as well as on the oxidation states of protective manganese oxides ${ }^{[39-41]}$. The mechanism by which $\mathrm{MnO}_{4}{ }^{-}$inhibits corrosion on AA2024 has been shown to be the growth of manganese oxides over copper-rich intermetallic particles ${ }^{[41]}$. The oxidation state of the manganese deposits is difficult to determine with no definitive proof of the valence state in the literature ${ }^{[41,42]}$. Little electrochemical information has been provided with regards to the effect of the manganese deposits or ions on the corrosion electrochemistry of AA2024-T351. It is useful to review the electrochemical and chemical dynamics of the aqueous-manganese system that apply to $\mathrm{MnO}_{4}^{-}$in treatments on AA2024-T351.

The inhibition of localized corrosion (pitting) on AA2024 by permanganate has been studied through anodic potentiodynamic pitting scans by Danilidis ${ }^{[43]}$. However, the effect of manganese oxides on the ORR has not been reported in the literature. Moreover, a fundamental understanding of the mechanism that leads to anodic inhibition is lacking. Release and transport of manganese species for active inhibition has not been studied. The manganese system has multiple valence states between +7 and +2 which, in a similar way to the chromate system, may provide mechanisms for storage release and transport of the soluble oxidizing inhibiting species to sites for additional reduction leading to possible self-healing capabilities. Initially, soluble permanganate, manganese in the +7 valence state, can be electrochemically deposited on $\mathrm{Cu}$ rich intermetallic sites. The $\mathrm{pH}$ will rise when a cathodic reaction occurs above a finite rate over a $\mathrm{Cu}$-rich site. $\mathrm{MnO}_{2}$ and $\mathrm{Mn}(\mathrm{OH})_{2}$ 
are relatively insoluble ${ }^{[44]}$. Moreover, incomplete $\mathrm{MnO}_{4}^{-}$reduction could lead to a reservoir of the $\mathrm{Mn}$ (VII) species trapped in a lower valence state oxide. Release of the $\mathrm{Mn}^{2+}$ species is also possible from the oxide caps if a solution is present at a $\mathrm{pH}$ lower than approximately eight. The $\mathrm{Mn}^{2+}$ could again be chemically deposited by a homogeneous chemical reaction forming $\mathrm{Mn}(\mathrm{OH})_{2}$ over the active cathodic site. In this manner the manganese species might protect actively corroding areas on AA2024-T351. However, prevailing oxide states after $\mathrm{MnO}_{4}^{-}$treatments are unclear. XPS, Raman, and XANES studies on platinum, gold, and additional aluminum alloy substrates have been conducted, mainly to elucidate oxide stoichiometry ${ }^{[42,43,45-50]}$. These papers illustrate the difficulty in defining oxide valence states in manganese oxides.

In this chapter we attempt to provide a more comprehensive electrochemical understanding of the mechanisms of permanganate inhibition. We seek to understand its viability as a stand-alone ionic inhibitor in $\mathrm{NaCl}$ solution under full immersion conditions. We examined both pretreatments with permanganate, to examine the protective manganese oxide deposits, and exposures in the presence of permanganate ions to distinguish the effects of the soluble ion from such manganese oxides. Open circuit full immersion exposure experiments were conducted. Surface chemical compositions and deposit morphologies were also investigated. Copper replating was investigated to indirectly explore the inhibition of $\mathrm{Cu}$ release and replating. Pitting potentials were determined and ORR kinetics were extracted using the Koutecky-Levich correction ${ }^{[51]}$. Theoretical modeling with both Gueshi and membrane models was performed to analyze ORR kinetics obtained from rotating disk electrode (RDE) experiments $[12,52,53]$. Electron transport through the solid/electrolyte-interface was also characterized. Raman spectroscopy and galvanostatic 
reductions were used to attempt to characterize the valence states of protective manganese oxides.

\subsection{Experimental Procedures}

\subsubsection{Materials and Test Electrolytes}

AA2024-T351 (1 mm sheet) and -T4 (rod) precipitation age hardened aluminum alloys, copper $(99.994 \%)$ and aluminum $(99.9996 \%)$ were investigated. Specific chemical compositions (wt \%) of the aluminum alloy can be found in Table 2.1. Composition was determined by ICP-OES. All sheet metal samples were tested on their L-T surfaces, rod samples were tested on their S-T surfaces. Manganese (99.99\% purity) was used as a control in Raman spectroscopy studies. S-phase ingots $\left(\mathrm{Al}_{2} \mathrm{CuMg}\right)$ from Sandia National Laboratory were examined ${ }^{[11]}$. Sample preparation varied by experimental technique depending on surface roughness requirements. These preparations fall into two categories; refinished and polished. The refinishing process consisted of a single grinding step using 600 grit silicon-carbide paper and was intended to restore the specimens to as-received condition. Polishing was carried out in 18.2 $\mathrm{M} \Omega$ deionized water. The samples were subsequently rinsed in 18.2 $\mathrm{M} \Omega$ deionized water, methanol, and again in 18.2 $\mathrm{M} \Omega$ deionized water for degreasing before being dried with cotton lab tissue. When a finer surface roughness was desired to resolve constituent particles the samples were ground sequentially to 1200 grit $\mathrm{Si}-\mathrm{C}$ paper resulting in a partially reflective surface on AA2024 and high purity copper, followed by the same degreasing procedure. 
The test solutions were prepared with 18.2 M $\Omega$ deionized water. Solutions were prepared with (in wt $\%$ ) sodium chloride from Fisher $(99.8 \% \mathrm{NaCl})$, potassium permanganate supplied by Fisher Scientific $\left(99.5 \% \mathrm{KMnO}_{4}\right)$. Sodium tetraborate decahydrate $(101.3 \%$ assay) was obtained from Fisher Scientific, while boric acid (99.99\% purity) was obtained from Alfa Aesar. Manganese chloride (99.99\% purity) was supplied by Sigma Aldrich. All solutions were aerated and at room temperature unless otherwise noted. Several solution preparations were used. Pretreatment solutions consisted of chloride free permanganate solutions with concentrations of $0.0 \mathrm{M}, 0.005 \mathrm{M}, 0.05 \mathrm{M}$, and $0.1 \mathrm{M}$. The naturally occurring $\mathrm{pH}$ of the solutions ranged from 5.8 for the $0.005 \mathrm{M}$ permanganate solution to 7.4 for the $0.1 \mathrm{M}$ concentration. Post-pretreatment exposure was carried out in $0.05 \mathrm{M}$ sodium chloride at its natural $\mathrm{pH}$ of six. Solutions used for exposure of AA2024-T351 in chloride and permanganate solutions consisted of $0.05 \mathrm{M}$ sodium chloride and $0.0 \mathrm{M}, 0.005$ $\mathrm{M}, 0.05 \mathrm{M}$, and $0.1 \mathrm{M}$ potassium permanganate. The $\mathrm{pH}$ of these solutions ranged from 5.8 to 7.4 .

Multiple phase synthesized ingots with large S-phase particles were examined at $-0.5 \mathrm{~V}$ (SCE), approximately the OCP of AA2024-T351 in permanganate and chloride solutions. Potentiostatic holds at $-0.5 \mathrm{~V}$ (SCE) were conducted for 200 seconds to determine the effect of permanganate on S-phase corrosion. The short time period was chosen to minimize damage to the singular S-phase ingot.

\subsubsection{Open Circuit Measurements and Electrochemical Impedance Spectroscopy}

OCP measurements were used to study the effect of the inhibitor on corrosion events when anodic and cathodic reactions were taking place at naturally-occurring rates on AA2024- 
T351. The potential measurements were carried out in three ways. One hour OCP measurements were taken while the sample was exposed to an aerated $0.05 \mathrm{M}$ sodium chloride solution in the presence of the inhibitor in the previously discussed concentrations. OCP measurements were also taken in permanganate-free $0.05 \mathrm{M}$ sodium chloride solution after a 24 hour pretreatment with permanganate at room temperature. The pretreatment consisted of immersion in aerated potassium permanganate solution with the concentrations previously discussed for 24 hours at room temperature and OCP. Care was taken to insure that none of the faces of the sheet AA2024 were allowed to rest against the walls or bottom of the chloride free glass beakers. After 24 hours the pretreated samples were rinsed thoroughly in $18.2 \mathrm{M} \Omega$ deionized water, blotted dry with cotton lab tissue, and attached to a standard three electrode cell within five minutes. After attachment the sample was thoroughly dried with compressed nitrogen to ensure that only the desired area was exposed to electrolyte.

The third measurement was a one hour OCP measurement at the beginning and end of a 24 hour continuous OCP exposure to $0.05 \mathrm{M}$ sodium chloride solution with varied inhibitor concentrations in-situ (0.0 M, 0.005 M, 0.05 M, and 0.1 M permanganate solutions). EIS data were collected at the end of the exposure. EIS measurements ranged from $10^{6}$ to $10^{-3}$ $\mathrm{Hz}$ taking six data points per decade with an RMS voltage amplitude of $20 \mathrm{mV}$.

\subsubsection{Microscopic Analysis after Open Circuit Exposures}

Scanning electron microscopy was used to examine samples after open circuit exposures, pretreatment, and pretreatment followed by exposure. Examination focused on intermetallic trenching and general pitting of the aluminum matrix as well as oxide growth 
on the sample. Manganese deposits were removed by a hard bristled brush to expose the underlying constituent particles. Multiple-phase synthesized ingots with large S-phase regions were examined at $-0.5 \mathrm{~V}$ (SCE), approximately the OCP of AA2024-T351, in permanganate and chloride solutions. Potentiostatic holds at $-0.5 \mathrm{~V}$ (SCE) were conducted for 200 seconds to determine the effect of permanganate on S-phase attack. The short time period was chosen to minimize damage to the singular S-phase ingot.

\subsubsection{Anodic and Cathodic Cyclic Potentiodynamic Polarization Scans}

Upward anodic scans were run immediately following the OCP measurements described above. The samples were scanned from $-0.05 \mathrm{~V}$ vs. OCP to $1.0 \mathrm{~V}$ vs. SCE or until the current density reached $5.0 \times 10^{-3} \mathrm{~mA} / \mathrm{cm}^{2}$. The scan then reversed and proceeded to $-0.5 \mathrm{~V}$ vs. OCP. These anodic scans were repeated between 5 and 10 times on replicate specimens to give a statistical distribution of the pitting potential. Pitting potential was defined as the value at a current density of $10^{-4} \mathrm{~A} / \mathrm{cm}^{2}$.

Cathodic reaction kinetics were studied using the rotating disk electrode (RDE) method. Samples were examined with permanganate and chloride in the test solution and also after a pretreatment in permanganate with the test scan solution consisting of aerated $0.05 \mathrm{M}$ sodium chloride or a $\mathrm{pH} 8.4$ borate buffer solution using a saturated calomel reference electrode. The pretreated samples were scanned in $\mathrm{pH} 8.4$ borate buffer using a $\mathrm{Hg} / \mathrm{Hg}_{2} \mathrm{SO}_{4}$ reference electrode. Rotation rates of 100, 500, 1000, 1500, 2000, and $2500 \mathrm{rpm}$ were used. Samples included high purity copper and AA2024-T4. The RDE samples were mounted in PTFE with BONDiT B-4811 from Reltek ${ }^{\mathrm{TM}}$. This epoxy is resistant to harsh chemical environments and is needed in the presence of permanganate, a strong oxidizer. 
Galvanostatic reduction experiments were performed on high purity copper samples that were both untreated and pretreated in a $0.1 \mathrm{M} \mathrm{KMnO}_{4}$ solution at a potential of $-0.5 \mathrm{~V}$ (SCE). These experiments were conducted to observe valence state changes in the electrodeposited manganese oxide on the pretreated copper samples. The galvanostatic tests were conducted in $\mathrm{pH} 10$ borate buffer at $-20 \mu \mathrm{A} / \mathrm{cm}^{2}$.

\subsubsection{Cyclic Voltammetry Tests to Assay Copper Redeposition}

Cyclic voltammetry (CV) experiments were performed on exposed, pretreated, and pretreated then exposed AA2024-T351 to assay the surface copper redeposition process after inhibitor pretreatment ${ }^{[25]}$. CV experiments were performed after transferring specimens to $\mathrm{pH} 8.4$ borate buffer solutions in cells free of chloride and copper (I and II) ions using $\mathrm{Hg} / \mathrm{Hg}_{2} \mathrm{SO}_{4}$ reference electrodes. The procedure for the test can be found in the literature $^{[25]}$. The anodic current that resulted from the scans is dependent on the surface coverage of copper and thus the area coverage of the active $\mathrm{Cu}$-rich cathodic reaction sites. Metallic copper on the surface can be oxidized to $\mathrm{Cu}_{2} \mathrm{O}$ and $\mathrm{CuO}$. There can be interference with this assay if $\mathrm{Mn}(\mathrm{OH})_{2}$ is oxidized to $\mathrm{Mn}_{2} \mathrm{O}_{3}$ during the potential sweep but this has been avoided by choosing the $\mathrm{pH} 8.4$ buffer solution such that the reversible potential for the $\mathrm{Mn}(\mathrm{II})$ to $\mathrm{Mn}(\mathrm{III})$ reaction is -0.117 vs. $\mathrm{Hg} / \mathrm{Hg}_{2} \mathrm{SO}_{4}$. Several manganese oxidation reduction reactions could potentially occur between -0.1 and $-1.6 \mathrm{~V} \mathrm{Hg} / \mathrm{Hg}_{2} \mathrm{SO}_{4}$. However, none of them occur near the reduction peak for $\mathrm{Cu}$ near of around $-0.4 \mathrm{~V}$ vs. $\mathrm{Hg} / \mathrm{Hg}_{2} \mathrm{SO}_{4}$. 


\subsubsection{Raman Spectroscopy}

Raman spectroscopy experiments were conducted on copper, pretreated copper, AA2024T351, pretreated AA2024-T351, manganese metal, and a series of manganese oxide powders used as standards from Alfa Aesar including $\mathrm{MnO}_{2}(99.999 \%), \mathrm{Mn}_{2} \mathrm{O}_{3}(98 \%)$, and $\mathrm{Mn}_{3} \mathrm{O}_{4}(99.9 \%)$. The Raman laser had a wavelength of $514.53 \mathrm{~nm}$ from an argon laser source. The machine was equipped with a single-axis spectrometer, a notch filter (Jobin Yvon Horiba, T64000) and a charge couple device detector. A resolution of $600 \mathrm{grit} / \mathrm{mm}$ was used in all experiments. Copper was pretreated in $0.1 \mathrm{M} \mathrm{KMnO}_{4}$ at a potential of -0.5 $\mathrm{V}$ (SCE) to mimic the galvanic couple potential of the intermetallic particles embedded in AA2024-T351 exposed to a similar solution. Manganese metal was held at a series of potentials ranging from $-1.84 \mathrm{~V}$ to $1.0 \mathrm{~V}$ (SCE) in $\mathrm{pH} 10$ borate buffer solution for 24 hours to grow stable manganese oxides on the surface of the metal. Raman spectroscopy was taken in-situ for the manganese metal, pretreated copper, and pretreated AA2024-T351. Raman spectroscopy was conducted ex-situ for the manganese oxide powders.

\subsection{Results}

\subsubsection{Effects of Permanganate during OCP exposures of AA2024 in $0.05 \mathrm{M} \mathrm{NaCl}$ Solution}

Figure 2.2 shows as-polished AA2024-T351 and the subsequent surface damage caused by 24 hour exposure to $0.05 \mathrm{M} \mathrm{NaCl}$ solution. The type of damage observed consisted of trenching and pitting attack which corresponds well with literature evidence ${ }^{[1,4]}$. Figure 
2.3(a) shows the distribution of intermetallic particles (the lighter regions, higher $\mathrm{Z}$ number) on bare AA2024-T351. Figure 2.3(b) shows the effects of pretreatment with 0.1 $\mathrm{M} \mathrm{KMnO}_{4}$ on the same region of intermetallic constituent particles as Figure 2.3(a). The particles studied by energy dispersive spectroscopy (EDS) are indicated in the secondary electron image shown in Figure 2.3 ( $\mathrm{a}$ and $\mathrm{b}$ ). Table 2.2 summarizes the qualitative EDS chemical analysis of the intermetallic particles before and after pretreatment. Manganese oxide growth was apparent on many of the intermetallics and was deposited specifically on the $\mathrm{Cu}$ rich phases as opposed to the aluminum matrix phase. Qualitative chemical data from EDS shows coverage of $\mathrm{Al}-\mathrm{Cu}, \mathrm{Al}-\mathrm{Cu}-\mathrm{Mg}$, and $\mathrm{Cu}-\mathrm{Mn}-\mathrm{Fe}-\mathrm{Al}$ type phases by a manganese oxide of unknown valence state. Permanganate formed thick oxide deposits over copper rich intermetallics in AA2024-T351 when pretreated. The deposition of permanganate was characterized by spherical caps over small $(\sim 0.5 \mu \mathrm{m})$ intermetallics and conformal layers with terraces over larger particles $(5 \mu \mathrm{m})$ (Figure 2.3(b)).

Figure 2.4 shows the result of 24 hours of pretreatment in chloride-free permanganate environment. As the concentration of permanganate increased, the size of the intermetallic cap decreased. Figure 2.5 shows the results of subsequent exposure to $0.05 \mathrm{M}$ sodium chloride solution after 24 hour pretreatment in potassium permanganate. Intermetallic trenching was inhibited at high concentrations of permanganate $(0.1 \mathrm{M})$ while the lower concentrations did not produce similar inhibition.

Figure 2.6 shows the results of exposure of unprotected AA2024 to 0.05 M sodium chloride solution with the inhibitor in-situ at the previously stated concentrations. The morphology of the manganese oxide coverage changed as the concentration of $\mathrm{MnO}_{4}{ }^{-}$decreased. At the 
$0.005 \mathrm{M}$ concentration the manganese oxide covered the aluminum matrix as well as the constituent particles. At 0.1 and $0.05 \mathrm{M}$ concentrations the manganese oxide mainly covered the intermetallic particles. Figure 2.7 shows the intact aluminum matrix surrounding the intermetallic particles after the manganese oxide caps have been removed post exposure revealing intact protected constituent particles.

EIS was performed on pretreated AA2024-T351 and on AA2024-T351 that had been exposed to $0.05 \mathrm{M} \mathrm{NaCl}$ with $0.1 \mathrm{M} \mathrm{KMnO}_{4}$ in solution. The results are shown in Figure 2.8. In both cases the impedance increased over the course of 24 hours. In the pretreated case an increase of almost 2 orders of magnitude was seen in the low frequency impedance data indicating an improvement in the barrier film. A simplified Randles circuit was used to model the system. Values for fit data for untreated AA2024 and for AA2024 pretreated in $0.1 \mathrm{M} \mathrm{KMnO}_{4}$ respectively after 24 hours where $\mathrm{R}_{\mathrm{s}}$ is the solution resistance, $\mathrm{R}_{\mathrm{p}}$ is barrier layer resistance, and $C$ is the capacitance were, $R_{s}: 320$ and $810 \mathrm{ohm}-\mathrm{cm}^{2}, R_{\mathrm{p}}: 7.3 \times 10^{4}$ and $4.9 \times 10^{6} \mathrm{ohm}-\mathrm{cm}^{2}, \mathrm{C}: 1.9 \times 10^{-6}$ and $5.7 \times 10^{-7} \mathrm{~F} / \mathrm{cm}^{2}$. The phase angle changes indicated a decrease in the capacitance due to the deposition of manganese oxides.

OCP data were collected for 1 hour after a 24 hour exposure to $0.05 \mathrm{M}$ sodium chloride solution with permanganate in solution (Figure 2.9). The OCP for all cases with in-situ permanganate were slightly higher than for AA2024-T351 in 0.05 M sodium chloride solution (-0.53 V vs SCE). The average OCP for 6 samples from the highest permanganate concentration to the lowest was as follows: $-0.46 \mathrm{~V},-0.48 \mathrm{~V}$, and $-0.49 \mathrm{~V}$ (SCE). The OCP rose but only slightly as expected given the oxidizing capability of $\mathrm{MnO}_{4}{ }^{-}$. 
The OCP was also measured for one hour after pretreatment for 24 hours in potassium permanganate but without $\mathrm{MnO}_{4}^{-}$in the OCP test solution. The samples were removed from pretreatment, rinsed thoroughly, and open circuit was measured in ambient aeration $0.05 \mathrm{M}$ sodium chloride solution. As Figure 2.10 shows, the OCP after such a pretreatment decreases to lower potentials slowly over time. This may be the result of residual permanganate, stored in the oxide, being reduced. As the source is depleted the reduction reaction slows and the OCP drops/stabilizes. Quite negative potentials were reached after this process had occurred. However, the Al alloy was not attacked. This was shown by the anodic upward scan after the open circuit measurement and will be discussed further in the section on anodic polarization behavior.

\subsubsection{Inhibition of Copper Replating}

Copper replating was also investigated on AA2024-T351 surfaces after OCP exposures using cyclic voltammetry $(\mathrm{CV})$ to assay the copper coverage. Cyclic voltammetric analyses of AA2024-T351 etched in for 24 hours in $0.05 \mathrm{M} \mathrm{NaCl}$ with and without inhibitor in solution and as a pretreatment were performed (Figure 2.11 (a and b)).

As expected, the AA2024 etched with no inhibitor in solution showed large current peaks reflecting the presence of replated copper and the change in surface copper valence state from (0) to (I) and then to (II). The etched AA2024-T351 showed a marked increase as compared to the freshly polished AA2024 (not shown). The anodic peaks reflect the surface coverage of deposited copper brought about by corrosion and thus were an indication of 
copper replating ${ }^{[25]}$. The results of the CV tests are summarized in the bar plots of Figure 2.12 with comparison to uncorroded AA2024 and high purity copper with essentially $100 \%$ copper surface coverage. Permanganate solution phase additions were the most effective at reducing $\mathrm{Cu}$ release by corrosion and/or $\mathrm{Cu}$ replating. The suppression of copper replating increased with inhibitor concentration in solution.

\subsubsection{Anodic Inhibition by Permanganate Ions}

\subsubsection{Localized Corrosion Behavior on Pretreated 2024-T351}

Figure 2.13(a-c) shows anodic E-logi behavior for AA2024-T351 in $0.05 \mathrm{M}$ sodium chloride solution during cyclic potentiodynamic scans. AA2024-T351 samples were first pretreated for 24 hours in a chloride free environment with various inhibitor concentrations and then exposed to $0.05 \mathrm{M} \mathrm{NaCl}$ solution without inhibitor.

Pretreated samples exhibited a decrease in the OCP which uncovered a passive region. The lower OCP consistently occurred at the lowest inhibitor concentration; at higher concentrations the OCP was statistically distributed. Only a small increase in pitting potential was observed for pretreatment in $0.005 \mathrm{M}$ potassium permanganate solution. The 0.05 and $0.1 \mathrm{M}$ concentrations showed no increase in the pitting potential. In summary, pretreatments with low concentrations showed the largest effect on the $\Delta \mathrm{E}$ defined as $\mathrm{E}_{\mathrm{pit}^{-}}$ EOCP. However, the effect was mainly associated with lowering the OCP. 


\subsubsection{Localized Corrosion Behavior of AA2024-T351 with Permanganate in Solution}

Figure 2.14 shows the behavior of AA2024-T351 in $0.05 \mathrm{M}$ sodium chloride solution with the inhibitor as an in-situ addition. Aluminum 2024-T351 was exposed for 24 hours with the inhibitor in solution prior to initiating the scan.

When the in-situ $\mathrm{MnO}_{4}^{-}$inhibitor was investigated at $0.1 \mathrm{M}$ concentration as shown in Figure 2.14(a), little to no anodic inhibition was observed. This lack of anodic inhibition was further exacerbated by the fact that the OCP was raised slightly because of the oxidizing nature of permanganate ions while the pitting potential was minimally affected. At lower concentrations $(0.005 \mathrm{M})$, the inhibitor significantly raised the pitting potential of AA2024-T351. Interestingly, the experiments in which an increase in pitting potential was observed all had elevated OCP levels. This could be the result of deposition of a high valence state oxide with a high redox potential covering the surface of the AA2024. When the OCP remained at $-0.5 \mathrm{~V}$ vs SCE, no increase in pitting potential was observed. Microscopy on samples that experienced very high pitting potentials showed substantial manganese oxide coverage of the entire surface.

Images of synthesized $\mathrm{Al}_{2} \mathrm{CuMg} \mathrm{S}$-phase held at $-0.5 \mathrm{~V}$ (SCE) in both $0.05 \mathrm{M}$ chloride solution and 0.1 $\mathrm{M} \mathrm{KMnO}_{4}$ and $0.05 \mathrm{M}$ chloride solution are shown in Figure 2.15. Figure 2.16 shows images of an AA2024 sample that exhibited a very high pitting potential. The manganese oxide covered both the intermetallic particles and the aluminum matrix. The manganese oxide caps over intermetallic particles were removed revealing areas of pitting but also generally intact intermetallic particles. 
Anodic polarization scans were also carried out in $0.025 \mathrm{M}$ manganese (II) chloride solution to determine if the reduced form of manganese effected the pitting potential of AA2024-T351. The concentration of $0.025 \mathrm{M}$ was chosen so that the total chloride concentration was the same as in the previous tests. No increase in the pitting potential was seen with $0.025 \mathrm{M} \mathrm{Mn}^{2+}$ in solution.

Figure 2.17 presents the cumulative probability distribution of pitting potentials as a function of potential for both pretreatment and in-situ solution phase inhibitor studies. Figure 2.18 presents the average of the difference between the pitting potential and the open circuit for both pretreatment and solution phase permanganate procedures on AA2024-T351. No significant increase in pitting potential was observed for pretreated AA2024-T351 tested in $0.05 \mathrm{M} \mathrm{NaCl}$ solution except at $0.005 \mathrm{M} \mathrm{KMnO}_{4}$. In-situ $\mathrm{MnO}_{4}{ }^{-}$ additions at low concentrations in the presence of an activating ion, like $\mathrm{Cl}^{-}$, produced a statistically significant increase in $\mathrm{E}_{\mathrm{pit}}$ and $\Delta \mathrm{E}$.

\subsubsection{Investigation of Cathodic Inhibition with $\mathrm{KMnO}_{4}$}

The rotating disk electrode method was used to examine the net cathodic reaction kinetics of both AA2024-T4 and pure copper in $\mathrm{pH} 8.4$ borate buffer solution to limit $\mathrm{pH}$ changes during the test (Figure 2.19). Rotating disk experiments were also conducted in $0.05 \mathrm{M}$ sodium chloride for comparison (not shown). Cathodic polarization curves were collected as a function of rotation rate as seen in Figure 2.19. AA2024-T4 exposed in $0.5 \mathrm{M} \mathrm{NaCl}$ showed mixed charge transfer - mass transfer controlled ORR from OCP to approximately 
$-0.9 \mathrm{~V}(\mathrm{SCE})$ at which point diffusion limited control dominated until the hydrogen evolution reaction (HER) dominated the total cathodic current density around $-1.3 \mathrm{~V}$ (SCE). High purity copper showed slightly different behavior in borate buffer than in sodium chloride solution. In borate buffer, the mixed charge transfer- mass transfer control region extended to $-0.8 \mathrm{~V}$ (SCE) with mass transfer control of the ORR dominating to -1.3 $\mathrm{V}$ (SCE) followed by the HER. In $0.05 \mathrm{M}$ sodium chloride solution, the oxygen reduction mass transfer control region was slightly larger and operated in the range -0.6 to $-1.3 \mathrm{~V}$ (SCE). As shown previously, the AA2024-T4 did not follow the Levich prediction because of the nature of its array of local fast cathodic reaction sites distributed in a less reactive $\mathrm{Al}$ matrix [51].

Figure 2.20 shows the cathodic polarization data collected using the rotating disk electrode method to examine the effects of potassium permanganate $\left(\mathrm{KMnO}_{4}\right)$ on the ORR kinetics on AA2024-T4. Two approaches were used to examine the effect of the inhibitor on the ORR; an inhibitor pretreatment and inhibitor addition in-situ (Figure 2.20, (a) and (b) respectively). It should be noted that $\mathrm{KMnO}_{4}$ provided an additional strong cathodic reactant with a very high redox potential in addition to dissolved $\mathrm{O}_{2}$ and $\mathrm{H}_{2} \mathrm{O}$ as discussed in the introduction. In the in-situ experiments, there is an increase in cathodic current density resulting from this additional cathodic reaction compared to either the pretreated or the inhibitor free condition.

Potassium permanganate changed the regions of mass and charge transfer control on AA2024-T4. In the pretreatment case, a mass transfer controlled region began around 0.2 $\mathrm{V}$ (SCE) and likely included residual $\mathrm{MnO}_{4}{ }^{-}$reduction. Figure 2.21 shows the cathodic polarization curve of a high purity copper sample pretreated in $0.05 \mathrm{M} \mathrm{KMnO}_{4}$ at $-0.5 \mathrm{~V}$ 
SCE, the open circuit potential of AA2024-T351 in the same solution. The reduction of the manganese oxide was evident in the increased cathodic current density compared to a copper sample with no pretreatment followed by a region of decreased cathodic current density after the oxides have been reduced. The reduction of deposited manganese oxide complicated the analysis of oxygen reduction on copper rich intermetallic particles in AA2024-T351 seen in Figure 2.20 that follows.

In Figure 2.20 (a) the HER was dominant between -0.75 and $-1.1 \mathrm{~V}$ (SCE) because the ORR was suppressed. When the inhibitor is used in-situ, mass transport control occurred from $-0.6 \mathrm{~V}$ to $-1.25 \mathrm{~V}(\mathrm{SCE})$ at which point the HER began to dominate (Figure 2.20(b)).

Plots of $1 / i_{\text {Tot }}$ vs $\omega^{-1 / 2}$ at $-1.0 \mathrm{~V}$ (SCE) (a potential likely dominated by oxygen reduction mass transfer control) showed that uninhibited AA2024-T4 has a lower current density than high purity copper (Figure 2.22(a)). This was due to the array of active electrodes surrounded by inactive aluminum matrix as described by the Gueshi model ${ }^{[51,52]}$. The Koutecky-Levich method ${ }^{[54]}$, as shown in Equation [13], was used to extract $i_{D}$ from $i_{T}$ (Figure 2.22) at $-1.0 \mathrm{~V}(\mathrm{SCE})$, where $i_{D}$ is the diffusion limited cathodic current density and $i_{c t}$ is the charge transfer limited cathodic current density. At this potential, $i_{H E R}$ is much lower than $i_{O R R}$.

$$
\frac{1}{i_{T o t}}=\frac{1}{i_{c t}}+\frac{1}{i_{D}}
$$

Where $i_{D}$ is given by 


$$
i_{D}=0.62 n F D^{2 / 3} v^{1 / 6} C_{O} \omega^{1 / 2}
$$

The results on high purity copper can be compared with the Levich description of masstransport limited $\mathrm{O}_{2}$ reduction given by Equation [14] (Figure 2.22(b)). Using a value for the diffusion of $\mathrm{O}_{2}$ in water, $\mathrm{D}=1.9 \times 10^{-5} \mathrm{~cm}^{2} / \mathrm{s}$, a value of $\mathrm{n}=4$, the concentration of $\mathrm{O}_{2}$ in solution, $C_{O}=7-9 \mathrm{ppm}$, and the kinematic viscosity, $v=1.0 \times 10^{-2} \mathrm{~cm}^{2} / \mathrm{s}$, good agreement was found between theoretical values and experimental data ${ }^{[55]}$. This supports the notion that the current on bare AA2024 at -1.0 V (SCE) due to the HER was a small fraction of the ORR current.

The diffusion limited current density was extracted from the total current density (Figure 2.23) using the Koutecky-Levich method (Equation13) for the pretreated case at $-1.0 \mathrm{~V}$ (SCE). The extracted diffusion limited current density is shown in Figure 2.24. Pretreated AA2024 had a lower cathodic current density than untreated AA2024-T4 over all rotation rates with a weaker rotation rate dependency. In-situ inhibitor presence only suppressed the total current density at high rotation rates or low $\omega^{-1 / 2}$. At low rotation rates the additional oxidizer raises the total cathodic reaction rate during the in-situ tests. In contrast, when the inhibitor was used as a pretreatment and limited additional $\mathrm{MnO}_{4}{ }^{-}$cathodic reactant was present, the total current density was suppressed as much as two orders of magnitude at all rotation rates. This indicates cathodic inhibition by $\mathrm{MnO}_{4}^{-}$pretreatment.

The activation controlled cathodic current density was a linear function of the copper surface coverage described by Kaesche ${ }^{[56]}$ by Equation [15].

$$
i_{\text {act,heterogeneous }}=\Theta_{\text {Cu }}^{\text {Kaeshe }} i_{\text {homogeneous }} .
$$


Where $\theta$ is the copper surface coverage or $\mathrm{Cu}$ rich IMC coverage and $i_{\text {homogeneous }}$ is exponentially dependent on the cathodic potential. The extracted activation current density is plotted vs. potential in Figure 2.25, along with estimated copper coverage.

\subsubsection{Manganese Oxide Valence State Determination}

Galvanostatic reduction curves of electrodeposited manganese oxides on copper were conducted to probe the valence state of the manganese oxide (Figure 2.26). The multiple potential waves compared to untreated copper indicated the presence and reduction of an oxide from a high valence state. The reduction of $\mathrm{CuO}$ was observed on untreated copper [57]. The manganese reduction potential waves are indicative of changes in the oxide's valence state as the reduction proceeds ${ }^{[58]}$. The reversible potentials of several likely manganese reduction reactions are indicated in the figure by the broken lines. A break in the abscissa is necessary as the complete reduction of the manganese oxide required much more charge than the reduction of the copper oxide. All of the prominent features of the manganese oxide reduction are shown to the right of the break between 200 and 4000 seconds. The potential decays linearly until 5000 seconds have passed at which point two reduction waves occurred in quick succession.

Raman spectroscopy was conducted on high purity copper, pretreated AA2024-T351, high purity metallic manganese, and $\mathrm{MnO}_{2}$ powder. The spectra for a selection of the data are presented in Figure 2.27. The figure shows three common peaks shared between the copper treated in $0.1 \mathrm{M} \mathrm{KMnO}_{4}$ at $-.5 \mathrm{~V}(\mathrm{SCE})$ and the AA2024-T351 pretreated at OCP in $0.1 \mathrm{M}$ 
$\mathrm{KMnO}_{4}$. The first peak was centered on 506 wavenumbers $\left(\mathrm{cm}^{-1}\right)$. The second common peak was centered on 573 wavenumbers, and the third on 637 wavenumbers. The potential of the potentiostatic hold of the manganese metal was chosen with the intention of growing $\mathrm{MnO}_{2}$. The E-pH diagram in Figure 2.1 shows that at a $\mathrm{pH}$ of 10, the chosen potential is well within the $\mathrm{MnO}_{2} \mathrm{E}-\mathrm{pH}$ phase field. The Raman peaks shared by the manganese oxide/hydroxide deposited on the high purity copper and on the AA2024 indicated that they are the same species. However, the complexity of the peak structure did not allow the identification of any single manganese oxide/hydroxide phase.

The steady drop in potential in Figure 2.10 indicated the further reduction of deposited manganese oxide valence state on AA2024-T351. As the reduction from manganese (VI) to (III) to (II) occurs, the potential of the system dropped towards the Nernst potential of the lowest valence oxide until all of the oxide had been reduced. At this point the rate of the HER coupled with aluminum oxidation dominate the OCP of AA2024-T351.

\subsection{Discussion}

\subsubsection{Oxidation States of Manganese}

The oxidation state of the manganese oxide deposits is critical to the understanding of this inhibitor system. If the deposited Mn cap has a high valence state, the E-pH diagrams in Figure 2.1 suggest that the oxide deposits may be electrochemically active and eligible for further reduction at the open circuit of AA2024. This activity can manifest itself as the reduction of the oxide deposits to lower valence state oxides accompanied by aluminum oxidation. These oxide reactions may have some impact on the corrosion inhibition. It is 
useful for the discussion to give a brief example of our understanding of these manganese deposits. A galvanostatic reduction at $-20 \mu \mathrm{A} / \mathrm{cm}^{2}$, conducted in $\mathrm{pH} 10$ borate buffer is shown in Figure 2.26. The multiple potential waves indicated that the oxide was reduced from a high valence state ${ }^{[48]}$. Evidence of this is also shown in Figure 2.10 at OCP and 2 upon downward scan. The reversible potentials of several likely reactions are indicated in Figure 2.26. This reduction path is supported in literature concerning the reduction of manganese oxides $[47,48,58]$. Raman spectroscopy (Figure 2.27) suggested that the manganese species present on the $\mathrm{Cu}$ and analyzed by galvanostatic reduction is the same as that reduced onto AA2024-T351 at open circuit. It is reasonable to argue that the manganese oxide present on the surface of the AA2024 has a high valence state and further reduction of the oxide presents a possible mechanism for release of an $\mathrm{Mn}$ (III) ion which may present the possibility of self-healing. The literature supports this reduction path and also the ionic release of a $\mathrm{Mn}(\mathrm{III})$ species ${ }^{[58]}$. The further reduction of the oxide may play a role in active corrosion inhibition in a corrosive environment but this additional aspect is beyond the scope of this paper.

\subsubsection{Modes of $\mathrm{MnO}_{4}^{-}$Inhibition: Anodic}

While the ORR was inhibited by the presence of Mn-oxides that result from permanganate reduction over IMC, elevation of the pitting potential is speculated to require the presence of a manganese oxide film-barrier formed over the aluminum matrix as seen in Figure 2.16. 
Figure 2.18 shows the trends in the extent of the passive region $\left(\Delta E\right.$ where $\Delta E=E_{p i t}-E_{c o r r}$ ) as a function of inhibitor concentration. In both pretreatment and solution phase tests, the inhibitor raises $\Delta E$ as the inhibitor concentration was decreased. This is an interesting and somewhat counter-intuitive observation. The interpretation of this behavior is that various amounts of $\mathrm{KMnO}_{4}$ are reduced onto $\mathrm{Cu}$-rich sites during pretreatment. Lower $\mathrm{KMnO}_{4}$ levels may deposit more efficiently with less residual/unreduced Mn(VII). This can lower the $\mathrm{OCP}$ and increase $\triangle \mathrm{E}$ by lowering $\mathrm{E}_{\mathrm{Corr}}$. Higher concentrations may induce pitting prior to deposition and blockage of IMC sites. This speculation is supported by Figure 2.9. Insitu inhibitor at low concentrations in the presence of an activating ion such as $\mathrm{Cl}^{-}$produced a statistical increase in $\mathrm{E}_{\text {pit }}$. The principal reason for this increase is speculatively believed to be the protective manganese oxide covering the S-phase as seen in Figure 2.15. We have shown that both $\mathrm{MnO}_{4}^{-}$and $\mathrm{Mn}^{2+}$ are not effective anodic inhibitors when they are in solution as seen in Figure 2.13 and 2.16. In Figure 2.13, the reverse dependence of pitting potential on concentration suggests that the ionic species does not directly lead to anodic inhibition. Inhibition is not seen on AA2024-T351 samples that have been exposed to the permanganate-chloride solution for 1 hour (polarization curves not shown). The 24 hour exposure prior to the anodic polarization may enable the growth of a protective manganese oxide film on the aluminum matrix and the S-phase. This is supported by the lack of manganese species in pitted areas examined via EDS. 


\subsubsection{Modes of $\mathrm{MnO}_{4}$ - Inhibition: Cathodic}

Permanganate functions as a cathodic inhibitor by growing Mn oxide caps over copper rich intermetallic particles (Figure 2.3 and 2.7). This process occurs quickly at the natural $\mathrm{pH}$ of the permanganate solution and does not require any $\mathrm{Cl}^{-}$activation. The oxide growth by direct electrochemical reduction of permanganate over the copper-rich intermetallics greatly reduces the total cathodic current density $i_{T o t}$ as well as the diffusion limited current density $i_{D}$ when used as a pretreatment (Figure 2.20(a)). The $\mathrm{MnO}_{4}^{-}$ion is reduced providing an additional cathodic reactant that increases the cathodic current density when present in solution (Figure 2.20 (b)). We will now examine the pretreatment case in more detail.

Using a model first utilized by Jakab ${ }^{[12,51,56]}$ that combines the Gueshi and membrane models, we can examine the controlling variables that limit the reduction of dissolved oxygen on a planar electrode with an array of fast ORR sites. The diffusion limited current density $i_{D}{ }^{*}$ on a homogeneous planar electrode is given by

$$
i_{D}^{*}=\frac{n F D C^{o}}{\delta}
$$

where $n$ is the number of electrons in the electrochemical reaction, $F$ is the Faradays constant, $D$ is the diffusion coefficient of the reactant, $C^{o}$ is the bulk concentration of the reactant, and $\delta$ is the diffusion affected zone thickness. In an RDE experiment $\delta$ is proportional to $\omega^{-1 / 2}$ where $\omega$ is the rotation rate of the planar electrode ${ }^{[52]}$. The Gueshi and membrane models enable the extension of the description of $i_{D}{ }^{*}$ to heterogeneous 
electrodes with discrete fast reaction sites. Two effects can occur when oxide caps are deposited on the intermetallic particle. The first is a change in the geometry and spacing of active regions in the inactive matrix as described by Gueshi in Equation [17].

$$
i_{D}=\frac{i_{D}^{*}}{\sigma^{2}-1}\left[\sigma e^{-l t}-1+\sqrt{\pi \sigma^{2}} \sqrt{\frac{l t}{\sigma^{2}-1}} \exp \left(\frac{l t}{\sigma^{2}-1}\right)\left\{\operatorname{erf}\left(\sigma \sqrt{\frac{l t}{\sigma^{2}-1}}\right)-\operatorname{erf}\left(\sqrt{\frac{l t}{\sigma^{2}-1}}\right)\right\}\right]
$$

In Equation [17], $l$ is a geometric term describing the spacing of active-inactive array sites, and $\sigma$ is a term that relates to the coverage of aluminum matrix (shown in Figure 2.28), by active sites or the coverage of active intermetallic sites. As the caps grow, one effect is the exposure of the effective active region or IMCs is decreased. This can be seen in the SEM microscopy of the pretreated AA2024, Figure 2.4, as well as by examining the activation controlled current density, Figure 2.25.

The decrease in copper "surface coverage" decreases the size of the active sites and increases the spacing between active sites in the Gueshi model. By examining Figure 2.28 the effect of copper coverage on ORR can be clearly seen. The coverage of the copper intermetallics by manganese oxides accounts for some of the ORR decrease seen on pretreated samples.

The second possible effect is the decreased rate of oxygen diffusion through the oxide barrier as opposed to through the electrolytic solution to the heterogeneous active sites. The membrane model treats the situation of two phase diffusion involving the oxygen diffusion boundary layer and the oxide. The model is described by Equation [18] and was used by Ilevbare ${ }^{[12]}$, 


$$
i_{D}=n F C^{o}\left(\frac{1}{\left(1.61 D_{\text {sol }}^{\frac{1}{3}} v^{\frac{1}{6}} / D_{s o l} \omega^{\frac{1}{2}}\right)+\left(\chi_{o x} / D_{o x}\right)}\right)
$$

where $\chi_{o x}$ is the oxide thickness, $\mathrm{D}_{\text {sol }}$ is the diffusion of $\mathrm{O}_{2}$ in solution, $\mathrm{D}_{\mathrm{ox}}$ is the diffusion of $\mathrm{O}_{2}$ in the oxide, and $v$ is the kinematic viscosity of water. To examine the diffusion of oxygen through a porous manganese oxide film, manganese oxide films were grown over a highly pure copper rotating disk electrode at a potential of $-0.5 \mathrm{~V}$ SCE. This potential was chosen to replicate the environment of the $\mathrm{Cu}$-rich intermetallics on AA2024, which are held at the OCP of AA2024. Optical and scanning electron microscopy of the film show it to be homogeneous in thickness and coverage (not shown). A cross-section of the film was taken to determine its thickness. Rotation-rate-ramp experiments were performed at a potential of $-1.0 \mathrm{~V}(\mathrm{SCE})$ on the sample in borate buffer and $i_{D}$ from $i_{T}$ were extracted. The result can be seen in Figure 2.29 compared to the model results for of the system. High purity copper with a uniform manganese oxide film deposited on the surface exhibits a large reduction in the ORR after the $\mathrm{MnO}_{4}{ }^{-}$is reduced. A potentiodynamic scan of high purity copper with a manganese film electrodeposited over its surface (Figure 2.21) shows that at $-1.0 \mathrm{~V}$ the ORR has been suppressed. The diffusion coefficient through the oxide film was estimated to be $1.0 \times 10^{-8} \mathrm{~cm}^{2} / \mathrm{sec}$ using Equation 18 , the rotation rate dependent mass limited current densities collected from the manganese oxide coated high purity copper samples, the film thickness, and reasonable values for the remaining parameters. 


\subsection{Conclusions}

Low concentration pretreatment with permanganate leads to a slight increase in the pitting potential ( $E_{\mathrm{pit}}$ ) of AA2024 but only when partially oxidized manganese oxides were electrochemically deposited over the AA2024 aluminum matrix. Decreases in OCP accompanied the elevated $\mathrm{E}_{\text {pit. }}$ Permanganate increased cathodic reaction rates when it was present in solution owing to the addition of this oxidizing cathodic reactant. However, when permanganate was used as a pretreatment and was electrochemically deposited as a lower valence state oxide on the IMCs, it decreased cathodic reaction rates. Moreover, OCP was greatly reduced after pretreatment as copper rich sites were covered with manganese oxides. The slow reduction of high valence manganese oxides may account for the gradual decrease in the OCP. Copper replating during $\mathrm{NaCl}$ exposure was also decreased after pretreatments. However, this effect did not occur to the same extent as when permanganate was added as an in-situ solution phase inhibitor. The manganese oxide has been shown to be present initially in a high valence state, most likely a mixed oxide with some $\mathrm{MnO}_{2}$ present at least initially. The reduction of this oxide to lower valence compounds has a number of effects including acting as an additional cathodic reactant which has the effect of complicating the cathodic reaction kinetics. In summary, $\mathrm{MnO}_{4}^{-}$is a complex ionic inhibitor that is more beneficial when present as a pretreatment compared to an ionic species addition. 


\subsection{References}

1. G.O. Ilevbare, O. Schneider, R.G. Kelly, and J.R. Scully, "In situ confocal laser scanning microscopy of AA 2024-T3 corrosion metrology - I. Localized corrosion of particles," Journal of the Electrochemical Society 151, 8 (2004): p. B453.

2. O. Schneider, G.O. Ilevbare, J.R. Scully, and R.G. Kelly, "In situ confocal laser scanning microscopy of AA 2024-T3 corrosion metrology - II. Trench formation around particles," Journal of the Electrochemical Society 151, 8 (2004): p. B465.

3. J.R. Scully, T.O. Knight, R.G. Buchheit, and D.E. Peebles, "Electrochemical characteristics of the $\mathrm{Al}_{2} \mathrm{Cu}, \mathrm{Al}_{3} \mathrm{Ta}$ and $\mathrm{Al}_{3} \mathrm{Zr}$ intermetallic phases and their relevancy to the localized corrosion of Al alloys," Corrosion Science 35, 1-4 (1993): p. 185.

4. R.P. Wei, C.M. Liao, and M. Gao, "A transmission electron microscopy study of constituent-particle-induced corrosion in 7075-T6 and 2024-T3 aluminum alloys," Metall Mater Trans A 29, 4 (1998): p. 1153.

5. G.S. Chen, "Microconstituent-induced pitting corrosion in aluminum alloy 2024T3," CORROSION 52, 01 (1996).

6. D.G. Harlow and R.P. Wei, "A probability model for the growth of corrosion pits in aluminum alloys induced by constituent particles," Engineering Fracture Mechanics 59, 3 (1998): p. 305.

7. R.G. Buchheit, R.P. Grant, P.F. Hlava, B. Mckenzie, and G.L. Zender, "Local dissolution phenomena associated with $\mathrm{S}$ phase $\left(\mathrm{Al}_{2} \mathrm{CuMg}\right)$ particles in aluminum alloy 2024-T3," Journal of the Electrochemical Society 144, 8 (1997): p. 2621.

8. C.M. Reddy, Q.S. Yu, C.E. Moffitt, D.M. Wieliczka, W. Johnson, J.E. Deffeyes, and H.K. Yasuda, "Improved corrosion protection of aluminum alloys by system approach interface engineering: Part 1 - Alclad 2024-T3," Corrosion 56, 8 (2000): p. 819.

9. D. Zhu and W.J. van Ooij, "Corrosion protection of AA 2024-T3 by bis-[3(triethoxysilyl)propyl]tetrasulfide in neutral sodium chloride solution. Part 1: corrosion of AA 2024-T3," Corrosion Science 45, 10 (2003): p. 2163.

10. K.A. Yasakau, M.L. Zheludkevich, S.V. Lamaka, and M.G.S. Ferreira, "Mechanism of Corrosion Inhibition of AA2024 by Rare-Earth Compounds," The Journal of Physical Chemistry B 110, 11 (2006): p. 5515.

11. G.O. Ilevbare and J.R. Scully, "Mass-transport-limited oxygen reduction reaction on AA2024-T3 and selected intermetallic compounds in chromate-containing solutions," Corrosion 57, 2 (2001): p. 134.

12. G.O. Ilevbare and J.R. Scully, "Oxygen Reduction Reaction Kinetics on Chromate Conversion Coated $\mathrm{Al}-\mathrm{Cu}, \mathrm{Al}-\mathrm{Cu}-\mathrm{Mg}$, and $\mathrm{Al}-\mathrm{Cu}-\mathrm{Mn}-\mathrm{Fe}$ Intermetallic Compounds," Journal of The Electrochemical Society 148, 5 (2001): p. B196.

13. G.O. Ilevbare, J.R. Scully, J. Yuan, and R.G. Kelly, "Inhibition of pitting corrosion on aluminum alloy 2024-T3: Effect of soluble chromate additions vs chromate conversion coating," Corrosion 56, 3 (2000): p. 227.

14. M.W. Kendig and R.G. Buchheit, "Corrosion inhibition of aluminum and aluminum alloys by soluble chromates, chromate coatings, and chromate-free coatings," Corrosion 59, 5 (2003): p. 379. 
15. Y. Yoon and R.G. Buchheit, "Dissolution Behavior of $\mathrm{Al}_{2} \mathrm{CuMg}$ (S Phase) in Chloride and Chromate Conversion Coating Solutions," Journal of the Electrochemical Society 153, 5 (2006): p. B151.

16. W. Zhang, B. Hurley, and R.G. Buchheit, "Characterization of Chromate Conversion Coating Formation and Breakdown Using Electrode Arrays," Journal of The Electrochemical Society 149, 8 (2002): p. B357.

17. OSHA, IMIS Code Number 0689 Chromium (VI) (Hexavalent Chromium). 2006.

18. R.G. Buchheit, R.K. Boger, M.C. Carroll, R.M. Leard, C. Paglia, and J.L. Searles, "The electrochemistry of intermetallic particles and localized corrosion in Al alloys," Jom-J Min Met Mat S 53, 7 (2001): p. 29.

19. S. Kim, J.T. Burns, and R.P. Gangloff, "Fatigue crack formation and growth from localized corrosion in Al-Zn-Mg-Cu," Engineering Fracture Mechanics 76, 5 (2009): p. 651.

20. R.M. Pidaparti and R.R. Patel, "Correlation between corrosion pits and stresses in Al alloys," Mater Lett 62, 30 (2008): p. 4497.

21. K.K. Sankaran, R. Perez, and K.V. Jata, "Effects of pitting corrosion on the fatigue behavior of aluminum alloy 7075-T6: modeling and experimental studies," Mat Sci Eng a-Struct 297, 1-2 (2001): p. 223.

22. R.L. Twite and G.P. Bierwagen, "Review of alternatives to chromate for corrosion protection of aluminum aerospace alloys," Progress in Organic Coatings 33, 2 (1998): p. 91.

23. W.D. Robertson, "Molybdate and Tungstate as Corrosion Inhibitors and the Mechanism of Inhibition," Journal of The Electrochemical Society 98, 3 (1951): p. 94.

24. K.D. Ralston, S. Chrisanti, T.L. Young, and R.G. Buchheit, "Corrosion inhibition of aluminum alloy 2024-T3 by aqueous vanadium species," Journal of the Electrochemical Society 155, 7 (2008): p. C350.

25. M.A. Jakab, F. Presuel-Moreno, and J.R. Scully, "Critical Concentrations Associated with Cobalt, Cerium, and Molybdenum Inhibition of AA2024-T3 Corrosion: Delivery from Al-Co-Ce(-Mo) Alloys," CORROSION 61, 3 (2005): p. 246.

26. M. Iannuzzi and G.S. Frankel, "Inhibition of Aluminum Alloy 2024 Corrosion by Vanadates: An In Situ Atomic Force Microscopy Scratching Investigation," Corrosion 63, 7 (2007): p. 672.

27. M. Iannuzzi and G.S. Frankel, "Mechanisms of corrosion inhibition of AA2024-T3 by vanadates," Corrosion Science 49, 5 (2007): p. 2371.

28. M. Iannuzzi, J. Kovac, and G.S. Frankel, "A study of the mechanisms of corrosion inhibition of AA2024-T3 by vanadates using the split cell technique," Electrochimica Acta 52, 12 (2007): p. 4032.

29. M. Iannuzzi, T. Young, and G.S. Frankel, "Aluminum alloy corrosion inhibition by vanadates," Journal of the Electrochemical Society 153, 12 (2006): p. B533.

30. K.D. Ralston, T.L. Young, and R.G. Buchheit, "Electrochemical Evaluation of Constituent Intermetallics in Aluminum Alloy 2024-T3 Exposed to Aqueous Vanadate Inhibitors," Journal of the Electrochemical Society 156, 4 (2009): p. C135. 
31. E.A. Lizlovs, "Molybdates as Corrosion-Inhibitors in Presence of Chlorides," Corrosion 32, 7 (1976): p. 263.

32. A.H. Alsaffar, V. Ashworth, A.K.O. Bairamov, D.J. Chivers, W.A. Grant, and R.P.M. Procter, "Effect of Molybdenum Ion-Implantation on the General and Pitting Corrosion Behavior of Pure Aluminum and a High-Strength AluminumAlloy," Corrosion Science 20, 1 (1980): p. 127.

33. I. Puigdomenech, Hydra/Medusa Chemical Equilibrium Database and Plotting Software. 2004, freely downloadable software at www.kemi.kth.se/medusa/: KTH Royal Institute of Technology.

34. M. Pourbaix, Atlas of electrochemical equilibria in aqueous solutions. 2d English ed (Houston, Tex.: National Association of Corrosion Engineers, 1974). p. 644 p.

35. Y. Baek and G.S. Frankel, "Electrochemical quartz crystal microbalance study of corrosion of phases in AA2024," Journal of the Electrochemical Society 150, 1 (2003): p. B1.

36. J.W. Bibber, US Patent 48956081990.

37. J.W. Bibber, US Patent 55542311996.

38. ASTM, B-117 - Standard Practice for Operating Salt Spray (Fog) Apparatus, in ASTM. 2009.

39. J.W. Bibber, A Chrome Free Conversion Coating and Sealant for Aluminum and Its Alloys, in CORROSION 99. 1999, NACE International: San Antonio, Tx.

40. M. Crouse, K.L. Vasanth, A.E. Miller, and M.G. Pujar, Evaluation of Potassium Permanganate $\left(\mathrm{KMnO}_{4}\right)$ as a Green Corrosion Inhibitor/Sealant for Anodized Al 2024 and Al 6061 at Different pH Values, in CORROSION 2002. 2002, NACE International: Denver, Co.

41. S.A. Kulinich, M. Farzaneh, and X.W. Du, "Growth of corrosion-resistant manganese oxide coatings on an aluminum alloy," Inorg Mater+ 43, 9 (2007): p. 956.

42. I. Danilidis, A.J. Davenport, and J.M. Sykes, "Characterisation by X-ray absorption near-edge spectroscopy of $\mathrm{KMnO} 4-$ based no-rinse conversion coatings on $\mathrm{Al}$ and Al alloys," Corrosion Science 49, 4 (2007): p. 1981.

43. I. Danilidis, J. Hunter, G.M. Scamans, and J.M. Sykes, "Effects of inorganic additions on the performance of manganese-based conversion treatments," Corrosion Science 49, 3 (2007): p. 1559.

44. H.A. Swain, C. Lee, and R.B. Rozelle, "Determination of the solubility of manganese hydroxide and manganese dioxide at 25.deg. by atomic absorption spectrometry," Analytical Chemistry 47, 7 (1975): p. 1135.

45. F. Buciuman, F. Patcas, R. Craciun, and D.R.T. Zahn, "Vibrational spectroscopy of bulk and supported manganese oxides," Physical Chemistry Chemical Physics 1, 1 (1999): p. 185.

46. I. Danilidis, J. Hunter, G.M. Scamans, and J.M. Sykes, "Effect of silica nanoparticles on the performance of manganese-based conversion treatments," Corrosion Science 50, 11 (2008): p. 3038.

47. B.A.L. Demishima, T. Ohtsuka, H. Konno, and N. Sato, "XPS Study of the $\mathrm{MnO}_{2}$ Electrode in Borate Solution during the Discharge Process," Electrochimica Acta 36, 9 (1991): p. 1485. 
48. B.A.L. Demishima, T. Ohtsuka, and N. Sato, "A Study of the Discharge Process of Manganese Oxide in Borate Solution Using Insitu Techniques," Electrochimica Acta 38, 2-3 (1993): p. 341.

49. L.M. Gassa, H.T. Mishima, B.A.L. deMishima, and J.R. Vilche, "An electrochemical impedance spectroscopy study of electrodeposited manganese oxide films in borate buffers," Electrochimica Acta 42, 11 (1997): p. 1717.

50. W.H. Ho and S.K. Yen, "Characterization of Electrolytic Manganese Oxide Coating on Pt for Lithium Battery Applications," Journal of The Electrochemical Society 152, 3 (2005): p. A506.

51. M.A. Jakab, D.A. Little, and J.R. Scully, "Experimental and Modeling Studies of the Oxygen Reduction Reaction on AA2024-T3," Journal of The Electrochemical Society 152, 8 (2005): p. B311.

52. T. Gueshi, K. Tokuda, and H. Matsuda, "Voltammetry at partially covered electrodes: Part I. Chronopotentiometry and chronoamperometry at model electrodes," Journal of Electroanalytical Chemistry 89, 2 (1978): p. 247.

53. R.B. Bird, W.E. Stewart, and E.N. Lightfoot, Transport phenomena. 2nd, Wiley international ed (New York: J. Wiley, 2002). p. xii.

54. E. Gileadi, Electrode Kinetics for Chemists, Chemical Engineers and Materials Scientists: John Wiley and Sons, 1993.

55. F.J. Presuel-Moreno, M.A. Jakab, and J.R. Scully, "Inhibition of the oxygen reduction reaction on copper with cobalt, cerium, and molybdate ions," Journal of the Electrochemical Society 152, 9 (2005): p. B376.

56. M.A. Jakab, F. Presuel-Moreno, and J.R. Scully, "Effect of Molybdate, Cerium, and Cobalt Ions on the Oxygen Reduction Reaction on AA2024-T3 and Selected Intermetallics," Journal of The Electrochemical Society 153, 7 (2006): p. B244.

57. S. Nakayama, T. Kaji, M. Shibata, T. Notoya, and T. Osakai, "Which is easier to reduce, $\mathrm{Cu}_{2} \mathrm{O}$ or $\mathrm{CuO}$ ?," Journal of the Electrochemical Society 154, 1 (2007): p. C1.

58. A. Kozawa and J.F. Yeager, "The Cathodic Reduction Mechanism of Electrolytic Manganese Dioxide in Alkaline Electrolyte," Journal of The Electrochemical Society 112, 10 (1965): p. 959. 


\subsection{Tables}

Table 2.1. Chemical composition of AA2024-T351 (wt. \%) The T4 designation indicates slight cold working of T351.

\begin{tabular}{lrrrrrrrrrrr} 
AA2024 & $\mathrm{Al}$ & $\mathrm{Cu}$ & $\mathrm{Mg}$ & $\mathrm{Mn}$ & $\mathrm{Fe}$ & $\mathrm{Zn}$ & $\mathrm{Si}$ & $\mathrm{Ti}$ & $\mathrm{Cr}$ & $\mathrm{V}$ & $\mathrm{Ga}$ \\
\hline -T351 & Balance & 4.56 & 1.26 & 0.59 & 0.22 & 0.12 & 0.08 & 0.02 & 0.01 & 0.01 & $\mathrm{NA}$ \\
& & & & & & & & & & & \\
-T4 & Balance & 4.34 & 1.38 & 0.585 & 0.221 & 0.007 & 0.069 & 0.017 & 0.006 & 0.006 & 0.011
\end{tabular}

Table 2.2.Compilation of EDS data from AA2024-T351 intermetallic particles in Figure 2.3, before and after pretreatment in $0.1 \mathrm{M} \mathrm{KMnO}_{4}$.

\begin{tabular}{|c|c|c|c|c|}
\hline Intermetallic phase (EDS) & Particle \# & $\begin{array}{l}\text { Manganese } \\
\text { Cap }\end{array}$ & Oxide & $\begin{array}{l}\text { Mn enrichment } \\
\text { (EDS) }\end{array}$ \\
\hline $\mathrm{Al}-\mathrm{Cu}-\mathrm{Mg}$ & 5,6 & Yes & & Yes \\
\hline $\mathrm{Al}-\mathrm{Cu}-\mathrm{Mn}-\mathrm{Fe}-\mathrm{Si}$ & $1,2,3$ & Yes & & Yes \\
\hline $\mathrm{Al}-\mathrm{Cu}$ & 4 & Yes & & Yes \\
\hline $\mathrm{Al}-\mathrm{Cu}-\mathrm{Mn}-\mathrm{Fe}-\mathrm{Mg}$ & 7,8 & Yes & & Yes \\
\hline
\end{tabular}




\subsection{Figures}

a)

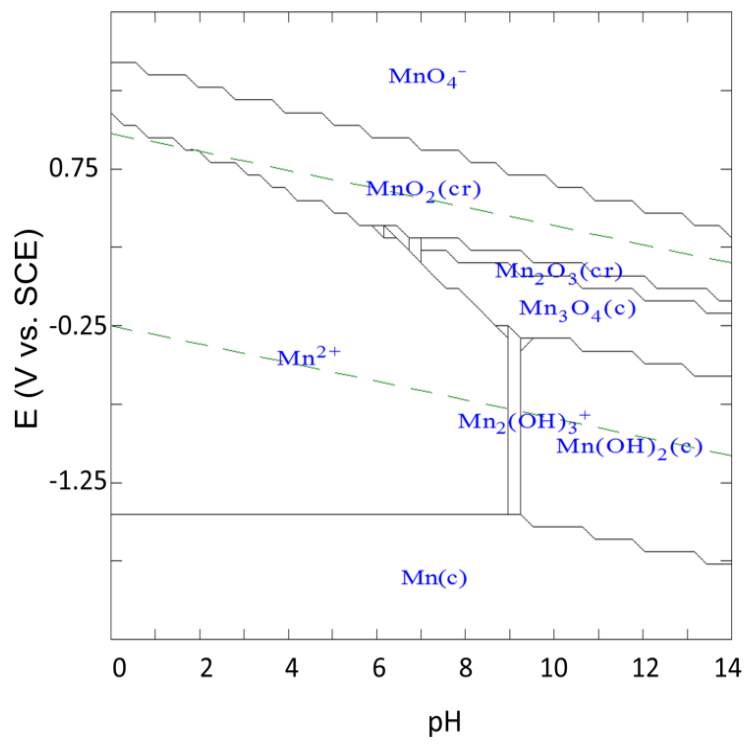

b)

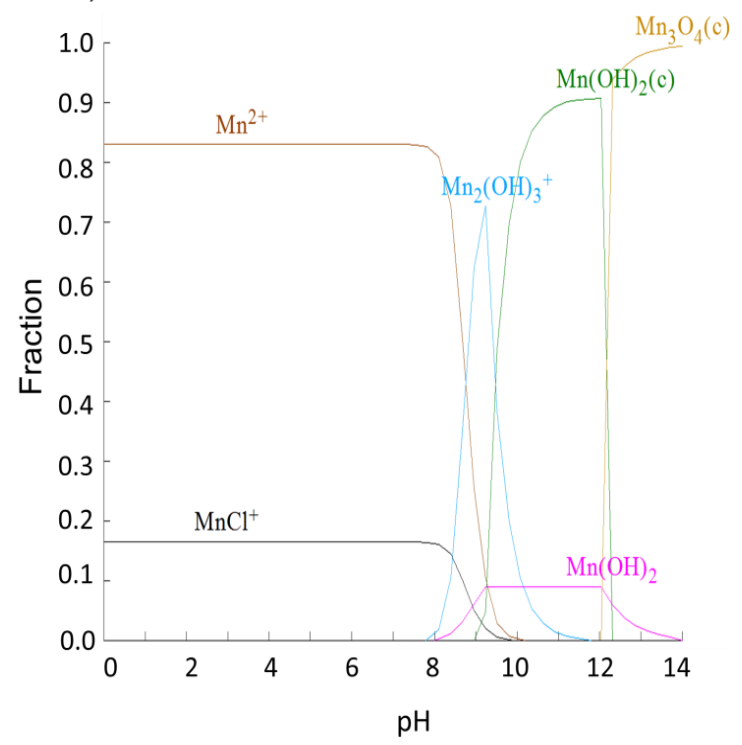

Figure 2.1.(a) E-pH diagram for $0.005 \mathrm{M}$ permanganate with addition of $0.05 \mathrm{M}$ chloride.

(b) Speciation diagrams for $0.005 \mathrm{M} \mathrm{MnO}_{4}^{-}+0.05 \mathrm{M} \mathrm{Cl}^{-}$at $-0.5 \mathrm{~V}_{\text {sce, }}$, the open circuit of AA-2024.AA2024. E-pH and speciation diagrams were calculated using Medusa software ${ }^{[1]}$.

a)

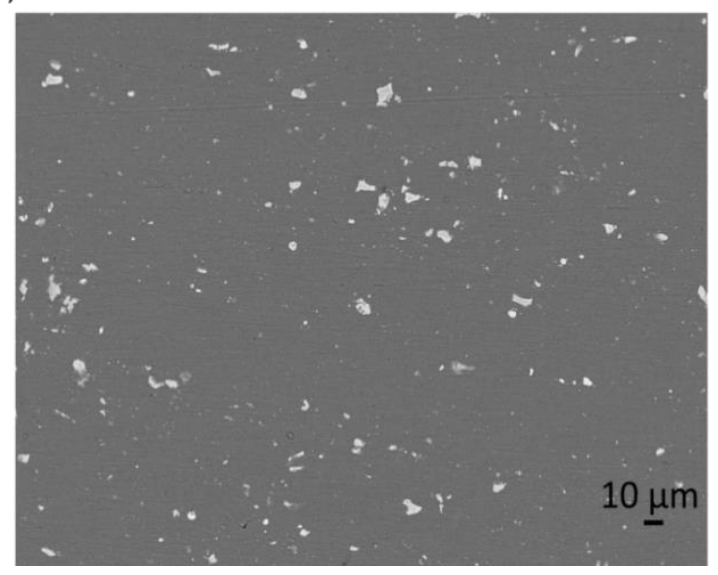

b)

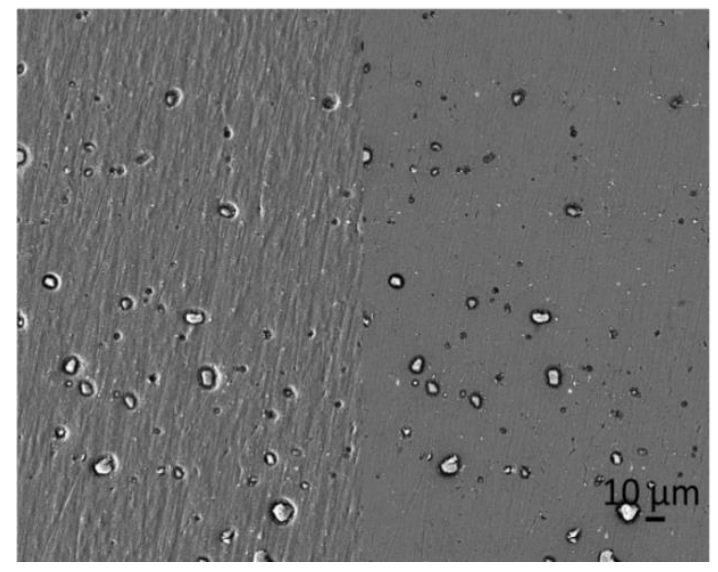

Figure 2.2. (a) As-polished (1200 grit) AA2024-T351 and subsequent surface damage caused by 24 hour exposure to aerated $0.05 \mathrm{M} \mathrm{NaCl}$ solution. (b) The damaged specimen image is distorted laterally but includes both a secondary electron image (left) and a backscattered electron image (right), which clearly shows intermetallic trenching. 
a)

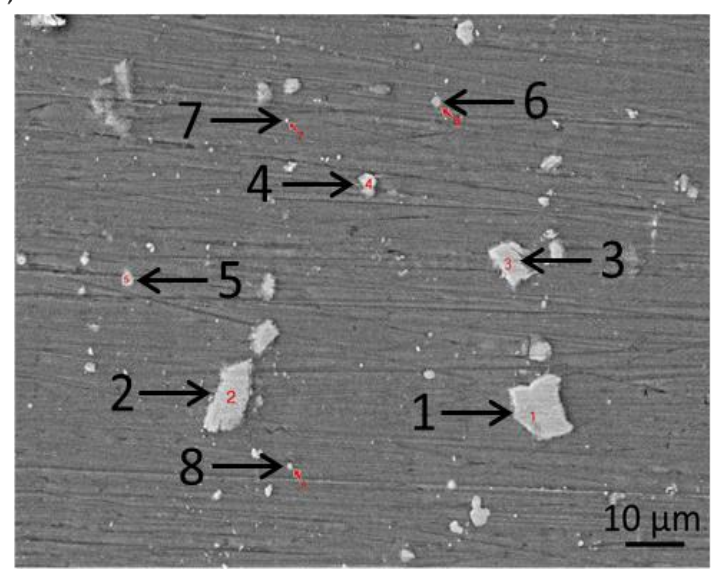

b)

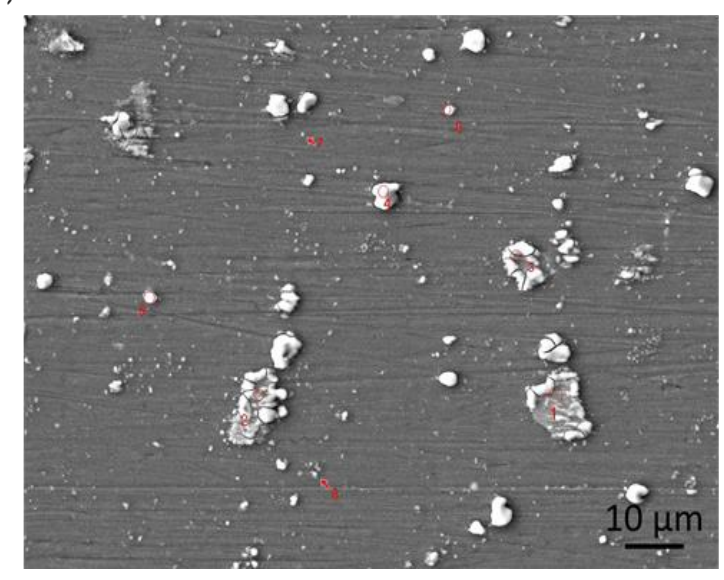

Figure 2.3. (a) AA2024-T351 polished to 1200 grit with specific EDS sites labeled 1-8 as reported in Table 2.2. (b) Same sample and area as (a) after pretreatment for 24 hours in $0.1 \mathrm{M} \mathrm{KMnO}_{4}$.

a)

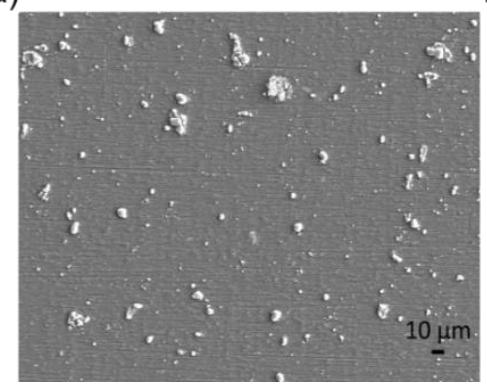

b)

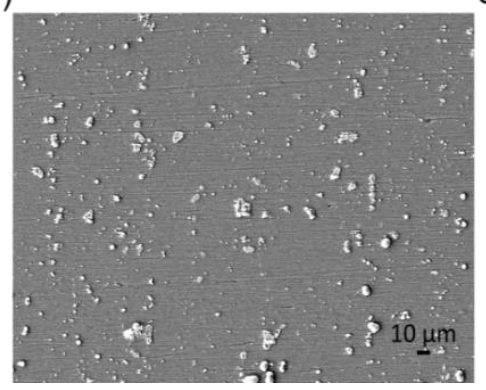

c)

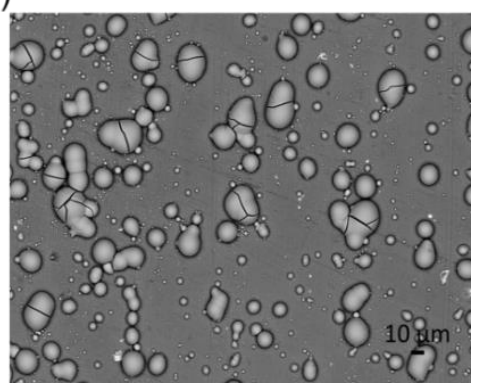

Figure 2.4. (a) AA2024-T351 LT surface pretreated with $0.1 \mathrm{M} \mathrm{KMnO}_{4}$ for 24 hours (secondary electron image). (b) AA2024-T351 pretreated with $0.05 \mathrm{M} \mathrm{KMnO}_{4}$ for 24 hours (secondary electron image). (c) AA2024-T351 pretreated with $0.005 \mathrm{M} \mathrm{KMnO}_{4}$ for 24 hours (backscattered image). 
a)

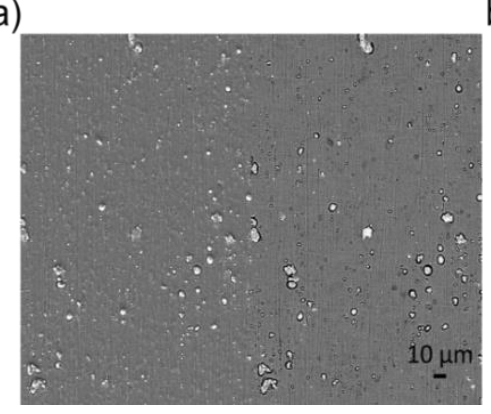

b)

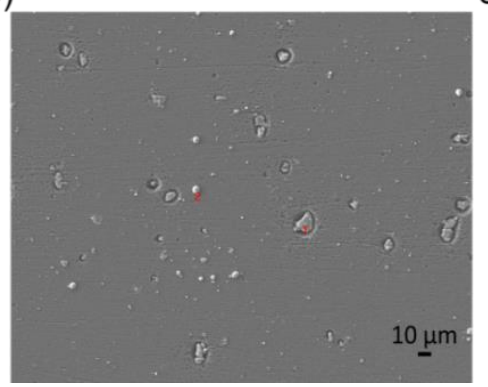

c)

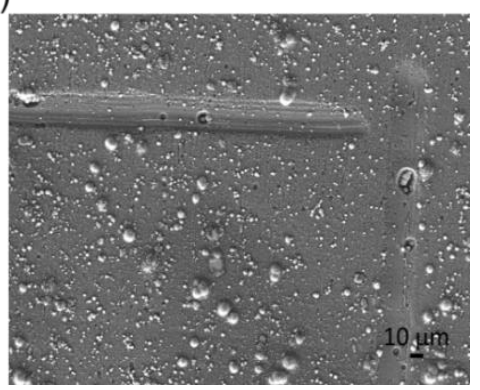

Figure 2.5. (a) AA2024-T351 LT surface exposed to aerated $0.05 \mathrm{M} \mathrm{NaCl}$ for 24 hours after pretreatment in $0.1 \mathrm{M} \mathrm{KMnO}_{4}$ for 24 hours. (b) AA2024-T351 exposed to $0.05 \mathrm{M}$ $\mathrm{NaCl}$ for 24 hours after pretreatment in $0.05 \mathrm{M} \mathrm{KMnO}_{4}$ for 24 hours. (c) AA2024-T351 exposed to $0.05 \mathrm{M} \mathrm{NaCl}$ for 24 hours after pretreatment in $0.005 \mathrm{M} \mathrm{KMnO}_{4}$ for 24 hours.

a)

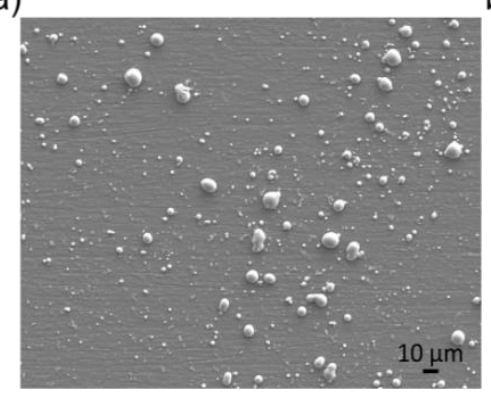

b)

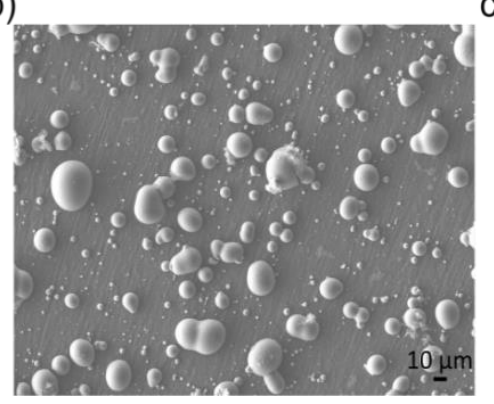

c)

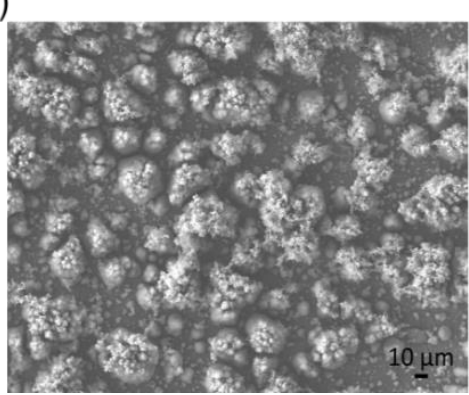

Figure 2.6. (a) AA2024-T351 LT surface exposed to aerated $0.05 \mathrm{M} \mathrm{NaCl}$ with $0.1 \mathrm{M}$ $\mathrm{KMnO}_{4}$ in-situ for 24 hours. (b) AA2024-T351 exposed to $0.05 \mathrm{M} \mathrm{NaCl}$ with $0.05 \mathrm{M}$ $\mathrm{KMnO}_{4}$ in-situ for 24 hours. (c) AA2024-T351 exposed to $0.05 \mathrm{M} \mathrm{NaCl}$ with $0.005 \mathrm{M}$ $\mathrm{KMnO}_{4}$ in-situ for 24 hours. 
a)

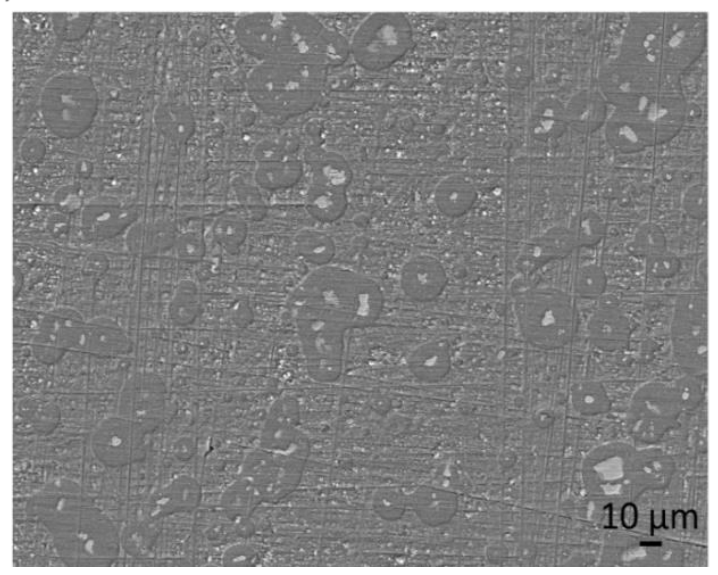

b)

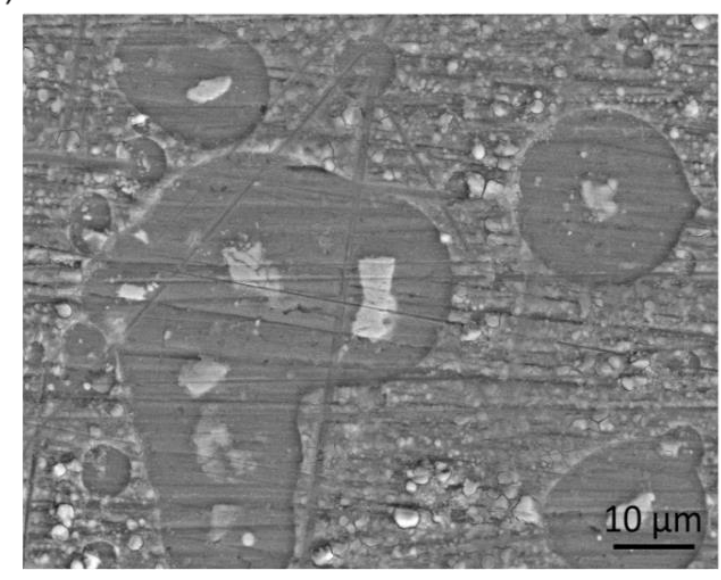

Figure 2.7. AA2024-T351 LT surface exposed to aerated $0.05 \mathrm{M} \mathrm{NaCl}$ solution with 0.05 $\mathrm{M} \mathrm{KMnO}_{4}$ in-situ for 24 hours. The manganese oxide caps were removed after the exposure with a stiff bristled brush.

a)

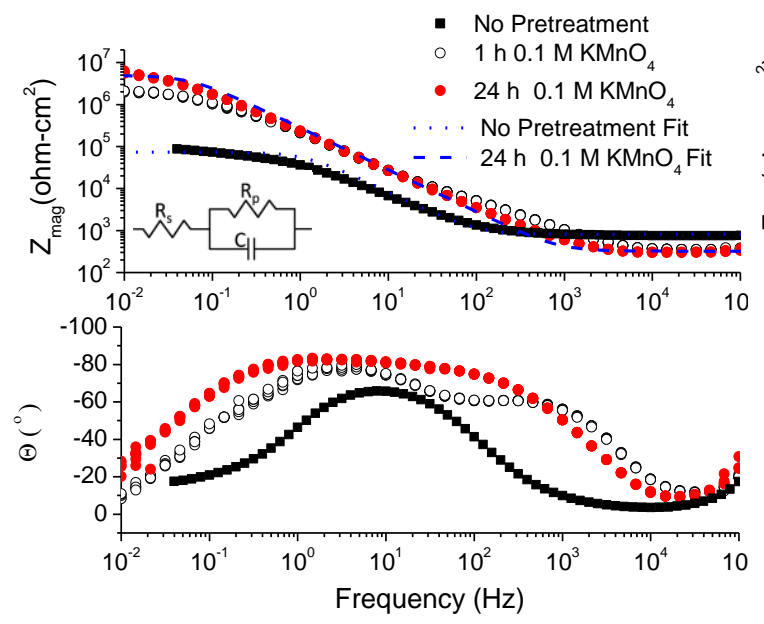

b)

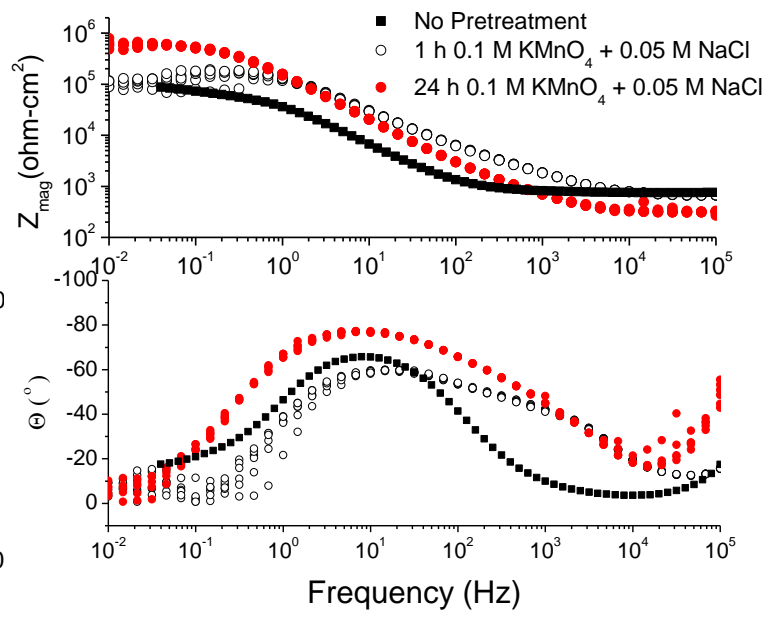

Figure 2.8. EIS of AA2024-T351 pretreated with $0.1 \mathrm{M} \mathrm{KMnO} 4$ for 1 and 24 hours (a) and exposed to $0.05 \mathrm{M} \mathrm{NaCl}$ with $0.1 \mathrm{M} \mathrm{KMnO} 4$ in solution for 1 and 24 hours (b). 


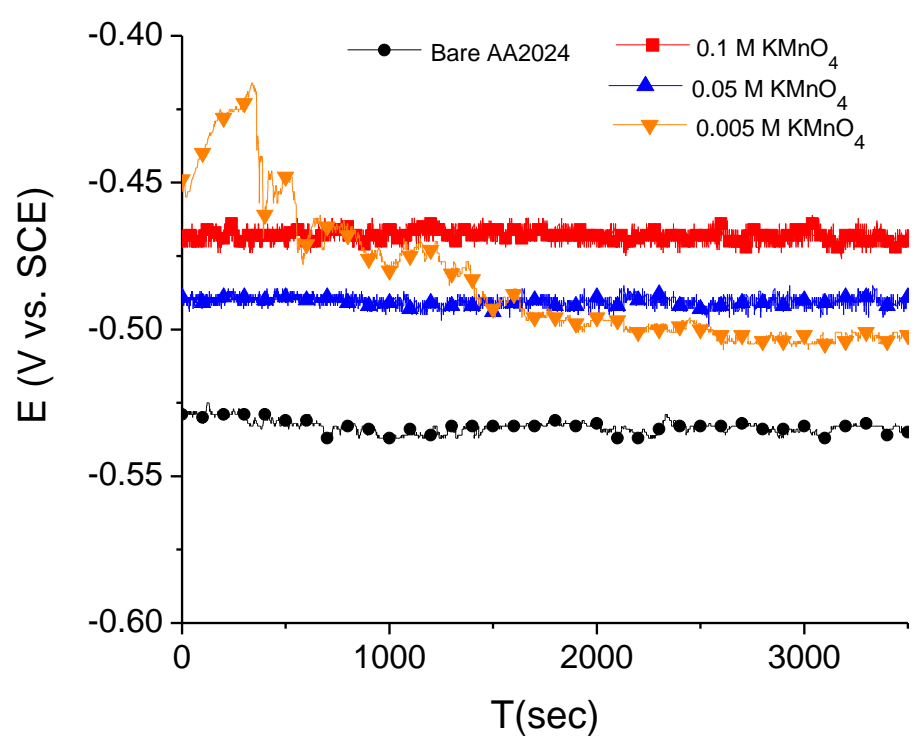

Figure 2.9. The OCP was recorded for one hour after 24 hours at OCP in $0.05 \mathrm{M}$ sodium chloride solution with inhibitor added in-situ at the concentrations reported.

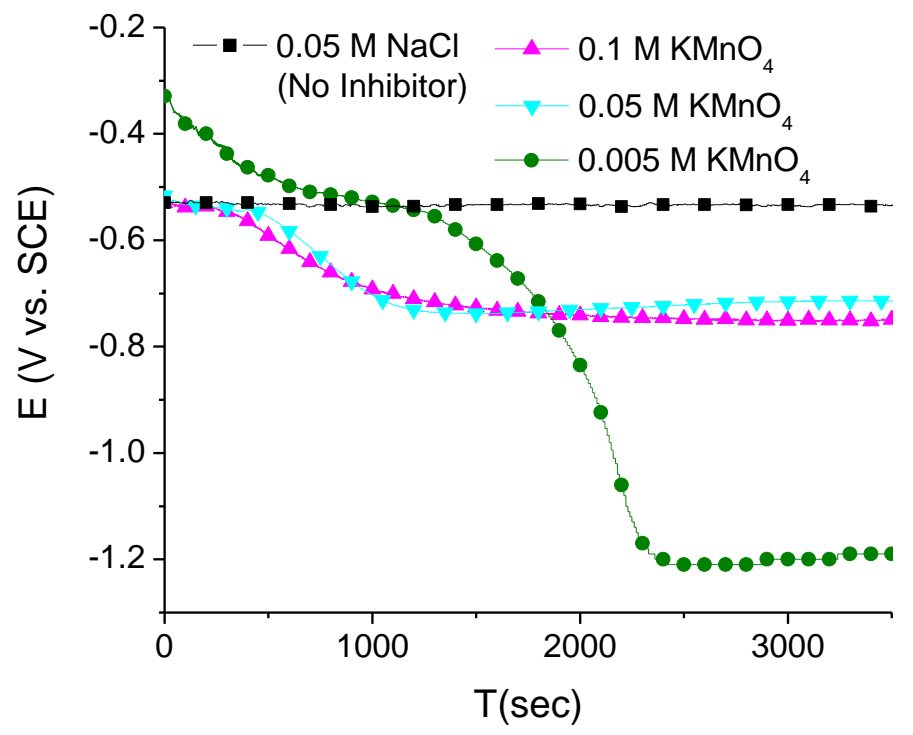

Figure 2.10. OCP measurements for pretreated AA2024-T351 after treatment for 24 hours in varying concentrations of $\mathrm{KMnO}_{4}$. OCP measured in $0.05 \mathrm{~m} \mathrm{NaCl}$ solution. Experiments have been run 10 times and show great reproducibility. 
a)

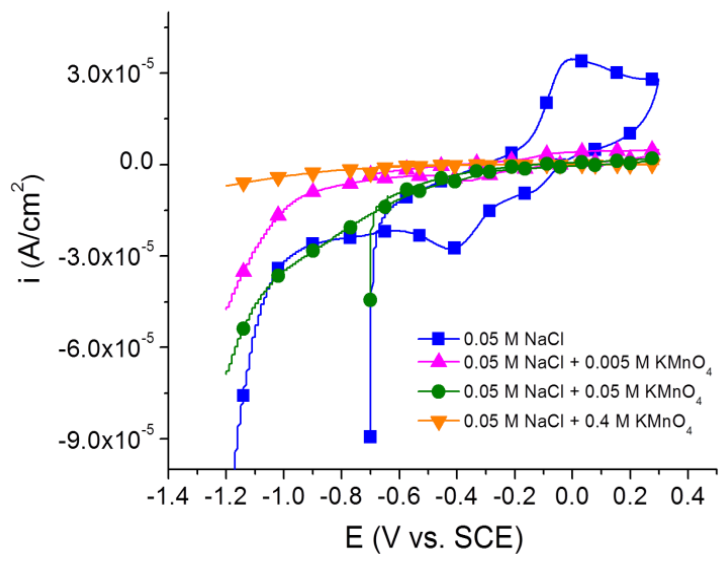

b)

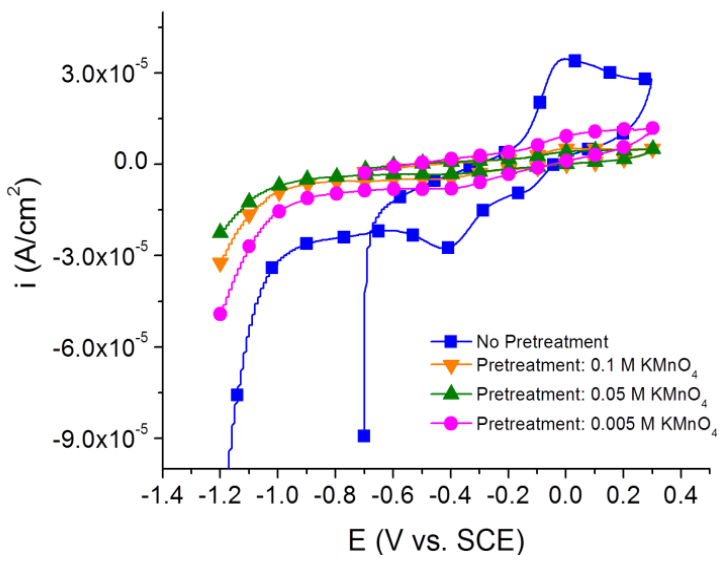

Figure 2.11. (a) CVs of AA2024-T351 etched for 24 hours in $0.05 \mathrm{M} \mathrm{NaCl}$ solution with varying inhibitor concentration in solution, $0.4 \mathrm{M}, 0.05 \mathrm{M}, 0.005 \mathrm{M} \mathrm{KMnO}_{4}$, and no inhibitor. (b) CVs of AA2024-T351 etched in $0.05 \mathrm{M} \mathrm{NaCl}$ solution for 24 hours after pretreatment for 24 hours in varying inhibitor concentration: $0.1 \mathrm{M}, 0.05 \mathrm{M}, 0.005 \mathrm{M}$ $\mathrm{KMnO}_{4}$.

a)

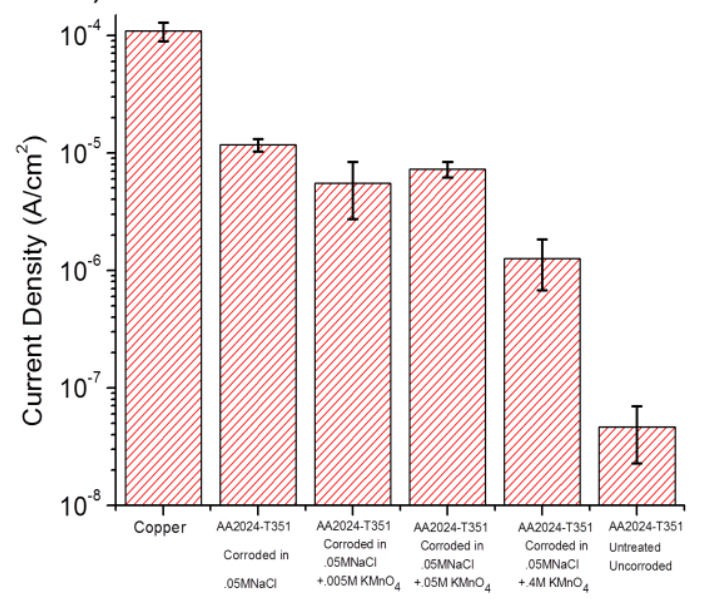

b)

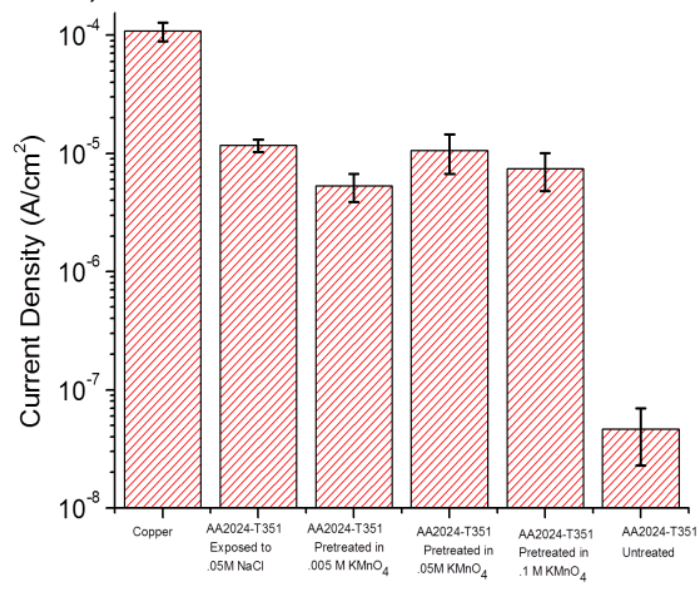

Figure 2.12. The relative intensities of the $\mathrm{Cu}^{1+}$ reduction peak on the AA2024-T351 surface when corroded with the $\mathrm{MnO}_{4}{ }^{-}$ion in solution for 24 hours in $0.05 \mathrm{M} \mathrm{NaCl}$ (a) and with $\mathrm{MnO}_{4}^{-}$inhibitor pretreatment followed by exposure for 24 hours in $0.05 \mathrm{M}$ $\mathrm{NaCl}$ (b). All CV tests run 5 times. Error bars indicate one standard deviation. 

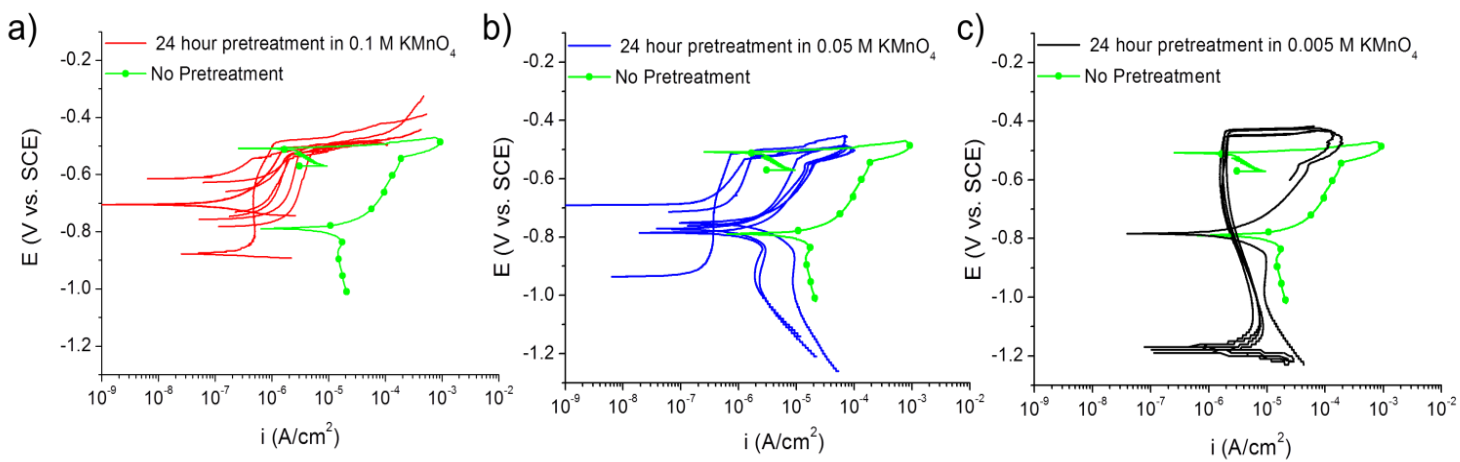

Figure 2.13. The anodic polarization behavior for AA2024-T351 after pretreatment in $\mathrm{KMnO}_{4}$ for 24 hours. Scan run in $0.05 \mathrm{M}$ sodium chloride solution. (a) Pretreatment in $0.1 \mathrm{M} \mathrm{KMnO}_{4}$, (b) Pretreatment in $0.05 \mathrm{M} \mathrm{KMnO}_{4}$, (c) Pretreatment in $0.005 \mathrm{M} \mathrm{KMnO}_{4}$.

a)

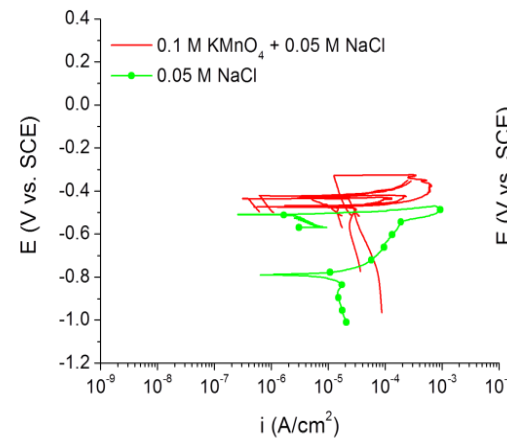

b)

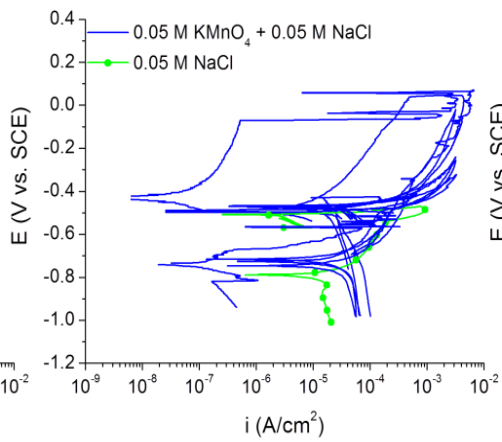

c)

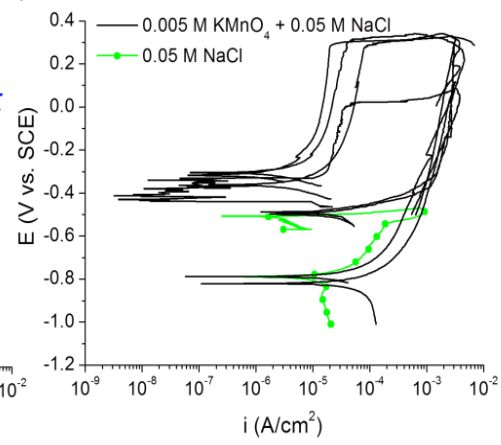

Figure 2.14. The anodic polarization behavior for AA2024-T351 after 24 hours of exposure to $0.05 \mathrm{M}$ sodium chloride solution with the inhibitor in-situ. Scans conducted in $0.05 \mathrm{M}$ sodium chloride solution with varied inhibitor concentrations of (a) $0.1 \mathrm{M}$ $\mathrm{KMnO}_{4}$, (b) $0.05 \mathrm{M} \mathrm{KMnO}_{4}$, and (c) $0.005 \mathrm{M} \mathrm{KMnO}_{4}$. 
a)

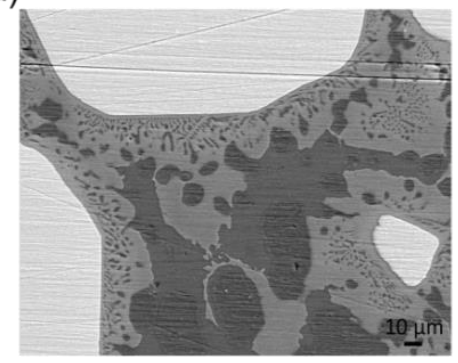

b)

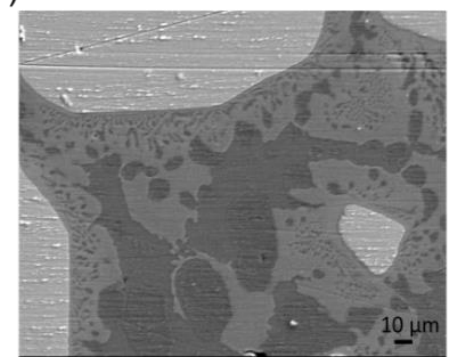

c)

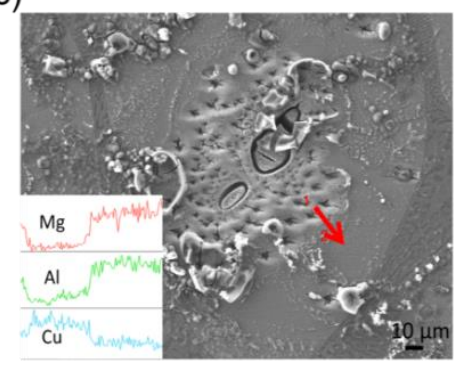

Figure 2.15. SEM backscattered image of multiple phase ingot with large S-phase regions. The large light colored regions of the micrographs are S-phase and indicate the higher copper content and corresponding higher Z. (a) Sample as polished. (b) S-phase held potentiostatically in $0.1 \mathrm{M} \mathrm{MnO}_{4}{ }^{-}$and $0.05 \mathrm{M} \mathrm{Cl}^{-}$solution for 200 seconds. Note the growth of manganese oxide over the S-phase particles. (c) S-phase held potentiostatically in $0.05 \mathrm{M} \mathrm{Cl}^{-}$solution for 200 seconds. While (a) and (b) represent the same region of the ingot, (c) does not. However, the region of severe damage is in the center of an S-phase particle. Insert of EDS line scan shows the enrichment of copper in proximity of visible S-phase damage.

a)

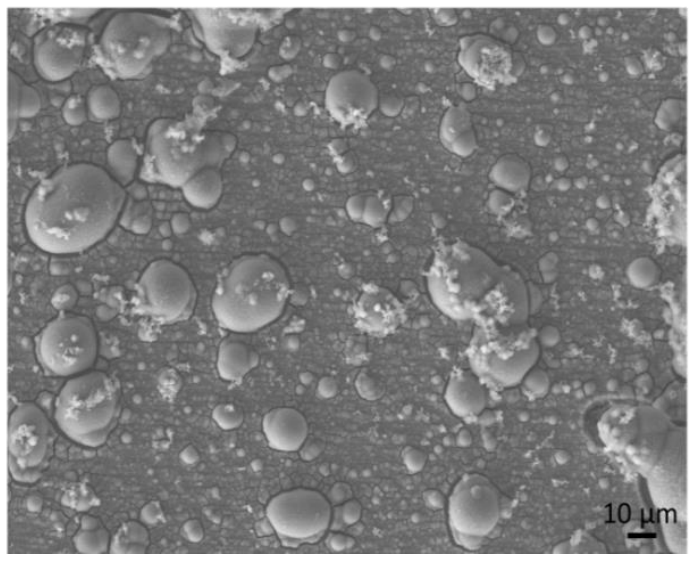

b)

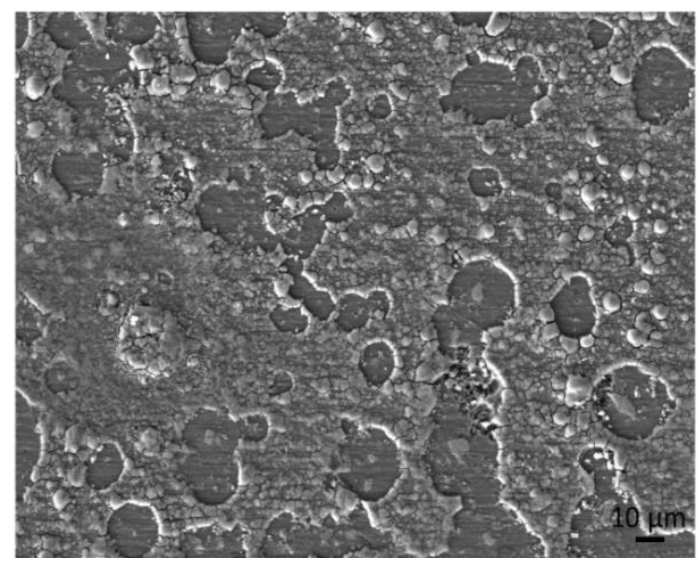

Figure 2.16. AA2024 exposed to $0.005 \mathrm{M} \mathrm{KMnO}_{4}+0.05 \mathrm{M} \mathrm{NaCl}$ for 24 hours and then anodically polarized to potentials above the pitting potential ( $0.3 \mathrm{~V}$ vs. SCE) in fresh $0.005 \mathrm{M} \mathrm{KMnO}_{4}+0.05 \mathrm{M} \mathrm{NaCl}$ solution. 

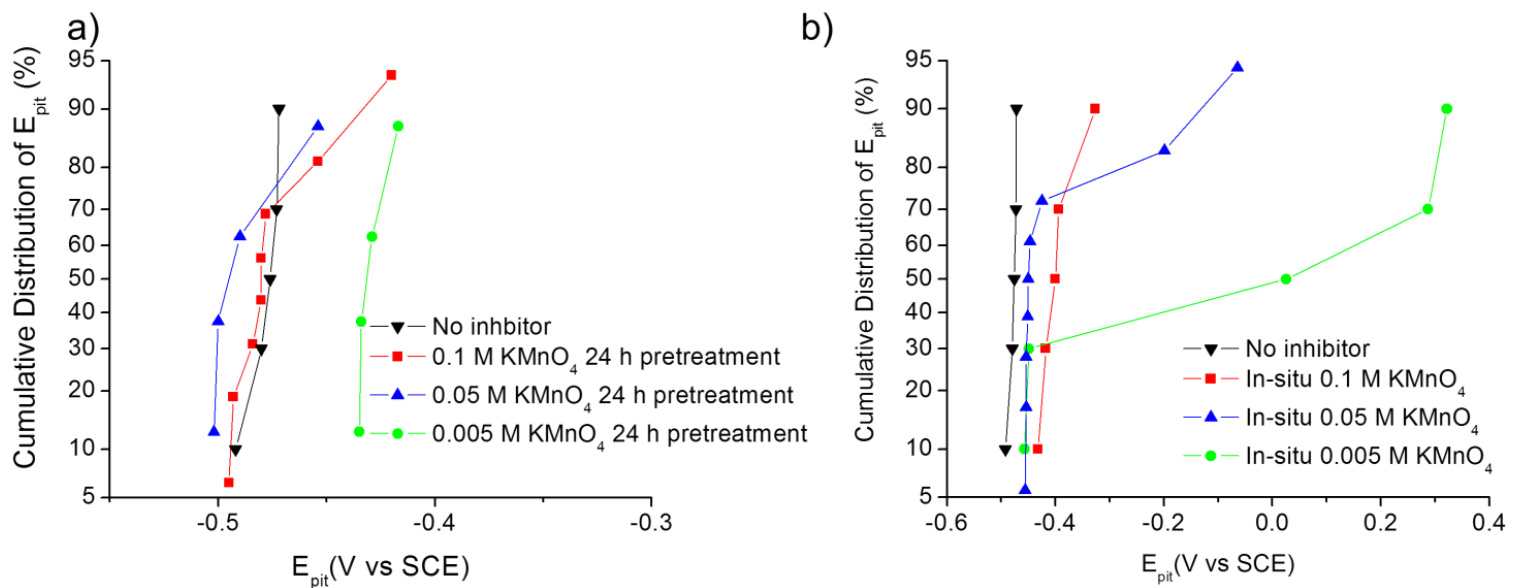

Figure 2.17. Cumulative probability of pitting as a function of potential for both pretreatment (a) and in-situ solution phase (b) permanganate inhibitor concentration. Probability of Pitting in $0.05 \mathrm{M} \mathrm{NaCl}$.
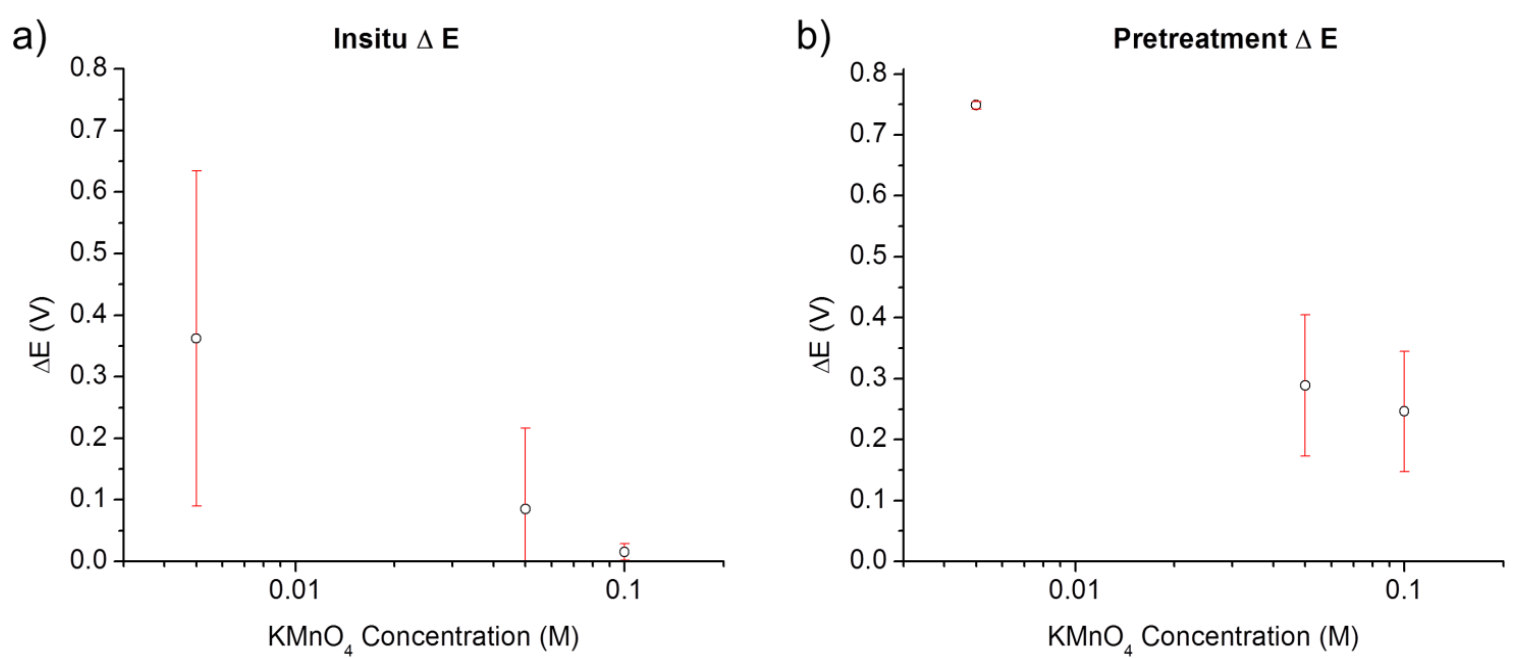

Figure 2.18. The average difference between pitting and OCP for Mn pretreatment (a) and in-situ solution phase additions (b), as a function of inhibitor concentration. The error bars indicate one standard deviation. 
a)

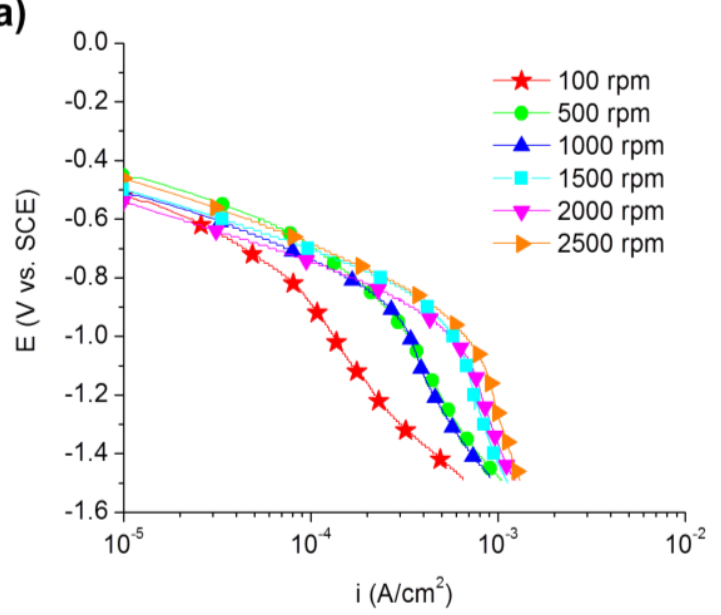

b)

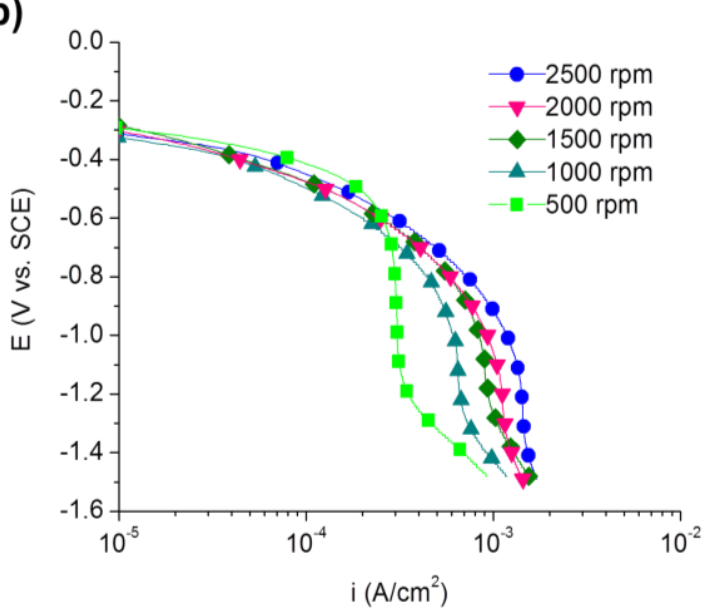

Figure 2.19. Cathodic E vs $\log (i)$ polarization curves for AA2024-T4 (a) and high purity copper (b) samples measured at various rotation rates from $100 \mathrm{rpm}$ to 2500 in $\mathrm{pH} 8.4$ borate buffer. Solutions were aerated naturally.

a)

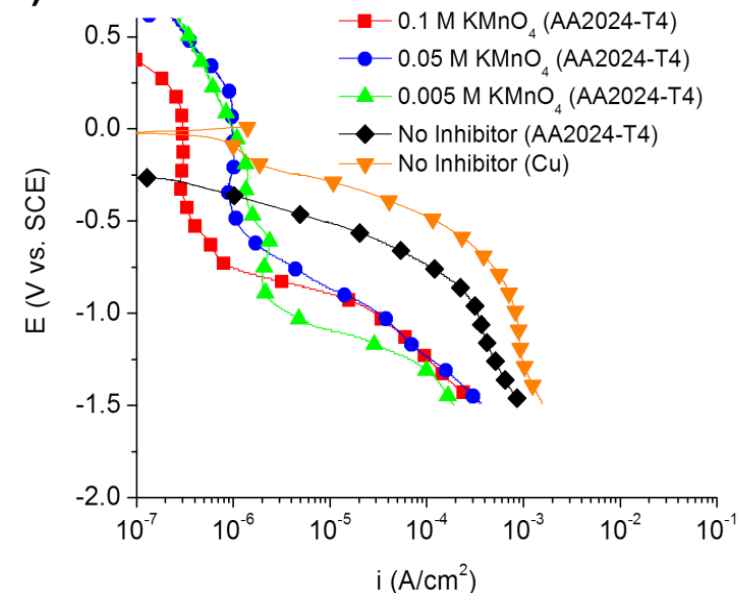

b)

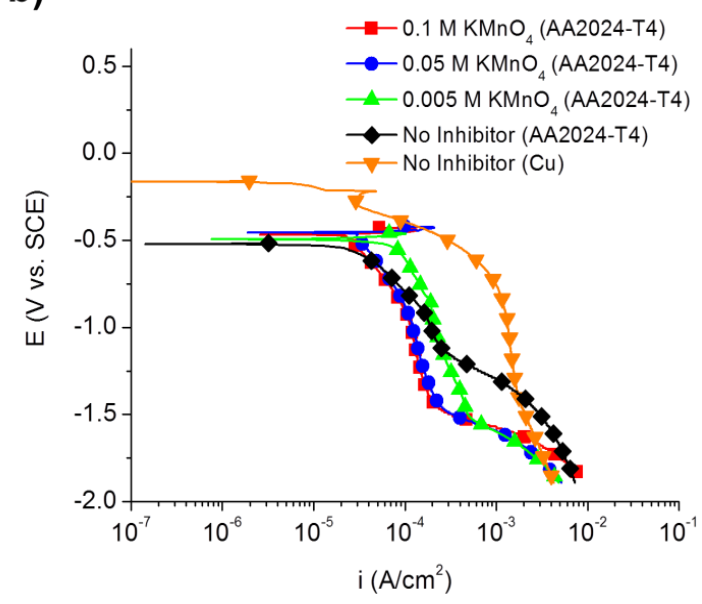

Figure 2.20. Cathodic polarization behavior of AA2024-T4 and high purity copper samples ground to 1200 grit, tested at $1000 \mathrm{rpm}$ in $\mathrm{pH} 8.4$ borate buffer solution after $100 \mathrm{~h}$ pretreatment (a) and run at $1500 \mathrm{rpm}$ in $0.05 \mathrm{M} \mathrm{NaCl}$ solution with in-situ inhibitor additions (b). Rotation rate has been kept constant and inhibitor concentration is tested at different levels. All data collected under natural aeration. 


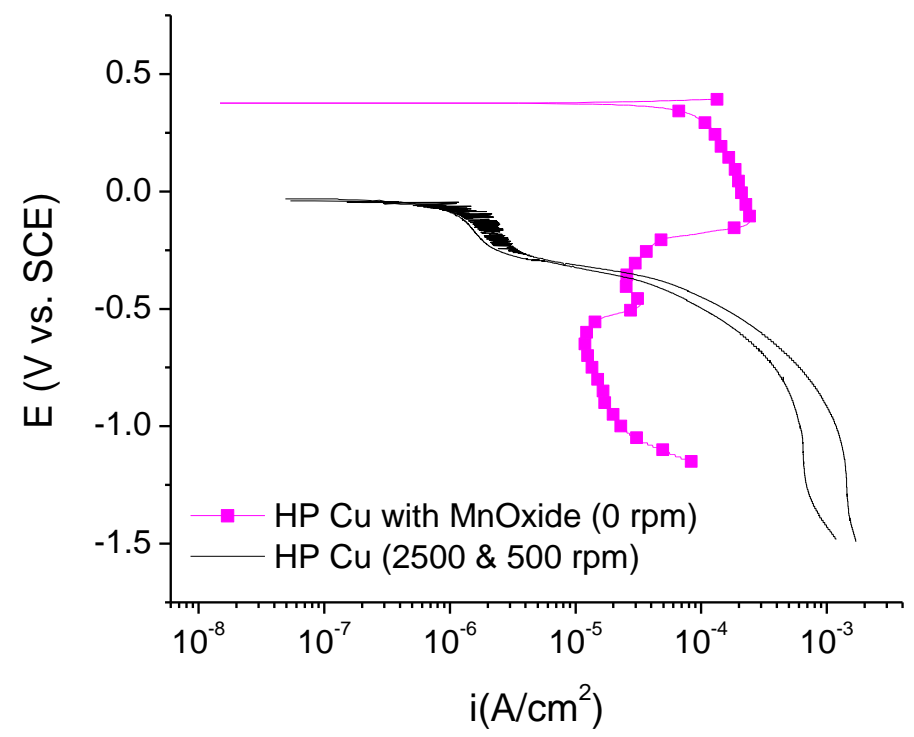

Figure 2.21. Potentiodynamic scan of high purity copper with Mn-oxide deposited at a potential of $-0.5 \mathrm{~V}$ (SCE) run in $\mathrm{pH} 8.4$ borate buffer.

a)

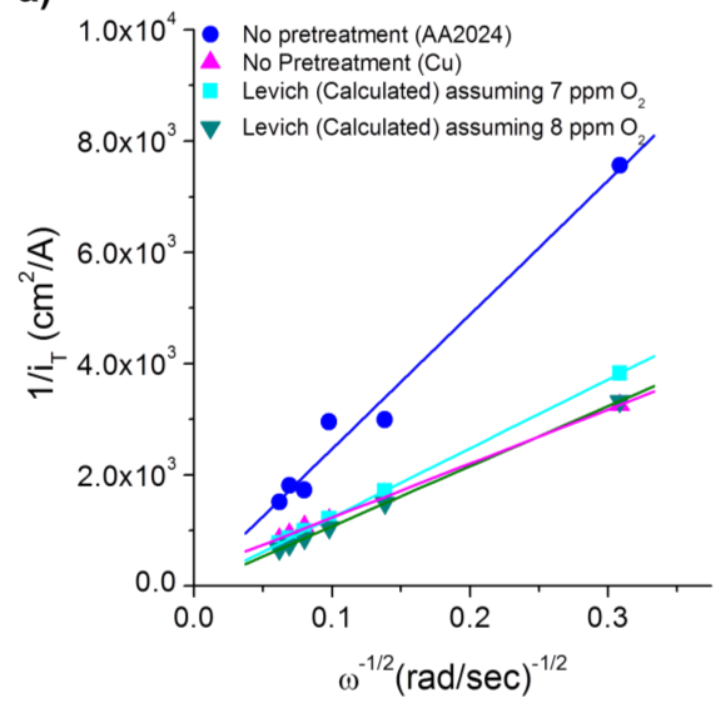

b)

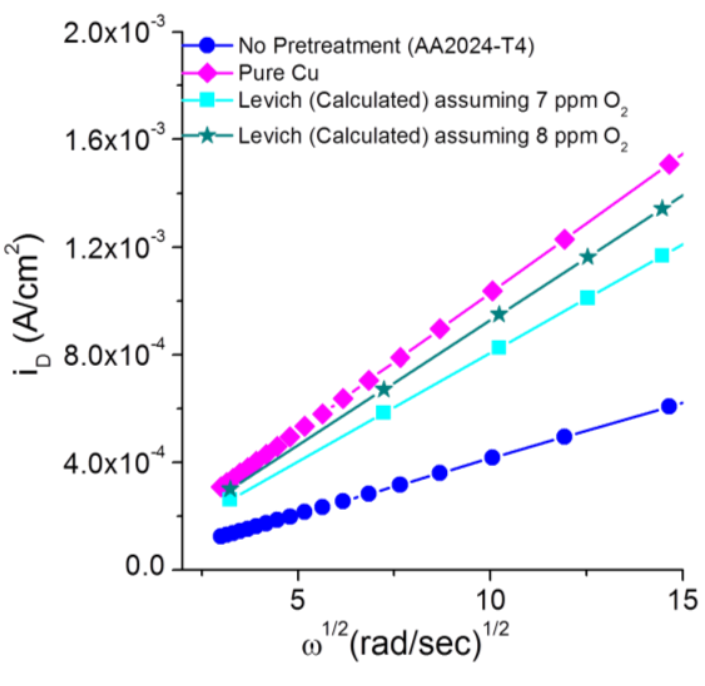

Figure 2.22. (a) Total current at $-1.0 \mathrm{~V}$ (SCE) for AA2024-T4 and pure copper compared with the Levich prediction $\left(i_{d}\right)$ in $\mathrm{pH} 8.4$ borate buffer solution. (b) Diffusion limited current extracted from $i_{\text {total }}$ at $-1.0 \mathrm{~V}$ (SCE) for AA2024-T4 and pure copper compared with the Levich prediction in $\mathrm{pH} 8.4$ borate buffer. Data extracted from Figure 2.20. 
a)

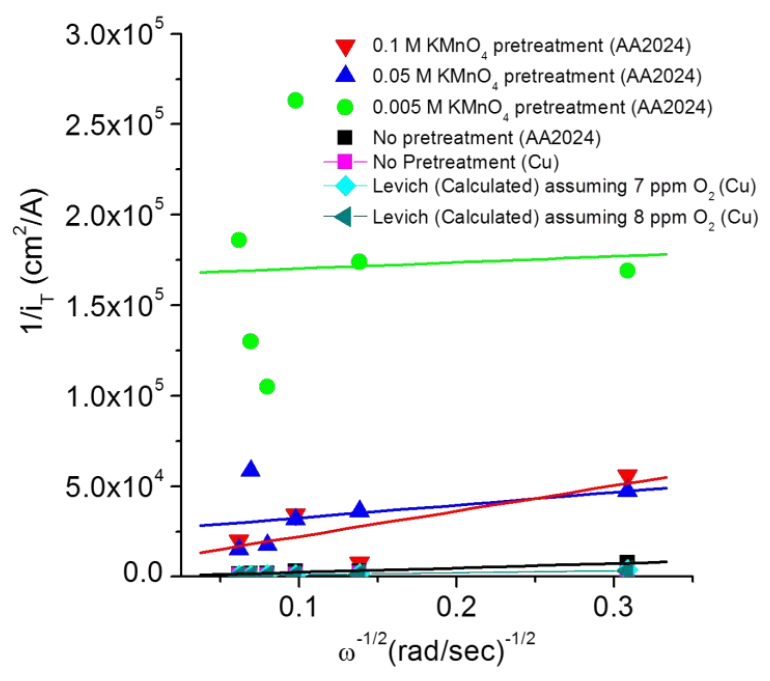

b)

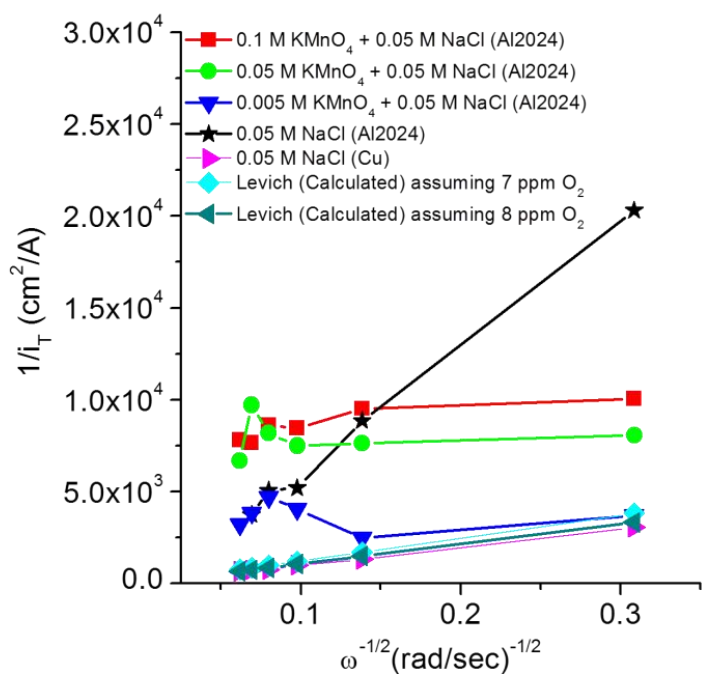

Figure 2.23. Total cathodic current at $-1.0 \mathrm{~V}$ (SCE) for AA2024-T4 for the inhibitor pretreatment tests in inhibitor free environment (a) and with the inhibitor present in-situ (b). Note that (a) and (b) have an order of magnitude difference in the ordinate. Total current densities for pure copper, bare AA2024-T4 and the Levich prediction $i_{d}$ values are included for comparison. Data extracted from Figure 2.20.

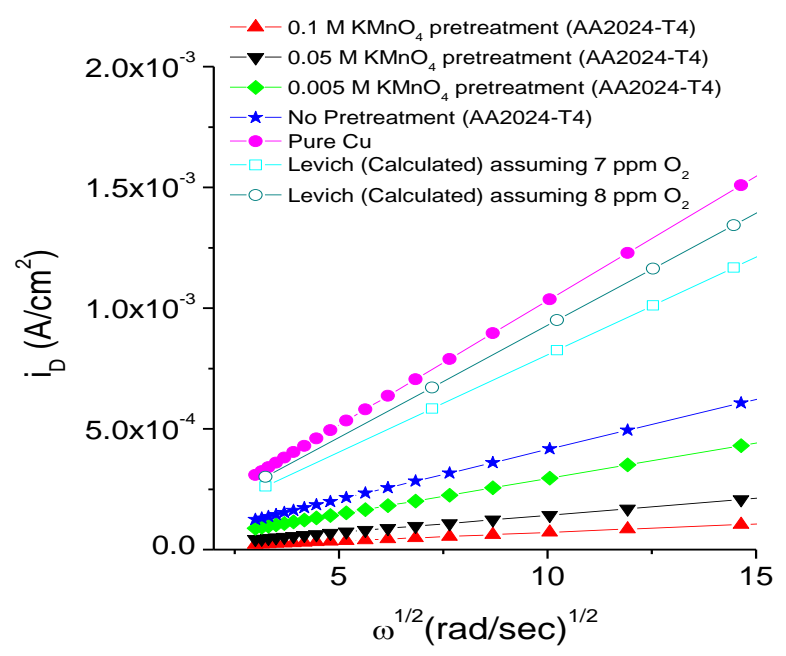

Figure 2.24. Diffusion limited current density extracted from $i_{\text {total }}$ at $-1.0 \mathrm{~V}$ (SCE) for AA2024-T4 after pretreatment at various inhibitor concentration compared with pure copper, bare AA2024-T4, and the Levich prediction. Tests were conducted in borate buffer solution at pH 8.4. Data extracted from Figure 2.23. 
a)

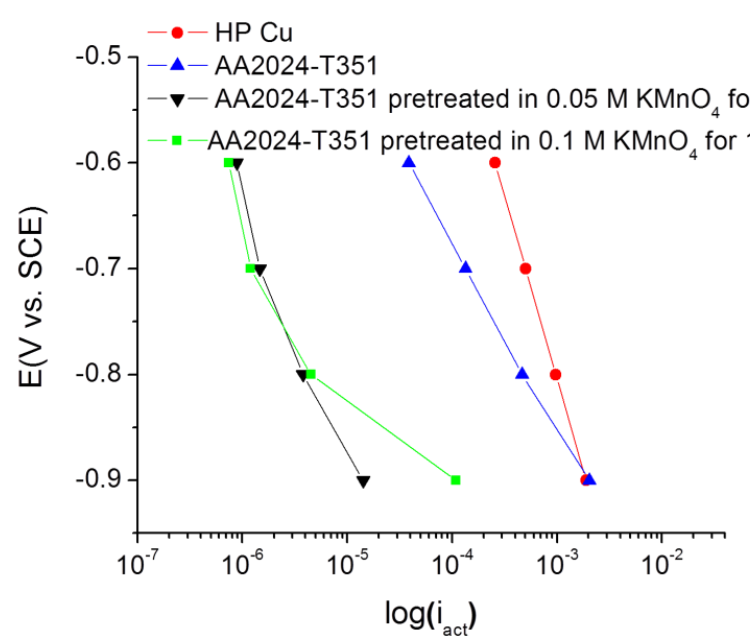

b)

4
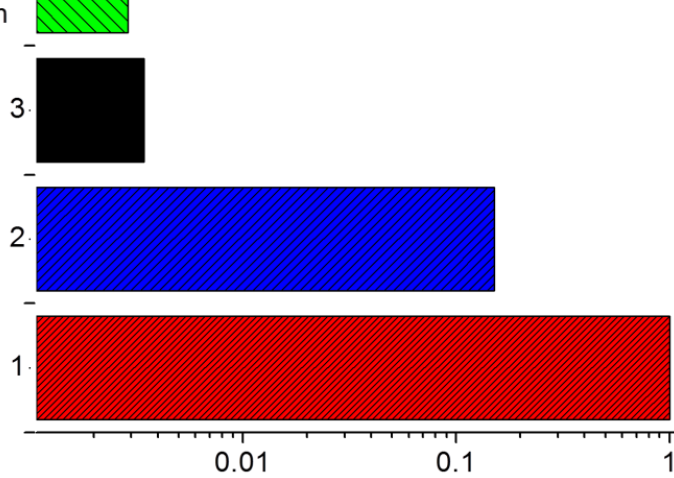

$\Theta_{\mathrm{Cu}}($ Copper Coverage)

Figure 2.25. (A) Extracted activation controlled current density over a range of potentials for high purity $\mathrm{Cu}$, AA2024-T351, and AA2024-T351 pretreated in $0.05 \mathrm{M}$ $\mathrm{MnO}_{4}^{-}$and $0.1 \mathrm{M} \mathrm{MnO}_{4}^{-}$. (B) Calculated copper coverage, $\boldsymbol{\Theta}_{\mathbf{C u}}^{\mathrm{Ka}}{ }^{-}$, at $-0.6 \mathrm{~V}$ using Equation [15]. $\mathrm{Cu}(1), \mathrm{AA} 2024-\mathrm{T} 351(2)$, and AA2024-T351 pretreated in $0.05 \mathrm{M} \mathrm{MnO}_{4}^{-}$ (3) and in $0.1 \mathrm{M} \mathrm{MnO}_{4}^{-}$(4). 


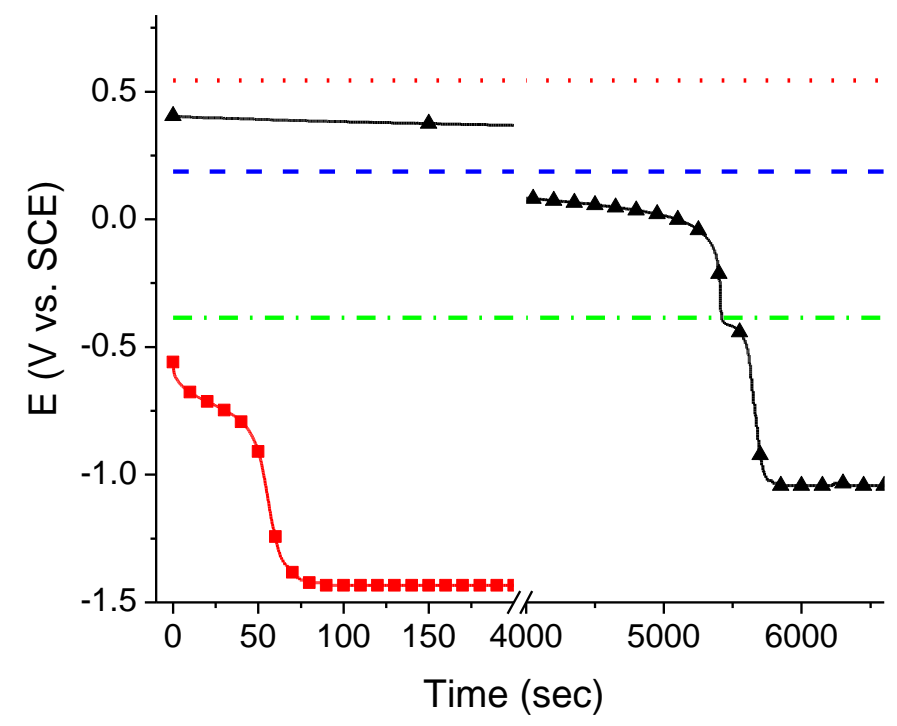

Figure 2.26. Constant current discharge potential vs. time plots on of electrodeposited manganese oxide coated copper (-А-) and uncoated copper (- $\mathbf{-}-)$. High purity Copper with Mn oxide deposited at -0.5 V SCE from $0.1 \mathrm{M} \mathrm{KMnO}_{4}$ with a pH of 6. Galvanostatic reduction performed at $-20 \mathrm{~mA} / \mathrm{cm}^{2}$ in $\mathrm{pH} 8.4$ borate buffer. The red dotted line $(\ldots)$ indicates the reversible potential of $\mathbf{M n O}_{\mathbf{4}}^{-}+\mathbf{4} \boldsymbol{H}^{+}+\mathbf{3} \boldsymbol{e}^{-} \leftrightarrow$ $\mathbf{M n O}_{2}+\mathbf{2} \mathbf{H}_{2} \mathbf{O}$ at $0.54 \mathrm{~V}$ vs SCE. The blue dashed (- - ) line indicates the reversible potential for $\mathbf{M n O}_{2}+\boldsymbol{H}_{2} \mathrm{O}+\boldsymbol{e}^{-} \leftrightarrow \mathbf{M n O O H}+\mathbf{O H}^{-}$at $0.187 \mathrm{~V}$ vs SCE. The green dash-dotted line (--) indicates the reversible potential for $\mathbf{M n O O H}+\mathrm{H}_{2} \boldsymbol{O}+\boldsymbol{e}^{-} \leftrightarrow$ $\boldsymbol{M n}(\boldsymbol{O H})_{2}+\boldsymbol{O H}^{-}$at $-0.385 \mathrm{~V}$ vs SCE. 
a)

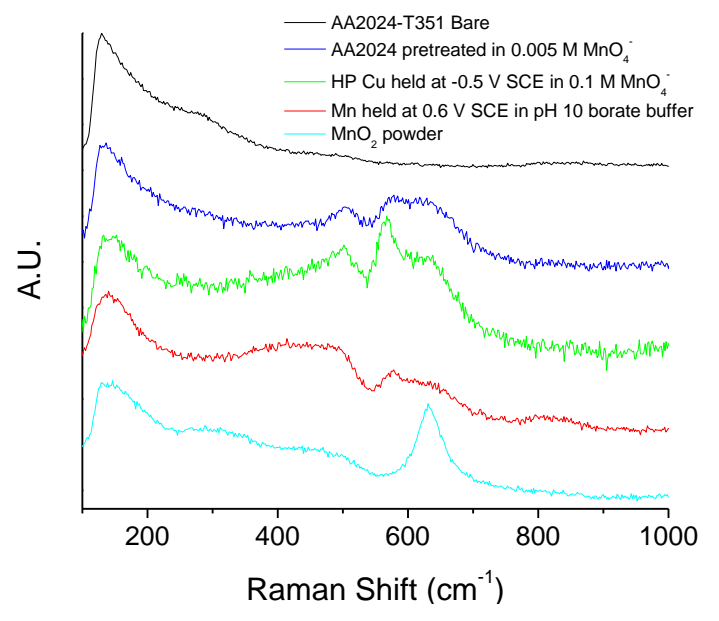

b)

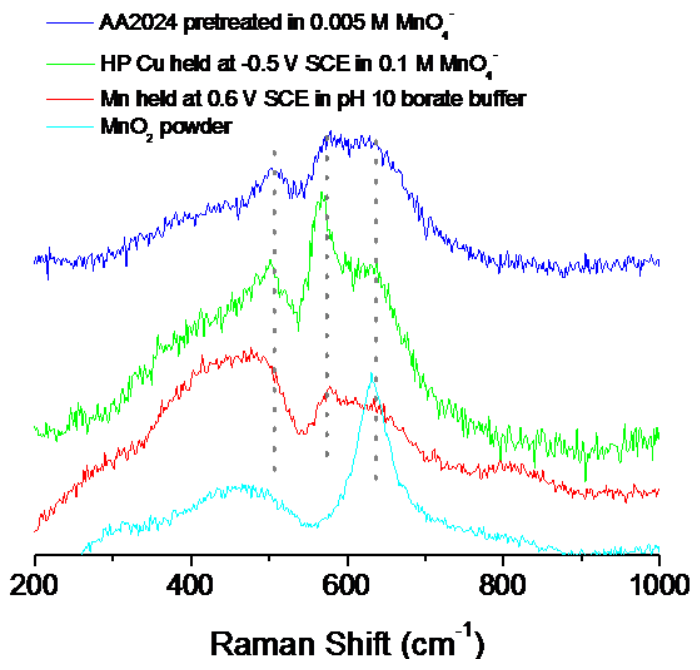

Figure 2.27. a) Raman spectra for AA2024-T351 (no Raman response), AA2024T351 pretreated in $0.005 \mathrm{M} \mathrm{KMnO}_{4}$, copper pretreated in $0.1 \mathrm{M} \mathrm{KMnO}_{4}$ at $-0.5 \mathrm{~V}$ (SCE), manganese metal held potentiostatically at $0.35 \mathrm{~V}$ (SCE), and $\mathrm{MnO}_{2}$ powder. b) Background subtracted peaks showing three peaks at 630,573 , and $506 \mathrm{~cm}^{-1}$.

a)

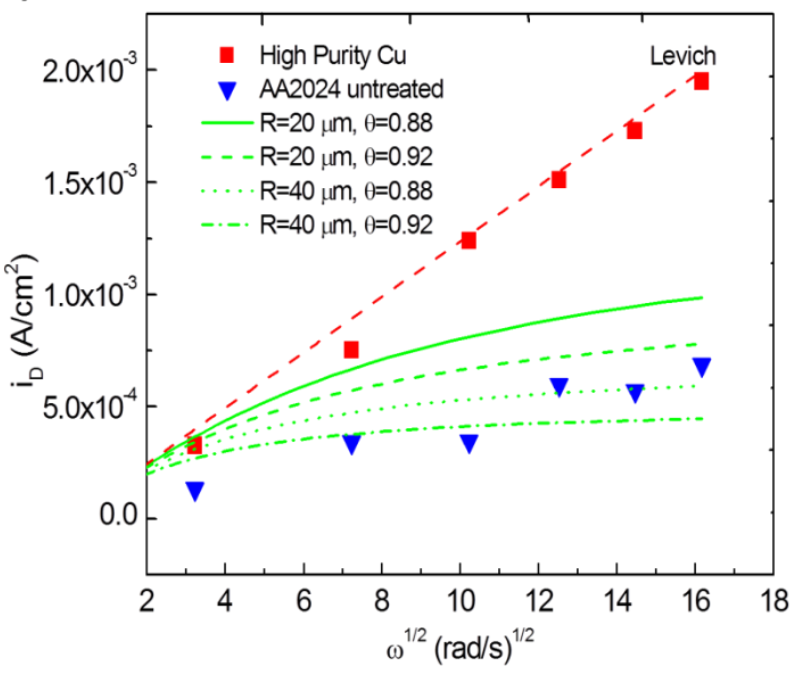

b)

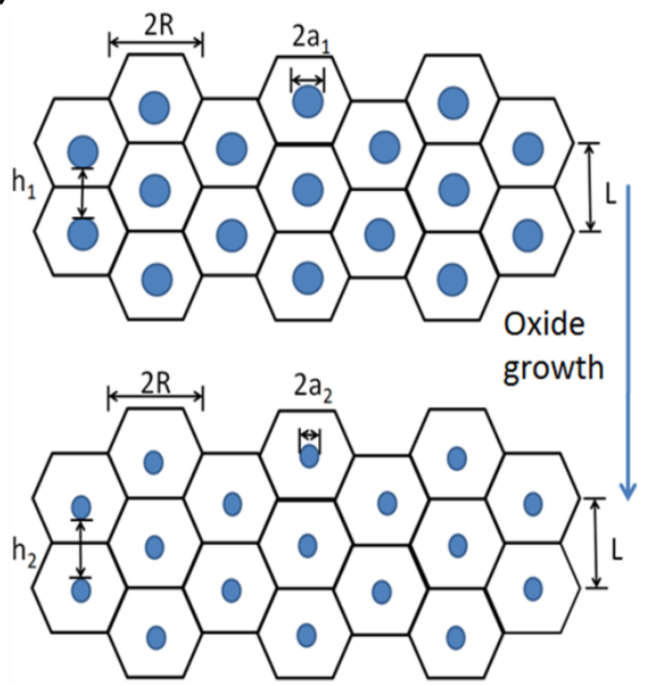

Figure 2.28. (a) Gueshi model showing effect of IMC special geometry in AA2024-T351 on modeled ORR kinetics and actual data values. (b) A schematic showing effect of decreasing active area in an inactive matrix. 
a)

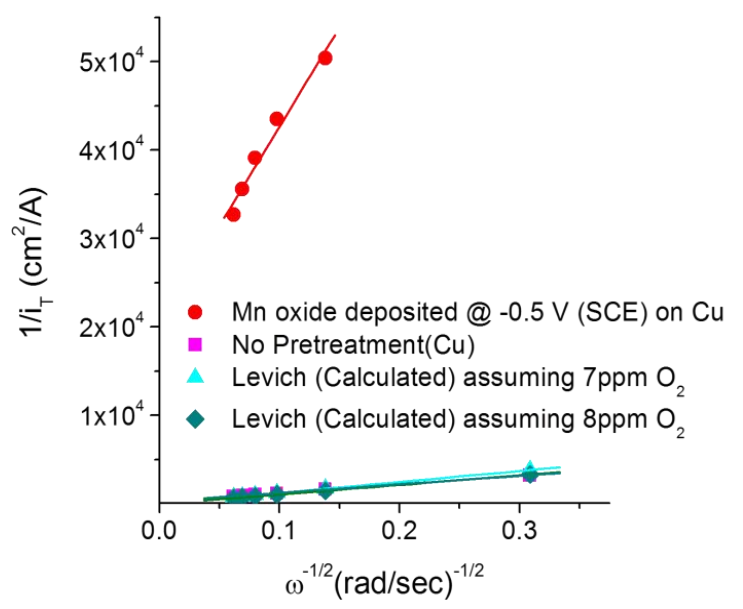

b)

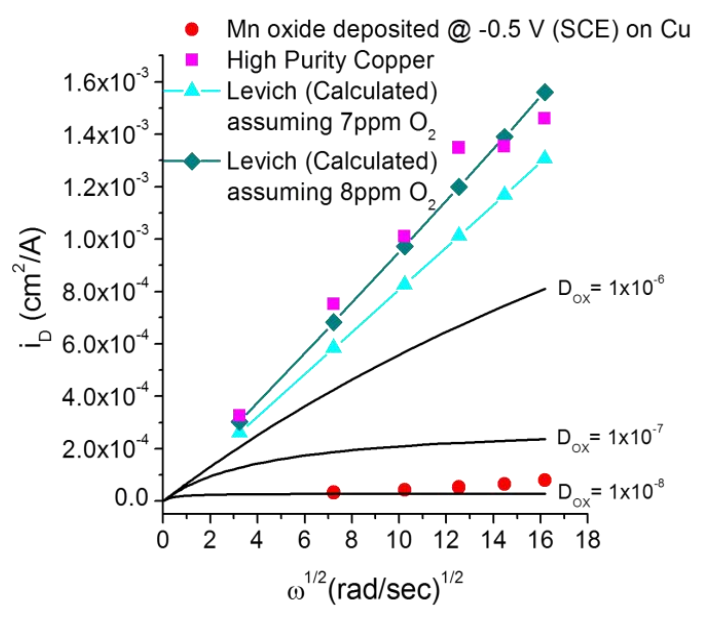

Figure 2.29. (a) Cathodic reaction rate on high purity copper with and without electrodeposited manganese oxide film. (b) Membrane modeling of cathodic reaction kinetics from Equation [18] in mass transport and mixed ORR regime for high purity copper with a manganese oxide film grown on its surface from $0.1 \mathrm{M} \mathrm{KMnO}_{4}$ solution at $-0.5 \mathrm{~V}(\mathrm{SCE})$.

1. I. Puigdomenech, Hydra/Medusa Chemical Equilibrium Database and Plotting Software. 2004, freely downloadable software at www.kemi.kth.se/medusa/: KTH Royal Institute of Technology. 


\section{Corrosion Inhibition of AA2024-T351 Using Cerium-Permanganate Combinations}

\subsection{Abstract}

The inhibition of corrosion on AA2024-T351 in NaCl solutions, mitigated by ceriumpermanganate combined inhibitor pretreatments was examined. Room temperature pretreatments were studied as a function of total inhibitor concentration from $10 \mathrm{mM}$ to 50 $\mathrm{mM}$. Pretreated samples were exposed to $50 \mathrm{mM} \mathrm{NaCl}$ solutions. The roles of the inhibitor during anodic and cathodic polarizations were investigated. Inhibition of open circuit corrosion under conditions where anodic and cathodic reactions are coupled was also examined. The Koutecky-Levich correction was applied to experimental cathodic E- $i$ data. The thermodynamics of the Ce-water and Mn-water systems was also considered over a range of concentrations. There was no indication of a combined species and no reference to such a species in the literature was found. The cerium-permanganate combination was determined to provide nearly the same level of cathodic inhibition seen in the single inhibitor application of permanganate. Cerium alone was inferior as a cathodic inhibitor. In addition to strong cathodic inhibition, the combination increased inhibition of open circuit corrosion compared to single inhibitor pretreatments as determined by copper redeposition. However, anodic inhibition was not observed with the inhibitors when used alone or when combined. 


\subsection{Background}

Precipitation hardened aluminum alloys containing copper and iron are highly susceptible to corrosion due to local galvanic coupling between intermetallic phases and the surrounding aluminum matrix ${ }^{[1-7]}$. Many forms of corrosion protection have been implemented to address this issue ${ }^{[8-10]}$. Hexavalent chromates are currently recognized as the most effective chemical species used to mitigate corrosion on alloys such as AA2024 ${ }^{[11-16]}$. Since 2006 the OHSA has imposed strict new Cr(VI) exposure limits due to their being a known human carcinogen ${ }^{[17]}$. The additional costs incurred providing an adequate working environment and allowing for the proper disposal of $\mathrm{CrO}_{4}^{-}$have led to efforts to find new non-toxic replacements.

Localized corrosion of alloys such as AA2024-T351 results from the presence of intermetallic compounds in a solute lean $\alpha$-Al matrix that are either noble with respect to their surrounding matrix or particles that tend to be initiation sites for pitting ${ }^{[1,4]}$. Particles such as $\mathrm{Al}_{\mathrm{s}} \mathrm{CuMg}$ and $\mathrm{Cu}_{2} \mathrm{Al}$ can also be detrimental to the corrosion resistance of the alloy because of their tendency to dealloy at the open circuit potential ${ }^{[7]}$. This process has many damaging consequences including the creation of catalytic high surface area copper enriched intermetallic remnants and the associated deposition of copper on the alloy surface $^{[18]}$, both of which result in increased oxygen reduction reaction rates. This increase in ORR supports increased aluminum matrix dissolution in the form of localized trenching around the intermetallics and pit initiation and growth that can produce nucleation sites for subsequent crack growth ${ }^{[19-21]}$. Effective inhibition of these constituent-particle-related damage modes in this class of alloys is of strong interest. 
One aspect of current research in the field of corrosion inhibition has focused on oxoanions of the form $\mathrm{MeO}_{4}{ }^{(\mathrm{n}-8)}$ (such as molybdates, tungstates, and vanadates) ${ }^{[22-32]}$, structural analogues of hexavalent chromium. Another is the use of cerium compounds such as cerium nitrate ${ }^{[33]}$, cerium dibutylphosphate ${ }^{[34]}$, and cerium acetate ${ }^{[25]}$. These cerium compounds are under consideration because of the stability of several cerium oxides that will be discussed in this study.

Combined inhibitors are of interest because of the possibility that a combination inhibitors will lead to enhanced corrosion protection. Three possibilities are envisioned. The combination can act independently, acting to fill gaps where the other inhibitor provides insufficient protection. They can have a supportive chemical role by altering the species of the other inhibitor, for instance by oxidizing a species to a more stable state leading to enhanced corrosion protection. Thirdly, there could be a new combined species of ion or oxide that involves both inhibitors that provides corrosion protection in an expanded manner. In this study, permanganate $\left(\mathrm{MnO}_{4}{ }^{-}\right)$has been paired with cerium with the goal of oxidizing cerium hydroxide to the higher valence state cerium oxide in order to take advantage of the larger range of thermodynamic stability.

The E-pH speciation predictions for the cerium-water system can be seen in Figure 3.1(b). These speciation diagrams indicate predominante species ratios at $-0.5 \mathrm{~V}_{\text {sce. }}$ This potential is approximatly the value of the observed OCP for AA2024-T351 in 0.05 M sodium chloride solution with $\mathrm{Ce}^{3+}$ or the permanganate ion in solution. The stable cerium oxide is $\mathrm{CeO}_{2}$ above a $\mathrm{pH}$ of 9.5 and $\mathrm{Ce}(\mathrm{OH})_{3}$ between a $\mathrm{pH}$ of 7.3 and 9.5 . When cerium acetate is added to solutions in contact with AA2024-T351, the species will undergo a chemical reaction near high $\mathrm{pH}$ regions such as at IMC where the ORR occurs. This behavior 
produces a precipitate oxide of $\mathrm{Ce}(\mathrm{OH})_{3}$ at low potentials such as those seen on AA2024T351 at $-0.5 \mathrm{~V}$ (SCE). The chemical reaction can be seen below.

$$
\mathrm{Ce}^{3+}+3 \mathrm{OH}^{-} \leftrightarrow \mathrm{Ce}(\mathrm{OH})_{3}
$$

The E-pH speciation predictions for the manganese-water system can be seen in Figure 3.2(b). The stable $\mathrm{Mn}(\mathrm{II})$ species is $\mathrm{Mn}(\mathrm{OH})_{2}$ above a $\mathrm{pH}$ of 8.5 at $-0.5 \mathrm{~V}$ (SCE). Permanganate $\left(\mathrm{MnO}_{4}^{-}\right)$is a very strong oxidizer due to its high potential as seen in Figure 3.2(a). When permanganate is added to solution in contact with AA2024-T351 at a lower potential, it has been shown to be reduced through serveral possible electrochemical reactions ${ }^{[35]}$. Over all ranges of $\mathrm{pH}, \mathrm{MnO}_{4}^{-}$in the +7 valence state will likely first be reduced to manganese (IV) dioxide by the following electrochemical $1 / 2$ cell reaction ${ }^{[36]}$. $\mathrm{E}^{\mathrm{o}}$ for this reaction at $\mathrm{pH} 8.4$ is $0.54 \mathrm{~V}(\mathrm{SCE})$.

$$
\mathrm{MnO}_{4}^{-}+4 \mathrm{H}^{+}+3 e^{-} \leftrightarrow \mathrm{MnO}_{2}+2 \mathrm{H}_{2} \mathrm{O}
$$

Several additional electrochemical reactions can further reduce the oxide to $\mathrm{Mn}(I I I)$, a mixed valence oxide, and finaly the $\mathrm{Mn}(I I) \operatorname{state}^{[36]}$. 


$$
\begin{gathered}
2 \mathrm{MnO}_{2}+4 \mathrm{H}^{+}+2 e^{-} \leftrightarrow \mathrm{Mn}_{2} \mathrm{O}_{3}+2 \mathrm{H}_{2} \mathrm{O} \\
3 \mathrm{Mn}_{2} \mathrm{O}_{3}+4 H^{+}+2 e^{-} \leftrightarrow 2 \mathrm{Mn}_{3} \mathrm{O}_{4}+2 \mathrm{H}_{2} \mathrm{O} \\
\mathrm{Mn}_{3} \mathrm{O}_{4}+2 H^{+}+2 e^{-} \leftrightarrow 3 M n O+\mathrm{H}_{2} \mathrm{O}
\end{gathered}
$$

At low $\mathrm{pH}$ and low potential the oxides may disolve as $\mathrm{Mn}^{2+}$. However, at higher $\mathrm{pH}$ above 8 a stable hydroxide exists in an insoluble form ${ }^{[36]}$. Half-cell reactions [3.1] through [3.5] substantiate that $\mathrm{Mn}(\mathrm{II})$ could be deposited electrochemically from $\mathrm{MnO}_{4}^{-}$and remain stable at high $\mathrm{pH}$ cathodic sites such as present over copper rich constituent particles ${ }^{[11,37]}$.

Of interest in this paper is the interaction between the initial petreatment that leads to chemically deposited $\mathrm{Ce}(\mathrm{OH})_{3}$ and the high valence state permangante ion used in the second step of the pretreatment. It is hypothesized that the cerium hydroxide will be oxidized to cerium oxide by the permanganate ion resulting in a cerium oxide coating that is stable over a larger range of $\mathrm{pH}$ than a cerium hydroxide coating Figure 3.1(a). The concentrations of the cerium species present at the recox potential of Equation [3.1] is shown in Figure 3.3. A literature search shows no documentation of an ionic species or oxide that consistes of both cerium and manganese. The resulting manganese oxides are also expected to provide corrosion protection in this dual inhibitor scheme.

This paper attempts to provide an understanding of the mechanisms of ceriumpermanganate inhibition. It seeks to understand the interaction between the two inhibitors in full immersion. In the paper both pretreatments with cerium and sequential pretreatments with permanganate are examined. Permanganate is also studied as a single inhibitor to 
examine the effects of the individual oxide barriers. Open circuit full immersion exposure experiments were conducted. Surface chemical compositions and deposit morphologies were also investigated. Copper replating was investigated to indirectly explore the inhibition of $\mathrm{Cu}$ release and replating. Pitting potentials were determined and oxygen reduction reaction (ORR) kinetics were extracted using the Koutecky-Levich correction [38].

\subsection{Experimental Procedures}

\subsubsection{Materials and Test Electrolytes}

AA2024-T351 (1 mm sheet) and T4 (rod) precipitation age hardened aluminum alloys, and copper $(99.994 \%)$ were investigated. Specific chemical compositions (wt \%) of the aluminum alloy can be found in Table 3.1. All sheet metal samples were tested on their LT surfaces, rod samples were tested on their S-T surfaces. Sample preparation varied by experimental technique depending on surface roughness requirements for imagining. These preparations fall into two categories; refinished and polished. The refinishing process consisted of a single polishing step using 600 grit silicon-carbide paper and was intended to restore the specimens to as-received condition. Polishing was carried out in $18.2 \mathrm{M} \Omega$ deionized water. The samples were subsequently rinsed in 18.2 $\mathrm{M} \Omega$ deionized water, methanol, and again in 18.2 $\mathrm{M} \Omega$ deionized water for degreasing before being dried on cotton lab tissue. When a finer surface roughness was desired to resolve constituent particles at micrometer scale resolution, the samples were ground sequentially to 1200 grit 
Si-C paper resulting in a partially reflective surface on AA2024 and high purity copper. The degreasing procedure was identical.

The test solutions were prepared with 18.2 M $\Omega$ deionized water. Solutions were prepared with (in wt $\%$ ) sodium chloride from Fisher $(99.8 \% \mathrm{NaCl})$, potassium permanganate supplied by + Fisher Scientific $\left(99.5 \% \mathrm{KMnO}_{4}\right)$ and cerium acetate $(99.99 \%)$ from Sigma Aldrich. Sodium Tetraborate Decahydrate $101.3 \%$ was obtained from Fisher Scientific, while Boric Acid, 99.99\% was obtained from Alfa Aeser. All solutions were aerated and at room temperature unless otherwise noted. Several solution preparations were used. Pretreatment solutions consisted of chloride free cerium or permanganate solutions with concentrations of $0 \mathrm{mM}, 10 \mathrm{mM}, 25 \mathrm{mM}$, and $40 \mathrm{mM}$, and $50 \mathrm{mM}$. The naturally occurring $\mathrm{pH}$ of the solutions ranged from 5.8 for the $5 \mathrm{mM}$ permanganate solution to 7.4 for the 100 $\mathrm{mM}$ concentration. Post-pretreatment exposure was carried out in $50 \mathrm{mM}$ sodium chloride at its natural $\mathrm{pH}$ of 6 in an ambient environment.

\subsubsection{Open Circuit Measurements During Open Circuit Exposure Periods}

Open circuit potential measurements were used to study the effect of the inhibitor on corrosion events when anodic and cathodic reactions were allowed to occur at uncontrolled rates on AA2024-T351.Open circuit potential measurements were taken in permanganate and cerium free $50 \mathrm{mM}$ sodium chloride after a pretreatment that consisted of sequential cerium and then permanganate treatments. The first step of the pretreatment was a 100 hour treatment in a cerium solutions with a concentration stated above. The next step was a 24 
hour treatment with permanganate in at a concentration that when added with the prior treatment in cerium came to a total inhibitor concentration of $50 \mathrm{mM}$. As an example, if the first treatment was in $10 \mathrm{mM}$ Ce-acetate, the second treatment was in $40 \mathrm{mM}$ permanganate. If the first treatment was in $25 \mathrm{mM}$ cerium-acetate, the second treatment was in $25 \mathrm{mM}$ permanganate. All treatments were carried out at room temperature and at the natural open circuit potential. Care was taken to insure that none of the faces of the sheet AA2024 were allowed to rest against the walls or bottom of the chloride free glass beakers. After the pretreatment the samples were rinsed thoroughly in $18.2 \mathrm{M} \Omega$ deionized water, blotted dry with cotton lab tissue, and attached to a standard 3 electrode cell within 5 minutes. After attachment the sample was thoroughly dried with compressed nitrogen to ensure that only the desired area was exposed to electrolyte. Following the pretreatment, the OCP was measured for 1 hour in $50 \mathrm{mM}$ sodium chloride solution.

\subsubsection{Microscopic Analysis after Open Circuit Exposures}

Scanning electron microscopy was used to examine samples after pretreatment, and pretreatment followed by exposure. Examination focused on intermetallic trenching and general pitting of the aluminum matrix as well as oxide growth on the sample. Images were recorded using the secondary electron LEI detector at $5 \mathrm{kV}$ and a working distance of 6 mm. EDS analysis was carried out at $15 \mathrm{kV}$ accelerating voltage with a working distance of $15 \mathrm{~mm}$. The K-alpha characteristic emission was recorded for all species with the exception of cerium whose L and M-alpha characteristic emissions were recorded. 


\subsubsection{Anodic and Cathodic Cyclic Potentiodynamic Polarization Scans}

Upward anodic scans were run immediately following the OCP measurements described above. The samples were scanned from $-0.05 \mathrm{~V}$ vs OCP to $1 \mathrm{~V}$ vs SCE or until the current density $(i)$ reached $5.0 \times 10^{-3} \mathrm{~mA} / \mathrm{cm}^{2}$. The scan was then reversed in direction and preceded to $-0.5 \mathrm{~V}$ vs OCP. These anodic scans were repeated on replicate specimens to give a statistical probability of pitting reported in cumulative probability plots. All measurements were taken with respect to a saturated calomel electrode. Pitting potentials were taken when the current density reached $10^{-4} \mathrm{~A} / \mathrm{cm}^{2}$.

Cathodic reaction kinetics were studied using the rotating disk electrode (RDE) method. Samples were examined after a pretreatment described above with the test scan solution consisting of $\mathrm{pH} 8.4$ borate buffer solution. The reference electrode used was mercury/mercury sulfate $\left(\mathrm{Hg} / \mathrm{Hg}_{2} \mathrm{SO}_{4}\right)$. However, all data has been converted to the saturated calomel scale for ease of use. Two types of scans were carried out. The first type was a potentiodynamic scan carried out at $2500 \mathrm{RPM}$ from $0.05 \mathrm{~V}$ above OCP to $-1.5 \mathrm{~V}$ (SCE). The second type of cathodic kinetics experiment was a potentiostatic hold at $-1.0 \mathrm{~V}$ (SCE). This potential was in the ORR dominated region of the cathodic reaction path. Rotation rate was varied during the potentiostatic hold at the following rpm: 100, 500, 1000, 1500, 2000, and 2500. Samples included high purity copper and AA2024-T4. The RDE samples were mounted in PTFE with BONDiT B-4811 from Reltek ${ }^{\mathrm{TM}}$. This epoxy is resistant to harsh chemical environments and is needed in the presence of permanganate, a strong oxidizer. 


\subsubsection{Cyclic Voltammetry Tests to Assay Copper Redeposition}

Cyclic voltammetry (CV) experiments were performed on pretreated samples that had been exposed to $50 \mathrm{mM} \mathrm{NaCl}$ for 24 hours to assay the surface copper redeposition process after inhibitor pretreatment ${ }^{[25]}$. CV experiments were performed after transferring specimens to $\mathrm{pH} 8.4$ borate buffer solutions in chloride and copper (I and II) free cells using $\mathrm{Hg} / \mathrm{Hg}_{2} \mathrm{SO}_{4}$ reference electrodes. All potentials have been reported on the saturated calomel scale for ease of use. The procedure for the test can be found in the literature ${ }^{[25]}$. The anodic current that resulted from the scans is dependent on the surface coverage of copper and thus the area coverage of the active $\mathrm{Cu}$-rich cathodic reaction sites. Metallic copper on the surface can be oxidized to $\mathrm{Cu}_{2} \mathrm{O}(\mathrm{Cu} \mathrm{I})$ and $\mathrm{CuO}(\mathrm{Cu}$ II). There can be interference with this assay if $\mathrm{Mn}(\mathrm{OH})_{2}$ is oxidized to $\mathrm{Mn}_{2} \mathrm{O}_{3}$ during the potential sweep but this has been avoided by choosing the $\mathrm{pH} 8.4$ buffer solution such that the reversible potential for the $\mathrm{Mn}$ (II) to $\mathrm{Mn}(\mathrm{III})$ reaction is $-0.117 \mathrm{vs} \mathrm{Hg} / \mathrm{Hg}_{2} \mathrm{SO}_{4}$. Several manganese oxidation reduction reactions could potentially occur between -0.1 and $-1.6 \mathrm{~V} \mathrm{Hg} / \mathrm{Hg}_{2} \mathrm{SO}_{4}$. However, none of them occur near the reduction peak for $\mathrm{Cu}$ seen in our experiments of around $-0.4 \mathrm{~V} \mathrm{Hg} / \mathrm{Hg}_{2} \mathrm{SO}_{4}$. 


\subsection{Results}

\subsubsection{Effects of Cerium-Permanganate Pretreatment on OCP Exposures of AA2024 in 50 mM NaCl Solution}

Figure 3.4 shows as-polished AA2024-T351 and the surface damage caused by a subsequent 24 hour exposure to $0.05 \mathrm{M} \mathrm{NaCl}$ solution. Figure 3.5 shows the effect of the pretreatment of AA2024-T351 with the cerium and permanganate solutions. In all pretreatment regimes where cerium was present above $10 \mathrm{mM}$ there appears to be a thick deposit that covers a large percentage of the surface of the AA2024-T351. This is in contrast to the permanganate pretreatment (Figure 3.6). The permanganate is electrochemically reduced over copper rich intermetallic particles, the active cathodic sites on AA2024-T351. Previous studies show that this mitigates corrosion ${ }^{[35]}$.

The manganese oxide particles detected by energy dispersive spectroscopy can be seen in the secondary electron image shown in Figure 3.6 ( $a$ and $b$ ). Figure 3.7 show EDS point scans of two intermetallic particles before and after pretreatment. Qualitative chemical analyses of the intermetallic particles before and after pretreatment are summarized in Table 3.2. Manganese oxide growth is apparent on many of the intermetallics and is shown to be deposited specifically on the various types of $\mathrm{Cu}$ rich phases as opposed to the aluminum rich matrix phase. Qualitative chemical data from EDS shows coverage of $\theta, S$, and $\mathrm{Cu}-\mathrm{Mn}-\mathrm{Fe}-\mathrm{Al}$ phases by a manganese oxide of unknown valence state. An example of this is shown in Figure 3.7. Permanganate forms thick oxide deposits over copper rich intermetallics in AA2024-T351 when pretreated. The deposition is characterized by 
spherical caps over small $(\sim 0.5 \mu \mathrm{m})$ intermetallics and conformal layers with terraces over larger particles $(5 \mu \mathrm{m})$.

The cerium-permanganate dual inhibitor pretreatments produced a wide array of surface features as see in Figure 3.5. In order to analyze the chemical composition of the complex oxide structures observed, EDS line scans for oxygen, magnesium, aluminum, chloride, manganese, iron, copper, and cerium were performed. Manganese is the only element that is found both in the pretreatment solution, and the alloy. The alloy contains $0.6 \%$ manganese by weight as shown in Table 3.1. This complicates the analysis of the manganese EDS results because the element is concentrated in certain intermetallic compounds in the alloy. However, manganese enrichment is clearly visible in the microscopy due to the unique oxide formations that have been observed.

EDS line scans are shown in Figure 3.8 for AA2024-T351 in one of the pretreated states used. There are three oxide formation structures visible for cerium and one structure visible for manganese. When the $10 \mathrm{mM} \mathrm{Ce}^{3+}+40 \mathrm{mM} \mathrm{MnO}_{4}^{-}$pretreatment was examined (Figure 3.5(a) and Figure 3.8(b)), long cerium rich oxide needles were observed while manganese oxides appeared to deposit over intermetallic particles as seen in the permanganate only pretreatment (Figure 3.6). When the $25 \mathrm{mM} \mathrm{Ce}^{3+}+24 \mathrm{mM} \mathrm{MnO}_{4}^{-}$pretreatment was examined (Figure 3.5(a) and (b)) cerium oxides no longer were in the form of long needles. The cerium deposits for this pretreatment regime are pellet shaped, clearly seen in Figure 3.8(b). Some permanganate deposits remain over the intermetallic particles. When the 40 $\mathrm{mM} \mathrm{Ce}{ }^{3+}+10 \mathrm{mM} \mathrm{MnO}_{4}^{-}$pretreatment was examined, cerium enrichment appears to be more widespread suggesting a more homogeneous cerium oxide or hydroxide covering the surface of the AA2024-T351. Manganese oxide deposits are still present but they appear 
to be smaller and less distinct. Finally, when a $50 \mathrm{mM}$ cerium pretreatment is conducted, general cerium enrichment is observed over the surface of the AA2024-T351 as seen in Figure 3.5(d) and Figure 3.8(d). In all cases the cerium and permanganate enrichment location indicated in EDS line scans does not coincide indicating that they are not present in a single phase. In both the $10 \mathrm{mM} \mathrm{Ce}^{3+}+40 \mathrm{mM} \mathrm{MnO}_{4}{ }^{-}$and the $25 \mathrm{mM} \mathrm{Ce}^{3+}+24 \mathrm{mM}$ $\mathrm{MnO}_{4}{ }^{-}$pretreatments the enrichment of cerium on the surface, as detected by EDS (Figure 3.8 (a) and (b)) appears to be minimal whereas the pretreatments with higher cerium concentrations (Figure 3.8 (c) and (e)) maintain some level of cerium enrichment covering the entire surface of the AA2024-T351.

Figure 3.9 shows the effects of a subsequent exposure of the pretreated samples to $0.05 \mathrm{M}$ $\mathrm{NaCl}$ for 24 hours. The effect of the inhibitor pretreatment on visible exposure damage is significant. After the pretreated samples were exposed to $50 \mathrm{mM} \mathrm{NaCl}$ for 24 hours the morphology of the surfaces changes significantly. Figure 3.10 shows EDS line scans of pretreated AA2024-T351 that had been exposed to $50 \mathrm{mM} \mathrm{NaCl}$ for 24 hours.

The corrosion inhibition of each pretreatment regime can be visually assessed by observing the extent of intermetallic trenching and pitting that has occurred on the surface of the AA2024-T351. Figure 3.9 and Figure 3.10 indicates that as the concentration of cerium increases the pretreatment appears to be more effective at decreasing the extent of damage surrounding the intermetallic particles. In Figure 3.9(a), the $10 \mathrm{mM} \mathrm{Ce}^{3+}+40 \mathrm{mM} \mathrm{MnO}_{4}^{-}$ pretreatment has slightly greater damage than any other cerium treated sample. As the concentration of cerium increases the visible damage decreases and is no longer observed once the $40 \mathrm{mM}$ concentration is reached albeit without cleaning. In order to determine the extent of surface damage hidden by cerium deposits the cerium oxide formed during 50 
$\mathrm{mM}$ Ce pretreatment was abrasively removed (Figure 3.11). When the oxide is removed it becomes apparent that the surface has been heavily damaged during the exposure. The dark spots in the microscopy indicate the presence of pervasive pitting attack to the sample surface below the cerium oxide layer. These pits can be seen to a lesser extent in Figure 3.9 D due to the presence of the cerium oxide layer. However, none are seen in Figure 3.9, $\mathrm{B}$ or $\mathrm{C}$, two samples there were also pretreated with permanganate.

This is in contrast to the $50 \mathrm{mM}$ permanganate pretreatment which is shown in Figure 3.9(e). There does appear to be trenching around the intermetallic particles. However, there is a lack of heavy pitting of the aluminum matrix as seen in the $50 \mathrm{mM}$ cerium pretreatment. In summary $\mathrm{MnO}_{4}^{-}$alone is more effective than $\mathrm{Ce}^{3+}$ with $\mathrm{Ce}-\mathrm{Mn}$ combinations intermediate.

\subsubsection{Open Circuit Behavior of Pretreated AA2024-T351}

Open circuit potential data was collected for 1 hour in ambient aerated $0.05 \mathrm{M} \mathrm{NaCl}$ solution after the dual inhibitor pretreatments were performed (Figure 3.12). The samples were removed from pretreatment, rinsed thoroughly, and the open circuit was measured in 0.05 M sodium chloride solution. The specimens that were treated with higher levels of permanganate tend to have lower OCP when compared with no pretreatment. This effect has been discussed in a previous publication ${ }^{[35]}$. Briefly, it is believed that the manganese oxide deposits initially contain some residual permanganate, which over the course of the first hour is reduced providing an additional cathodic reactant. As the residual permanganate is reduced the OCP drops as the result of the blockage of the intermetallic particles for fast ORR. As the manganese oxide deposits over the intermetallic caps 
degrade or dissolve the open circuit recovers to the open circuit of the un-treated AA2024T351 substrate. Figure 3.12 and Figure 3.9(e) show this behavior. A significant result is that $\mathrm{MnO}_{4}^{-}$is much more effective at lowering OCP than $\mathrm{Ce}^{+3}$ suggesting greater ORR inhibition.

\subsubsection{Inhibition of Copper Replating}

Copper replating was also investigated on pretreated AA2024-T351 surfaces after OCP exposures in $50 \mathrm{mM} \mathrm{NaCl}$ using the cyclic voltammetry $(\mathrm{CV})$ method to assay the copper coverage. Figure 3.13 shows the cyclic voltammetric analysis of pretreated AA2024-T351, and AA2024-T351 etched in .05 M NaCl solution for 24 hours. The charge associated with anodic $\mathrm{Cu}(\mathrm{I})$ to $\mathrm{Cu}(\mathrm{II})$ reaction peaks reflect the surface coverage of copper brought about by corrosion and are indicative of copper replating ${ }^{[25]}$. The results of the $\mathrm{CV}$ tests are summarized in the bar plots in Figure 3.14. These suggest that cerium-permanganate pretreatments reduce copper redeposition the most.

\subsubsection{Anodic Inhibition of AA2024-T351 by Cerium-Permanganate Pretreatments}

Figure 3.15 (a-e) shows anodic E-logi behavior for pretreated AA2024-T351 in $0.05 \mathrm{M}$ sodium chloride solution during upward cyclic potentiodynamic scans. Ceriumpermanganate pretreatments had very little effect on the pitting potential of AA2024-T351. A very slight increase in the pitting potential is observed but it is not significant. As shown

previously ${ }^{[35]}$, the samples pretreated with permanganate alone experience a significantly suppressed OCP that exposes a passive region. However, the pitting potential of the exposed passive film is not affected. However $\Delta \mathrm{E}=\mathrm{E}_{\mathrm{pit}}-\mathrm{E}_{\mathrm{OCP}}$ is significantly improved. 
Cerium pretreatments alone show little effect on the pitting potential or EocP. Therefor $\Delta \mathrm{E}$ is not reduced.

Figure 3.16 presents the cumulative probability of pitting as a function of potential for pretreated AA2024-T351. No significant increase in pitting potential is observed for pretreated AA2024-T351 tested in $50 \mathrm{mM} \mathrm{NaCl}$ solution.

\subsubsection{Investigation of Cathodic Inhibition with Cerium-Permanganate Pretreatments}

Figure 3.18 shows the cathodic polarization data collected using the rotating disk electrode method to examine the effects of cerium-permanganate pretreatments on the ORR kinetics on AA2024-T4 in pH 8.4 borate buffer. The E- $i$ behavior of untreated AA2024-T351 and high purity copper were run with traditional potentiodynamic control to illustrate the effect of rotation rate over all potentials. This can be seen in Figure 3.17. Figure 3.18 shows the results of potentiodynamic downward scans at a set rotation rate of $2500 \mathrm{rpm}$. Ceriumpermanganate pretreatments change the regions of mass and charge transfer control on AA2024-T4. Cerium-permangante inhibitor combinations show very similar behavior to that of permanganate pretreated samples. This begins with an elevated open circuit potential in $\mathrm{pH} 8.4$ borate buffer. The elevated potential can be explained by considering the half-cell reaction potentials for manganese oxide species at $\mathrm{pH}$ 8.4. As the potential is decreased these oxides are reduced resulting in the cathodic current density shoulder observed from $0.5 \mathrm{~V}$ (SCE) to $-0.5 \mathrm{~V}$ (SCE). As the potential continues downward the oxygen reduction reaction begins to dominate the current density and becomes mass transport limited starting around -0.6 V (SCE). This cathodic kinetic behavior is not evident 
for two pretreatment systems: $10 \mathrm{mM} \mathrm{Ce}^{3+}+40 \mathrm{mM} \mathrm{MnO}_{4}^{-}$and $25 \mathrm{mM} \mathrm{Ce}^{3+}+24 \mathrm{mM}$ $\mathrm{MnO}_{4}^{-}$. These two systems show remarkable inhibition of the oxygen reduction reaction. The current density for these two systems is two and a half orders of magnitude lower than untreated AA2024-T351. The remaining E-logi scans show the onset of HER dominated current density between -1.2 and $-1.5 \mathrm{~V}$ (SCE).

A second rotating disk electrode method was used to examine the ORR further. The working electrode was held at a potential of $-1.0 \mathrm{~V}(\mathrm{SCE})$ and the rotation rate of the sample was varied. The results can be seen in Figure 3.19. The primary finding is that ceriumpermanganate is more effective at cathodic inhibition than cerium alone. The variable rotation rate results for AA2024-T351 pretreated in cerium-permanganate dual inhibitor pretreatments in Figure 3.19(a), suffer from the presence of an additional source of cathodic current, the reduction of manganese. The rotation rate dependent cathodic current density changes manifest themselves as near instantaneous steps in current density that correlate exactly with the change in rotation rate. The ORR rotation dependence can be extracted by observing the instantaneous jump in current density that occurs when the rotation rate is increased after accounting for the additional manganese reduction current.

The additional cathodic current from the reduction of manganese is not seen when pretreatments were conducted on high purity copper that was potentiostatically pretreated at the open circuit of the AA2024-T351 system (-0.5 V (SCE)) in cerium and permanganate solutions. These pretreatments showed limited inhibition (Figure 3.19).

Plots of $1 / i_{\text {Tot }} \mathrm{Vs} \omega^{-1 / 2}$ at $-1.0 \mathrm{~V}(\mathrm{SCE})$ (a potential dominated by oxygen reduction mass transfer control) show that uninhibited AA2024-T4 has a slightly lower current density 
than high purity copper (Figure 3.20(a)). This is due to the array of active electrodes surrounded by inactive aluminum matrix as described by the Gueshi model ${ }^{[38,39]}$. The Koutecky-Levich method ${ }^{[40]}$, as shown in Equation [3.6], was used to extract $i_{D}$ from $i_{T}$ at $-1.0 \mathrm{~V}$ (vs SCE), where $i_{D}$ is the diffusion limited cathodic current density and $i_{c t}$ is the charge transfer limited cathodic current density (Figure 3.20(b)). At this potential $i_{H E R}$ is much lower than $i_{O R R}$.

$$
\frac{1}{i_{T o t}}=\frac{1}{i_{c t}}+\frac{1}{i_{D}}
$$

Where $i_{D}$ is given by

$$
i_{D}=.62 n F D^{2} / 3 v^{1 / 6 C} \omega^{1 / 2}
$$

The results on high purity copper can be compared with the Levich description of masstransport limited $\mathrm{O}_{2}$ reduction given by Equation [3.7]. Using a value for the diffusion of $\mathrm{O}_{2}$ in water, $\mathrm{D}=1.9 \times 10^{-5} \mathrm{~cm}^{2} / \mathrm{s}$, a value of $\mathrm{n}=4$, the concentration of $\mathrm{O}_{2}$ in solution, $\mathrm{C}=$ 7-9 ppm, and the kinematic viscosity, $v=1 \times 10^{-2} \mathrm{~cm}^{2} / \mathrm{s}$. Good agreement is found between theoretical values and experimental data ${ }^{[41]}$. This suggests that at $-1.0 \mathrm{~V}$ (SCE) the current due to the HER is a small fraction of the ORR current.

The diffusion limited current density was extracted from the total current density using the Koutecky-Levich method for the pretreated case at -1.0 V (SCE). The extracted diffusion limited current density is shown in Figure 3.21(b). Pretreated AA2024 has a lower cathodic current density than untreated AA2024-T4 over all rotation rates with a weaker rotation rate dependency. Permanganate and Cerium-permanganate pretreatments suppress the 
diffusion limited current density significantly. Permanganate alone affords the best reduction in cathodic current on AA2024.

\subsection{Discussion}

\subsubsection{Open circuit and Anodic Inhibition by Cerium Permanganate Pretreatments}

All inhibitor combinations show some decrease in current densities attributed to copper reduction. The suppression of copper replating as indicated by CV (Figure 3.14) correlates with the reduced damage observed in our OCP microscopy experiments when the cerium deposits are removed compared to untreated (Figure 3.9, 3, and 3). However, when permanganate is not used in the pretreatment, pitting attack through a cerium oxide layer occurs resulting in only a small reduction in current during the surface copper assay. The attack of the substrate through the observed cerium film appears to be mitigated by manganese deposits when dual inhibitor pretreatments are used and this is reflected in the $\mathrm{CV}$ data (Figure 3.14). When permanganate is used alone the reduction in current during the surface copper assay is almost an order of magnitude lower than when cerium is used alone. This is also supported by $\Delta \mathrm{E}=\mathrm{E}_{\text {pit }}-\mathrm{E}_{\mathrm{OCP}}$. The greatest reduction in $\Delta \mathrm{E}$ is for permanganate or cerium-permanganate combinations. Cerium alone is less effective than permanganate possibly due to homogeneous chemical precipitation vs. direct permanganate reduction which effectively blocks IMC electrochemical $\mathrm{MnO}_{4}{ }^{-}$deposition. The oxidation state of the cerium oxide/hydroxide is unclear and further study needs to be done to determine if permanganate has oxidize the cerium deposits from cerium hydroxide to cerium oxide. 


\subsubsection{Diffusion Limited Current Density Inhibition by Cerium-Permanganate Pretreatments}

Figure 3.19 (a) shows the true total cathodic current density on single inhibitor pretreatments of cerium and permanganate as well as combinations of the two. Cerium clearly shows a higher cathodic current density. This may result from the chemical precipitation reaction which can't be site specific that leads to the observed irregular cerium rich oxide or hydroxide film seen in Figure 3.5 (d). The cerium-permanganate combination pretreatments and the permanganate single inhibitor pretreatment have lower total cathodic current densities. The rotation rate dependence of the cathodic current density is not immediately obvious as it is in the cerium pretreatment. The reduction of manganese has a linear relationship with time and when subtracted out, reveals the rotation rate dependence seen in Figure 3.20 (a). The manganese reduction is removed from the diffusion limited current density analysis so that a better understanding of the oxygen reduction reaction can be obtained leading to more information about the blockage of cathodic reaction sites. In Figure 3.20 (a) $1 / \mathrm{i}_{\text {Total }}$ is plotted against one over the square root of rotation rate $(\omega)$. Here $i_{\text {Total }}$ is the total current seen in Figure 3.19(a) minus the cathodic current due to manganese reduction. High rotation rates are on the left of the graph. The permanganate pretreated sample shows the lowest current density while the cerium pretreated samples shows the highest cathodic current density. The two step inhibitor pretreatments also show strong cathodic current inhibition. However, it is not as strong as permanganate pretreatment as a single inhibitor. 
When the K-L correction is applied to the data, seen in Figure 3.20(b), the inhibition of diffusion limited cathodic current density due to permanganate pretreatment shows the highest inhibition, while cerium shows the lowest levels of inhibition. The homogeneous chemical reaction that leads to cerium deposition cannot be site specific and may leave gaps in coverage of constituent particles, the primary cathodic reaction sites. In contrast, the reduction reaction that leads to manganese oxide coverage is targeted to fast reacting cathodic sites and complete as it is the result of a cathodic reaction (Figure 3.22). Sites where cathodic reactions happen quickly will be sites were permanganate is reduced quickly, leading to blockage and deactivation of the cathodic reaction site. When ceriumpermanganate dual inhibitor pretreatments are conducted the cerium may act to block this direct, targeted deposition leading to a slight decrease in the permanganate cathodic current inhibition efficiency.

\subsection{Conclusions}

High concentrations of cerium in the pretreatment package can lead to a homogeneous cerium enriched oxide surface. However, such a treatment shows little indication of being passive or suppressing corrosion. Cerium-permanganate pretreatments show insignificant anodic inhibition in pitting scans but reduced copper replating during open circuit corrosion. Permanganate pretreatments are effective at increasing $\Delta \mathrm{E}\left(\mathrm{E}_{\mathrm{pit}}-\mathrm{E}_{\mathrm{OCP}}\right)$ mainly through reducing the EOCP. Permanganate pretreatments are shown to have strong cathodic inhibition properties. Cerium-permanganate pretreatments show promise as cathodic inhibitors, in some cases reducing cathodic current density two and a half orders of 
magnitude when compared to AA2024-T351. However, no indication of a combined species was found and there is no reference to such a species in the literature. Cerium can act detrimentally to block permanganate oxide formation over copper rich intermetallic particles. 


\subsection{References}

1. G.O. Ilevbare, O. Schneider, R.G. Kelly, and J.R. Scully, "In situ confocal laser scanning microscopy of AA 2024-T3 corrosion metrology - I. Localized corrosion of particles," Journal of the Electrochemical Society 151, 8 (2004): p. B453.

2. O. Schneider, G.O. Ilevbare, J.R. Scully, and R.G. Kelly, "In situ confocal laser scanning microscopy of AA 2024-T3 corrosion metrology - II. Trench formation around particles," Journal of the Electrochemical Society 151, 8 (2004): p. B465.

3. J.R. Scully, T.O. Knight, R.G. Buchheit, and D.E. Peebles, "Electrochemical characteristics of the $\mathrm{Al}_{2} \mathrm{Cu}, \mathrm{Al}_{3} \mathrm{Ta}$ and $\mathrm{Al}_{3} \mathrm{Zr}$ intermetallic phases and their relevancy to the localized corrosion of Al alloys," Corrosion Science 35, 1-4 (1993): p. 185.

4. R.P. Wei, C.M. Liao, and M. Gao, "A transmission electron microscopy study of constituent-particle-induced corrosion in 7075-T6 and 2024-T3 aluminum alloys," Metall Mater Trans A 29, 4 (1998): p. 1153.

5. G.S. Chen, "Microconstituent-induced pitting corrosion in aluminum alloy 2024T3," CORROSION 52, 01 (1996).

6. D.G. Harlow and R.P. Wei, "A probability model for the growth of corrosion pits in aluminum alloys induced by constituent particles," Engineering Fracture Mechanics 59, 3 (1998): p. 305.

7. R.G. Buchheit, R.P. Grant, P.F. Hlava, B. Mckenzie, and G.L. Zender, "Local dissolution phenomena associated with $\mathrm{S}$ phase $\left(\mathrm{Al}_{2} \mathrm{CuMg}\right)$ particles in aluminum alloy 2024-T3," Journal of the Electrochemical Society 144, 8 (1997): p. 2621.

8. C.M. Reddy, Q.S. Yu, C.E. Moffitt, D.M. Wieliczka, W. Johnson, J.E. Deffeyes, and H.K. Yasuda, "Improved corrosion protection of aluminum alloys by system approach interface engineering: Part 1 - Alclad 2024-T3," Corrosion 56, 8 (2000): p. 819.

9. D. Zhu and W.J. van Ooij, "Corrosion protection of AA 2024-T3 by bis-[3(triethoxysilyl)propyl]tetrasulfide in neutral sodium chloride solution. Part 1: corrosion of AA 2024-T3," Corrosion Science 45, 10 (2003): p. 2163.

10. K.A. Yasakau, M.L. Zheludkevich, S.V. Lamaka, and M.G.S. Ferreira, "Mechanism of Corrosion Inhibition of AA2024 by Rare-Earth Compounds," The Journal of Physical Chemistry B 110, 11 (2006): p. 5515.

11. G.O. Ilevbare and J.R. Scully, "Mass-transport-limited oxygen reduction reaction on AA2024-T3 and selected intermetallic compounds in chromate-containing solutions," Corrosion 57, 2 (2001): p. 134.

12. G.O. Ilevbare and J.R. Scully, "Oxygen Reduction Reaction Kinetics on Chromate Conversion Coated $\mathrm{Al}-\mathrm{Cu}, \mathrm{Al}-\mathrm{Cu}-\mathrm{Mg}$, and $\mathrm{Al}-\mathrm{Cu}-\mathrm{Mn}-\mathrm{Fe}$ Intermetallic Compounds," Journal of The Electrochemical Society 148, 5 (2001): p. B196.

13. G.O. Ilevbare, J.R. Scully, J. Yuan, and R.G. Kelly, "Inhibition of pitting corrosion on aluminum alloy 2024-T3: Effect of soluble chromate additions vs chromate conversion coating," Corrosion 56, 3 (2000): p. 227. 
14. M.W. Kendig and R.G. Buchheit, "Corrosion inhibition of aluminum and aluminum alloys by soluble chromates, chromate coatings, and chromate-free coatings," Corrosion 59, 5 (2003): p. 379.

15. Y. Yoon and R.G. Buchheit, "Dissolution Behavior of $\mathrm{Al}_{2} \mathrm{CuMg}$ (S Phase) in Chloride and Chromate Conversion Coating Solutions," Journal of the Electrochemical Society 153, 5 (2006): p. B151.

16. W. Zhang, B. Hurley, and R.G. Buchheit, "Characterization of Chromate Conversion Coating Formation and Breakdown Using Electrode Arrays," Journal of The Electrochemical Society 149, 8 (2002): p. B357.

17. OSHA, IMIS Code Number 0689 Chromium (VI) (Hexavalent Chromium). 2006.

18. R.G. Buchheit, R.K. Boger, M.C. Carroll, R.M. Leard, C. Paglia, and J.L. Searles, "The electrochemistry of intermetallic particles and localized corrosion in $\mathrm{Al}$ alloys," Jom-J Min Met Mat S 53, 7 (2001): p. 29.

19. S. Kim, J.T. Burns, and R.P. Gangloff, "Fatigue crack formation and growth from localized corrosion in Al-Zn-Mg-Cu," Engineering Fracture Mechanics 76, 5 (2009): p. 651.

20. R.M. Pidaparti and R.R. Patel, "Correlation between corrosion pits and stresses in Al alloys," Mater Lett 62, 30 (2008): p. 4497.

21. K.K. Sankaran, R. Perez, and K.V. Jata, "Effects of pitting corrosion on the fatigue behavior of aluminum alloy 7075-T6: modeling and experimental studies," Mat Sci Eng a-Struct 297, 1-2 (2001): p. 223.

22. R.L. Twite and G.P. Bierwagen, "Review of alternatives to chromate for corrosion protection of aluminum aerospace alloys," Progress in Organic Coatings 33, 2 (1998): p. 91.

23. W.D. Robertson, "Molybdate and Tungstate as Corrosion Inhibitors and the Mechanism of Inhibition," Journal of The Electrochemical Society 98, 3 (1951): p. 94.

24. K.D. Ralston, S. Chrisanti, T.L. Young, and R.G. Buchheit, "Corrosion inhibition of aluminum alloy 2024-T3 by aqueous vanadium species," Journal of the Electrochemical Society 155, 7 (2008): p. C350.

25. M.A. Jakab, F. Presuel-Moreno, and J.R. Scully, "Critical Concentrations Associated with Cobalt, Cerium, and Molybdenum Inhibition of AA2024-T3 Corrosion: Delivery from Al-Co-Ce(-Mo) Alloys," CORROSION 61, 3 (2005): p. 246.

26. M. Iannuzzi and G.S. Frankel, "Inhibition of Aluminum Alloy 2024 Corrosion by Vanadates: An In Situ Atomic Force Microscopy Scratching Investigation," Corrosion 63, 7 (2007): p. 672.

27. M. Iannuzzi and G.S. Frankel, "Mechanisms of corrosion inhibition of AA2024-T3 by vanadates," Corrosion Science 49, 5 (2007): p. 2371.

28. M. Iannuzzi, J. Kovac, and G.S. Frankel, "A study of the mechanisms of corrosion inhibition of AA2024-T3 by vanadates using the split cell technique," Electrochimica Acta 52, 12 (2007): p. 4032.

29. M. Iannuzzi, T. Young, and G.S. Frankel, "Aluminum alloy corrosion inhibition by vanadates," Journal of the Electrochemical Society 153, 12 (2006): p. B533.

30. K.D. Ralston, T.L. Young, and R.G. Buchheit, "Electrochemical Evaluation of Constituent Intermetallics in Aluminum Alloy 2024-T3 Exposed to Aqueous 
Vanadate Inhibitors," Journal of the Electrochemical Society 156, 4 (2009): p. C135.

31. E.A. Lizlovs, "Molybdates as Corrosion-Inhibitors in Presence of Chlorides," Corrosion 32, 7 (1976): p. 263.

32. A.H. Alsaffar, V. Ashworth, A.K.O. Bairamov, D.J. Chivers, W.A. Grant, and R.P.M. Procter, "Effect of Molybdenum Ion-Implantation on the General and Pitting Corrosion Behavior of Pure Aluminum and a High-Strength AluminumAlloy," Corrosion Science 20, 1 (1980): p. 127.

33. M.L. Zheludkevich, R. Serra, M.F. Montemor, K.A. Yasakau, I.M.M. Salvado, and M.G.S. Ferreira, "Nanostructured sol-gel coatings doped with cerium nitrate as pretreatments for AA2024-T3: Corrosion protection performance," Electrochimica Acta 51, 2 (2005): p. 208.

34. D. Ho, N. Brack, J. Scully, T. Markley, M. Forsyth, and B. Hinton, "Cerium Dibutylphosphate as a Corrosion Inhibitor for AA2024-T3 Aluminum Alloys," Journal of The Electrochemical Society 153, 9 (2006): p. B392.

35. S. Madden, Investigation of Permanganate as an Environmentally Friendly Inhibitor of Corrosion on Aluminum 2024-T351. In preparation for submission to JECS as of October, 2011.

36. M. Pourbaix, Atlas of electrochemical equilibria in aqueous solutions. 2d English ed (Houston, Tex.: National Association of Corrosion Engineers, 1974). p. 644 p.

37. Y. Baek and G.S. Frankel, "Electrochemical quartz crystal microbalance study of corrosion of phases in AA2024," Journal of the Electrochemical Society 150, 1 (2003): p. B1.

38. M.A. Jakab, D.A. Little, and J.R. Scully, "Experimental and Modeling Studies of the Oxygen Reduction Reaction on AA2024-T3," Journal of The Electrochemical Society 152, 8 (2005): p. B311.

39. T. Gueshi, K. Tokuda, and H. Matsuda, "Voltammetry at partially covered electrodes: Part I. Chronopotentiometry and chronoamperometry at model electrodes," Journal of Electroanalytical Chemistry 89, 2 (1978): p. 247.

40. E. Gileadi, Electrode Kinetics for Chemists, Chemical Engineers and Materials Scientists: John Wiley and Sons, 1993.

41. F.J. Presuel-Moreno, M.A. Jakab, and J.R. Scully, "Inhibition of the oxygen reduction reaction on copper with cobalt, cerium, and molybdate ions," Journal of the Electrochemical Society 152, 9 (2005): p. B376. 


\subsection{Tables}

Table 3.1.Chemical composition of AA2024-T351 (wt. \%) The T4 designation indicates slight cold working of T351.

\begin{tabular}{lrrrrrrrrrrr} 
AA2024 & $\mathrm{Al}$ & $\mathrm{Cu}$ & $\mathrm{Mg}$ & $\mathrm{Mn}$ & $\mathrm{Fe}$ & $\mathrm{Zn}$ & $\mathrm{Si}$ & $\mathrm{Ti}$ & $\mathrm{Cr}$ & $\mathrm{V}$ & $\mathrm{Ga}$ \\
\hline -T351 & Balance & 4.560 & 1.260 & 0.590 & 0.220 & 0.120 & 0.080 & 0.020 & 0.010 & 0.010 & $\mathrm{NA}$ \\
-T4 & Balance & 4.34 & 1.38 & 0.585 & 0.22 & 0.007 & 0.07 & 0.02 & 0.006 & 0.006 & 0.011
\end{tabular}

Table 3.2. Compilation of EDS data for AA2024-T351 intermetallic particles in Figures 1 and 2, before and after pretreatment in .1 $\mathrm{M} \mathrm{KMnO}_{4}$.

Intermetallic phase Particle \# Manganese Oxide Cap Mn enrichment (EDS)

\begin{tabular}{lrcc}
\hline $\mathrm{AlCuMg}$ & 5,6 & Yes & Yes \\
$\mathrm{Al}(\mathrm{Cu}, \mathrm{Mn}, \mathrm{Fe}, \mathrm{SI})$ & $1,2,3$ & Yes & Yes \\
$\mathrm{AlCu}$ & 4 & Yes & Yes \\
$\mathrm{Al}(\mathrm{Cu}, \mathrm{Mn}, \mathrm{Fe}, \mathrm{Mg})$ & 7,8 & Yes & Yes
\end{tabular}




\subsection{Figures}

a)

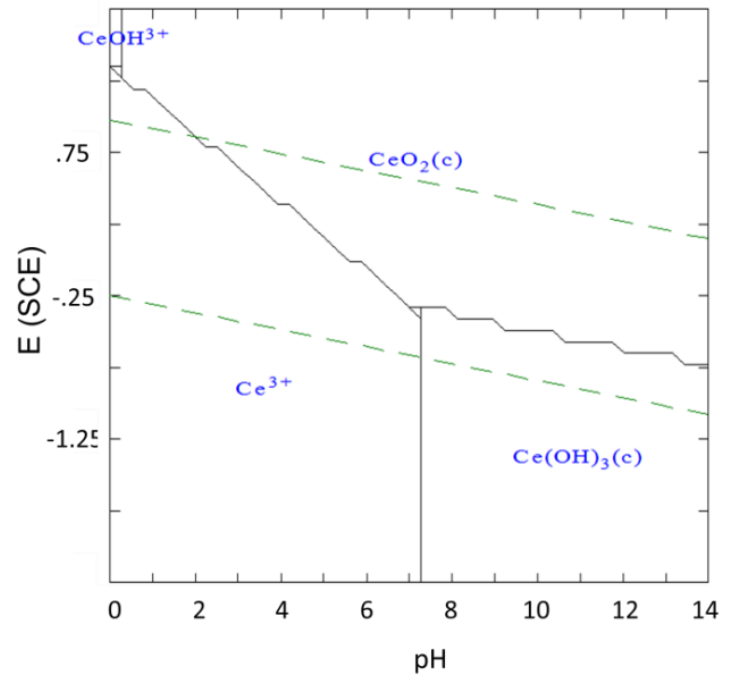

b)

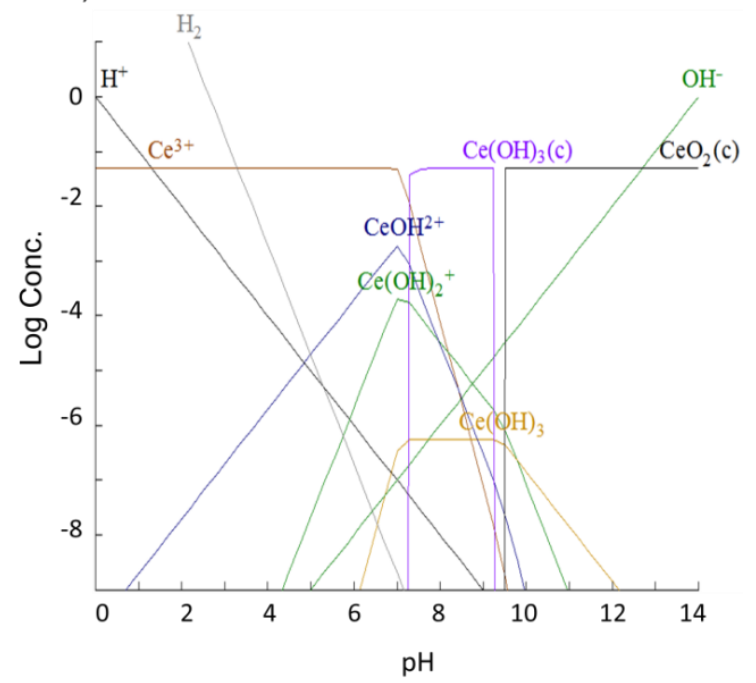

Figure 3.1.(a) The E-pH behavior of $.05 \mathrm{M} \mathrm{Ce}^{3+}$ in aqueous solution. (b) The concentrations of cerium species in the cerium- $\mathrm{H}_{2} \mathrm{O}$ system at $-0.5 \mathrm{~V} \mathrm{SCE}$, near the open circuit potential of AA2024. 

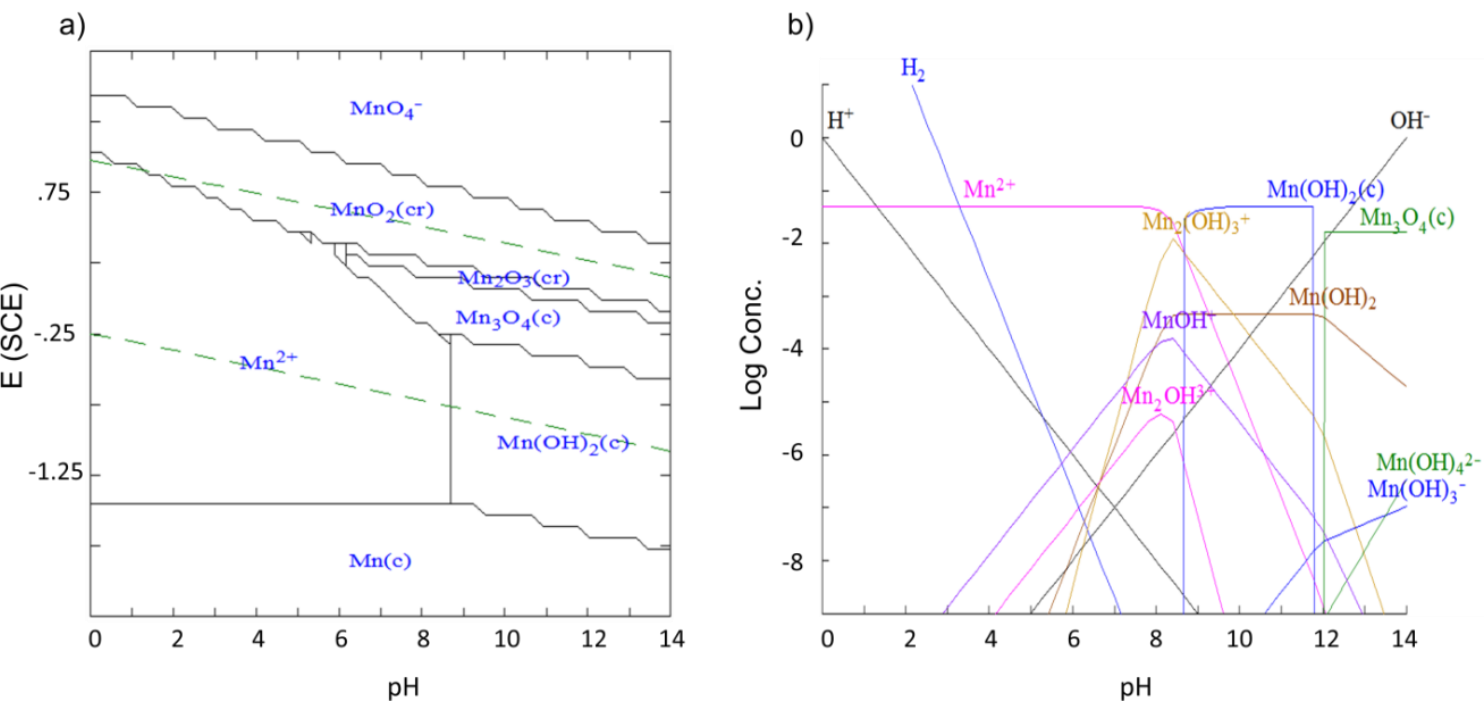

Figure 3.2. (a) The E-pH behavior of $0.05 \mathrm{M} \mathrm{MnO}_{4}^{-}$in aqueous solution. (b) The concentrations of manganese species in the manganese- $\mathrm{H}_{2} \mathrm{O}$ system at $-0.5 \mathrm{~V}$ SCE, near the open circuit potential of AA2024. 


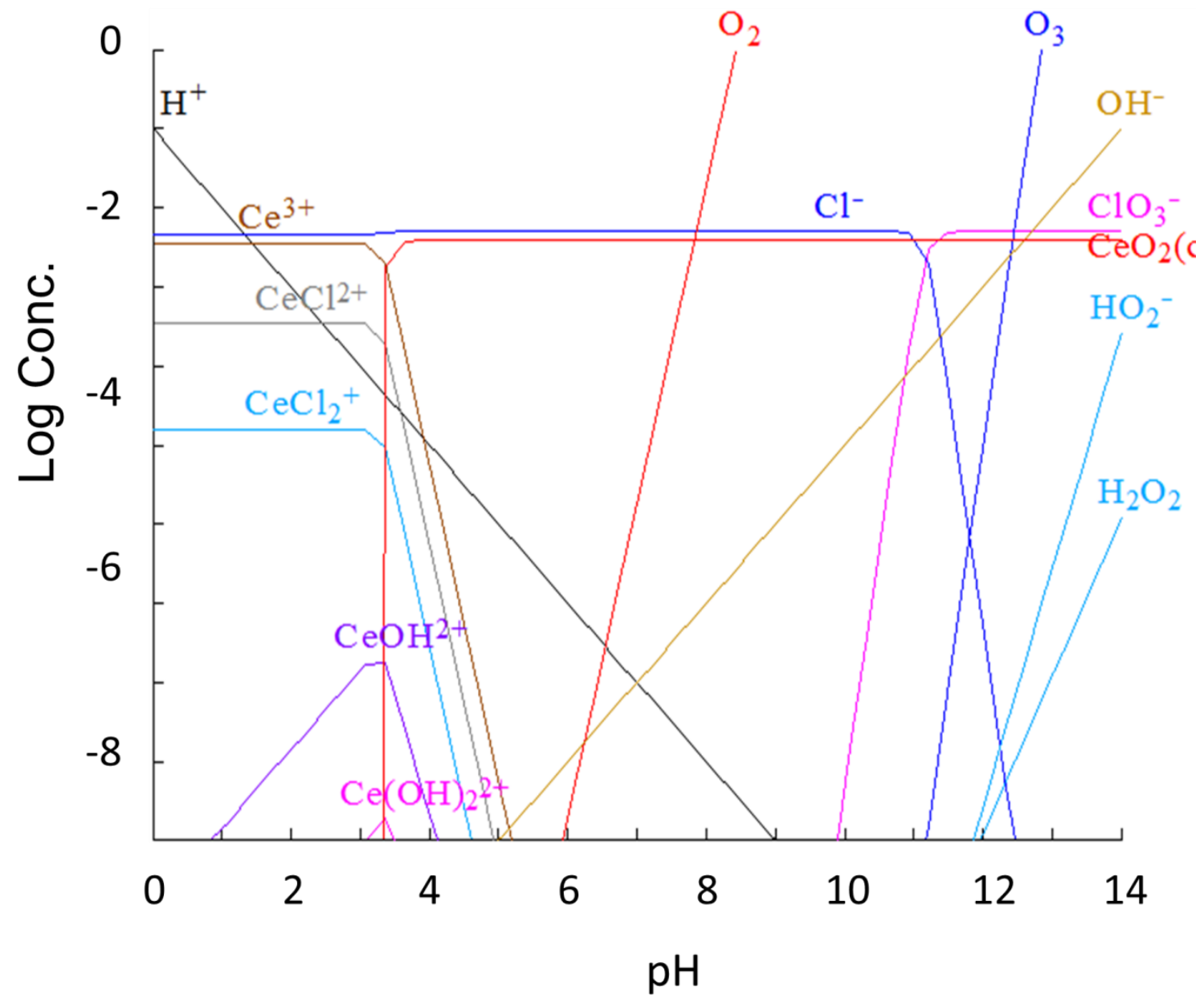

Figure 3.3. Concentrations of species in the cerium-chloride- $\mathrm{H}_{2} \mathrm{O}$ system at $0.78 \mathrm{~V}$ (SCE), the $\mathrm{E}^{\mathrm{o}}$ of Equation [3.2]. The cerium oxide species $\left(\mathrm{CeO}_{2}\right)$ is stable above $\mathrm{pH} 3.3$ at this potential. 
a)

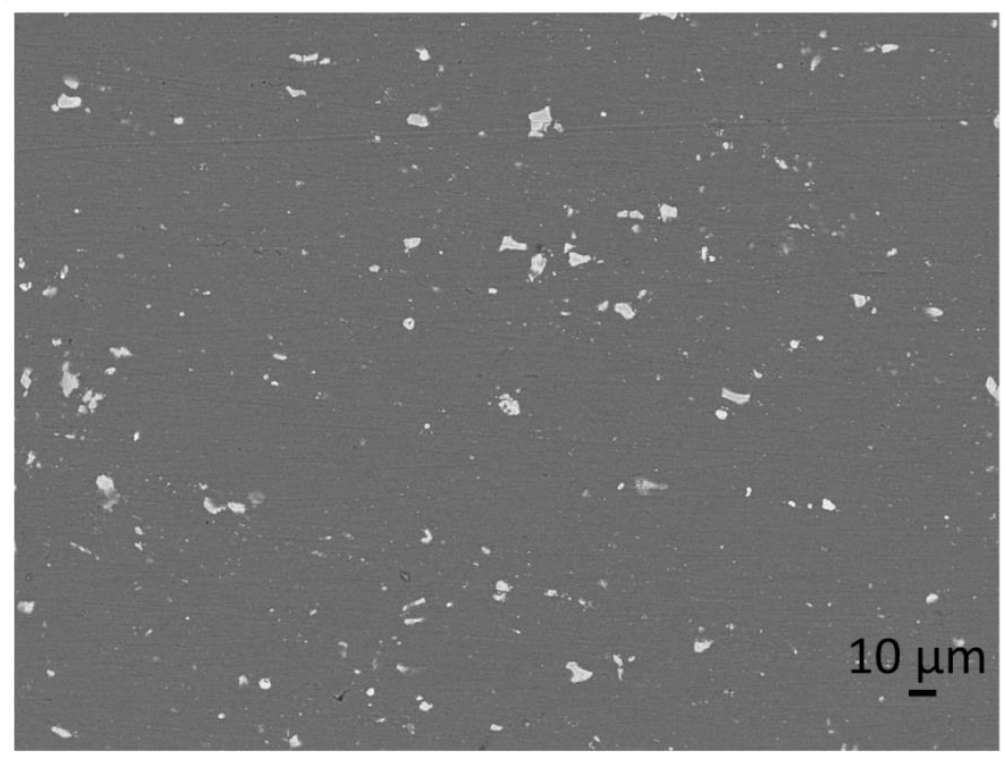

b)

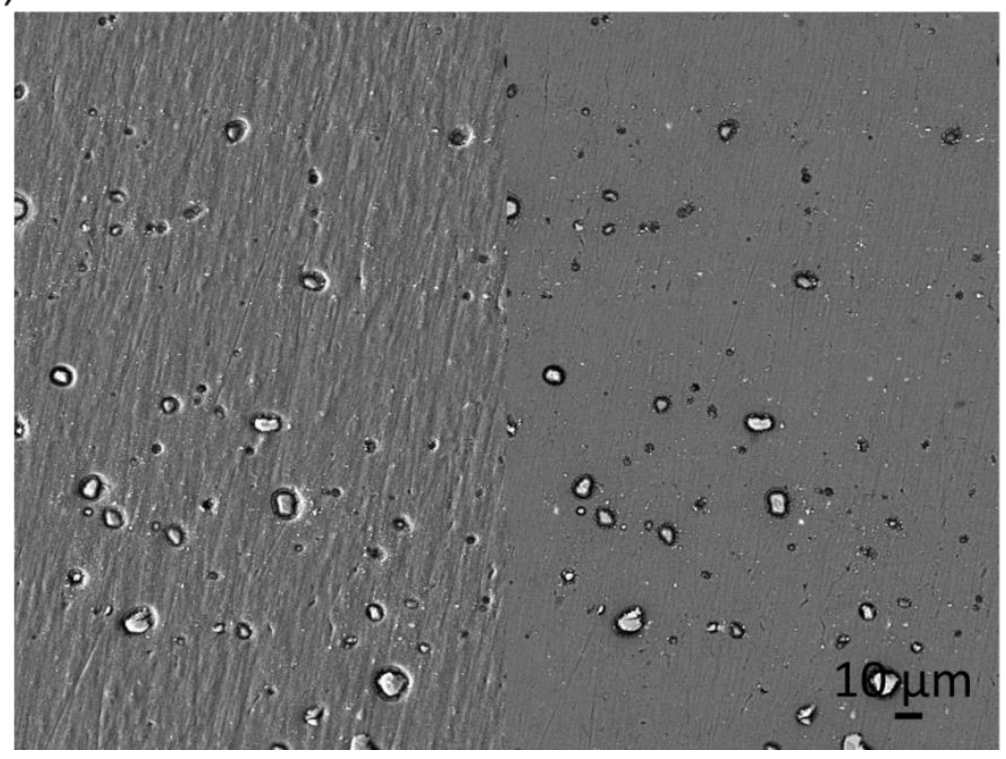

Figure 3.4. (a) The as-polished (1200 grit) AA2024-T351 and the subsequent surface damage caused by 24 hour exposure to $0.05 \mathrm{M} \mathrm{NaCl}$ solution in a naturally aerated state at room temperature. (b) The damaged specimen image is compressed horizontally to include a secondary electron and backscattered electron image. 
a)

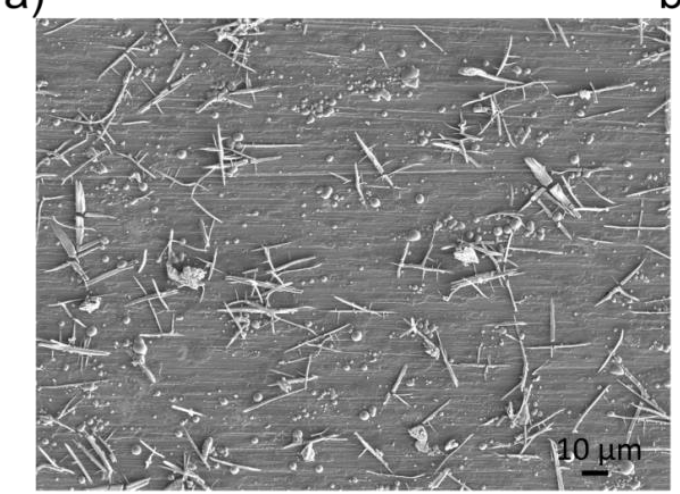

b)

c)

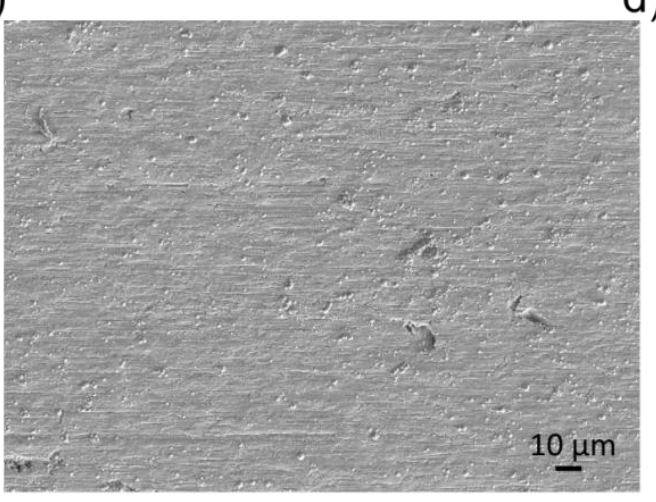

d)

e)

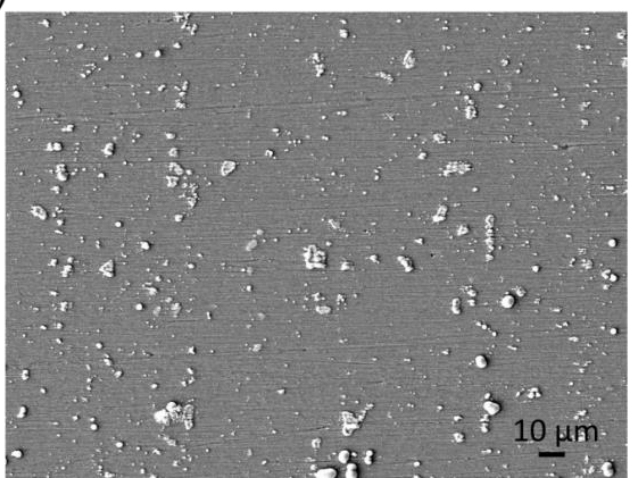

Figure 3.5. (a) AA2024-T351 pretreated with $0.01 \mathrm{M} \mathrm{Ce}^{3+}$ for $100 \mathrm{hr}$ and $0.04 \mathrm{M} \mathrm{MnO}_{4}{ }^{-}$ for $24 \mathrm{hr}$ (secondary electron image). (b) Same pretreatment times with inhibitor concentrations of $0.025 \mathrm{M} \mathrm{Ce}^{3+}$ and $0.025 \mathrm{MnO}_{4}^{-}$. (c) Same pretreatment times with inhibitor concentrations of $0.04 \mathrm{M} \mathrm{Ce}^{3+}$ and $0.01 \mathrm{MnO}_{4}^{-}$. (d) Same pretreatment times with inhibitor concentrations of $0.05 \mathrm{M} \mathrm{Ce}^{3}$. (e) Same pretreatment time with inhibitor concentration of $0.05 \mathrm{MnO}_{4}^{-}$. 
a)

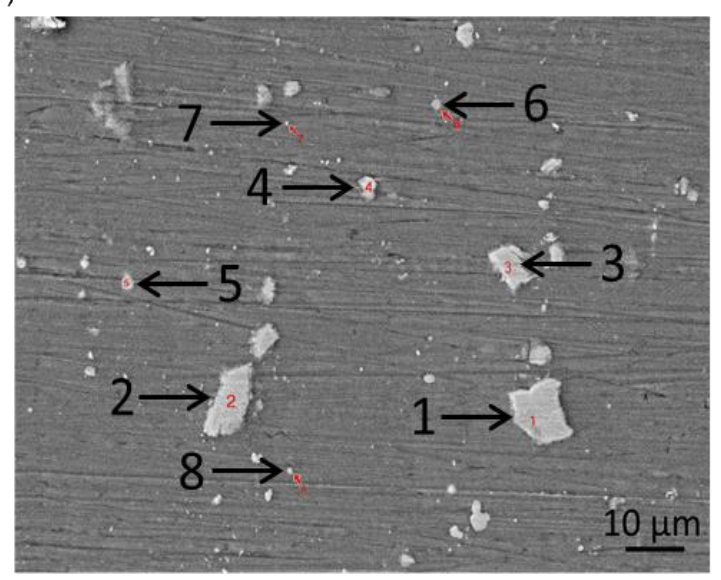

b)

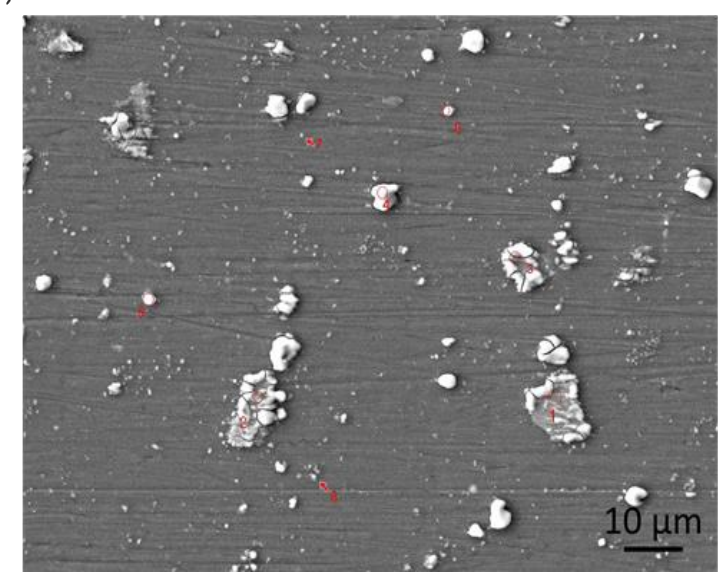

Figure 3.6. (a) AA2024-T351 with indication of EDS sites labeled 1-8. (b) Same sample and area as (a) Sample has been pretreated for 24 hours in $0.1 \mathrm{M} \mathrm{KMnO}_{4}$. Indication of EDS sites labeled 1-8. 

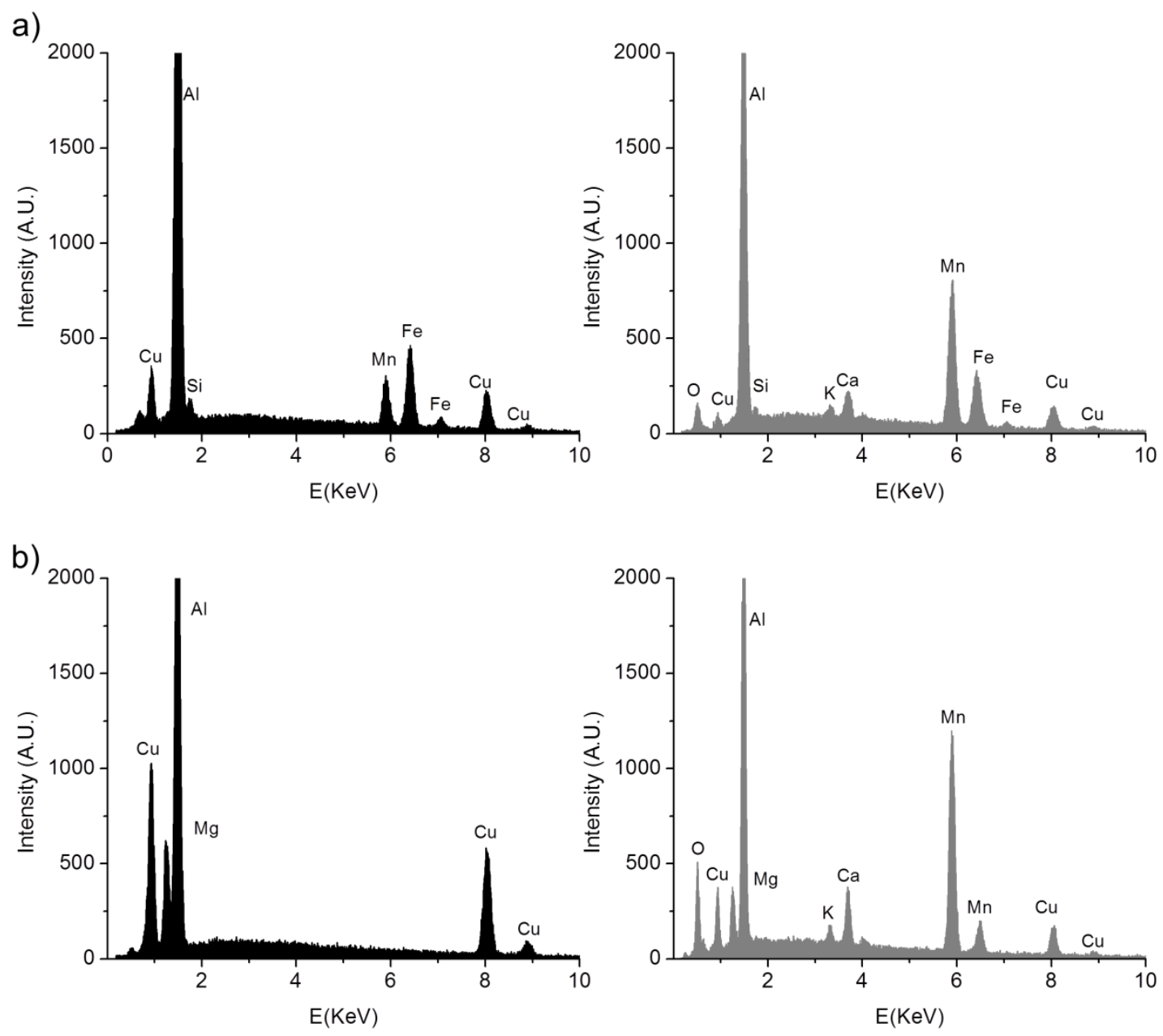

Figure 3.7. (a) Intermetallic particle 1 from Figure 3.6 (b). Intermetallic particle 5 from Figure 3.6. The left spectrum is taken from an untreated sample, the right, a pretreated sample. 


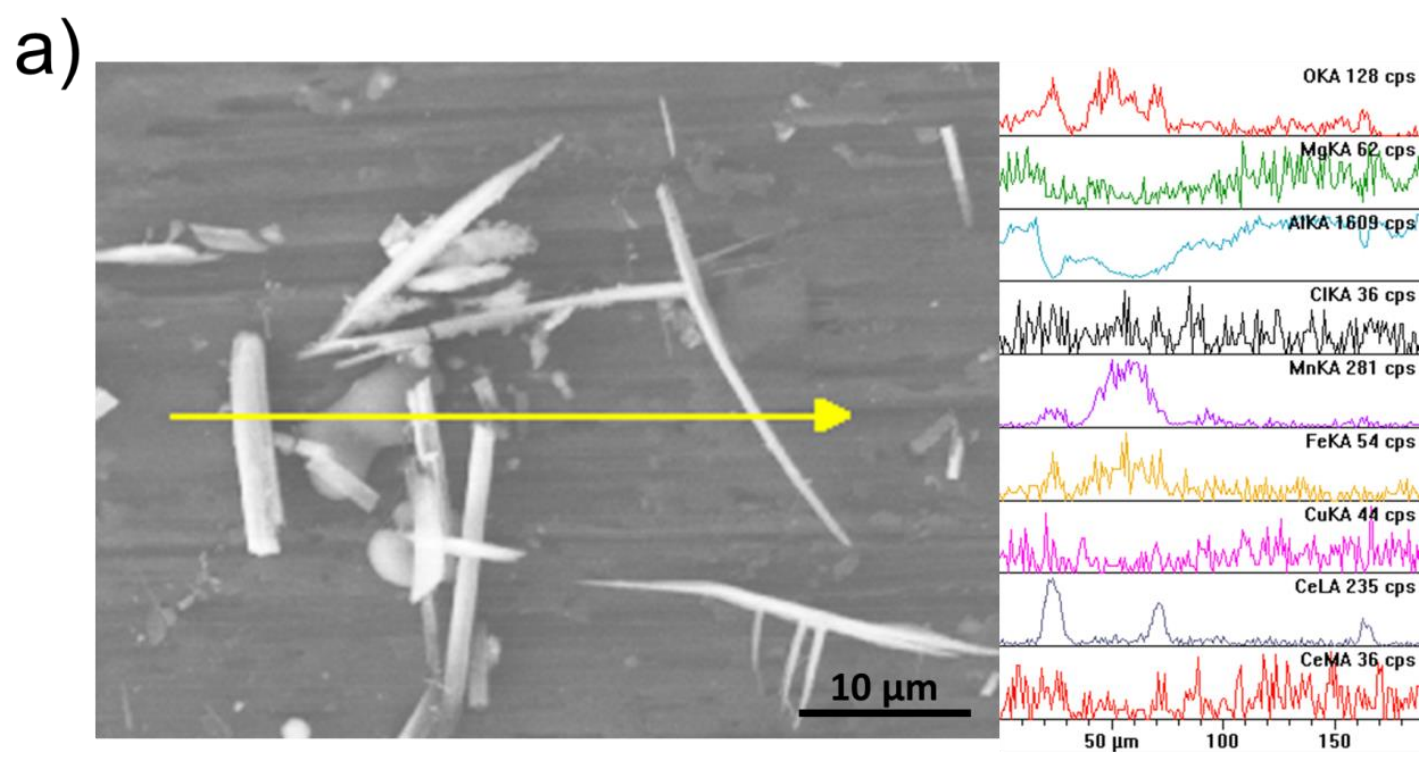

b)

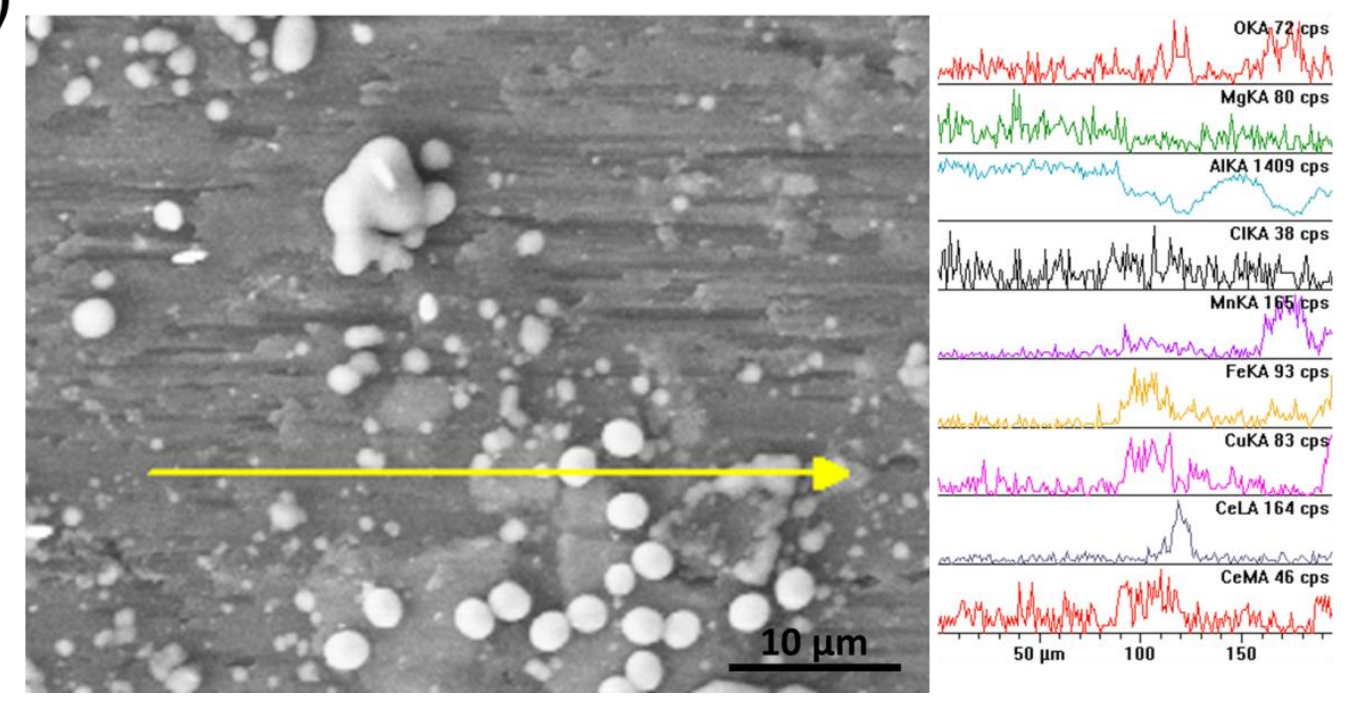

Figure 3.8. EDS at $15 \mathrm{kV}$ accelerating potential of AA2024-T351 pretreated in $10 \mathrm{mM}$ $\mathrm{Ce}^{3+}+40 \mathrm{mM} \mathrm{MnO}_{4}^{-}(\mathrm{a}), 25 \mathrm{mM} \mathrm{Ce}^{3+}+24 \mathrm{mM} \mathrm{MnO}_{4}^{-}$(b), $40 \mathrm{mM} \mathrm{Ce}^{3+}+10 \mathrm{mM} \mathrm{MnO}_{4}^{-}$ . All cerium pretreatments lasted for 100 hours. All permanganate pretreatments lasted for 24 hours. 

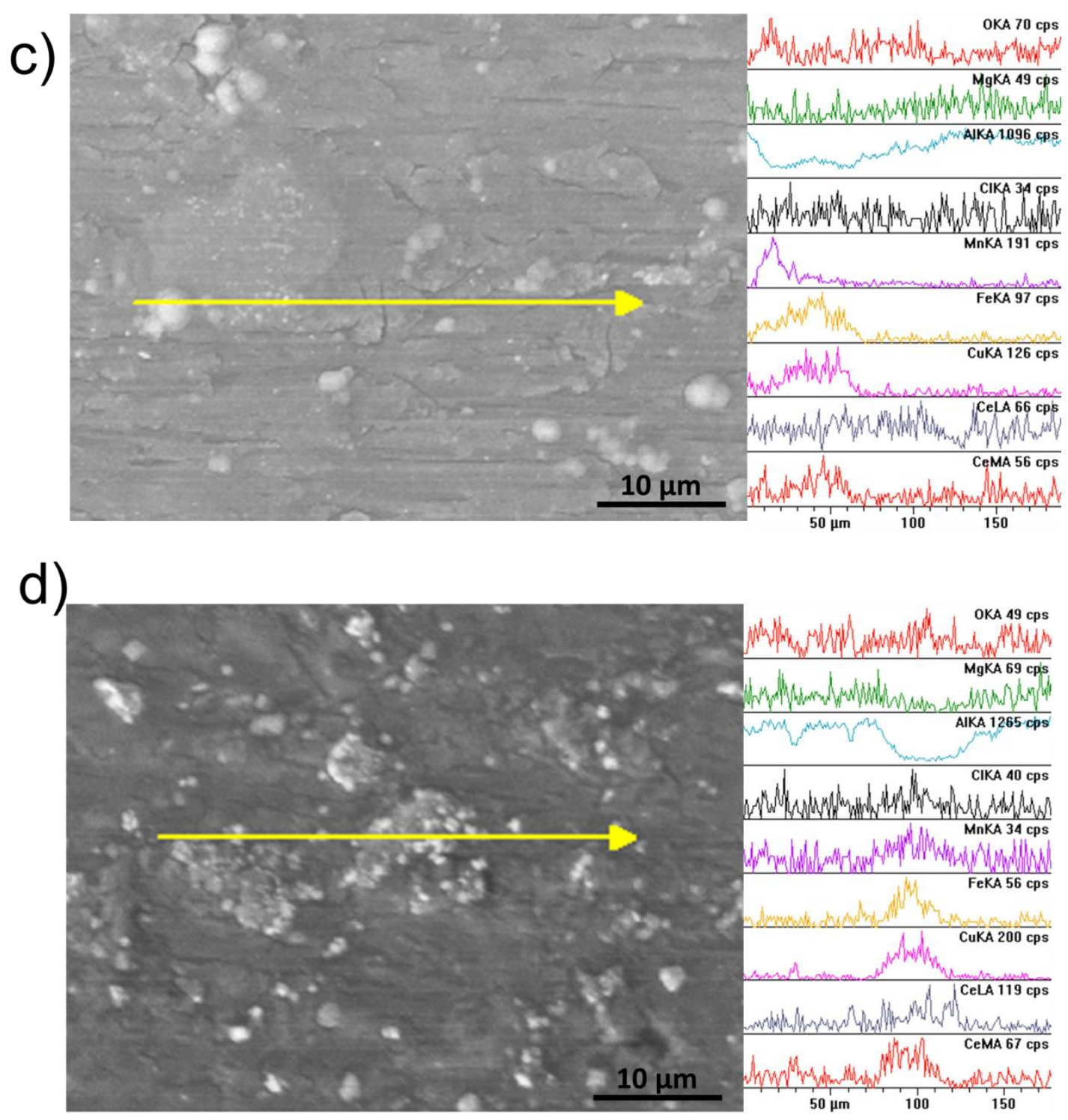

Figure 3.8. EDS at $15 \mathrm{kV}$ accelerating potential of AA2024-T351 pretreated in $40 \mathrm{mM}$ $\mathrm{Ce}^{3+}+10 \mathrm{mM} \mathrm{MnO}_{4}^{-}$(c), and $50 \mathrm{mM} \mathrm{Ce}^{3+}(\mathrm{d})$. All cerium pretreatments lasted for 100 hours. All permanganate pretreatments lasted for 24 hours. 


\section{a)}

b)
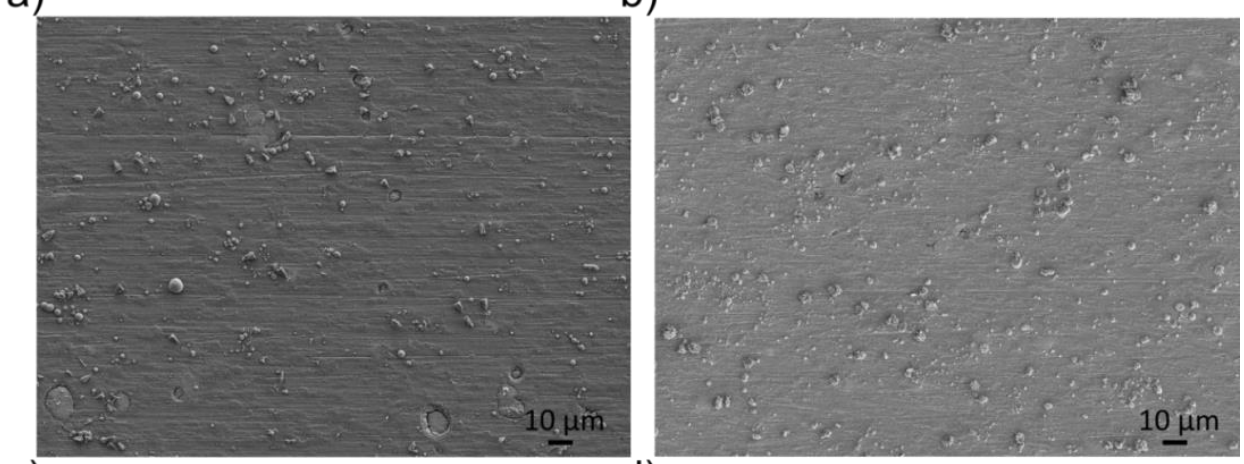

c)

d)
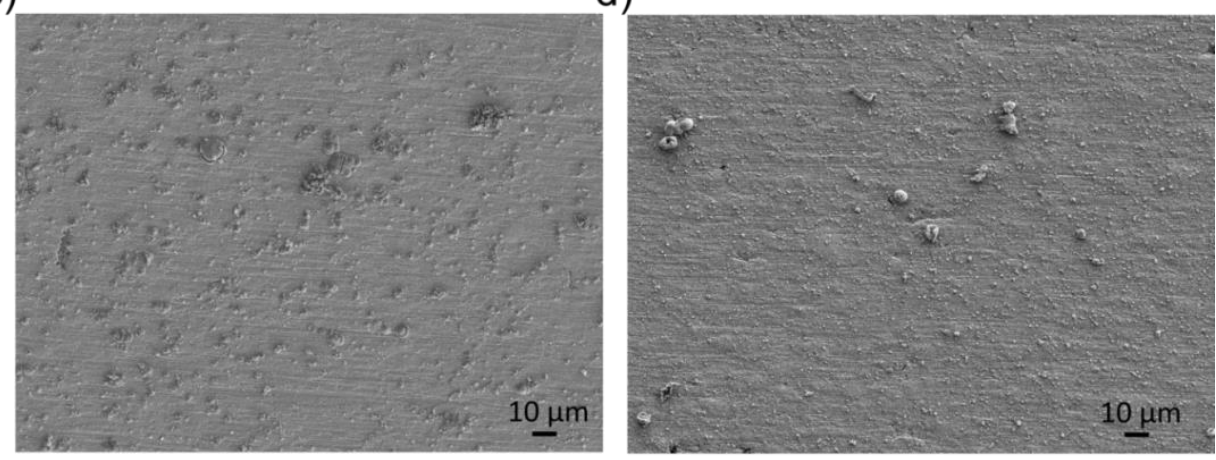

e)

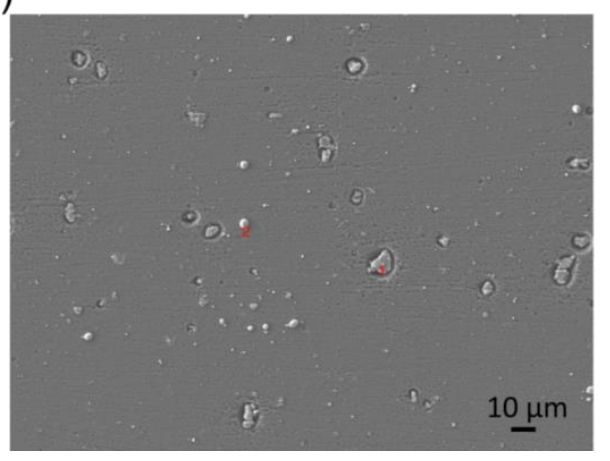

Figure 3.9. (a) AA2024-T351 pretreated with $0.01 \mathrm{M} \mathrm{Ce}^{3+}$ for $100 \mathrm{hrs}$ and $0.04 \mathrm{M} \mathrm{MnO}_{4}{ }^{-}$ for 24 hours and exposed to $0.05 \mathrm{M} \mathrm{Cl}^{-}$for $24 \mathrm{~h}$ (secondary electron image). (b) Same pretreatment with inhibitor concentrations of $0.025 \mathrm{M} \mathrm{Ce}^{3+}$ and $0.025 \mathrm{MnO}_{4}^{-}$and exposed to $0.05 \mathrm{M} \mathrm{Cl}^{-}$for $24 \mathrm{~h}$ (c) Same pretreatment with inhibitor concentrations of $0.04 \mathrm{M} \mathrm{Ce}^{3+}$ and $0.01 \mathrm{MnO}_{4}{ }^{-}$and exposed to $0.05 \mathrm{M} \mathrm{Cl}^{-}$for $24 \mathrm{~h}$. (d) Same pretreatment with inhibitor concentrations of $0.05 \mathrm{M} \mathrm{Ce}^{3+}$ and exposed to $0.05 \mathrm{M} \mathrm{Cl}^{-}$for $24 \mathrm{~h}$.(e) Same pretreatment with inhibitor concentrations of $0.05 \mathrm{MnO}_{4}{ }^{-}$and exposed to $0.05 \mathrm{M} \mathrm{Cl}^{-}$for $24 \mathrm{~h}$. Samples were not cleaned post exposure. 
a)
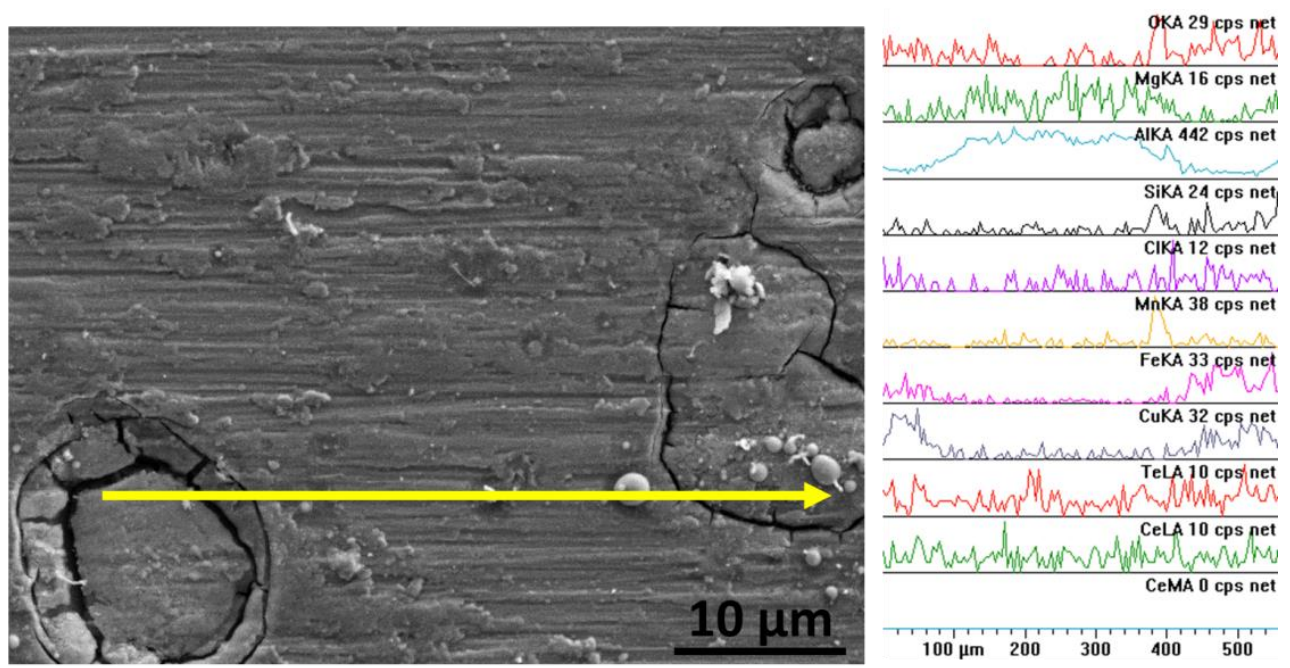

b)
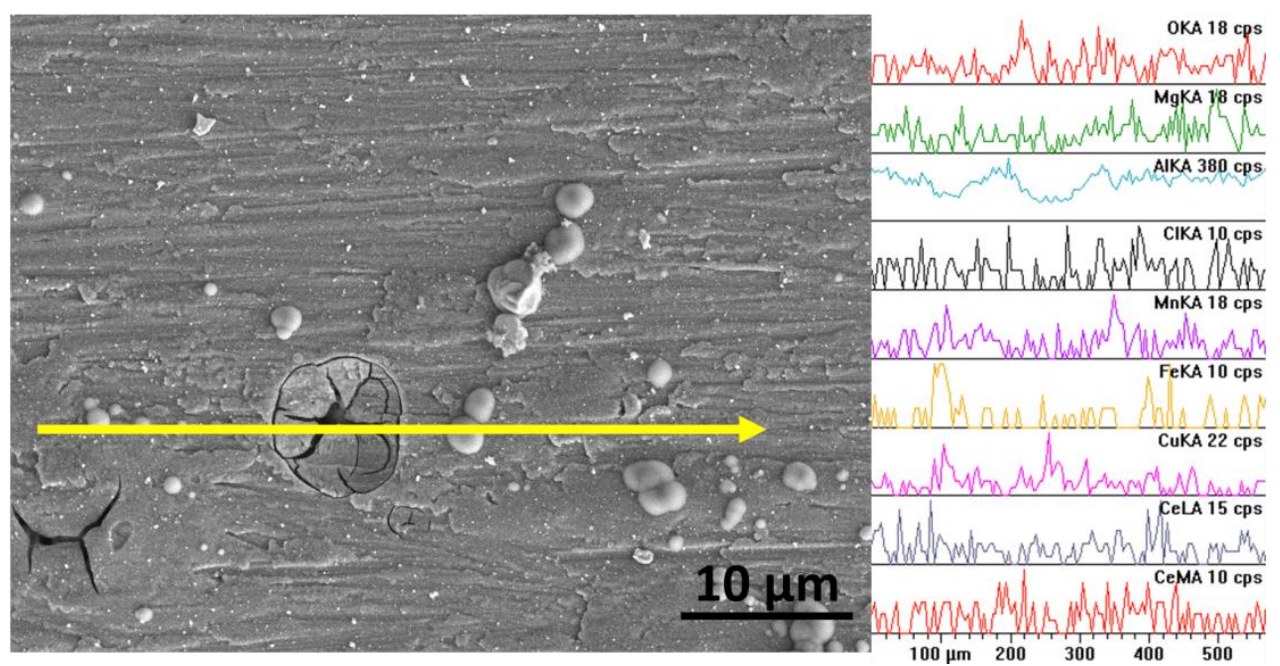

Figure 3.10. EDS at $15 \mathrm{kV}$ accelerating potential of AA2024-T351 exposed for 24 hours in $50 \mathrm{mM} \mathrm{NaCl}$ after pretreatment in $10 \mathrm{mM} \mathrm{Ce} 3++40 \mathrm{mM} \mathrm{MnO} 4-(\mathrm{a}), 25 \mathrm{mM} \mathrm{Ce} 3++$ $24 \mathrm{mM}$ MnO4- (b). All cerium pretreatments lasted for 100 hours. All permanganate pretreatments lasted for 24 hours. 


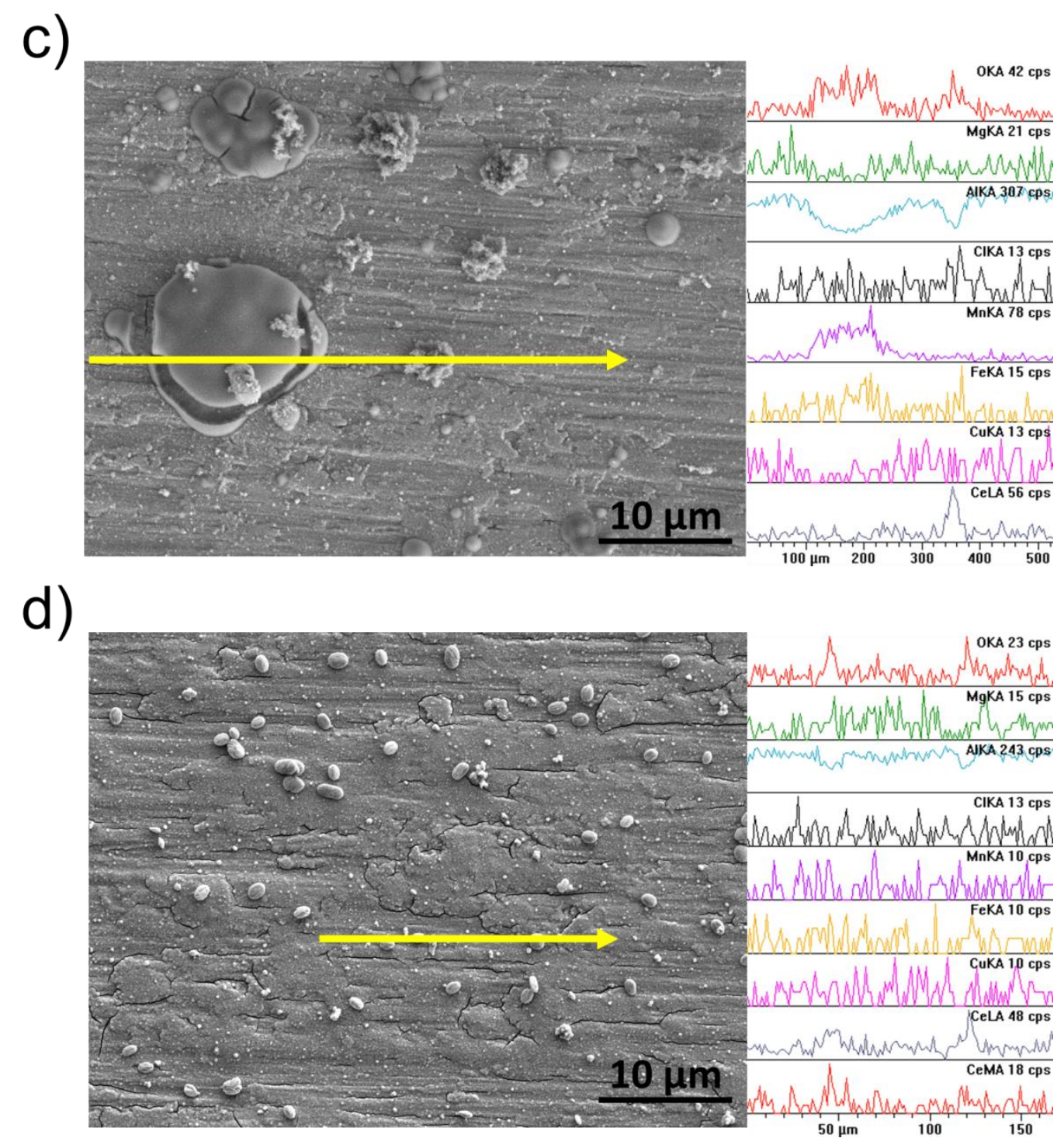

Figure 3.10. EDS at $15 \mathrm{kV}$ accelerating potential of AA2024-T351 exposed for 24 hours in $50 \mathrm{mM} \mathrm{NaCl}$ after pretreatment in $40 \mathrm{mM} \mathrm{Ce}^{3+}+10 \mathrm{mM} \mathrm{MnO}_{4}^{-}(\mathrm{c})$, and $50 \mathrm{mM} \mathrm{Ce}^{3+}$ (d). All cerium pretreatments lasted for 100 hours. All permanganate pretreatments lasted for 24 hours. 


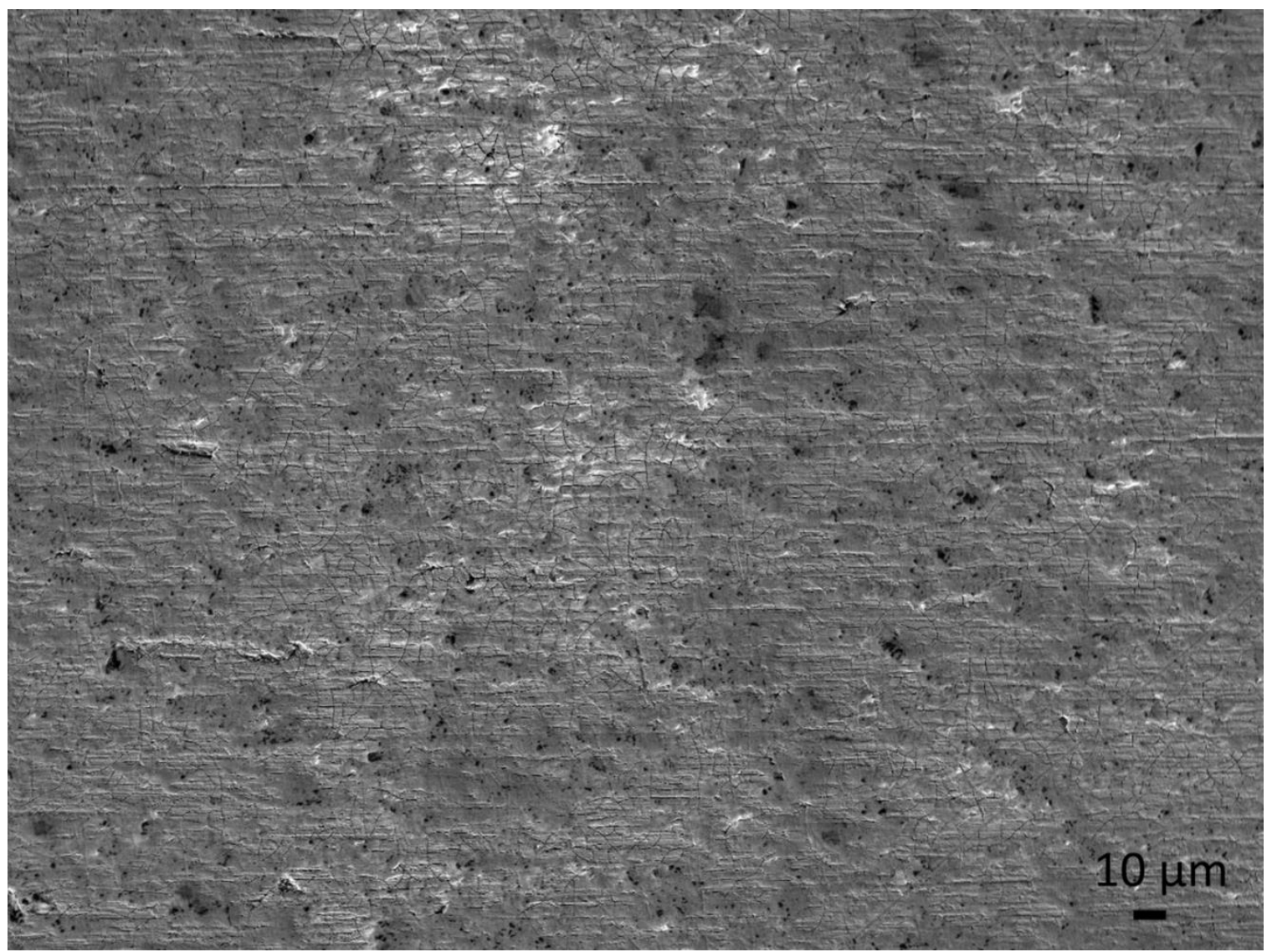

Figure 3.11. Surface damage revealed by abrasive removal of cerium oxide after pretreatment in $0.05 \mathrm{M} \mathrm{Ce}^{3+}$ for $100 \mathrm{~h}$ and subsequent exposure to $0.05 \mathrm{M} \mathrm{NaCl}$ for 24 hours. 


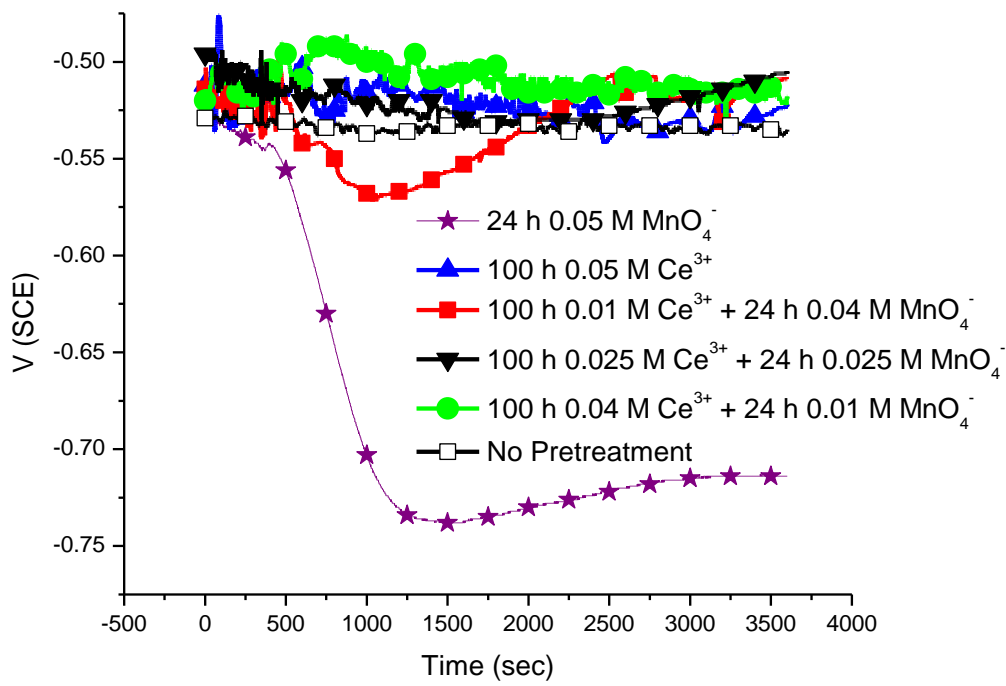

Figure 3.12. Open circuit test of pretreated AA2024-T351 in $50 \mathrm{mM} \mathrm{NaCl}$ solution with ambient aeration. Test included $100 \mathrm{~h}$ pretreatment in $\mathrm{Ce}^{3+}$ followed by $24 \mathrm{~h}$ pretreatment in $\mathrm{MnO}_{4}{ }^{-}$at the indicated concentrations. Pretreatments are $\mathrm{Cl}-$ free.

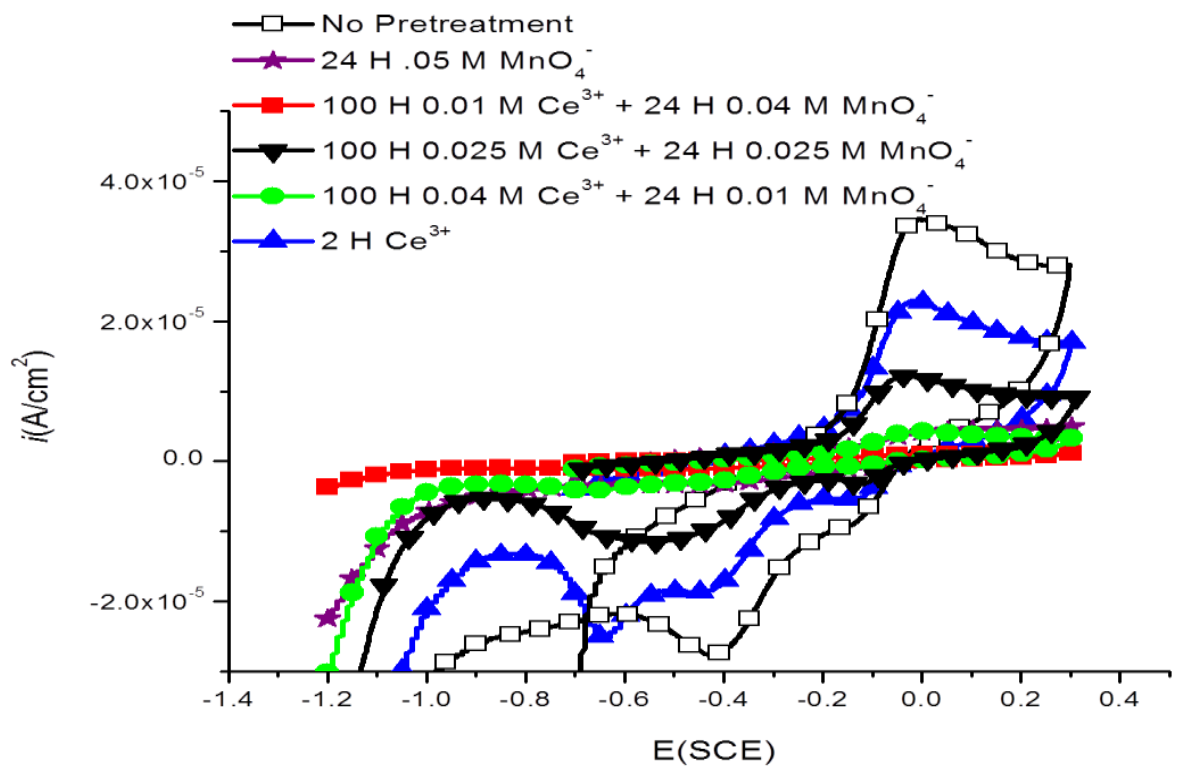

Figure 3.13. CVs of pretreated AA2024-T351 after a 24 hour exposure in $50 \mathrm{mM} \mathrm{NaCl}$ solution. 


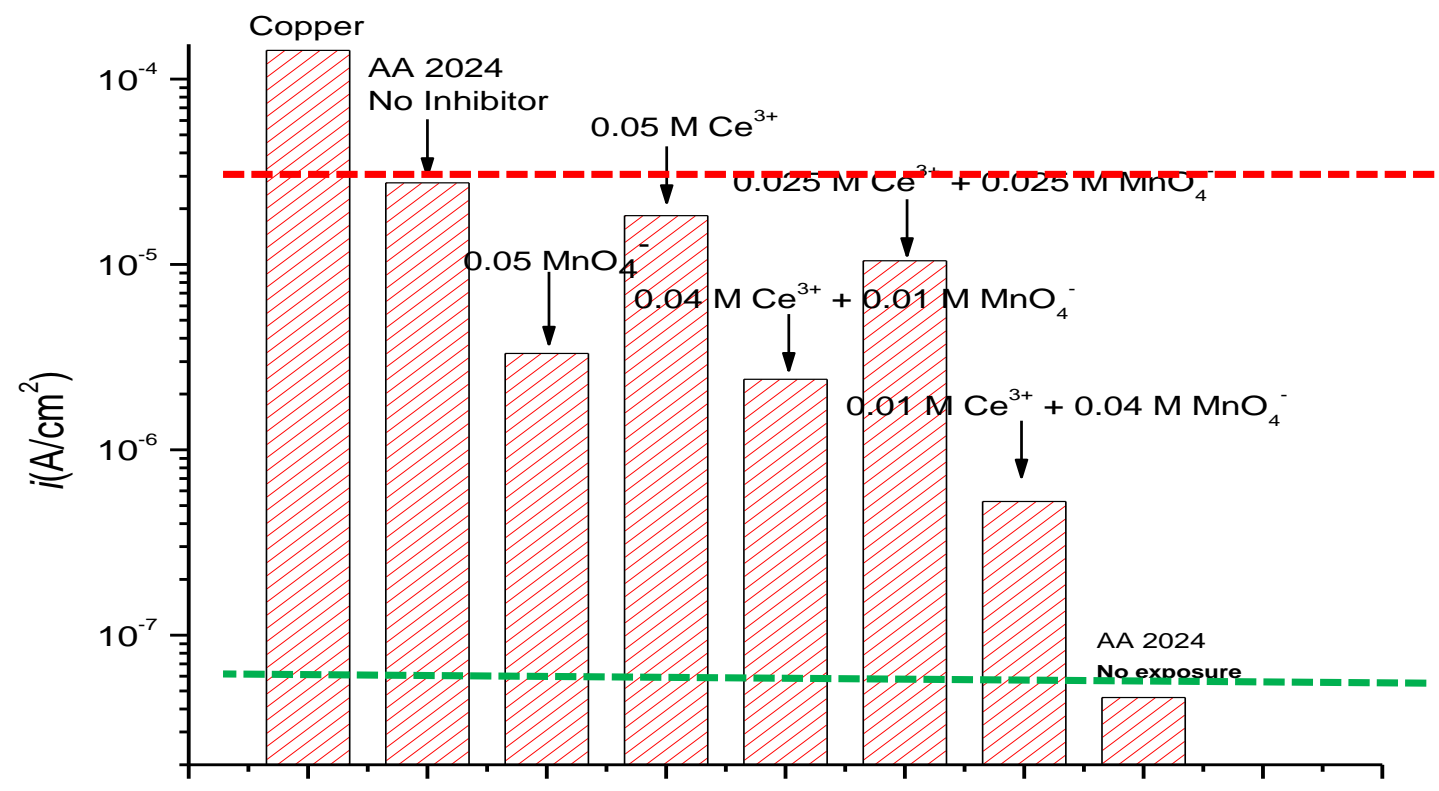

Figure 3.14. The relative intensities of the $\mathrm{Cu}^{1+}$ reduction peak on the AA2024-T351 surface pretreated in cerium for $100 \mathrm{~h}$ and subsequently in permanganate for $24 \mathrm{~h}$ followed by exposure for $24 \mathrm{~h}$ in $0.05 \mathrm{M} \mathrm{NaCl}$ are shown. Combined inhibitor concentrations tested had ratios of cerium to permanganate of 0.2 and 0.8 with a total inhibitor concentration of $0.05 \mathrm{M}$. Results are compared to permanganate pretreatments and cerium pretreatments. A large decrease in the copper surface coverage is evident for the combined inhibitor pretreatment over either single inhibitor pretreatment. 

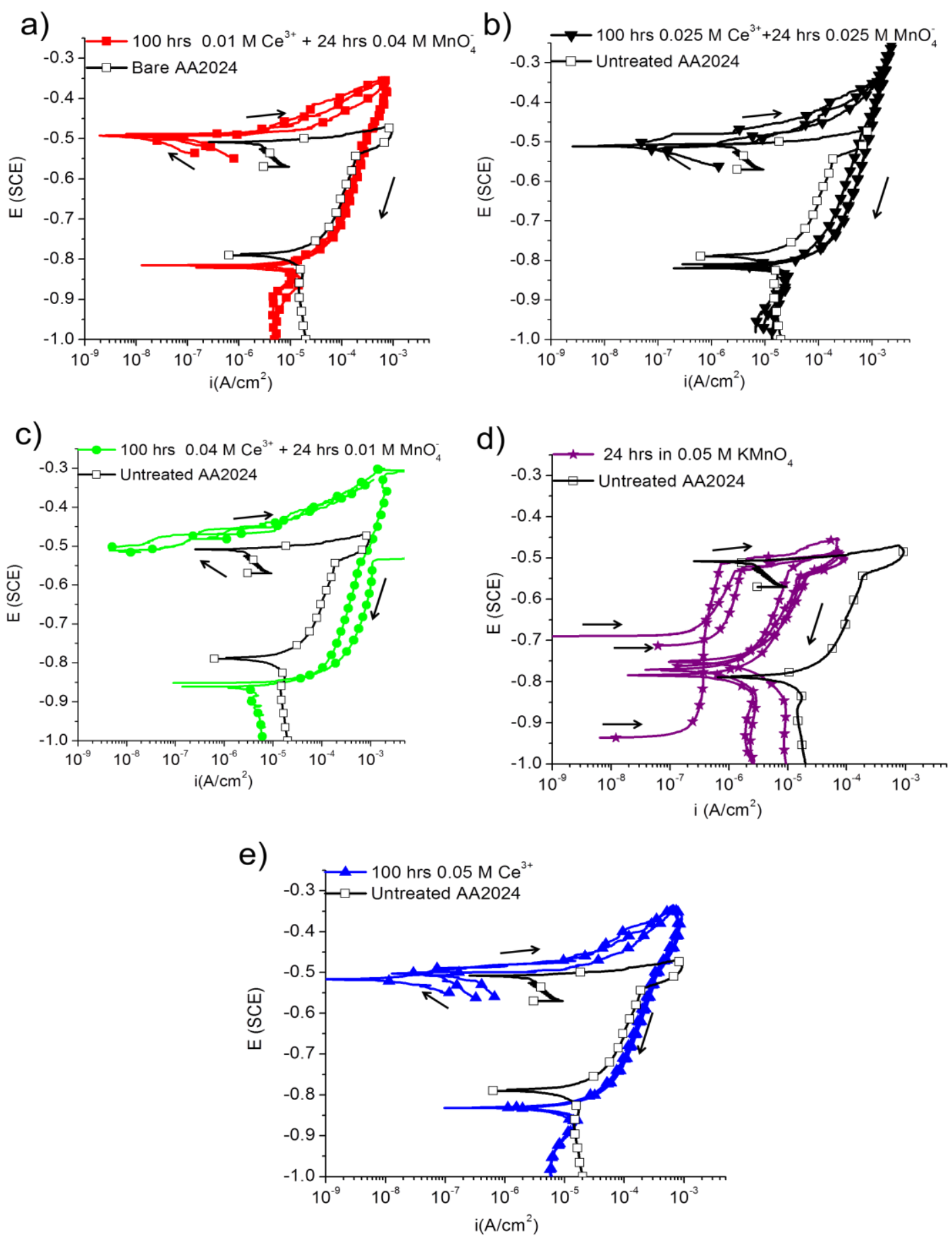

Figure 3.15. Anodic E-logi behavior of AA2024 with cerium-permanganate pretreatments at stated concentrations tested in $5 \mathrm{mM}$ sodium chloride. 


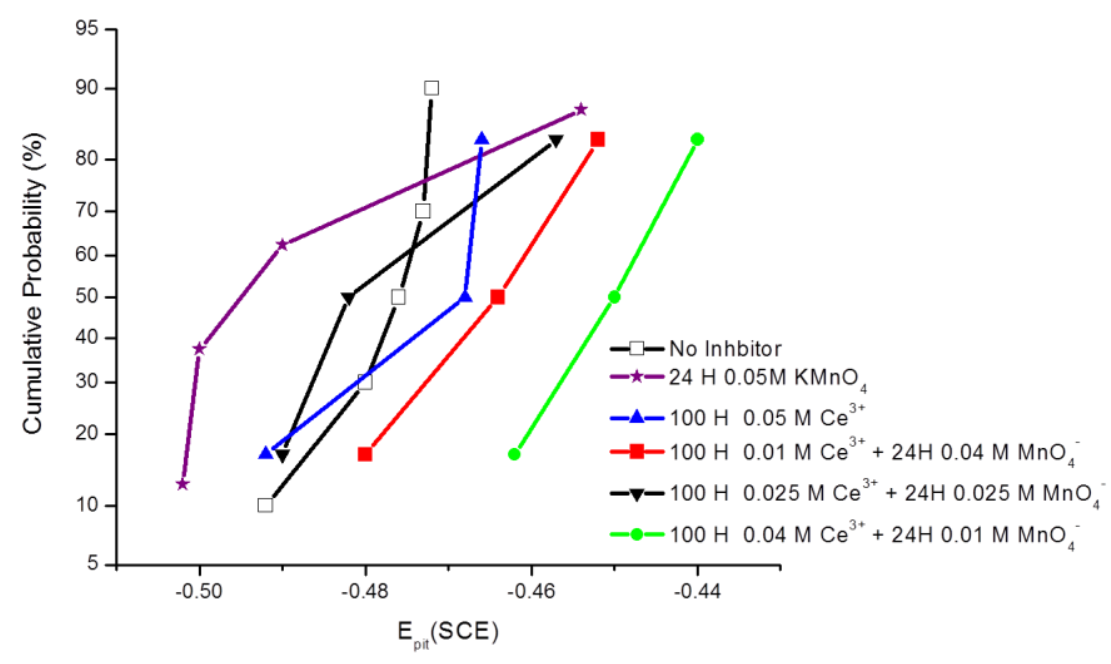

Figure 3.16. Cumulative probability of pitting on AA 2024-T351 as a function of potential for both permanganate- cerium combinations and in-situ solution phase permanganate inhibitor concentration. Combined inhibitor concentrations tested had ratios of cerium to permanganate of $0, .2, .5, .8$, and 1 with a total inhibitor concentration of $0.05 \mathrm{M}$. Cumulative probability of pitting in $0.05 \mathrm{M} \mathrm{NaCl}$ solution. 
a)

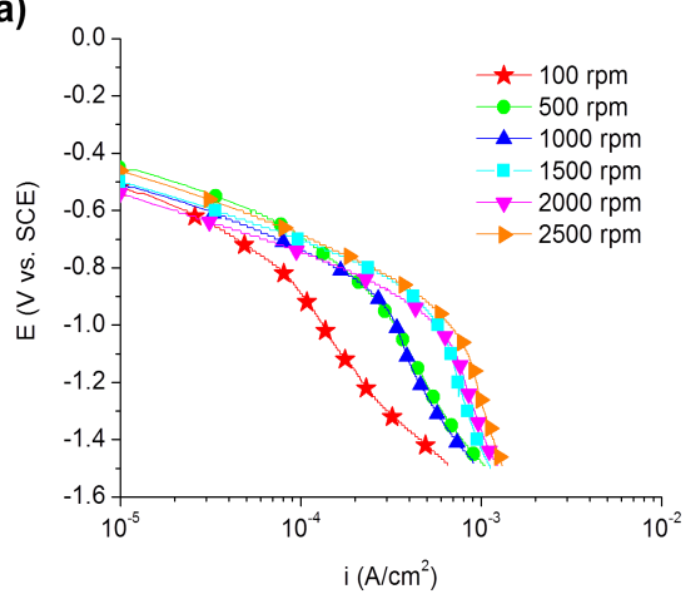

b)

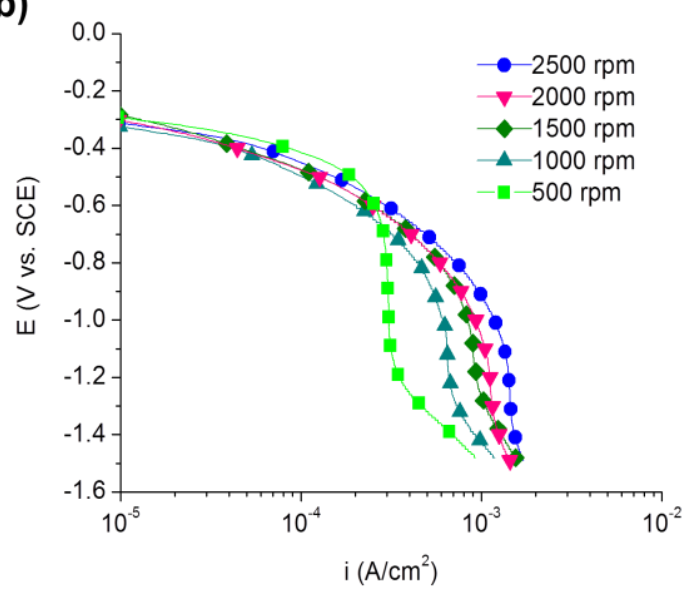

Figure 3.17. Cathodic E vs $\log (i)$ data for AA2024-T4 (a) and high purity copper (b) samples run at various rotation rates from $100 \mathrm{rpm}$ to 2500 in $\mathrm{pH} 8.4$ borate buffer. Solutions were aerated naturally and no pretreatment was performed.

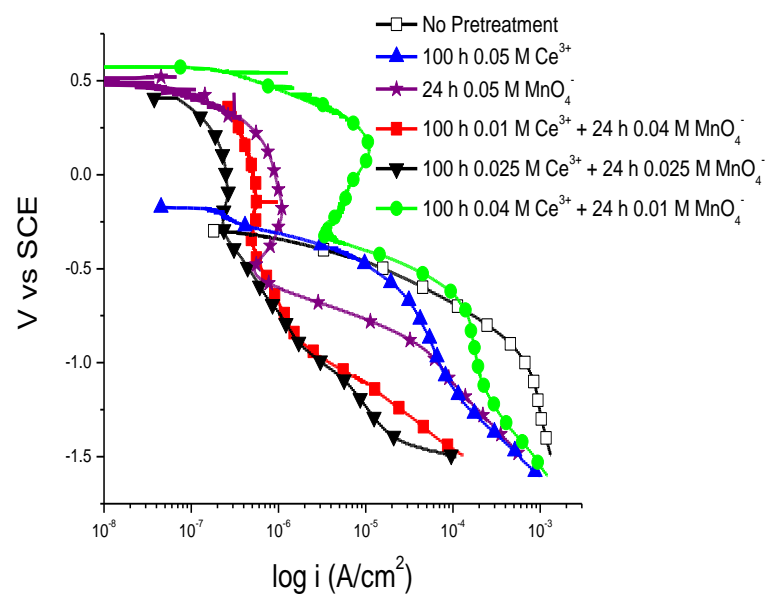

Figure 3.18. Rotating disk cathodic polarizations experiments on AA2024-T351 at a rotation rate of $2500 \mathrm{rpm}$ in $\mathrm{pH} 8.4$ borate buffer. Combined inhibitor concentrations tested had ratios of cerium to permanganate of $0.2,0.5$, and 0.8 with a total inhibitor concentration of $0.05 \mathrm{M}$. Total current densities for bare AA2024, cerium treated, and permanganate treated have been included for reference. 

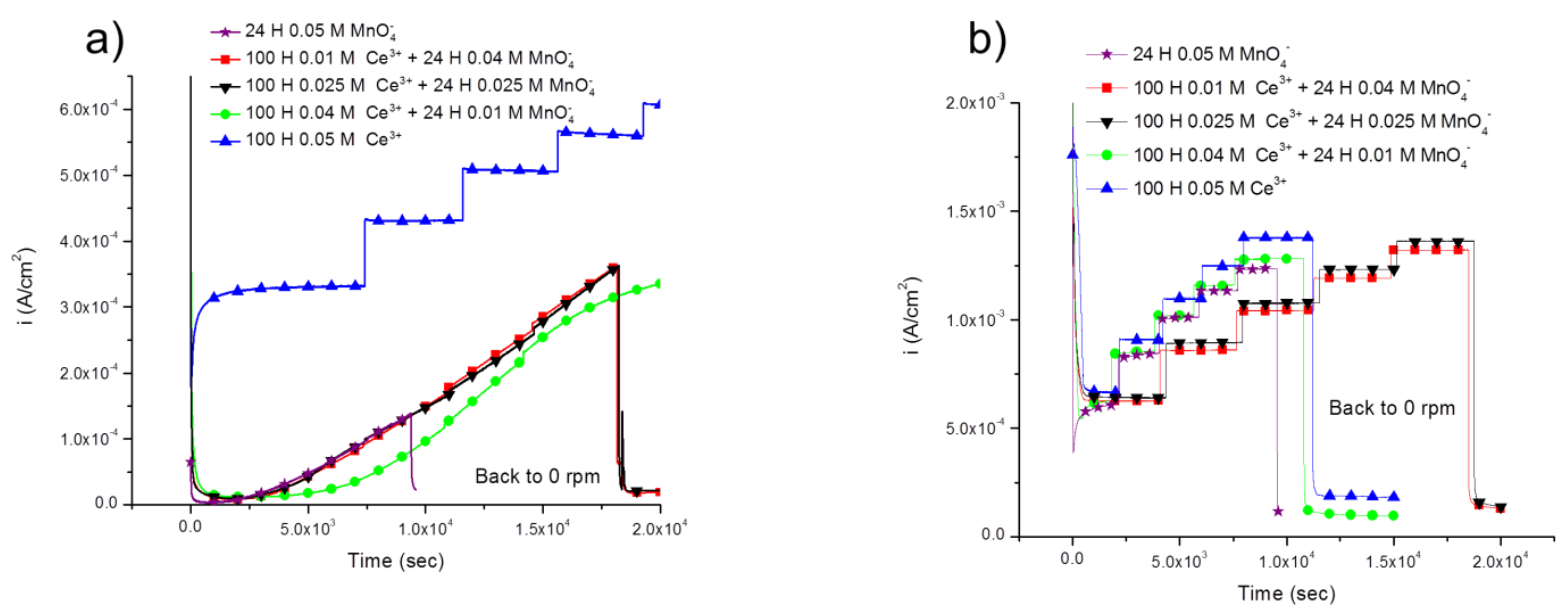

Figure 3.19. (a) RPM Ramp 500 to 2500 (increments of 500) of AA2024-T351 in pH 8.4 borate buffer after stated pretreatments When manganese deposits are present it reacts electrochemically effecting the current density at each rotation rate. (b) RPM Ramp 500 to 2500 (increments of 500) of high purity copper in $\mathrm{pH} 8.4$ borate buffer after stated pretreatments. 
a)

b)

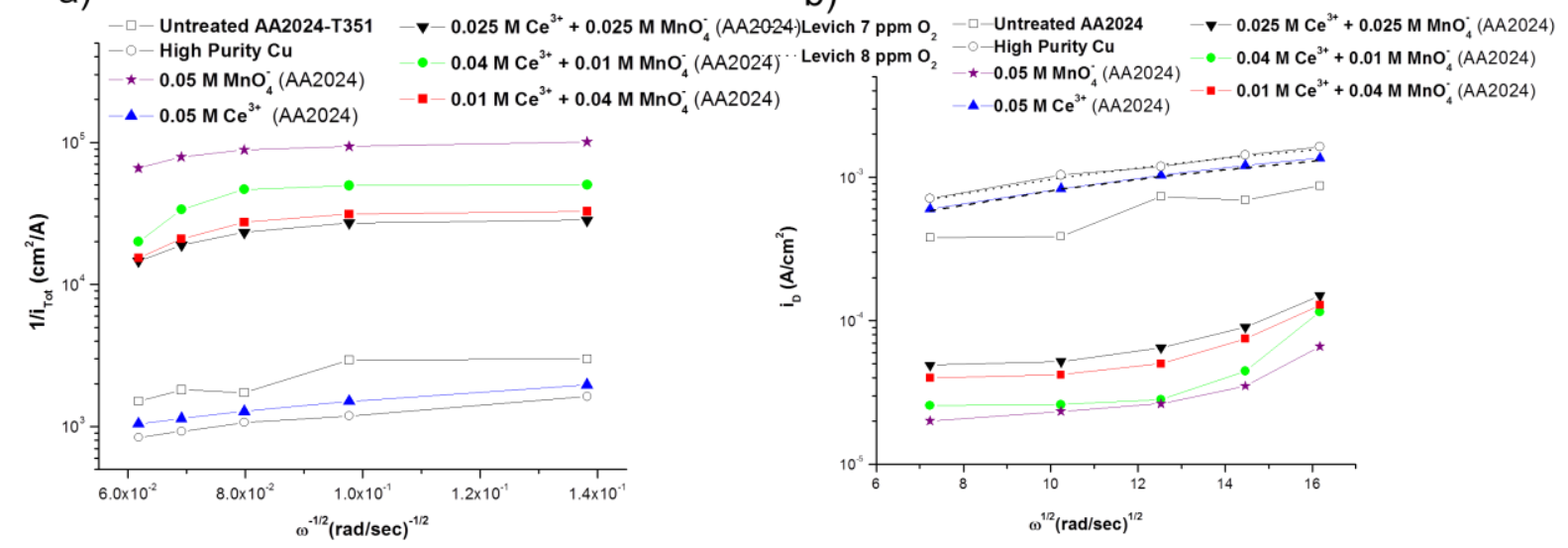

Figure 3.20. Diffusion limited current density on high purity copper compared to AA2024-T351 at rotation rates from 500 to $2500 \mathrm{rpm}$ in $\mathrm{pH} 8.4$ borate buffer. Combined inhibitor concentrations tested had ratios of cerium to permanganate of 4,1 , and 0.2 with a total inhibitor concentration of $0.05 \mathrm{M}$.

a)

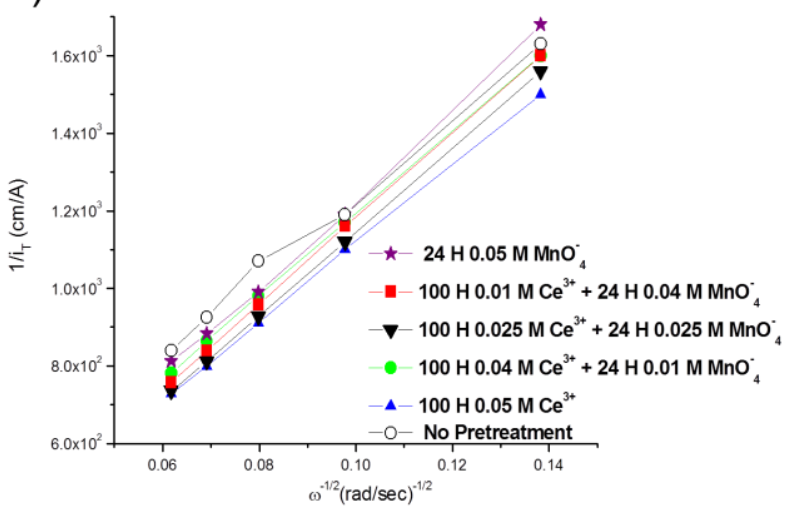

b)

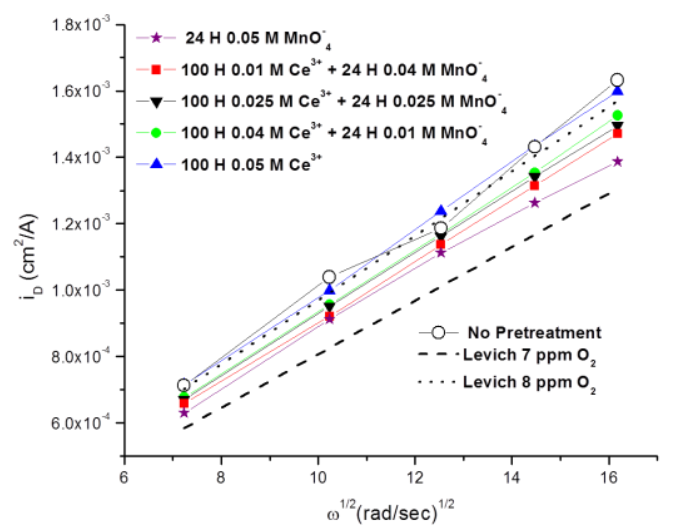

Figure 3.21. Diffusion limited current density on high purity copper at rotation rates from 500 to $2500 \mathrm{rpm}$ in $\mathrm{pH} 8.4$ borate buffer. Combined inhibitor concentrations tested had ratios of cerium to permanganate of 4,1 , and 0.2 with a total inhibitor concentration of $.05 \mathrm{M}$. 


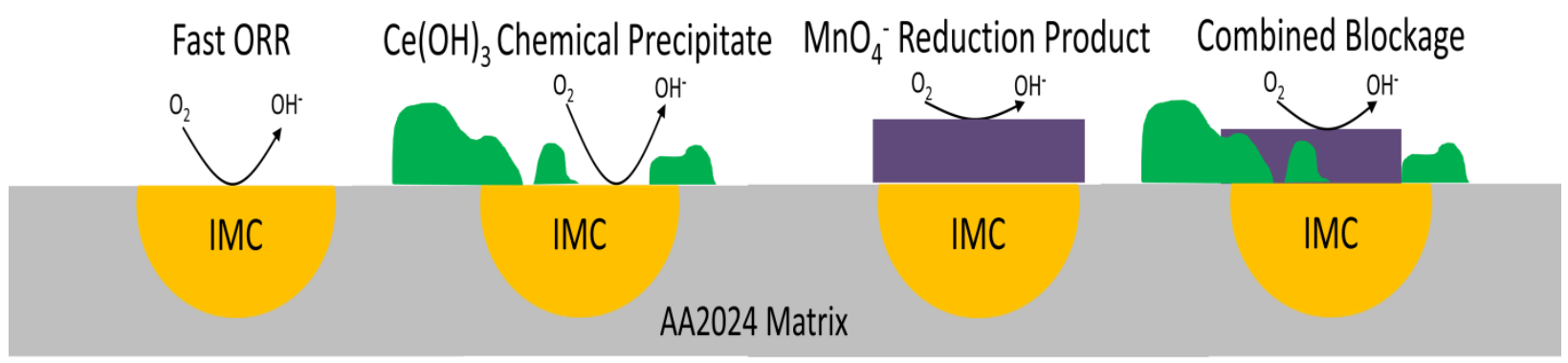

Figure 3.22. Schematic of different cathodic reaction inhibition mechanisms for the reduction of permanganate, the chemical precipitation of cerium hydroxide, and the effect of dual inhibitor pretreatment. 


\section{Effects of Chromate and Molybdate Ions on Scratch Repassivation Behavior of Precipitation Hardened Aluminum Alloys}

\subsection{Abstract}

The scratch depassivation technique was utilized on AA2024-T351, AA7075-T6, and 99.999\% aluminum in molybdate $\left(\mathrm{MoO}_{4}{ }^{2-}\right)$ permanganate $\left(\mathrm{MnO}_{4}^{-}\right)$and chromate $\left(\mathrm{CrO}_{4}{ }^{2-}\right)$ containing $\mathrm{NaCl}$ solutions to examine electrochemical repassivation kinetics over a range of applied potentials and inhibitor concentrations. Current vs time and single frequency impedance measurements were taken in the region of frequency response dominated by surface capacitance. Current decay and high frequency impedance measurements enabled the observation of the effects of inhibitor additions on film formation and oxide growth as protective oxide films formed on the scratched electrode surface. Impedance measurements overcame partial inventory of anodic currents typical of aluminum repassivation. Chromate was found to suppress scratched electrode current transients at high potentials on both AA2024-T351 and AA7075-T6. Capacitance measurements suggested the rapid formation and growth of an oxide at enhanced rates when $\mathrm{CrO}_{4}{ }^{2-}$ was in solution as compared to an uninhibited $\mathrm{NaCl}$ solution. Molybdate did not suppress transient current density in AA2024-T351 or AA7075-T6. Impedance measurements in the presence of molybdate do not indicate the enhanced formation and growth of a passive film. The observed EFCP inhibition due to chromate may be due to enhanced repassivation and limited hydrogen production and uptake. The observed ECFP reduction with $\mathrm{MoO}_{4}{ }^{2-}$ is discussed in light of the observed lack of effect on repassivation kinetics. The buffer capacity of molybdate may 
affect local solution chemistry which in turn may lead to EFCP inhibition. Permanganate was found to suppress the transient current density of AA7075 after scratch depassivation but not AA2024. $\mathrm{MnO}_{4}^{-}$was also found to positively affect barrier impedance for both AA7075 and AA2024. These findings suggest some promise for the inhibition of EFCP with chromate, molybdate, and permanganate albeit by different processes.

\subsection{Introduction}

Crack tip film rupture events are thought to lead to both hydrogen production and anodic dissolution in aerospace aluminum alloy crack tips ${ }^{[1,2]}$. The ability of a stable film to regrow after film rupture is a critical factor because it reduces the production of atomic hydrogen at the crack tip by limiting coupled dissolution, hydrolysis of metal ions, and subsequent hydrogen evolution (HER) ${ }^{[1,2]}$. The rate of hydrogen evolution at the crack tip is exacerbated by the decrease in potential due to IR voltage drop down the length of the crack. If the dissolution current density is reduced, the high hydrogen overpotential brought about by the hydrolysis acidification and IR voltage drop at the crack tip are both lowered $^{[3]}$. HER occurs at faster rates on bare aluminum compared to oxidized aluminum so the growth of a protective oxide barrier is critical to controlling the local environment and hydrogen production rate ${ }^{[4]} \cdot \mathrm{Al}_{2} \mathrm{O}_{3}$ oxide is also a hydrogen permeation barrier which can limit hydrogen ingress ${ }^{[5]}$. The occlusion at the crack tip also leads to increased concentration of $\mathrm{Al}^{3+}$ and $\mathrm{H}^{+}$due to the tortuous diffusion of ionic species down the crack tip where they can be diluted by a bulk solution. In order to maintain electroneutrality at the crack tip, anions migrate into the crack creating high concentrations of $\mathrm{Cl}^{-}$. Anionic inhibitor species present in the bulk solution can also migrate to the crack tip subject to 
lower ionic mobility ${ }^{[3,6]}$. These inhibitors typically will have a lower equivalent conductance $^{[6]}$. An inhibitor can reduce atomic hydrogen production on the surface of the metal by adsorption and assisting in the formation and growth of an oxide permeation barrier or film based competitive adsorption as well as by the reduction in surface reactions such as the oxidation of aluminum and the reduction of $\mathrm{H}^{+[7]}$. This decreases the surface hydrogen concentration, the hydrogen flux, and $C(x, t)$ of embrittling, absorbed atomic hydrogen in the fracture process zone. Here $C(x, t)$ is the concentration of hydrogen as a function of time and position. Once hydrogen is produced and adsorbed it arrives in the fracture process zone by solid state diffusion in response to chemical potential gradient and stress assisted diffusion. Once $\mathrm{C}_{\mathrm{H}}(\mathrm{x}, \mathrm{t})$ is greater than some $\mathrm{C}_{\text {crit }}$ over a critical microstructural length scale a fracture event may occur ${ }^{[8]}$. A crack tip schematic illustrating these processes is shown in Figure 4.1 ${ }^{[3,9]}$.

Understanding inhibitor effects on repassivation is crucial to understanding the effects of inhibitors on cyclic or static load environmental crack growth. Subcritical crack growth including stress corrosion cracking (SCC), intergranular stress corrosion cracking (IGSCC), and corrosion fatigue are important modes of degradation in these alloys all of which do not occur in dry gas environments ${ }^{[10,11]}$. With the introduction of water vapor enhanced subcrititcal crack growth is observed indicting that an aqueous environment, that can be the source of atomic hydrogen from cathodic reduction of water, enables these modes of degradation ${ }^{[11,12]}$. Chloride content in aqueous solutions further increases the rate of subcritical crack growth in $\mathrm{Al}-\mathrm{Cu}-\mathrm{Mg}$ and $\mathrm{Al}-\mathrm{Zn}-\mathrm{Mg}-\mathrm{Cu}$ alloys likely through an increase in anodic dissolution rate coupled to HER ${ }^{[8]}$. These facts indicate that the electrochemical behavior of these alloys at the crack tip in the relevant environments is 
critical to understanding subcritical crack growth. Furthermore, the important phenomenon likely take place at the crack tip due to the predominance of crack tip local hydrogen production and uptake.

Intergranular stress corrosion cracking (IGSCC) is one common mode of degradation in AA7075 and AA2024 that occurs due to susceptible microstructural features along the grain boundaries. There are many indications that cracking is identical in hydrogen producing environments like hot water vapor and humid salty air suggesting that the important aspect of an anionic inhibitor would be to reduce hydrogen production and uptake. Furthermore, bulk hydrogen uptake from all external surfaces is likely improbable due to the open circuit of these materials being higher than the reversible potential for hydrogen and the presence of intact oxide layers which act as hydrogen permeation barriers. This points towards the importance of the processes at the crack tip in IGSCC. The features unique to the grain boundaries include the higher likelihood of heterogeneously nucleated precipitates rich in copper along the grain boundary regions and the accompanying solute depletion in the surrounding matrix. This microstructural circumstance leads to a strong galvanic couple between the particles and the solute lean matrix leading to the selective dissolution of these regions and the accompanying increase in hydrogen production.

Environmental fatigue crack propagation (EFCP) is another important mode of degradation in these alloy systems ${ }^{[11,13]}$. However, unlike IGSCC, this mode of cracking tends to occur with no relationship to high angle grain boundary direction and actually tends to follow an intersubgranular or transgranular path ${ }^{[8,11,13]}$. There is a body of research on the effects of several inhibitors on the EFCP of AA7075 ${ }^{[8,14]}$. The key results of the cited works as they 
relate to ionic inhibition of EFCP can be found in Figures 4.2 and 4.3. The increase in $d a / d N$ over vacuum levels in full immersion sodium chloride environments is attributed to the effects of crack tip hydrogen production and ingress leading to hydrogen embrittlement (HE) on the fracture process zone. In order for this mechanism to be feasible hydrogen from the crack environment be produced and absorbed, and diffuse the critical distance, $x_{c r i t}$, over a period of $1 / f$, where $f$ is the loading frequency, to reduce the local cohesive strength $^{[8]}$. The significance of $1 / f$ is that it is presumed to be the time period of facile $\mathrm{H}$ production and entry ${ }^{[8]}$. In order for cracking to advance, the hydrogen concentration over this zone must be above a critical concentration at which the cohesive strength of the material is reduced sufficiently ${ }^{[8]}$. An ionic inhibitor capable of lowering the surface concentration of hydrogen $\left(\mathrm{C}_{\mathrm{s}}\right)$ either directly or indirectly, would effectively decrease the $C(x, t)$ function which enables the hydrogen to reach the critical concentration over $x_{c r i t}$, through diffusion. An effective inhibitor could also affect the anodic repassivation speed and lower the total charge of dissolution which is coupled to hydrogen production, reducing the time available for hydrogen production and uptake to occur with a fixed $\mathrm{C}_{\mathrm{s}}$. This first process has been modeled using the one dimensional semi-infinite diffusion, Equation 4.1

$$
C(x, t)=C_{S}\left(1-\operatorname{erf}\left(\frac{x}{2 \sqrt{D_{H, e f f} * t}}\right)\right)
$$

and is shown in Figure 4.4, where $C_{s}$ was varied from 0.5 to 0.1 hydrogen atoms per solvent atom, $D_{H, e f f}$ was $2.3 \times 10^{-7} \mathrm{~cm}^{2} / \mathrm{s}^{[15,16]}$, and $t$ was 0.1 seconds. Lowering $\mathrm{C}_{\mathrm{s}}$ or increasing the repassivation rate, which is inversely proportional to $t$, would lead to inability to satisfy $C_{c r i t}$ over $x_{c r i t}$ leading to smaller crack growth increments and lowering $d a / d N$. Thus, it is the goal of the remaining chapters to explore the repassivation process in some detail with 
and without selected ionic inhibitors in simulated crack tip environments to explore the effect of ionic inhibitors on processes that would ultimately effect crack tip hydrogen concentrations.

The growth of oxides at the crack tip has been rationalized to be responsible for the frequency dependence of fatigue crack growth rate, da/dN, in the low frequency regime by Gasem and Warner ${ }^{[17,18]}$. The critical frequency upper bound $\left(f_{C r i t}\right)$ has been thought to indicate the frequency at which an oxide barrier is disrupted faster than it can repassivate or regrow to prevent $\mathrm{Al}^{3+}$ dissolution and/or the absorption of $\mathrm{H}_{\text {ads }}$ into the lattice. At low to moderate frequencies and up to the critical frequency upper bound, $f_{C r i t U B}$, inhibitors such as chromate and molybdate have been shown to reduce da/dN to levels below those seen in moist air and/or full immersion chloride containing environments ${ }^{[17,18]}$.

To understand how and why molybdate and chromate can act as effective inhibitors of environmental fatigue crack propagation (EFCP) at both the molecular and the electrochemical level, a brief overview of their thermodynamics is required. Molybdate and chromate exist as high valence state oxoanions in aqueous solutions ${ }^{[19]}$. One mechanism, through which they inhibit corrosion and EFCP more specifically, is by being reduced from a soluble high valence state anion to lower valence state oxide with lower solubility, creating an oxide barrier that protects the underlying aluminum ${ }^{[20-22]}$. Another is through the competitive adsorption of these unreduced ionic inhibitors with chloride ${ }^{[7]}$. The adsorption of anionic inhibitor species has a complex relationship with the potential of zero charge (EPZC $)$ of the surface on which adsorption occurs ${ }^{[7]}$. For aluminum in a 0.01 $\mathrm{M} \mathrm{Cl}^{-}$solution the potential of zero charge is reported to be $-0.761 \mathrm{~V}(\mathrm{SCE})^{[7]}$. At potentials higher that EPZC, anions will be adsorbed to metal surface. At potentials below EPZC, cations 
will be adsorbed. When the potential favors anion adsorption a competitive adsorption of anions at the metal surface can occur such that a compound such as $\mathrm{MoO}_{4}{ }^{2-}$ and $\mathrm{Cl}^{-}$would both compete and occupy adsorption sites on the metal surface. This would effectively reduce the concentration of the aggressive chloride species and presumably decrease the rate of dissolution. The E-pH behavior of chromate is shown in Figure 4.5. Chromate can be reduced to a $\mathrm{Cr}^{3+}$ oxide that is stable over a much wider range of potential and $\mathrm{pH}$ in comparison to aluminum oxide. Aluminum oxide is only stable in a narrow band while $\mathrm{Cr}_{2} \mathrm{O}_{3}$ is stable at $-0.8 \mathrm{~V}_{\mathrm{SCE}}$ from $\mathrm{pH} 4$ to 14 as shown in the speciation diagram in Figure 4.5(b). Figure 4.6 a shows molybdenum's E-pH behavior with its stable $\mathrm{Mo}^{4+}$ oxide over all $\mathrm{pH}$ values. At $-0.8 \mathrm{~V}_{\mathrm{SCE}}$ the oxide is stable from a $\mathrm{pH}$ of 4 to 12 as shown in Figure 4.5(b). At low $\mathrm{pH}$, molybdenum oxides exhibit a region of thermodynamic stability that extends to high potentials. The stability of the inhibitor oxides may be expected to have a significant effect on the repassivation of the alloy surface. This type of information gives a preliminary indication of the E-pH range of oxide stability. In summary, the function of anionic inhibitors at crack tips must be investigated using methods which allow some of the factors discussed above to be examined.

\subsubsection{Methods to Study Repassivation}

One method used to examine repassivation kinetics in the past has been mechanical depassivation of metal surfaces. There are many methods that have been used to examine repassivation behavior including fractured thin film ${ }^{[23-25]}$, fractured tensile bar $^{[26]}$, laser depassivation $^{[27]}$, guillotined wire ${ }^{[28]}$, continuous abrasion ${ }^{[29]}$, and scratch depassivation ${ }^{[30-}$ 
32]. Electrochemical net current is collected often at fast data acquisition rates. Scratch testing has been demonstrated in the literature to be useful in determining rates of metal dissolution, proton reduction, pitting potential, and oxide growth on many substrates including aluminum and aluminum alloys ${ }^{[33-37]}$, titanium ${ }^{[38]}$, and steels ${ }^{[32,39,40]}$. Many of the complications that arise with the use of the scratch depassivation method such as capacitive current contribution $^{[41,42]}$, stylus contact effects ${ }^{[23]}$, and maximum current discrepancies ${ }^{[43]}$ have been addressed in detail leading to a number of considerations taken into account during the development of the testing method.

Scratch depassivation has been used extensively in the study of repassivation kinetics. Ford and Burstein examined the repassivation of an Al-Mg alloy with 7 weight percent $\mathrm{Mg}^{[33]}$. They found that the rate of dissolution of the alloy and hydrogen evolution reaction were increased by several orders of magnitude on electrodes from which the oxide barrier had been removed. The found that the dissolution kinetics of the aluminum alloy followed Tafel's law independent of $\mathrm{pH}$ and chloride with a Tafel slope, $\mathrm{b}_{\mathrm{a}}$, of $118 \mathrm{mV}$ between 1.8 and $-1.4 \mathrm{~V}$ (SCE). They concluded that the bare surface reaction rate was determined by the single electron transfer steps as follows

$$
\mathrm{Al}+\mathrm{H}_{2} \mathrm{O} \rightarrow \mathrm{AlOH}_{\text {ads }}+\mathrm{H}^{+}+e^{-}
$$

due to the independence of the rate on either chloride or $\mathrm{pH}$. They also found that the oxide reforms quickly on the alloy in chloride solutions causing the current to decay as

$$
i_{\text {anodic }}(t)=i_{\text {anodic } \max } \exp \left(-a t^{n}\right)
$$

where $n=1$ initially but changes to 0.5 after some time where the change is a function of both chloride and potential. They explain the change in current decay by rationalizing a 
change in the oxide growth characteristics. When $n=1$ they hypothesize a relatively defect free oxide monolayer stage of growth followed by a thickening of the oxide and the incorporation of defects and a change to $n=0.5$. They found a typical anodic maximum current density $\left(i_{a m}\right)$ of $1 \mathrm{~A} / \mathrm{cm}^{2}$ for a scratched $\mathrm{Al}-\mathrm{Mg}$ alloy in $1 \mathrm{M} \mathrm{NaCl}$ with a $\mathrm{pH}$ of 6 when held potentiostatically at $-0.9 \mathrm{~V}(\mathrm{SCE})$.

\subsubsection{Repassivation Studies on Aluminum with Ionic Inhibiting Species}

Rudd and J.C. Scully ${ }^{[34]}$ conducted scratched electrode experiments on high purity aluminum in the presence of several inhibiting species. The inhibitors that they examined included nitrate, phosphate, citrate, tartrate, benzoate, and acetate in $0.085 \mathrm{M}$ sodium chloride solution. Scratch current transients were modeled with a similar relationship to the one reported by Ford and Burstein ${ }^{[33]}$ in Equation 4.2. No variation in $\mathrm{n}$ was seen. $n$ was equal to one throughout their analysis. The constant $a$ (reported as $\beta$ in their manuscript) was used to rank inhibitors and found that the trend correlated with the trend observed in pitting potential in the presence of inhibitors. The dependence of $\beta$ on potential in the reported solutions can be seen in Figure 4.7. The most effective inhibitor was nitrate which had a much larger (and unreported) $\beta$ followed by phosphate with acetate being the least effective. At $-0.6 \mathrm{~V}$ (SCE) they report values of $\beta$ for phosphate and acetate of $1.1 \times 10^{5}$ and $4.6 \times 10^{3}$ respectively. They suggest that dependence of $\beta$ on inhibitor species exists due to the production of different complex-forming, blocking and oxidizing inhibitor effects on repassivation kinetics. They observed pit growth at high potentials in the scratch wake. 
They did not explore the relationship between repassivation kinetics and EAC, nor did they examine inhibitors relevant to the EFCP literature.

Devicharan and Clayton ${ }^{[37]}$, Schmutz and Frankle ${ }^{[36]}$, and Cinderey and Burstein ${ }^{[35]}$ all explored the effect of chromate on scratch repassivation of aluminum. Devicharan and Clive conducted one scratch test after an exposure to hexavalent chromate to observe migration of hexavalent chrome from the surrounding oxide into the freshly depassivated scratch surface. They observed the OCP during the scratch; however, no control was conducted without chromate so the test provides very little insight. They claim chromate was capable of migrating into the scratch based on secondary ion mass spectroscopy (SIMS) and synchrotron infrared spectroscopy (SIRMS) mapping. However, the evidence provided is very weak and no electrochemical information of any merit is provided. Schmutz and Frankel examined the effect of chromate on the process of rastering the tip of an AFM across the surface of the alloy in a contact/scratching mode ${ }^{[36]}$. They found that in the absence of chromate rastering of the tip resulted in local dissolution of the underlying alloy. With the addition of small concentrations of chromate the corrosion morphology changed to localized corrosion in the rastering zone which they attributed to an increase in cathodic reactant, either chromate or oxygen. In the presence of higher concentrations of chromate, above $5 \mathrm{mM}$, they found that the surface was impervious to the previously aggressive chloride containing solution, suggesting the formation of a more protective oxide layer in the presence of the chromate anion. This included the protection of the Al$\mathrm{Cu}-\mathrm{Mg}$ constituent particles. Several anodic polarization scans were conducted during the rastering procedure showing the effect of cell geometry factors; however, no transient repassivation data was collected during scratching. 
Cinderey and Burstein ${ }^{[35]}$ conducted scratch repassivation of high purity aluminum in chloride and chromate solutions using the guillotined electrode technique. They examined high purity aluminum guillotined in $0.1 \mathrm{M} \mathrm{NaCl}$ with $0.01 \mathrm{M}$ or $1 \mathrm{M}$ chromate added to the aqueous solutions. They monitored the open circuit potential of the electrode as repassivation occurred after guillotine and noted a moderation of the maximum negative potential observed after depassivation when chromate anions were present in solution. This is significant because of the decrease in hydrogen evolution overpotential with the addition of chromate. Their results can be found in Figure $4.8^{[35]}$. For $0.01 \mathrm{M} \mathrm{Na}_{2} \mathrm{CrO}_{4}$ and $1.0 \mathrm{M}$ $\mathrm{Na}_{2} \mathrm{CrO}_{4}$ in $0.1 \mathrm{M} \mathrm{NaCl}$ solution (unbuffered, $\mathrm{pH}$ not reported) they found a minimum potential $\left(\mathrm{E}_{\min }\right)$ of $-1.7 \mathrm{~V}$ and $-1.5 \mathrm{~V}$ (SCE), respectively, as compared with $-1.8 \mathrm{~V}$ (SCE) in $1.0 \mathrm{M} \mathrm{NaCl}$ without chromate addition. They attributed this to enhanced repassivation in the presence of chromate, noting that the cathodic reduction of chromate was responsible for a change in the observed slope of the $\mathrm{E} / \log t$ behavior. The slope $(\partial \mathrm{E} / \partial \log t)$ of the potential transient for $0.01 \mathrm{M} \mathrm{Na}_{2} \mathrm{CrO}_{4}$ was $0.076 \mathrm{~V} /$ decade for $\mathrm{t}<0.5$ seconds and 0.129 V/decade for $\mathrm{t}>0.5$ to $\mathrm{t}=125$ second. For and $1.0 \mathrm{M} \mathrm{Na}_{2} \mathrm{CrO}_{4} \partial \mathrm{E} / \partial \log t$ was 0.048 $\mathrm{V} /$ decade for $\mathrm{t}<12$ seconds. They also note that the potential transient takes on a curved nature that they attribute to the cathodic reduction of the aqueous chromate species. This can be seen in lines $b$ and $c$ of Figure 4.8.

Kolman and Scully performed scratch tests on high purity titanium ${ }^{[30]}$. They modelled current decay with a logarithmic expression shown in Equation 4.3 where $i$ is current density, $i_{o}$ is peak bare surface current density, $t$ is time elapsed for $t>t_{o}$ or $t=t_{o}$ for $t<t_{o}$

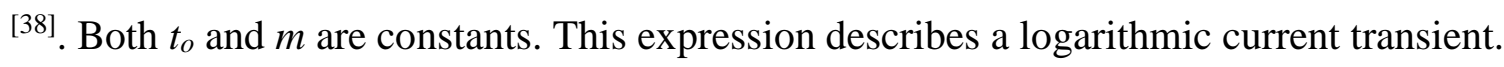




$$
i=i_{o}\left(\frac{t}{t_{o}}\right)^{-m}
$$

This expression differs from the exponential decay found by Ford and Burnstein ${ }^{[33]}$ which was reviewed in the introduction and shown in Equation 4.2. The expression in Equation 4.3 was observed by Kolman and Scully in high purity titanium scratch repassivation experiments ${ }^{[30]}$. Kolman and Scully also observed the current density plateau at short times ${ }^{[30]}$. They found that the onset of current density plateau decay was related to the depassivation speed.

There is a very limited body of research addressing the influence of corrosion inhibitors such as chromate and molybdate on the processes that lead to the repassivation of scratched electrodes ${ }^{[34,36,37,44]}$. Isolated metrics such as OCP, $a$, and $i_{\max }$ have been observed but never systematically combined into a unifying study.

\subsubsection{Finite Cathodic Limited Repassivation Studies - The Role of Inhibitors on the Crack Flank}

During a crack advancement event the crack tip rupture repassivation event is electrochemically coupled to its local crack flank surroundings and depends on them for the current needed to anodically dissolve the metal exposed during the rupture. The electrochemical couple behavior depends on the rate of the cathodic reactions occurring on the crack flank which acts as a finite cathode. This is distinct from a potentiostat repassivation experiment where the cathode can supply infinite current to support the anode. If the rate of the cathodic reaction is limited, it may influence the anodic reaction 
rate at the crack tip. This relationship deserves some attention, however, no literature could be found relating the effects of cathodic inhibition to repassivation. An experimental setup utilizing a split cell design was developed to explore the electrochemical couple between the crack tip and the crack flank to examine the effects of cathodic inhibition on the cathodic reaction on the crack flank distinct from anodic repassivation of the tip.

\subsubsection{Complications in Scratch Repassivation Testing}

One difficulty with analyzing scratched electrode experiments is the presence of sizable local cathodic reactions which obscure the true anodic reaction rate when recording the net current density as seen in Equation 4.4. In Equation 4.4, $i_{\text {net }}$ is the total current, $i_{\text {crack }}^{\text {anodip }}$ is the true anodic current generated from the dissolution of aluminum, the cathodic terms represent the current from the reduction of oxygen, the evolution of hydrogen, or the

reduction of an inhibitor, and the $C \frac{d V}{d t}$ term represents the capacitive current generated from the rapid changes in potential, $V$. Here $\mathrm{C}$ is the capacitance of adjacent surface next to the scratch.

$$
i_{\text {net }}=i_{\text {crack tip }}^{\text {anodic }}-\left(i_{\text {crack tip }}^{\text {cathodic }}+i_{\text {passive surface }}^{\text {cathodic }}\right) \pm C \frac{d V}{d t}
$$

The addition of inhibitors to the repassivation process creates additional difficulty in analyzing current transients because of additional cathodic current resulting from possible 
inhibitor reduction reactions. Therefore the addition of oxidizing inhibitors can add greatly to the cathodic components of Equation $4.4^{[45]}$. In this study $i_{\text {net }}$ was monitored. OCP experiments were also conducted to assess the potential excursions during scratching both uncoupled and coupled to a large cathode.

\subsubsection{Novel Method for Inhibitor Testing During Scratch Repassivation Testing}

To address the ambiguity inherent to the scratched electrode current transient due to the difficulty extracting the true anodic current from the measured net current, a complimentary technique to examine oxide capacitance is utilized in this chapter. The wellknown relationship between oxide thickness $\left(d_{o x}\right)$ and capacitance $\left(C_{o x}\right)$ can be used to monitor oxide growth with an assumption regarding oxide permittivity. Several stages of formation and growth are predicated after scratching a metallic electrode, as shown in Figure 4.9. These include the nucleation of oxide on the bare metallic surface, complete coverage, and thickening. Surface capacitance can be exploited as an indicator of repassivation because it is affected by the capacitance described by Equation 4.5, where $\theta$ is the fraction of bare metallic electrode.

$$
c_{\text {total }}=\theta C_{\text {bare }}+(1-\theta) C_{o x}
$$

The method relies on the fact that $C_{b a r e}$ is much larger than $C_{o x}$ for aluminum ${ }^{[46]}$. $C_{b a r e}$ will be on the order of the double layer or Helmholtz layer capacitance which is between 40 and $100 \mu \mathrm{F} / \mathrm{cm}^{2}{ }^{[46-48]}$ while the $\mathrm{Al}_{2} \mathrm{O}_{3}$ oxide capacitance is on the order of $1-2 \mu \mathrm{F} / \mathrm{cm}^{2}{ }^{[49]}$. 
Therefore, $\mathrm{C}_{\text {total }}$ during repassivation should decrease by at least an order of magnitude as the electrode repassivates.

The measurement of the capacitance will be enabled through the use of a fast single frequency impedance measurement (200-250 impedance measurements per second) in the capacitive region of frequency response. Using Equation 4.6, the capacitance can be calculated assuming that the frequency applied is in the capacitance dominated region of the frequency response of the working electrode impedance.

$$
C=1 / 2 \pi f\left|Z_{m a g}\right|
$$

Figure 4.10 presents a theoretical Bode impedance plot under passive and mechanically depassivated conditions. These calculations were used to determine the single frequency at which impedance is dominated by capacitance for the duration of the repassivation event. The capacitive data would be easily extractable from $|Z|, Z$ ', Z', and $\theta$ data. A frequency of $1000 \mathrm{~Hz}$ was selected because it was well within the capacitive region of both the passive and depassivated surfaces. The capacitive region also has a negative slope with a value close to -1 on the Bode impedance plot shown in Figure $4.11^{[50,51]}$. Relationship 4.6 will hold true only in the region where the slope of $\log |\mathrm{Z}|$ vs $\log \omega$ is close to -1 or when $\theta$ is equal to $-90^{\circ}$. If solution resistance is prohibitive it can be subtracted. The nature of the impedance and meeting this criterion can also be monitored by the phase angle, $\theta$, shown in Figure 4.11. The phase angle is defined as the arctangent of the imaginary component of impedance, Z", over the real component of impedance, Z'. When Z" is much larger than Z' the phase angle approaches $-90^{\circ}$ and the impedance is dominated by the imaginary component which is indicative of the capacitive nature of the oxide. In the capacitive 
dominated region, the impedance magnitude will be inversely proportional to the oxide thickness according to the following relationship

$$
d=\frac{\kappa \epsilon_{o} A}{C}
$$

where $\kappa$ is the dielectric constant, $\epsilon_{\mathrm{o}}$ is the permittivity of free space, $A$ is the electrode surface area, and $d$ is the charge separation or thickness of the dielectric oxide. In this region of capacitance the phase angle $(\theta)$ should be large and approaching $-90^{\circ}$. The relationship between $|\mathrm{Z}|$ and the phase angle is shown in Figure $4.11^{[51]}$ where the real and imaginary components of impedance are Z' and Z" respectively. The relationships between impedance magnitude and phase angle, and barrier oxide properties will allow the effects of inhibitors to be assessed. The dielectric constant of the oxide as it is formed in an aqueous environment is likely to be different than what would be reported in the literature for the high purity dry aluminum oxide ${ }^{[52]}$. The presence of inhibitors whether they are doped in the aluminum oxide or present as a separate oxide species will also affect the dielectric constant of the full oxide structure. Some uncertainty in this value is expected. 


\subsubsection{Objective}

This paper will present recent experimental evidence that test the notion that inhibition of $\mathrm{EFCP}$ is due to the effects of chromate and molybdate in enhancing repassivation rate and oxide barrier growth after fracture or mechanical depassivation. Permanganate will also be analyzed for its repassivating inhibitor characteristics. The regrowth of oxide films over freshly bared aluminum surfaces in the presence of aqueous inhibitors and chloride is examined. The following information is obtained by direct measurement; $i_{\text {net, }}|\mathrm{Z}|, Z^{\prime}, Z^{\prime}$, and phase angle, as well as the effects of inhibitors on a simulated crack-tip galvanically coupled to a simulated crack flank. The net charge $\mathrm{Q}$, where $Q=\int i_{\text {repass }} d t$, is determined and $\mathrm{d}_{\mathrm{ox}}$ is calculated when appropriate using assumptions for $\epsilon_{\mathrm{o}}$. Furthermore, alternative mechanisms through which inhibitors can reduce da/dN such as buffering the $\mathrm{pH}$ of crack tip solutions are discussed.

\subsection{Experimental}

\subsubsection{Net Current Collection for Scratch Depassivation}

The general procedure for conducting a scratch depassivation experiment has been reported elsewhere ${ }^{[53]}$. AA2024-T351, AA7075-T6, and 99.999\% aluminum were cut and ground to $0.03 \mathrm{~cm}$ width and $0.8 \mathrm{~cm}$ length. Chemical compositions for the alloys are reported in Table 4.1. Samples were bonded with highly chemically resistant epoxy to polytetrafluoroethylene (PTFE) mounts that had been etched with an activated sodium 
solution. Electrical connection was made with heavy gauge copper wire through the back of the mount using high silver content epoxy. The sample mounts were inserted into a three electrode cell so that the face of the sample was vertical (Figure 4.12). A saturated calomel electrode (SCE) and platinized niobium mesh counter-electrode were used throughout all electrochemical experiments. The reference electrode was placed far enough away from the working electrode to be considered at an infinite distance to facilitate the calculation of the theoretically limited ohmic current density.

The scratch was made with a diamond-tip scribing stylus. Scratches were examined with an optical 3D tiling microscope to determine the width, depth, and area of the scratch (Figure 4.13). Scratches were found to be uniform in width and length owing to the small width of the mounted sample and the blunt nature of the diamond scribe. Typical width measurements were 60 micrometers, while the length of the scratch was controlled by the grinding of the sample prior to mounting. Sample width was on the order of $0.3 \mathrm{~mm}$. The nature of the scratched surface can be seen in Figure 4.13. Several constituent particles are visible in the SEM image which allows for comparison with overall scratch geometry. Given the length of the scratch, the probability of scratching through a large number of constituent particles in every experiment was assured reducing the variation in current from scratch to scratch. The average nearest neighbor distance for constituent particles larger than $0.3 \mu \mathrm{m}$ in AA7075-T6 and AA2024-T351 sheet was $3 \mu \mathrm{m}$ and $5 \mu \mathrm{m}$ respectively.

The exposed sample face was lightly coated with vacuum grease prior to the scratch to eliminate faradaic and capacitive current contributions from adjacent areas as indicated in Equation $4.4^{[53]}$. Scratches were examined with an optical microscope after every scratch to ensure that there was no vacuum grease or Teflon contamination. The width of the 
sample across (the length of the scratch) was small to minimize the time that the stylus was in contact with the sample ${ }^{[45]}$. The rise time for current transients was on the order of 0.5 ms. The scratch speed was approximately $60 \mathrm{~cm} / \mathrm{s}$.

Solutions investigated include aqueous molybdate, permanganate, and chromate with concentrations ranging from $0.1 \mathrm{mM}$ to $0.1 \mathrm{M}$ in $\mathrm{NaCl}$ solution concentrations from 0.05 $\mathrm{M}$ to $1.0 \mathrm{M}$. All solutions were prepared with $18.2 \mathrm{M} \Omega$ water and chemicals procured from Alfa-Aesar. All solutions were adjusted to $\mathrm{pH} 6.5$ with concentrated $\mathrm{NaOH}$ and $\mathrm{HCl}$. All solutions were deaerated with high purity nitrogen for one hour prior to testing and an $\mathrm{N}_{2}$ blanket was maintained over the cell.

The working electrodes were held potentiostatically at applied potentials ranging from -0.9 $\mathrm{V}$ to $-0.2 \mathrm{~V}$ (SCE). A PAR 273A potentiostat was used to apply potentials and collect net current. The current data was sampled through the potentiostat's analog outputs and collected with an NI SCXI-1140 DAQ and a SCXI-1600 chassis with sampling capability of 200 kilosamples per second $(\mathrm{kS} / \mathrm{s})$. Auto-ranging of the potentiostat was disabled. Successive scratches were performed until a current range was found at which peak currents could be captured. Auto-ranging was then enabled and short time data was sacrificed when long term data was collected. The rise time for the ranges used was less than $3 \mu$ s and the range accuracy was below $0.2 \%$ of the range limit. For example if the 1 $\mathrm{mA}$ range was selected the accuracy was $2 \mu \mathrm{A}$. Current below $2 \mu \mathrm{A}$ would not be measurable. 


\subsubsection{Finite Cathodic Coupled Crack tip and Crack Flank}

A spit cell was used to examine the effects of cathodic inhibition on the repassivation characteristics of AA7075-T6 when coupled to a finite cathode. A schematic of the cell and wiring diagram can be seen in Figure 4.14. The galvanic current is measured with a potentiostat operating as a zero resistance ammeter (ZRA), while the potential of the scratch depassivated surface coupled through the salt bridge to the cathode cell is monitored with a saturated calomel reference electrode. The area of the scratched AA7075T6 surface was $2 \times 10^{-4} \mathrm{~cm}^{2}$ produced according to the standard scratch repassivation test procedure, while the cathode AA7075-T6 surface area was $0.78 \mathrm{~cm}^{2}$ and was ground to 1200 grit with $\mathrm{SiC}$ paper. The ratio of ruptured surface area in an actual crack tip to the surrounding couple crack flank is not known precisely but will certainly be very small. The crack flank cell was quiescent while the crack tip cell was deaerated with high purity nitrogen to simulate the deaerated conditions at the crack tip.

\subsubsection{Impedance Measurement of the Scratch Depassivated Surface}

A Gamry G300 series potentiostat was used when single frequency impedance was collected to hold the sample potentiostatically and perform the impedance measurements. A fast single frequency impedance script was developed in collaboration with Gamry Instruments, INC to increase the sampling rate capabilities of the standard EIS software from 1-2 data points per second to 200 to 250 data points a second. The software suite used to make the measurement was the ESA410 package. The following lines of code must be 
entered in the Gamry6.ini file, which can be found at the following path (C:IProgramDatalGamry Instruments $\backslash$ Framework $\backslash$ Gamry6.ini), in order to enable the debug mode of the software, where the experimental user interface (UI) is located. A new section in the code should be added as follows.

[ESA410]

\section{DEBUG=TRUE}

Once this is entered into the Gamry6.ini file, a full expansion of the UI will reveal the experimental sections needed to run the experiment. This procedure has been completed and is available for use with the REF600 when used with the multichem dongle (software license dongle USB) in WDF315.

Warning: you can break the instrument if you do not follow these directions carefully. Figure 4.15 shows the standard ESA410 UI. Figure 4.16 shows the debug mode of the ESA410 software which contains the parameters needed to conduct the experiment. The first steps involve setting up the standard UI in Figure 4.15. The following settings are required and should be followed in order. Current Device should be set to an equipped REF600 potentiostat. Control Mode should be set to potentiostatic. Autoscaling Y axis should be unchecked. The potential for the potentiostatic experiment should be entered in Bias Voltage in volts. Important - The IE range should be set to a predetermined current range with which to measure the peak current densities. The correct range should be determined using the standard $i_{n e t}$ scratch procedure outlined above. The Auto Up box that controls auto ranging of the current range should not be checked. The gain change (Vch Range and Ich Range) should be in auto mode by checking the auto box corresponding to 
the gain setting. Output Frequency should then be set to $4000 \mathrm{~Hz}$. Auto Filter should not be adjusted. This concludes the standard UI settings.

In debug mode first change the Voltage Channel Filter and Current Channel Filter to 200 kHz. Then set the Control Amplifier to medium. The next step is to set the amplitude and frequency of the applied sine wave. Amplitude should be set to $25 \mathrm{mV}$ and frequency should be set to $1000 \mathrm{~Hz}$. After this is complete the Apply Sine Wave? and Use DDS? boxes should be checked. At this point, go back to the standard UI and click Scale Now. Finally, select the duration of the measurement (no more than 4 minutes), select a file path to save your data, and under the Setup directory click Create Secondary ASCII Datafile. The experiment will now be ready to run. Click Record Data and the single frequency measurement will begin. Do not adjust any other settings in Debug Mode.

The following description of the issues with standard EIS analysis and the improvements made to the EIS measurements conducted in this work are taken from an edited personal correspondence with Burak Ulgut of Gamry Instruments Inc. [54] "A typical impedance measurement is iterative in nature. The measuring algorithm optimizes gains, offsets, and analog and digital ranges, successively, in order to achieve a measurement that meets the signal to noise criterion. This optimization starts from the settings of the previous successful measurement. In most standard cases the required hardware settings do not change drastically from one measurement to the next.

A scratch repassivation experiment is very different compared to the typical impedance spectroscopy measurement. The measured system is very dynamic in both the DC offsets and the AC amplitudes. In the pre-scratch phase, both the DC current and the AC current 
are very low. At the time of the scratch both the DC current and the AC current increase by approximately 3 orders of magnitude. The DC current quickly decays to zero whereas the AC current decays back to the levels reflecting a repassivating aluminum surface. This process evolves quickly within the first second. Even though all three phases of the system are easily measurable with state-of-the-art instrumentation, they all require different hardware settings.

In order to accomplish the scratch measurement, the settings were divided into two. The first group, the analog settings, are settings that take a relatively long time to settle. These settings were optimized to cover the most significant portions of the scratch and repass processes and then kept constant throughout the measurement. The second group, the digital settings, are the faster settling offsets and gains that are varied continuously throughout. With these settings, the prescratch impedance measurements through the grease barrier do not yield any signal beyond the noise base of the instrument.

The optimal sampling time was decided to be $4 \mathrm{kHz}$. Any faster sampling compromises the performance of the offset tracking algorithm and any slower sampling cannot faithfully reconstruct the $1 \mathrm{kHz}$ signal.

Once the time domain signal was acquired, it was divided into 16ms blocks (64points). Each block was then individually Fourier transformed to calculate the impedance value at $1 \mathrm{kHz} . "$

The Fourier transform was conducted in Mathcad with a script written by Dr. Ulgut and edited by the author of this manuscript. The full script can be seen in Figure $4.17^{[54]}$. 
The frequency applied was $1000 \mathrm{~Hz}$ with an amplitude of $20 \mathrm{mV}$ RMS. The measurements were taken at an applied potential between -0.8 and $-0.6 \mathrm{~V}_{\mathrm{SCE}}$ unless otherwise stated. These tests were conducted in $1 \mathrm{M} \mathrm{NaCl}$ with various concentrations of molybdate, permanganate, and chromate as indicated. Solutions were all adjusted to $\mathrm{pH} 6.5$ with $\mathrm{NaOH}$ or $\mathrm{HCl}$ as needed.

\subsubsection{Additional Methods}

OCP measurement during scratch depassivation was monitored with a Gamry Ref600 potentiostat at a data acquisition rate of $10 \mathrm{~Hz}$ with the standard Gamry OCP collection parameters used. Otherwise, the experimental setup was the same as the net current collection.

Buffer capacity was measured using $200 \mathrm{~mL}$ of the relevant solution, titrated with a burette, while the $\mathrm{pH}$ was measured with an Accumet XL.

Pitting potentials and cathodic kinetics were determined with a scan rate of $0.1 \mathrm{mV} / \mathrm{sec}$ in a standard three electrode flat cell, utilizing a saturated calomel reference electrode. The surface area of the exposed electrode was $0.78 \mathrm{~cm}^{2}$. All solutions were $\mathrm{pH}$ adjusted where noted with $\mathrm{NaOH}$ or $\mathrm{HCl}$. Open circuit potential was allowed to stabilize for half an hour before the tests were conducted. 


\subsection{Results}

\subsubsection{The Inhibition of Pitting Potential as Determined by Epit}

AA2024-T351 and AA7075-T6 are especially prone to pitting attack due to their alloying constituents and microstructure ${ }^{[55,56]}$. Molybdate and chromate are among the most effective inhibitors at raising the pitting potential of aluminum alloys ${ }^{[55,57]}$. Figure 4.18 illustrates the increase in pitting potential for AA2024-T351 that can result from solution additions of molybdate and chromate. The selected potentials for scratch repassivation tests are referenced against potentiodynamic scans to investigate the effects of inhibitors on oxide stability in Figure 4.18. Permanganate has been shown to be an effective inhibitor of pitting potential in AA2024-T351 under select conditions and has been studied here due to its strong oxidizing nature, which may affect oxide film formation ${ }^{[20]}$. Molybdate and chromate were chosen for this study because of the evidence that suggests they are potent inhibitors of $\mathrm{EFCP}^{[8,14]}$ and while permanganate was selected for its demonstrated stabilizing effects on barrier oxides and because of the highly oxidizing nature ${ }^{[20]}$.

An investigation of inhibition of pitting on AA7075 over a range of pHs relevant to the crack tip was documented for chromate, molybdate, and permanganate. Figure 4.19 and Figure 4.20 show the effect of $0.1 \mathrm{M} \mathrm{CrO}_{4}{ }^{2-}$ addition to $1 \mathrm{M} \mathrm{NaCl}$ at $\mathrm{pH} 3$ and 6.5, respectively. At these chloride levels there is little to no evidence of pitting inhibition. However, $\mathrm{i}_{\text {corr }}$ does appear to be slightly reduced along with a decrease in fluctuations in the passive region which may be interpreted as metastable pitting events ${ }^{[58]}$. Additionally, two breakdown potentials are evident for the AA7075-T6. This may be an effect of 
polishing this particular alloy or the breakdown and corrosion of specific phases or microstructural regions ${ }^{[59-62]}$. Upon polishing AA7075-T6 with 1- $\mu$ m diamond paste, Frankel found an absence of small hardening precipitates in a thin surface layer characterized by nano-grain boundaries ${ }^{[59]}$. Frankel suggests that attack of the nano-grain boundaries may result in the first breakdown potential and that this first breakdown is purely the result of a polishing artifact. Maitra suggests that the second breakdown potential results from the local breakdown of the solute $(\mathrm{Zn}$ and $\mathrm{Mg}$ ) enriched grain boundaries $^{[62]}$. Regardless, $0.1 \mathrm{M}$ chromate in $1 \mathrm{M} \mathrm{NaCl}$ was not able to affect the breakdown at either $\mathrm{pH} 3$ or $\mathrm{pH} 6.5$.

Figure 4.21, 4.22 and 4.23 examine the effect of $0.1 \mathrm{M}$ molybdate addition to $1 \mathrm{M} \mathrm{NaCl}$ at pH 3, 6.5 and 9, respectively. At pH 3 and 9 molybdate has little effect on the breakdown of passivity. However, at pH 6.5 a significant increase in the pitting potential is observed, approximately $75 \mathrm{mV}$. This increase in breakdown potential obscures the presence of the second breakdown potential suggesting that molybdate is effectively preventing the breakdown of the hypothesized surface fine grain boundary regions. It should be noted that $\mathrm{pH} 9$ is the natural $\mathrm{pH}$ of the $\mathrm{MoO}_{4}{ }^{2-}$ solution as prepared and $1 \mathrm{~N} \mathrm{HCl}$ was used to adjust to the lower $\mathrm{pH}$ values. The buffering capacity of molybdate will be discussed in the following section.

Figure $4.24,4.25$, and 4.26 show the effect of the addition of permanganate to $1 \mathrm{M} \mathrm{NaCl}$ solutions with $\mathrm{pH}$ of $3,6.5$, and 9 respectively. At all $\mathrm{pH}$ values the introduction of permanganate lead to an increased open circuit potential and the obfuscation of the second breakdown potential as well and as passive region. The increase in $i_{\text {corr }}$ suggests an increase in the cathodic reaction kinetics and the increase in OCP likely results in an increase in 
anodic dissolution rate. However, if the pitting potential is defined as the open circuit potential in the absence of a passive region then there is a small increase in the pitting potential over all cases. Molybdate is shown to be the most promising anodic inhibitor among the three selected inhibitors, increasing the pitting potential at neutral $\mathrm{pH}$ in $1 \mathrm{M}$ $\mathrm{NaCl}$ by $75 \mathrm{mV}$ (Figure 4.22). Chromate is shown to affect metastable pitting without having an appreciable effect on the onset of pitting at the concentration explored (Figure 4.20). Permanganate is found to increase the OCP and $\mathrm{i}_{\text {corr }}$ accompanied by a small increase in pitting potential (Figure 4.24). Caution is necessary in that a complete study of every possible $\mathrm{C}_{\mathrm{inh}}+\mathrm{C}_{\mathrm{NaCl}}$ was not undertaken.

\subsubsection{The Inhibitor Buffer Capacity}

The buffering capacity of several molybdate concentration have been reported in the literature and are shown in Figure $4.27^{[63]}$. At high concentrations of molybdate the buffer capacity below pH 6 is large owing to the pKa for $\mathrm{HMoO}_{4}{ }^{-} / \mathrm{MoO}_{4}{ }^{2-}$ reaction. The limited buffer capacity of the $\mathrm{NaCl}$ solution is hypothesized to be the result of atmospheric $\mathrm{CO}_{2}$ and the $\mathrm{H}_{2} \mathrm{CO}_{3} / \mathrm{HCO}_{3}{ }^{-} / \mathrm{CO}_{3}{ }^{2-}$ equilibrium of carbonate. The buffer capacity of chromate has been reported in the literature and is shown in Figure $4.28^{[3]}$. The solution of $0.5 \mathrm{M} \mathrm{CrO}_{4}{ }^{2-}$ $+0.05 \mathrm{M} \mathrm{NaCl}$ shows a large buffering capacity.

Further titrations were performed to investigate the effect of each inhibitor on simulated crack tip type solutions. Figure 4.29 shows the titration curves of $0.1 \mathrm{M} \mathrm{Na}_{2} \mathrm{Cr}_{2} \mathrm{O}_{7}$ and 0.1 $\mathrm{M} \mathrm{Na}_{2} \mathrm{MoO}_{4}$ in an aqueous $0.1 \mathrm{M} \mathrm{AlCl}_{3}$ solution. The titration curves are very similar for both salts. The starting $\mathrm{pH}$ for the chromate solution plus $\mathrm{AlCl}_{3}$ was 3.15 while the starting 
$\mathrm{pH}$ for the molybdate solution plus $\mathrm{AlCl}_{3}$ was slightly higher at 3.87 . Both titrations demonstrate two half-equivalence points (smallest $\partial p H / \partial$ Voladded base) and two equivalence

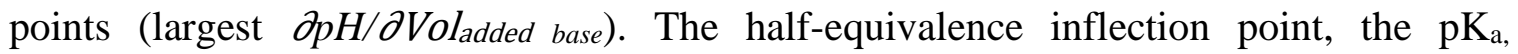
represents the solutions $\mathrm{pH}$ value where the conjugant acid and base are present in solution in equal proportion. The chromate solution had a $\mathrm{pKa}_{1}$ of 3.95 and a $\mathrm{pKa}_{2}$ of 6.58 , respectively. These values correspond to 27.7 and $73.7 \mathrm{~mL}$ of $0.1 \mathrm{M} \mathrm{NaOH}$ added to 200 $\mathrm{mL}$ of $0.1 \mathrm{M} \mathrm{Na}_{2} \mathrm{Cr}_{2} \mathrm{O}_{7}$ and $0.1 \mathrm{M} \mathrm{AlCl}_{3}$. The molybdate solution had a $\mathrm{pKa}_{1}$ of 3.9 and a $\mathrm{pKa}_{2}$ of 6.59 . These values correspond to 22 and $57 \mathrm{~mL}$ of $0.1 \mathrm{M} \mathrm{NaOH}$ added to $200 \mathrm{~mL}$ of $0.1 \mathrm{M} \mathrm{Na}_{2} \mathrm{MoO}_{4}$ and $0.1 \mathrm{M} \mathrm{AlCl}_{3}$. It should be noted that the speciation of the $\mathrm{Cr}_{2} \mathrm{O}_{7}{ }^{2-}$ and $\mathrm{CrO}_{4}{ }^{2-}$ and their corresponding acids complicates the analysis as the acid-base reaction. When the $\mathrm{CrO}_{4}{ }^{2-}$ species is present the ability of the inhibitor to buffer the addition of the strong base will be doubled due to the stoichiometry of the acid/base pairs. This is also true of the molybdate and polymolybdate species where the ratio of hydrogen to molybdenum in the fully protonated acid ranges from $2 / 1$ for the $\mathrm{H}_{2} \mathrm{MoO}_{4}$ species to at least $1 / 7$ for the $\mathrm{HMo}_{7} \mathrm{O}_{24}{ }^{4-}$ species. Considering these factors it is still possible to compare the buffer capacity of each $\mathrm{Cr}$ and Mo regardless of specific speciation in this specific solution. The addition of $2.77 \times 10^{-3} \mathrm{~mol}$ of $\mathrm{NaOH}$ is needed to reach the pKa value for the sodium dichromate solution while $2.2 \times 10^{-3}$ mol of $\mathrm{NaOH}$ was needed to do the same with the sodium molybdate solution. Given the factor of two differences in stoichiometry it is clear that for every $2.0 \times 10^{-2} \mathrm{~mol}$ of $\mathrm{Cr}, 1.385 \times 10^{-3} \mathrm{~mol}$ of $\mathrm{NaOH}$ is needed while $2.2 \times 10^{-3} \mathrm{~mol}$ is needed in the case of the Mo showing that the molybdate buffer has a much larger buffer capacity. 
Figure 4.29 also shows the effect of titrating $0.1 \mathrm{M} \mathrm{AlCl}_{3}$ into $0.1 \mathrm{M} \mathrm{MoO}_{4}{ }^{2-}$. This better illustrates the effects of molybdate on a crack tip type solution. However, quantitative buffering information is not available as the $\mathrm{pH}$ change relies on hydrolysis and not a strong acid or base. The experiment illustrates the effective $\mathrm{pH}$ control that molybdate can provide in a crack tip like environment, high in $\mathrm{Al}^{3+}$ and $\mathrm{Cl}^{-}$.

The buffering attributes of both chromate and molybdate are explained by the presence of several high potential soluble species such as chromic acid, the acid chromate ion, and chromate $\left(\mathrm{H}_{2} \mathrm{CrO}_{4}, \mathrm{HCrO}_{4}^{-}\right.$, and $\left.\mathrm{CrO}_{4-}{ }^{2-}\right)$ and molybdic acid, the acid molybdate ion as well as the polymolybdate ions that can acquire $\mathrm{H}^{+}$in acid solutions, and the molybdate ion $\left(\mathrm{H}_{2} \mathrm{MoO}_{4}, \mathrm{HMoO}_{4}{ }^{-}\right.$, and $\left.\mathrm{MoO}_{4-}{ }^{2-}\right)$ which can be seen in Figure 4.5 and Figure 4.6. It should be noted that the complex polymerization of these inhibitors can have a drastic effect on their ability to buffer. However, in a simulated crack tip solution molybdate has a significantly higher capacity to buffer the solution than chromate. Permanganate is not a buffer due to the absence of any conjugate acid/base reaction pairs.

\subsubsection{The Effect of Inhibitors on Open Circuit Potential during Scratch Repassivation}

Figure 4.30 shows the effect of solution $\mathrm{pH}$ and chloride concentration on the OCP transient of the scratch only after scratch depassivation in $\mathrm{N}_{2}$ purged aqueous chloride containing solutions. The effect of $\mathrm{pH}$ (Figure 4.30A) had a marked effect on $d E / d t$ as well as affecting the steady state OCP at longer times. Both low and high $\mathrm{pH}$ appeared to lower the OCP at longer times below that of the $\mathrm{pH} 6.5$ experiment. However, the $\mathrm{pH} 10$ resting 
OCP was almost $200 \mathrm{mV}$ below the $\mathrm{pH}$ 4. There was little effect of $\mathrm{Cl}^{-}$concentration (Figure 4.30B) on the open circuit potential of scratched AA2024 in aqueous solutions.

Figure 4.31 shows the effect of $0.05 \mathrm{M} \mathrm{CrO}_{4}{ }^{2-}, \mathrm{MoO}_{4}{ }^{2-}$, and $\mathrm{MnO}_{4}{ }^{-}$on the open circuit potential of scratched AA2024 in $\mathrm{pH} 6.5$ aqueous $0.05 \mathrm{M} \mathrm{NaCl}$. The data can be compared to $0.05 \mathrm{M} \mathrm{NaCl}$ without the inhibitor. The inhibitors mediate the extent of potential drop that occurs on the electrode after the surface oxide has been removed. The effect ranks generally in accordance with the strength of the oxidizing nature of each ion with the highest potentials reached (less severe minimum) by permanganate, followed by molybdate and then chromate. However, film growth also contributes. The relevant reduction reactions are shown in Equations 4.8, 4.9, and 4.10 where concentration was taken to be $0.1 \mathrm{M}$ and the $\mathrm{pH}$ was 6.5 .

$$
\begin{aligned}
& 2 \mathrm{CrO}_{4}^{2-}+10 \mathrm{H}^{+}+6 e^{-} \leftrightarrow \mathrm{Cr}_{2} \mathrm{O}_{3}+5 \mathrm{H}_{2} \mathrm{O}\left(E_{0}=0.44 \mathrm{VSCE}\right) \\
& \mathrm{MoO}_{4}^{2-}+4 \mathrm{H}^{+}+2 e^{-} \leftrightarrow \mathrm{MoO}_{2}+2 \mathrm{H}_{2} \mathrm{O}\left(E_{0}=-0.37 \mathrm{~V} \mathrm{SCE}\right) \\
& \mathrm{MnO}_{4}^{-}+4 \mathrm{H}^{+}+3 e^{-} \leftrightarrow \mathrm{MnO}_{2}+2 \mathrm{H}_{2} \mathrm{O}\left(E_{0}=0.958 \mathrm{~V} \mathrm{SCE}\right)
\end{aligned}
$$

Figure 4.32 shows the effect of inhibitor concentration on the OCP transient after scratch depassivation. There is no discernable trend of inhibitor concentration over the concentrations probed. 


\subsubsection{Crack Flank - Crack Tip Galvanic Couple Behavior and The Inhibition of Cathodic Reactions Critical to Crack Chemistry}

Figure 4.33 shows the effect of having a finite cathode coupled to a scratch depassivated electrode. The addition of chromate to the cathode cell in the dual cell setup reduces the potential of the scratched electrode which likely has two effects. The first is that the current passed between the simulated crack tip and the simulated crack flank is reduced. This is likely due to a decrease in the supporting cathodic reaction rate on the finite cathode cell, which effectively decreases the open circuit potential. However, there is an argument that the decrease in potential also raises the hydrogen overpotential which likely leads to an increase in hydrogen evolution.

The molybdate appears to have the opposite effect. The open circuit potential recovers more quickly when molybdate is added to the coupled cathode cell than even the chloride solution in the absence of any inhibitor addition. The origin of this behavior can be seen in the open circuit data just prior to scratch depassivation also shown in Figure 4.33. When the scratch occurs, the open circuit potential of the large cathode plays an important role in the behavior of the repassivating electrode.

Figure 4.34 shows the effect of $0.1 \mathrm{M} \mathrm{CrO}_{4}{ }^{2-}, \mathrm{MoO}_{4}{ }^{2-}$, and $\mathrm{MnO}_{4}{ }^{2-}$ on the cathodic $\mathrm{E}-\log i$ behavior of AA7075 in aqueous $1 \mathrm{M} \mathrm{Cl}^{-}$with an adjusted $\mathrm{pH}$ of 6.5 and 3. Chromate is shown to be the most potent cathodic inhibitor on AA7075, reducing the cathodic current density in both the mass transport and charge transport dominated cathodic regions. The inhibition of cathodic reaction kinetics for molybdate and permanganate appear to be 
dependent on $\mathrm{pH}$. At a $\mathrm{pH}$ of 6.5 molybdate shows periodic inhibition of cathodic reaction rate. There are two regions of drastic cathodic reaction retardation, once at -1.04 V (SCE) and once at $-1.2 \mathrm{~V}$ (SCE). Two scans were performed to validate the observed behavior. All current density values correspond to cathodic current indicating that a potential dependent mechanism of inhibition is occurring. However, at $\mathrm{pH} 3$ molybdate is shown to be detrimental to the cathodic reaction rate in the mass transport limited regime, increasing it by an order of magnitude. Permanganate demonstrates the opposite behavior to chromate as well. At $\mathrm{pH} 6.5$ permanganate increases the cathodic reaction rate by an order of magnitude in the mass transport limited regime while at $\mathrm{pH} 3$ it reduces the charge transport regime of the hydrogen evolution reaction (HER). Permanganate has little effect on the mass transport limited oxygen reduction reaction (ORR). The results for chromate are consistent with the scratch split cell experiment. The greater potential drop corresponds to lower cathodic current density.

\subsubsection{The Inhibition of Net Current Density During Scratch Repassivation}

Figure 4.35 shows a typical repassivation event conducted on $99.999 \%$ pure aluminum. The repassivation shows a plateau at early times before the first millisecond when plotted on a $\log i-\log t$ scale. Interestingly, this behavior was described by Kolman and Scully for high purity titanium depassivation experiments ${ }^{[30]}$. The plateau evident in the study presented appears to be slightly shorter lived than those found with scratch tests by Kolman and Scully indicating that the depassivation speed is slightly faster than was used in their experiments. They claim a rise time of 1-2 ms while the rise time observed in our scratch 
tests was typically less than $1 \mathrm{~ms}$. The decay expression reported by Kolman and Scully ${ }^{[30]}$ in Equation 4.3 was modified for our purposes. The maximum current, $i_{o}$, deviated from the logarithmic expression that describes the majority of the transient so it was not included in the expression and $t_{o}$ was accounted for in the definition of $t$. The new expression is shown in Equation 4.11

$$
i=n(t)^{-m}
$$

where $i$ is current density, $t$ is time since peak current, and $n$ and $m$ are constants. The constant $m$ is the gradient $-\partial \log i / \partial \log t$, while $n$ represents the current density at time zero which is not a physically significant parameter due to the deviation of current from logarithmic behavior. The transients were fit between $1 \mathrm{~ms}$ and $50 \mathrm{~ms}$, after which the current decays into a region of lower precision due to the high current range selection of the potentiostat and the $0.2 \%$ of range limitation on accuracy. When the current transient in Figure 4.35 is fit logarithmically the value of $m$ was 0.69 .

Figure 4.36 shows the applied potential corrected for ohmic drop for an applied potential of $-0.8 \mathrm{~V}(\mathrm{SCE})$ at the working electrode relative to the reversible hydrogen potential (SCE

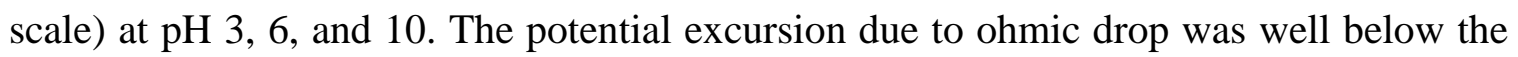
hydrogen evolution potential for all scratch tests due to the standard testing $\mathrm{pH}$ of between 6.5 and 3. Given the potential excursions in Figure 4.36 the hydrogen evolution reaction overpotential would be $0.582 \mathrm{~V}$ at $\mathrm{pH} 3$ and $0.379 \mathrm{~V}$ for $\mathrm{pH} 6$.

Figure 4.37 shows repassivation transients for $99.999 \%$ aluminum in $1 \mathrm{M}, 0.5 \mathrm{M}$, and 0.05 $\mathrm{M} \mathrm{NaCl}$ solution, indicating the effect of chloride concentration and potential on repassivation. In Figure 4.37(a), increased potential leads to onset of persistent 
depassivated anodic behavior in high purity aluminum. The scratch begins to pit or active intermetallic phases never repassivate. This behavior is seen in the figures as the formation of a plateau in the current density at longer time after initial peak scratch current decay described by Equation 4.3 has occurred. The nature of the dissolution of the scratched substrate during this plateau has been reported as localized pitting in high purity aluminum ${ }^{[34]}$ and is assumed to be local pitting of the alloy when the potential is sufficiently positive. The time of onset and the current density of the post-transient behavior are affected by the applied potential. As the chloride level decreases, the depassivated anodic behavior is suppressed revealing repassivation transients of the form expressed by Equation 4.3. Table 4.2 lists the fitting parameters for the high purity aluminum scratch transients in Figure 4.37(d). The onset of initial plateau decay appears to be chloride concentration or solution resistance dependent as seen in Figure 4.37 (d). As the sodium chloride concentration and thus the solution conductivity decreases the theoretically IR limit decreases. This may result in the decrease in the current maximum. Potential does not appear have as strong of an effect as chloride concentration on the current maximum but does have a sizable effect at longer times with the onset of pitting.

Chloride concentration clearly has an effect on the onset of the persistent active behavior at long times. It also appears to have an effect on the maximum peak current at short times after the scratch. Figure 4.38 relates the maximum current density observed for each chloride-potential combination in relation to the theoretical ohmically limited current density for each solution, calculated using Equations 4.12 and 4.13 for a rectangular band [38]. 


$$
R_{s}=\left(\frac{1}{2 \pi \kappa b}\right) \ln \left(\frac{4 b}{a}\right)
$$

where $R_{s}$ is the solution resistance $(\Omega), \kappa$ is the solution conductivity $(\Omega-\mathrm{cm}), a$ is one half of the bared areas width $(\mathrm{cm})$, and $b$ is one half of the bared areas length $(\mathrm{cm})$. The ohmic current density was calculated.

$$
i_{\text {ohmic }}=\frac{E_{\text {app }}-E_{\text {bare oCP }}}{\left(R_{S}\right)(\text { Area })}
$$

In Equation 4.13, $i$ is the theoretical ohmically limited current density, $E_{a p p}$ is the applied potential, and $E_{\text {bare }, O C P}$ is the open circuit potential of the bare metal taken to be $-1.8 \mathrm{~V}_{\text {SCE }}$. The data suggests that at all chloride concentrations the peak current density is approaching the theoretical ohmically limited current density as seen in Figure 4.38. This would suggest that at very short times the ohmic influence on the current density might be substantial in addition to any scratch depassivation speed effects. Therefore, $i_{o}$ may not be the most important parameter to consider as the details of Equation 4.11 and 4.12 differ at the crack tip.

Figure 4.39 shows the scratched electrode current density transients for both AA2024-T351 and AA7075-T6 at two chloride levels and over multiple potentials. The dependence of maximum current density and initial plateau to logarithmic decay on chloride concentration also remain evident for the two alloy systems. However, the effect of potential on the sustained or persistent active plateau observed after incomplete logarithmic decay is drastically different between the two alloy systems. AA7075 suffers higher sustained active plateau levels at more negative applied potentials than AA2024 at both chloride levels. For 
instance at $-0.6 \mathrm{~V}(\mathrm{SCE})$ in $1 \mathrm{M} \mathrm{NaCl} \mathrm{AA7075} \mathrm{has} \mathrm{a} \mathrm{sustained} \mathrm{active} \mathrm{current} \mathrm{density}$ plateau that is more than an order of magnitude greater than AA2024. This suggests that once depassivation has occurred, it is difficult to repassivate 7075 either due to matrix pitting or attack at constituent particles.

Figure 4.40 shows the effects of $0.1 \mathrm{M}$ chromate and $0.1 \mathrm{M}$ molybdate on the repassivation behavior of AA7075-T6 in aqueous $0.05 \mathrm{M} \mathrm{NaCl}$ solution with $\mathrm{pH}$ 6.5. A relatively low concentration of chloride and a large concentration of molybdate in a neutral solution result in no inhibition of either peak current density or depassivated anodic behavior onset. However, the onset of persistent active current behavior is shifted from $-0.7 \mathrm{~V}$ to $-0.4 \mathrm{~V}_{\mathrm{SCE}}$ when chromate is used. That is to say, repassivation occurs over a larger potential range. Even at $-0.4 \mathrm{~V}$ the current density at 1 second is less than the uninhibited solution at -0.6 $\mathrm{V}_{\text {SCE. }}$ Peak current densities are summarized in Figure 4.40(b). Chromate and molybdate also both raise the theoretical ohmic limit due to increased conductivity of the solutions. The solution conductivity of $0.05 \mathrm{M} \mathrm{NaCl}$ was $8 \mathrm{mS} / \mathrm{cm}$ while the addition of $0.1 \mathrm{M} \mathrm{CrO}_{4}{ }^{2-}$ and $0.1 \mathrm{MoO}_{4}{ }^{2-}$ led to solution conductivities of $11.5 \mathrm{mS} / \mathrm{cm}$ and $18.8 \mathrm{mS} / \mathrm{cm}$, respectively. Figure 4.41 shows integration of the current density over several time periods for all investigated potentials. Chromate shows inhibition of net anodic charge $\left(\mathrm{Q} / \mathrm{cm}^{2}\right)$ passed over all integration periods while molybdate shows no inhibition of net anodic charge after scratching of AA7075.

Figure 4.42 shows the effects of $0.1 \mathrm{M}$ molybdate and $0.1 \mathrm{M}$ chromate additions to aqueous $0.05 \mathrm{M} \mathrm{NaCl}$, with a $\mathrm{pH}$ 6.5, on AA2024-T351. No inhibition of either peak current density or active long term anodic behavior onset is observed for molybdate additions. Chromate inhibits the onset of active long term persistent depassivated type current behavior, 
increasing onset of persistent depassivation from $-0.7 \mathrm{~V}$ to $-0.2 \mathrm{~V}_{\mathrm{SCE}}$. Peak current densities are summarized in Figure 4.42(b) with respect to the ohmic limit of each solution. Chromate and molybdate both raise the theoretical ohmic limit due to the increased conductivity of the solutions. Figure 4.43 shows integration of the current density over several time periods since scratch depassivation for all investigated potentials. Chromate shows inhibition in charge passed over all integration periods since scratch depassivation while molybdate shows no inhibition of charge passed for AA2024-T351. The inhibition efficiency of chromate is greater for AA2024 then for AA7075.

Additional scratch depassivation experiments were carried out in $1 \mathrm{M} \mathrm{NaCl}$ to better simulate crack tip conditions in anodically polarized cracks. Figure 4.44 shows the effects of $0.1 \mathrm{M}$ molybdate, $0.1 \mathrm{M}$ chromate, and $0.1 \mathrm{M}$ permanganate additions to aqueous $1.0 \mathrm{M}$ $\mathrm{NaCl}$, with an adjusted $\mathrm{pH}$ 6.5, on AA7075-T6. No inhibition of either peak current density or active long term persistent anodic behavior onset is observed for molybdate or chromate additions. Permanganate showed inhibition of current density at $-0.8 \mathrm{~V}$ (SCE) by two orders of magnitude. Figure 4.45 shows the effects of $0.1 \mathrm{M}$ molybdate, $0.1 \mathrm{M}$ chromate, and $0.1 \mathrm{M}$ permanganate additions to aqueous $1.0 \mathrm{M} \mathrm{NaCl}$, with an adjusted $\mathrm{pH} 6.5$, on AA2024-T351. No inhibition of either peak current density or active long term persistent anodic behavior onset is observed for molybdate permanganate, or chromate additions in $1 \mathrm{M} \mathrm{NaCl}$ at the inhibitor levels investigated. 


\subsubsection{The Effect of Chromate, Molybdate, and Permanganate on Surface Impedance Changes During Scratch Repassivation in NaCl Solutions}

Figure 4.46 and 4.47 show the capacitive nature of scratched AA2024 at pH 3 and $\mathrm{pH} 6.5$. The impedance in the capacitive region is investigated with $\mathrm{CrO}_{4}{ }^{2-}, \mathrm{MoO}_{4}{ }^{2-}$, and $\mathrm{MnO}_{4}{ }^{-}$ inhibitor additions. In addition to tracking the impedance magnitude $|\mathrm{Z}|$ of the surface, the phase angle of the frequency response is recorded. Recall that a $-90^{\circ}$ phase angle indicates purely capacitive behavior. This is the first instance of fast single frequency impedance measurement after a scratch depassivation event known to the author.

The initial findings indicate that there are large differences in impedance magnitude and phase angle with the addition of inhibitor species. Figure 4.46 A shows the impedance magnitude, $|\mathrm{Z}|$, and phase angle of AA2024 scratched in $\mathrm{pH} 6.5$ aqueous $1 \mathrm{M} \mathrm{NaCl}$. The average impedance magnitude and phase angle response is potential dependent. However, they do not show any sustained increase in magnitude during the length of the test implying that repassivation is difficult in $1 \mathrm{M} \mathrm{NaCl}$. Figure $4.46 \mathrm{~B}$ shows the impedance magnitude, $|\mathrm{Z}|$, and phase of AA2024 scratched in $\mathrm{pH} 6.5$ aqueous $1 \mathrm{M} \mathrm{NaCl}$ with the addition of 0.1 $\mathrm{M} \mathrm{CrO}_{4}{ }^{2-}$. In contrast to the chloride solution, the chromate containing solution show a steady increase in impedance magnitude and phase angle at -0.7 and $-0.8 \mathrm{~V}$ (SCE) from values of $7 \Omega-\mathrm{cm}^{2}$ and $-40^{\circ}$ to $30 \Omega-\mathrm{cm}^{2}$ and $-80^{\circ}$. At $-0.6 \mathrm{~V}$ (SCE) the impedance is similar to the uninhibited solution with values of $2 \Omega-\mathrm{cm}^{2}$. Figure $4.46 \mathrm{C}$ shows the impedance and phase of AA2024 scratched in $\mathrm{pH} 6.5$ aqueous $1 \mathrm{M} \mathrm{NaCl}$ with the addition of $0.1 \mathrm{M} \mathrm{MoO}_{4}{ }^{2-}$. At short times the impedance is lower than in the uninhibited solution. However, over time the impedance increases to levels higher than the uninhibited solution. At 1000 seconds the impedance and phase angle are $2 \Omega-\mathrm{cm}^{2}$ and $10^{\circ}$ at $-0.6 \mathrm{~V}$ (SCE) to 
$20 \Omega-\mathrm{cm}^{2}$ and $60^{\circ}$ at $-0.8 \mathrm{~V}(\mathrm{SCE})$. Figure $4.46 \mathrm{D}$ shows the impedance and phase of AA2024 scratched in $\mathrm{pH} 6.5$ aqueous $1 \mathrm{M} \mathrm{NaCl}$ with the addition of $0.1 \mathrm{M} \mathrm{MnO}_{4}^{-}$. For permanganate the impedance and magnitude of the phase angle increase steadily over time at all measured potentials, indicating some formation and increase in barrier film properties over the uninhibited solution.

Figure 4.47 shows similar impedance response at $\mathrm{pH} 3$ to that found at $\mathrm{pH}$ 6.5. Again, chromate, molybdate, and permanganate show increases in impedance magnitude and phase magnitude. The only drastic difference in response is for the chromate containing solution at $-0.6 \mathrm{~V}(\mathrm{SCE})$. The impedance response in this case is so poor that the measurement is an order of magnitude below the uninhibited solution at short times.

Figure 4.48 and 4.49 show the impedance at $1000 \mathrm{~Hz}$ of scratched AA7075 at pH 6.5 and $\mathrm{pH} 3$, respectively. The impedance in the capacitive region is investigated with $\mathrm{CrO}_{4}{ }^{2-}$, $\mathrm{MoO}_{4}{ }^{2-}$, and $\mathrm{MnO}_{4}{ }^{-}$inhibitor additions. At $\mathrm{pH} 6.5$ both chromate and permanganate show high impedance magnitude at $1000 \mathrm{~Hz}$ of $20-30 \Omega-\mathrm{cm}^{2}$ at $-0.8 \mathrm{~V}(\mathrm{SCE})$ and chromate maintains a high phase angle of $-70^{\circ}$ after only a tenth of a second. Permanganate takes almost 10 seconds to reach a phase angle bellow $-60^{\circ}$. Molybdate does not show much improvement over the uninhibited solution, only showing a slight increase in the phase angle at $-0.8 \mathrm{~V}$ (SCE). At $\mathrm{pH} 3$ little to no effect of inhibitor is observed on the impedance magnitude or phase angle with values recovering from around $5-10 \Omega-\mathrm{cm}^{2}$ to $30-40 \Omega-\mathrm{cm}^{2}$ for the inhibited solutions at $-0.8 \mathrm{~V}$ (SCE). The uninhibited solution did show a breakdown in recovered impedance at longer times. There are still large differences from -0.8 to -0.6 V (SCE) but these differences are largely independent of inhibitor presence. 


\subsection{Discussion}

\subsubsection{Effect of Selected Inhibitor additions on Current Transient Behavior of AA2024 and AA7075}

The transient current behavior is characterized by an exponential constant $m$ obtained from Equation 4.11 and reported in Figure 4.50. The significance of this is best understood by examining the constant $m$ in light of the theoretical high-field ion conduction kinetics and direct logarithmic film growth kinetics model. In the high field model one of the governing assumptions is that all current is consumed in the growth of the nucleating and thickening oxide. The derivation can be found in the literature ${ }^{[64-68]}$. This assumption is critical and it constrains the allowable values of $\partial \log i / \partial \log t$ transient behavior, or $m$, to between -0.8 and $-0.92^{[64]}$. In the case of the logarithmic models for oxide growth, the assumption leads to a slope of -1, exactly ${ }^{[64]}$. In the case of AA2024 and AA7075 large deviation from either of the models allowable constants suggests that the assumption that current can be attributed solely to oxide growth is not one that can be made. In Figure 4.50 the value of $m$ vs potential with and without inhibiting species show several interesting trends. If one assumes that values of $m$ approaching -1 indicate that anodic current density is being utilized more efficiently to nucleate and thicken the barrier oxide then the effect of the inhibitors on this process can be analyzed. For the chromate ion, the value of $m$ is more negative over the vast majority of potentials when compared to either $0.05 \mathrm{M} \mathrm{NaCl}$ or the molybdate inhibited solutions (Figure 4.48). The value of $m$ is -0.99 for the chromate containing solution compared to -0.79 for the $0.05 \mathrm{~m} \mathrm{NaCl}$ solution as seen in Figure 4.48. This suggests that chromate is effecting the anodic oxide film growth in a beneficial manner. 
The molybdate ion does not appear to have any drastic effect on the oxide growth kinetics as determined by analysis of scratch repassivation net current characteristics. Whether there is any benefit over the uninhibited solution conditions is debatable as judged from $m$. Beneficial inhibitor effects manifest themselves as a trend of $m$ values towards the bottom right of the graphs or -1 , indicating better repassivation. In the case of AA2024, molybdate and the chloride solutions appear to occupy the same plane in regards to the trend while the chromate ion occupies the area to the lower right. In the case of AA7075 there does appear to be some improvement with the addition of molybdate but not to the extent of the chromate anion.

The initial stages of current density decay in Figure 4.35, 4.37, 4.39, 4.4 , 4.42, 4.44, and 4.45 deviate from logarithmic behavior, showing instead a plateau with - $\partial \log i / \partial \log t$ rising through values that are far above unity. This plateau has two origins; the first is the current limited by ohms law and ohmic potential drop ${ }^{[64]}$. The finite electrolyte resistant leads to the aforementioned voltage drop shown in Figure 4.36. In addition to the ohmic potential drop effects on the current, there is also the effect of scratch speed. During the initial depassivation event, the scribe passes along the surface of the electrode rupturing the oxide film. The oxide immediately begins to nucleate and grown in the scribe wake. During this short time period while the scribe is in contact with the electrode, oxide growth in the scribe wake will lead to a moderation in the peak current density ${ }^{[30]}$. 


\subsubsection{Comparison of Current Transient Behavior of AA2024 and AA7075}

AA2024-T351 shows an onset of sustained depassivated current densities offset towards higher potentials than AA7075-T6. This may result from the beneficial effect of higher copper content in the alloy. A higher level of soluble copper in solid solution in the aluminum matrix has been shown to have a positive effect on pitting potential and it is reasonable to believe the effect may also be seen in repassivation behavior in scratched

electrode experiments ${ }^{[69,70]}$. AA7075 is richer in magnesium $(2.5 \mathrm{wt} \%)$ and zinc $(5.6 \mathrm{wt} \%)$ then AA2024 which may contribute to the observed onset of high depassivated current densities at most potentials indicating greater difficulty of repassivation.

Figure 4.51 and 4.52 demonstrate the effect of chloride concentration on the E-logi behavior of high purity aluminum, AA7075, and AA2024. The data used to construct these E-logi maps was obtained from the scratch transient data Figure 4.37 and 4.39. For each potential, current densities over several decades of repassivation time were plotted versus the applied potential. Again the effect of solid solution copper can be seen in the differences between AA2024 and AA7075 where AA2024 shows decreases by orders of magnitude after 1 and 10 seconds at $-0.6 \mathrm{~V}$ (SCE) as compared to AA7075.

\subsubsection{Effect of Chloride Concentration, $\mathrm{pH}$, and Potential}

Figure 4.53 and 4.54 show the effect of molybdate and chromate on the reconstructed E$\log i$ behavior of AA2024 and AA7075. Figure 4.53 shows a large decrease in current density with the addition of chromate to solution as soon as $10 \mathrm{~ms}$ after depassivation for 
AA7075. Likewise, the same is seen for AA2024 in Figure 4.54 where even at potentials as high as $-0.3 \mathrm{~V}$ (SCE) chromate has a drastic effect in current density as compared to the uninhibited solution.

Figure 4.55 shows the effect of increasing the ratio of chloride to inhibitor anion. For all inhibitor species no change in the current density is observed. The difficulty of inhibiting $1 \mathrm{M} \mathrm{NaCl}$ is shown in the anodic polarization data in Figure 4.19 through 4.26 as well as the scratch transient data in Figure 4.44 and 4.45. Some evidence of increases in barrier properties in the presence of inhibitors in the impedance data in Figure 4.46 through 4.49 where increases in both $|\mathrm{Z}|$ and the capacitive nature of the phase angle were apparent at potentials of -0.8 and -0.7 .

Equation 4.6 and 4.7 were used to calculate the oxide thickness vs. time for AA7075-T6 scratch depassivated in $1.0 \mathrm{M} \mathrm{NaCl}$ with $0.1 \mathrm{M} \mathrm{CrO}_{4}{ }^{2-}$ solution shown in Figure 4.56.

\subsubsection{Effects Relevant to EFCP and Implications Toward Understanding of EFCP}

The reciprocal of the critical frequency $\left(f_{c r i t}\right)$ shown in Figure 4.2 and 4.3 during fatigue crack growth ranges from 0.1 to 30 seconds for molybdate and 0.01 to 1 second for chromate ${ }^{[17]}$. At the short times the inhibitor is no longer effective. These critical frequencies are hypothesized to be related to a repassivation time constant. The EFCP data for molybdate in Figure 4.2 was collected for a solution that had a concentration of chloride that was an order of magnitude lower than the solution from which the chromate EFCP data in Figure 4.3 was produced. The critical frequency for molybdate 
was also an order of magnitude lower for the molybdate inhibitor as compared to chromate. However, from the data collected it would appear that molybdate does not possess the same ability as chromate to rapidly influence oxide film formation and growth. Assumptions about the nature of the crack tip need to be made in order to compare the electrochemical data compiled here to the critical frequencies found in the EFCP measurements. If, as has been discussed, the chloride concentration at the crack tip approaches $1 \mathrm{M}$ there is little evidence that any significant decrease in current density would occur over the time window relevant to EFCP as shown in Figure 4.55. However, if the chloride concentration was closer to $0.05 \mathrm{M}$ significant decreases in the current density of the freshly bared surface might be expected as shown in Figure 4.53. In the case of chromate, in the window of 0.01 to 10 seconds there is clear evidence of some inhibitor effect on the E-logi behavior of depassivated AA7075. Figure 4.50 also suggests that in the presence of chromate, significant inhibitor effects on the stability of the repassivating oxide are present as indicated by the trend in the logarithmic constant $m$ (Figure 4.50). Perhaps the most encouraging indication that chromate has an effect on oxide barrier properties even at high chloride concentrations is seen in the impedance data presented in Figure 4.48. AA7075 at $-0.8 \mathrm{~V}$ (SCE) shows a very clear distinction in both the impedance magnitude, $|\mathrm{Z}|$, and the accompanying phase angle. Starting at 0.05 seconds an order of magnitude difference in $|\mathrm{Z}|$ can be seen and the phase angle at 0.05 seconds has gone from approximately $0^{\circ}$ in the uninhibited solution to almost $60^{\circ}$ in the chromate containing solution. The fact that these time scales align quite well with the frequency dependence of the EFCP behavior is encouraging. 
The effect of molybdate on the repassivation kinetics of AA7075 is not as well documented as chromate. There is an argument that molybdate is not as strong an inhibitor of EFCP to begin with[14]. There is no impedance evidence to support the improvement of barrier properties with the addition of molybdate. Neither impedance magnitude or phase angle are changed drastically with molybdate addition to chloride solutions at pH 6.5 or 3 as shown in Figure 4.46 through 4.49. Nor is there evidence that anodic reaction kinetics are effected at high chloride concentrations There is some evidence that at lower concentrations of chloride there is a inhibiting effect of $\mathrm{MoO}_{4}{ }^{2-}$ on the repassivation anodic current density. This is reflected in Figure 4.50. The anodic inhibition at longer times is evident through the subtle changes in the anodic polarization data shown in Figure 4.22. Thus, at lower chloride concentrations the results here support the notion that EFCP might be inhibited through improved oxide stability.

Permanganate, while not tested in the EFCP literature, does exhibit promise in the form of suppressed transient current densities in strong chloride solutions as shown in Figure 4.44 as well as moderate improvement in $|\mathrm{Z}|$ and phase angle data as seen in Figure 4.48 and 4.49. Additionally, the strong oxidizing nature of permanganate acts to moderate the potential drop during film rupture events. This has the effect of reducing the hydrogen evolution over potential which could decrease the amount of adsorbed atomic hydrogen available for diffusion into the fracture process zone. 


\subsubsection{Alternative Mechanisms of EFCP Inhibition}

There are other possibilities that can account for the benefit by molybdate as seen in Figure $4.2^{[14]}$ besides the improvement of the repassivation characteristics. The first is that molybdate acts as a buffer in the crack tip. The buffer properties of molybdate are shown in Figure 4.29. It shows more buffer capacity than chromate in simulated crack tip solution $^{[63]}$. The connection between buffer capacity and critical frequency is not direct and may be complex making a mechanistic explanation for the frequency dependence of the EFCP inhibition of molybdate more difficult to test. It is possible that a buffer's ability to moderate $\mathrm{pH}$ at the crack tip could be overwhelmed at high frequency by the rapid dissolution and hydrolysis of the exposed aluminum at the crack tip.

\subsubsection{Remaining Concerns for Repassivation as it Relates to SCC and EFCP}

No direct evidence of incorporation of $\mathrm{Cr}$, Mo, or Mn into repassivated oxide films has yet been demonstrated. Additionally there remains the question of how this repassivation data relates to actual hydrogen concentration below repassivated oxide layers. Answering these questions is critical to fully understanding the effect of these selected inhibitors on crack tip repassivation mechanisms.

\subsection{Conclusions}

The effect of inhibitor concentration, chloride concentration, and applied potential on the repassivation of AA2024, AA7075, and high purity aluminum has been investigated. 
Results to date have indicated that chromate is an inhibitor of net anodic scratch current densities as well as total charge after depassivation. This result is evident for both AA2024 and AA7075 at a neutral $\mathrm{pH}$ of 6.5. The net anodic charge passed in the presence of chromate is two orders of magnitude lower than uninhibited solution for AA2024 and one order of magnitude for AA7075. A protective oxide barrier is observed through a variation on the scratched electrode technique that exploits high frequency impedance measurements to observe oxide regrowth. Chromate reveals an order of magnitude increase in impedance magnitude and phase angle during repassivation in chromate inhibited vs. uninhibited chloride solutions at $\mathrm{pH}$ 6.5. Scratch current density reduction by molybdate of either AA2024 or AA7075 was not observed. Nor was molybdate observed to affect the impedance magnitude or phase angle of film growth after a rupture event. Acid-base titration has shown that in simulate crack tip solution molybdate can act as a strong buffer with a capacity 1.6 times higher than chromate. These findings are related to $1 / f$ in EFCP observations. This suggests that there are multiple mechanisms through which EFCP inhibition occurs as both chromate and molybdate inhibit crack growth rates ${ }^{[17]}$. However, it is unclear how $\mathrm{MoO}_{4}{ }^{2-}$ results in a similar $1 / f$ EFCP dependency.

\subsection{Acknowledgements}

This work was supported by SERDP project \# WP-1621 and the Office of the Secretary of Defense (UCC) and Gamry INST. 


\subsection{References}

1. T.H. Nguyen and R.T. Foley, "The Chemical Nature of Aluminum Corrosion: III . The Dissolution Mechanism of Aluminum Oxide and Aluminum Powder in Various Electrolytes," Journal of the Electrochemical Society 127, 12 (1980): p. 2563.

2. N.J.H. Holroyd and G.M. Scamans, "Stress Corrosion Cracking in Al-Zn-Mg-Cu Aluminum Alloys in Saline Environments," Metall and Mat Trans A (2012): p. 1.

3. K.R. Cooper, Chemistry and Electrochemistry of Environment-assisted Cracking of an $\mathrm{Al}-\mathrm{Zn}-\mathrm{Mg}-\mathrm{Cu}$ Alloy: University of Virginia, 2001.

4. G.O. Ilevbare and J.R. Scully, "Mass-transport-limited oxygen reduction reaction on AA2024-T3 and selected intermetallic compounds in chromate-containing solutions," Corrosion 57, 2 (2001): p. 134.

5. G.A. Young Jr and J.R. Scully, "The diffusion and trapping of hydrogen in high purity aluminum," Acta Materialia 46, 18 (1998): p. 6337.

6. J.S. Newman and K.E. Thomas-Alyea, Electrochemical systems. 3rd ed (Hoboken, N.J.: J. Wiley, 2004). p. xx.

7. E. McCafferty, Introduction to corrosion science (New York: Springer, 2010). p. xvi.

8. Z.M. Gasem, Frequency dependant environmental fatigue crack propagation in the 7XXX alloy/aqueous chloride system., in MSE PhD Thesis. 1999, University of Virginia: Charlottesville.

9. J.R. Rice, "Some mechanics research topics related to the hydrogen embrittlement of metals," CORROSION 32, 1 (1976): p. 22.

10. M.O. Speidel, "Stress-Corrosion Cracking of Aluminum-Alloys," Metall Trans A 6, 4 (1975): p. 631.

11. J. R. Davis \& Associates. and ASM International. Handbook Committee., Aluminum and aluminum alloys. ASM specialty handbook (Materials Park, $\mathrm{OH}$ : ASM International, 1993). p. iii.

12. R.P. Gangloff and M.B. Ives, eds. Environment-Induced Cracking of Metals. 1989, NACE: Houston, TX. 55.

13. A. Handbook, "Volume 13B," Corrosion: Materials (2005).

14. J.S. Warner, Inhibition of environmental fatigue crack propagation in agehardenable aluminum alloys, in MSE PhD Thesis. 2010, University of Virginia: Charlottesville.

15. J.H. Ai and J.R. Scully, "Hydrogen Diffusivity During Corrosion of High-Purity Aluminum," Corrosion 69, 8 (2013): p. 752.

16. J.R. Scully, R.P. Gangloff, and B.P. Somerday, 19. Hydrogen embrittlement of aluminum and aluminum-based alloys, in Gaseous hydrogen embrittlement of materials in energy technologies. 2012, Woodhead Publishing: Cambridge, UK ; Phildelphia, PA.

17. J.S. Warner, S. Kim, and R.P. Gangloff, "Molybdate inhibition of environmental fatigue crack propagation in $\mathrm{Al}-\mathrm{Zn}-\mathrm{Mg}-\mathrm{Cu}$," International Journal of Fatigue 31, 11-12 (2009): p. 1952. 
18. Z.M. Gasem and R.P. Gangloff, "Effect of temper on environmental fatigue crack propagation in 7000-series aluminum alloys," Mater Sci Forum 331-3, (2000): p. 1479.

19. M. Pourbaix, Atlas of electrochemical equilibria in aqueous solutions. 2d English ed (Houston, Tex.: National Association of Corrosion Engineers, 1974). p. 644 p.

20. S.B. Madden and J.R. Scully, "Inhibition of AA2024-T351 Corrosion Using Permanganate," Journal of The Electrochemical Society 161, 3 (2014): p. C162.

21. O. Lopez-Garrity and G.S. Frankel, "Corrosion Inhibition of Aluminum Alloy 2024-T3 by Sodium Molybdate," Journal of The Electrochemical Society 161, 3 (2014): p. C95.

22. M.W. Kendig and R.G. Buchheit, "Corrosion inhibition of aluminum and aluminum alloys by soluble chromates, chromate coatings, and chromate-free coatings," Corrosion 59, 5 (2003): p. 379.

23. D.G. Kolman and J.R. Scully, "Limitations of Potentiostatic Repassivation Techniques and Their Relationship to the Applicability of the High-Field Approximation to the Repassivation of Titanium," Journal of the Electrochemical Society 142, 7 (1995): p. 2179.

24. G.S. Frankel, C.V. Jahnes, V. Brusic, and A.J. Davenport, "Repassivation Transients Measured with the Breaking-Electrode Technique on Aluminum ThinFilm Samples," Journal of the Electrochemical Society 142, 7 (1995): p. 2290.

25. G.S. Frankel, B.M. Rush, C.V. Jahnes, C.E. Farrell, A.J. Davenport, and H.S. Isaacs, "Repassivation Transients Measured with Thin Film Breaking Electrodes," Journal of The Electrochemical Society 138, 2 (1991): p. 643.

26. R.P. Frankenthal, J. Kruger, and Electrochemical Society., Passivity of metals : (proceedings of the fourth International Symposium on Passivity). The Corrosion monograph series (Princeton, N.J.: Electrochemical Society, 1978). p. xviii.

27. R. Oltra and G.M. Indrianjafy, "Depassivation Experiments by Laser-PulseInduced Spallation," Journal of The Electrochemical Society 139, 9 (1992): p. L78.

28. G.T. Burstein and R.J. Cinderey, "Evolution of the corrosion potential of repassivating aluminium surfaces," Corrosion Science 33, 3 (1992): p. 475.

29. N.D. Tomashov and L.P. Vershinina, "Kinetics of some electrode processes on a continuously renewed surface of solid metal," Electrochimica Acta 15, 4 (1970): p. 501.

30. D.G. Kolman and J.R. Scully, "On the repassivation behavior of high-purity titanium and selected alpha, beta, and beta+alpha titanium alloys in aqueous chloride solutions," Journal of the Electrochemical Society 143, 6 (1996): p. 1847.

31. G.T. Burstein and D.H. Davies, "The Electrochemical Behavior of Scratched Iron Surfaces in Aqueous Solutions," Journal of The Electrochemical Society 128, 1 (1981): p. 33.

32. G.T. Burstein and P.I. Marshall, "Growth of Passivating Films on Scratched 3041 Stainless-Steel in Alkaline-Solution," Corrosion Science 23, 2 (1983): p. 125.

33. F.P. Ford, G.T. Burstein, and T.P. Hoar, "Bare Surface Reaction Rates and Their Relation to Environment Controlled Cracking of Aluminum Alloys," Journal of The Electrochemical Society 127, 6 (1980): p. 1325. 
34. W.J. Rudd and J.C. Scully, "The function of the repassivation process in the inhibition of pitting corrosion on aluminium," Corrosion Science 20, 5 (1980): p. 611.

35. R.J. Cinderey and G.T. Burstein, "The effects of chromate on the transient repassivation potential of aluminium in chloride solution," Corrosion Science 33, 3 (1992): p. 493.

36. P. Schmutz and G.S. Frankel, "Influence of dichromate ions on corrosion of pure aluminum and AA2024-T3 in NaCl solution studied by AFM scratching," Journal of the Electrochemical Society 146, 12 (1999): p. 4461.

37. C. Devicharan, M.J. Vasquez, P.H. Gary, and R.C. Clive, "Studies on the repassivation behavior of aluminum and aluminum alloy exposed to chromate solutions," Surface and Interface Analysis 35, 2 (2003): p. 226.

38. D.G. Kolman and J.R. Scully, "On the Repassivation Behavior of High-Purity Titanium and Selected alpha, beta, and beta + alpha Titanium Alloys in Aqueous Chloride Solutions," Journal of The Electrochemical Society 143, 6 (1996): p. 1847.

39. R.S. Lillard, G. Vasquez, and D.F. Bahr, "The Kinetics of Anodic Dissolution and Repassivation on Stainless Steel 304L in Solutions Containing Nitrate," Journal of the Electrochemical Society 158, 6 (2011): p. C194.

40. G.T. Burstein and D.H. Davies, "The Effects of Anions on the Behavior of Scratched Iron Electrodes in Aqueous-Solutions, "Corrosion Science 20, 10 (1980): p. 1143.

41. G.T. Burstein and G. Gao, "Verification of the Validity of Peak Bare Surface Current Densities Obtained from the Scratched Electrode," Journal of the Electrochemical Society 138, 9 (1991): p. 2627.

42. R.P. Wei and M. Gao, "Further Observations on the Validity of Bare Surface Current Densities Determined by the Scratched Electrode Technique," Journal of the Electrochemical Society 138, 9 (1991): p. 2601.

43. R.G. Kelly and R.C. Newman, "Confirmation of the Applicability of Scratched Electrode Techniques for the Determination of Bare Surface Current Densities," Journal of the Electrochemical Society 137, 1 (1990): p. 357.

44. H.C. Lee, F. Xu, C.S. Jeffcoate, and H.S. Isaacs, "Cyclic polarization behavior of aluminum oxide films in near neutral solutions," Electrochem Solid St 4, 10 (2001): p. B31.

45. D.G. Kolman and J.R. Scully, "An assessment of the crack tip potential of betatitanium alloys during hydrogen environmentally assisted crack propagation based on crack tip and passive surface electrochemical measurements," Corrosion Science 42, 11 (2000): p. 1863.

46. J.R. Scully, D.C. Silverman, and M.W. Kendig, Electrochemical Impedance: Analysis and Interpretation. Stp (Philadelphia: ASTM, 1993.

47. J.R. Scully, "Characterization of the Corrosion of Aluminum Thin Films using Electrochemical Impedance Methods " Electrochemical Impedance: Analysis and Interpretation ASTM STP 1188, (1993): p. 276.

48. C.H. Hamann, A. Hamnett, and W. Vielstich, Electrochemistry (Weinheim ; New York: Wiley-VCH, 1998). p. xvii. 
49. J.R. Scully, R.P. Frankenthal, K.J. Hanson, D.J. Siconolfi, and J.D. Sinclair, "Localized Corrosion of Sputtered Aluminum and Al-0.5-Percent $\mathrm{Cu}$ Alloy ThinFilms in Aqueous Hf Solution .2. Inhibition by $\mathrm{CO}_{2}$," Journal of the Electrochemical Society 137, 5 (1990): p. 1373.

50. G.W. Walter, "A review of impedance plot methods used for corrosion performance analysis of painted metals," Corrosion Science 26, 9 (1986): p. 681.

51. J.M. W.J. Eggers, J.R. Scully, R. Spotnitz, S.R. Taylor A Short Course on Electrochemical Impedance Spectroscopy (Charlottesville University of Virginia, 2002.

52. J.H. Jun, H.J. Kim, and D.J. Choi, "Effect of the hydration on the properties of an aluminum oxide film," Journal of Ceramic Processing Research 9, 1 (2008): p. 75.

53. P.D. Bastek, R.C. Newman, and R.G. Kelly, "Measurement of Passive Film Effects on Scratched Electrode Behavior," Journal of the Electrochemical Society 140, 7 (1993): p. 1884.

54. B. Ulgut, Personal Correspondence concerning Scratch Test Impedance, S.B. Madden, Editor. 2013: Charlottesville

55. G.O. Ilevbare, J.R. Scully, J. Yuan, and R.G. Kelly, "Inhibition of pitting corrosion on aluminum alloy 2024-T3: Effect of soluble chromate additions vs chromate conversion coating," Corrosion 56, 3 (2000): p. 227.

56. G.O. Ilevbare, O. Schneider, R.G. Kelly, and J.R. Scully, "In situ confocal laser scanning microscopy of AA 2024-T3 corrosion metrology - I. Localized corrosion of particles," Journal of the Electrochemical Society 151, 8 (2004): p. B453.

57. M.A. Jakab, F. Presuel-Moreno, and J.R. Scully, "Critical Concentrations Associated with Cobalt, Cerium, and Molybdenum Inhibition of AA2024-T3 Corrosion: Delivery from Al-Co-Ce(-Mo) Alloys , March 2005," CORROSION 61, 03 (2005).

58. S.T. Pride, J.R. Scully, and J.L. Hudson, "Metastable Pitting of Aluminum and Criteria for the Transition to Stable Pit Growth," Journal of the Electrochemical Society 141, 11 (1994): p. 3028.

59. Z. Zhao and G.S. Frankel, "On the first breakdown in AA7075-T6," Corrosion Science 49, 7 (2007): p. 3064.

60. Z.J. Zhao and G.S. Frankel, "The effect of temper on the first breakdown in AA7075," Corrosion Science 49, 7 (2007): p. 3089.

61. Y. Liu, A. Laurino, T. Hashimoto, X. Zhou, P. Skeldon, G.E. Thompson, G.M. Scamans, C. Blanc, W.M. Rainforth, and M.F. Frolish, "Corrosion behaviour of mechanically polished AA7075-T6 aluminium alloy," Surface and Interface Analysis 42, 4 (2010): p. 185.

62. S. Maitra and G.C. English, "Mechanism of localized corrosion of 7075 alloy plate," MTA 12, 3 (1981): p. 535.

63. S.M. Al-Ghamdi, Thesis: The effect of aqueous phase inhibitors on mitigating potential-dependant hydrogen environmental assisted cracking of an ultra-high strength steel, in Materials Science and Engineering. May, 2010 University of Virginia: Charlottesville.

64. G.T. Burstein and A.J. Davenport, "The Current-Time Relationship during Anodic Oxide Film Growth under High Electric Field," Journal of The Electrochemical Society 136, 4 (1989): p. 936. 
65. M.M. Lohrengel, "Thin anodic oxide layers on aluminium and other valve metals: high field regime," Materials Science and Engineering: R: Reports 11, 6 (1993): p. 243.

66. E. Verwey, "Electrolytic conduction of a solid insulator at high fields The formation of the anodic oxide film on aluminium," Physica 2, 1 (1935): p. 1059.

67. P. Schmuki, "From Bacon to barriers: a review on the passivity of metals and alloys," Journal of Solid State Electrochemistry 6, 3 (2002): p. 145.

68. F.P. Fehlner and N.F. Mott, "Low-temperature oxidation," Oxid Met 2, 1 (1970): p. 59.

69. G.S. Chen, "Microconstituent-induced pitting corrosion in aluminum alloy 2024T3," CORROSION 52, 01 (1996).

70. D.A. Little, B.J. Connolly, and J.R. Scully, "An electrochemical framework to explain the intergranular stress corrosion behavior in two $\mathrm{Al}-\mathrm{Cu}-\mathrm{Mg}-\mathrm{Ag}$ alloys as a function of aging," Corrosion Science 49, 2 (2007): p. 347. 


\subsection{Tables}

Table 4.1. Chemical composition of AA2024-T351 and AA7075-T6 (wt. \%). AA2024 chemical composition measured with ICP-OES. AA7075 ingot metallurgy provided by Alcoa.

\begin{tabular}{lrrrrrrrr} 
Material & $\mathrm{Al}$ & $\mathrm{Cu}$ & $\mathrm{Cr}$ & $\mathrm{Mg}$ & $\mathrm{Mn}$ & $\mathrm{Fe}$ & $\mathrm{Zn}$ & $\mathrm{Si}$ \\
\hline AA2024-T351 & Balance & 4.5 & $<0.1$ & 1.2 & 0.6 & 0.2 & 0.1 & 0.1 \\
AA7075-T6 & Balance & 1.4 & 0.2 & 2.5 & $<0.1$ & 0.3 & 5.6 & 0.1 \\
$99.999 \% \mathrm{Al}$ & Balance & $<0.1$ & $<0.1$ & $<0.1$ & $<0.1$ & $<0.1$ & $<0.1$ & $<0.1$
\end{tabular}

Table 4.2. Fitting parameters for $99.999 \%$ aluminum at $-0.8 \mathrm{~V}$ (SCE)

\begin{tabular}{rr}
$\mathrm{Cl}^{-}(\mathrm{M})$ & $m$ \\
\hline 1.0 & 0.69 \\
0.5 & 0.51 \\
0.05 & 0.67
\end{tabular}




\subsection{Figures}

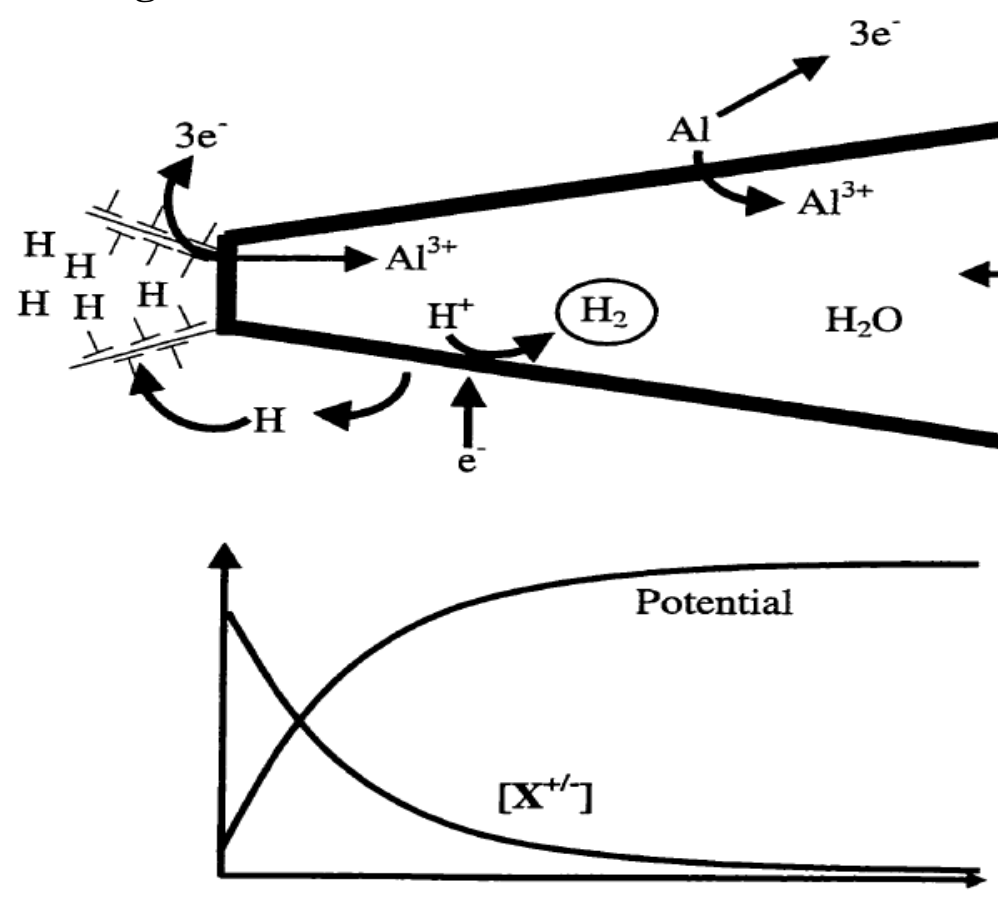

Distance from Crack Tip

$$
\begin{array}{ll}
\hline \text { Anodic Dissolution: } & \mathrm{Al} \rightarrow \mathrm{Al}^{3+}+3 e^{-} \\
\text {Hydrolysis: } & \mathrm{Al}^{3+}+y \mathrm{H}_{2} \mathrm{O} \rightarrow \mathrm{Al}(\mathrm{OH})_{y}{ }^{(3-y)+}+y \mathrm{H}^{+} \\
\text {Cathodic Reactions: } & \mathrm{H}^{+}+e^{-} \rightarrow \mathrm{H} \\
& \mathrm{H}_{2} \mathrm{O}+e^{-} \rightarrow \mathrm{H}+\mathrm{OH}
\end{array}
$$

Figure 4.1. Diagram illustrating crack tip processes that lead to the embrittlement of precipitation age hardened aluminum alloys in the fracture process zone through the uptake of hydrogen from proton and water reduction. Hydrolysis of dissolving aluminum can exacerbate hydrogen production and uptake by increasing $\mathrm{H}^{+}$concentration and raising $\mathrm{E}_{\mathrm{H}+} / \mathrm{E}_{\mathrm{H} 2}$. Inhibitors are thought to act through the passivation of the fractured surfaces, producing a decrease in dissolution and subsequently hydrolysis while providing a hydrogen permeation barrier. Buffering effects may also influence the crack tip environment.

Used with permission of UVA 


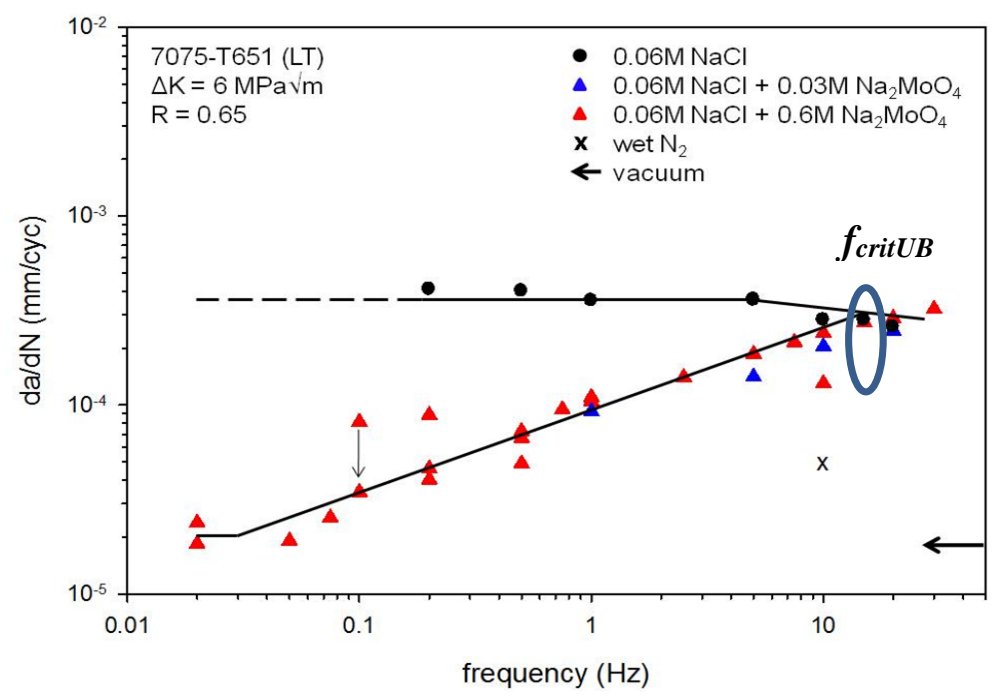

Figure 4.2. Crack growth rates for full immersion molybdate containing sodium chloride solutions as compared to sodium chloride alone $f_{\text {Crit }}$ is on the order of $10 \mathrm{~Hz}$.

Used with permission of UVA

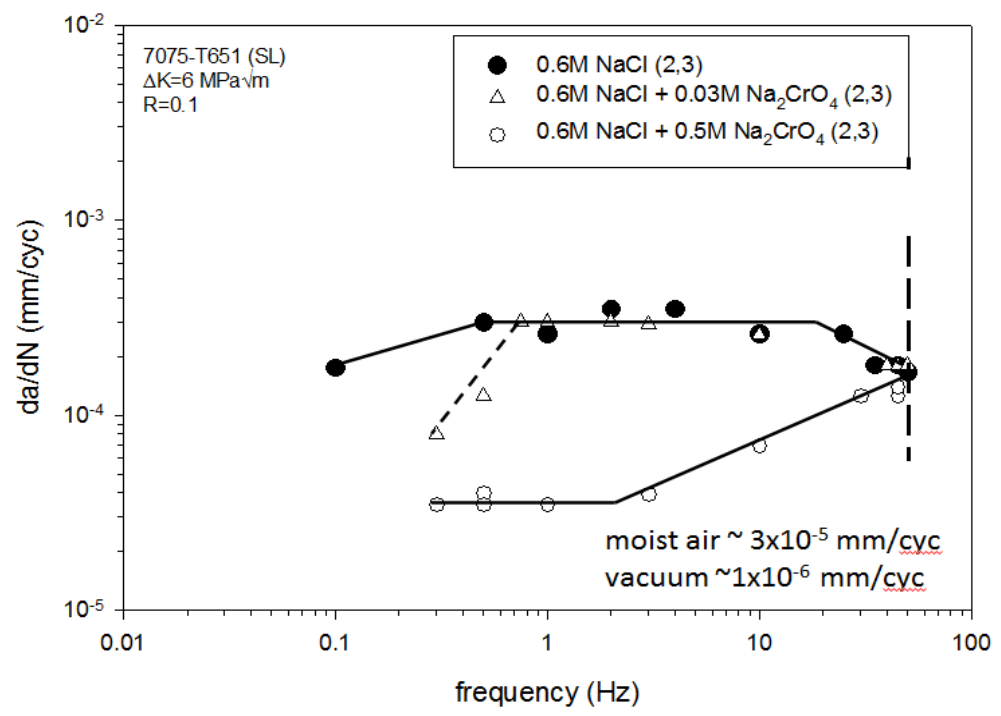

Figure 4.3. Crack growth rates for full immersion chromate containing sodium chloride solutions as compared to sodium chloride alone. $f_{C r i t}$ is on the order of $100 \mathrm{~Hz}$. Used with permission of UVA 


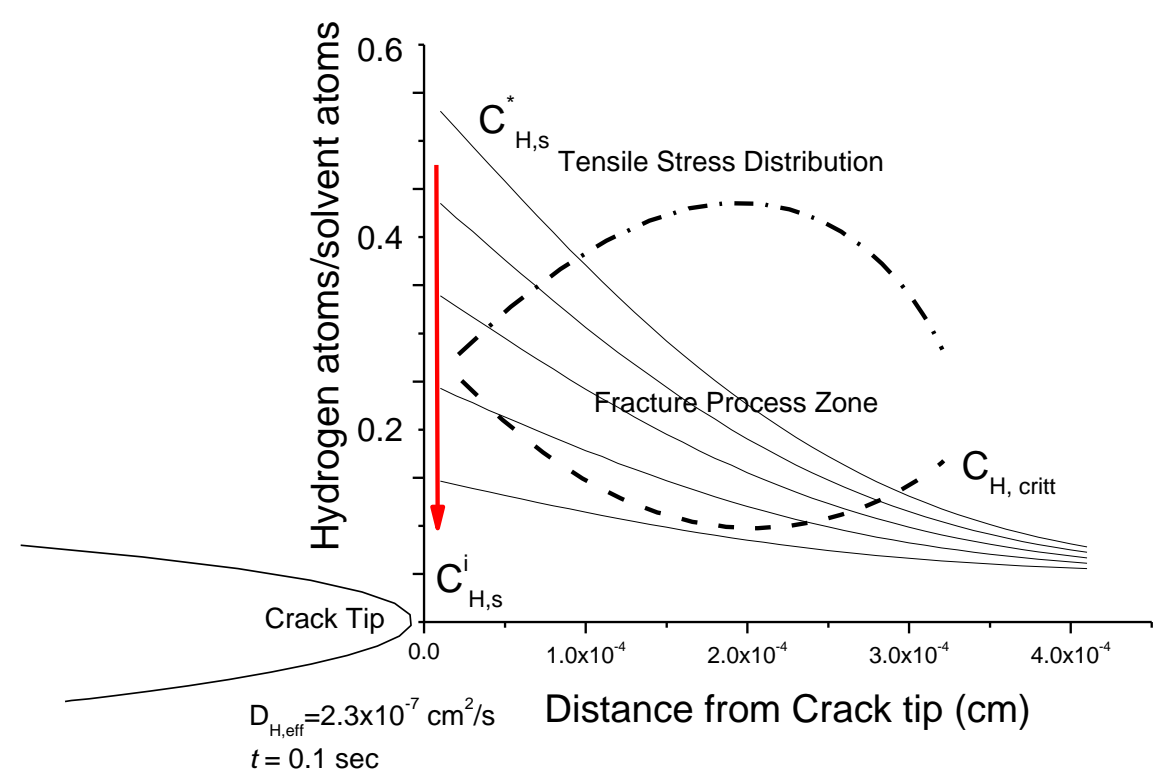

Figure 4.4. Effect of surface concentration from 0.5 to 0.1 hydrogen atoms per solvent atom on concentration gradient of hydrogen in a semi-infinite plate provided to show the possibility of lowering the internal hydrogen concentration bellow the critical concentration through the use of an inhibiting ion which lowers $\mathrm{C}_{\mathrm{H}, \mathrm{s}}^{*}$. 
a)

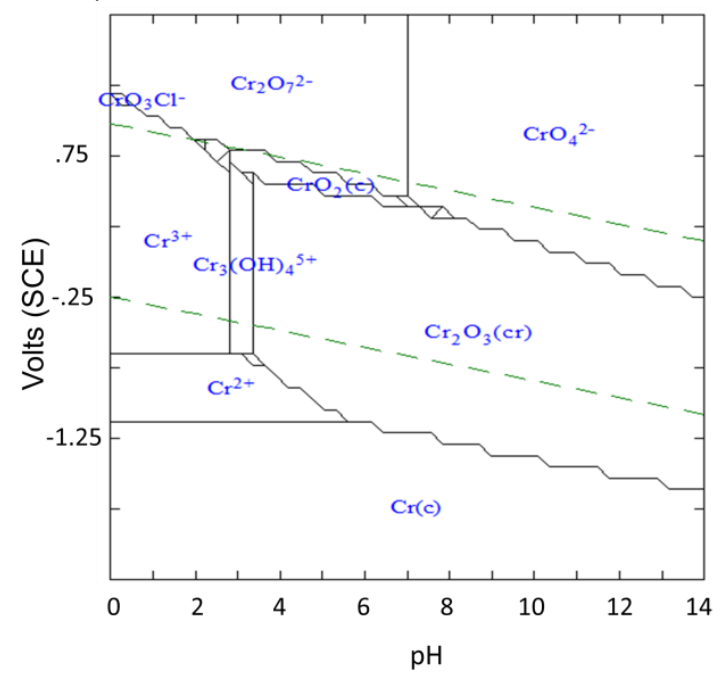

b)

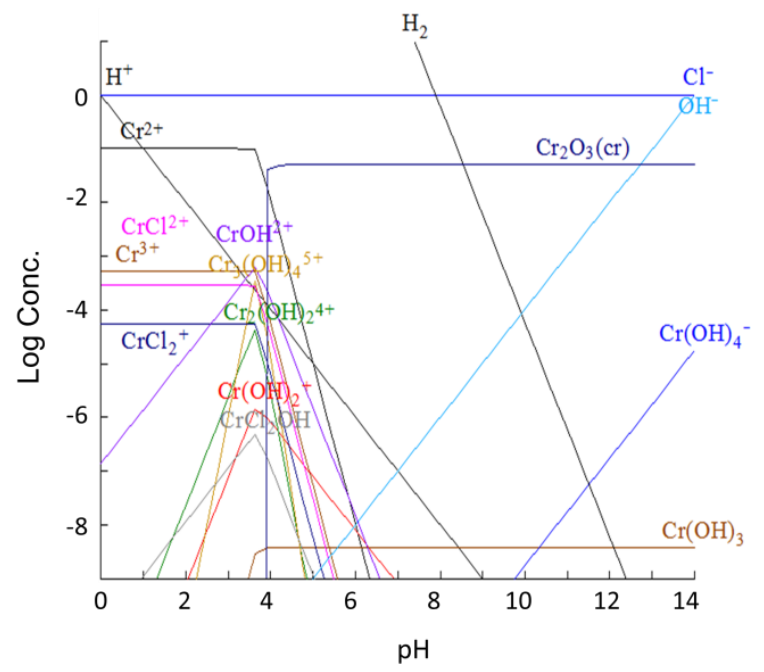

Figure 4.5. (a) E-pH diagram in $\mathrm{H}_{2} \mathrm{O}$ at $25^{\circ} \mathrm{C}$ for $0.1 \mathrm{M}$ chromate and $1 \mathrm{M}$ chloride showing the stability of chromium oxide over a large region of potential and $\mathrm{pH}$. (b) Speciation of chromium-chloride system at previously stated concentrations at $-0.8 \mathrm{~V}$ (SCE).
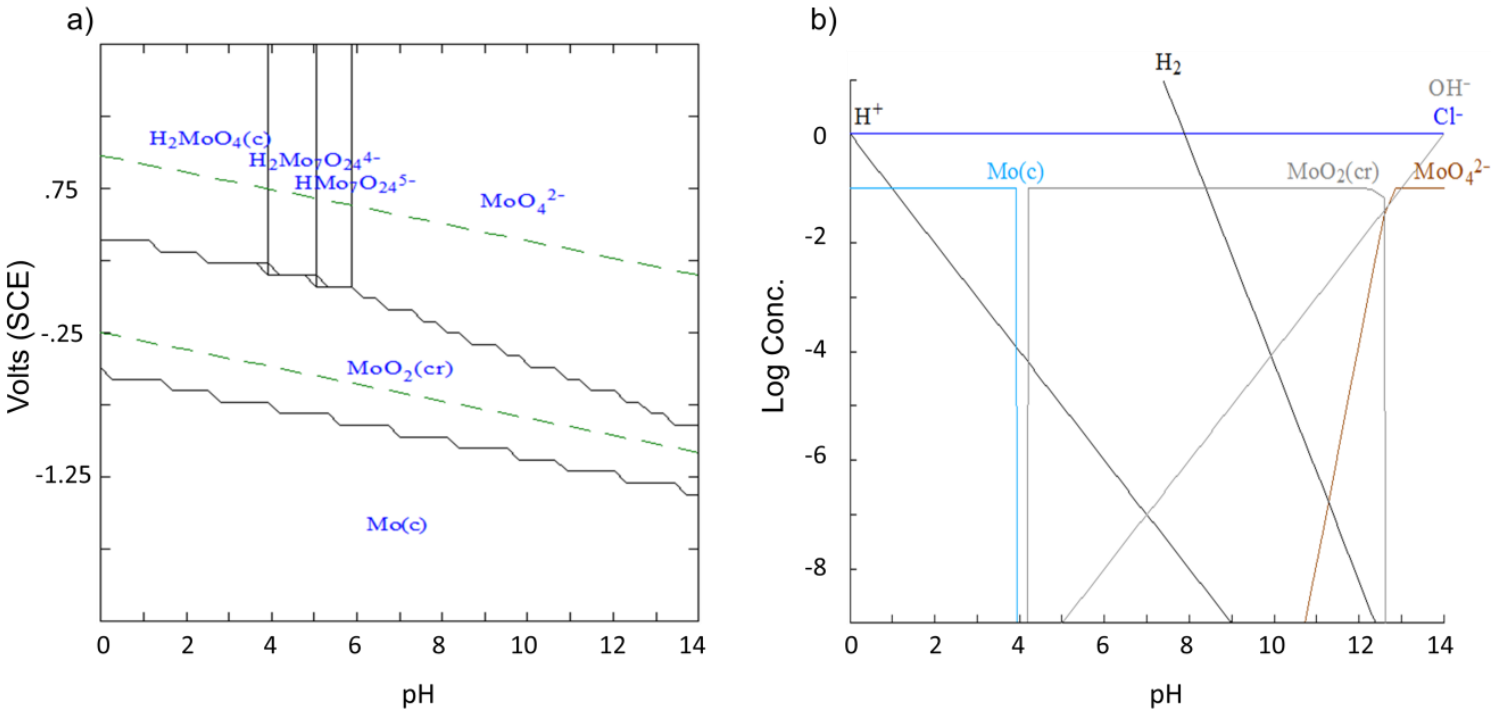

Figure 4.6. (a) E-pH diagram for 0.1 M molybdate and $1 \mathrm{M}$ chloride solution showing the stability of molybdenum oxide over a large region of potential and $\mathrm{pH}$ with high potential stability in the low $\mathrm{pH}$ region. (b) Speciation of molybdate-chloride system at previously stated concentrations at $-0.8 \mathrm{~V}$ (SCE). 


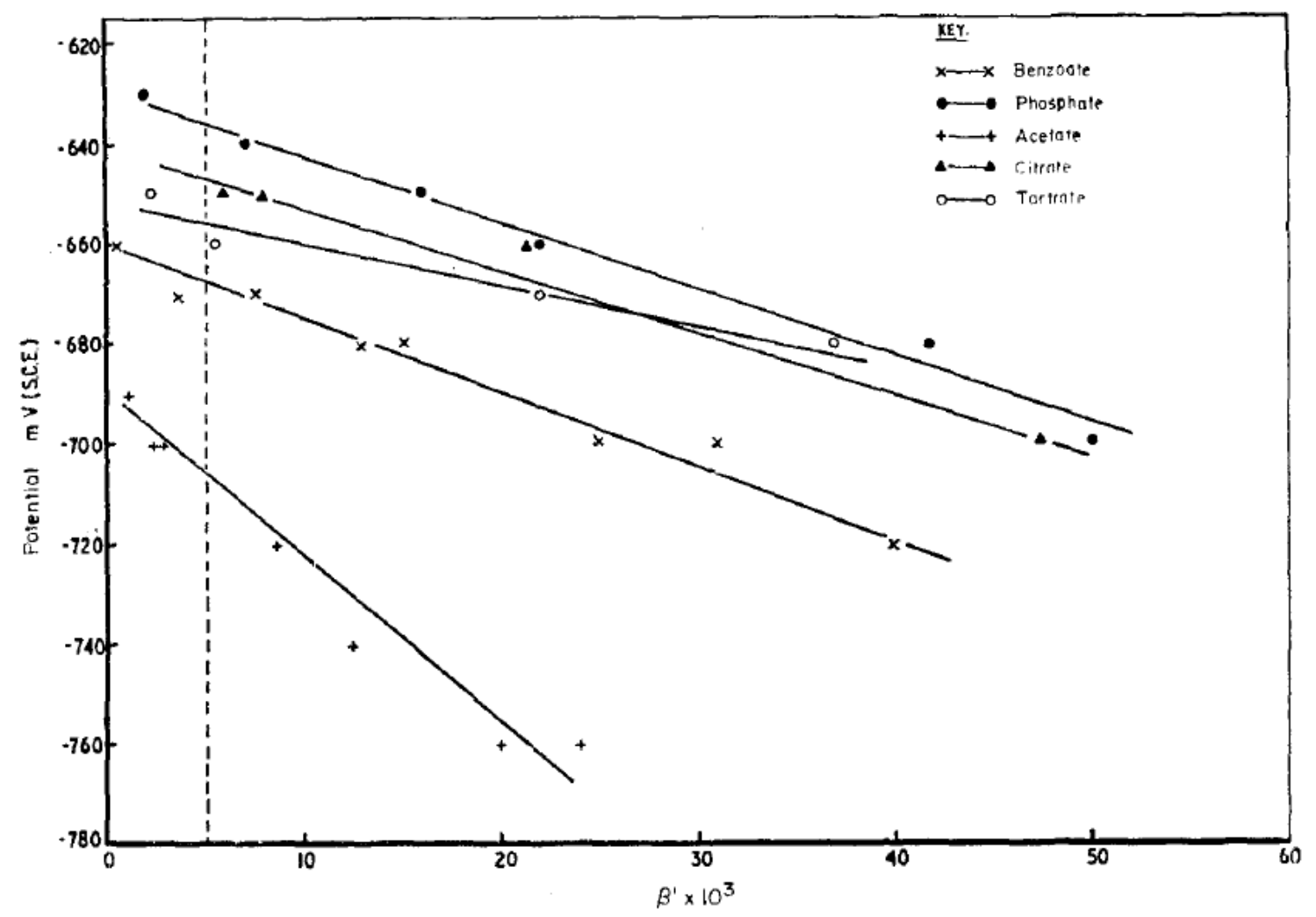

Figure 4.7. The effect of potential on the transient exponential behavior of anodic current decay as characterized by the constant $\beta$ on the high purity aluminum scratched in 0.085 $\mathrm{M} \mathrm{NaCl}$ with the addition of reported inhibitors with a concentration of $0.08 \mathrm{M}$. ( $\beta$ $\left.=2.3 \beta^{\prime}\right)$ 


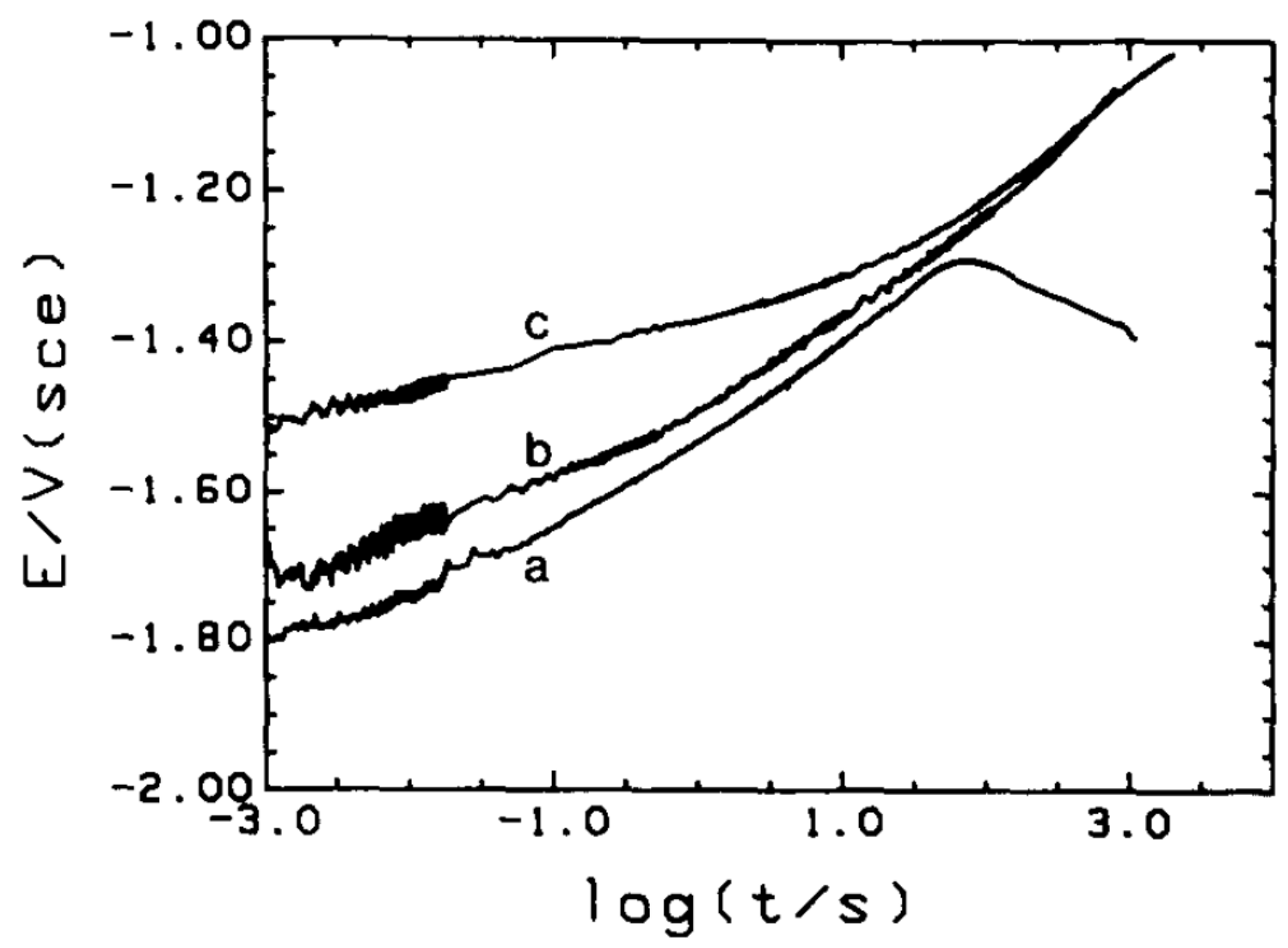

Figure 4.8. Effect of chromate on the open circuit potential of aluminum after it was depassivated in a guillotine experiment. Line $a$ was conducted in $0.1 \mathrm{M} \mathrm{NaCl}$ solution while $b$ and $c$ were conducted in 0.01 and $1.0 \mathrm{M} \mathrm{Na}_{2} \mathrm{CrO}_{4}$ solutions, respectively. Reproduced with permission of Corrosion Science 


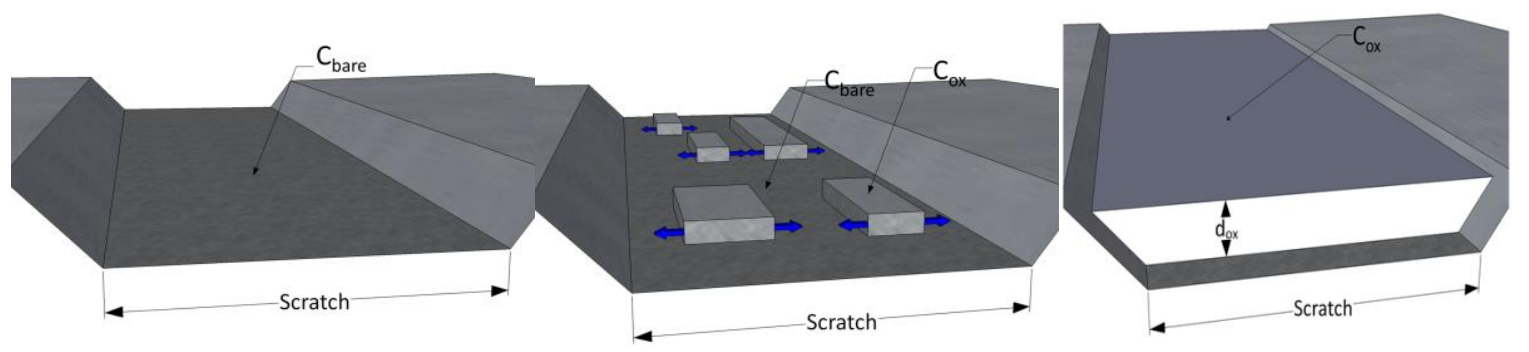

Figure 4.9. Schematic of an oxide covered surface after a scratch. The bare metallic electrode undergoes oxide nucleation and an increase in oxide surface coverage, followed by thickening of the oxide film as repassivation occurs. The thickness of the oxide layer is $\mathrm{d}_{\mathrm{ox}}$. 


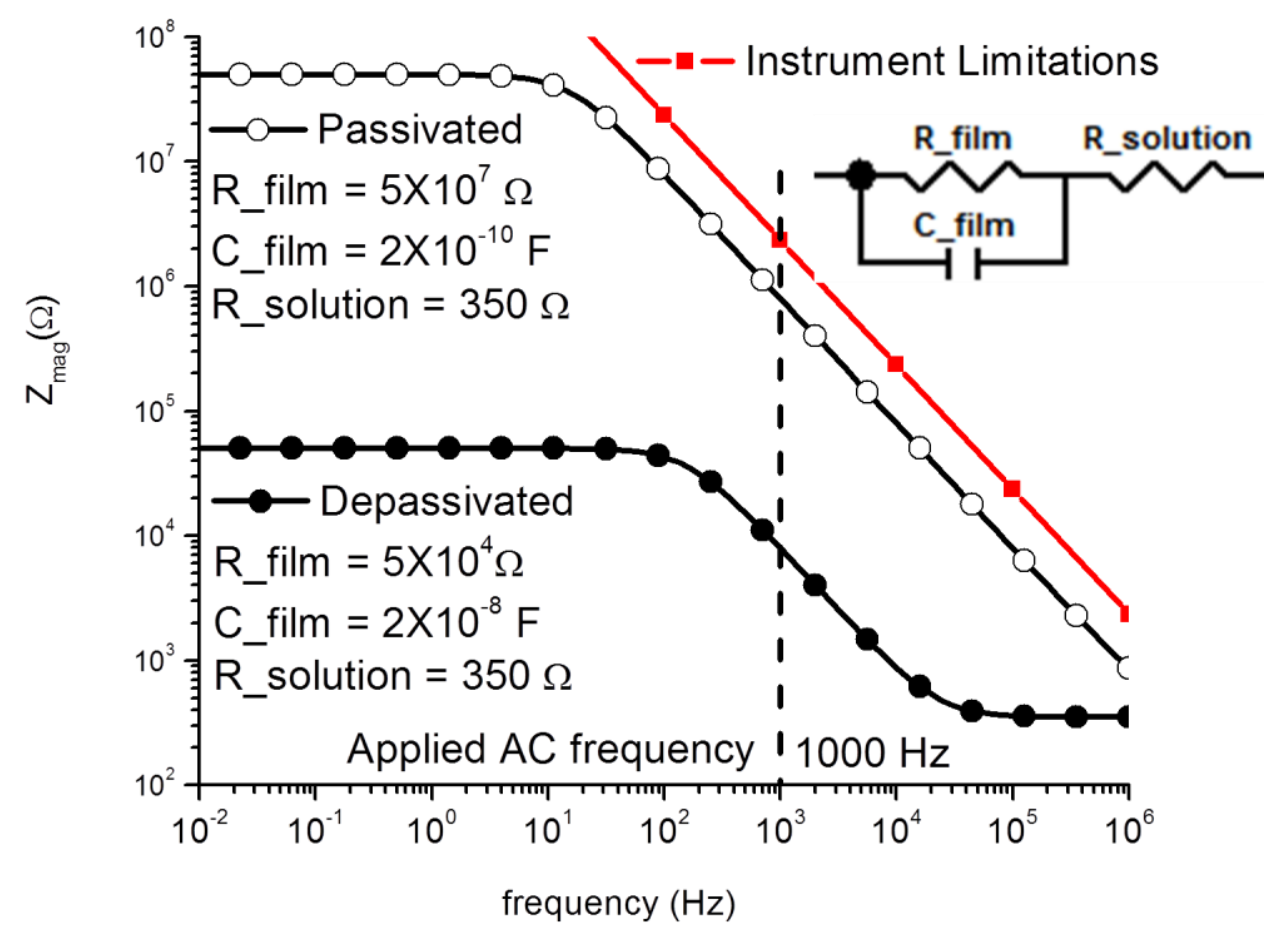

Figure 4.10. Theoretical model Bode impedance for depassivated and passive aluminum electrodes. Values were determined assuming a passivated $R_{\text {film }}$ value of $10^{4} \Omega-\mathrm{cm}^{2}$, depassivated $R_{\text {film }}$ of $10 \Omega-\mathrm{cm}^{2}$, and $R_{\text {solution }}$ of $350 \Omega$. Capacitance of $1 \mu \mathrm{F} / \mathrm{cm}^{2}$ and 100 $\mu \mathrm{F} / \mathrm{cm}^{2}$ were used for the passivated and bare electrodes, respectively. Values corrected for electrode surface area of $2 \times 10^{-4} \mathrm{~cm}^{2}$. 

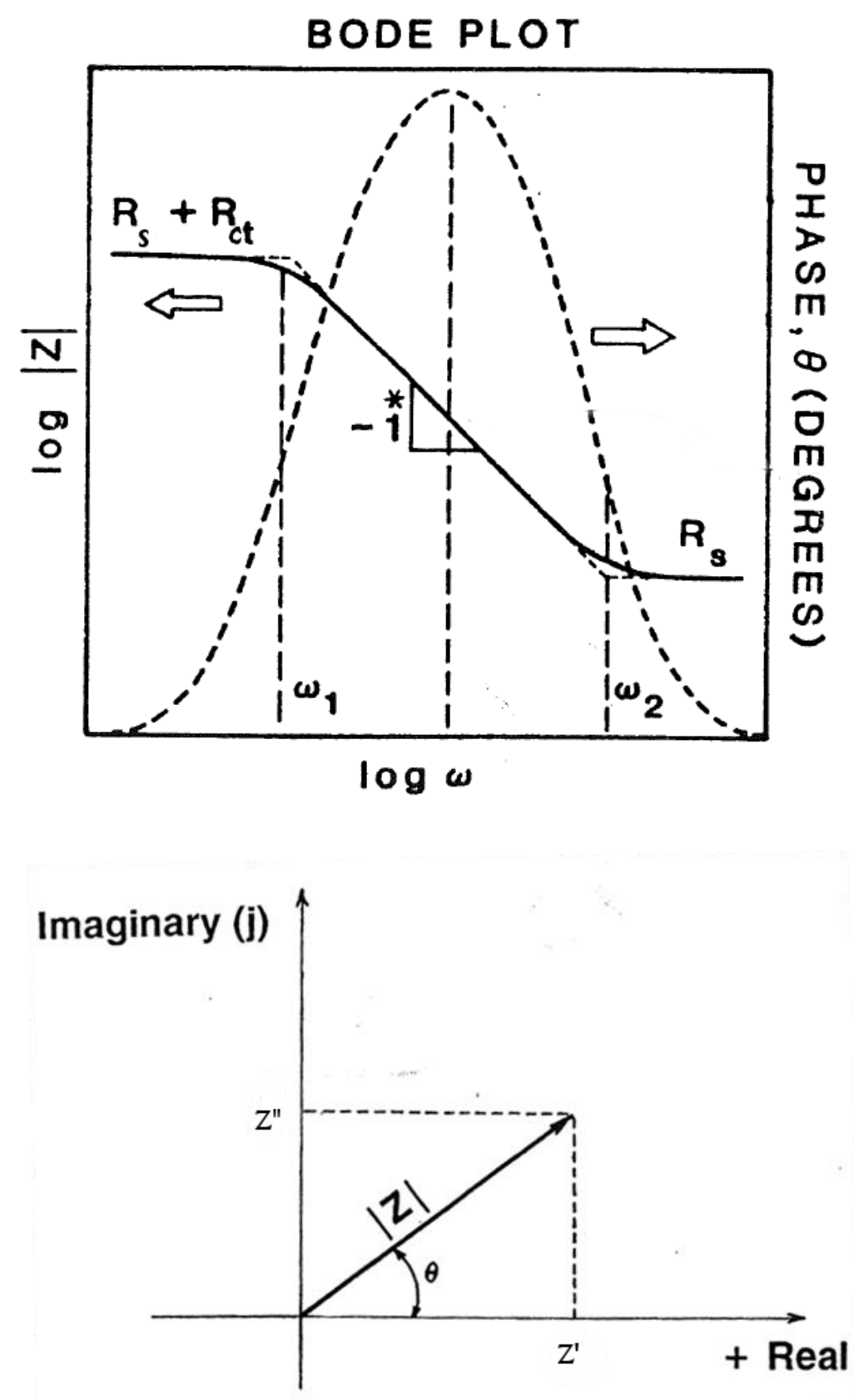

Figure 4.11. In the region where the slope of $\log |\mathrm{Z}|$ vs $\log \omega$ is equal to -1 the relationship $C=1 / 2 \pi f|Z|$ holds true. 


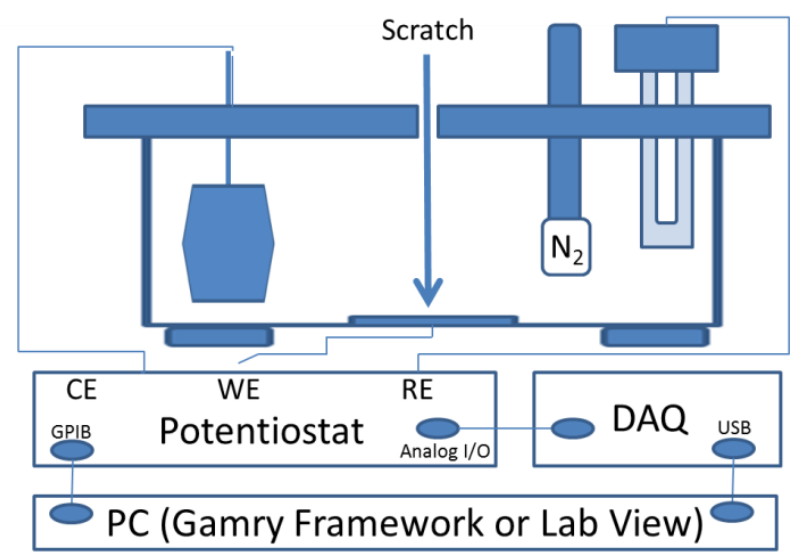

Figure 4.12. Schematic of scratched electrode cell with wiring diagram for data capture devices. A PAR 273A was used with corrware for easy control of range changes. A SCE was used unless otherwise noted. Data acquisition rate of 10,000 to 20,000 points a second was utilized. DAQ was a National Instruments rack mounted PXI Express module. 

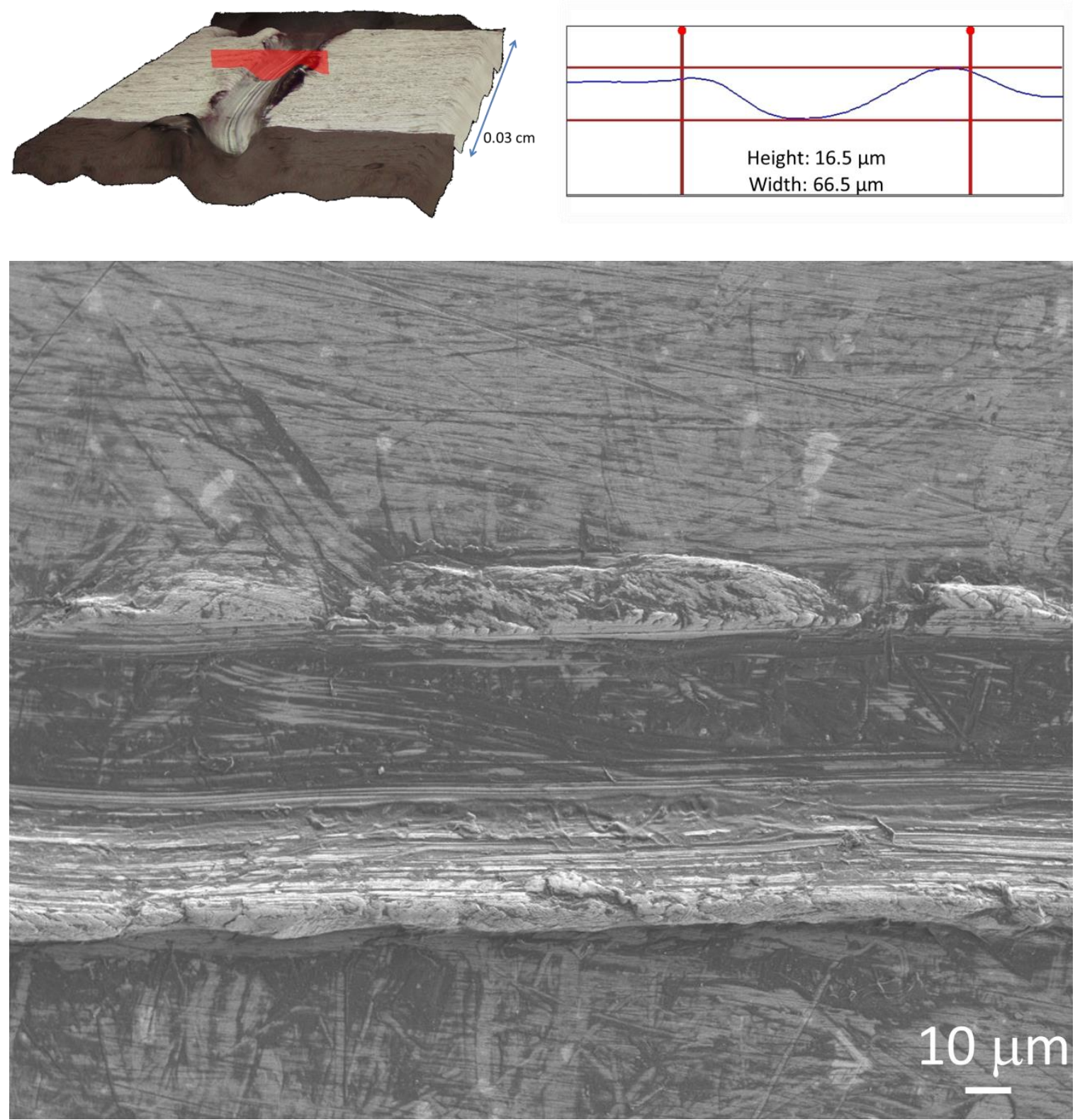

Figure 4.13. Detailed 3-D scratch topography for calculating area of exposed electrode during scratched electrode testing. SEM image of AA2024 scratched in aqueous $0.5 \mathrm{M}$ $\mathrm{CrO}_{4}{ }^{2-}+0.05 \mathrm{M} \mathrm{NaCl}$ with a $\mathrm{pH}$ of 6.5 . Accelerating voltage of $2 \mathrm{kV}$ was used in secondary electron mode with a working distance of $11 \mathrm{~mm}$. 
Reference Electrode

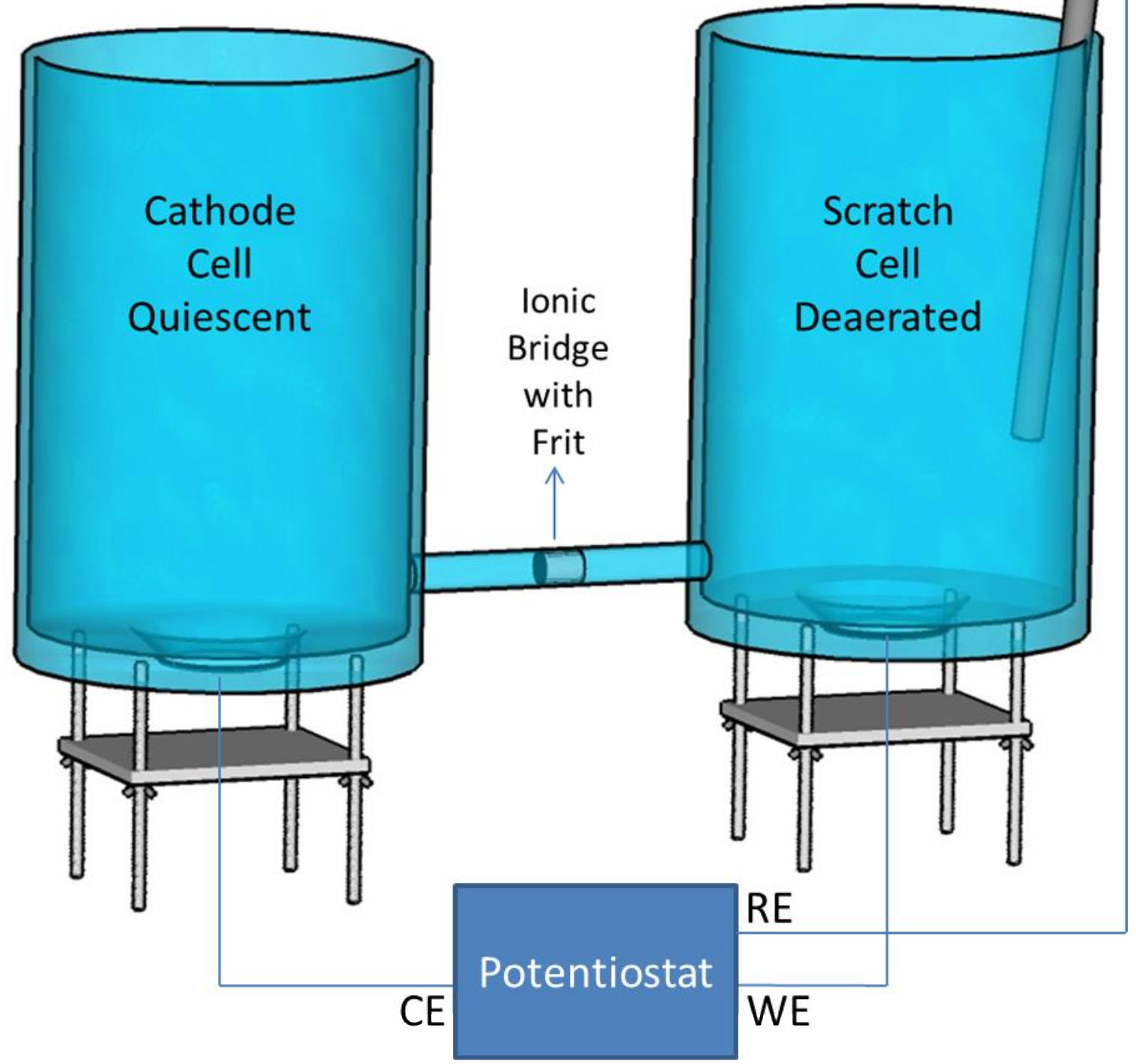

Figure 4.14. Schematic of split cell design. Galvanic current is measured with a zero resistance ammeter (ZRA) while the potential of the scratch depassivated surface coupled through the salt bridge to the cathode cell is monitored with a saturated calomel reference electrode. 


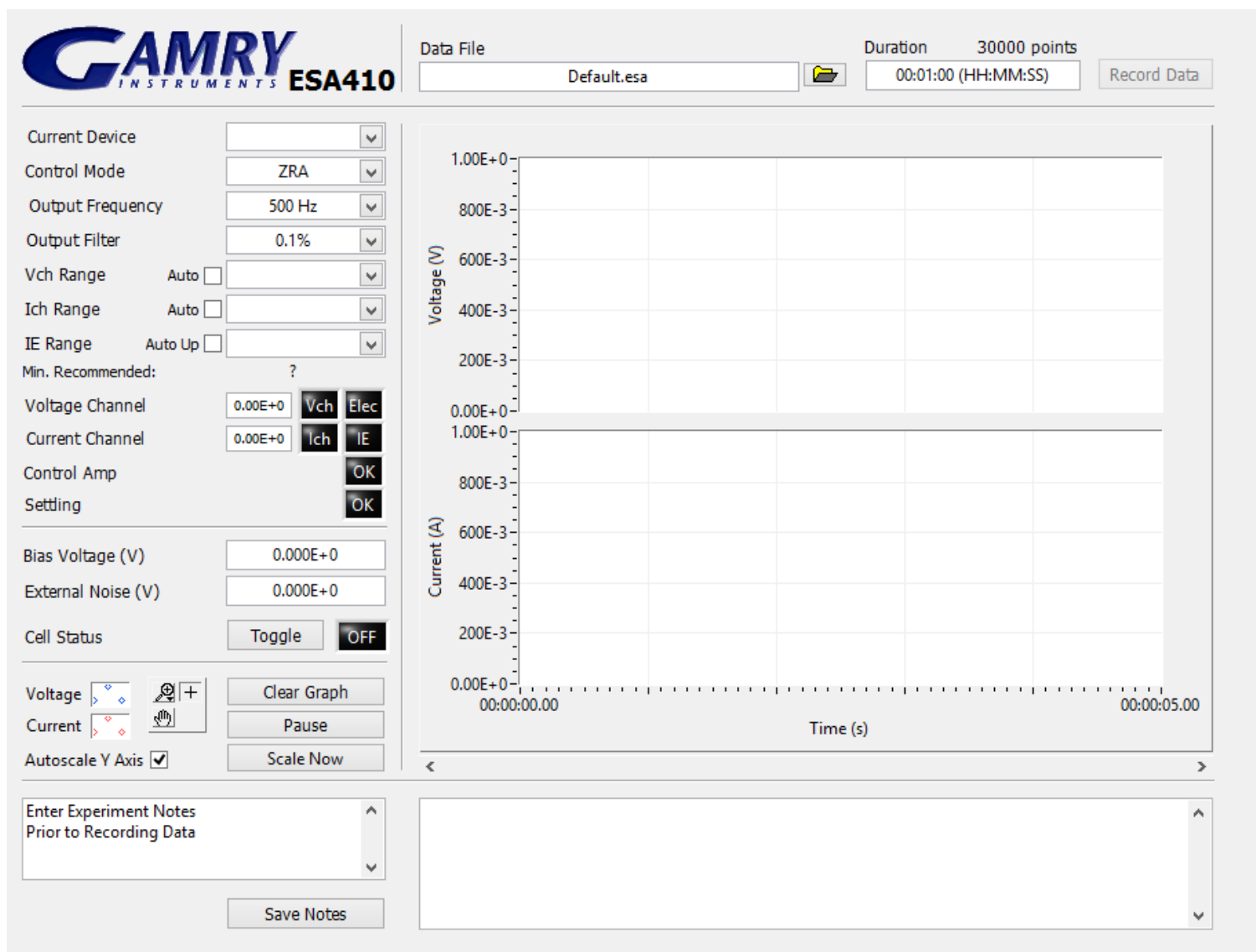

Figure 4.15. The standard UI for the ESA400 software suite from Gamry Instruments, Inc. 


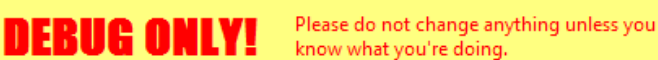

\begin{tabular}{|c|c|c|}
\hline \multicolumn{3}{|c|}{ I/E Converter } \\
\hline Stability & Normal & $v$ \\
\hline \multicolumn{3}{|c|}{ Voltage Channel } \\
\hline Offset & \multicolumn{2}{|l|}{$0.000 E+0 V$} \\
\hline Filter & & $\checkmark$ \\
\hline \multicolumn{3}{|c|}{ Current Channel } \\
\hline Offset & \multicolumn{2}{|l|}{$0.000 \mathrm{E}+0 \mathrm{~V}$} \\
\hline Filter & & $v$ \\
\hline \multicolumn{3}{|c|}{ Control Amplifier } \\
\hline Speed & & $v$ \\
\hline \multicolumn{3}{|c|}{ Do not set to FAST in ZRA mode! } \\
\hline Ground & Unknown & \\
\hline
\end{tabular}

\begin{tabular}{|c|c|c|}
\hline \multicolumn{2}{|c|}{ Apply Sine Wave? } & $\square$ \\
\hline \multicolumn{2}{|l|}{ Use DDS? } & $\square$ \\
\hline Amplitude & $0.000 \mathrm{E}+0$ & \\
\hline Frequency & $0.000 \mathrm{E}+0$ & \\
\hline Phase & $0.000 \mathrm{E}+0$ & \\
\hline
\end{tabular}

Pstat Error Dtaq Erro

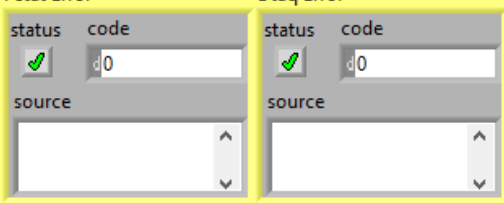

Overloads

CA Overload

v Overload

0

I ADC Railed

V ADC Railed

$O$

Hardware Settling

O

Software Settling

0

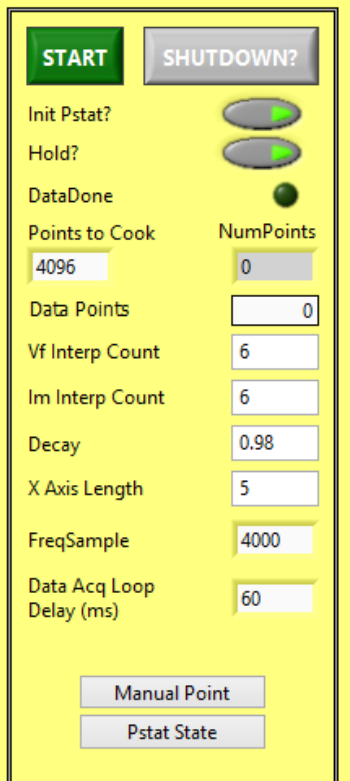

Active Setup Fil

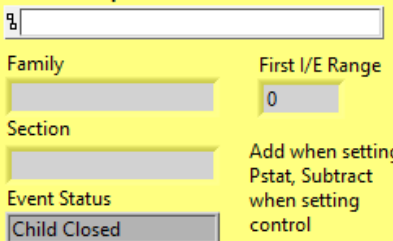

OldDevicelndex NewDevicelndex

$\begin{array}{lll}-1 & -1\end{array}$

Acq Semaphore Timeout (ms) $=10000$

FilterDelay

Orig. Duration

Time Elapsed

current time

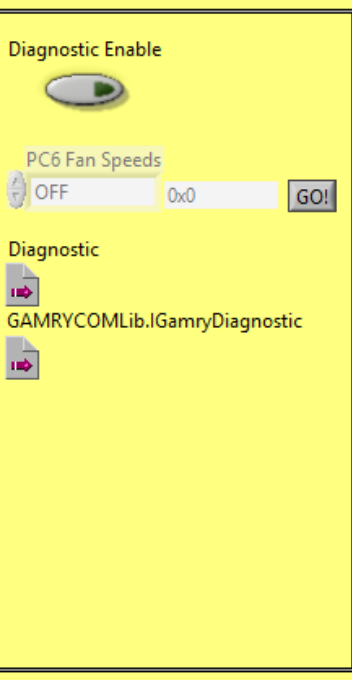

0

RuntimeSetup Cluster Points/Block

$512 \checkmark$

Acq Frequency

$500.000 \mathrm{E}+0 \mathrm{~Hz}$

Data Filename

q. Default.esa

Bias

0

Control Mode

ZRA V

Electrode Configuration

2 Electrode

Notes

Default

Initial Delay

0

Filter Precision

$0.1 \% \quad v$

Noise Amplitude

Duration

00:00:00.000 PM $\mathrm{MM} / \mathrm{DD} / \mathrm{YVYY}$

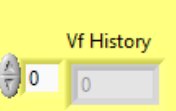

Data Cluster

Waveform Chart$$
\text { VF }
$$

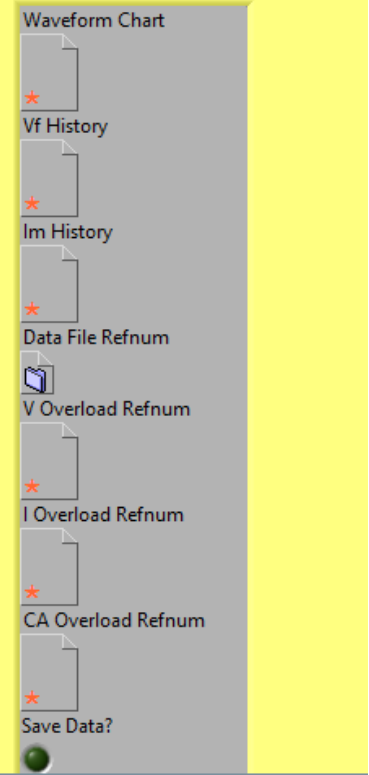

Figure 4.16. The Debug UI for the modified ESA400 software suite. 

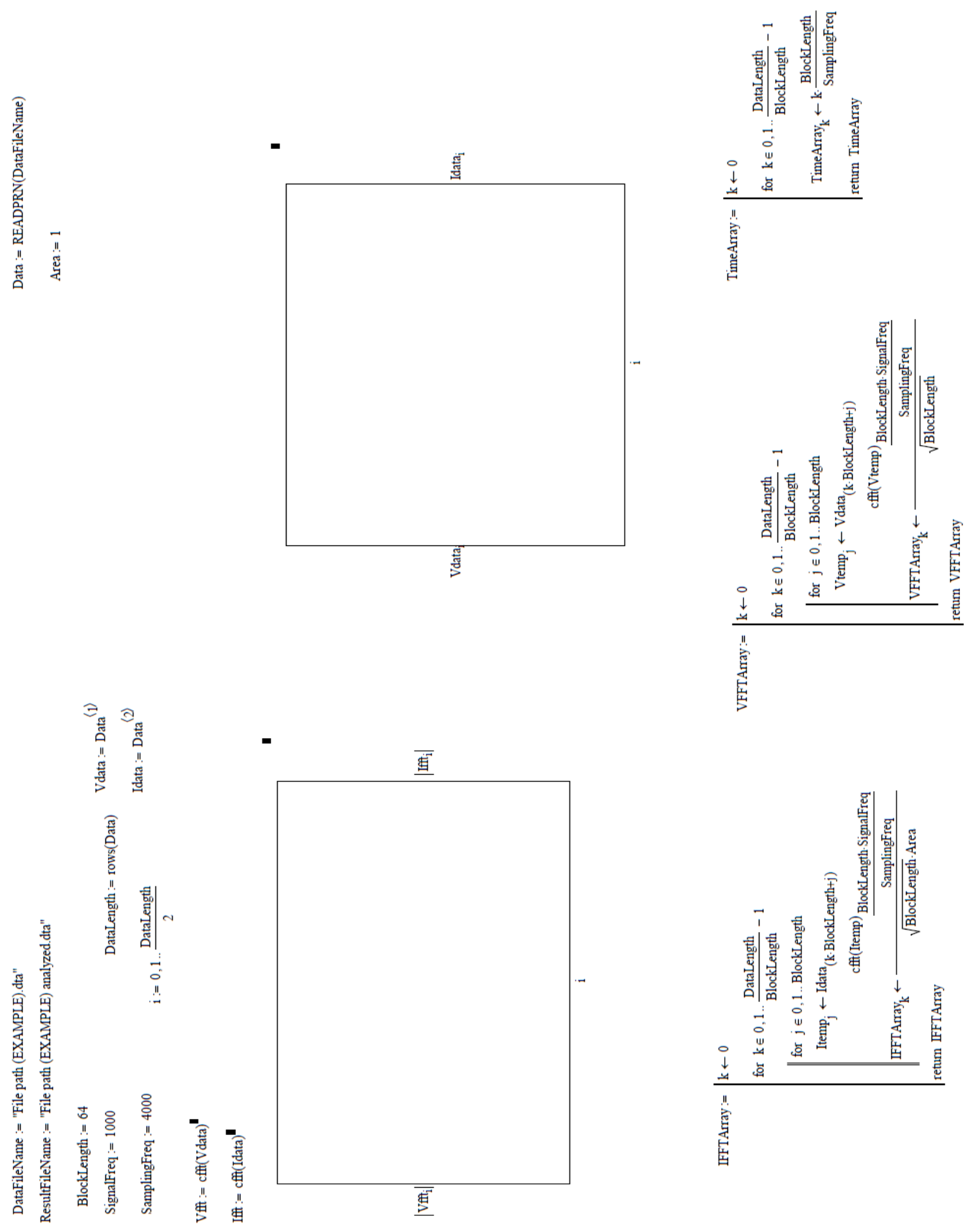

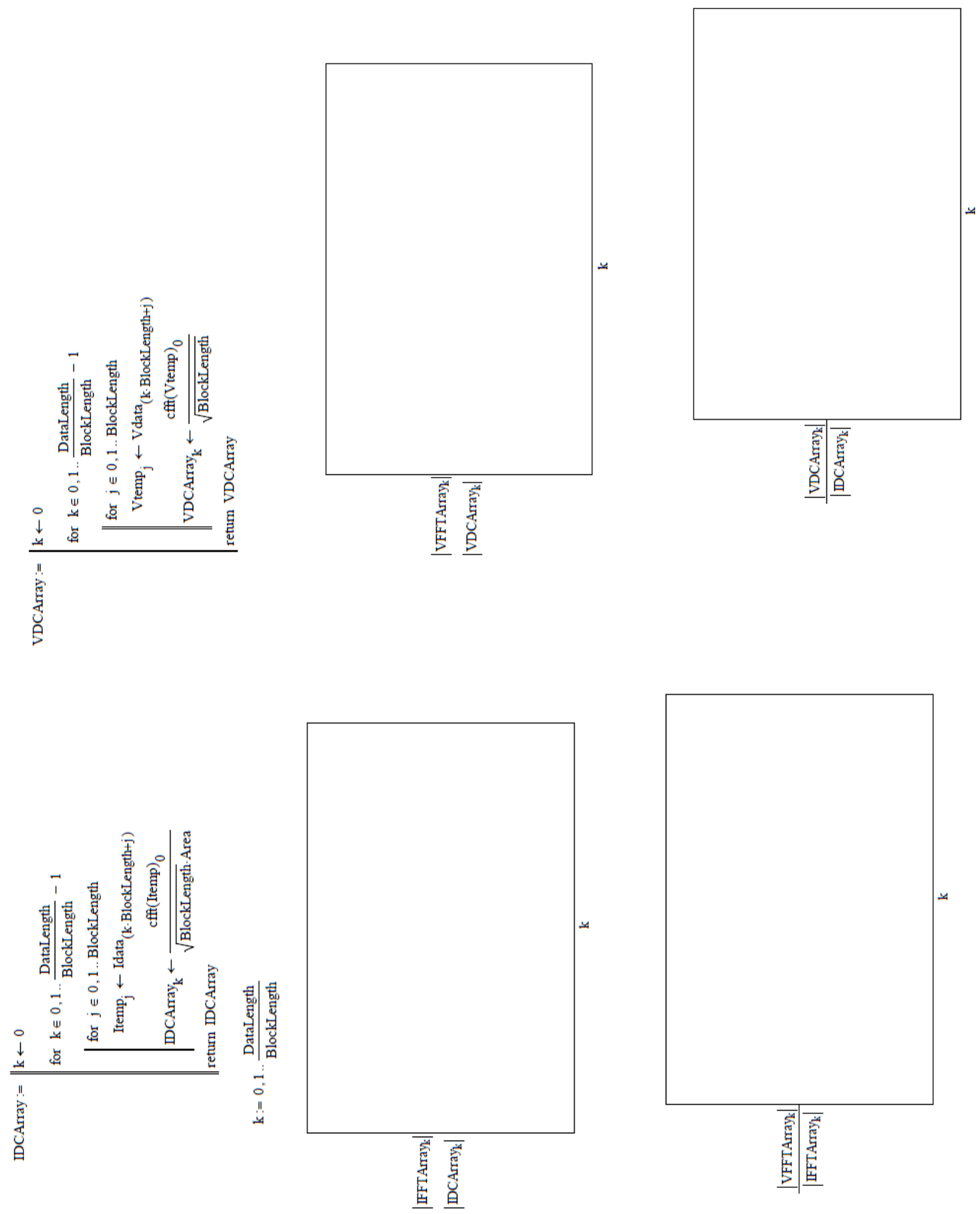

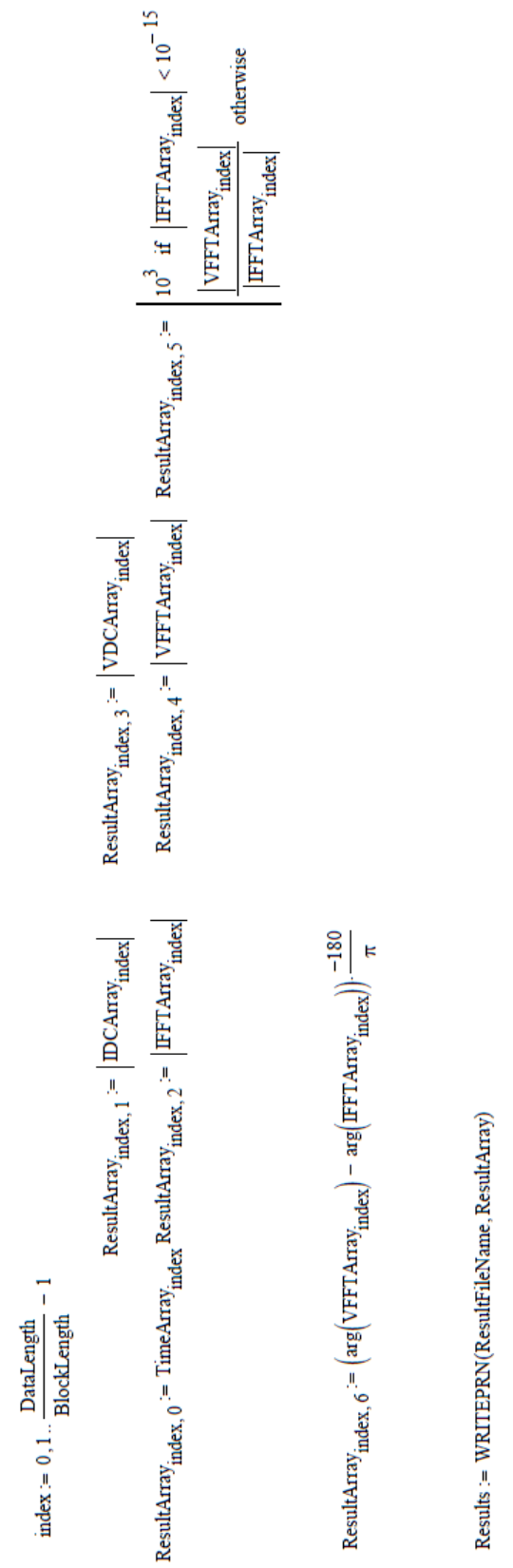

Figure 4.17. Mathcad Fourier transforms script for analysis of single frequency impedance measurements written by Burak Ulgut and edited by the author. 


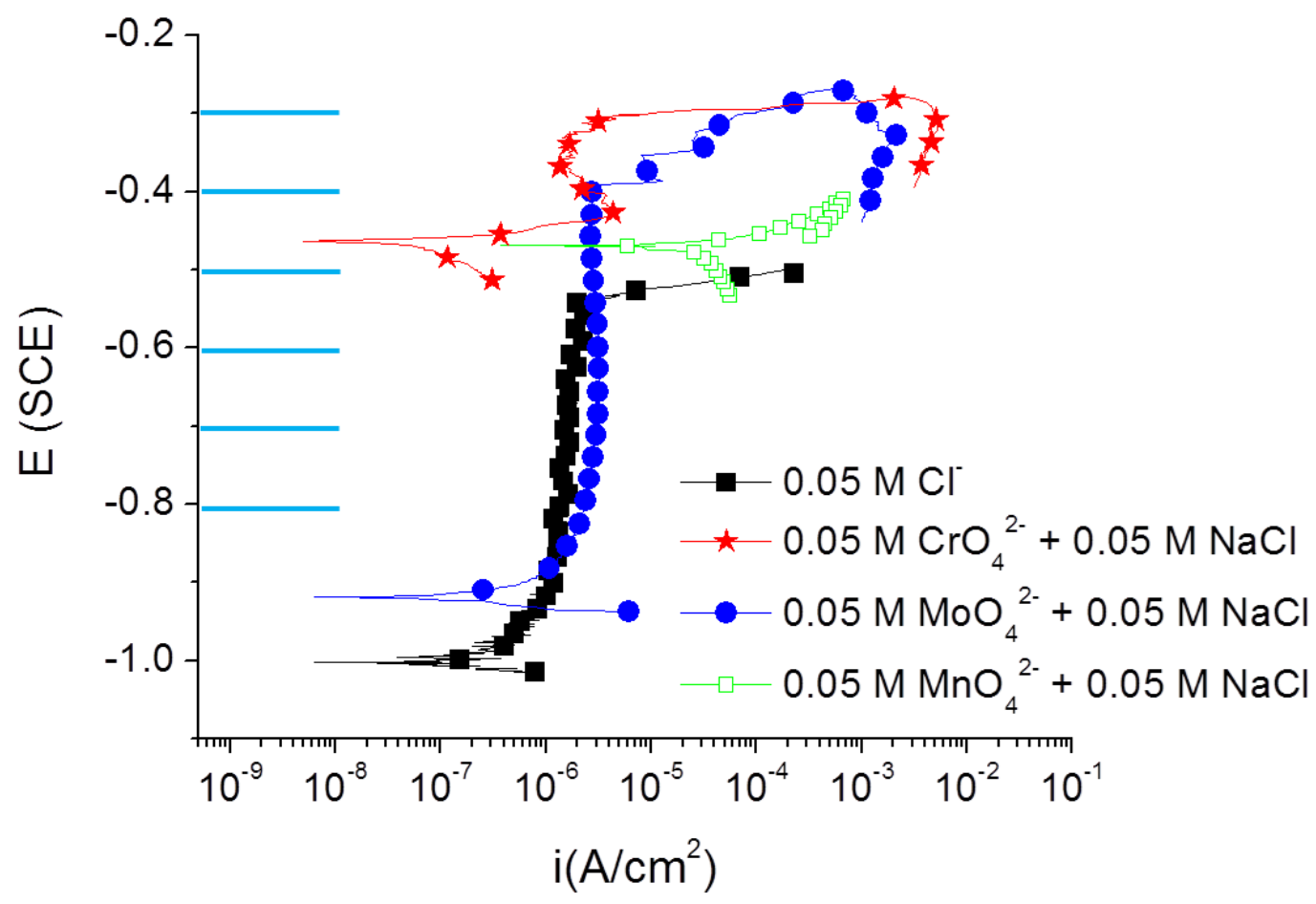

Figure 4.18. Anodic inhibitors of interest for EFCP inhibition. Potentiodynamic scan of AA2024-T351 in molybdate, permanganate, and chromate solutions containing $0.05 \mathrm{M}$ $\mathrm{NaCl}$ solution. Lines to the left of polarization curves indicate scratch potentials investigated ranging from $-0.8 \mathrm{~V}$ to $-0.3 \mathrm{~V}$ (SCE). Solutions were deaerated. 


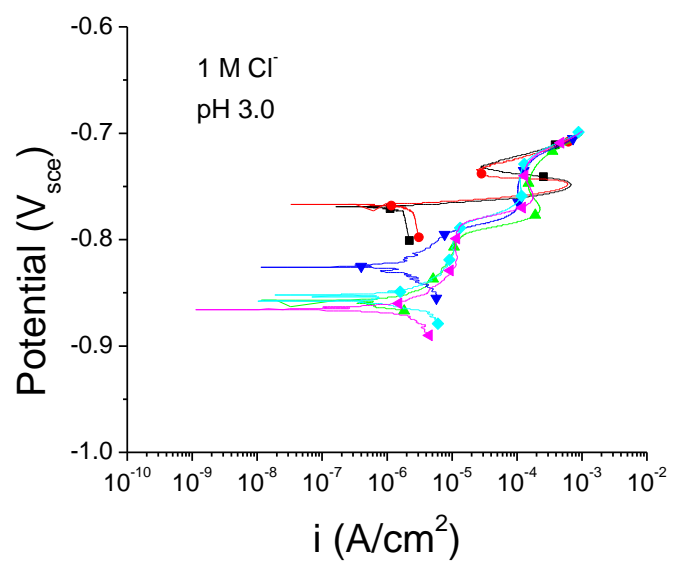

A

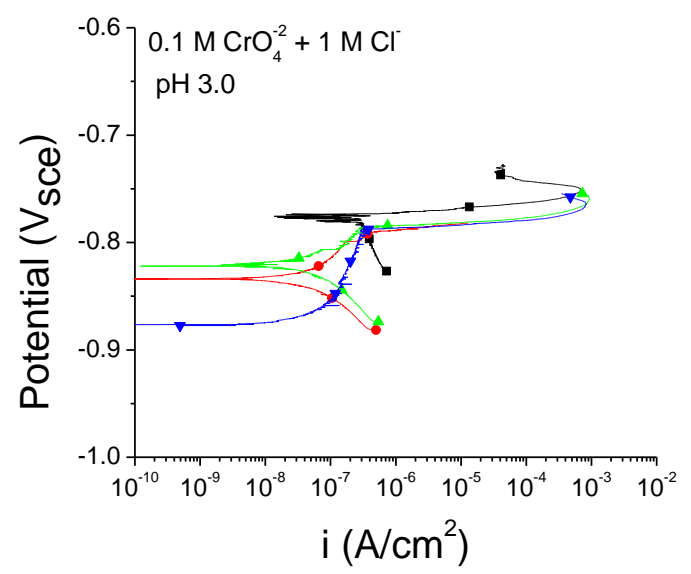

B

Figure 4.19. Effect of $0.1 \mathrm{M} \mathrm{CrO}_{4}{ }^{2-}$ on the anodic E-logi behavior of AA7075 in aqueous $1 \mathrm{M} \mathrm{Cl}^{-}$with an adjusted $\mathrm{pH}$ of 3 . All runs in duplicate.

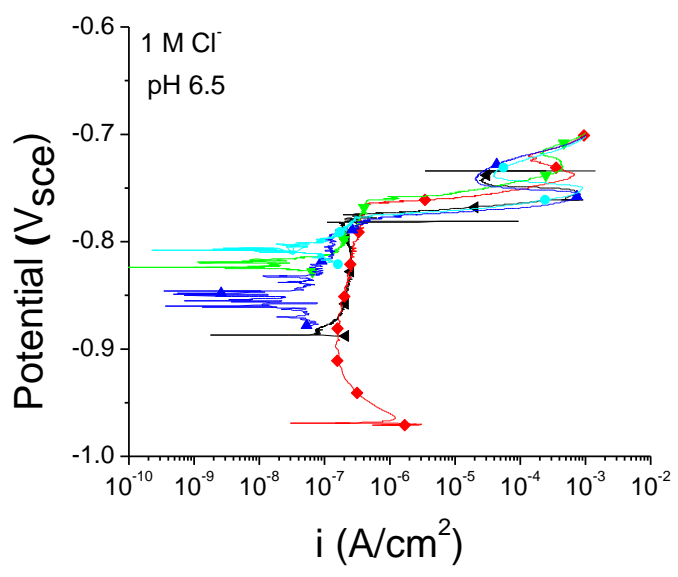

A

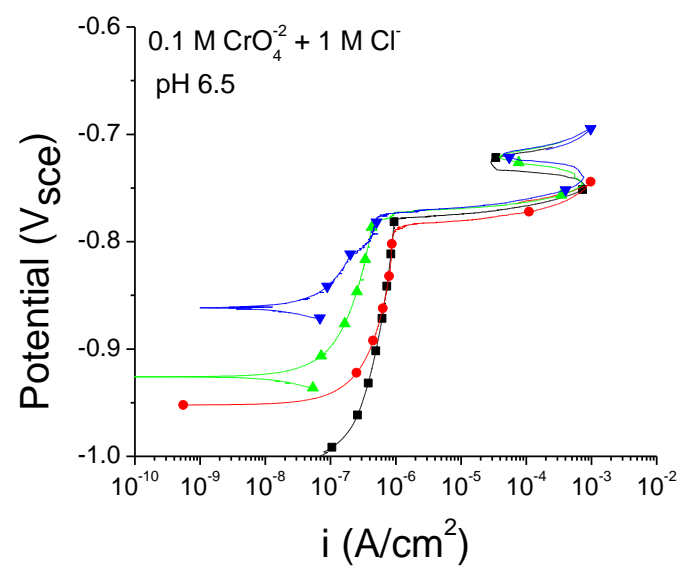

B

Figure 4.20. Effect of $0.1 \mathrm{M} \mathrm{CrO}_{4}{ }^{2-}$ on the anodic E-logi behavior of AA7075 in aqueous $1 \mathrm{M} \mathrm{Cl}^{-}$with an adjusted $\mathrm{pH}$ of 6.5. All runs in duplicate. 


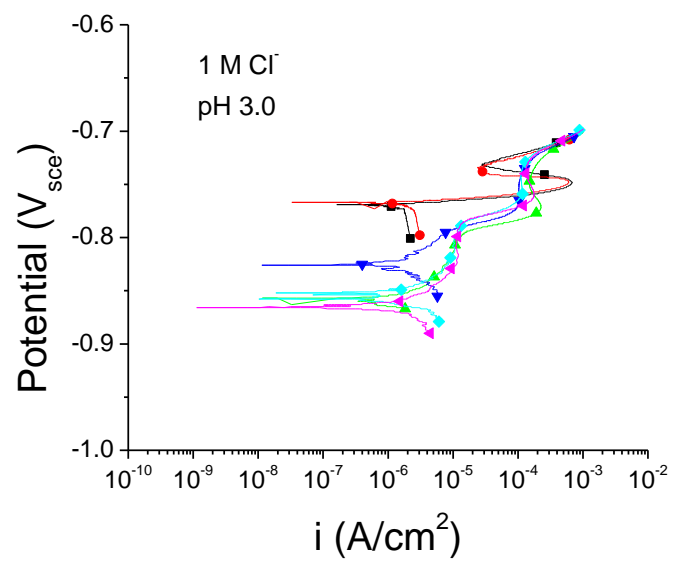

A

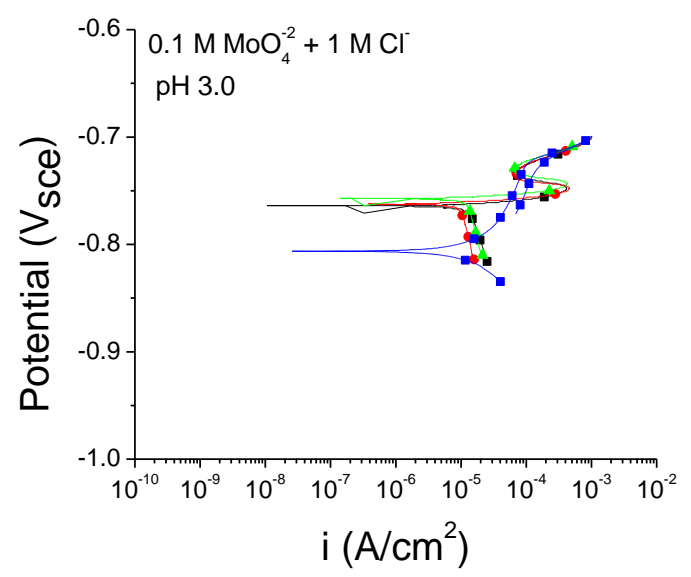

B

Figure 4.21. Effect of $0.1 \mathrm{M} \mathrm{MoO}_{4}{ }^{2-}$ on the anodic E-logi behavior of AA7075 in aqueous $1 \mathrm{M} \mathrm{Cl}^{-}$with an adjusted $\mathrm{pH}$ of 3 . All runs in duplicate.

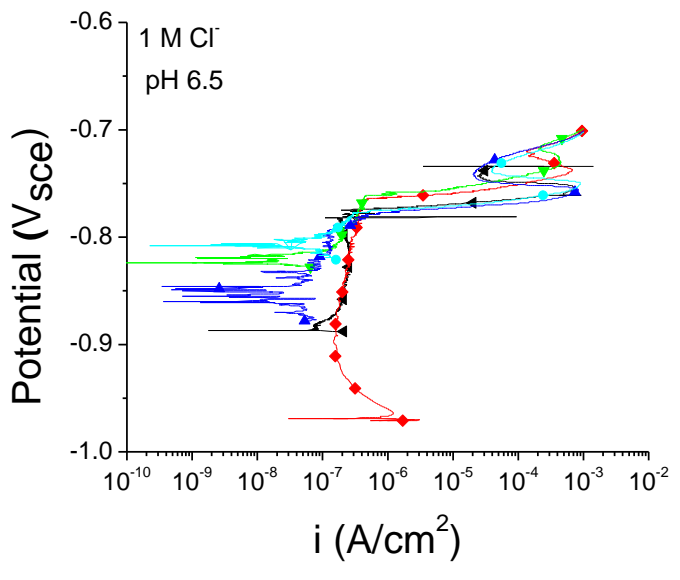

A

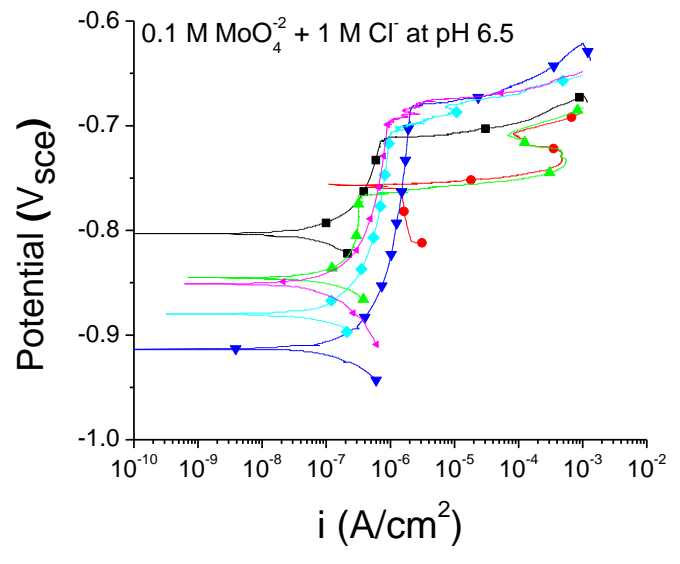

B

Figure 4.22. Effect of $0.1 \mathrm{M} \mathrm{MoO}_{4}{ }^{2-}$ on the anodic E-logi behavior of AA7075 in aqueous $1 \mathrm{M} \mathrm{Cl}^{-}$with an adjusted $\mathrm{pH}$ of 6.5. All runs in duplicate. 


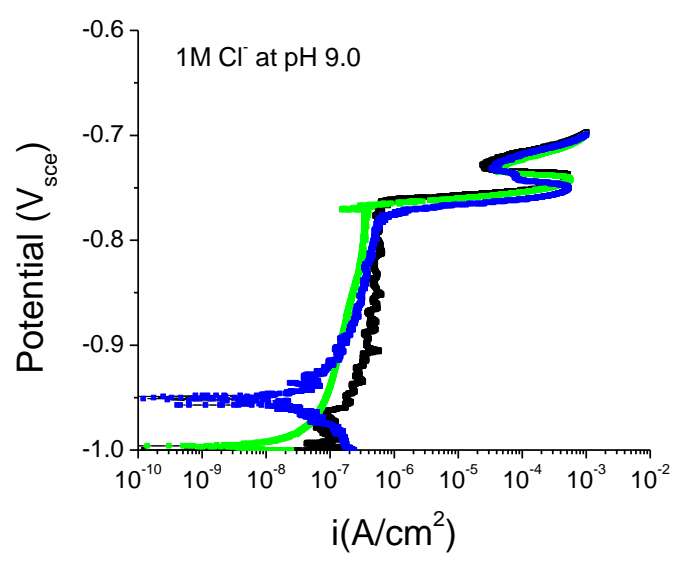

A

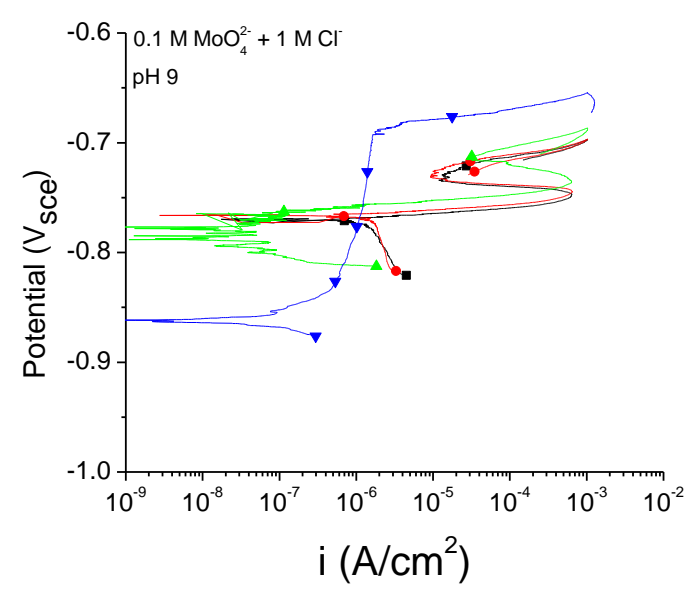

B

Figure 4.23. Effect of 0.1 $\mathrm{M} \mathrm{MoO}_{4}{ }^{2-}$ on the anodic E-logi behavior of AA7075 in aqueous $1 \mathrm{M} \mathrm{Cl}^{-}$with an adjusted $\mathrm{pH}$ of 9 , the natural $\mathrm{pH}$ of the molybdate solution as prepared. All runs in duplicate.

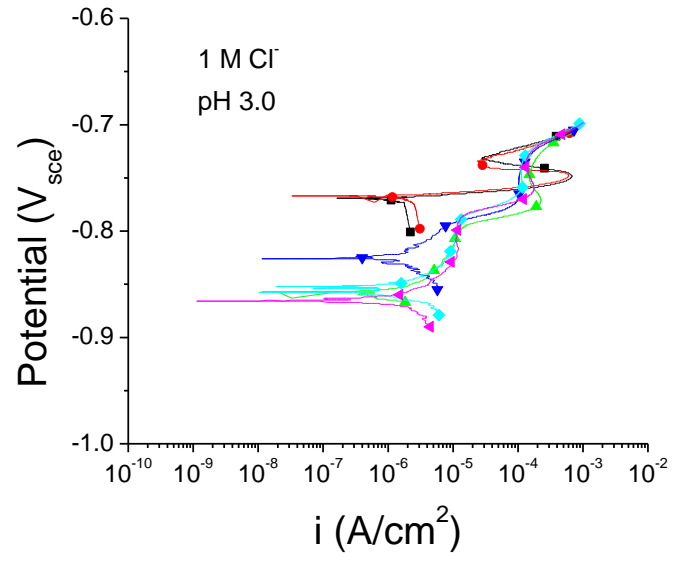

A

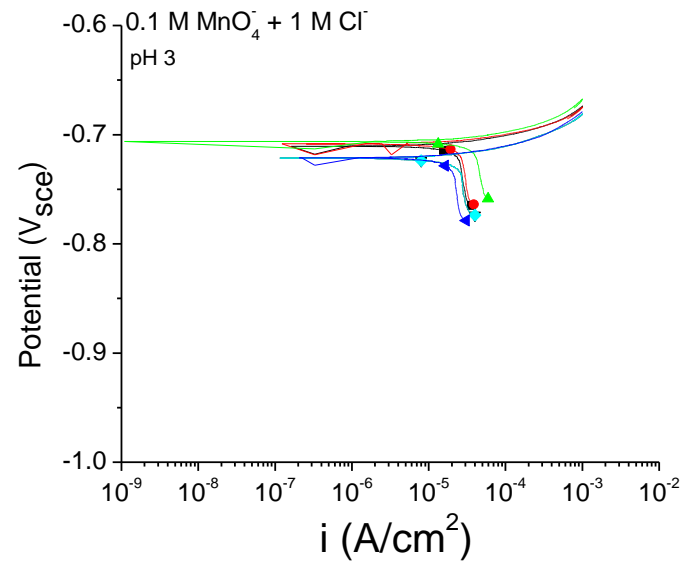

B

Figure 4.24. Effect of $0.1 \mathrm{M} \mathrm{MnO}_{4}^{-}$on the anodic E-logi behavior of AA7075 in aqueous $1 \mathrm{M} \mathrm{Cl}^{-}$with an adjusted $\mathrm{pH}$ of 9 . All runs in duplicate. 


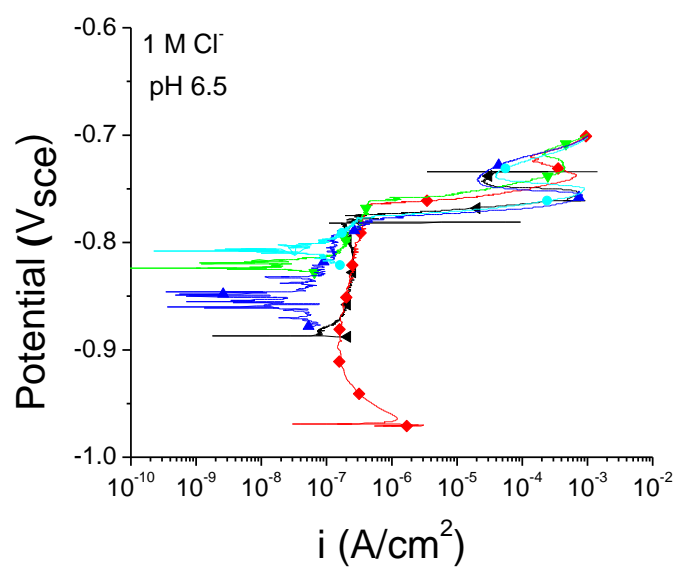

A

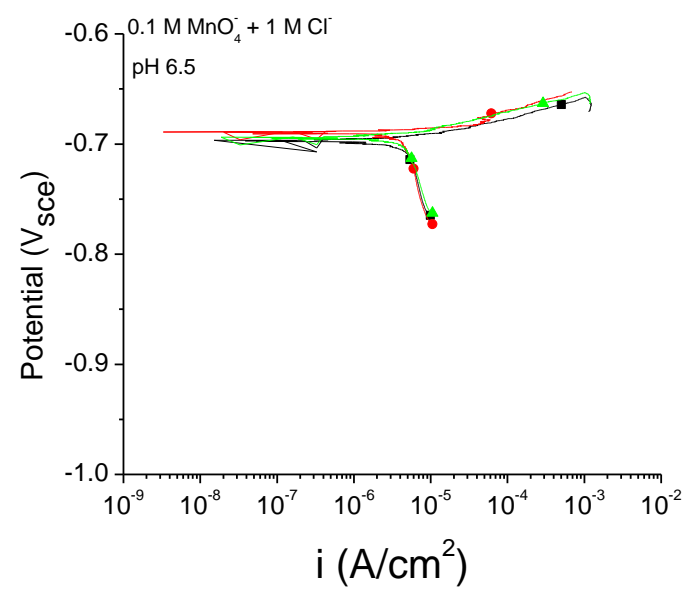

B

Figure 4.25. Effect of $0.1 \mathrm{M} \mathrm{MnO}_{4}^{-}$on the anodic E-logi behavior of AA7075 in aqueous $1 \mathrm{M} \mathrm{Cl}^{-}$with an adjusted $\mathrm{pH}$ of 6.5. All runs in duplicate.

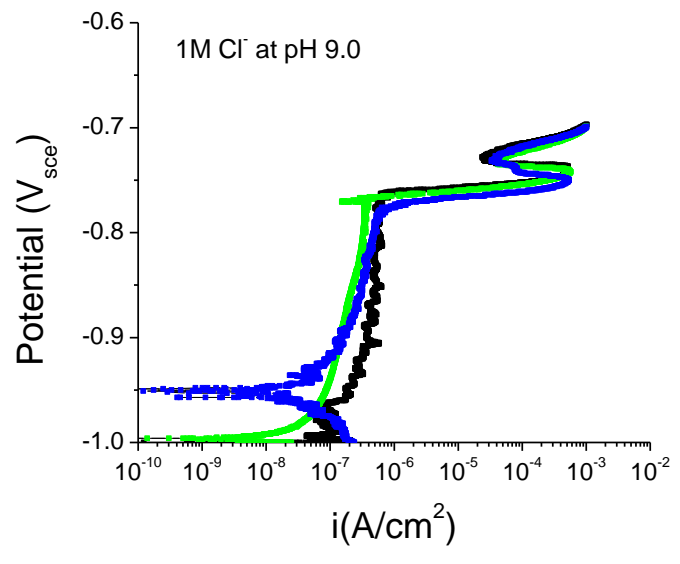

A

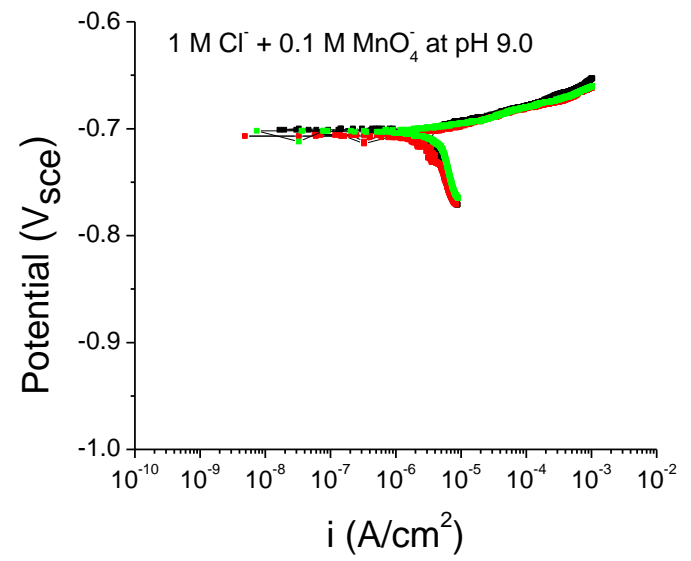

B

Figure 4.26. Effect of $0.1 \mathrm{M} \mathrm{MnO}_{4}^{-}$on the anodic E-logi behavior of AA7075 in aqueous $1 \mathrm{M} \mathrm{Cl}^{-}$with an adjusted $\mathrm{pH}$ of 9 . 


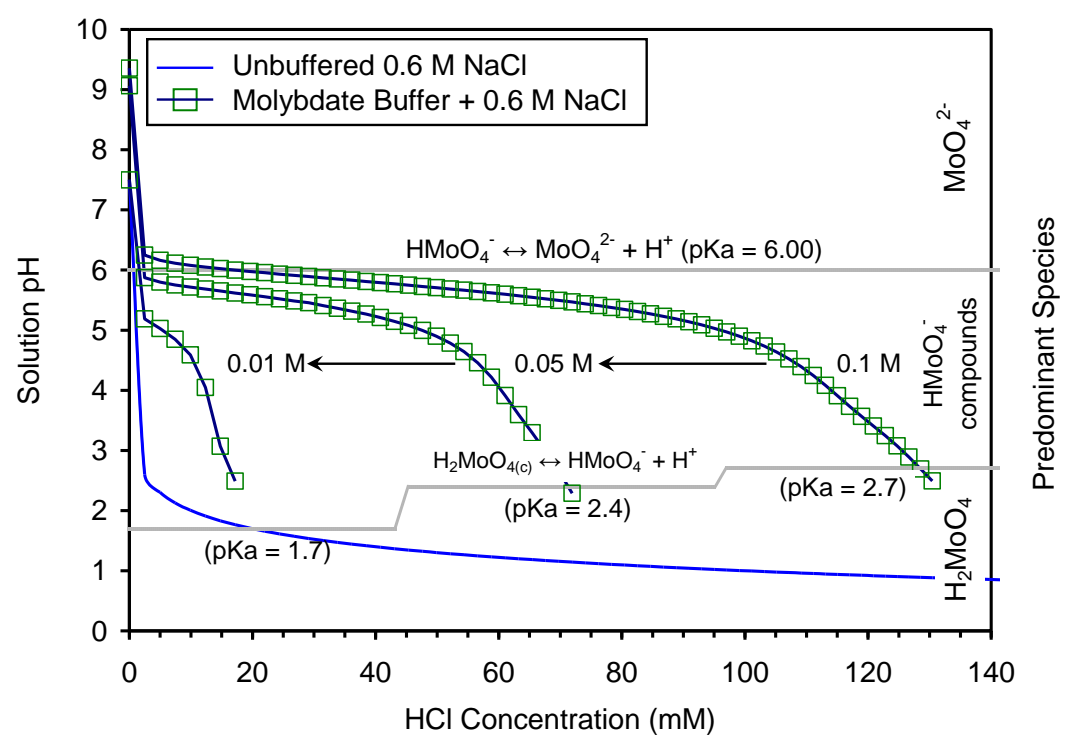

Figure 4.27. Molybdate buffer capacity as determined by Al-Ghamdi. Initial pH was 9.3 for $0.1 \mathrm{M} \mathrm{MoO}_{4}{ }^{2-}$ solutions. "Solution $\mathrm{pH}$ from titration of $(0.01 \mathrm{M}, 0.05 \mathrm{M}$, and $0.1 \mathrm{M})$ sodium molybdate $+0.6 \mathrm{M} \mathrm{NaCl}$ with $0.25 \mathrm{~mL}$ of $1 \mathrm{M} \mathrm{HCl}$ reported as $\mathrm{HCl}$ concentration in $\mathrm{mM}$. Molybdate concentration varied from 0.1 to $0.01 \mathrm{M}$ and results are compared against uninhibited $0.6 \mathrm{M} \mathrm{NaCl}$. Molybdate chemical reactions are shown with relevant predominant species in the measured pH range." 


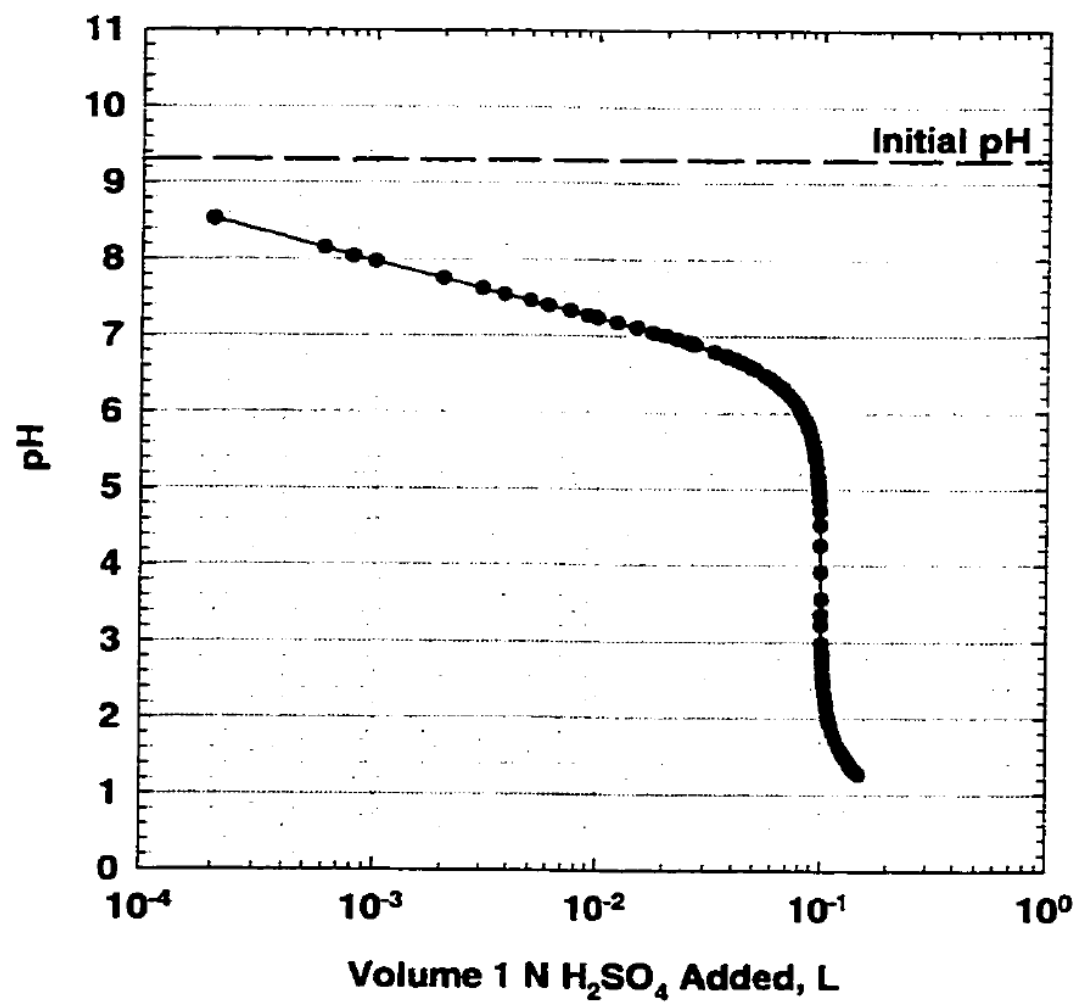

Figure 4.28. Chromate buffer capacity. The capacity of $0.5 \mathrm{M} \mathrm{CrO}_{4}^{2-}+0.05 \mathrm{M} \mathrm{NaCl}$ to buffer $\mathrm{pH}$ with addition of $\mathrm{H}_{2} \mathrm{SO}_{4} .0 .20 \mathrm{~L}_{\text {of }} 0.5 \mathrm{M} \mathrm{CrO}_{4}{ }^{2-}+0.05 \mathrm{M} \mathrm{NaCl}$ solution were titrated with $1 \mathrm{~N} \mathrm{H}_{2} \mathrm{SO}_{4}$. 

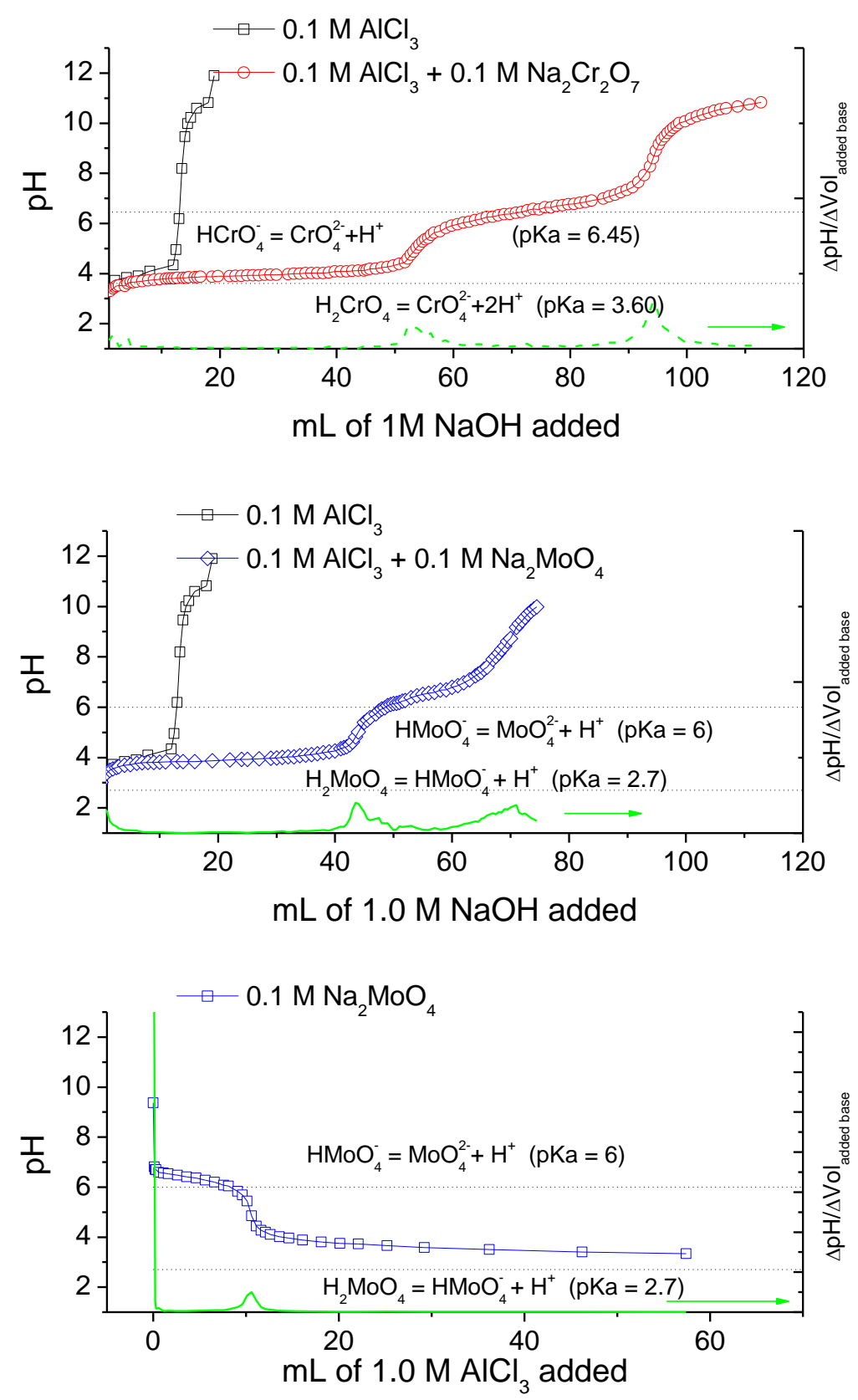

Figure 4.29. Buffer capacity of $200 \mathrm{~mL}$ of $0.1 \mathrm{M} \mathrm{AlCl}_{3}+0.1 \mathrm{M} \mathrm{Na}_{2} \mathrm{Cr}_{2} \mathrm{O}_{7}$ or $0.1 \mathrm{M}$ $\mathrm{Na}_{2} \mathrm{MoO}_{4}$. Solution was titrated with $0.1 \mathrm{M} \mathrm{NaOH}$. Values of $\mathrm{kPa}$ are taken from the literature. Actual kPa, calculated from inflection of titration curve, was pKa 1 of 3.95 and a $\mathrm{pKa}_{2}$ of 6.58 for chromate and $\mathrm{pKa}_{1}$ of 3.9 and a $\mathrm{pKa}_{2}$ of 6.59 for molybdate. Also shown, the titration of $0.1 \mathrm{M} \mathrm{AlCl}_{3}$ into $200 \mathrm{ml}$ of $1.0 \mathrm{M} \mathrm{MoO}_{4}{ }^{2-}$. 

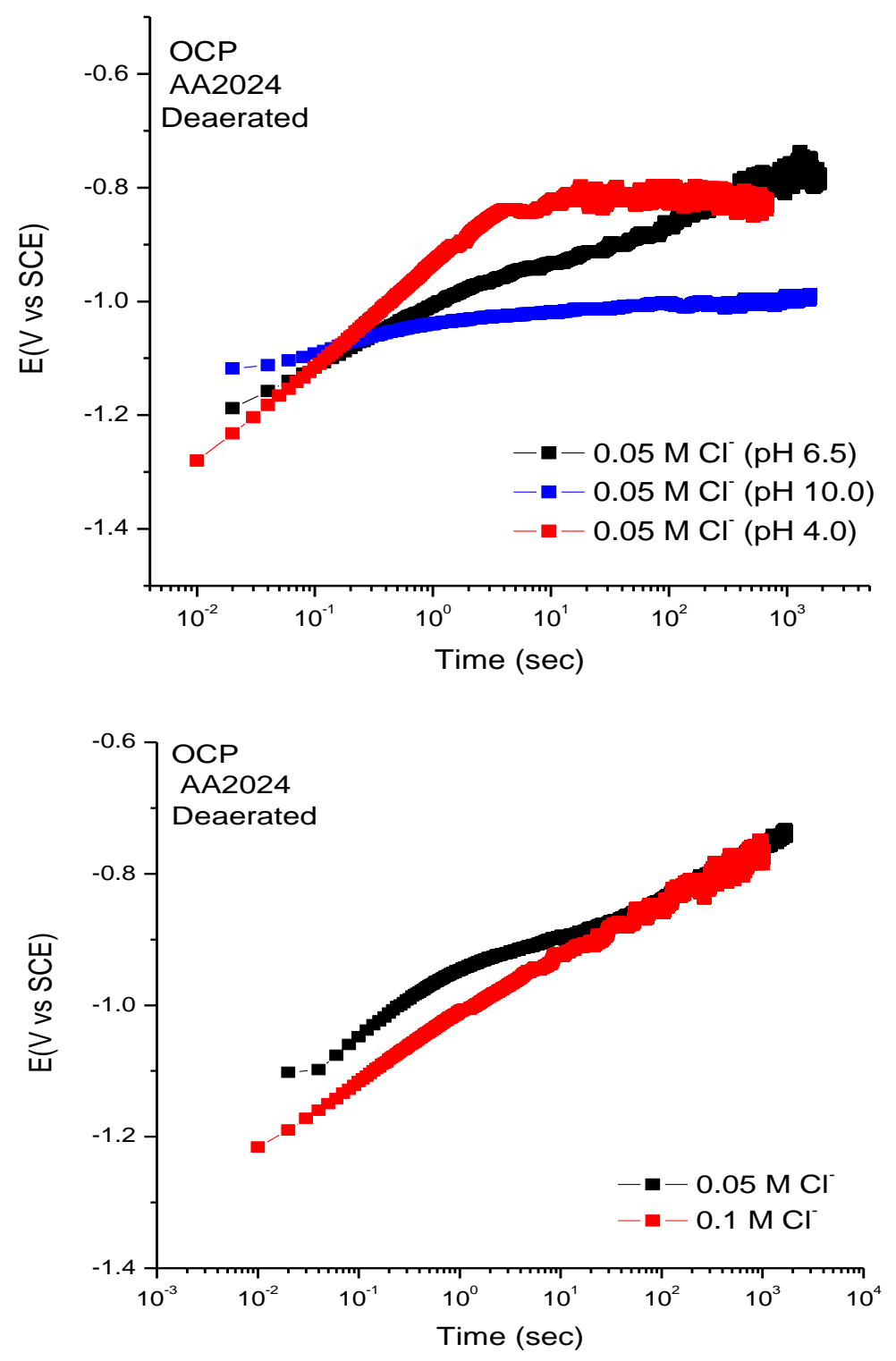

Figure 4.30. The effect of $\mathrm{pH}(\mathrm{A})$ and $\mathrm{Cl}^{-}$concentration (B) on the open circuit potential of scratched AA2024 in aqueous $0.05 \mathrm{M} \mathrm{NaCl}$. Chloride concentration had little effect on the transient recovery of the OCP while $\mathrm{pH}$ had a marked effect on $d E / d t$ as well as affecting the steady state OCP at longer times. Both low and high $\mathrm{pH}$ appeared to lower the OCP at longer times below that of the $\mathrm{pH} 6.5$ experiment. However, the $\mathrm{pH} 10$ resting OCP was almost $200 \mathrm{mV}$ below the $\mathrm{pH} 4$. 


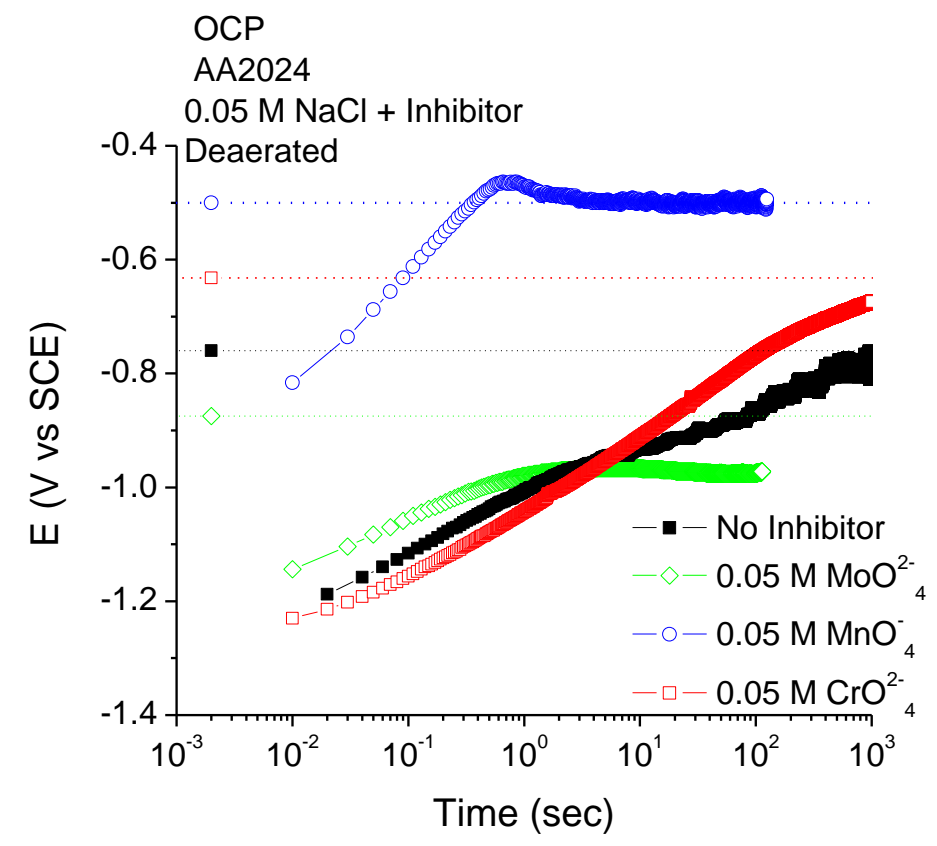

Figure 4.31. The effect of $0.05 \mathrm{M} \mathrm{CrO}_{4}{ }^{2-}, \mathrm{MoO}_{4}{ }^{2-}$, and $\mathrm{MnO}_{4}{ }^{-}$on the open circuit potential of scratched AA2024 in $\mathrm{pH} 6.5$ aqueous $0.05 \mathrm{M} \mathrm{NaCl}$. The inhibitors mediate the potential drop that occurs on the electrode after the surface oxide has been removed. The effect ranks with the strength of the oxidizing nature of each ion with the highest potentials reached by permanganate, followed by chromate and then molybdate. Open circuit potentials of passivated surfaces in the deaerated solutions are indicated by dotted lines. 

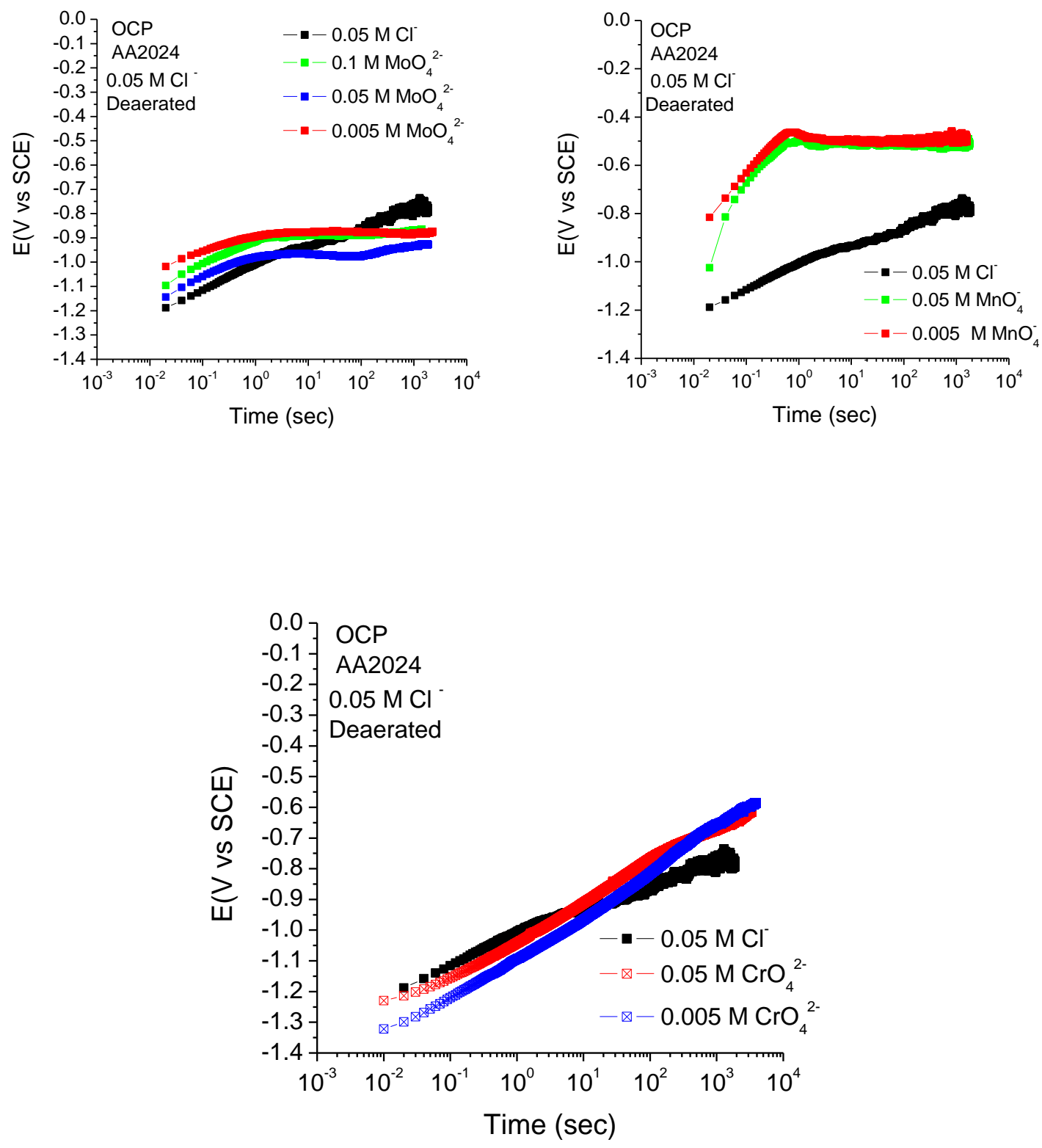

Figure 4.32. The effect of $\mathrm{CrO}_{4}{ }^{2-}, \mathrm{MoO}_{4}{ }^{2-}$ and $\mathrm{MnO}_{4}{ }^{-}$concentration on the open circuit potential of scratched AA2024 in $\mathrm{pH} 6.5$ aqueous $0.05 \mathrm{M} \mathrm{NaCl}$. The inhibitors mediate the potential drop that occurs on the electrode after the surface oxide has been removed. 

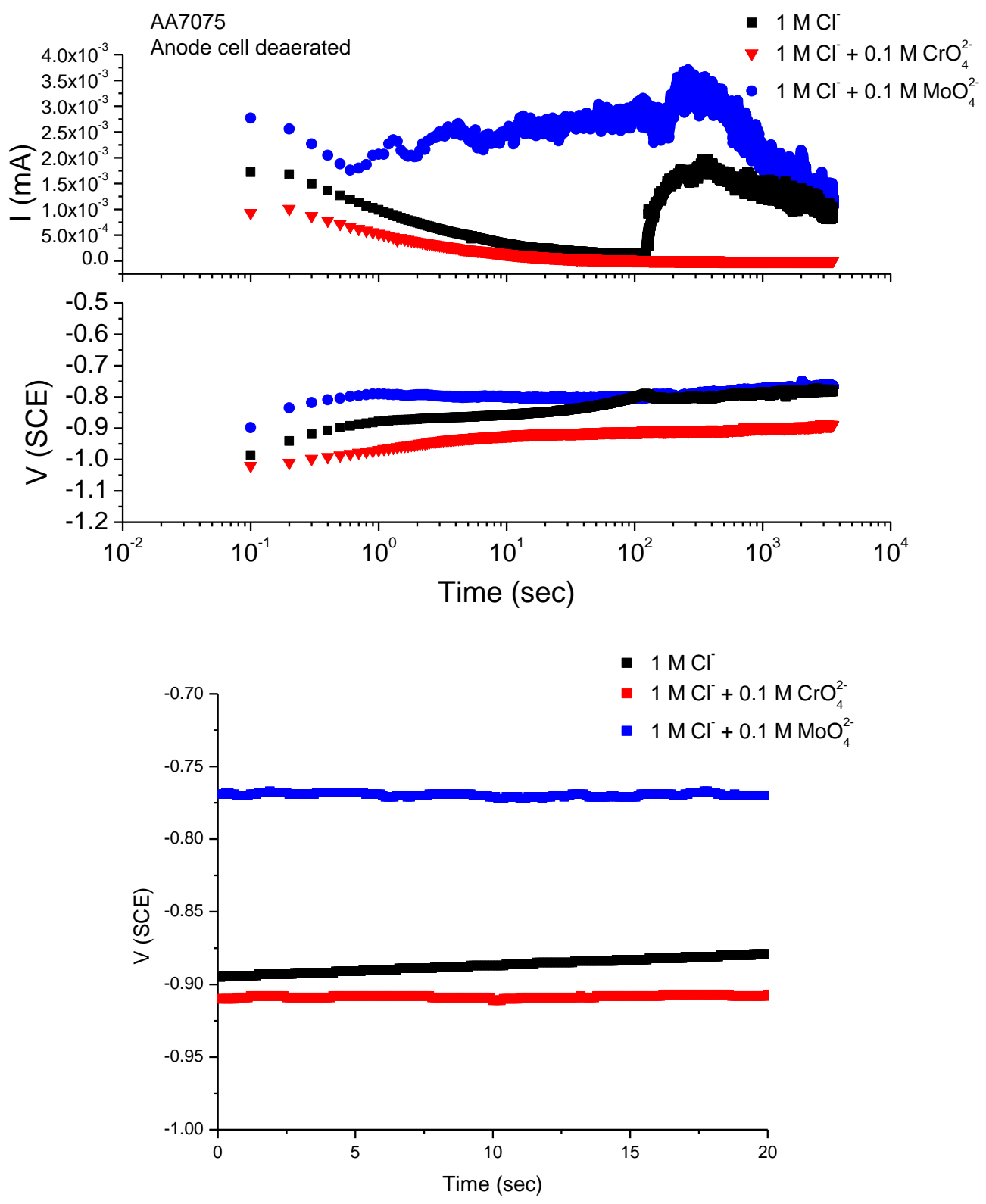

Figure 4.33. The effect of $0.1 \mathrm{M} \mathrm{CrO}_{4}{ }^{2-}$ and $\mathrm{MoO}_{4}{ }^{2-}$ on the scratch repassivation behavior of AA7075 when the inhibitors are confined to a much larger cathode in aqueous $1 \mathrm{M}$ $\mathrm{NaCl}$ with an adjusted $\mathrm{pH}$ 6.5. Also shown, is the OCP of the passivated 7075 electrolyte system moments before the scratch occurs. 

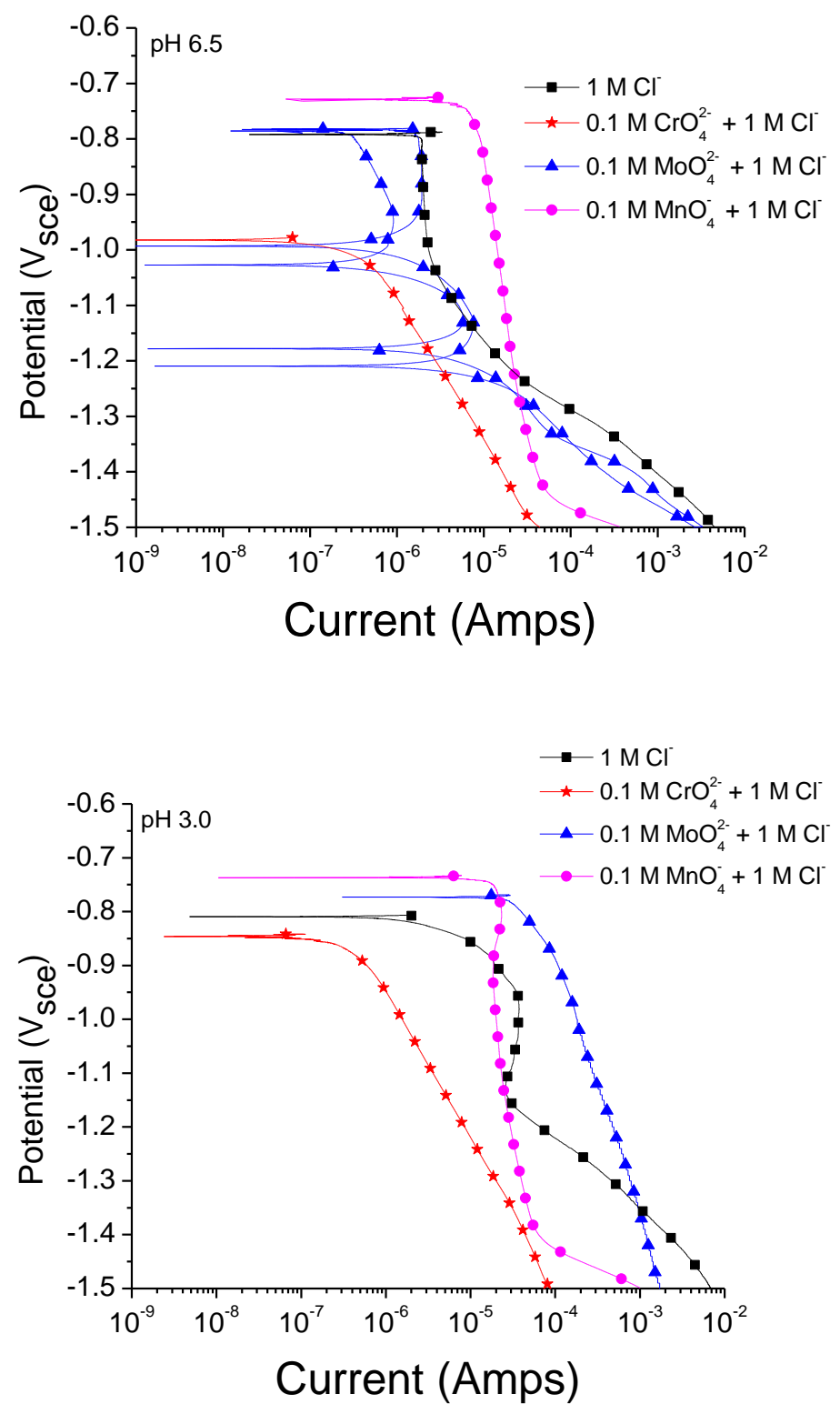

Figure 4.34. Effect of $0.1 \mathrm{M} \mathrm{CrO}_{4}{ }^{2-}, \mathrm{MoO}_{4}{ }^{2-}$, and $\mathrm{MnO}_{4}{ }^{2-}$ on the cathodic E-logi behavior of AA7075 in aqueous $1 \mathrm{M} \mathrm{Cl}^{-}$with an adjusted $\mathrm{pH}$ of 3 and 6.5. 

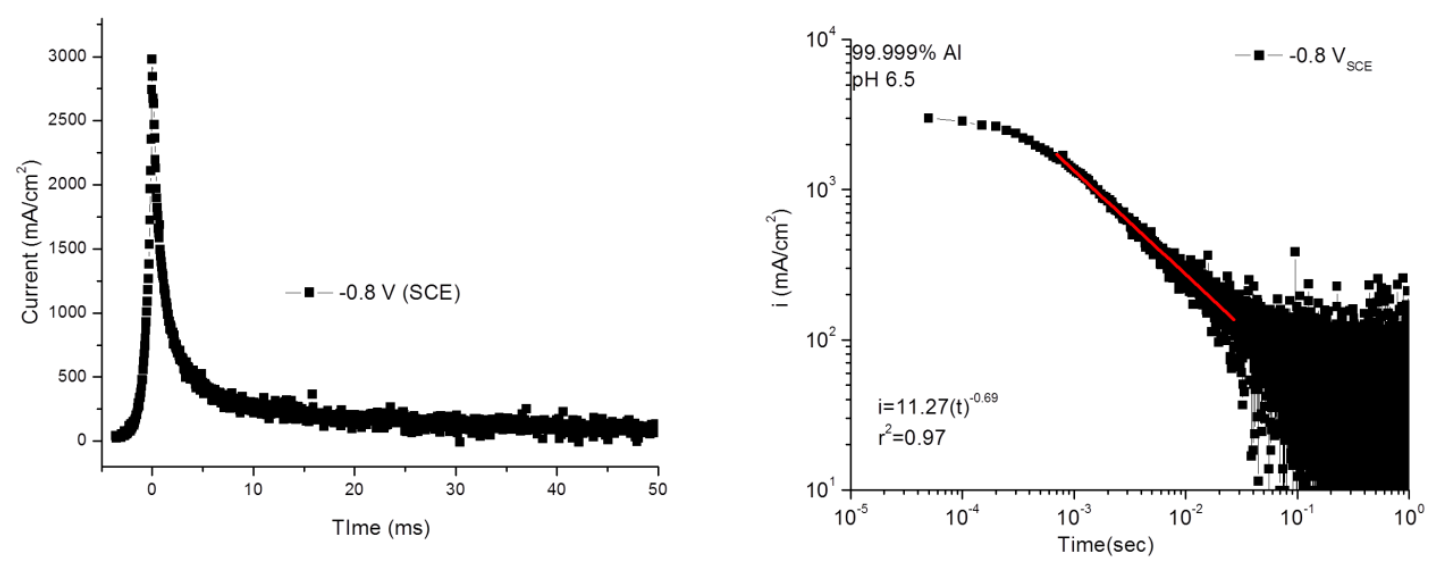

Figure $4.35 . i_{\text {net }}$ vs. time behavior of $99.999 \% \mathrm{Al}$ in $1 \mathrm{M} \mathrm{NaCl}$ at $\mathrm{pH} 6.5$ potentiostatically held at $-0.8 \mathrm{~V} \mathrm{SCE}$. The same data is plotted in both formats.

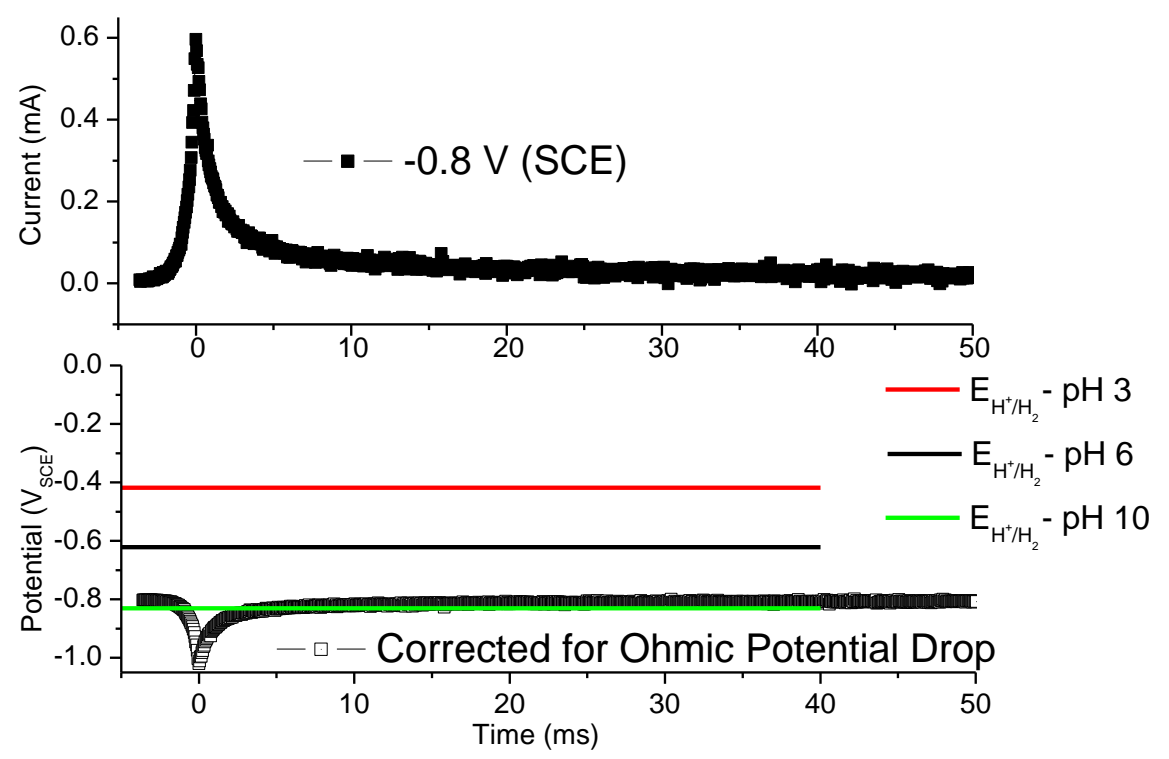

Figure 4.36. Scratch of $99.999 \% \mathrm{Al}$ in $1 \mathrm{M} \mathrm{NaCl}$ at $\mathrm{pH} 6.5$ potentiostatically held at $0.8 \mathrm{~V}$ SCE. The maximum ohmic potential drop at the working electrode is $0.3 \mathrm{~V}$ increasing the hydrogen over-potential. 
a)

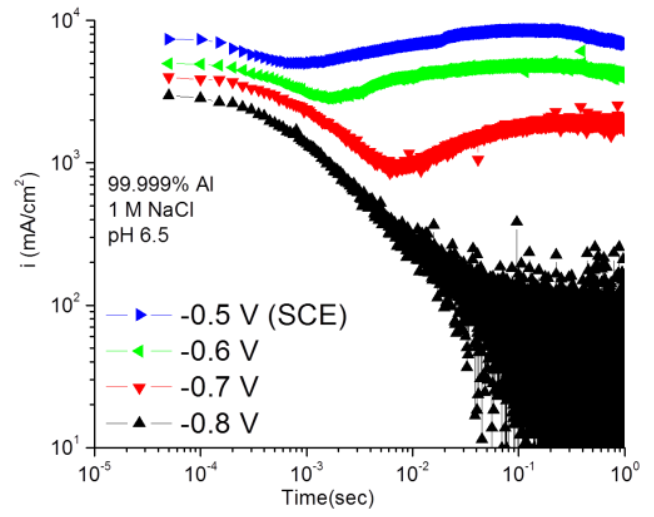

c)

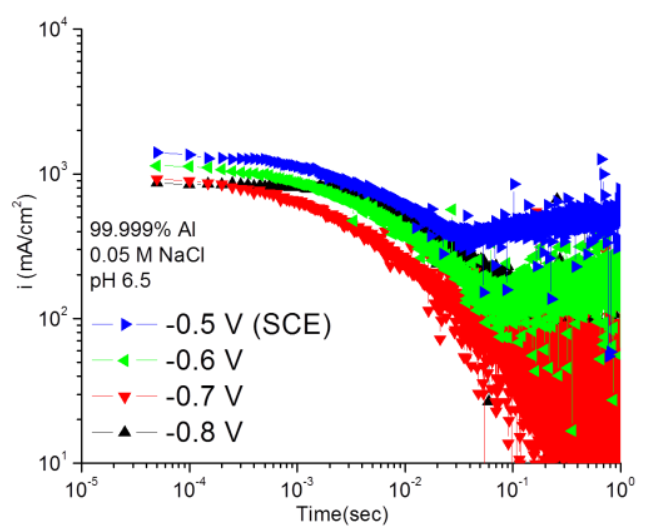

b)

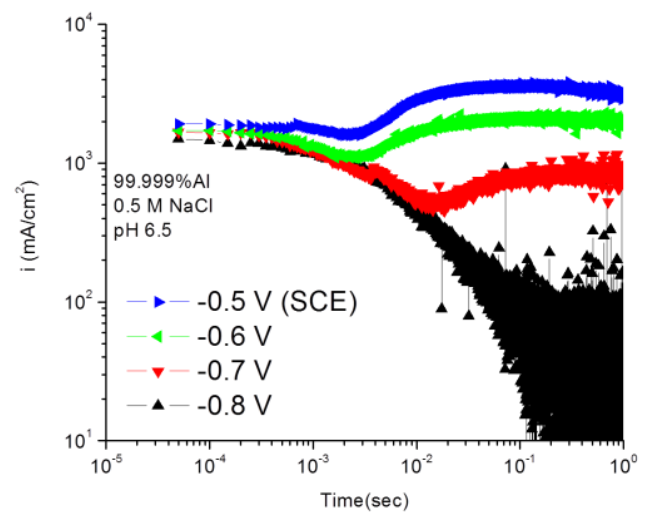

d)

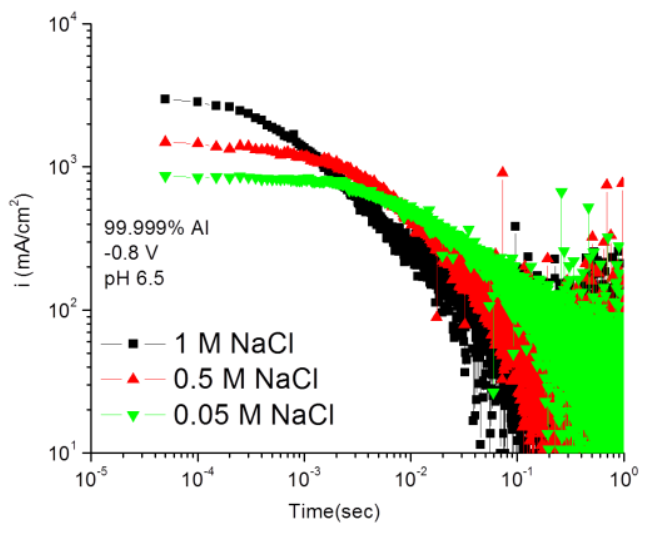

Figure 4.37. Current transients from 99.999\% aluminum scratched electrodes. Potentials from -0.5 to $-0.8 \mathrm{~V}$ (SCE) are shown at three chloride concentrations from 0.05 to $1 \mathrm{M}$. The $\mathrm{pH}$ for all tests was 6.5 . 


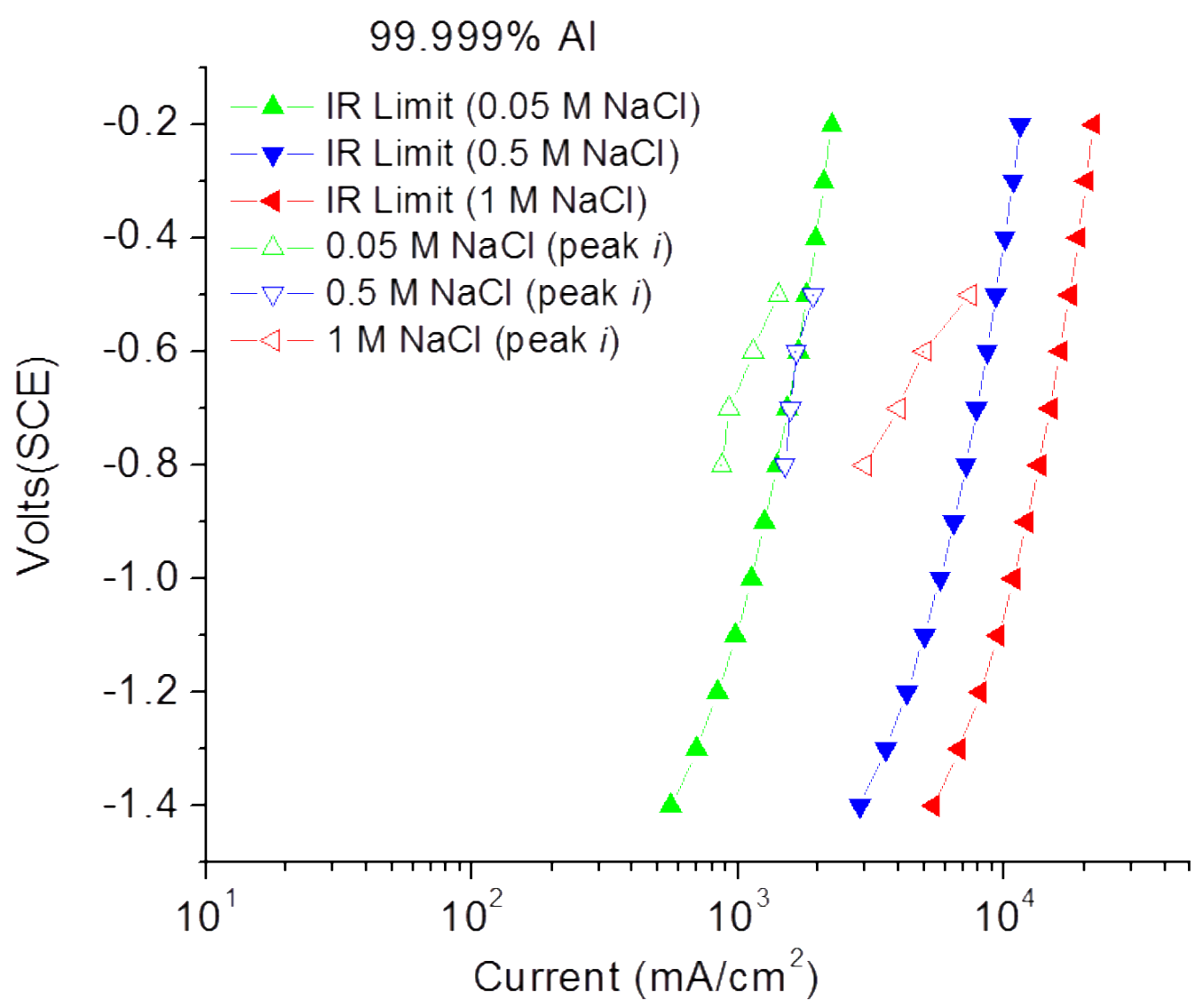

Figure 4.38. Calculated IR limit as compared to peak net current density of scratched 99.999\% aluminum electrodes in various chloride concentrations at time zero after scratch. 
a)

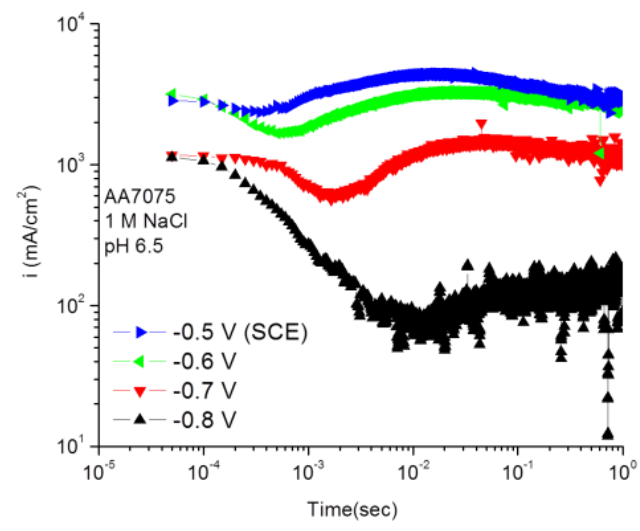

c)

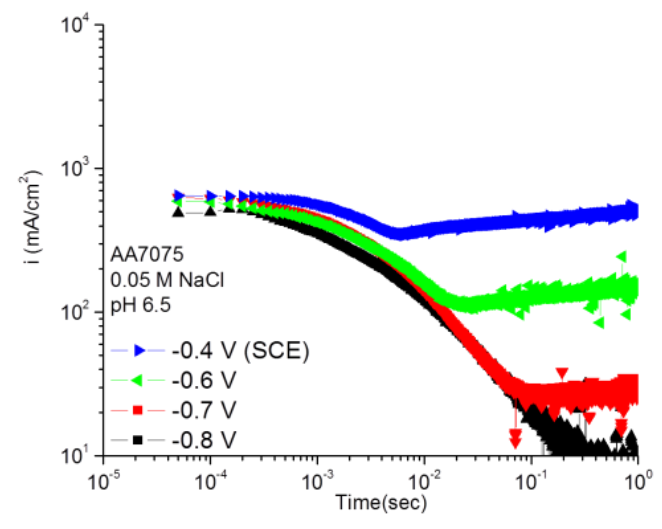

b)

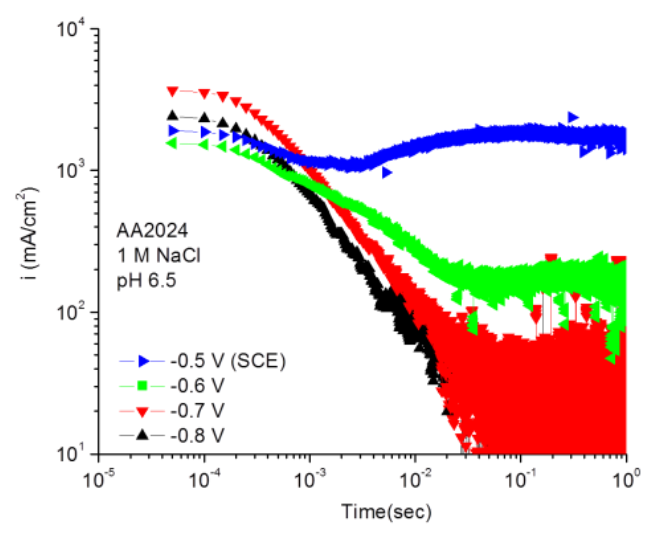

d)

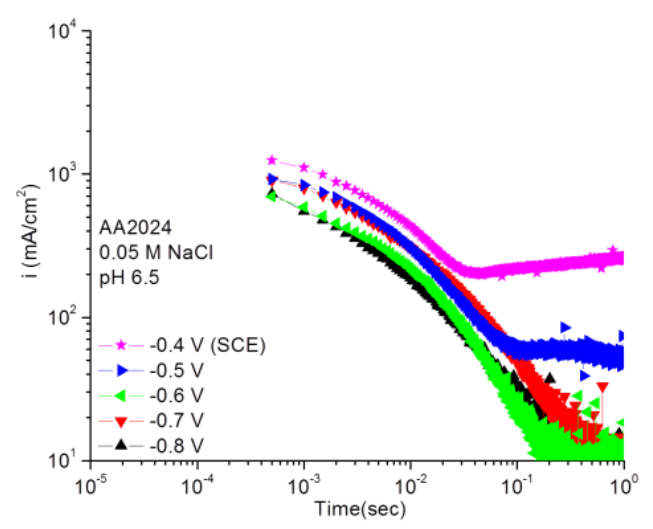

Figure 4.39. Net current transients from AA7075 (a) and AA2024 (b) in aqueous 1 M $\mathrm{NaCl}$ with $\mathrm{pH}$ adjusted to 6.5 with $\mathrm{NaOH}$. Scratch potentials vary from -0.5 to $-0.8 \mathrm{~V}$ (SCE). Current transients from AA7075 (c) and AA2024 (d) in aqueous $1 \mathrm{M} \mathrm{NaCl}$ with $\mathrm{pH}$ adjusted to 6.5. Scratch potentials vary from -0.5 to $-0.8 \mathrm{~V}$ (SCE) for (c) and -0.4 to $-0.8 \mathrm{~V}$ (SCE) for (d). 
a)

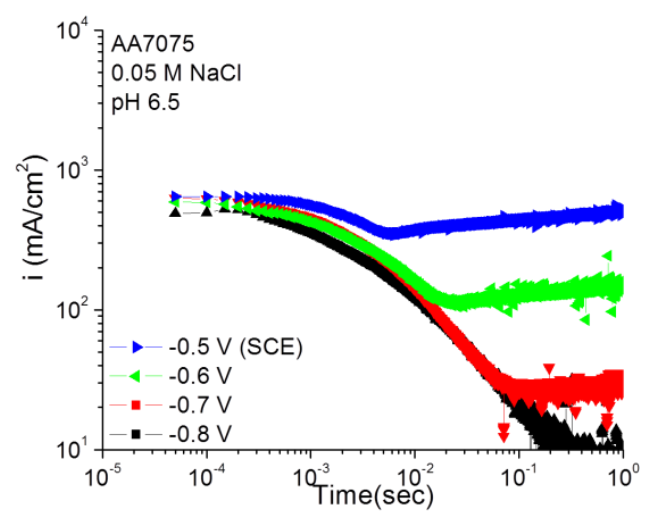

c)

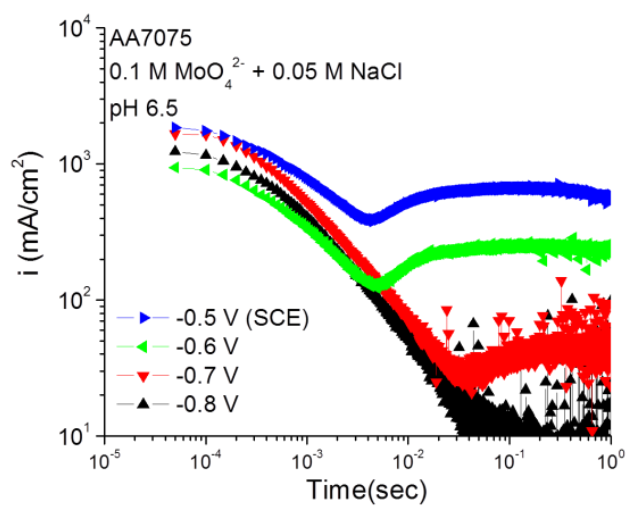

b)

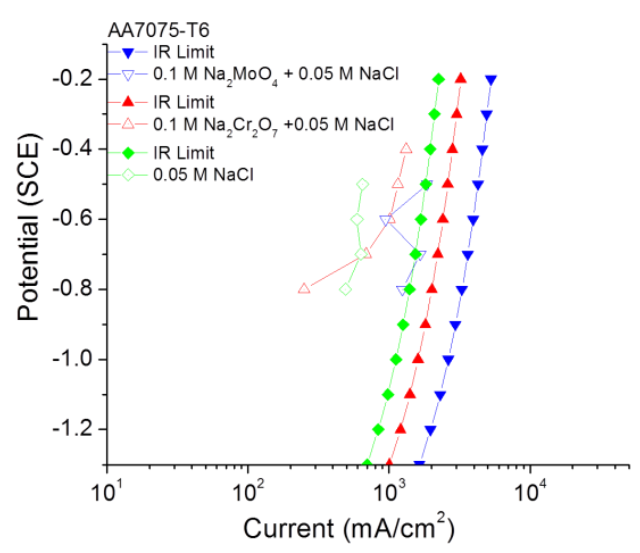

d)

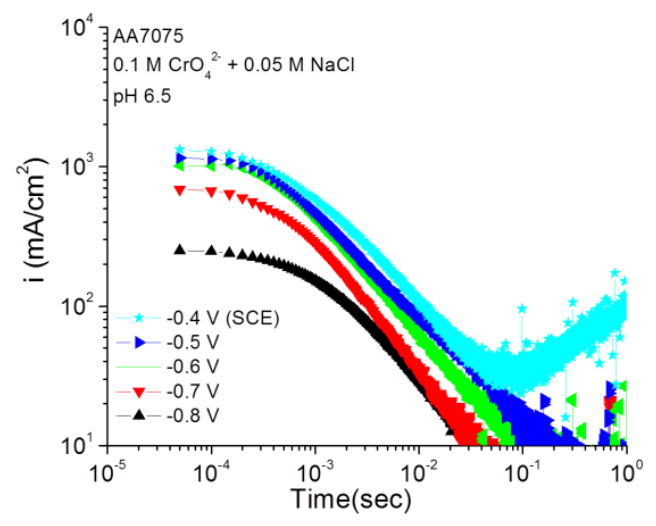

Figure 4.40. (a) Scratched electrode net anodic current densities for AA7075-T6 in 0.05 $\mathrm{M} \mathrm{NaCl}$ solution with $\mathrm{pH}$ 6.5. (b) Calculated IR limit as compared to peak current density of scratched AA7075 electrodes at time zero after scratch. IR limit is calculated for an aqueous $0.05 \mathrm{M} \mathrm{NaCl}$ solution, a $0.1 \mathrm{M} \mathrm{CrO}_{4}{ }^{2-}$ and $0.05 \mathrm{M} \mathrm{NaCl}$ solution, and $0.1 \mathrm{M}$ $\mathrm{MoO}_{4}{ }^{2-}$ and $0.05 \mathrm{M} \mathrm{NaCl}$ solution. (c) and (d) show scratched electrode current transients for AA7075-T6 with $0.1 \mathrm{M} \mathrm{MoO}_{4}{ }^{2-}$ and $0.1 \mathrm{M} \mathrm{CrO}_{4}{ }^{2-}$ additions, respectfully. 

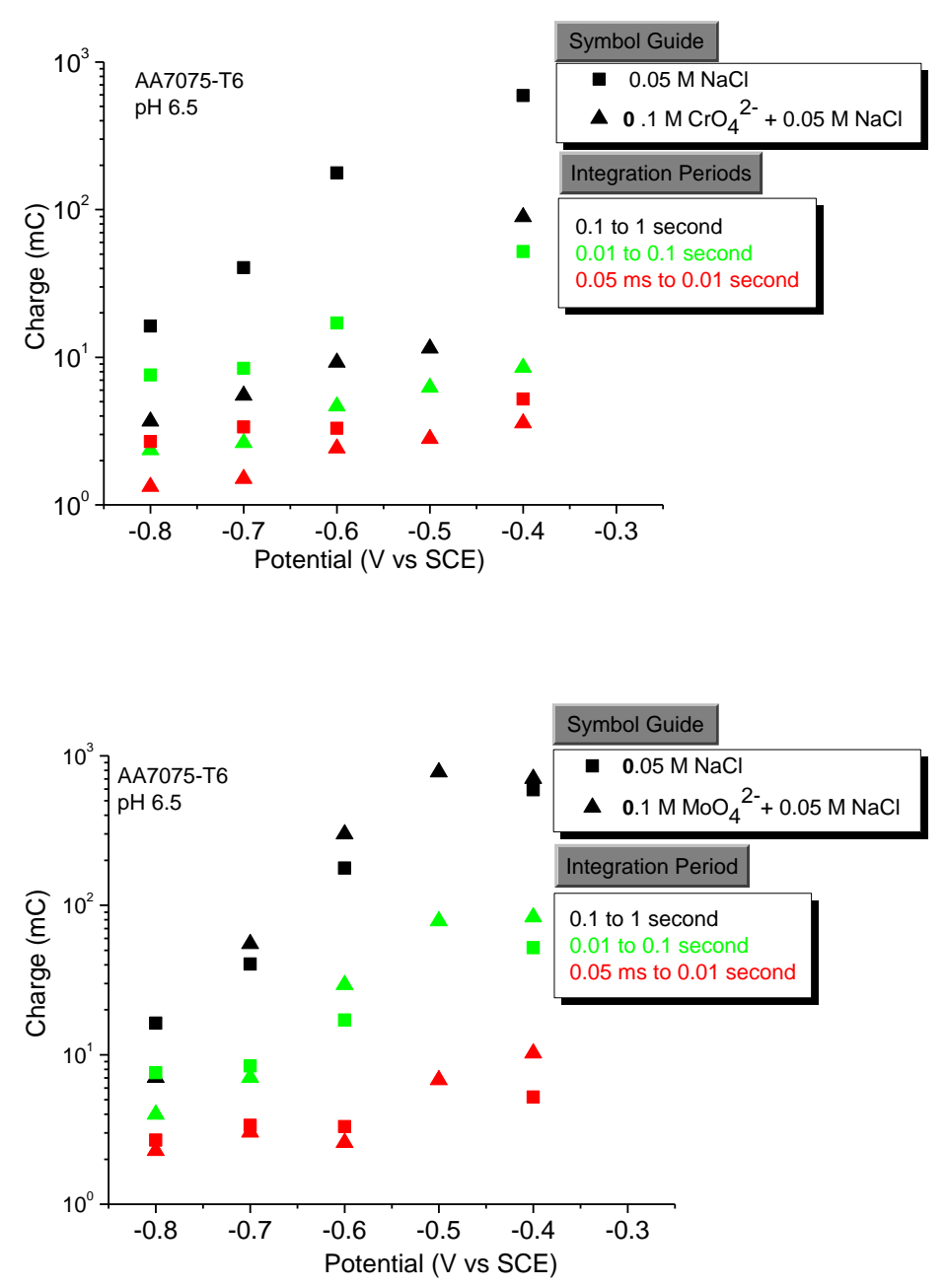

Figure 4.41. (a) Scratched electrode charge passed for AA7075-T6 in $0.05 \mathrm{M} \mathrm{NaCl}$ solution with a pH of 6.5 and $0.1 \mathrm{M} \mathrm{CrO}_{4}{ }^{2-}$ and $0.05 \mathrm{M} \mathrm{NaCl}$ solution. (b) Scratched electrode charge passed for AA2024-T351 in $0.05 \mathrm{M} \mathrm{NaCl}$ solution with a pH of 6.5 and $0.1 \mathrm{M} \mathrm{Na}_{2} \mathrm{MoO}_{4}$ and $0.05 \mathrm{M} \mathrm{NaCl}$ solution. Integration of current over three periods from 0.05 milliseconds to 0.01 second, 0.01 to 0.1 second, and 0.1 to 1 second. 
a)

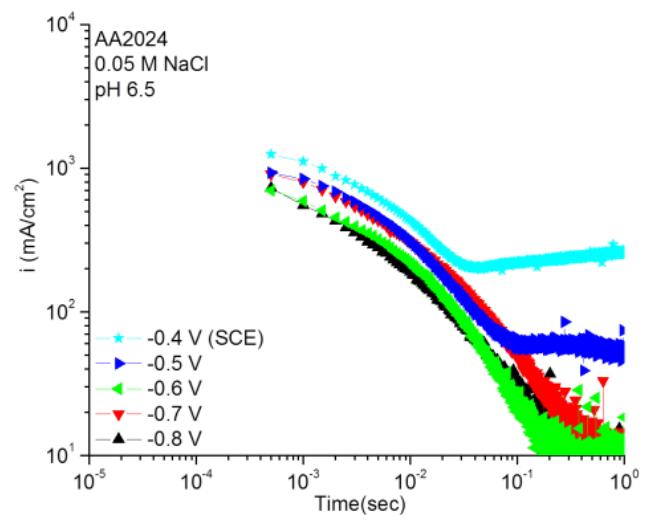

c)

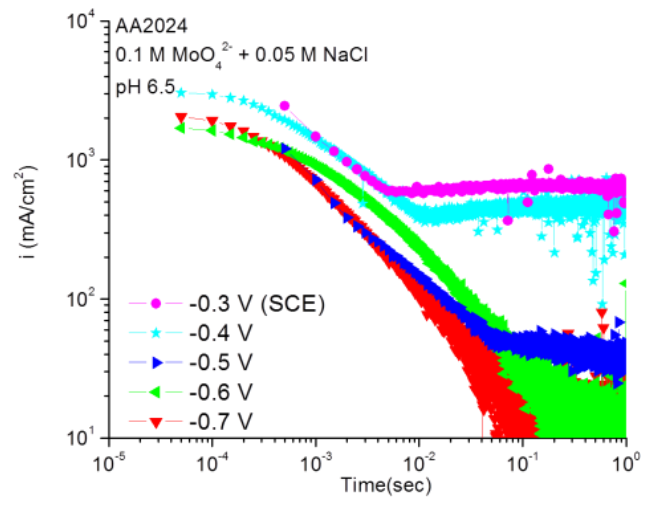

b)

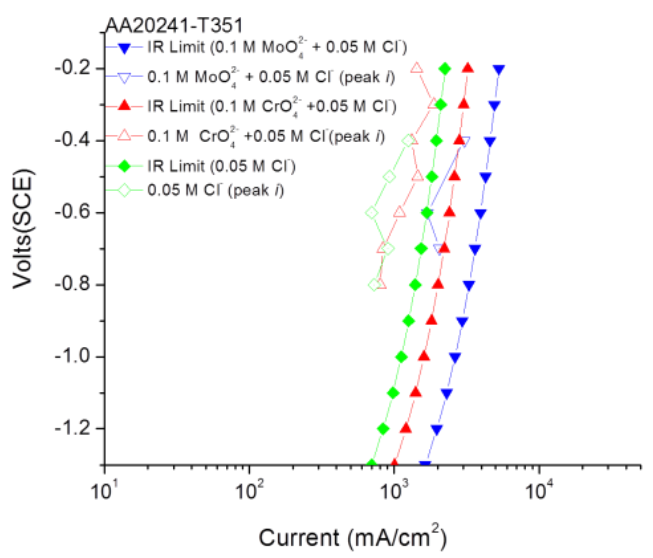

d)

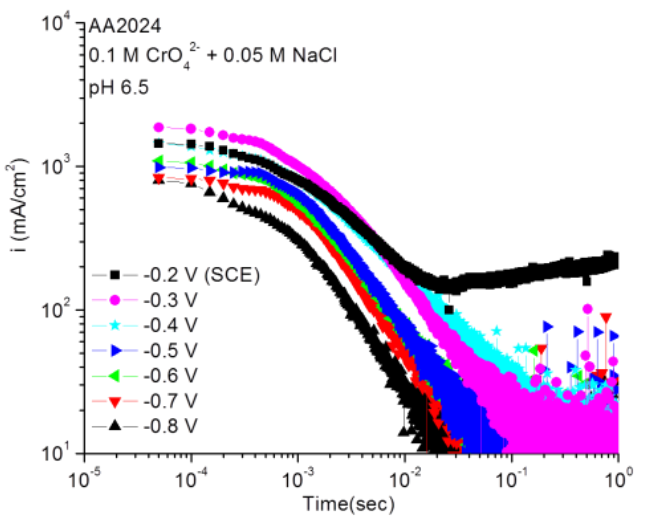

Figure 4.42. (a) Scratched electrode net anodic current densities for AA2024-T6 in 0.05 $\mathrm{M} \mathrm{NaCl}$ solution with $\mathrm{pH}$ 6.5. (b) Calculated IR limit as compared to peak current density of scratched AA2024 electrodes at time zero after scratch. IR limit is calculated for an aqueous $0.05 \mathrm{M} \mathrm{NaCl}$ solution, $0.1 \mathrm{M} \mathrm{CrO}_{4}{ }^{2-}$ and $0.05 \mathrm{M} \mathrm{NaCl}$ solution, and $0.1 \mathrm{M}$ $\mathrm{MoO}_{4}{ }^{2-}$ and $0.05 \mathrm{M} \mathrm{NaCl}$ solution. (c) and (d) show scratched electrode current transients for AA2024-T351 with $0.1 \mathrm{M} \mathrm{MoO}_{4}{ }^{2-}$ and $0.1 \mathrm{M} \mathrm{CrO}_{4}{ }^{2-}$ additions, respectfully. 

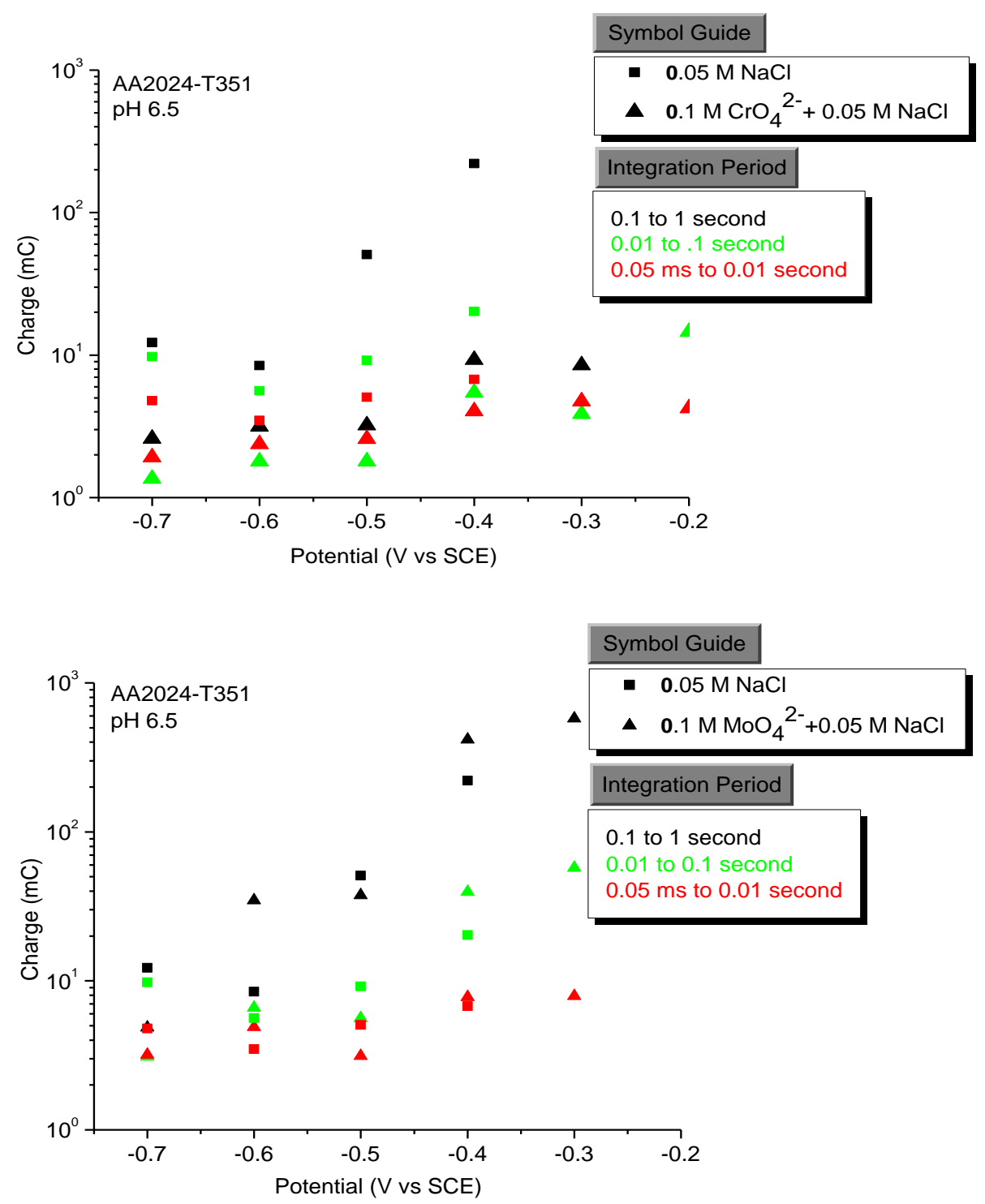

Figure 4.43. (a) Scratched electrode charge passed for AA2024-T351 in $0.05 \mathrm{M} \mathrm{NaCl}$ solution with a pH of 6.5 and $0.1 \mathrm{M} \mathrm{CrO}_{4}{ }^{2-}$ and $0.05 \mathrm{M} \mathrm{NaCl}$ solution. (b) Scratched electrode charge passed for AA2024-T351 in $0.05 \mathrm{M} \mathrm{NaCl}$ solution with a pH of 6.5 and $0.1 \mathrm{M} \mathrm{MoO}_{4}{ }^{2-}$ and $0.05 \mathrm{M} \mathrm{NaCl}$ solution. Integration of current over three periods from 0.05 milliseconds to 0.01 second, 0.01 to 0.1 second, and 0.1 to 1 second. 


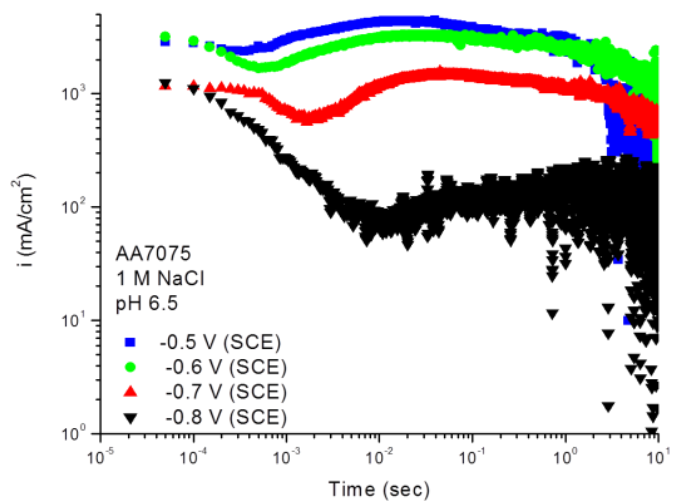

A

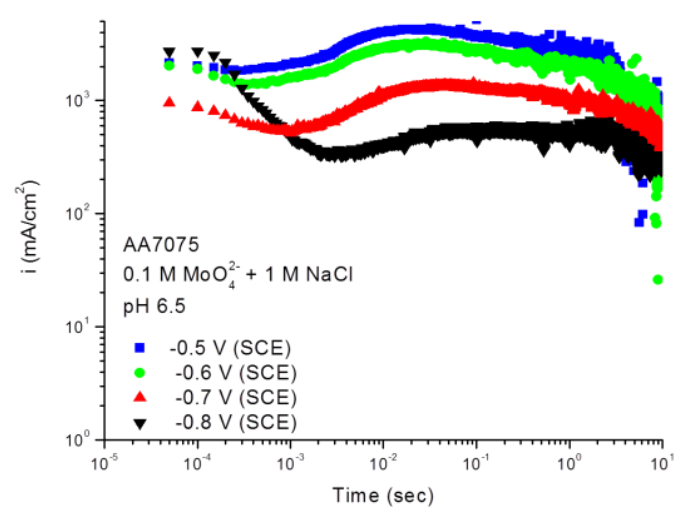

C

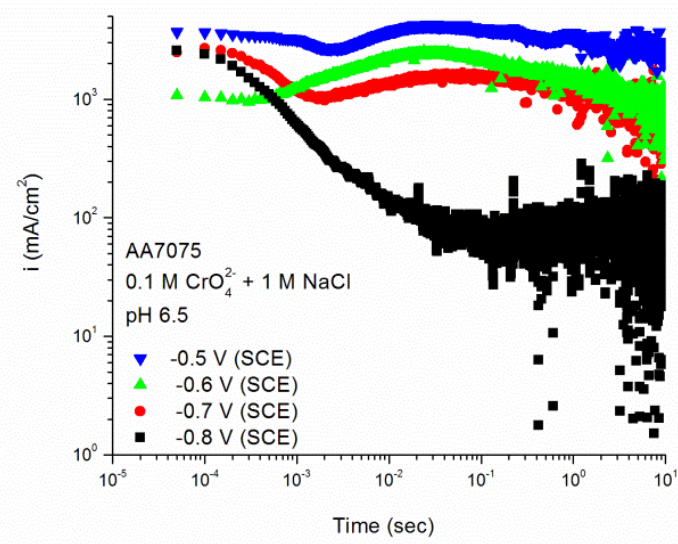

B

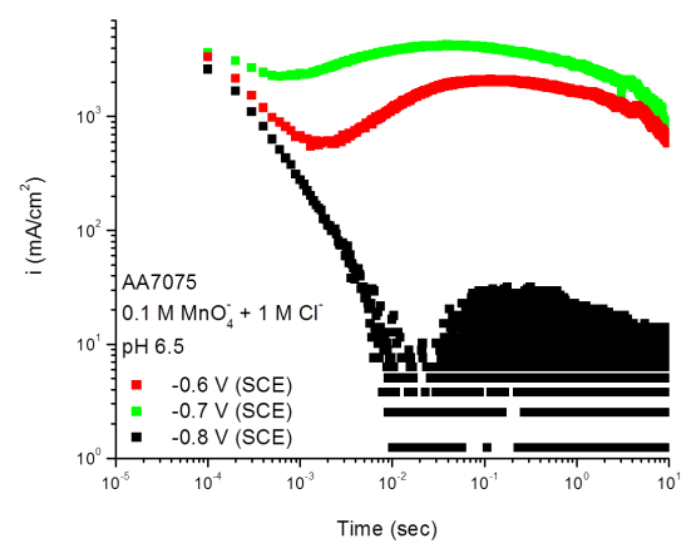

D

Figure 4.44. (a) Scratched electrode net anodic current densities for AA7075-T6 in $1 \mathrm{M}$ $\mathrm{NaCl}$ solution with $\mathrm{pH}$ 6.5. Effect of addition of $0.1 \mathrm{M} \mathrm{MoO}_{4}{ }^{2-}, 0.1 \mathrm{M} \mathrm{MnO}_{4}^{-}$, and $0.1 \mathrm{M}$ $\mathrm{CrO}_{4}{ }^{2-}$ additions are also presented. 


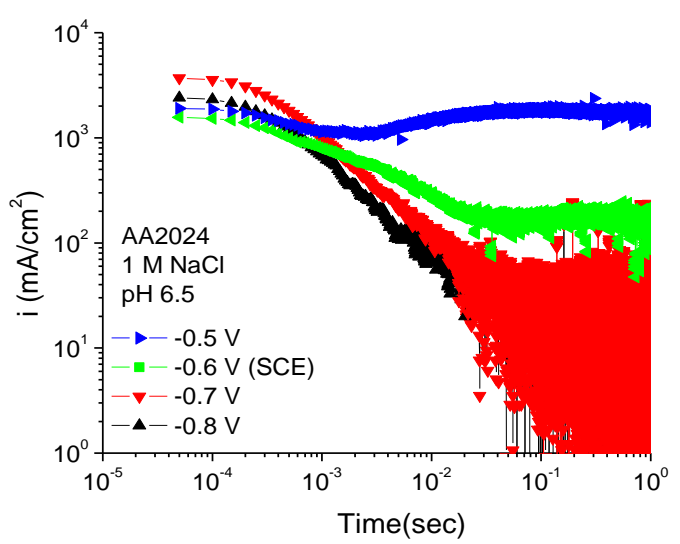

A

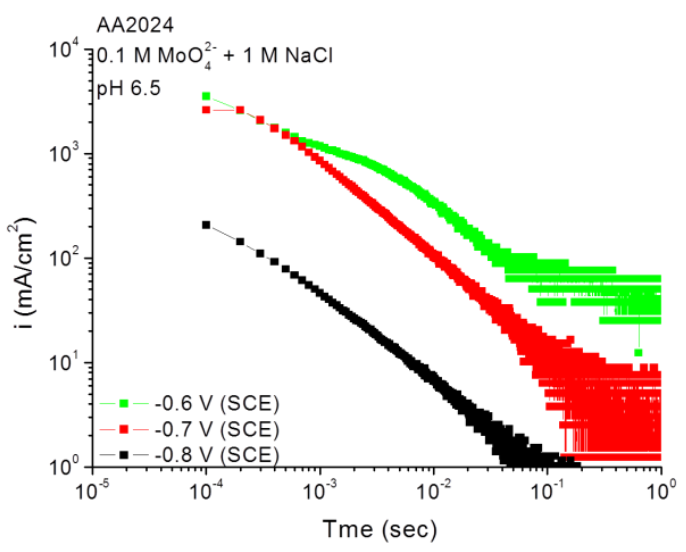

C

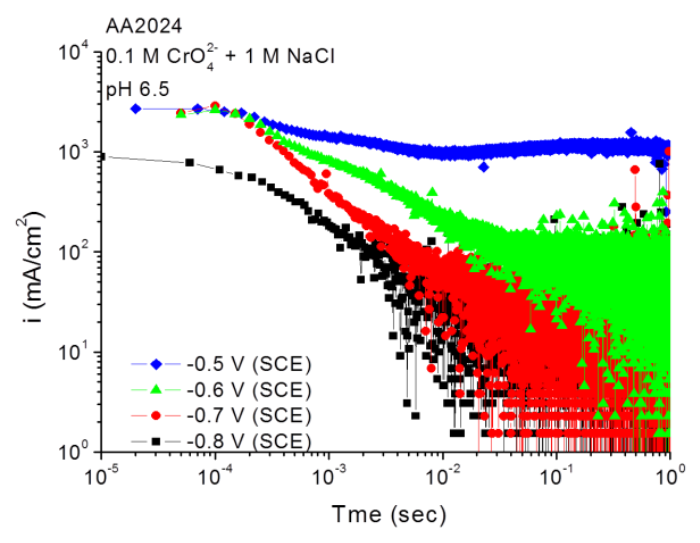

B

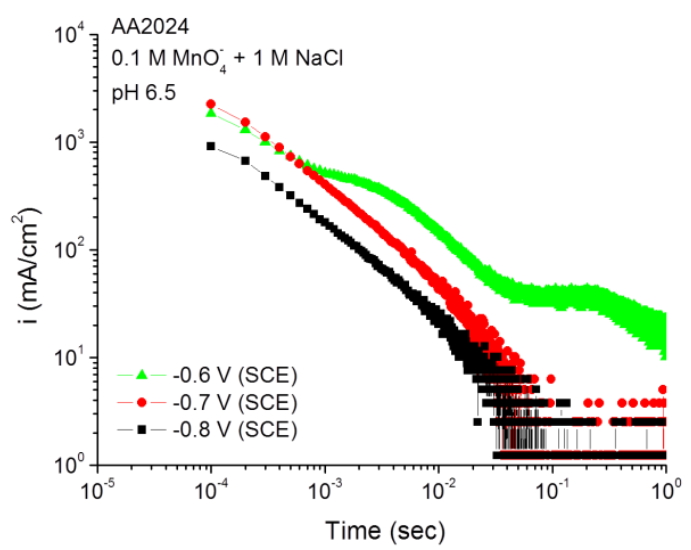

C

Figure 4.45. (a) Scratched electrode net anodic current densities for AA2024-T351 in 1 $\mathrm{M} \mathrm{NaCl}$ solution with $\mathrm{pH}$ 6.5. (b) (c) and (d) show scratched electrode current transients for AA7075-T6 with $0.1 \mathrm{M} \mathrm{CrO}_{4}{ }^{2-}, 0.1 \mathrm{M} \mathrm{MoO}_{4}{ }^{2-}$, and $0.1 \mathrm{M} \mathrm{MnO}_{4}^{-}$additions, respectfully. 

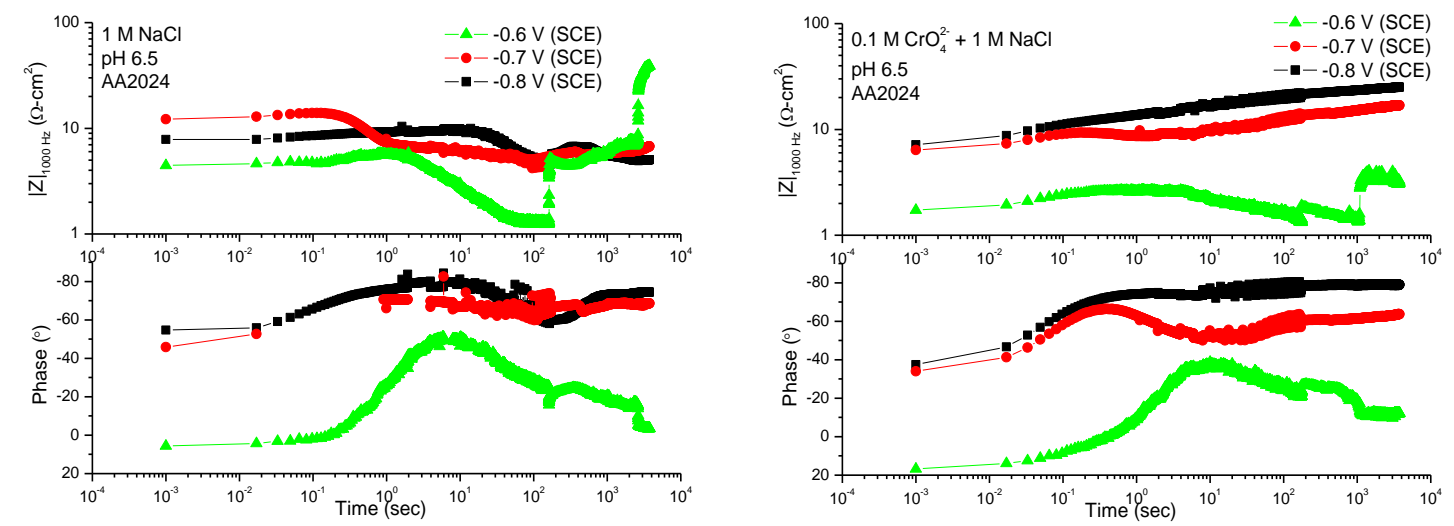

A

B
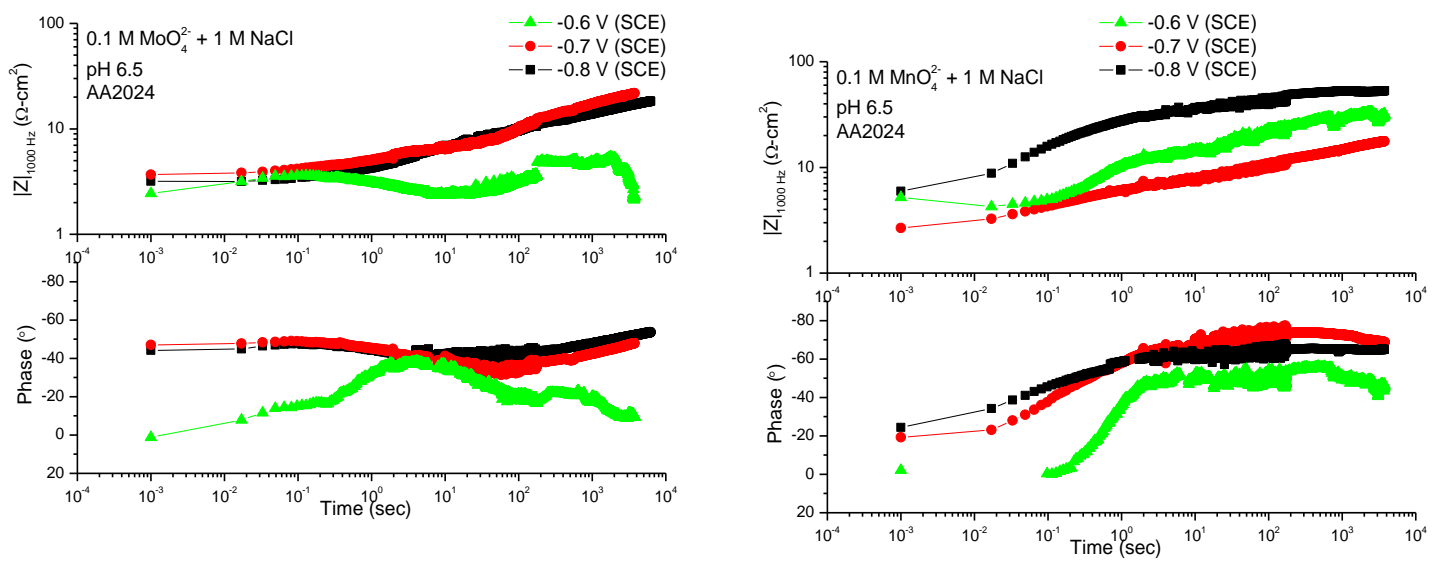

C

$\mathrm{D}$

Figure 4.46 Single frequency impedance at $1000 \mathrm{~Hz}$ and phase angle data for scratched AA2024 in $1 \mathrm{M} \mathrm{NaCl}$ with and without the addition of inhibiting $\mathrm{CrO}_{4}{ }^{2-}, \mathrm{MoO}_{4}{ }^{2-}$, and $\mathrm{MnO}_{4}{ }^{-}$anions with the concentration of $0.1 \mathrm{M}$. $\mathrm{pH}$ was adjusted to 6.5 for all solutions. 

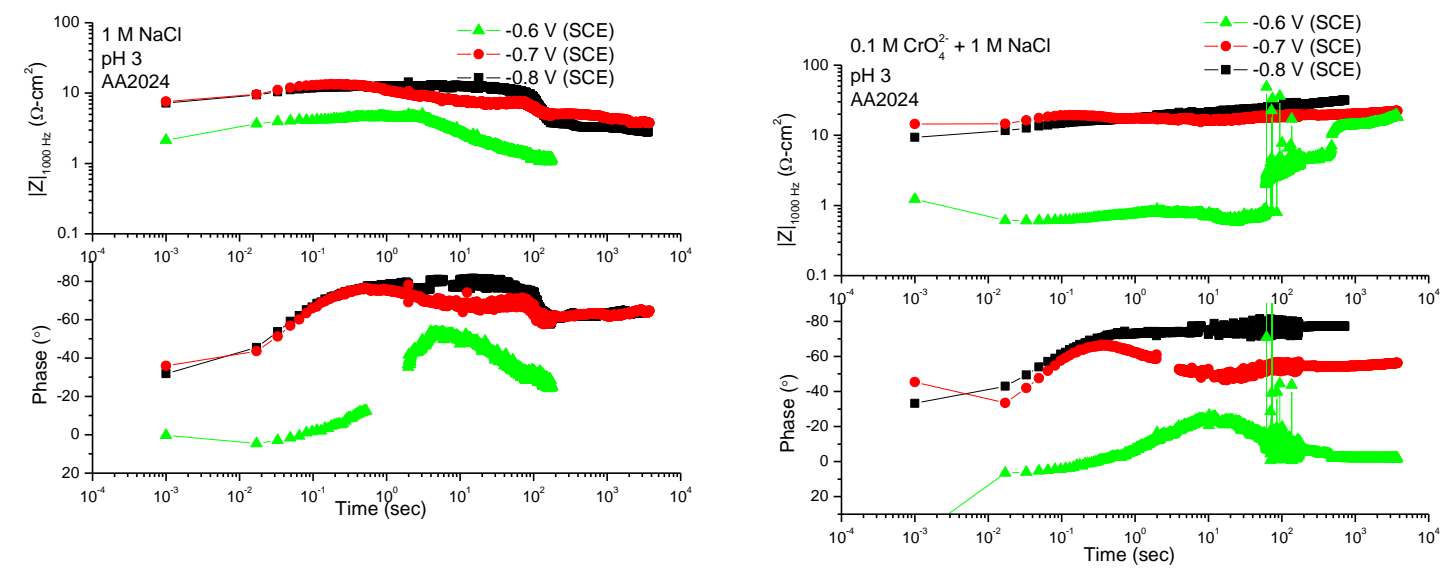

A

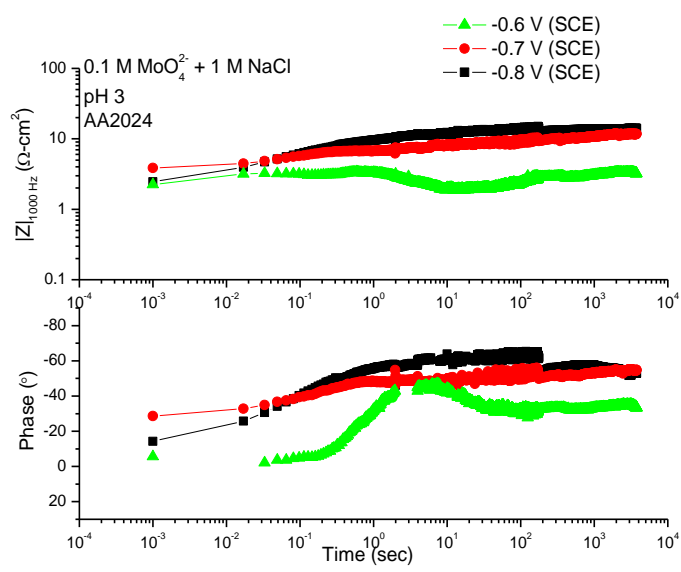

$\mathrm{C}$

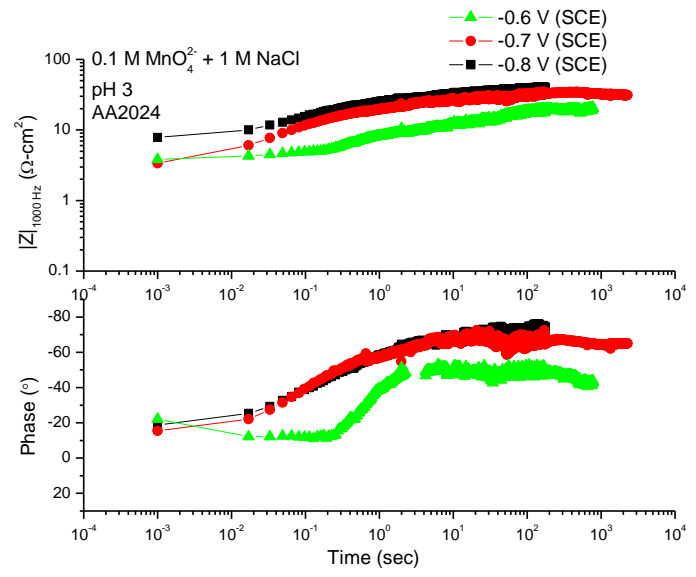

$\mathrm{D}$

Figure 4.47 Fast single frequency impedance and phase data for scratched AA2024 in 1 $\mathrm{M} \mathrm{NaCl}$ with and without the addition of inhibiting $\mathrm{CrO}_{4}{ }^{2-}, \mathrm{MoO}_{4}{ }^{2-}$, and $\mathrm{MnO}_{4}{ }^{-}$anions with the concentration of $0.1 \mathrm{M}$. $\mathrm{pH}$ was adjusted to 3 for all solutions. 


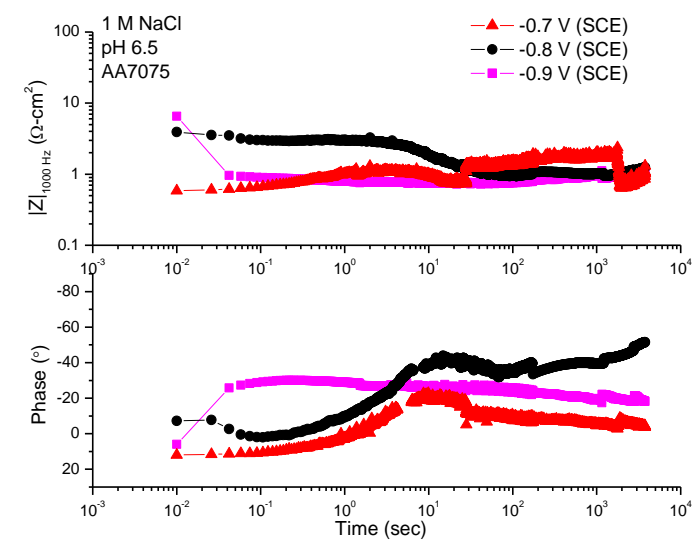

A

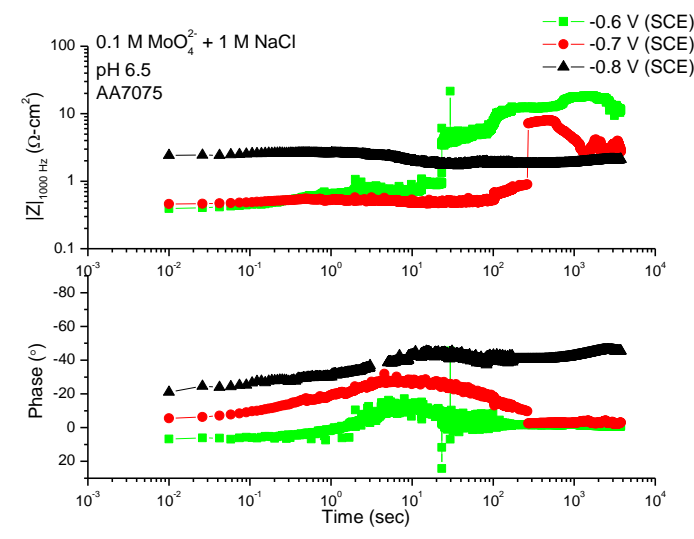

$\mathrm{C}$

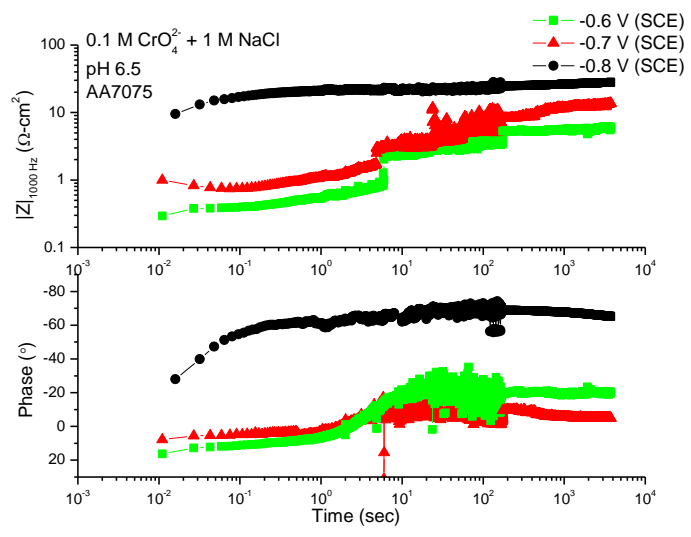

B

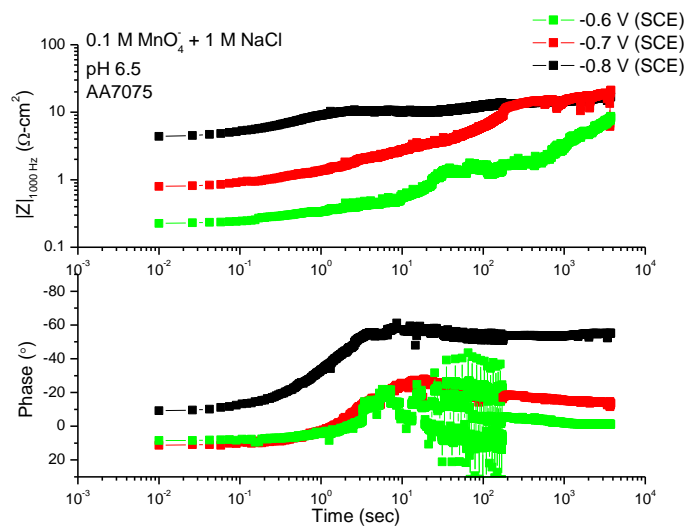

D

Figure 4.48 Fast single frequency impedance and phase data for scratched AA7075 in 1 $\mathrm{M} \mathrm{NaCl}$ with and without the addition of inhibiting $\mathrm{CrO}_{4}{ }^{2-}, \mathrm{MoO}_{4}{ }^{2-}$, and $\mathrm{MnO}_{4}{ }^{-}$anion with concentration of $0.1 \mathrm{M}$. $\mathrm{pH}$ was adjusted to 6.5 for all solutions. 

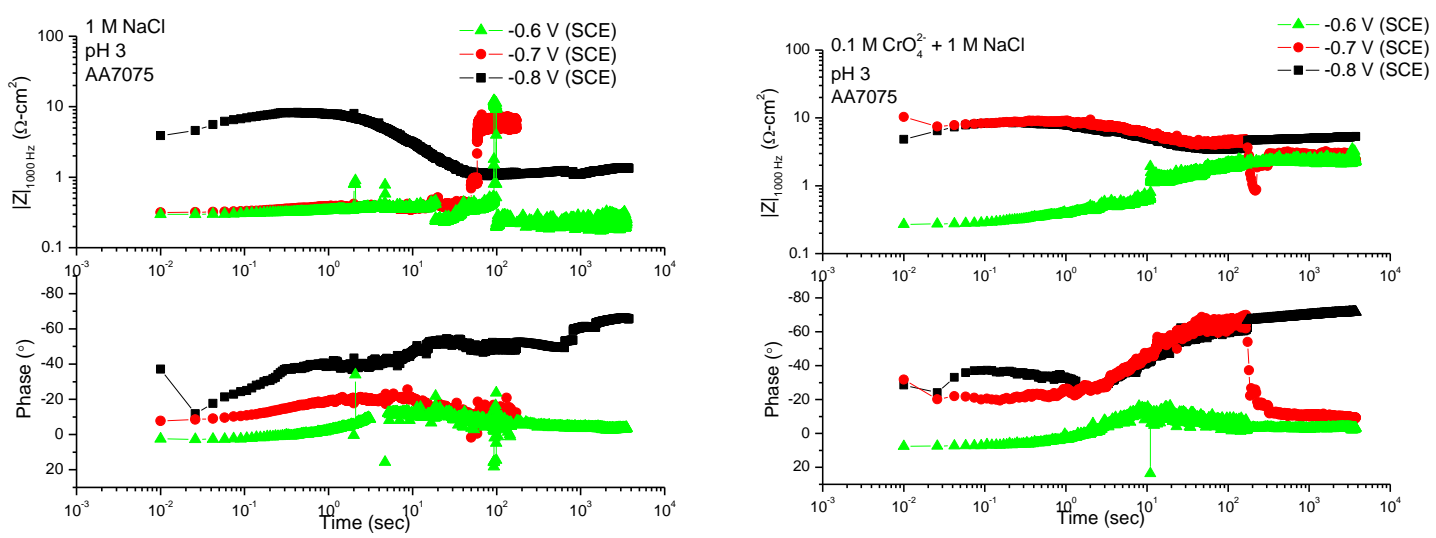

$\mathrm{B}$

A
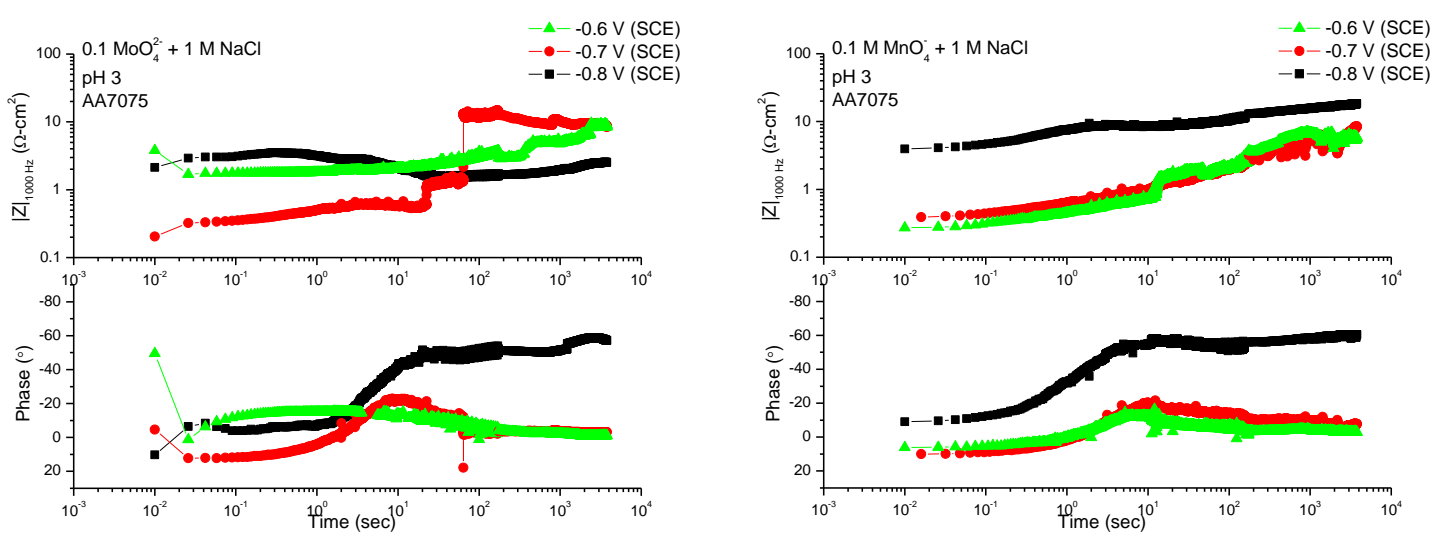

$\mathrm{C}$

Figure 4.49 Fast single frequency impedance and phase data for scratched AA7075 in 1 $\mathrm{M} \mathrm{NaCl}$ with and without the addition of inhibiting $\mathrm{CrO}_{4}{ }^{2-}, \mathrm{MoO}_{4}{ }^{2-}$, and $\mathrm{MnO}_{4}{ }^{-}$anion with concentration of $0.1 \mathrm{M}$. $\mathrm{pH}$ was adjusted to 3 for all solutions. 

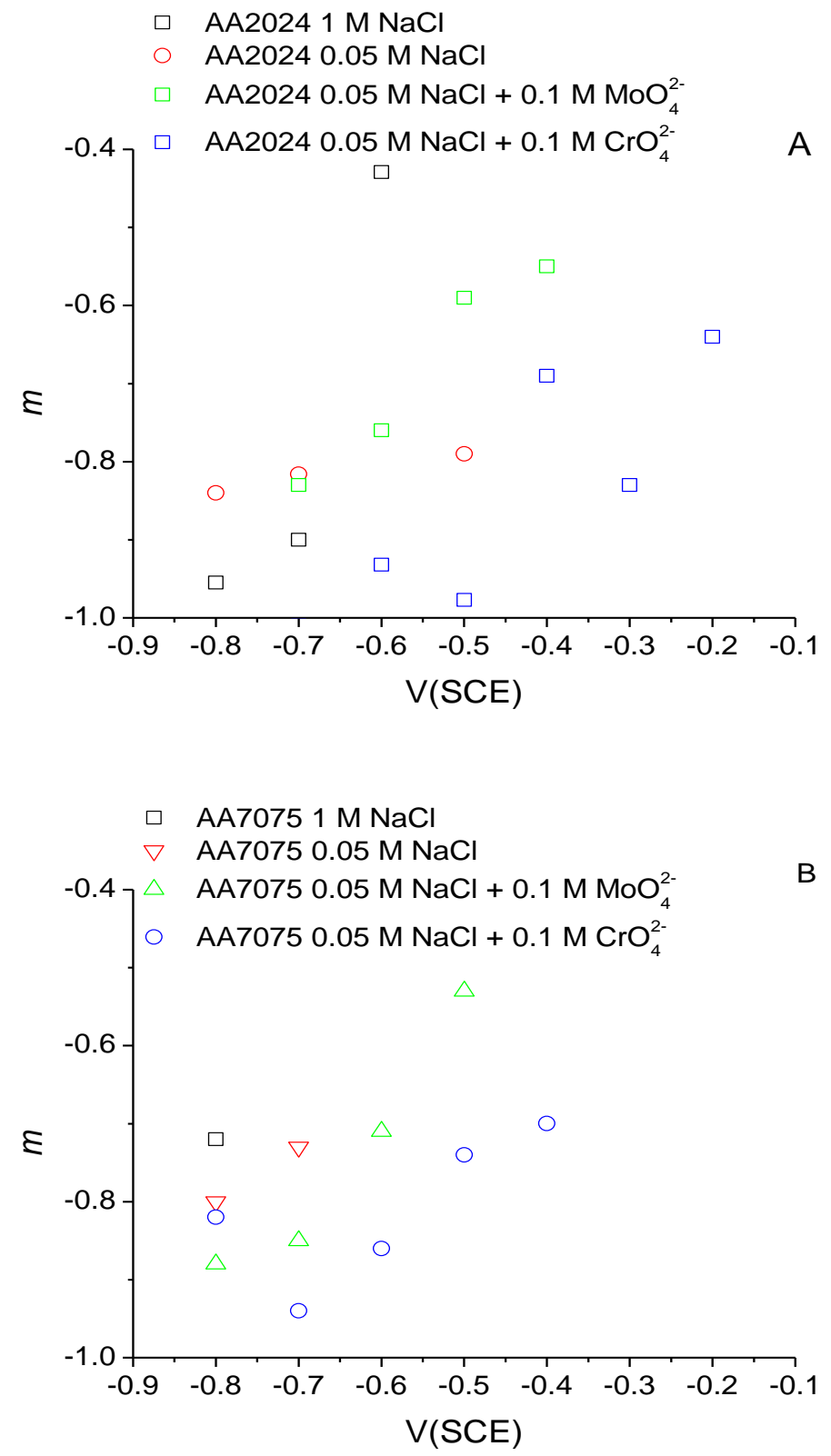

Figure 4.50 Logarithmic constant $m$ plotted vs potential for passivating oxide film growth on AA2024 (A) and AA7075 (B) in $1 \mathrm{M} \mathrm{NaCl}, 0.05 \mathrm{M} \mathrm{NaCl}$, and $0.05 \mathrm{M} \mathrm{NaCl}$ with $0.1 \mathrm{M} \mathrm{MoO}_{4}{ }^{2-}$ and $0.1 \mathrm{M} \mathrm{CrO}_{4}{ }^{2-}$ inhibitor additions. Constant $m$ is obtained by logarithmic modeling of current transients according to Equation 4.11 which was modified from Equation 4.3. 


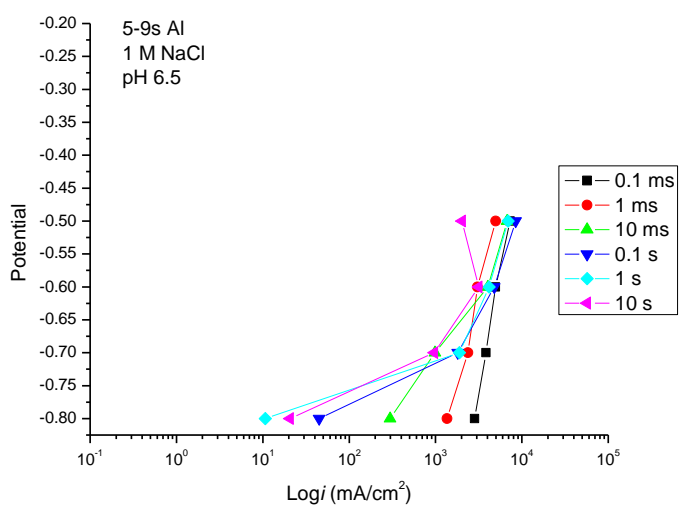

A

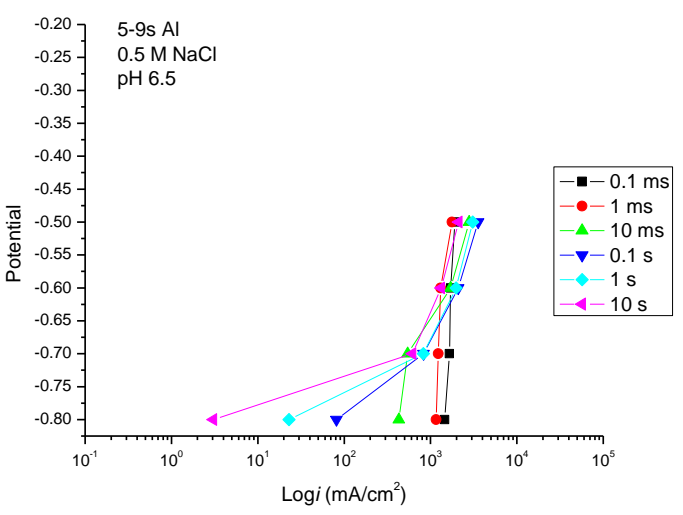

B

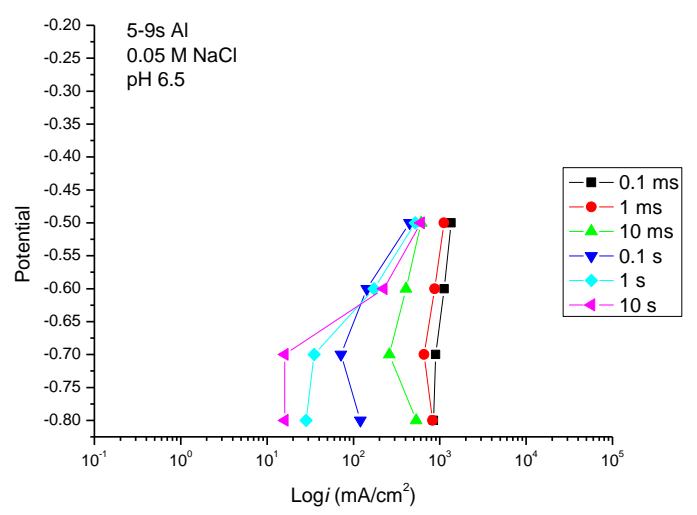

C

Figure 4.51. E-logi behavior of 99.999 wt \% aluminum as a function of time after a mechanical depassivation event. E-logi behavior in $1 \mathrm{M}, 0.5 \mathrm{M}$, and $0.05 \mathrm{M} \mathrm{NaCl}$ are shown in A, B, and C respectively. Data gathered from scratch transients shown in Figure 4.37 a-c. 


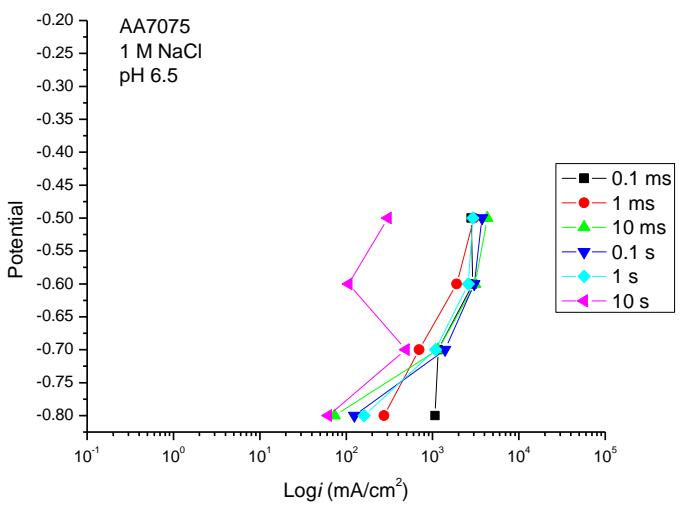

A

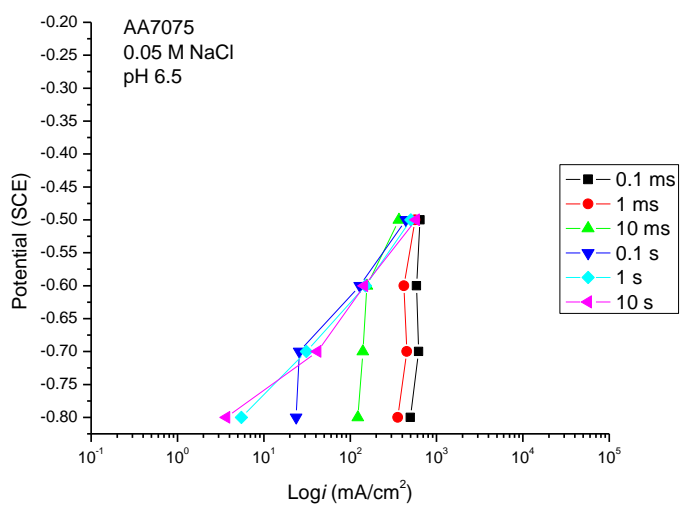

$\mathrm{C}$

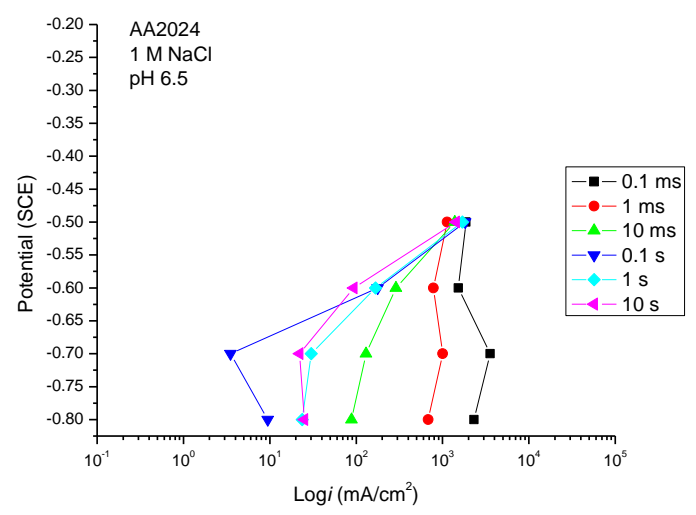

B

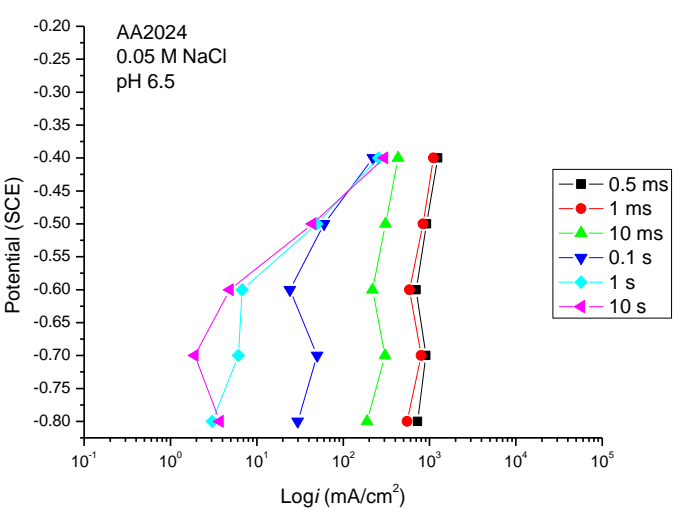

$\mathrm{D}$

Figure 4.52. E-logi behavior of AA7075 and AA2024 as a function of time after a mechanical depassivation event. AA7075 and A2024 E-logi behavior in $1 \mathrm{M} \mathrm{NaCl}$ is shown in $\mathrm{A}$ and $\mathrm{B}$ respectively and in $0.05 \mathrm{M} \mathrm{NaCl}$ in $\mathrm{C}$ and $\mathrm{D}$ respectively. Data gathered from scratch transients shown in Figure 4.39. 


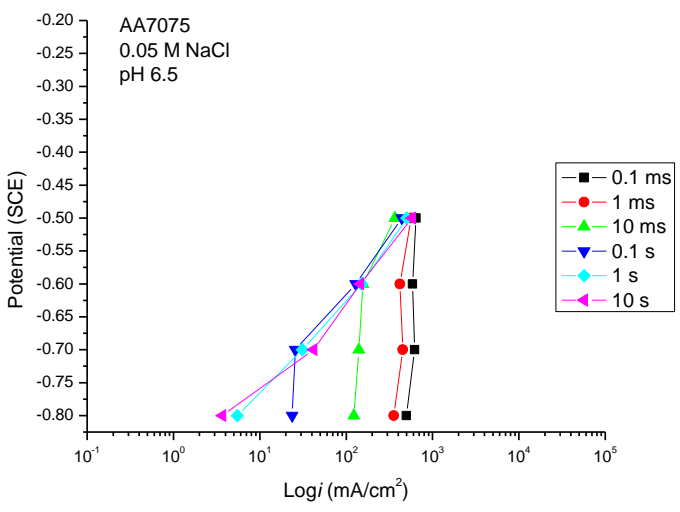

A

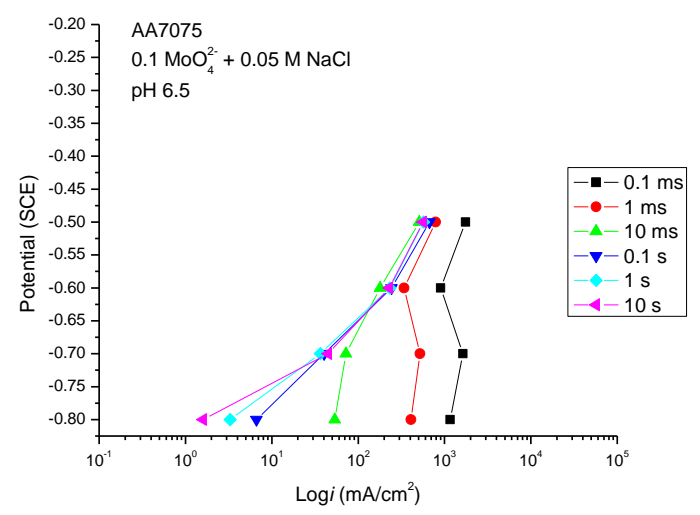

B

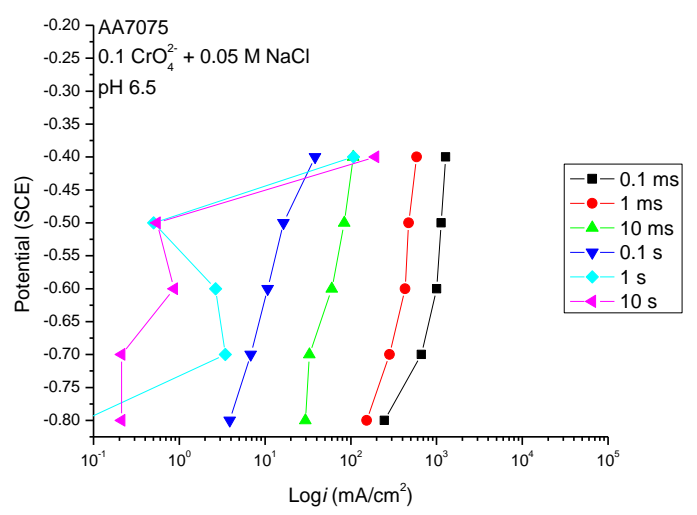

C

Figure 4.53. The effect to $0.1 \mathrm{M} \mathrm{MoO}_{4}{ }^{2-}$ and $\mathrm{CrO}_{4}{ }^{2-}$ on the E-logi behavior of AA7075 as a function of time after a mechanical depassivation event. AA7075 E-logi behavior in $0.1 \mathrm{MoO}_{4}{ }^{2-}$ and $0.05 \mathrm{M} \mathrm{NaCl}$ is shown in $\mathrm{B}$ and in $0.1 \mathrm{CrO}_{4}{ }^{2-}$ and $0.05 \mathrm{M} \mathrm{NaCl}$ in $\mathrm{C}$. Data gathered from scratch transients shown in Figure 4.40. 


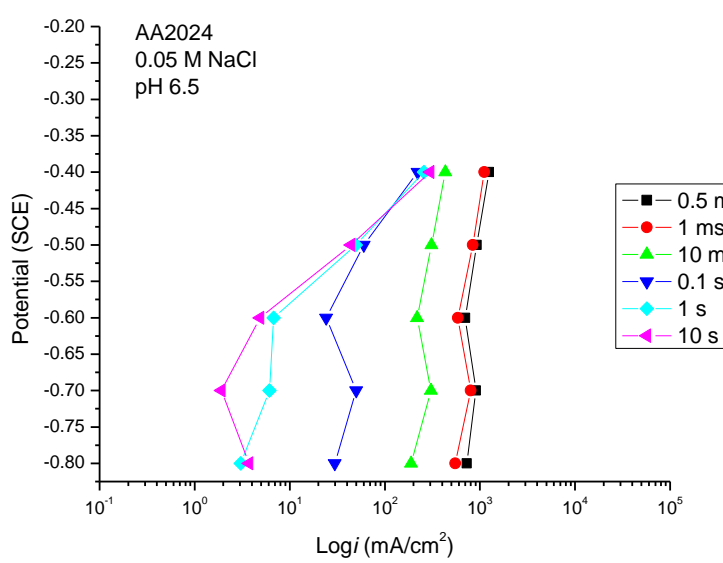

A

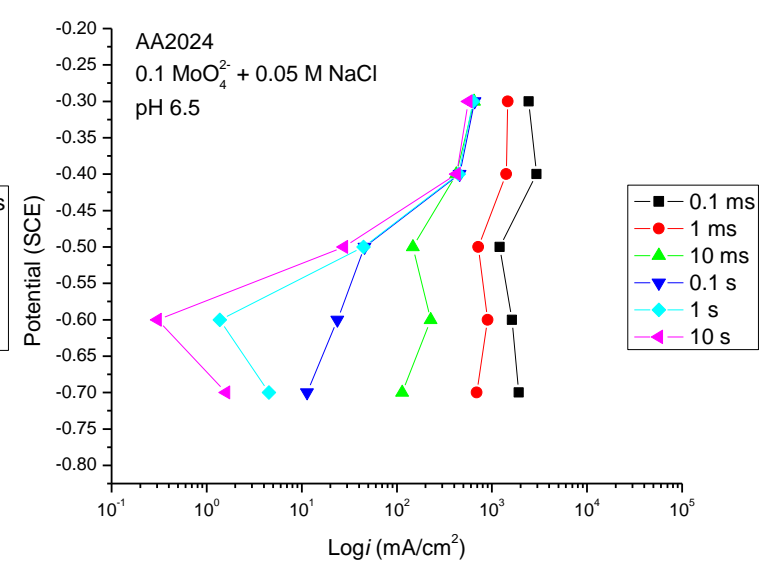

B

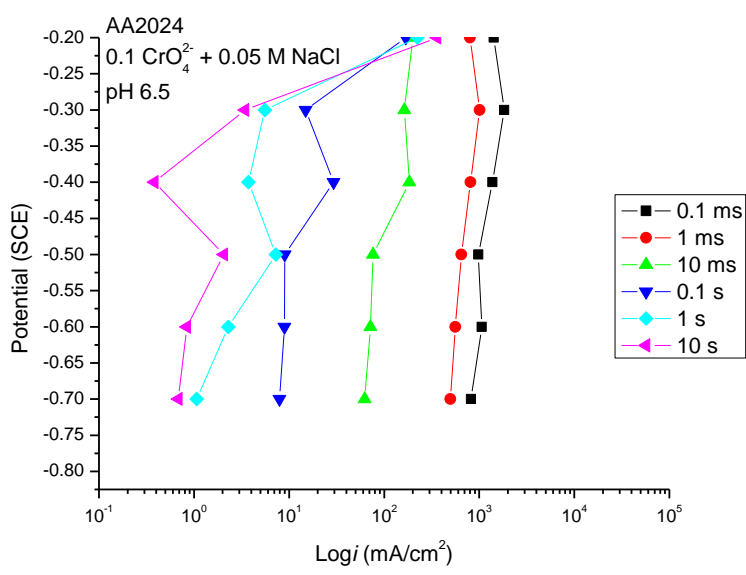

C

Figure 4.54. The effect to $0.1 \mathrm{M} \mathrm{MoO}_{4}{ }^{2-}$ and $\mathrm{CrO}_{4}{ }^{2-}$ on the E-logi behavior of AA2024 as a function of time after a mechanical depassivation event. AA2024 E-logi behavior in $0.1 \mathrm{MoO}_{4}{ }^{2-}$ and $0.05 \mathrm{M} \mathrm{NaCl}$ is shown in $\mathrm{B}$ and in $0.1 \mathrm{CrO}_{4}{ }^{2-}$ and $0.05 \mathrm{M} \mathrm{NaCl}$ in $\mathrm{C}$. Data gathered from scratch transients shown in Figure 4.42. 


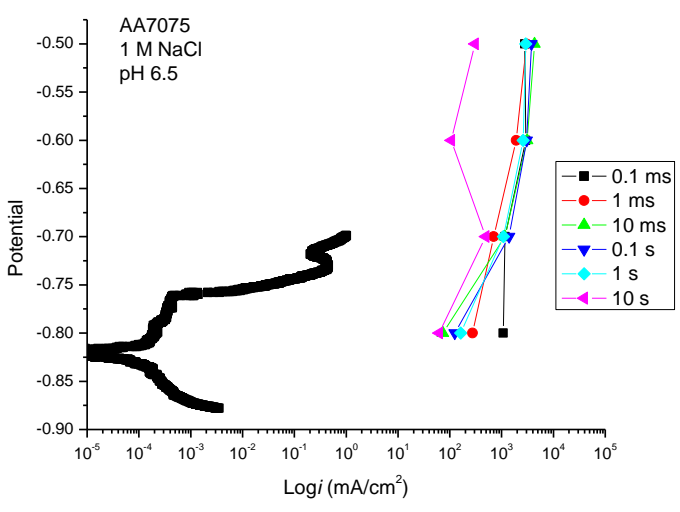

A

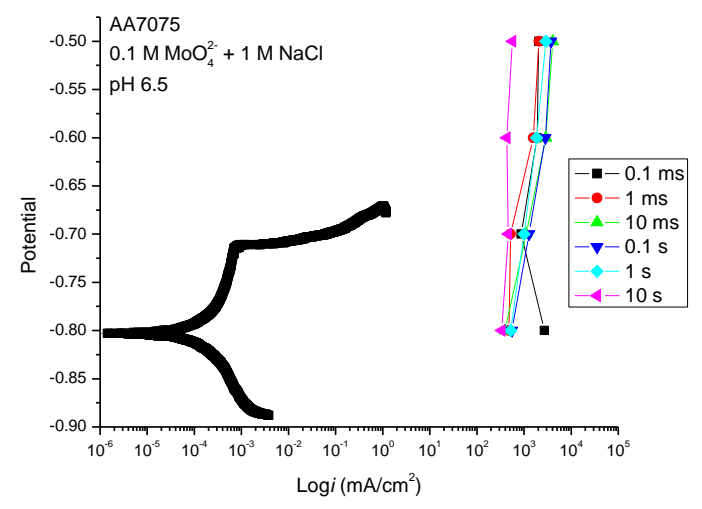

B

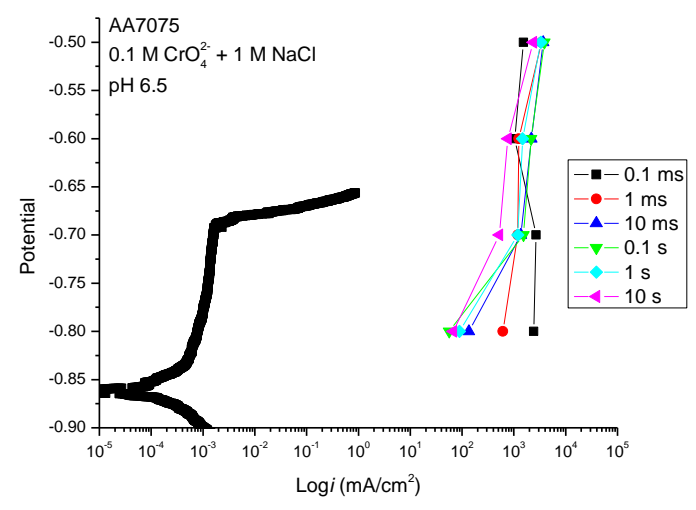

C

Figure 4.55. The effect to $0.1 \mathrm{M} \mathrm{MoO}_{4}{ }^{2-}$ and $\mathrm{CrO}_{4}{ }^{2-}$ on the E-logi behavior of AA7075 as a function of time after a mechanical depassivation event. AA7075 E-logi behavior in $0.1 \mathrm{MoO}_{4}{ }^{2-}$ and $0.1 \mathrm{M} \mathrm{NaCl}$ is shown in $\mathrm{B}$ and in $0.1 \mathrm{CrO}_{4}{ }^{2-}$ and $0.1 \mathrm{M} \mathrm{NaCl}$ in C. Data gathered from scratch transients shown in Figure 4.42. Anodic potentiodynamic polarization curves show the high current densities that persist after a depassivation event. 


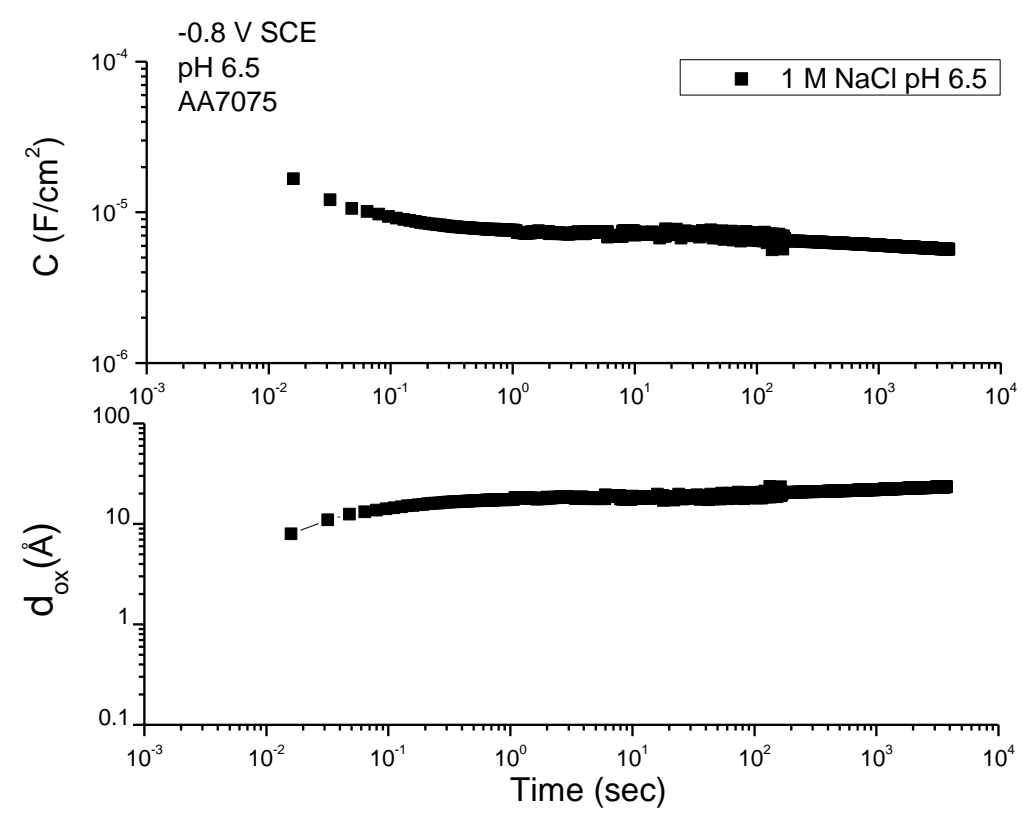

Figure 4.56. Oxide thickness calculated from impedance measurements shown in Figure 4.48 for AA7075-T6 scratch depassivated in $1.0 \mathrm{M} \mathrm{NaCl}$ and $0.1 \mathrm{M} \mathrm{CrO}_{4}{ }^{2-}$ solution at $0.8 \mathrm{~V}(\mathrm{SCE})$. 


\section{Direct Evidence for Enhanced Repassivation, Incorporation of Inhibitor Species, and Reduced Hydrogen Uptake in an Al-Zn-Mg- Cu Alloy with Selected Ionic Inhibitors}

\subsection{Abstract}

Single frequency impedance, Rutherford backscattering spectrometry (RBS) and elastic recoil detection (ERD) analysis of abrasion-repassivated Al-Mg-Zn-Cu alloy (AA7075T6) surfaces give the first direct measurement of enhanced repassivation, inhibitor species incorporation, followed by reduced hydrogen uptake into an $\mathrm{Al}-\mathrm{Zn}-\mathrm{Mg}-\mathrm{Cu}$ alloy in a single combined experiment. Repassivation was investigated with chromate, molybdate, and permanganate inhibitor additions to aqueous sodium chloride solutions during repassivation. Chromate $(0.1 \mathrm{M})$ was found to be an effective inhibitor of hydrogen uptake when added to $1.0 \mathrm{M} \mathrm{NaCl}$ solution. The evidence presented provides insight into the processes responsible for inhibition of cyclic fatigue crack propagation (EFCP) of AA7075 controlled by hydrogen ingress at depassivated crack tips. Moreover, these findings provide a new methodology through which corrosion inhibitors should be evaluated for SCC and EFCP inhibitor function.

\subsection{Introduction}

Chromate and molybdate ionic inhibitors added to full emersion environmental fatigue crack propagation (EFCP) tests has shown that these inhibitors are capable of retarding the 
growth rate per cycle of cracks. Such cracking inhibition is inhibitor concentration dependent. ${ }^{[1-3]}$ Moreover, the behavior is operative at low cycle frequencies but can be suppressed by fast frequencies. Crack tip film rupture events are thought to lead to hydrogen production, uptake, diffusion to the fracture process zone, and hydrogen embrittlement couple with anodic dissolution in the crack tip. ${ }^{[4]}$ In $7 x x x$ series aluminum alloys evidence for hydrogen controlled EAC is strong. ${ }^{[5,6]}$ The ability of a stable film to regrow rapidly after film rupture is critical because it reduces the production of atomic hydrogen at the crack tip by limiting metallic dissolution and hydrolysis of metal ions. The $\mathrm{Al}_{2} \mathrm{O}_{3}$ oxide is also a hydrogen permeation barrier which limits further hydrogen ingress. ${ }^{[7]}$ The reduction in anodic dissolution of $\mathrm{Al}$ results in a reduction in deposition of atomic hydrogen at crack tips, and the presence of a permeation barrier decreases the amount of embrittling absorbed atomic hydrogen in the fracture process zone. However, direct evidence is lacking for the combination of improved repassivation, incorporation of species in repassivated oxides, as well as reduced hydrogen uptake. Crack tip hydrogen concentrations have been estimated and predicted, even with inhibitors, but are rarely measured directly. ${ }^{[8-12]}$

The growth of oxides at the crack tip has also been rationalized to be responsible for the frequency dependence of $\mathrm{da} / \mathrm{dN}$ in the low frequency regime in fatigue testing at constant $\Delta \mathrm{K}$. At low to moderate frequencies up to the critical frequency upper bound $f_{C r i t U B}(f \approx 10$ $50 \mathrm{~Hz}$ ) electrochemical corrosion inhibitors such as chromate and molybdate have been shown to reduce da/dN to levels below those seen in moist air and/or full immersion chloride environments ${ }^{[1,2]}$. The existence of a critical frequency above which no inhibition of EFCP occurs is interpreted as the frequency at which an oxide barrier is disrupted faster 
than it can repassivate to prevent $\mathrm{Al}^{3+}$ dissolution, coupled hydrogen production, and absorption of $\mathrm{H}_{\mathrm{ads}}$ into the lattice. However, direct evidence of enhanced repassivation, inhibitor incorporation during repassivation, and subsequent reduced hydrogen uptake is lacking.

The objective of the research presented is to understand inhibitor assisted repassivation of high strength aluminum 7xxx-series alloys in an aqueous environment with $\mathrm{NaCl}$ nd to test the notion that inhibitor assisted repassivation actively reduces hydrogen ingress. The potential of chromate and molybdate and also permanganate, ionic corrosion inhibitors, to form, stabilize, or incorporate into such protective surface oxides in $\mathrm{NaCl}$ solution is examined. Investigation of repassivated oxide films grown in concentrated crack-tip-like solution will provide direct evidence of the relationship between the growth of a protective inhibitor-assisted repassivated oxide film and the reduction of crack growth rates by directly examining how repassivation changes the absorbed hydrogen concentration vs. depth profile. This will lead to greater understanding of the mechanisms through which inhibitors can effect static and cyclic crack growth.

\subsection{Materials and Methods}

Abrasion and scratch repassivation experiments were carried out on AA7075 with peak aged T6 temper (YS $503 \mathrm{MPa}$ ) and a composition of 94.1 at $\% \mathrm{Al}, 2.8$ at $\% \mathrm{Mg}, 2.4$ at $\% \mathrm{Zn}$, and 0.7 at $\% \mathrm{Cu}$. The tests were performed in full immersion at an applied potential of -0.8 $\mathrm{V}$ (SCE) in 1.0 M aqueous chloride solution to approximate the crack tip conditions found 
in 7XXX series alloys during corrosion fatigue crack growth. ${ }^{[13-17]} \mathrm{A} \mathrm{pH}$ of 6.5 was selected to approximate the experimentally confirmed mild acidity during the corrosion fatigue crack propagation in a $7 \times x x$ series alloy at a rate of between $10^{-6}$ and $5 \times 10^{-5} \mathrm{~mm} / \mathrm{s}$ while experiencing enhanced migration of electrolyte species in the crack tip due to turbulent flushing at high frequencies. ${ }^{[18]}$ This crack growth rate is consistent with the growth rate seen during fatigue testing of AA7075 and in the presence of inhibitor species based on data collected by Gasem and Warner. ${ }^{[1,3]}$

The repassivation of the mechanically depassivated surface was examined using a novel scratch test experiment incorporating both net current and fast single frequency electrochemical impedance measurements ${ }^{[19]}$. The current and impedance of the barrier film were followed after mechanical depassivation with a diamond tipped scribe. The rise time for current transients was on the order of $0.5 \mathrm{~ms}$. The scratch speed was approximately $60 \mathrm{~cm} / \mathrm{s}$. Sample width was on the order of $0.3 \mathrm{~mm}$ and the scratch area was $2 \times 10^{-4} \mathrm{~cm}^{2}$. A thin layer of vacuum grease was used to control capacitive contributions from the sample surface not depassivated during scratching. ${ }^{[20]}$ The impedance method relies on the measurement in the optimal frequency range to assess the total capacitance of the repassivating oxide film, $C_{\text {total }}$ where $C_{\text {total }}=\theta C_{\text {bare }}+(1-\theta) C_{o x}$ and $C_{\text {bare }}$ is typically much larger than $C_{o x}$ for aluminum. ${ }^{[21]} C_{b a r e}$ will be on the order of the double layer or Helmholtz capacitance which is between 40 and $100 \mu \mathrm{F} / \mathrm{cm}^{2}{ }^{[22-24]}$ and the $\mathrm{Al}_{2} \mathrm{O}_{3}$ oxide capacitance is on the order of $1-2 \mu \mathrm{F} / \mathrm{cm}^{2}$ for a dielectric constant of 10 for $\mathrm{Al}_{2} \mathrm{O}_{3}{ }^{[25]}$. The passive and active interfacial impedance of aluminum are modelled in Figure 5.1 A using well established aluminum impedance data in aqueous solutions. The limits of the instrument are clearly marked. The spectra indicates that for the equivalent circuit values 
appropriate for the circumstances described impedance is dominated by interfacial capacitance at all times during repassivation at an applied AC frequency of $1000 \mathrm{~Hz}$. Figure 5.1 B shows an electrochemical impedance spectroscopy (EIS) measurement 15 minutes after scratch depassivation of the electrode. As the repassivation progresses, the impedance measured at $1 \mathrm{kHz}$ should follow the trend depicted by the red arrow.

In addition to observing the barrier properties of the oxide during repassivation, post abrasion analysis of oxide chemical composition was conducted using Rutherford backscattering spectroscopy. In order to obtain a full chemical analysis including hydrogen concentrations elastic recoil detection (ERD) was also conducted on the same test coupon.

One centimeter square samples of $1.5 \mathrm{~mm}$ thick AA7075-T6 were ground to 800 grit with a homemade dremel attachment with $\mathrm{SiC}$ grinding paper for 5 seconds while submerged in $1 \mathrm{M}$ chloride solution, with and without the addition of selected corrosion inhibitors. The sample was held potentiostatically during the grinding and during repassivation for $1 \mathrm{~h}$ at $-0.8 \mathrm{~V}$ (SCE) to mimic the crack tip potential. ${ }^{[8,16]}$ The inhibitors that were selected included $0.05 \mathrm{M} \mathrm{Na}_{2} \mathrm{Cr}_{2} \mathrm{O}_{7}$ and $0.1 \mathrm{M} \mathrm{Na}_{2} \mathrm{MoO}_{4}$. All solutions were adjusted to a $\mathrm{pH}$ of 6.5 with $\mathrm{HCl}$ and $\mathrm{NaOH}$ and were quiescent without deaeration.

SEM imaging and EDS mapping of an as-received sample and samples abraded in the presence of inhibitors were conducted after compositional analysis of the surfaces was first completed. Imaging was conducted in both secondary electron and backscattered electron mode with an accelerating voltage of $15 \mathrm{kV}$ and a working distance of 12 or $10 \mathrm{~mm}$.

Two additional AA7075-T6 samples were prepared for comparison. One AA7075-T6 sample was ground in high purity ethanol (Fisher) to 800 grit and exposed briefly to humid 
lab air to serve as a control while another was exposed to $0.1 \mathrm{M} \mathrm{NaOH}$ for 10 hours after being ground in ethanol to 800 grit. This $\mathrm{NaOH}$ sample was prepared for comparison with work in the literature. ${ }^{[26]}$ The first was tested and used as a baseline with minimal hydrogen while the second was expected to have a very high concentration of hydrogen with respect to sample depth without the need for transient depassivation. ${ }^{[26]}$

Rutherford backscattering (RBS) and elastic recoil detection (ERD) analysis of the resulting oxide chemistry and thickness as well as hydrogen depth profiles were conducted at the Michigan Ion Beam Laboratory (MIBL). The samples were shipped on frozen carbon dioxide which was expected to decrease the diffusion coefficient by 2 orders of magnitude during shipping, and experiments were conducted within 48 hours of preparation. The 1.7 MV Tandetron, solid state, gas insulated, high frequency accelerator was used in the measurements. The incident ion used for both RBS and ERD was ${ }^{4} \mathrm{He}$ accelerated to energy of $3000 \mathrm{keV}$. The geometry of the RBS experiments was as follows. The incident angle was 75 degrees, the exit angle was 55 degrees, and the scattering angle was 160 degrees. The geometry for the ERD was as follows. The incident angle was 75 degrees, the exit angle was 75 degrees and the scattering angle was 30 degrees. A schematic of the experimental setup is provided in Figure 5.2. A polyethylene terephthalate (PET) range foil with a thickness of $13 \mu \mathrm{m}$ was used to filter out the ${ }^{4} \mathrm{He}$. The detector is a solid state device with a resolution of $20 \mathrm{keV}$. The beam line contained a quadrupole magnet for focusing, an analyzer magnet, a raster-scanner and a steerer. The detection limit for hydrogen is half of one atomic percent. The chamber pressure was in the range of $10^{-7}$ Torr. Data analysis was performed with the program SIMNRA v6.06 ${ }^{[27]}$. 
RBS and ERD were also collected at the University of Western Ontario for the purpose of showing reproducibility. The 1.7 MV Tandetron, solid state, gas insulated, high frequency accelerator was used in the measurements. The incident ion used for RBS was ${ }^{4} \mathrm{He}$ accelerated to energy of $3050 \mathrm{keV}$. This is slightly higher than the beam energy at MIBL to take advantage of an oxygen resonance to obtain higher resolution in the oxygen peak. The geometry of the RBS experiments was as follows. The incident angle was 75 degrees, the exit angle was 55 degrees, and the scattering angle was 160 degrees. The detector is a solid state device with a resolution of $15 \mathrm{keV}$ The incident ion used for ERD was ${ }^{4} \mathrm{He}$ accelerated to energy of $2900 \mathrm{keV}$ The geometry for the ERD was as follows. The incident angle was 75 degrees, the exit angle was 75 degrees and the scattering angle was 30 degrees. The range film used to filter out the ${ }^{4} \mathrm{He}$ consisted of high purity aluminum with a thickness of $5 \mathrm{~nm}$ deposited on a $10 \mu \mathrm{m}$ PET film. The detector is a solid state device with a resolution of $20 \mathrm{keV}$. The beamline contained a quadrupole magnet for focusing, an analyzer magnet, a raster-scanner and a steerer. The detection limit for hydrogen is less than a tenth of one atomic percent. The chamber pressure was in the range of $10^{-7}$ Torr. Data analysis was performed with the program SIMNRA v6.06 ${ }^{[27]}$.

For each data set there are two collected spectrum per identical experiment; an experimental RBS spectrum and an experimental ERD spectrum. The RBS spectrum indicates heavy element chemistry and provides surface layer depth-composition information while the ERD spectrum provides the ${ }^{1} \mathrm{H}$ concentration depth profile. Chemical depth profiles were modelled using both data sets in conjunction to accurately reproduce the chemical composition depth profile that matches the experimental data. 


\subsection{Results}

Scratch depassivated current-time data for a $\mathrm{Al}-\mathrm{Zn}-\mathrm{Mg}-\mathrm{Cu}$ alloy in chloride containing electrolyte with and without chromate, permanganate, and molybdate are shown in Figure 5.3. Chromate, and to a lesser extent permanganate and molybdate, lowered net scratch current densities. Strong increases in impedance as well as the capacitive nature of the film, reflected in the phase angle approaching $-90^{\circ}$, were found when chromate was present in the solution. The changes in barrier film properties upon addition of chromate to the test solution resulted in an order of magnitude increase in the impedance of the film. Permanganate also showed a strong increase in impedance magnitude. However, the phase angle of the film remained suppressed for almost 10 seconds before recovering to a level just below that of chromate. Molybdate had the least effect on the impedance of any of the inhibitors.

SEM and EDS mapping of the samples was conducted to examine the condition of the sample surface after potentiostatically holding the samples during an abrasion and repassivation event. Imaging was conducted after the samples were analyzed with the RBS and ERD techniques. Figure 5.4 and 5.5 show the SEM imaging and EDS mapping of an as-received sample ground with 1200 grit $\mathrm{SiC}$ paper. Imaging was conducted with a backscattered electron detector to highlight the presence of coarse constituent particles. The EDS mapping shows the presence of $\mathrm{Mg}, \mathrm{Fe}$, and $\mathrm{Cu}$ rich particles.

Figure 5.6 and 5.7 show the SEM image and EDS map of AA7075 held potentiostatically at $-0.8 \mathrm{~V}$ (SCE) and abraded with 800 grit $\mathrm{SiC}$ paper in $0.1 \mathrm{M} \mathrm{CrO}_{4}{ }^{2-}+1 \mathrm{M} \mathrm{NaCl}$ solution. The sample was then held potentiostatically at $-0.8 \mathrm{~V}$ (SCE) for $1 \mathrm{~h}$ after abrasion. Imaging 
was conducted with a secondary electron detector. One interesting thing to note is the presence of general corrosion on the sample surface. This was the result of handling during return shipping, the corrosion was clearly visible to the naked eye and was not present before the sample was placed on dry ice. The EDS mapping again shows $\mathrm{Mg}, \mathrm{Fe}$, and $\mathrm{Cu}$ constituent particles. The X-ray signature for chromate is weak indicating that a thick $\mathrm{Cr}$ rich oxide may not have formed.

Figure 5.9 and 5.1 show the SEM imaging and EDS mapping of AA7075 held potentiostatically during and after grinding at $-0.8 \mathrm{~V}$ (SCE) and abraded with 800 grit $\mathrm{SiC}$ paper in $0.1 \mathrm{M} \mathrm{MoO}_{4}{ }^{2-}+1 \mathrm{M} \mathrm{NaCl}$ solution. The sample was then held potentiostatically at $-0.8 \mathrm{~V}$ (SCE) for $1 \mathrm{~h}$ after abrasion. Imaging was conducted with a secondary electron detector. No significant corrosion damage is visible on the sample surface. The EDS mapping shows $\mathrm{Mg}, \mathrm{Fe}$, and $\mathrm{Cu}$ constituent particles are visable. However, the $\mathrm{X}$-ray signature for molybdenum is much more significant than that of the sample abraded in chromate.

Figure 5.10 and 5.11 show the SEM imaging and EDS mapping of AA7075 held potentiostatically at $-0.8 \mathrm{~V}(\mathrm{SCE})$ and abraded with 800 grit $\mathrm{SiC}$ paper in $0.1 \mathrm{M} \mathrm{MnO}_{4}^{-}+$ $1 \mathrm{M} \mathrm{NaCl}$ solution. The sample was then held potentiostatically at $-0.8 \mathrm{~V}$ (SCE) for $1 \mathrm{~h}$ after abrasion. Imaging was conducted with a secondary electron detector. No significant corrosion damage was visible on the surface. Again, $\mathrm{Mg}, \mathrm{Fe}$, and $\mathrm{Cu}$ constituent particles are seen in the EDS mapping, however, their intensity is diminished. The reason for this can be seen in the manganese map which shows a bright and slightly irregular presence of manganese on the sample surface. 
The RBS results for the abraded and as received samples can be seen in Figure 5.12 with more detailed examinations of inhibitor laden surface oxide seen in Figure 5.13. ERD spectra measurements of hydrogen response can be seen in Figure 5.14. The modeled target simulations shown as the blue line overlay the experimental data. The model simulation is constructed in SIMNRA. ${ }^{[27]}$ The model fits account for each element, the beam energy, the chamber geometry, and the energy loss as the beam is backscattered from within the specimen. The models were calculated with multiple iterative simulations so that the RBS and ERD physical model are one and the same when complete. The RBS magnesium edge was obscured by the aluminum edge due to the relatively low concentration of magnesium, their similar recoil energies, and the fact that they experience similar concentration variations with depth. Also, the similar Rutherford backscattering surface energy of zinc and copper, similar bulk concentration, and their like concentration profile with depth makes it difficult to distinguish an observed experimental edge for either element. The theoretical backscattering energy of each element on the surface of the sample (i.e., without energy loss due to the stopping cross-section) is reported in each figure for reference.

The target composition vs depth plots from which the simulations of RBS intensity vs., channel data were generated can be seen in Figure 5.15 and 5.16. Only the aluminum, oxygen and hydrogen concentration profiles are reported with the exception of the as received specimen. Zinc, magnesium, and copper agreed with and didn't show any significant deviation from bulk concentrations at depths below the oxide/metal interface. The layered model seen in Figure 5.15 (a), the as received samples, shows an oxygen rich surface layer containing hydrogen followed abruptly by the thick aluminum substrate with a chemical composition very similar to the bulk Al-Zn-Mg-Cu composition. The oxide 
contains $10-20 \%$ hydrogen probably as $\mathrm{H}_{2} \mathrm{O}$ or as a hydroxide. Beneath this there is an aluminum rich layer with is a slightly enriched level of hydrogen ( 2 atomic percent) in the first 1000 atom layers $\left(10^{15}\right.$ atoms $\left./ \mathrm{cm}^{2}\right)$ which corresponds to roughly $150 \mathrm{~nm}$ of $\mathrm{Al}-\mathrm{Zn}$ $\mathrm{Mg}-\mathrm{Cu}$ given its FCC structure. This enrichment drops to the hydrogen detection limit of the ERD system beyond 1000 atom layers $\left(10^{15}\right.$ atoms $\left./ \mathrm{cm}^{2}\right)$, approximately 0.5 atomic percent hydrogen.

Figure 5.15 (b) shows the modeled compositional depth profile for the Al- $\mathrm{Zn}-\mathrm{Mg}-\mathrm{Cu}$ sample exposed to $0.1 \mathrm{M} \mathrm{NaOH}$ for 10 hours $^{[26]}$. The layered model indicates a very different oxide structure as compared to (a), as well as elevated hydrogen content throughout the bulk of the sample and some enrichment at the surface. The highly oxygen enriched surface layer has a similar oxygen/hydrogen ratio and a similar thickness, around 200 atom layers $\left(10^{15}\right.$ atoms $\left./ \mathrm{cm}^{2}\right)$. After this surface layer there is a complex transition to the aluminum bulk chemistry. In this transition layer, the oxygen content tapers off while the aluminum content increases steadily. Important to note is the elevated bulk hydrogen concentration which is estimated from the simulation to be around 2 atomic percent throughout the observable thickness of the $\mathrm{NaOH}$ treated specimen well beyond the oxygen layer. This can be interpreted as atomic hydrogen in the $\mathrm{Al}-\mathrm{Zn}-\mathrm{Mg}-\mathrm{Cu}$ alloy. It is consistent with levels reported elsewhere. ${ }^{[10,28]}$

Figure 5.15(c) show the modelled compositional depth profile of the sample ground for 5 seconds in $1 \mathrm{M} \mathrm{NaCl}$. Of importance is the deep levels of oxygen associated with slow inefficient repassivation (Figure 5.3) and the elevated concentration of hydrogen throughout this mixed oxide layer and into the bulk alloy. The hydrogen concentration does return to baseline levels unlike the sample exposed to sodium hydroxide. However, the 
depth of hydrogen ingress is $2300\left(10^{15}\right.$ atoms $\left./ \mathrm{cm}^{2}\right)$ or double the as received level and beyond the oxygen rich region.

Figure 5.16 (a) show the details of the sample ground in $1 \mathrm{M} \mathrm{NaCl}$ and $0.1 \mathrm{M} \mathrm{CrO}_{4}{ }^{2-}$. The chromate limited the oxygen overlayer formed by more rapid repassivation (Figure 5.3) and greatly decreased the concentration of hydrogen observed near the surface of the sample. The compact nature of the oxide is notable. The RBS shows a very abrupt transition from oxide like compositions to bulk $\mathrm{Al}-\mathrm{Zn}-\mathrm{Mg}$-Cu composition in the first 400 atomic layers $\left(10^{15}\right.$ atoms $\left./ \mathrm{cm}^{2}\right)$. The oxide shows $\mathrm{Cr}$ levels in the 7 atomic percent range indicating that the oxide is unlikely to be a simple $\mathrm{CrO}_{3}$ or $\mathrm{Cr}(\mathrm{OH})_{3}$ layer. More likely, it is a dense aluminum oxide with some incorporation of $\mathrm{Cr}$. The hydrogen atomic concentration just after this oxide-metal transition is around 2 atomic percent and very quickly decreases to below the detection limit over 700 atomic layers $\left(10^{15}\right.$ atoms $\left./ \mathrm{cm}^{2}\right)$ or approximately 100 $\mathrm{nm}$ of bulk $\mathrm{Al}-\mathrm{Zn}-\mathrm{Mg}-\mathrm{Cu}$ alloy.

Figure 5.16 (b) shows the details of the sample ground in $1 \mathrm{M} \mathrm{NaCl}$ and $0.1 \mathrm{M} \mathrm{MoO}_{4}{ }^{2-}$. There is a large molybdenum rich oxide structure with large concentrations of hydrogen in the mixed oxide zone. Figure 5.16 (c) shows the details of the sample ground in $1 \mathrm{M} \mathrm{NaCl}$ and $0.1 \mathrm{M} \mathrm{MnO}_{4}^{-}$. There is a very thick manganese rich oxide structure with large concentrations of hydrogen in the mixed oxide zone and continuing into the bulk $\mathrm{Al}-\mathrm{Zn}$ $\mathrm{Mg}-\mathrm{Cu}$ alloy.

Figure 5.17 shows a summary plot of the hydrogen depth profiles for the as received, $\mathrm{NaOH}$ exposed, uninhibited $\mathrm{Cl}^{-}$, and inhibited $\mathrm{Cl}^{-}$solutions. There is substantial evidence of chromate, permanganate, and molybdate enrichment in the surface oxides for the sample 
ground in the presence of inhibitors. Each species is indicated by a peak at the appropriate energy for Cr, Mn, and Mo. The peak is justified because the concentration of inhibitor is only at the surface when incorporated during repassivation. In the case of chromate, this also corresponds to a large reduction in the hydrogen concentration observed in the surface beyond the oxygen rich region as seen in Figure 5.17 (c). In the case of molybdate, the reduction in hydrogen is not seen to the same extent. The molybdenum enrichment at the surface is much larger and thicker than the enrichment of chromium. This may suggest a thick porous film which is ineffective as a hydrogen permeation barrier (Figure 5.13). Permanganate was shown to increase the repassivation rate and repassivated oxide film impedance (Figure 5.3). However, this did not correspond to a decrease in the absorbed hydrogen reflected in the hydrogen concentration profile (Figure 5.17).

Replicate spectra taken at the University of Western Ontario are shown in Figure 5.18 through 5.21. The $1 \mathrm{M} \mathrm{NaCl}$ sample was contaminated with molybdate and is not shown. The following trends are reproduced. Chromate again shows a thin oxide structure with molybdate being slightly thicker and permanganate being substantially thicker. The hydrogen concentration depth profile is consistent with the MIBL experimental data set with the exception of the $0.1 \mathrm{M} \mathrm{NaOH}$ sample, where the increased bulk hydrogen concentration is not observed. The UWO accelerator was slightly more sensitive to hydrogen atomic percentage which revealed a lower background hydrogen concentration in the as received samples. 


\subsection{Discussion}

The elemental distribution vs. depth of the oxides grown after abrasion repassivation in $\mathrm{NaCl}$ solution and chromate, molybdate, and permanganate solutions with $\mathrm{NaCl}$ show several interesting characteristics. The first is that the oxide film formed in the presence of chromate is much more compact with sharp interfaces compared to the oxide formed in $\mathrm{NaCl}$ solutions with or without molybdate or permanganate. The chromate containing oxide layer is contained within the first 50-75 nm of the surface. The concentration of $\mathrm{Cr}$ in the oxide film is $2-3$ atomic percent. In contrast to this, permanganate forms a thicker oxide layer $250 \mathrm{~nm}$ thick with $\mathrm{Mn}$ present at concentrations from 30 to 2 atomic percent through the depth of the layer. Molybdate forms an intermediate thickness oxide of $150 \mathrm{~nm}$ with Mo concentrations of between 20 and 2 atomic percent through the oxide layer. The interface between oxide and metal was more diffuse in the case of Mn and Mo.

The effect that these oxide films have on the concentration of hydrogen in the bulk alloy is complex. When chromate is added to a $\mathrm{NaCl}$ solution during potentiostatic abrasion it reduces the concentration of absorbed hydrogen in comparison to the $\mathrm{NaCl}$ control or the $\mathrm{NaCl}$ with addition of molybdate or permanganate. This decrease in hydrogen concentration depth profile can be seen in Figure 5.17 and 5.21. The hydrogen concentration profile is reduced almost to the levels seen in the ethanol/humid air control sample.

Figure 5.22 shows simulated hydrogen concentration profiles based on simplistic one dimensional diffusion in a semi-infinite plate during a repassivation event assuming fixed surface concentrations of 10 and 2 at\% hydrogen, diffusion controlled transport in the 
absence of stress assisted effects, and a conservative diffusion coefficient $\left(\mathrm{D}_{\mathrm{H}, \mathrm{eff}}\right)$ of 2.3 $\mathrm{x} 10^{-9} \mathrm{~cm}^{2} / \mathrm{s}$. This value is justified as discussed below. The drop in $\mathrm{C}_{\mathrm{s}, \mathrm{H}}$ from $10 \%$ to $2 \%$ is justified in the case of chromate by increased repassivation speeds as seen in Figure 5.17. The decrease in repassivation time constant is also consistent with Figure 5.3. The profiles produced suggest that for a fixed surface concentration of hydrogen, a fixed relatively slow diffusion coefficient, and a short time period reflecting the critical time corresponding to $f_{c r i t}$, the hydrogen diffuses over the region probed with ERD and falls to levels less than 0.1 at $\%$ within $0.4 \mu \mathrm{m}$. However, since the hydrogen concentration reported is considerable for the case of aluminum another factor must be at play. The high concentrations of hydrogen observed in this study, up to 20 at $\%$, suggest that interstitial hydrogen solubility in the perfect lattice is not energetically favorable. ${ }^{[7,28]}$ Hydrogen present at concentration far above those predicted for interstitial occupancy strongly suggests that vacancies are present in the local surface region. These vacancies are likely injected during the rapid corrosion of the surface after depassivation ${ }^{[28]}$. This process is shown in Figure $5.23^{[7]}$. The existence of vacancy-hydrgen pairs is probable due to the reduced energy state when the two are combined ${ }^{[28]}$. This accounts for the high hydrogen concentration seen and is consistent with past nuclear reaction analysis (NRA) studies. Moreover, the self-diffusion of aluminum or vacancy diffusion is significantly slower than the perfect lattice interstitial diffusion rate of hydrogen in aluminum. Figure $5.23^{[7]}$ shows the effect of vacancy concentration on effective diffusion rates in high purity aluminum ${ }^{[7]}$. The results likely differ for AA7075 but the trend is similar. It is possible that a high vacancy concentration, due to vacancy injection in the near surface layers, could result in drastically slower hydrogen diffusion rates. While the exact diffusion rate of hydrogen through this vacancy 
zone is difficult to judge, slow diffusion may confine hydrogen to distances consistent with the ERD results. An additional hydrogen concentration profile, shown in Figure 5.23, constructed for the 3600 second abrasion repassivation experiment period suggests that the concentration profile may be fixed or frozen as hydrogen-vacancy complexes are slow to diffuse.

\subsection{Conclusions}

A combined repassivation and RBS detection of inhibitor elements incorporated in the repassivating oxide and ERD analysis of hydrogen was performed to provide direct assessment of inhibitor function. Formation of a thin chromium rich oxide film was observed after repassivation in $0.1 \mathrm{M} \mathrm{NaCl}$ with $0.1 \mathrm{M} \mathrm{CrO}_{4}{ }^{2-}$. Direct evidence for reduction of the hydrogen absorption into AA7075-T6 was shown where hydrogen was reduced to levels seen when abrasion was performed in ethanol. Formation of a thick molybdenum oxide film was observed, however, the reduction in hydrogen uptake was not observed, indicating that the film was not an effective barrier to hydrogen ingress when compared to chromate. A thick manganese rich oxide film was observed. However, the film did not reduce the concentration of absorbed hydrogen in the substrate.

Evidence of vacancy injection during repassivation was implicated by the high hydrogen concentrations present in the local surface regions. Vacancy controlled diffusion kinetics account for the steep hydrogen concentration depth profile observed long after the repassivation event. 
Chromate reduces $\mathrm{C}_{\mathrm{s}, \mathrm{H}}$ and reduces the amount of dissolution, coupled hydrogen production, and uptake. As clearly shown in Figure 5.17 repassivation lowers the time of diffusion. A steep hydrogen concentration profile persists due to vacancy hydrogen trapping.

This work is supported under SERDP project number WP-1621 and DoD Mandaree/ERDC. 


\subsection{References}

1. J.S. Warner, S. Kim, and R.P. Gangloff, "Molybdate inhibition of environmental fatigue crack propagation in Al- $\mathrm{Zn}-\mathrm{Mg}-\mathrm{Cu}$," International Journal of Fatigue 31, 11-12 (2009): p. 1952.

2. Z.M. Gasem, Rate-Limiting Process in Environmental Fatigue Crack Propagation in 7000-Series Aluminum Alloys in TMS-AIME, R.H. Jones, Editor. 2001: Warrendale PA. p. 501.

3. Z.M. Gasem, Frequency dependant environmental fatigue crack propagation in the 7XXX alloy/aqueous chloride system., in MSE PhD Thesis. 1999, University of Virginia: Charlottesville.

4. $\quad$ N.J.H. Holroyd and D. Hardie, "Factors controlling crack velocity in 7000 series aluminium alloys during fatigue in an aggressive environment," Corrosion Science 23, 6 (1983): p. 527.

5. R.P. Gangloff and M.B. Ives, eds. Environment-Induced Cracking of Metals. 1989, NACE: Houston, TX. 55.

6. J. R. Davis \& Associates. and ASM International. Handbook Committee., Aluminum and aluminum alloys. ASM specialty handbook (Materials Park, $\mathrm{OH}$ : ASM International, 1993). p. iii.

7. G.A. Young Jr and J.R. Scully, "The diffusion and trapping of hydrogen in high purity aluminum," Acta Materialia 46, 18 (1998): p. 6337.

8. K. Cooper, L. Young, R.P. Gangloff, and R. Kelly. The electrode potential dependence of environment-assisted cracking of AA 7050. in Materials science forum. 2000: Trans Tech Publ.

9. G.A. Young Jr and J.R. Scully, "The effects of test temperature, temper, and alloyed copper on the hydrogen-controlled crack growth rate of an Al-Zn-Mg-(Cu) alloy," Metall and Mat Trans A 33, 4 (2002): p. 1167.

10. P. Rozenak, B. Ladna, and H. Birnbaum, "SIMS study of deuterium distribution in chemically charged aluminum containing oxide layer defects and trapping sites," Journal of Alloys and Compounds 415, 1 (2006): p. 134.

11. L.J. Qiao, J.L. Luo, and X. Mao, "Hydrogen Evolution and Enrichment Around Stress Corrosion Crack Tips of Pipeline Steels in Dilute Bicarbonate Solution," CORROSION 54, 2 (1998): p. 115.

12. S.M. Al-Ghamdi, Thesis: The effect of aqueous phase inhibitors on mitigating potential-dependant hydrogen environmental assisted cracking of an ultra-high strength steel, in Materials Science and Engineering. May, 2010 University of Virginia: Charlottesville.

13. T.H. Nguyen, B.F. Brown, and R.T. Foley, "On the Nature of the Occluded Cell in the Stress-Corrosion Cracking of Aa-7075-T651 - Effect of Potential, Composition, Morphology," Corrosion 38, 6 (1982): p. 319.

14. A.H. Le and R.T. Foley, "Technical Note: On the Nature of the Occluded Cell in the Stress Corrosion Cracking of AA7075-T651-Analysis of the Solution inside the Crack," CORROSION 40, 4 (1984): p. 195.

15. A. Sedriks, J.A. Green, and D. Novak, "On the chemistry of the solution at tips of stress corrosion cracks in Al alloys," CORROSION 27, 5 (1971): p. 198. 
16. K. Cooper and R. Kelly. Measurement and Modeling of Crack Conditions During the Environment-Assisted Cracking of An Al-Zn-Mg-Cu Alloy. in Chemistry and Electrochemistry of Corrosion and Stress Corrosion Cracking: A Symposium Honoring the Contributions of RW Staehle (Materials Park, OH: TMS, 2001). 2001.

17. K.R. Cooper and R.G. Kelly, "Using capillary electrophoresis to study the chemical conditions within cracks in aluminum alloys," Journal of Chromatography A 850, 1-2 (1999): p. 381.

18. K.R. Cooper and R.G. Kelly, "Crack tip chemistry and electrochemistry of environmental cracks in AA 7050," Corrosion Science 49, 6 (2007): p. 2636.

19. S.B. Madden, D.J. Moosbauer, and J.R. Scully, "Effects of Chromate and Molybdate Ions on Scratch Repassivation Behavior of Precipitation Hardened Aluminum Alloys," ECS Transactions 50, 30 (2013): p. 57.

20. P.D. Bastek, R.C. Newman, and R.G. Kelly, "Measurement of Passive Film Effects on Scratched Electrode Behavior," Journal of the Electrochemical Society 140, 7 (1993): p. 1884.

21. J.M. W.J. Eggers, J.R. Scully, R. Spotnitz, S.R. Taylor A Short Course on Electrochemical Impedance Spectroscopy (Charlottesville University of Virginia, 2002.

22. J.R. Scully, D.C. Silverman, and M.W. Kendig, Electrochemical Impedance: Analysis and Interpretation. Stp (Philadelphia: ASTM, 1993.

23. ASTM, Characterization of the Corrosion of Aluminum Thin Films using Electrochemical Impedance Methods in Electrochemical Impedance: Analysis and Interpretation. 1993: Philadelphia. p. 276.

24. C.H. Hamann, A. Hamnett, and W. Vielstich, Electrochemistry (Weinheim ; New York: Wiley-VCH, 1998). p. xvii.

25. J.R. Scully, R.P. Frankenthal, K.J. Hanson, D.J. Siconolfi, and J.D. Sinclair, "Localized Corrosion of Sputtered Aluminum and Al-0.5-Percent $\mathrm{Cu}$ Alloy ThinFilms in Aqueous $\mathrm{Hf}$ Solution .2. Inhibition by $\mathrm{CO}_{2}$," Journal of the Electrochemical Society 137, 5 (1990): p. 1373.

26. J.H. Ai and J.R. Scully, "Hydrogen Diffusivity During Corrosion of High-Purity Aluminum," Corrosion 69, 8 (2013): p. 752.

27. M. Mayer. SIMNRA Home Page. 2011 Feb, 15, 2011 [cited 2014 Jan 20]; Available from: http://home.rzg.mpg.de/ mam/.

28. J.R. Scully, R.P. Gangloff, and B.P. Somerday, 19. Hydrogen embrittlement of aluminum and aluminum-based alloys, in Gaseous hydrogen embrittlement of materials in energy technologies. 2012, Woodhead Publishing: Cambridge, UK ; Phildelphia, PA. 


\subsection{Figures}

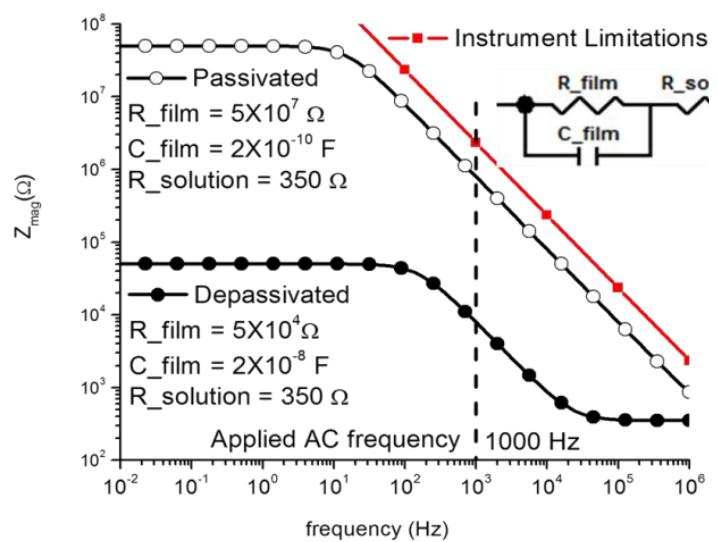

A
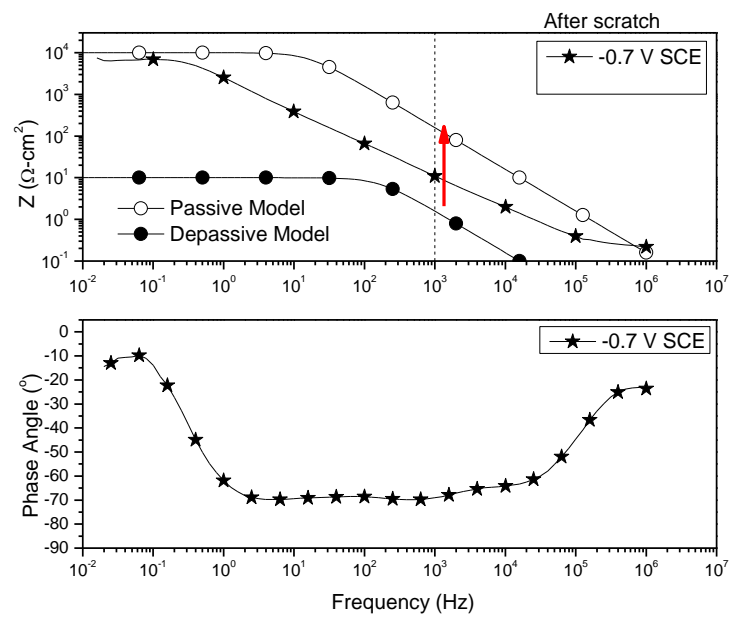

B

Figure 5.1. Theoretical electrochemical impedance spectroscopy of a completely passivated and completely active aluminum surface using well established data from the literature (A). Example of experimental electrochemical impedance spectroscopy conducted 15 minutes after scratch depassivation of an area of $8 \times 10^{-4} \mathrm{~cm}^{2}$. The red arrow indicated the trend expected of the single frequency impedance data collected during scratch testing. The phase data shows that at $1 \mathrm{kHz}$ after 15 minutes the frequency is well within the capacitive range. 


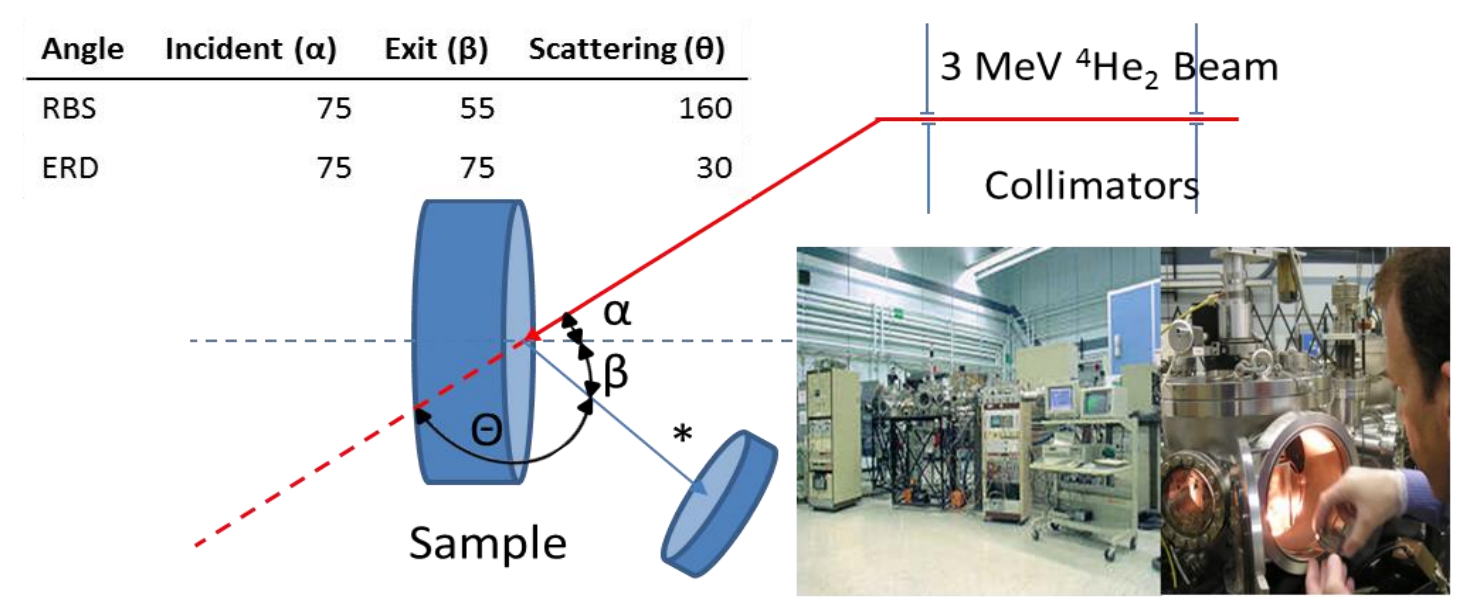

*A PET film is inserted before the detector to block ${ }^{4}$ He during ERD

\section{Michigan Ion Beam Laboratory}

Figure 5.2. Schematic of Michigan ion beam lab RBS chamber.
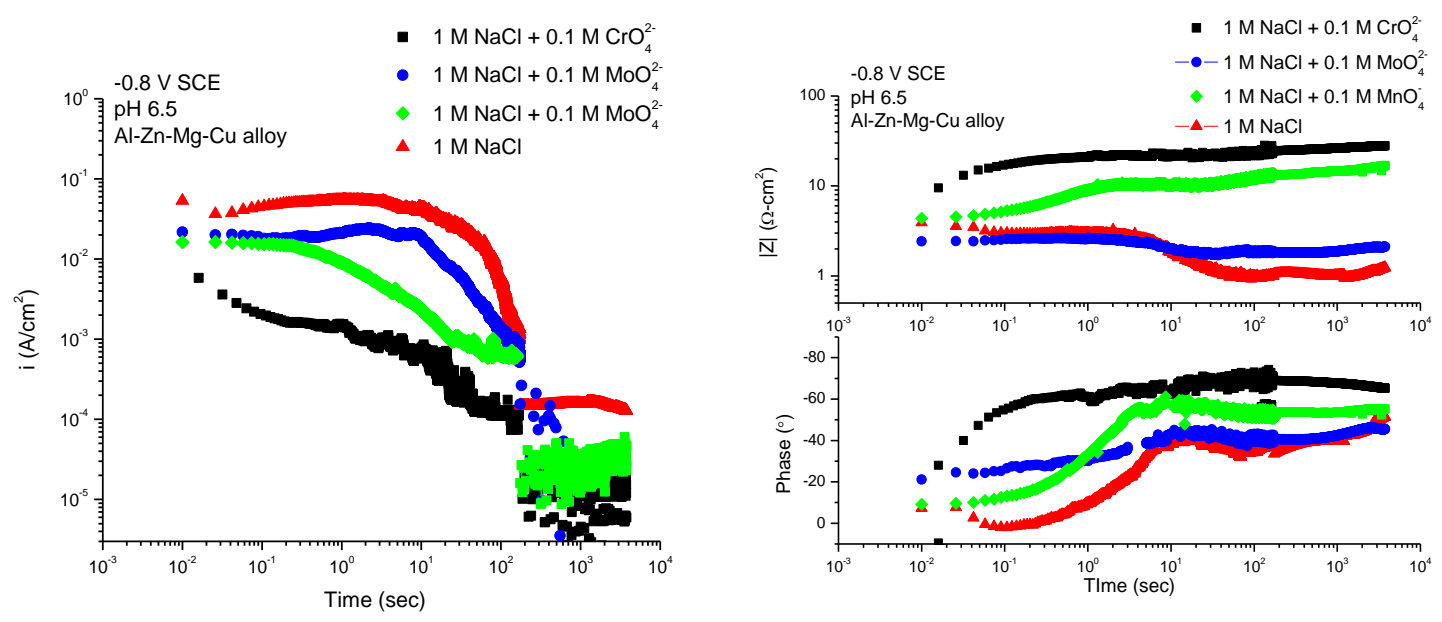

Figure 5.3. Al-Zn-Mg-Cu alloy depassivated in $1 \mathrm{M} \mathrm{NaCl}$ with and without $0.1 \mathrm{M} \mathrm{CrO}_{4}{ }^{2-}$ and $0.1 \mathrm{M} \mathrm{MoO}_{4}{ }^{2-}$ with diamond tip scribe showing lower $i_{n e t}$ vs.time and enhanced barrier properties of the oxide film in the presence of chromate and molybdate. 


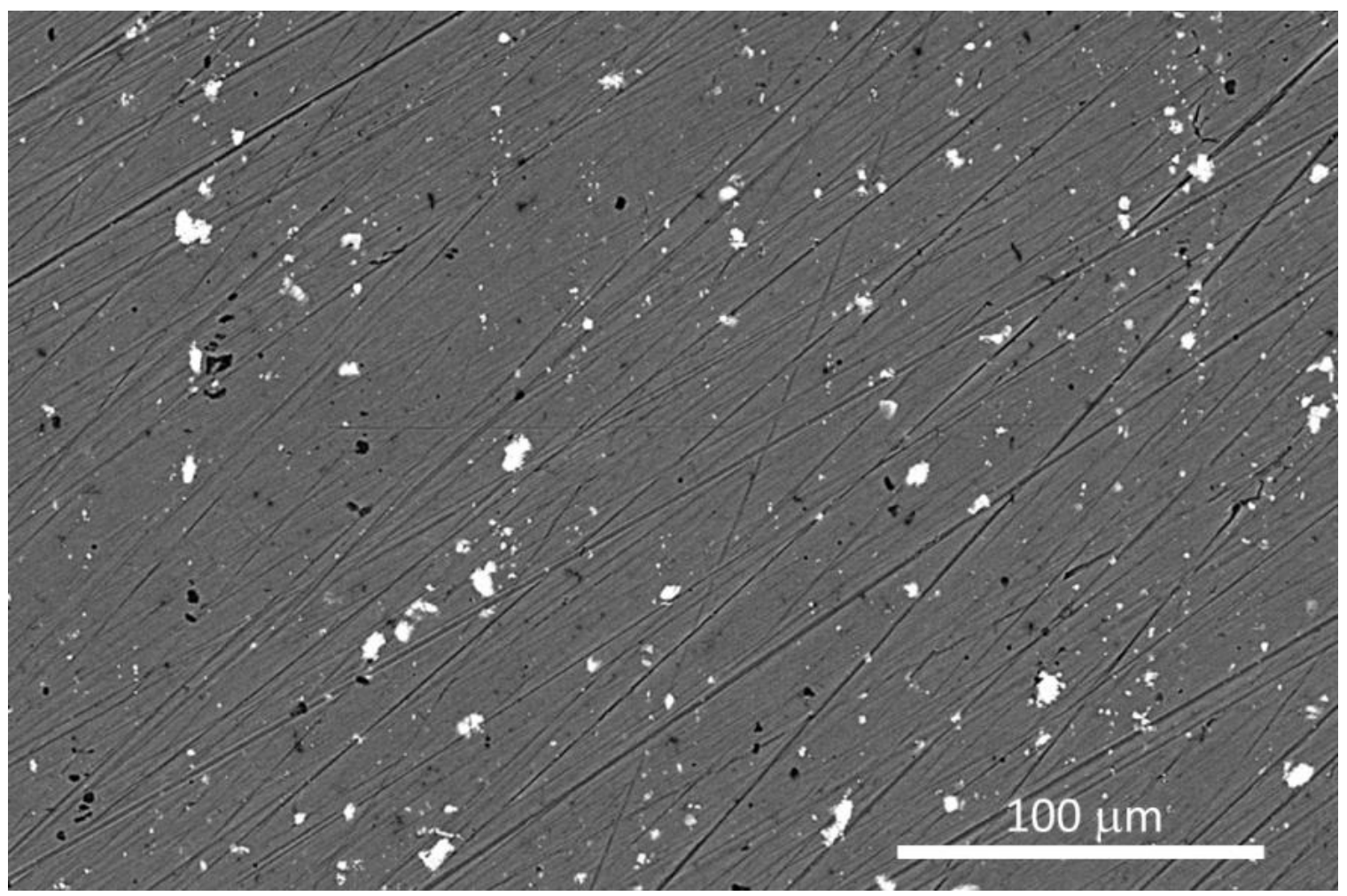

Figure 5.4. Backscattered electron image of AA7075 ground to 1200 grit in ethanol. Image taken with an accelerating voltage of $15 \mathrm{kV}$ and a working distance of $12 \mathrm{~mm}$. 

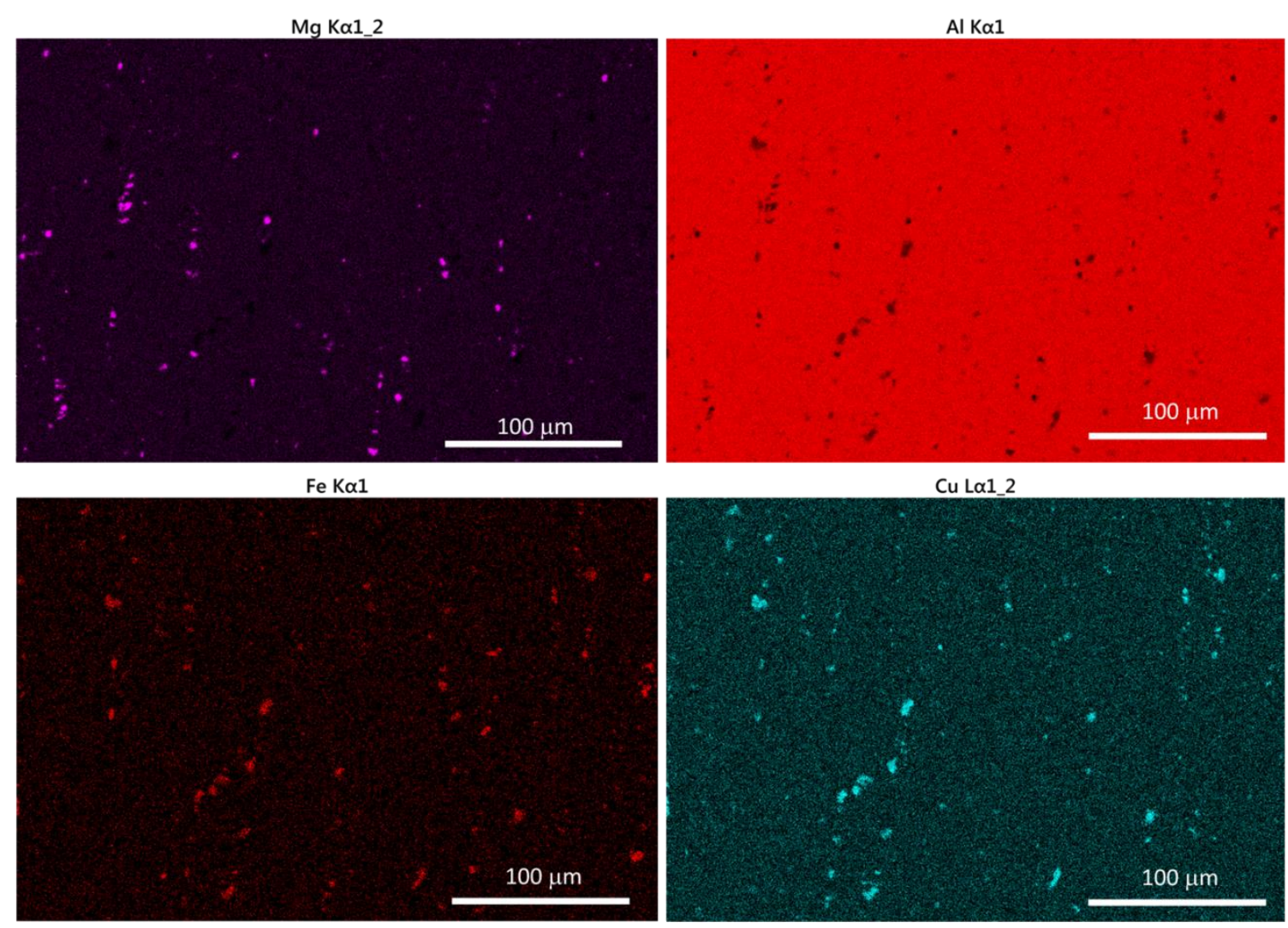

Cu L $\alpha 1 \_2$

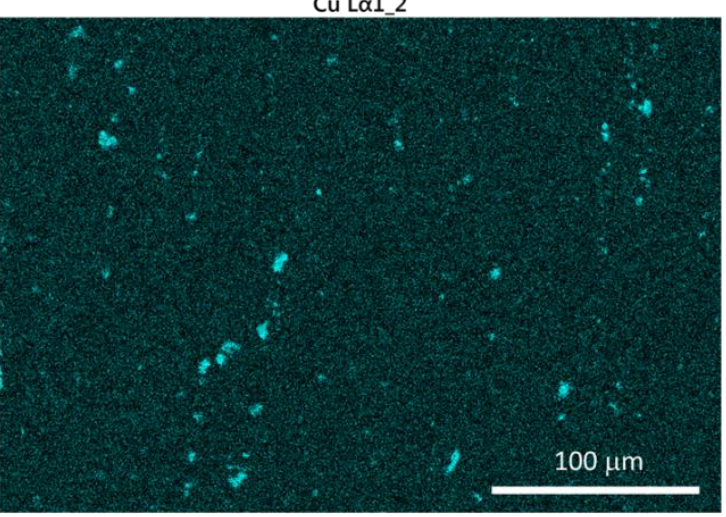

Zn L $\alpha 1 \_2$

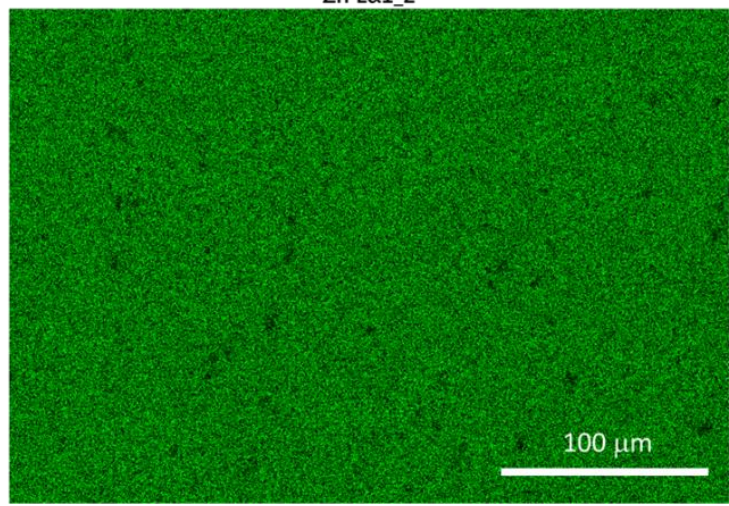

Figure 5.5. EDS map of AA7075 ground to 1200 grit in ethanol. An accelerating voltage of $15 \mathrm{kV}$ was used. 


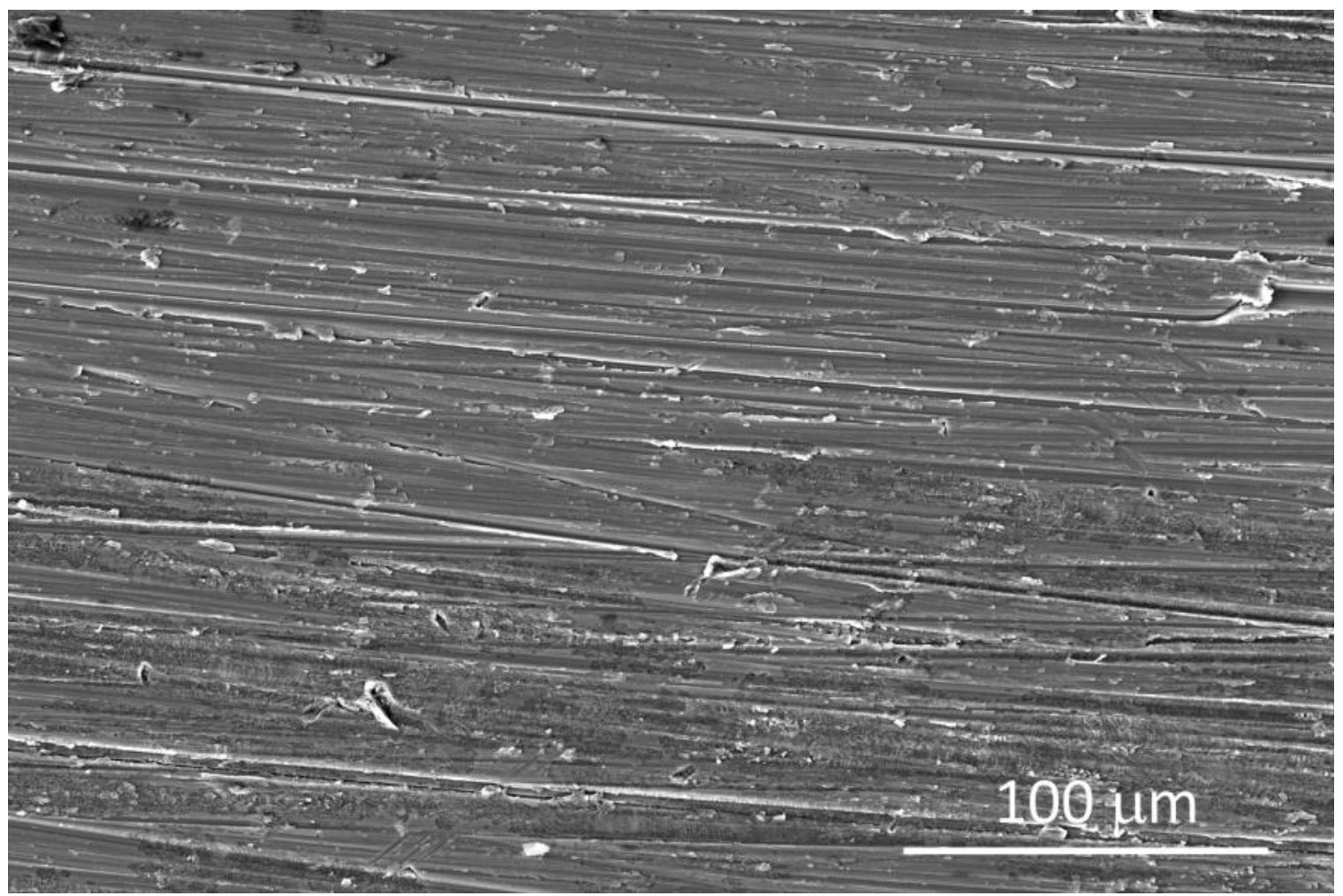

Figure 5.6. Secondary electron image of AA7075 held potentiostatically at -0.8 V (SCE) and abraded with 800 grit $\mathrm{SiC}$ paper in $0.1 \mathrm{M}$ chromate $+1 \mathrm{M}$ sodium chloride. The sample was then held potentiostatically at $-0.8 \mathrm{~V}$ (SCE) for $1 \mathrm{~h}$ after abrasion. Sample imaging occurred several weeks after RBS and ERD analysis upon return of the samples. Image taken with an accelerating voltage of $15 \mathrm{kV}$ and a working distance of $10 \mathrm{~mm}$. 


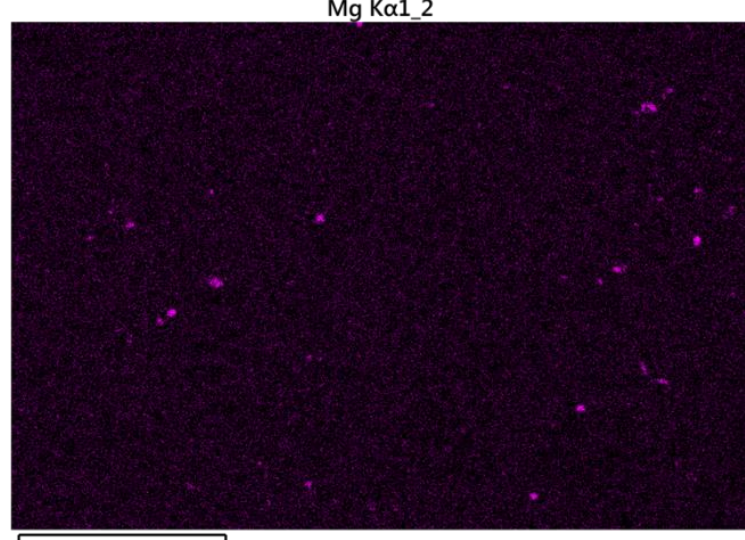

$100 \mu \mathrm{m}$

Fe $K \alpha 1$
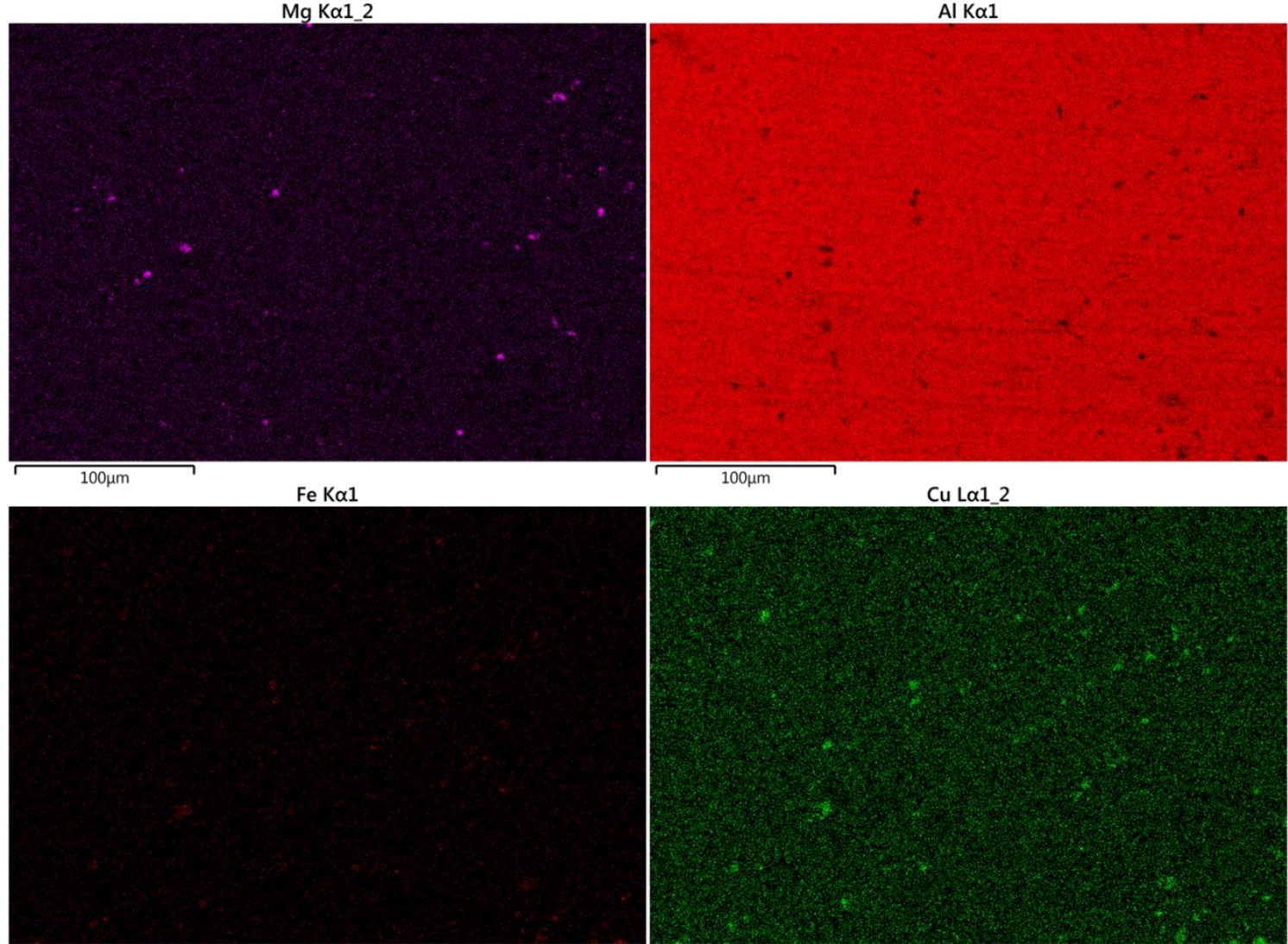

$100 \mu \mathrm{m}$

Cu L $\alpha 1 \_2$

$100 \mu \mathrm{m}$

$\mathrm{Cr} \mathrm{K} \alpha 1$
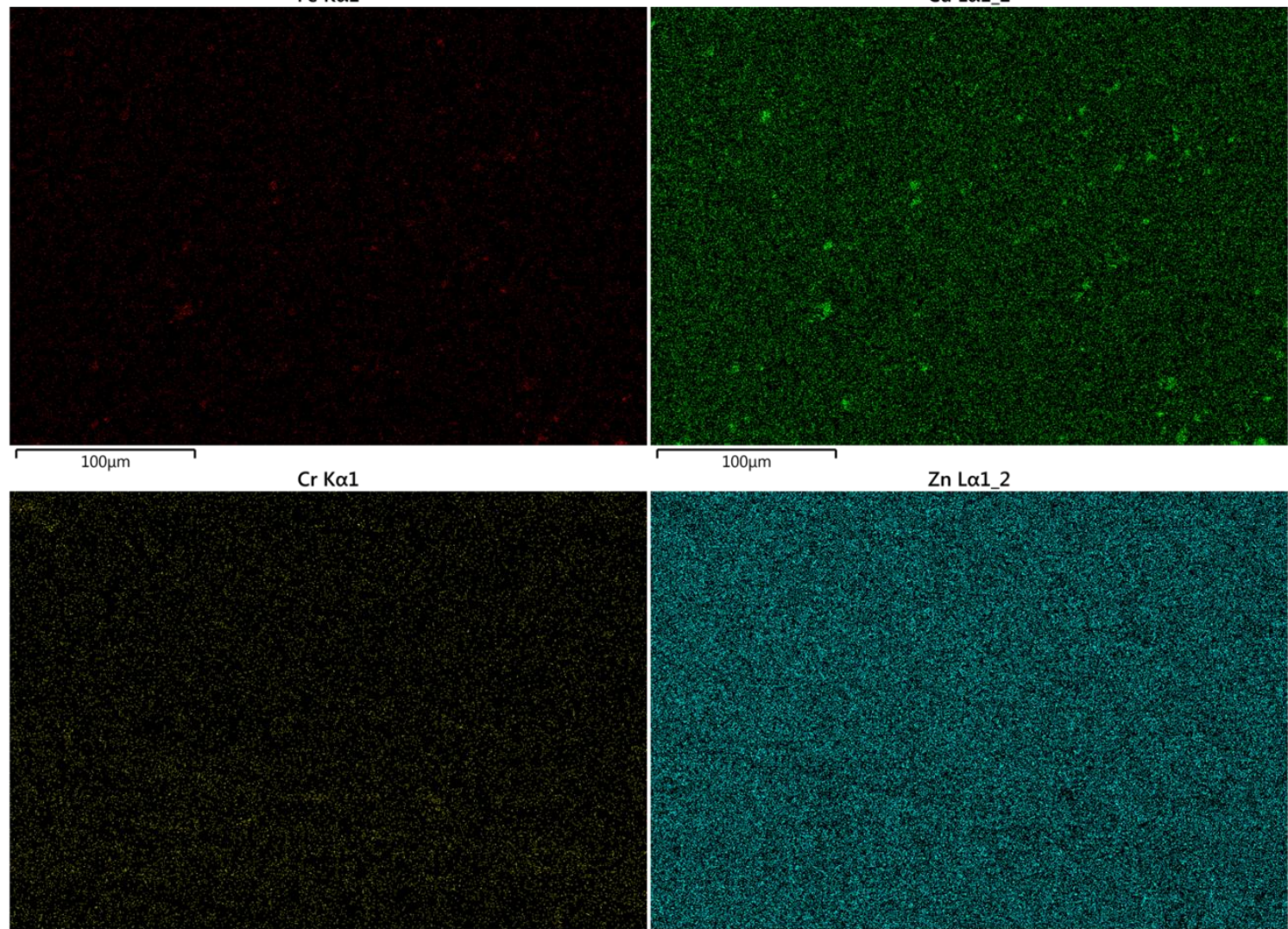

$100 \mu \mathrm{m}$

Zn L $\alpha 12$

$100 \mu \mathrm{m}$

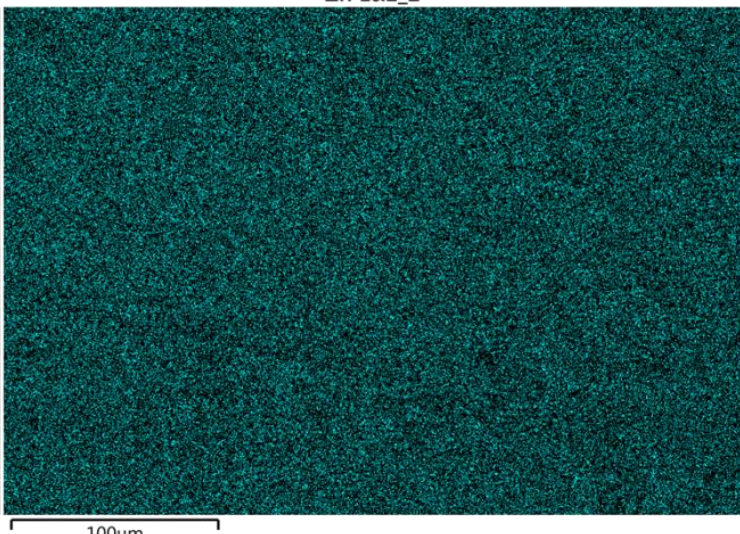

$100 \mu \mathrm{m}$

Figure 5.7. EDS map of AA7075 abraded in $0.1 \mathrm{M}$ chromate + $1 \mathrm{M}$ sodium chloride and held potentiostatically at $-0.8 \mathrm{~V}$ (SCE) for $1 \mathrm{~h}$. 


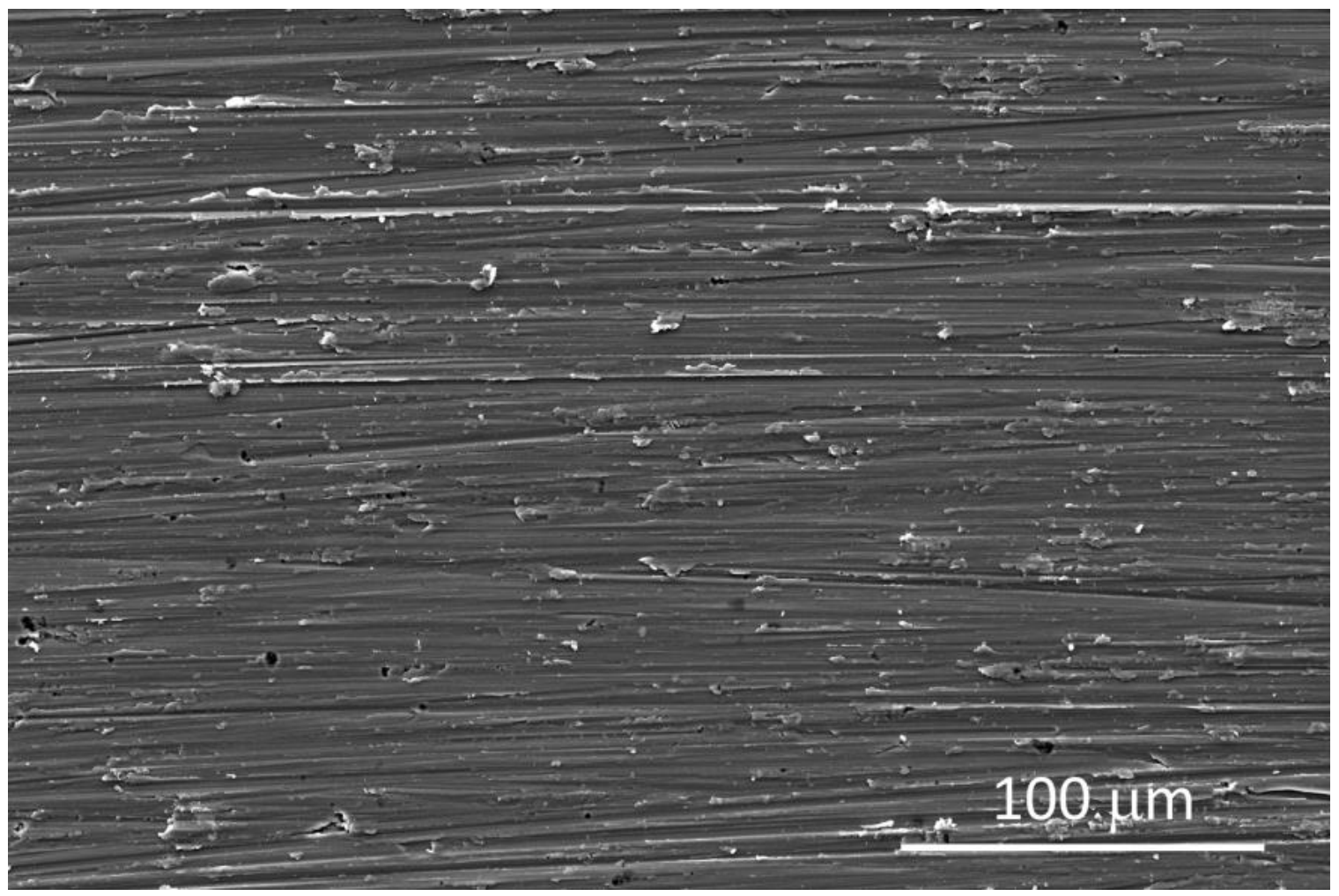

Figure 5.8. Secondary electron image of AA7075 held potentiostatically at -0.8 V (SCE) and abraded with 800 grit $\mathrm{SiC}$ paper in $0.1 \mathrm{M}$ molybdate $+1 \mathrm{M}$ sodium chloride. Held potentiostatically at $-0.8 \mathrm{~V}$ (SCE) for $1 \mathrm{~h}$ after abrasion. Sample imaging occurred several weeks after RBS and ERD analysis upon return of the samples. Image taken with an accelerating voltage of $15 \mathrm{kV}$ and a working distance of $10 \mathrm{~mm}$. 
$\mathrm{Mg} \mathrm{K} \alpha 1 \_2$
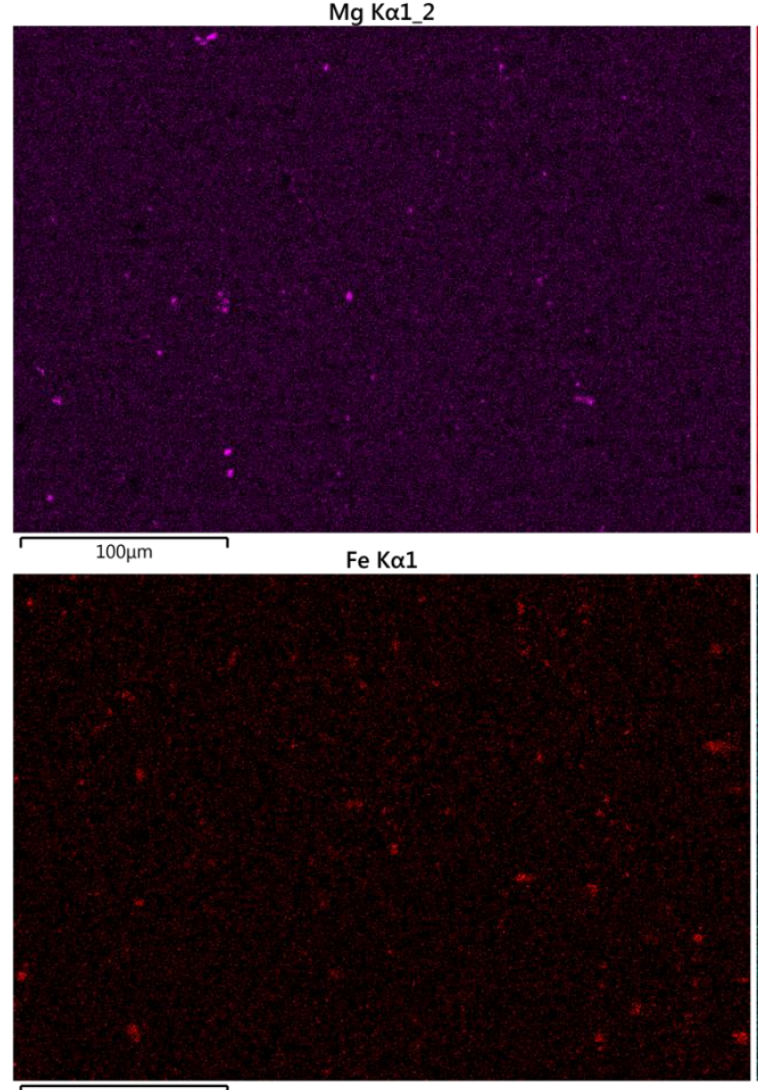

$100 \mu \mathrm{m}$

Zn L $\alpha 12$

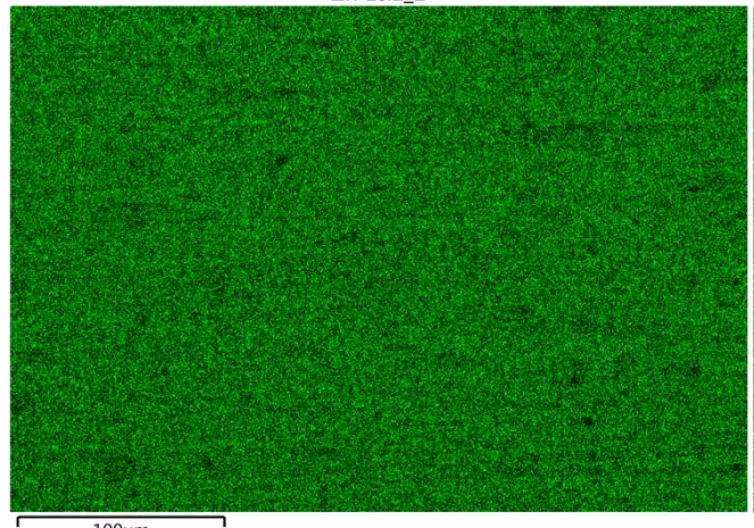

$100 \mu \mathrm{m}$
Al $K \alpha 1$

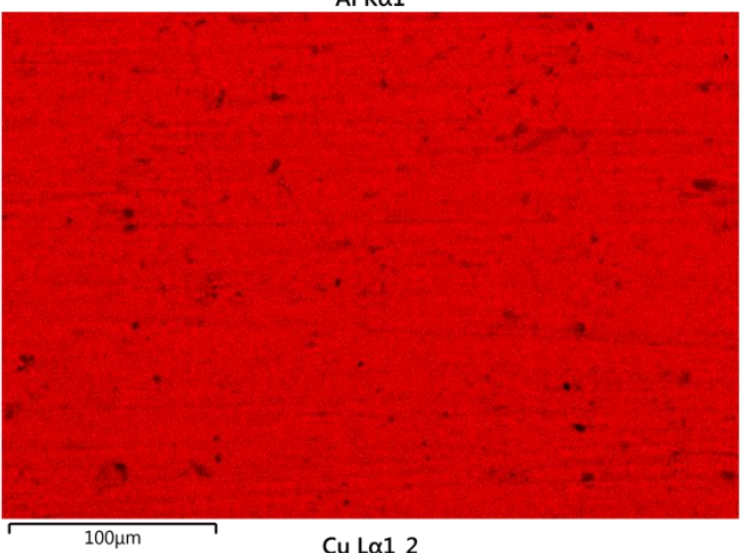

Cu L $\alpha 1 \_2$

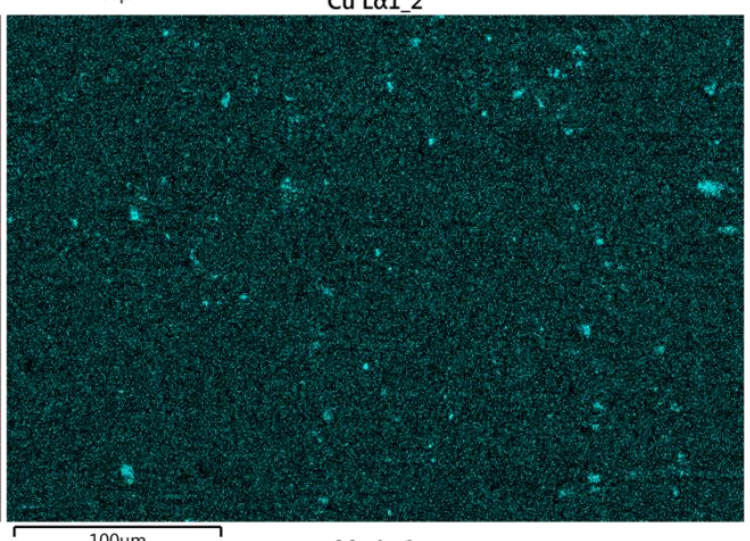

$100 \mu \mathrm{m}$

Mo L $\alpha 1$

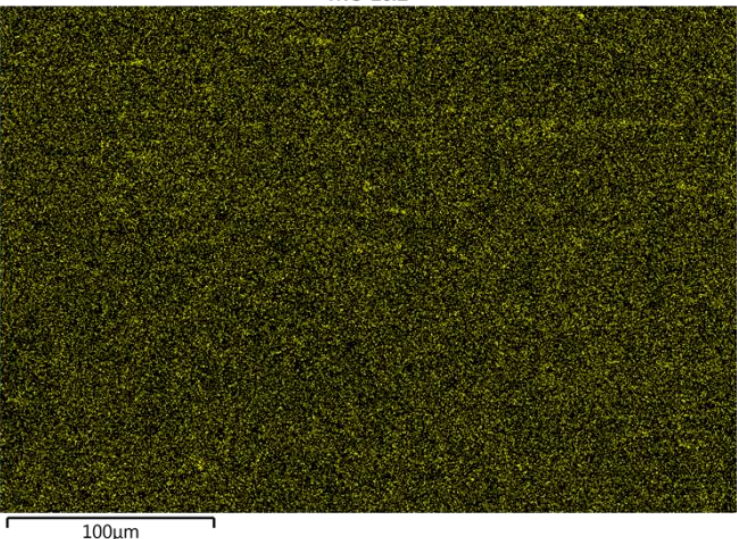

$100 \mu \mathrm{m}$

Figure 5.9. EDS map of AA7075 abraded in $0.1 \mathrm{M}$ molybdate $+1 \mathrm{M}$ sodium chloride and held potentiostatically at $-0.8 \mathrm{~V}$ (SCE) for $1 \mathrm{~h}$. 


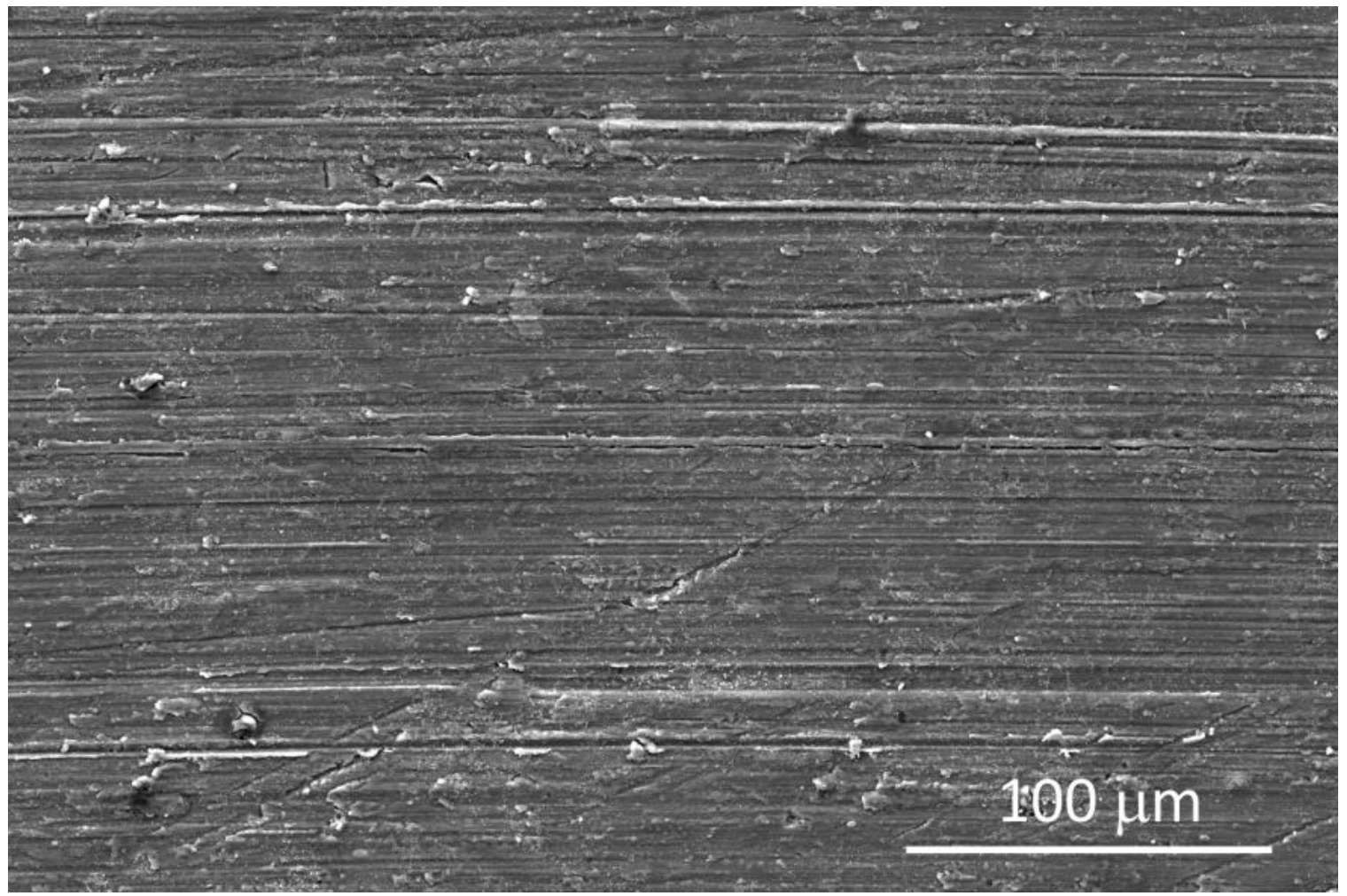

Figure 5.10. Secondary electron image of AA7075 held potentiostatically at $-0.8 \mathrm{~V}$ (SCE) and abraded with 800 grit $\mathrm{SiC}$ paper in $0.1 \mathrm{M}$ permanganate $+1 \mathrm{M}$ sodium chloride. Held potentiostatically at $-0.8 \mathrm{~V}$ (SCE) for $1 \mathrm{~h}$ after abrasion. Sample imaging occurred several weeks after RBS and ERD analysis upon return of the samples. Image taken with an accelerating voltage of $15 \mathrm{kV}$ and a working distance of $10 \mathrm{~mm}$. 

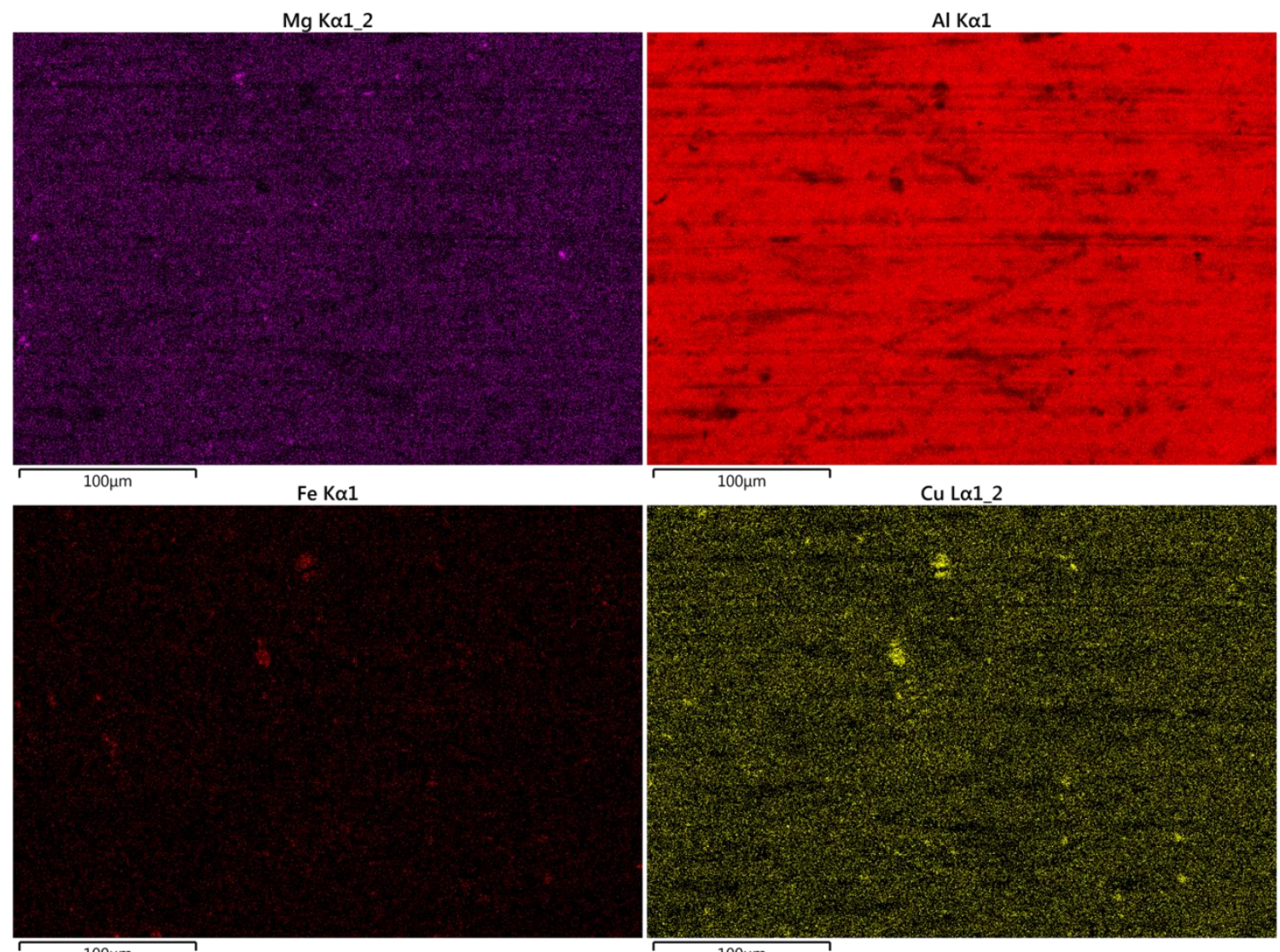

$100 \mu \mathrm{m}$
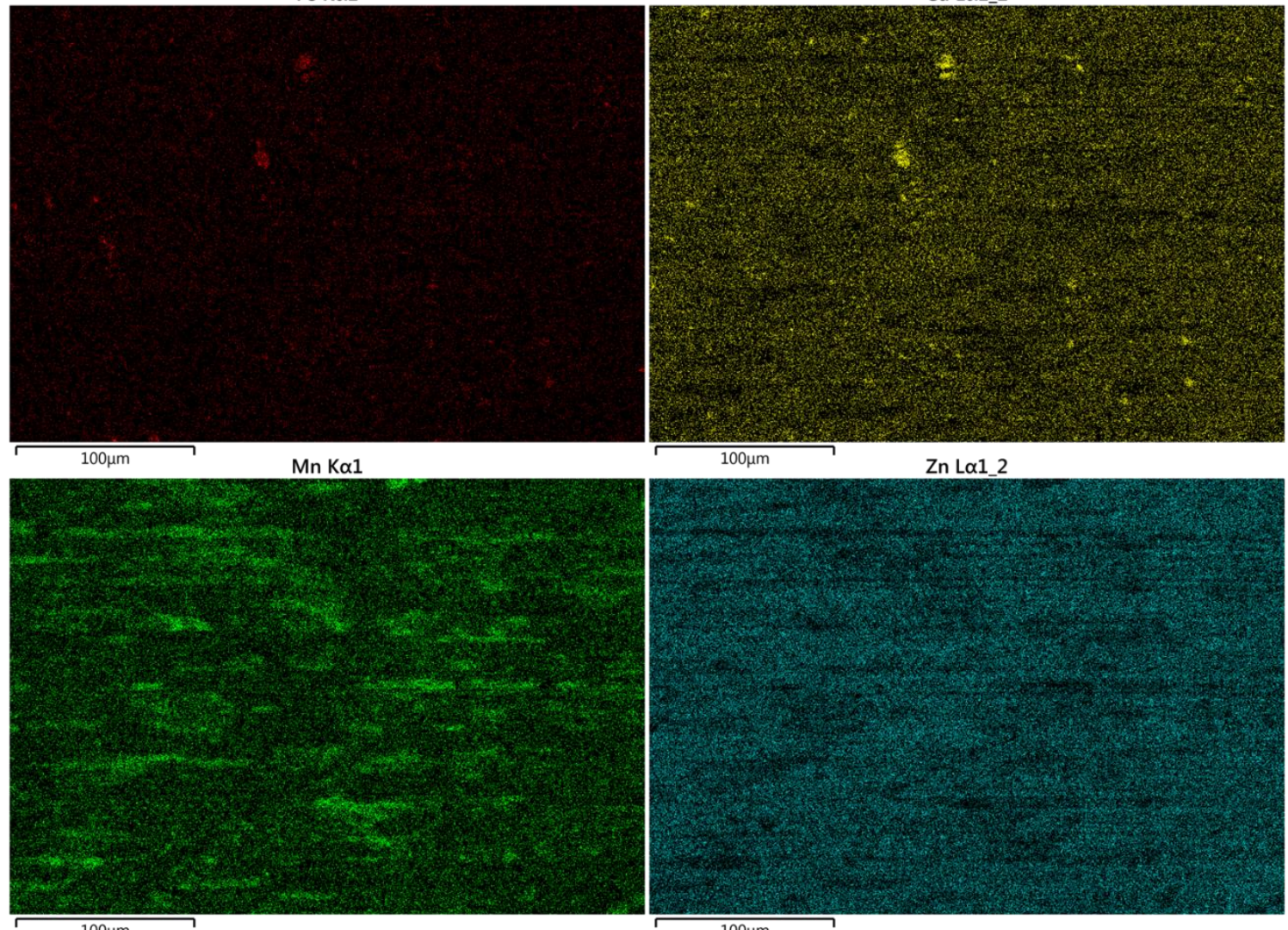

$100 \mu \mathrm{m}$

Zn L $11 \_2$

$100 \mu \mathrm{m}$

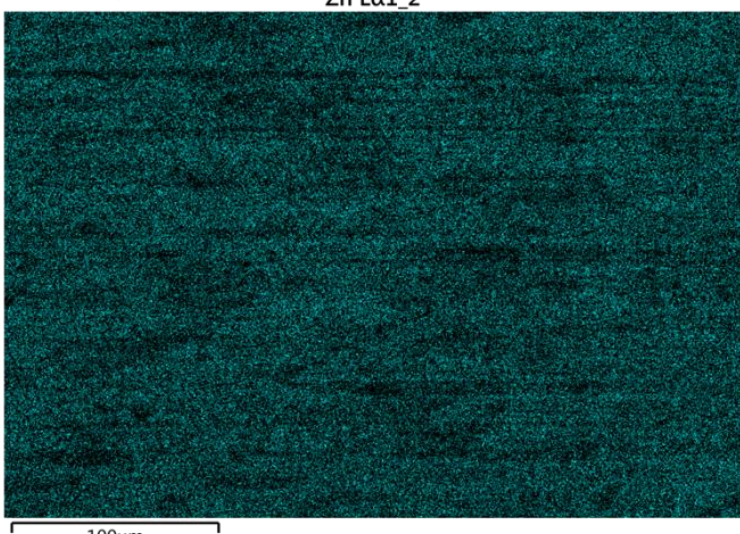

$100 \mu \mathrm{m}$

Figure 5.11. EDS map of AA7075 abraded in $0.1 \mathrm{M}$ permanganate $+1 \mathrm{M}$ sodium chloride and held potentiostatically at $-0.8 \mathrm{~V}$ (SCE) for $1 \mathrm{~h}$. 


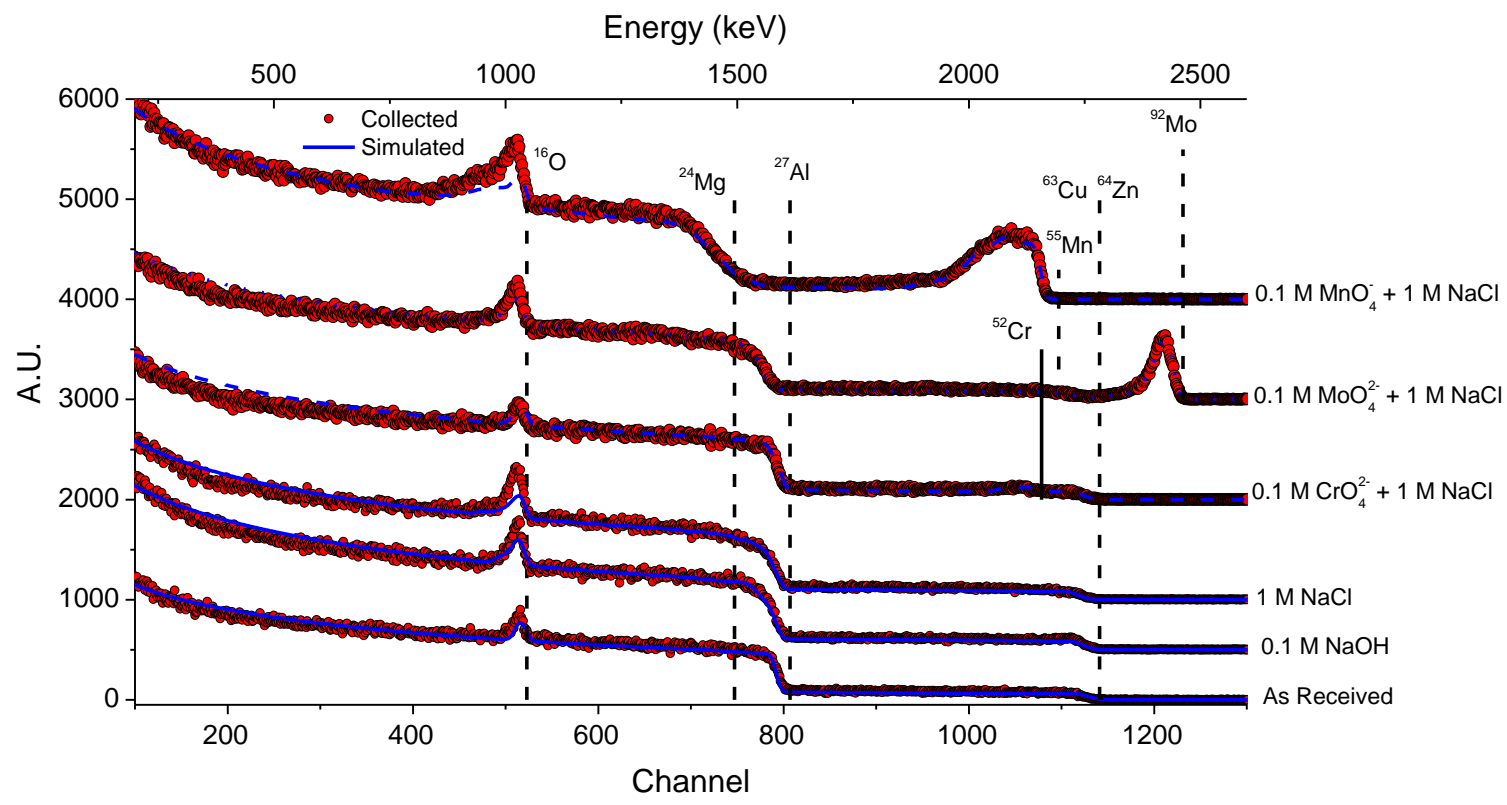

Figure 5.12. RBS spectra and simulation of $\mathrm{Al}-\mathrm{Zn}-\mathrm{Mg}-\mathrm{Cu}$ ground in aqueous $1 \mathrm{M}$ chloride solution with chromate, molybdate, and permanganate. As received and $10 \mathrm{~h}$ $0.1 \mathrm{M} \mathrm{NaOH}$ samples for reference. 

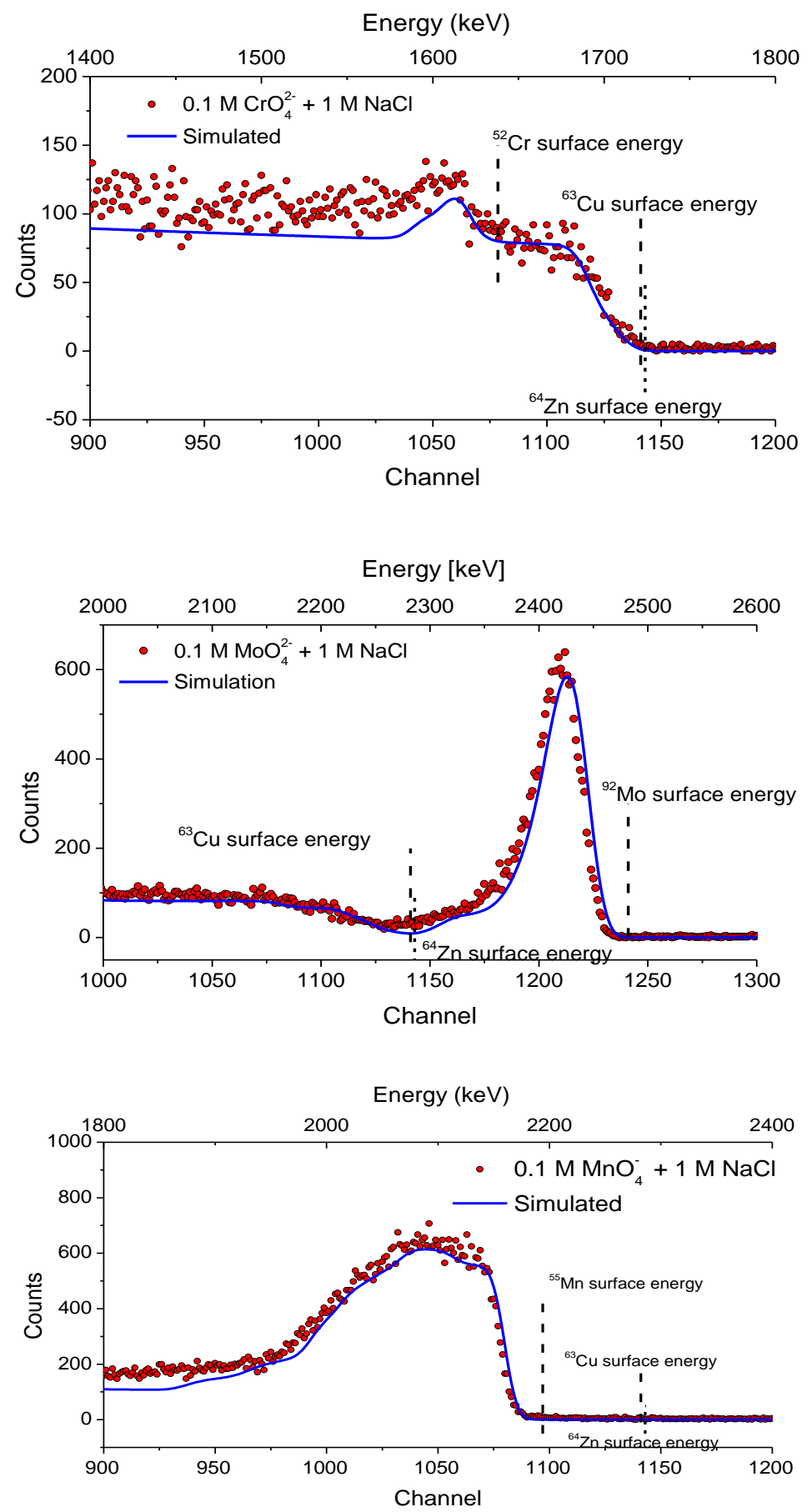

Figure 5.13. Detailed view of chromium, molybdenum, and manganese enriched oxides. 


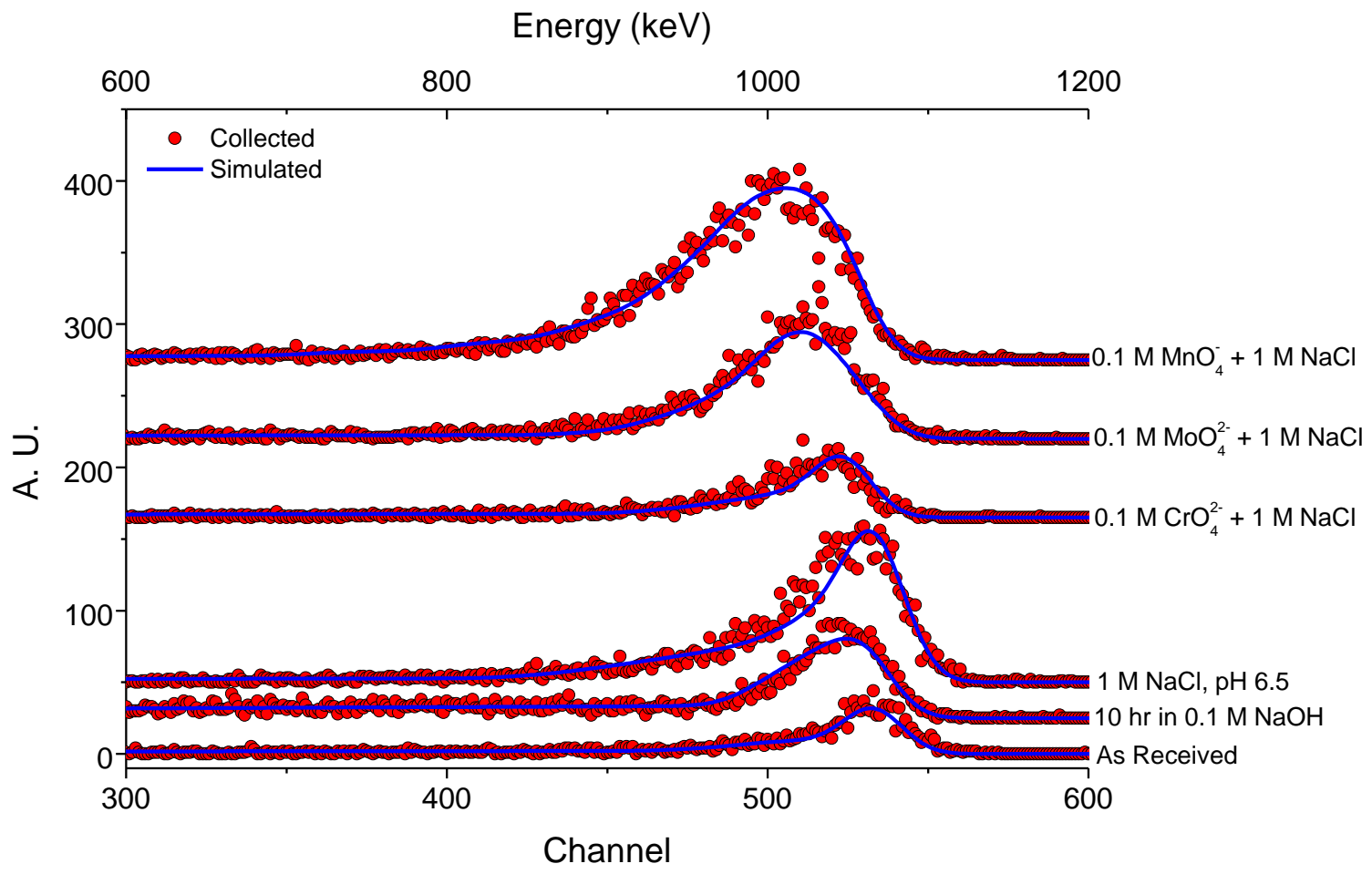

Figure 5.14. ERD spectra and simulation of $\mathrm{Al}-\mathrm{Zn}-\mathrm{Mg}-\mathrm{Cu}$ ground in aqueous $1 \mathrm{M}$ chloride solution with chromate and molybdate additions. As received and $10 \mathrm{~h} 0.1 \mathrm{M}$ $\mathrm{NaOH}$ samples for reference. 
A
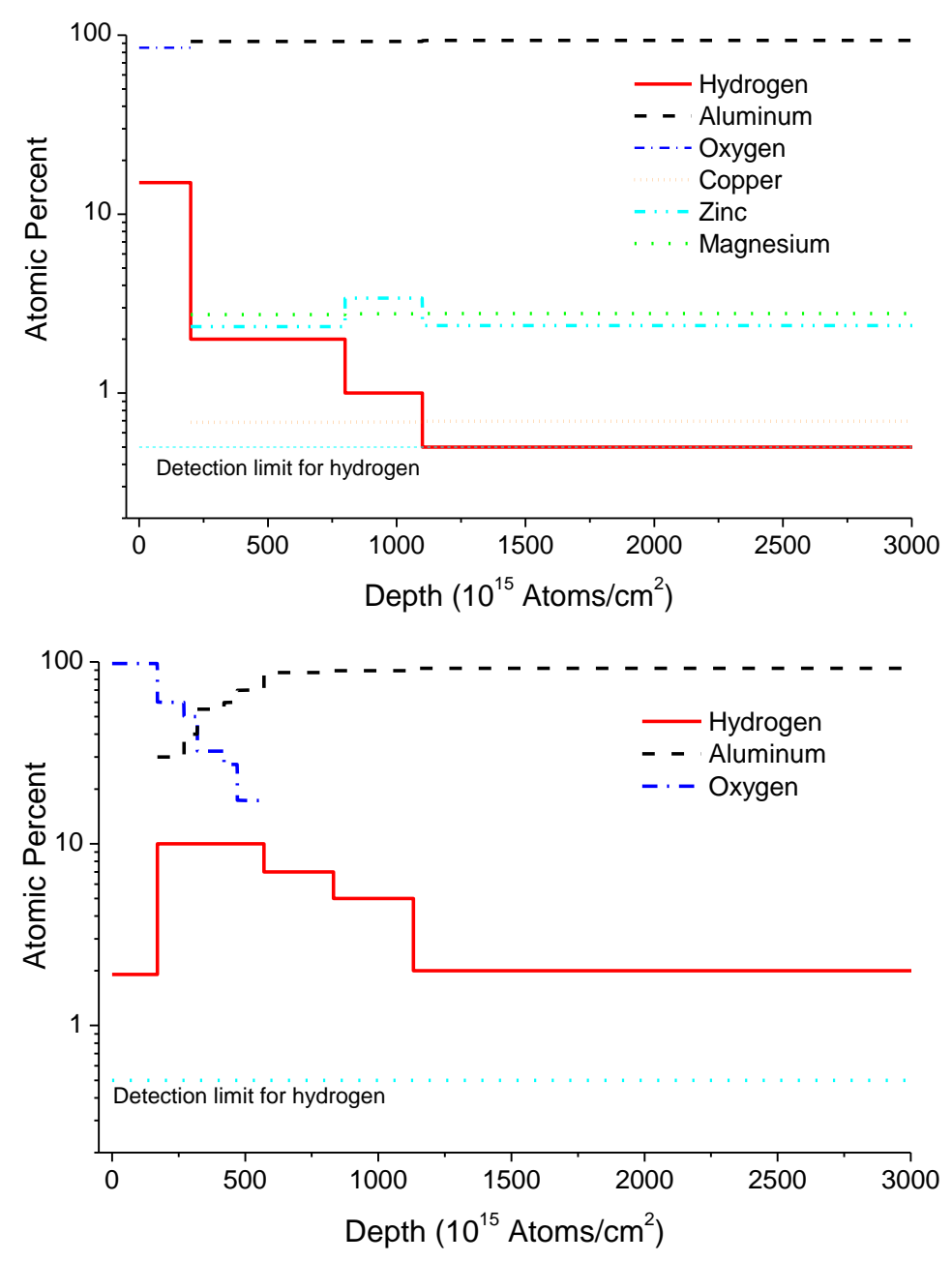

B

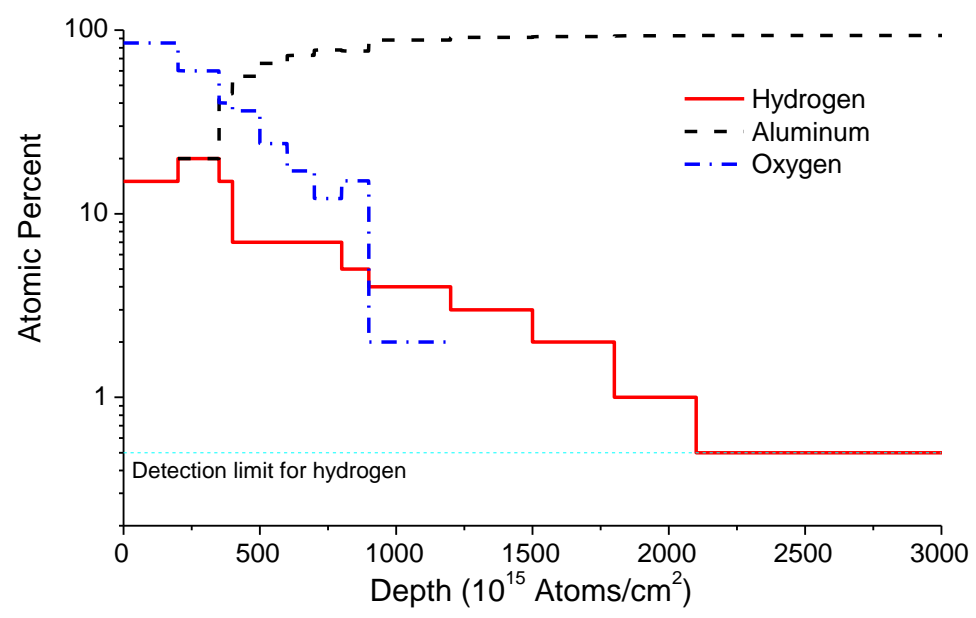

Figure 5.15. Model systems showing atomic concentration depth profiles for AA7075 as received exposed to ethanol and humid air (a), exposed for $10 \mathrm{~h}$ to $0.1 \mathrm{M} \mathrm{NaOH}$ (b), and ground in $1 \mathrm{M}$ chloride (c). 

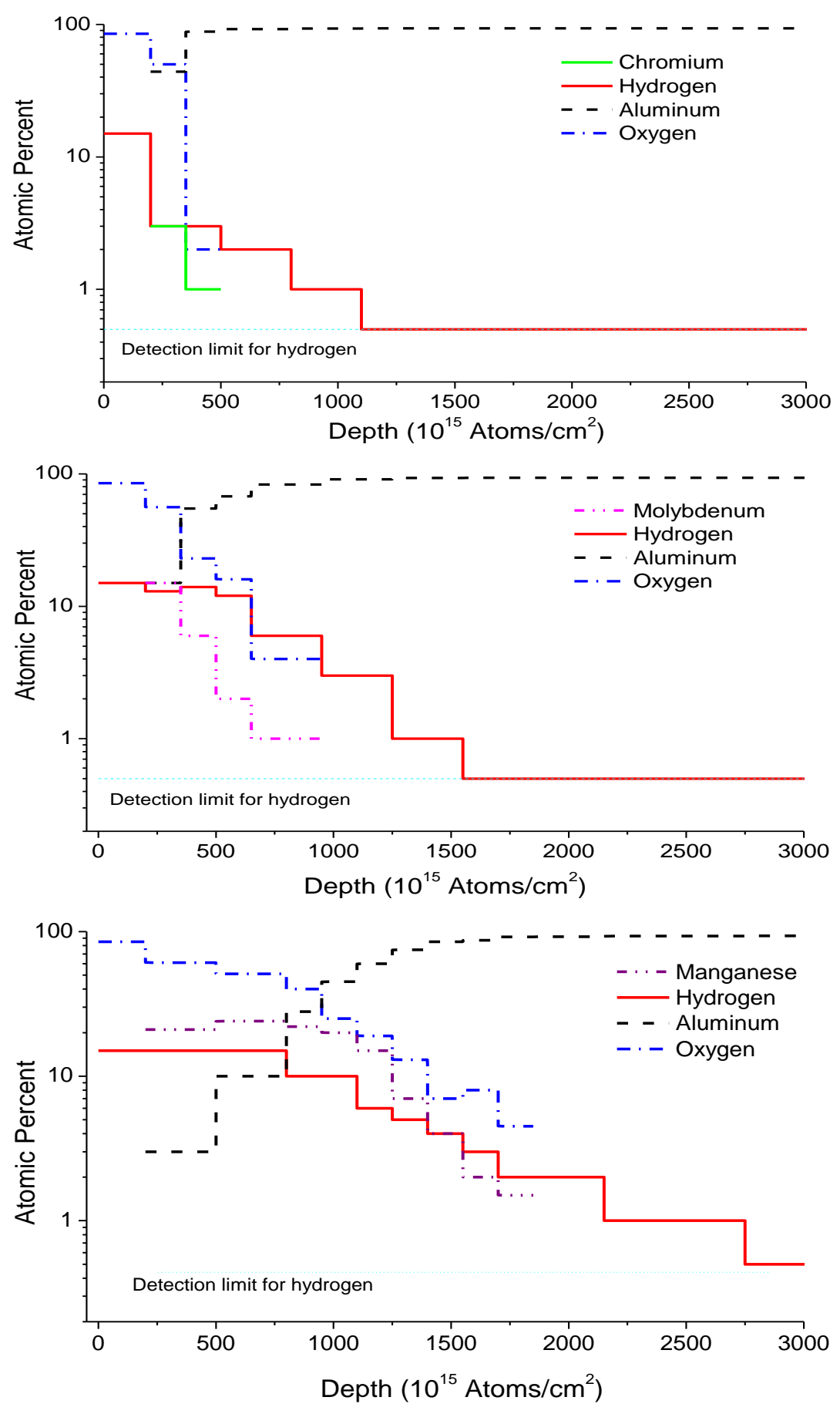

Figure 5.16. Model systems showing atomic concentration depth profiles for AA7075 ground in $1 \mathrm{M}$ chloride with $0.05 \mathrm{M} \mathrm{Cr}_{2} \mathrm{O}_{7}{ }^{2-}, 0.1 \mathrm{M} \mathrm{MoO}_{4}{ }^{2-}$ and $0.1 \mathrm{M} \mathrm{MnO}_{4}^{-}$. 


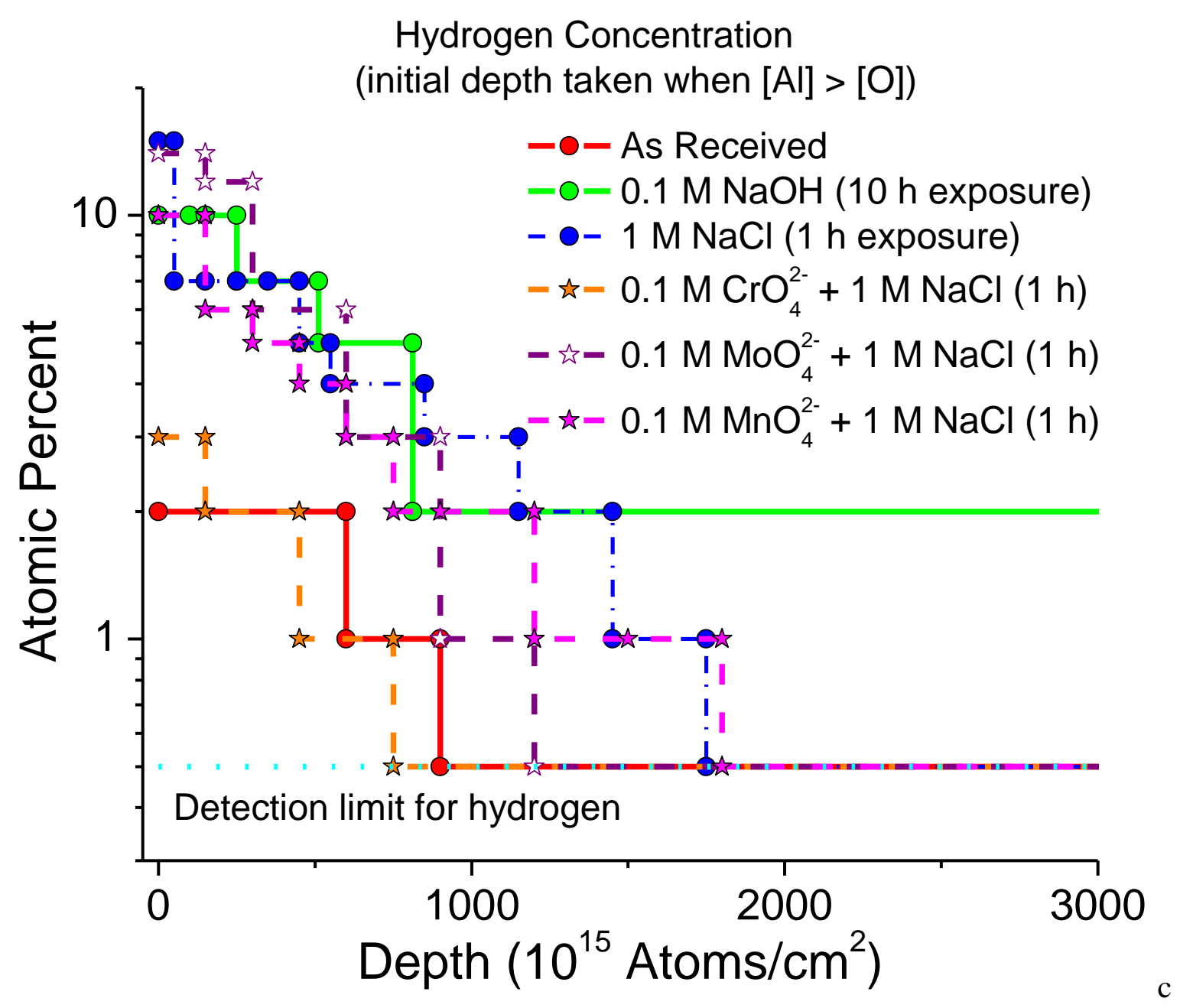

Figure 5.17. Hydrogen depth profile of all samples showing protective nature of chromate. Hydrogen concentration depth profiles for AA7075-T6 abraded in $1.0 \mathrm{M} \mathrm{NaCl}$ with $0.1 \mathrm{M} \mathrm{CrO}_{4}{ }^{2-}$ are reduced to level similar to those seen in a similar sample ground in ethanol and exposed to humid lab air. 

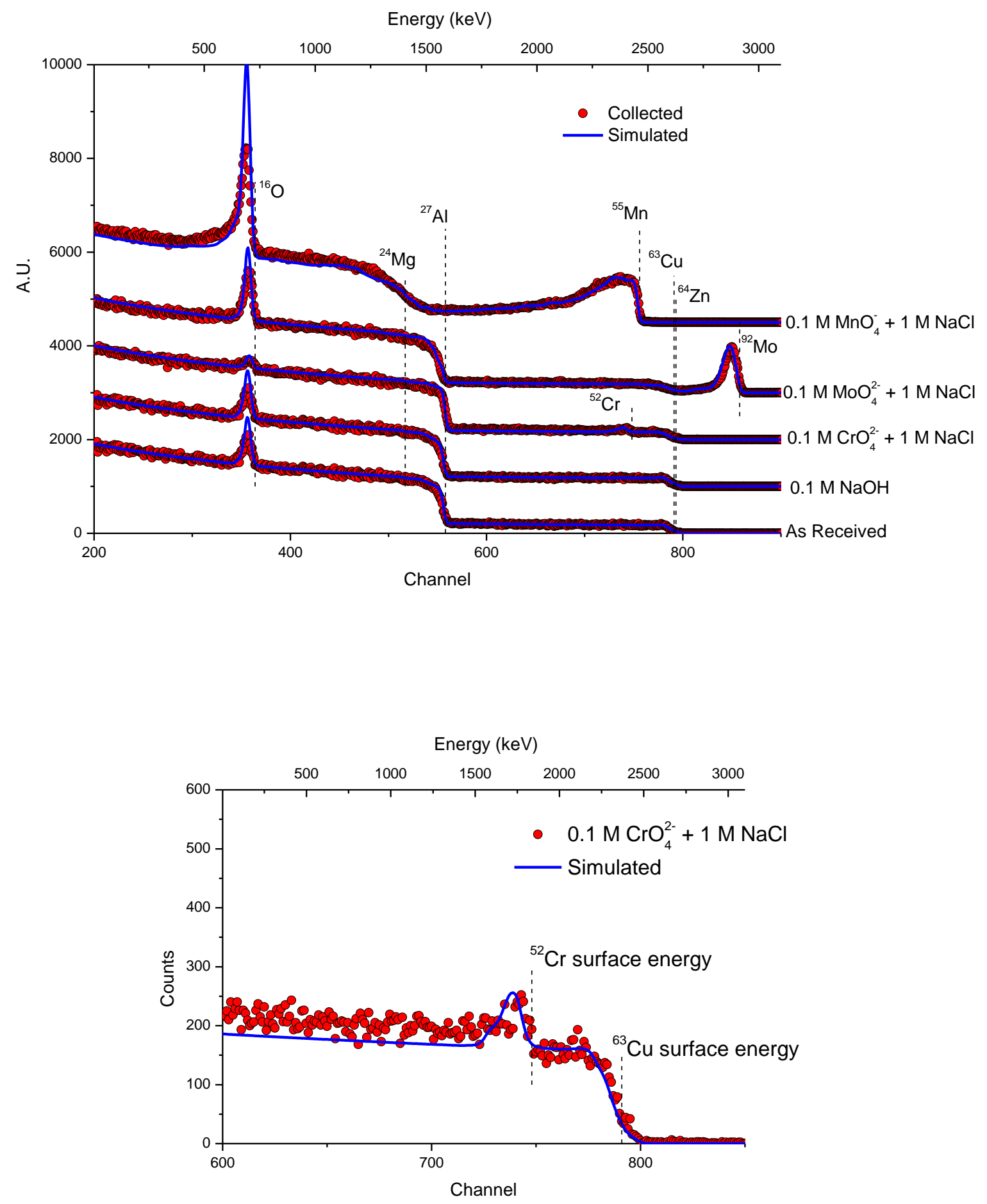

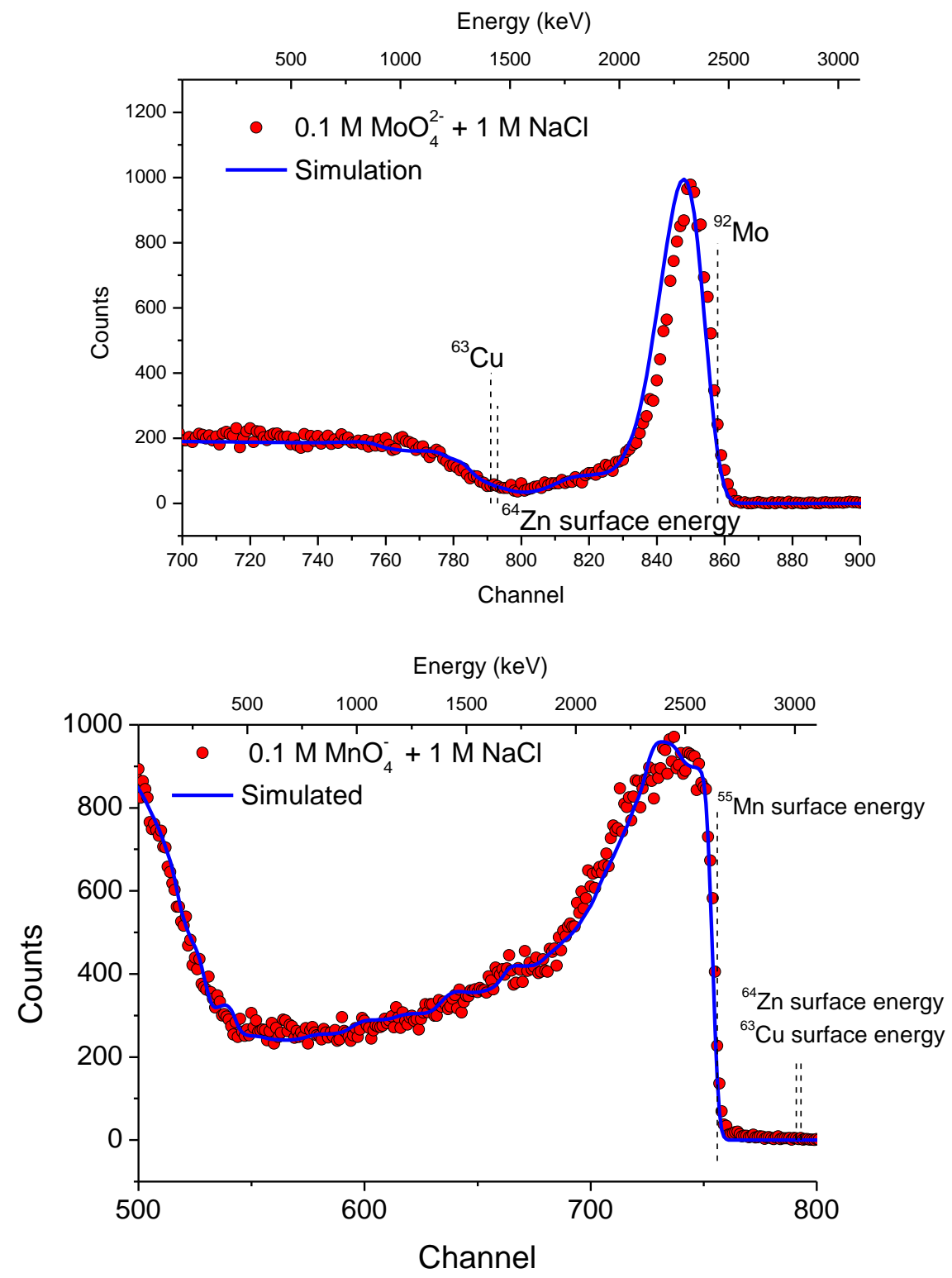

Figure 5.18. RBS spectra and simulation of $\mathrm{Al}-\mathrm{Zn}-\mathrm{Mg}-\mathrm{Cu}$ ground in aqueous $1 \mathrm{M}$ chloride solution with chromate, molybdate, and permanganate additions. As-received (ground in ethanol and exposed to humid lab air) and $10 \mathrm{~h} 0.1 \mathrm{M} \mathrm{NaOH}$ samples for reference. RBS results from the University of Western Ontario (UWO). Detailed view of chromium, molybdenum, and manganese enriched oxides. 


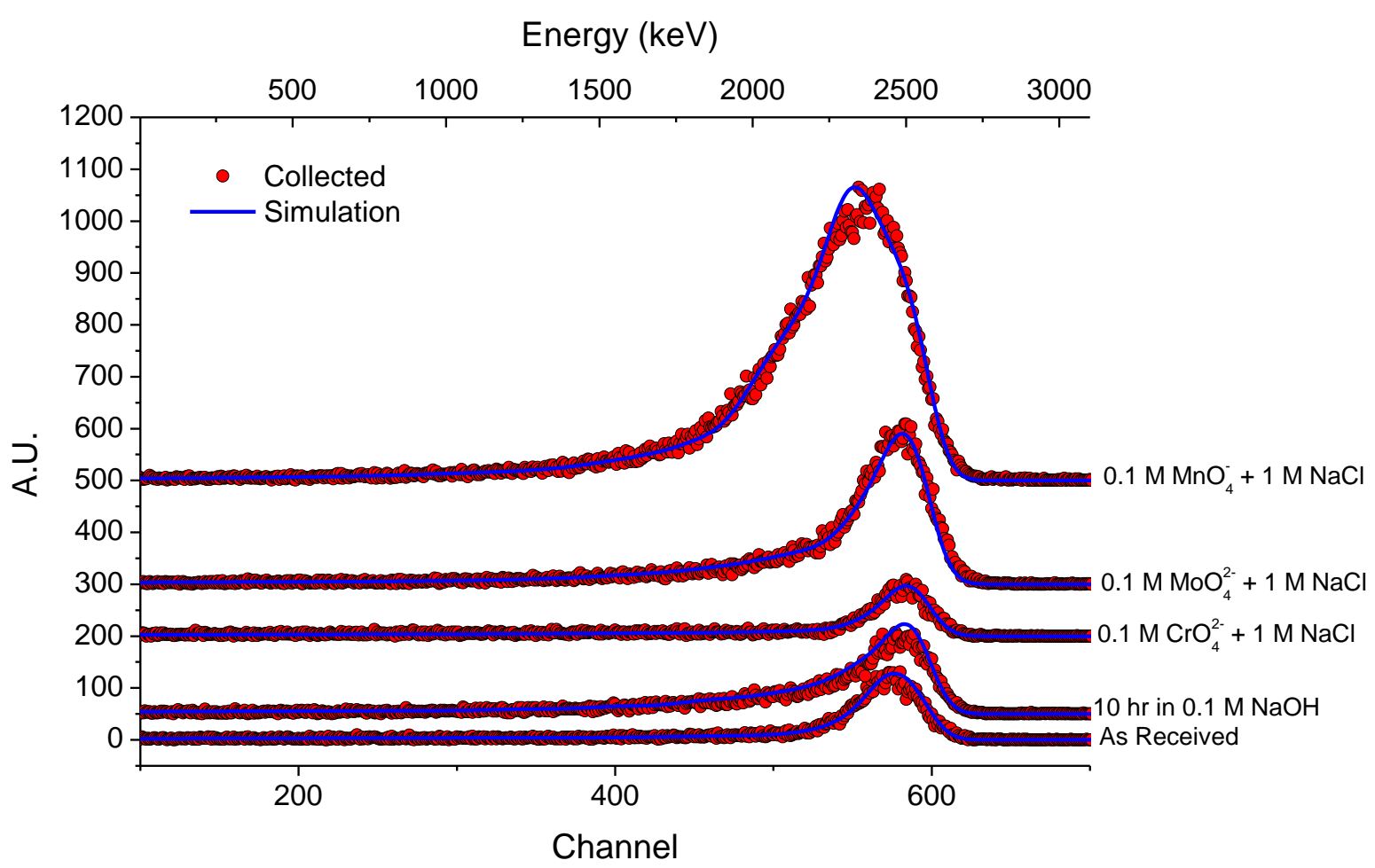

Figure 5.19. ERD spectra and simulation of $\mathrm{Al}-\mathrm{Zn}-\mathrm{Mg}-\mathrm{Cu}$ ground in aqueous $1 \mathrm{M}$ chloride solution with chromate, molybdate, and permanganate additions. As-received (ground in ethanol and exposed to humid lab air) and $10 \mathrm{~h} 0.1 \mathrm{M} \mathrm{NaOH}$ samples for reference. ERD from UWO. 

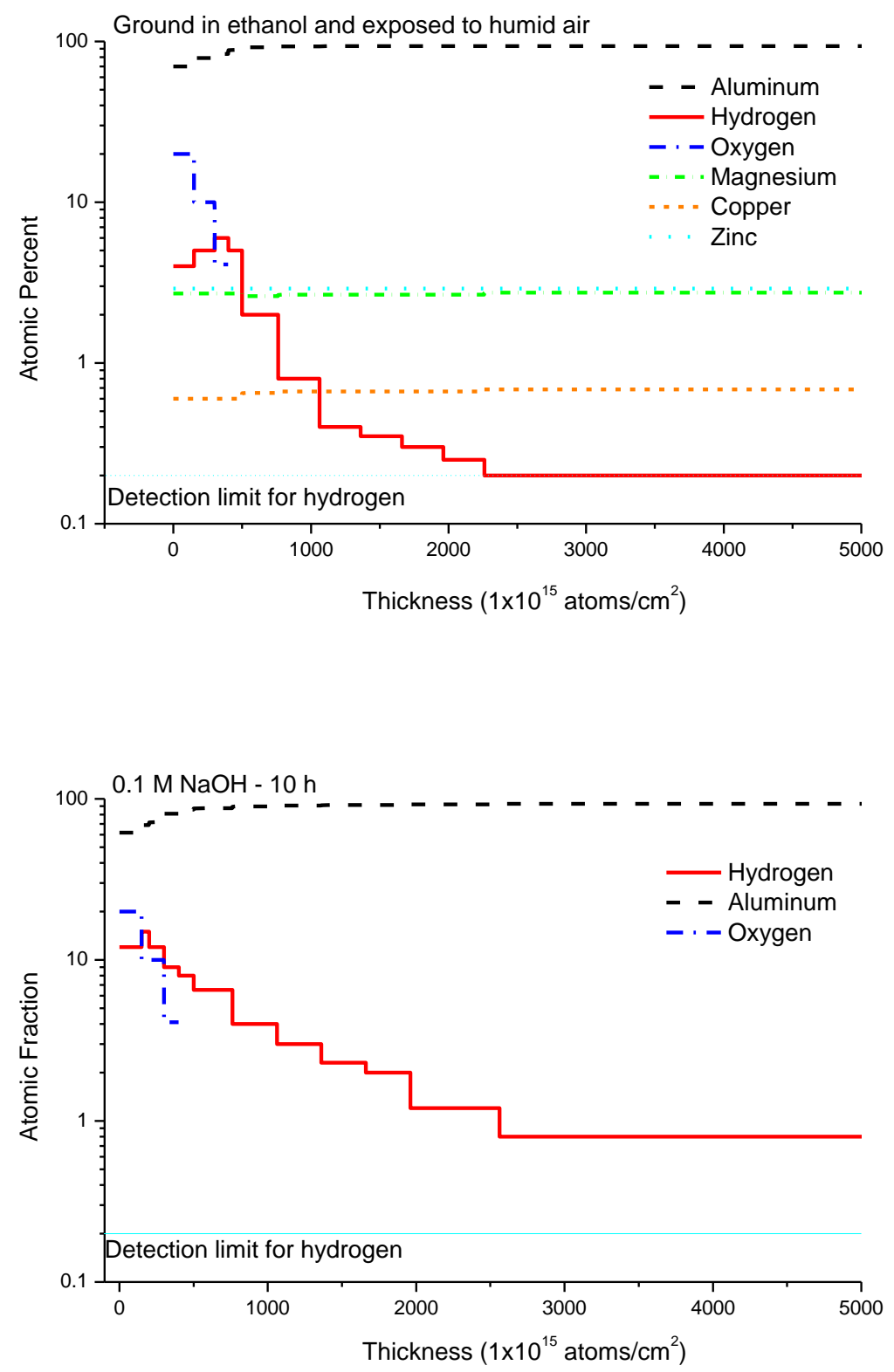

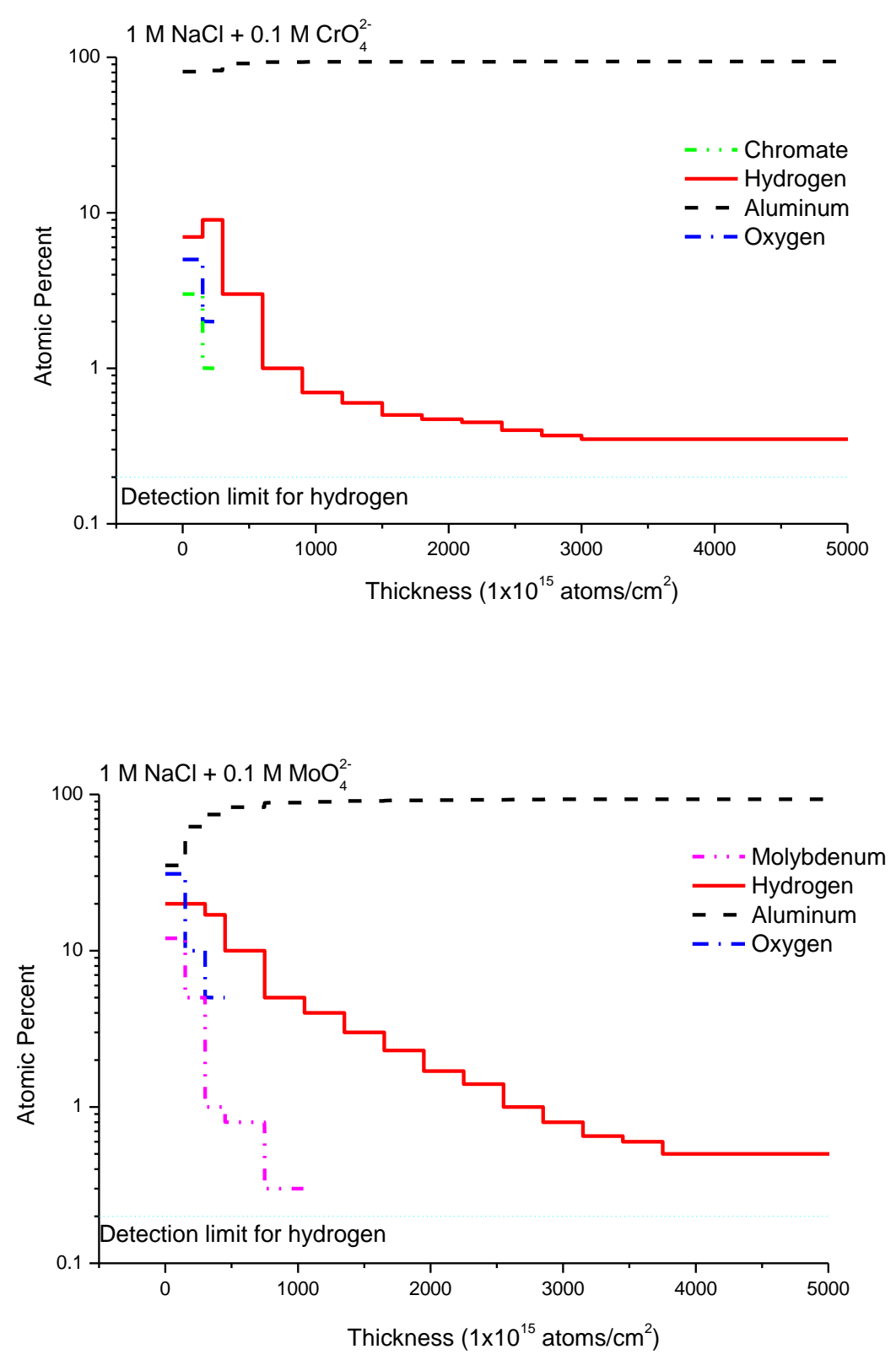


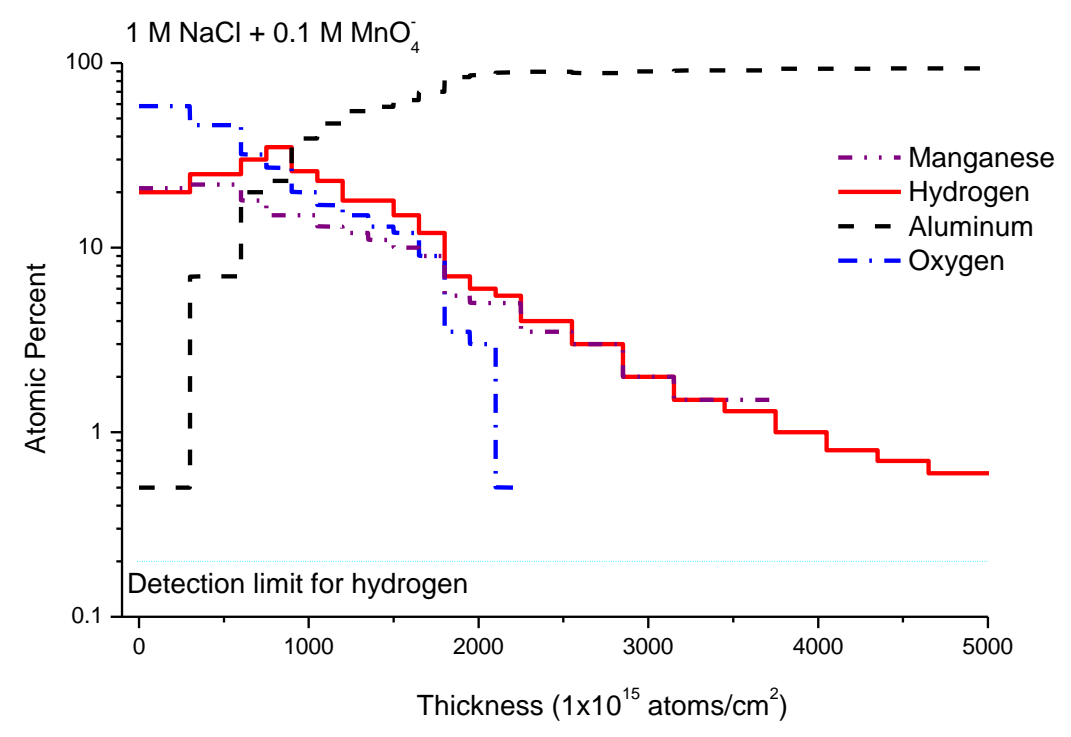

Figure 5.20. Model systems showing atomic concentration depth profiles for AA075 as received exposed to ethanol and humid air, exposed for $10 \mathrm{~h}$ to $0.1 \mathrm{M} \mathrm{NaOH}$, and ground in $1 \mathrm{M}$ chloride with $0.05 \mathrm{M} \mathrm{Cr}_{2} \mathrm{O}_{7}{ }^{2-}, 0.1 \mathrm{M} \mathrm{MoO}_{4}{ }^{2-}$ and $0.1 \mathrm{M} \mathrm{MnO}_{4}{ }^{-}$. 


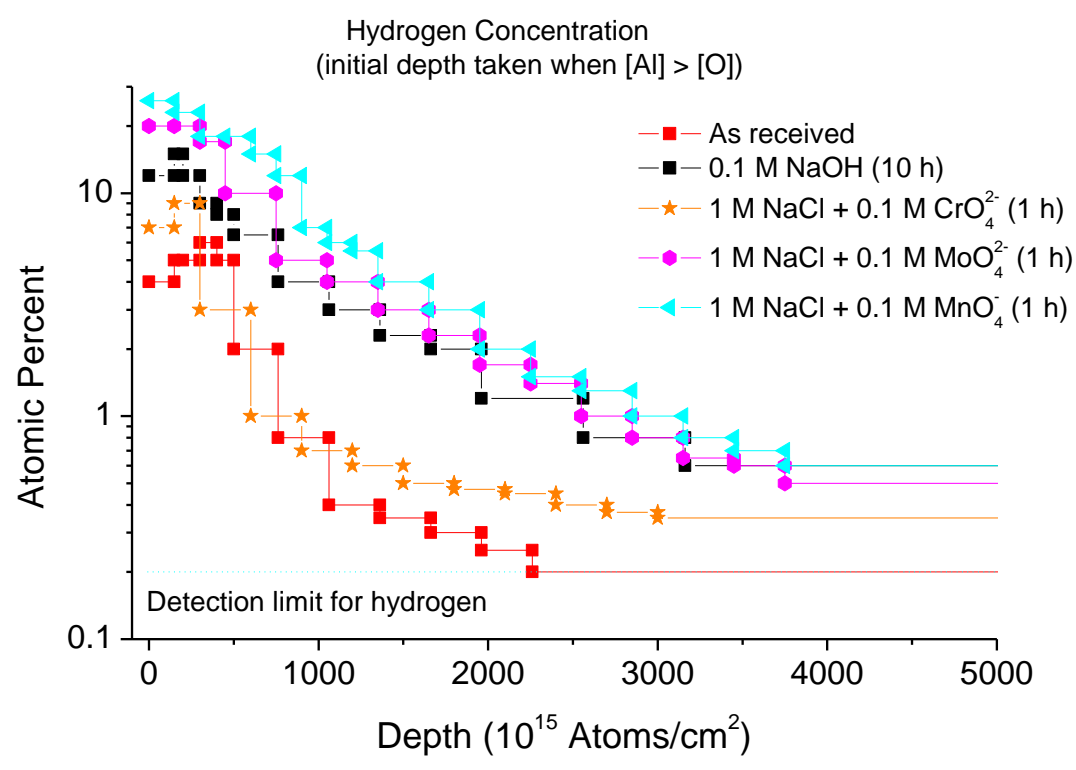

Figure 5.21. Hydrogen depth profile of all samples showing protective nature of chromate. Hydrogen concentration depth profiles for AA7075-T6 abraded in $1.0 \mathrm{M} \mathrm{NaCl}$ with $0.1 \mathrm{M} \mathrm{CrO}_{4}^{2-}$ are reduced to level similar to those seen in a similar sample ground in ethanol and exposed to humid lab air. 


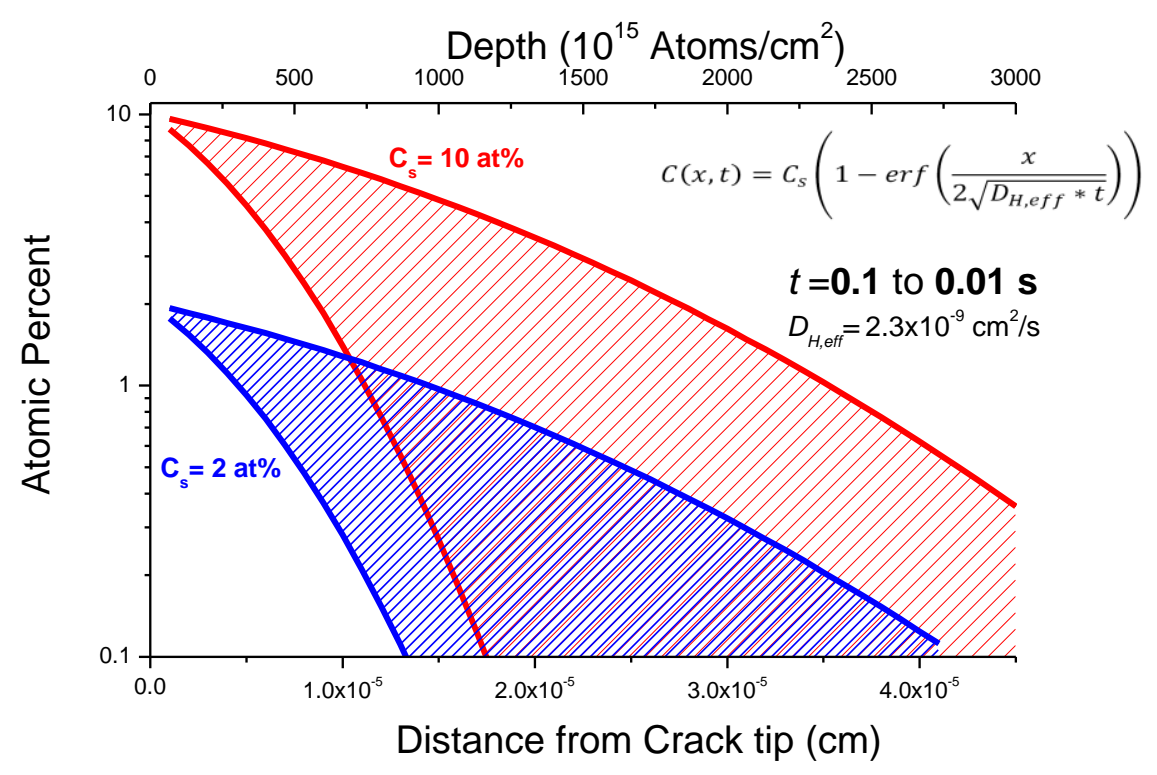

Figure 5.22. Projected diffusion concentration profiles for hydrogen diffusion through bulk AA7075.
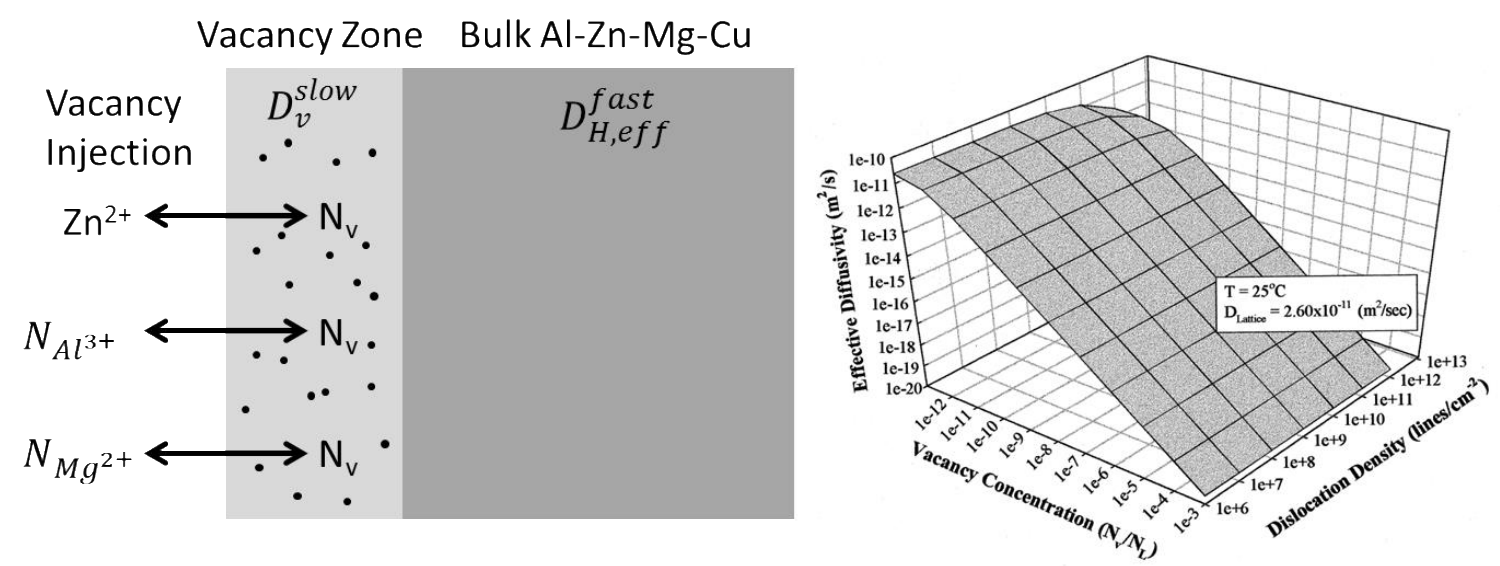

Figure 5.23. Effect of injected vacancy concentration on $D_{\text {eff }}$ in the vacancy effected zone. Used with permission of Acta Metallurgica 


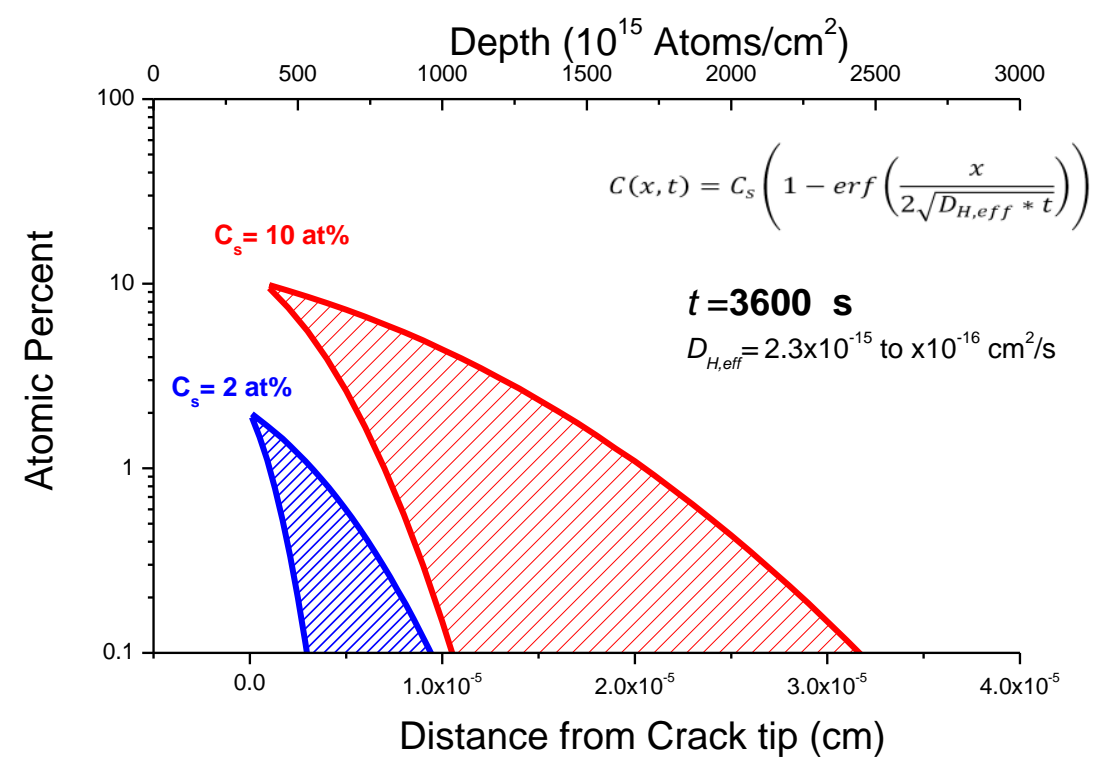

Figure 5.24. Projected diffusion concentration profiles for hydrogen-vacancy complexes through AA7075 over 3600 seconds. 


\section{Thesis Conclusions and Suggested Future Work}

\subsection{Conclusions}

Low concentration pretreatment with permanganate leads to a slight increase in the pitting potential $\left(\mathrm{E}_{\mathrm{pit}}\right)$ of AA2024 but only when partially oxidized manganese oxides were electrochemically deposited over the AA2024 aluminum matrix. Decreases in OCP accompanied the elevated $\mathrm{E}_{\text {pit. }}$ Permanganate increased cathodic reaction rates when it was present in solution owing to the addition of this oxidizing cathodic reactant. However, when permanganate was used as a pretreatment and was electrochemically deposited as a lower valence state oxide on the IMCs, it decreased cathodic reaction rates. Moreover, OCP was greatly reduced after pretreatment as copper rich sites were covered with manganese oxides. The slow reduction of high valence manganese oxides may account for the gradual decrease in the OCP. Copper replating during $\mathrm{NaCl}$ exposure was also decreased after pretreatments. However, this effect did not occur to the same extent as when permanganate was added as an in-situ solution phase inhibitor. The manganese oxide has been shown to be present initially in a high valence state, most likely a mixed oxide with some $\mathrm{MnO}_{2}$ present at least initially. The reduction of this oxide to lower valence compounds has a number of effects including acting as an additional cathodic reactant which has the effect of complicating the cathodic reaction kinetics. In summary, $\mathrm{MnO}_{4}^{-}$is a complex ionic inhibitor that is more beneficial when present as a pretreatment compared to an ionic species addition. 
For a cerium-permanganate binary inhibitor system high concentrations of cerium in the pretreatment package can lead to a homogeneous cerium enriched oxide surface. However, such a treatment shows little indication of increasing passivity or suppressing corrosion. Cerium-permanganate pretreatments show insignificant anodic inhibition in pitting scans but reduced copper replating during open circuit corrosion. Permanganate pretreatments are effective at increasing $\Delta \mathrm{E}\left(\mathrm{E}_{\mathrm{pit}}-\mathrm{E}_{\mathrm{OCP}}\right)$ mainly through reducing the Eocp. Permanganate pretreatments are shown to have strong cathodic inhibition properties. Ceriumpermanganate pretreatments show promise as cathodic inhibitors, in some cases reducing cathodic current density by two and a half orders of magnitude in chloride solution when compared to AA2024-T351 with no inhibitor pretreatment. However, no indication of a combined Ce- Mn molecular species was found and there is no reference to such a species in the literature. Cerium can act detrimentally to block permanganate oxide formation over copper rich intermetallic particles.

The effect of inhibitor concentration, chloride concentration, and applied potential on the repassivation of AA2024, AA7075, and high purity aluminum has was also investigated. Results to date have indicated that chromate is an inhibitor of net anodic scratch current densities as well as total charge after depassivation. This result is evident for both AA2024 and AA7075 at a neutral $\mathrm{pH}$ of 6.5. The net anodic charge passed in the presence of chromate is two orders of magnitude lower than in the uninhibited solution for AA2024 and one order of magnitude for AA7075. A protective oxide barrier is observed through a variation in the scratched electrode technique that exploits high frequency impedance measurements to observe oxide regrowth. Chromate reveals an order of magnitude increase in impedance magnitude and phase angle during repassivation in chromate inhibited vs. 
uninhibited chloride solutions at $\mathrm{pH}$ 6.5. Scratch current density reduction by molybdate of either AA2024 or AA7075 was not observed. Nor was molybdate observed to affect the impedance magnitude or phase angle of film growth after a rupture event. Acid-base titration has shown that in simulated crack tip solution molybdate can act as a strong buffer with a capacity 1.6 times higher than chromate. These findings are related to $1 / f$ in EFCP observations. This suggests that there are multiple mechanisms through which EFCP inhibition occurs as both chromate and molybdate inhibit crack growth rates ${ }^{[1]}$. However, it is unclear how $\mathrm{MoO}_{4}{ }^{2-}$ results in a similar $1 / f$, EFCP dependency.

A combined repassivation and RBS detection of inhibitor elements incorporated in the repassivating oxide and ERD analysis of hydrogen was performed to provide direct assessment of inhibitor function. Formation of a thin chromium rich oxide film was observed after repassivation in $0.1 \mathrm{M} \mathrm{NaCl}$ with $0.1 \mathrm{M} \mathrm{CrO}_{4}{ }^{2-}$. Direct evidence for reduction of the hydrogen absorption into AA7075-T6 was shown where hydrogen was reduced to levels seen when abrasion was performed in ethanol. Formation of a thick molybdenum oxide film was observed. However, the reduction in hydrogen uptake was not observed, indicating that the film was not an effective barrier to hydrogen ingress when compared to chromate. A thick manganese rich oxide film was observed. However, the film did not reduce the concentration of absorbed hydrogen in the alloy substrate.

Evidence of vacancy injection during repassivation was implicated by the high hydrogen concentrations present in the local surface regions. Vacancy controlled diffusion kinetics account for the steep hydrogen concentration depth profile observed long after the repassivation event. 
Chromate reduces $\mathrm{C}_{\mathrm{s}, \mathrm{H}}$ and reduces the amount of dissolution, coupled hydrogen production, and uptake as clearly shown in Chapter 4.

\subsection{Suggested Future Work}

Single and binary inhibitor systems would benefit from further valance state and speciation analysis. Future work should also be done to investigate other binary inhibitor combinations, specifically those found to be strongly synergistic in the work of Chambers $^{[2]}$. Further analysis of active inhibition for both single and binary inhibitor systems would benefit their development as well as efforts to incorporate the findings into intelligent coating designs.

Further studies of crack tip solutions should be conducted. While a notional investigation was conducted in this thesis, the work would benefit from a better understanding of actual inhibitor concentrations available at an occluded crack tip. A better understanding of cracktip chemistry in the presence of inhibitors would further the analysis of repassivation kinetics and hydrogen uptake.

Further study of the repassivated oxide interface with the alloy substrate and the vacancy concentration in the immediate sublayers would improve the understanding of hydrogen transport into the fracture process zone. 


\subsection{References}

1. J.S. Warner, S. Kim, and R.P. Gangloff, "Molybdate inhibition of environmental fatigue crack propagation in Al-Zn-Mg-Cu," International Journal of Fatigue 31, 11-12 (2009): p. 1952.

2. B.D. Chambers and S.R. Taylor, "The high throughput assessment of aluminium alloy corrosion using fluorometric methods. Part II - A combinatorial study of corrosion inhibitors and synergistic combinations," Corrosion Science 49, 3 (2007): p. 1597. 